\title{
Acoustical measurements on stages of nine U.S. concert halls
}

\section{Gade, Anders Christian; Bradley, J S}

\section{Published in:}

Acoustical Society of America. Journal

Link to article, DOI:

10.1121/1.406640

Publication date:

1993

\section{Document Version}

Publisher's PDF, also known as Version of record

Link back to DTU Orbit

\section{Citation (APA):}

Gade, A. C., \& Bradley, J. S. (1993). Acoustical measurements on stages of nine U.S. concert halls. Acoustical Society of America. Journal, 93(4), 2266-2266. https://doi.org/10.1121/1.406640

\section{General rights}

Copyright and moral rights for the publications made accessible in the public portal are retained by the authors and/or other copyright owners and it is a condition of accessing publications that users recognise and abide by the legal requirements associated with these rights.

- Users may download and print one copy of any publication from the public portal for the purpose of private study or research.

- You may not further distribute the material or use it for any profit-making activity or commercial gain

- You may freely distribute the URL identifying the publication in the public portal

If you believe that this document breaches copyright please contact us providing details, and we will remove access to the work immediately and investigate your claim. 
The 125th Meeting of the Acoustical Society of America

\author{
Château Laurier Hotel • Ottawa, Canada • 17-21 May 1993
}

NOTE: All Journal articles and Letters to the Editor are peer reviewed before publication. Program abstracts, however, are not reviewed before publication, since we are prohibited by time and schedule.

\title{
Session 1aAA
}

\section{Architectural Acoustics and Musical Acoustics: New Auditorium Acoustics Measurements-Results and Comparisons I}

\author{
Leo L. Beranek, Chair \\ 975 Memorial Drive, Suite 804, Cambridge, Massachusetts 02138
}

Chair's Introduction-9:00

\section{Invited Papers}

9:05

1aAA1. The acoustic survey of British auditoria. Michael Barron (School of Architecture and Bldg. Eng., Univ. of Bath, Claverton Down, Bath BA2 7AY, UK)

By 1980 the understanding of acousties for concert halls had reached an interesting stage. Two German studies at Göttingen and Berlin with dummy head recordings had gone a long way to determining the important subjective and objective dimensions. However the influence of architectural form on subjective acoustic conditions was much less well established. There were two possible routes: testing acoustic scale models or full-size auditoria. With access to sufficient full-size auditoria, the latter was the obvious choice since it also allowed true subjective assessment. The acoustic survey of British auditoria involved both objective measurements and subjective assessments in over $\mathbf{4 0}$ auditoria including concert halls, drama theaters, opera houses, and multipurpose spaces. The whole exercise has now been published [M. Barron, Auditorium Acoustics and Architectural Design (Chapman \& Hall, London, 1993)]. In concert halls a major conclusion of the subjective exercise was that halls with good reputations score well with regard to several attributes, such as reverberance, envelopment, and intimacy. Several novel observations on the effect of design details will also be discussed.

1aAA2. Effects of measurement procedure and equipment on gverage room acoustic measurements. A. C. Gade (Acoust. Lab., Tech. Univ. of Denmark, Bldg. 352, Lyngby, DK 28000 Denmark), John S. Bradley (Natl. Res. Council, Ottawa K1A 0R6, Canada), and Gary W. Siebein (Univ. of Florida, Gainesville, FL 32611)

This paper reports the results of a measurement tour of nine U.S. concert halls. Three measurements teams, from the University of Florida, the National Research Council of Canada, and the Technical University of Denmark, made parallel sets of measurements using their own equipment and procedures. In some of the halls measurements were repeated using the procedures of the other teams to make it possible to separate the effects of different equipment and different procedures. The paper will present position-averaged results from the three teams and will discuss reasons for the differences observed. [Work partially supported by the Concert Hall Research Group.]

1aAA3. Comparisons of auditorium acoustics measurements as a function of location in halls. John S. Bradley (M-27, IRC, Natl. Res. Council, Ottawa, ON K1A 0R6, Canada), A. C. Gade (Tech. Univ. of Denmark, Lyngby, DK 28000, Denmark), and Gary W. Siebein (Univ. of Florida, Gainesville, FL 32611)

In a measurement tour of nine U.S. concert halls measurements were made at 30 or more combinations of source and receiver position in each hall. Each of the three measurement teams (the University of Florida, the Danish Technical University, and the National Research Council of Canada) made parallel measurements of a number of modern room acoustics quantities using 
different equipment and measurement procedures. These results are compared on a seat-by-seat basis and the differences are explained in terms of earlier systematic studies of the effects of measurement procedure details. The measurement results were also used to examine the influence of different measurement equipment and measurement procedures on the within hall variations of the various acoustical quantities. [Work partially supported by the Concert Hall Research Group.]

10:20

1aAA4. Effects of measurement equipment and procedure on IACC measurements. Gary W. Siebein, Wei-hwa Chiang, Gary S. Madaras, Harold W. Doddington, Wilhelm K. Schwab (Architecture Technol. Res. Ctr., Univ. of Florida, 231 ARCH, Gainesville, FL 32611-2004), and John S. Bradley (Natl. Res. Council, Ottawa, ON K1A OR6, Canada)

A series of binaural acoustical measurements were taken at several source locations and multiple receiver locations in eight concert halls using the Acoustical Research Instrumentation for Architectural Spaces (ARIAS) system and manikin developed at the University of Florida and the BRAM software system developed at NRC Canada with a B\&K dummy head and torso. This paper will compare the resuits of room average data and several specific seat locations obtained from the two measurement systems with an analysis of the similarities and differences that arose. Correlations among various forms of IACC including

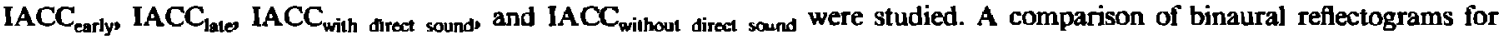
locations within each room were also made. Summary graphs showing ranges of each measurement within each of the rooms and discussion of similarities and differences among the groups of rooms will be presented. [Work supported by the National Science Foundation and Concert Hall Research Group.]

\section{0:45}

1aAA5. Microphone placement in a binaural hearing manikin and its effects on IACC, reflectograms, and head-related transfer functions. Gary S. Madaras, Gary W. Siebein, Wei-hwa Chiang, Harold W. Doddington, and Wilhelm K. Schwab (Architecture Technol. Res. Ctr., Univ. of Florida, 231 ARCH, Gainesville, FL 3261 1-2004)

A binaural hearing manikin was constructed and then tested by comparing its head-related transfer functions to those of a human subject. The manikin was used to record impulse responses in eight northeastern United States concert halls as part of the recent Concert Hall Research Group Study. The placement of the manikin's 1/2-in. Bruel \& Kjaer microphones varied between two locations: inside the head (truncating the auditory canal) and outside the head ( $20 \mathrm{~mm}$ from the canal opening). For most measurements, no statistically significant differences were found between octave band IACC values for the two microphone locations. The manikin's head-related transfer functions were measured in an anechoic chamber using both microphone positions and 324 source positions. Comparisons of reflectograms and head-related transfer functions show that the manikin's head and the human subject's head affect sound in similar ways. [Work supported by the National Science Foundation and Concert Hall Research Group.]

11:10

1aAA6. Acoustical measurements on stages of nine U.S. concert halls. A. C. Gade (Acoustics Lab., Tech. Univ. of Denmark, Bldg. 352, Lyngby, DK 28000, Denmark) and John S. Bradley (Natl. Res. Council, Ottawa, ON K1A OR6, Canada)

A measurement tour of nine U.S. concert halls included acoustical measurements on the stage of each hall. Two teams (from the National Research Council of Canada, and the Technical University of Denmark) made measurements of the acoustical quantities suggested by Gade: the "support" family of measures describing the acoustical conditions for the musicians on stage. This paper compares the results from the two measurement teams and discusses the influence of hall designs. [Work partially supported by the Concert Hall Research Group.]

\section{1:35}

1aAA7. Statistical relations among architectural features and objective acoustical measurements of concert halls. Gary W. Siebein, Wei-hwa Chiang, Gary S. Madaras, Harold W. Doddington, Wilhelm K. Schwab (Architecture Technol. Res. Ctr., Univ. of Florida, 231 ARCH, Gainesville, FL 32611-2004), John S. Bradtey (Natt. Res. Council, Ottawa, ON K1A 0R6, Canada), and A. C. Gade (Danish Tech. Univ., Lyngby, DK 28000, Denmark)

A statistical analysis of architectural features and detailed objective acoustical measurements made in eight concert halls and several multi-use rooms in their concert configuration will be presented. A method for evaluating the architectural features of rooms that affect their acoustical properties was developed. Architectural features of interest include both room average values and more-detailed subdivisions of surfaces including shape, volume, height, width, and sound absorption properties of materials. Regression modeling was performed for individual source-receiver paths as well as for entire rooms. Measurements data from all three teams were used in the models to assess the sensitivity of the models to expect variations in measurements. The results were compared to the previous work of Barron, Gade, and Hook among others. [Work supported by the National Science Foundation and Concert Hall Research Group.] 


\title{
Session 1aAO
}

\section{Acoustical Oceanography and Underwater Acoustics: Ocean Acoustics in Shallow Water: What are the Issues? I}

\author{
Mohsen Badiey, ${ }^{*}$ Cochair \\ University of Delaware, Graduate College of Marine Studies, Newark, Delaware 19716
}

*Current: Office of Naval Research, Ocean Acoustics, Code 11250A, 800 North Quincy Street, Arlington, Virginia 22217

Peter H. Rogers, Cochair

Georgia Institute of Technology, School of Mechanical Engineering, Atlanta, Georgia 30332

\author{
Chair's Introduction-8:00
}

Invited Papers

8:05

1aAO1. Critical propagation issues in shallow-water acoustics. David E. Weston (White Laird, 77 Wyke Rd., Weymouth, Dorset DT4 9QN, England)

Several sets of propagation problems in shallow-water acoustics are discussed, covering overlapping frequency ranges. At VLF (say below $300 \mathrm{~Hz}$ ) the bottom material is important, because energy travels in the bottom or because of attenuation associated with shear-wave coupling. At LF (say above $30 \mathrm{~Hz}$ ) the presence of gas in surficial sediments can change boundary conditions and bottom losses, and even allow a new type of interface wave. At MF ( $300 \mathrm{~Hz}$ to several $\mathrm{kHz}$ ) dispersed fish can raise the attenuation, typically by $1 \mathrm{~dB} / \mathrm{km}$. Fish have also been observed to dominate the echo structure or reverberation, out to $100 \mathrm{~km}$. At HF (say above several $100 \mathrm{~Hz}$ ) the sound-speed profile becomes important, partly because of channeling. At VHF (usually above several $\mathrm{kHz}$ ) the propagation mechanisms tend to change from boundary reflection to boundary scattering. In addition all these effects are greatly influenced by distance and by water depth.

\section{$8: 30$}

1aAO2. Shallow water active sonar environmental acoustic issues. William M. Carey (DARPA, 3701 North Fairfax Dr., Arlington, VA 22203), Peter G. Cable (BBN Systerns Technology, New London, CT 06320), and Henry Cox (Orincon, Arlington, VA 22202)

The propagation and reverberation of sound in shallow water under downward refracting conditions is governed by the geoacoustic and scattering properties of the bottom. The active bistatic sonar equation for this case is developed and it is shown that, when the transmission to the target and the bottom are approximately the same for reverberation-limited conditions, a simpler parametric form of this equation results. That is, the signal excess depends on the effective bottom backscattering strength, the coherent signal gain, and a reverberation area for a given target strength and threshold. A parametric analysis is presented that focuses on the frequency-dependent characteristics of the bottom backscattering strength and the effect of coherence on receiving array aperture. Recent reverberation and signal coherence measurements are reviewed and the needs for additional studies delineated. The coherence issue for the waveguide is stressed and the measurement problem of coherence is discussed and compared to the measurement of signal versus the receiver length in wavelengths.

\section{$8: 50$}

1aAO3. Ambient noise oceanography: A new remote sensing technique for shallow water. Michael J. Buckingham and Grant B. Deane (Marine Phys. Lab., Scripps Inst. of Oceanography, La Jolla, CA 92093-0213)

Ambient noise contains broadband information about the oceanic environment. In particular, in shallow water, because of the multiple boundary interactions, ambient noise contains information about the geoacoustic parameters of the bottom. To extract this information, it is necessary to identify stable, reliable features of the noise. (Noise intensity is neither stable nor reliable since it is influenced by numerous highly variable factors.) An example of a reliable feature of the shallow-water noise field is its vertical directionality, which is controlled by the bottom. In fact, in an isovelocity channel of uniform depth overlying a fast fluid bottom, the vertical structure of the noise can be inverted to obtain an accurate estimate of the sound speed in the bottom sediment [Buckingham and Jones, J. Acoust Soc. Am. 81, 938-946 (1987)]. Thus, in this example, a simple measurement of the vertical coherence of the noise in the water column yielded an important geoacoustic parameter of the sediment. Similarly, in shallow water overlying a sloping, penetrable bottom (i.e., a range-dependent channel supporting three-dimensional acoustic propagation) the noise shows a horizontal anisotropy that could be used to determine geometric and geoacoustic parameters of the channel. In such applications, "ambient noise oceanography" provides a remote sensing tool for interrogating the bottom, much as seismic reflection and refraction techniques are used at present. [Research supported by ONR.] 
1aA 04. Considerations for shallow water propagation experiments. T. Ewart, F. Henyey, Darrell R. Jackson, and Eric I. Thorsos (Appl. Phys. Lab., Univ. of Washington, Seattle, WA 98105)

When one examines the issues involved in propagation of sound in shallow water, the conclusion is that the ever-present ocean bottom, with its inhomogeneity and anisotropy, will dominate. A single model with a few parameters will not be appropriate for all bottom types. This is unlike studies of scattering in the random ocean volume and from the sea surface, where simply parametrized models are useful. The presence of very large numbers of multipaths blurs the distinction between stochastic and deterministic issues. It is clear that one needs to understand the angular and temporal behavior of the acoustic fields in order to understand the channel characteristics for given source(s)/receiver(s) combinations. This implies that large aperture and broadband experiments will be required. Several issues must be addressed before designing such experiments. (1) Will the present simulation capability allow one to design a good experiment for the case of a reasonably homogeneous bottom? (2) How many multipaths are there, and can they be separated? (3) If there are a large aperature and bandwidth, can matched field or backpropagation methods of source localization work? (4) What are the rest of the questions? This group will conduct an experiment in Knight Inlet, BC, which will include preliminary shallow water measurements, and the plans for this experiment will be discussed.

1aAO5. An issue (sediment volume fluctuations) and a nonissue (shear wave propagation) in shallow water acoustics. Fredrick D. Tappert and T. Yamamoto (Appl. Marine Phys., Univ. Miami/RSMAS, 4600 Rickenbacker Cswy., Miami, FL 33149)

A two-way full-wave PE reverberation model has been developed that provides predictions of mean reverberation levels in shallow water to long ranges (hundreds of water depths) due to sediment volume fluctuations as well as bottom and basement interface roughnesses. Using T. Yamamoto's tomographically measured sediment volume fluctuation spectra as one of the inputs, the PE reverberation model has been exercised at several locations on the shelf in the East China Sea over a broad range of frequencies. It has been found that predicted reverberation levels due to both mechanisms are generally comparable in magnitude, and that absolute levels are controlled mainly by total field ensonification of the sediment volume and boundaries, i.e., forward transmission losses. The question whether it is necessary to include shear wave propagation in shallow water acoustic models has been resolved: the answer is definitely negative, and this is no longer an issue. Measured shallow water transmission loss data over a broad range of frequencies with source and receiver in the water column has been successfully modeled using a full-wave range-dependent PE model that includes rough bottom forward scattering (necessary for good agreement), but treats shear wave effects as an equivalent fluid by augmenting the volume attenuation of compressional waves in the sediment layers. The conclusion that it is not necessary to include shear wave propagation when sources and receivers are in the water column has been further confirmed by inter-model and intra-model comparisons using realistic geoacoustic sediment properties as measured by $T$. Yamamoto's BSMP system [J. Acoust. Soc. Am. 90, 441-456 (1991)]. [Work supported by ONR, 1125OA.]

\section{9:50-10:10 Break}

10:10

1aAO6. Predictability of the acoustic field in a shallow water waveguide. George V. Frisk and Subramaniam D. Rajan (Dept. of Appl. Ocean Phys. \& Eng., Woods Hole Oceanographic Inst., Woods Hole, MA 02543)

The ability to predict the sound field magnitude and phase in a shallow water waveguide is critically dependent upon accurate knowledge of the acoustic properties of the environment. In this paper, attention is focused on the influence of the geoacoustic properties of the seabed on the predictability of the field. Emphasis is placed on the use of geoacoustic parameter estimates obtained from modal inversion techniques. Some of the research questions to be addressed include: (1) What is the measure of predictability? (2) What are the errors associated with various inversion methods and how do they affect predictability? (3) What is the effect of noise in the data? (4) How useful are geoacoustic parameter estimates obtained over a band of frequencies in predicting the sound field outside that band? Examples using both real and synthetic data will be discussed. [Work supported by ONR.]

\section{0:30}

1aAO7. Geoacoustic modeling of the seafloor-Recent experimental work. R. D. Stoll (Lamont-Doherty Earth Observatory, Palisades, NY 10964), J. Ewing (Woods Hole Oceanographic Inst., Woods Hole, MA 02543), and T. Akal (SACLANT Undersea Res. Ctr., LaSpezia, Italy)

Over the past decade a number of new techniques have been developed for measuring the geoacoustic properties of seafloor sediments. Several of these methods utilize sensors that are deployed on the seafloor and respond to seismic waves generated by different kinds of source including explosives and mechanical impact as well as the ambient pressure fluctuations generated by wave motion at the sea surface. Since the motion that is sensed at the seafloor is a combination of many different kinds of interface and body waves (i.e., Scholte waves, Love waves, $p, s_{v 0}$ and $s_{h}$ refractions and reflections, etc.), inversion to obtain a geoacoustic model is a complex task that must be tailored to each individual experiment taking into account the bandwidth of the signal and the interaction between the various types of wave. This paper summarizes some of the recent field experiments and the methods that are being used to interpret the data. [Work supported by ONR codes $11250 \mathrm{~A}$ and 1125GG.] 
1aA08. High-resolution shallow water 3-D survey and inversion for geophysical parameters. Mrinal K. Sen, Paul L. Stoffa, and James A. Austin, Jr. (Inst. for Geophys., Univ. of Texas at Austin, 8701 N. MoPac Expwy., Austin, TX 78759)

In the shallow water environment even in subsurface depths less than $30 \mathrm{~m}, 3-\mathrm{D}$ structures can affect wave propagation. A high-resolution ( $500-3500 \mathrm{~Hz}$ ) 3-D survey ( 53 lines; 5-km length, 10-m line spacing, 5-m shot spacing) using a Huntec deep-towed boomer source and STARFIX navigation was carried out offshore New Jersey in 1989. Additional 3-D acquisition along with vibra coring will be conducted in the same region in the summer of 1993. Processing and 3-D interpretation of the data record geologic features hundreds of meters across but only several meters in total depth, i.e., glacial meltwater channels prior to the most recent Wisconsin glaciation. Reducing spatial sampling even to $100-\mathrm{m}$ line spacing results in severe spatial aliasing of these features, making geologic interpretation erroneous. In addition to 3-D structure, local subsurface elastic properties need to be estimated quantitatively by inverse methods. The global optimization methods can be employed to derive earth models and their uncertainties. A major challenge is to combine 3-D acquisition, imaging, interpretation, and core data with the requirements of material property estimation such that a complete understanding of propagation in shallow water environment can be achieved.

11:10

1aAO9. Influence of internal solitons on sound propagation in shallow water with a summer strong thermocline. Ji-Xun Zhou, Xue-Zhen Zhang (School of Mech. Eng., Georgia Inst. of Technol., Atlanta, GA 30332 and Inst. of Acoust., Acad. Sinica, Beijing 100080, People's Republic of China), and Peter H. Rogers (Georgia Inst. of Technol., Atlanta, GA 30332)

Analysis of extensive data has shown that the internal waves in the coastal zone exhibit the properties of solitons. Using experimental data and numerical simulation results, this paper shows what happens to sound propagation if internal solitons are present in shallow water with a strong summer thermocline. The experiments were conducted in the Yellow Sea off the China coast during the summer, where strong thermoclines with more than $10^{\circ} \mathrm{C}$ temperature difference are often present. In order to provide a clear physical picture on the true problem, a carefully chosen simple ocean model is used to show the resonant interaction of sound waves with internal solitons, including signal frequency, soliton wavelength, and packet length resonances. Then, the numerical model is extended to include the classic characteristic properties of more realistic ocean soliton packets. Under the resonance condition acoustic mode coupling transfers a significant amount of energy from lower modes into higherorder modes that have much larger attenuation rates. This becomes a new attenuation mechanism for shallow water with a strong thermocline. The "resonancelike" behavior predicted by the PE analysis is consistent with mode coupling theory. [Work supported by ONR and IAAS.]

MONDAY AFTERNOON, 17 MAY 1993

BALLROOM, 1:00 TO 5:15 P.M.

Session 1pAO

Acoustical Oceanography and Underwater Acoustics: Ocean Acoustics in Shallow Water: What are the Issues? II

Mohsen Badiey,* Cochair

University of Delaware, Graduate College of Marine Studies, Newark, Delaware 19716

*Current: Office of Naval Research, Ocean Acoustics, Code 11250A, 800 North Quincy Street, Arlington, Virginia 22217

Peter H. Rogers, Cochair

Georgia Institute of Technology, School of Mechanical Engineering, Atlanta, Georgia 30332

Contributed Papers

1:00

1pAO1. Shallow water acoustics research issues. Hassan B. Ali, Robert L. Field, Jerald W. Caruthers, R. A. Wagstaff, and Stanley A. Chin-Bing (Naval Res. Lab., Stennis Space Center, MS 39529)

Propagation of sound in shallow water is complicated by the proximity of the boundaries and interferring noise sources and by environmental variability. Moreover, geomorphic and geoacoustic properties of the seabed play a major role in the character of the propagating signal. Propagation prediction is complicated by the need to use full wave field models versus simpler ray models. Roughness and inhomogeneities of the interfaces and seafloor are important to both forward and backscatter. Multiple interactions with the interfaces cause signal frequency, angle, and time spreads, coherence loss, and out-of-plane effects, and they exacerbate shear and interface wave problems. The practical effects of all this are complications in the reverberation and noise fields and in signal coherence. On the other hand, match-field processing in shallow water provides some benefits (essentially, by taking advantage of multiple images of the target reflected in the boundaries), but achieving a positive net gain is critically dependent on our basic understanding of the multiplicity of detrimental effects. These issues are discussed in terms of their relevancy to acquiring this understanding to support ongoing technology and advanced development programs in the Navy.

1:15

1pAO2. Issues in numerical modeling of shallow water seismoacoustics. Henrik Schmidt (Dept. of Ocean Eng., MIT, Cambridge, MA 02139)

Over the last couple of decades significant progress has been made in modeling seismoacoustic propagation in shallow water. Thus, for example, environmental features such as a stratified, elastic bottom is a standard feature in several propagation models, even range-dependent ones 
with the development of the elastic PE. The past emphasis on deterministic propagation modeling has been driven by the primary interest in passive sonar systems for shallow water. However, with the increased importance of active sonar scenarios in shallow water, as well as the significance of acoustics for underwater communication, a wide range of new issues face the acoustic modelers. Thus, at the higher frequencies involved, the traditional wave theory models are not particularly effcient, and classical approaches such as ray tracing should be re-visited and modified to properly account for bottom interaction in particular. In addition, the spatial and temporal coherence becomes an important issue, in particular in relation to the oceanographic and geophysical variability, raising a need for stochastic seismoacoustic models. Another important issue is reverberation from the rough and inhomogeneous, elastic bottom. The various issues are illustrated using simulated and real data, and the associated needs for new mathematical and numerical models are discussed.

\section{1:30}

1pAO3. Modeling acoustic propagation in shallow range-dependent environments. R. A. Stephen (Dept. of Geol. and Geophys., Woods Hole Oceanographic Inst., Woods Hole, MA 02543)

"Shallow" water means water depth less than an acoustic wavelength and implies by definition strong interaction with the bottom. However since most ocean bottoms are rough and laterally heterogeneous many of the assumptions used to simplify the elastodynamic equations are invalid. On the other hand, since the depths and ranges of interest are considerably less (in terms of wavelengths) than deep water problems, direct numerical solutions of shallow water problems are quite tractable. Finite difference approaches, for example, have the following advantages over alternative methods: (a) They include all shear wave (rigidity) effects in the bottom including body and interface waves. (b) They can be applied to pulse beams at low grazing angles (less than $20^{\circ}$ ). (Methods based on "infinite plane waves" have conceptual problems at low angles.) (c) Both forward scatter and backscatter are included in the solutions. (d) Multiple interactions between scatterers are included. (e) Arbitrary range-dependent topography and volume heterogeneity, can be treated simultaneously in the same formulation. (f) Problems are scaled to wavelengths and periods so that the results are applicable to a wide range of frequencies. (g) The method considers scattering from structures with length scales on the order of acoustic wavelengths.

\section{1:45}

1pAO4. Modeling issues for predicting sound propagation at moderate frequencies in bottom-limited sound channels. Frederick D. Tappert, Michael G. Brown, Harry A. DeFerrari, Charles Monjo, and Lan Nghiem-Phu (Div. of Appl. Marine Phys., RSMAS/U.M., 4600 Rickenbacker Cswy., Miami, FL 33149-1098)

Consider sound propagation in the moderate frequency range (depth to wavelength ratio, $H / \lambda$, between 20 and 100) in shallow water environments with bottom roughness. Measurements in the Straits of Florida have shown remarkable phase stability together with rapid (minutes-to-hours) unstable amplitude fluctuations that nevertheless yield stable envelopes when averaged over many hours. What physical effects should be contained in an acoustic model in order to predict the observed behavior? It is suggested that a full-wave model that is capable of multiple rough bottom forward scattering and includes temporally slowly varying range-dependent sound-speed profiles will yield observed results, when used together with coherent signal processing techniques. A broadband PE model will illustrate the assertion, at least qualitatively. Absolute level prediction is quite another matter that requires more extensive environmental inputs.

1pAO5. Sonar signal processing and shallow water acoustics. Arthur B. Baggeroer (Dept. of Ocean Eng., Rm. 5-204, MIT, Cambridge, MA 02139)
Sonars operating in shallow water usually encounter what signal processors term a reverberation-limited environment; higher signal levels simply lead to higher reverberation and not to improved performance. Consequently, the principal means of improving sonar performance is to use signals appropriate for the task and environment. This implies that robust environmental models are imperative. In both sonar and radar, reverberation, or scattering, is usually modeled to be a random linear system characterized by a scattering function, or some other second moment such as a two-frequency correlation function. Typically, an active sonar resolves a target or an environmental parameter by either range or time gating (high bandwidth signals) or Doppler gating (long duration signals). The resolution properties of a signal are specified by its ambiguity function and the interaction with the scattering function is the single most important factor in determining system performance. This talk will provide a brief overview of some of the signal processing issues for shallow water sonars.

\section{2:15}

1pAO6. Forward scatter and signal coherence in shallow water. Jerald W. Caruthers and Michael F. Werby (Naval Res. Lab., Stennis Space Center, MS 39529)

In shallow water it is difficult to separate the effects of propagation in a waveguide composed of layered media from those of scattering produced by roughness at the layer interfaces. Moreover, to properly account for these combined effects, three-dimensional, fully elastic propagation and scattering models must be used, if a fundamental understanding of shallow water propagation is to be attained. Very few forward propagation studies include scattering effects on forward propagating signal properties such as amplitude and coherence. In shallow water such effects are critical to the condition of the signal when it reaches a target and when it returns to the monostatic receiver. The importance of these effects on the signal properties is magnified for bistatic receivers. Boundary scatter, including out-of-plane scatter, in shallow water significantly affects time and angle (as well as frequency) spreads that lead to degradation of signal coherence. The requirements to deal with scattering in three dimensions and to include full wave field physics in propagation models introduce the need for extensive computer code and computational power. While there are no simple solutions to the problems these requirements impose, the advance of computational technologies and the development of advanced propagation and scattering codes will allow comprehensive and innovative approachs. These issues are addressed and an approach to solving this significant part of the shallow water acoustics problem is discussed.

\section{2:30}

1pAO7. Fluctuations and predictability of sound propagation at moderate frequencies in bottom-limited sound channels. Harry A. DeFerrari, Michael G. Brown, Frederick D. Tappert, Charles Monjo, and Lan Nghiem-Phu (Div. of Appl. Marine Phys., RSMAS/U.M., 4600 Rickenbacker Cswy., Miami, FL 33149-1098)

Sound propagation in the moderate frequency range (depth to wavelength ratio, $h / \lambda$, between 20 and 100 ) in shallow water environments with bottom roughness is extremely complicated, variable, and poorly understood. Multipaths are generally neither resolvable (assuming $Q>1$ ) nor stable. Fluctuations are dominated by a combination of continual formation and destruction of multipaths as well as by perturbations to otherwise stable paths-as is the case in deep water. Propagation characteristics observed in the Straits of Florida for several ranges have consistent features. Sound fields are slowly varying and phase coherent. Large fluctuations with periodicities of a few minutes to a few hours can be qualitatively accounted for as a slowly modulated complicated caustic structure. The quantitative description of these phenomena is beyond the domain of validity of existing theories because variables do not separate and rays behave chaotically. Advances in both theory and experiments are required to improve understanding of these propagation issues. 
nally, the impact of this forward modeling study on possible inverse problem studies for the oceanography is discussed.

1pA08. Some features of ambient noise in shallow tropical waters. Douglas H. Cato (Maritime Operations Div., Mater. Res. Lab., Defence Sci. \& Technol. Organisation, P.O. Box 44, Pyrmont, NSW, 2009, Australia)

This paper discusses the main features of ambient noise in shallow tropical waters near Australia and the implications for shallow water acoustics. Noise from biological sources is usually the dominant component of the ambient, in the absence of high winds and heavy rain. Noise levels fluctuate by more than $30 \mathrm{~dB}$ (commonly by about $20 \mathrm{~dB}$ ) as a result of biological activity. Diurnally varying biological choruses produce the highest noise levels and are evident in the frequency range $40 \mathrm{~Hz}$ to $5 \mathrm{kHz}$. Snapping shrimp noise, extending to frequencies beyond $200 \mathrm{kHz}$, is the dominant component of ultrasonic noise where habitats are favorable. Biological transients (signals detectable from individual animals, usually fish and whales) are also important. Traffic noise varies from high levels near ports to negligible in areas remote from ports and where propagation is poor.

\section{3:00-3:15 Break}

\section{3:15}

1pA09. The issue of time-varying oceanographic conditions as related to acoustics in shallow water. Peter J. Stein (Scientific Solutions, Inc., 47 Aster Ct., Nashua, NH 03062) and James K. Lewis (Atlantic Applied Research Corp., Longbeach, MS 39560)

Here evidence is shown that time-varying oceanographic conditions are an important consideration in shallow water acoustic propagation. In most shallow water conditions there can be substantial temperature and salinity variations. Coastal jets and freshwater outflows can effect regions out to $40-50 \mathrm{~km}$ offshore. Sound-speed profiles, and thus the acoustic propagation conditions, can be highly variable in both space and time. This is shown by looking at a few examples. Oceanographic modeling was conducted using a three-dimensional numerical model for predicting the time- and range-varying conditions. The results from the oceanographic model were input into the Navy standard PE model to predict the acoustic propagation through these model test regions. The results show substantial differences in the transmission loss over time changes on the order of hours. Other issues such as bottom absorption, surface roughness effects, and reflections from a sloping bottom will also be addressed. Further modeling efforts that couple advanced oceanographic models to advanced range-dependent acoustic models are suggested.

\section{3:30}

1pAO10. How well do physical oceanographic (P.O.) models and routine P.O. measurements help predict acoustic propagation in shallow water? James F. Lynch and Timothy F. Duda (Dept. of Appl. Ocean Phys. \& Eng., Woods Hole Oceanographic Inst., Woods Hole, MA 02543)

In trying to answer the question posed in this title, the following outline is followed. First, the dominant oceanographic processes that effect shallow water (coastal) acoustic propagation in the $50-\mathrm{Hz}$ to $2-\mathrm{kHz}$ band is identified. Next, how well these ocean processes are understood and modeled, and how the models are constrained by standard input data is investigated. The limits of predictability of the ocean field (spatially and temporally) are then examined. The issue of the sensitivity of the acoustic field to the particular ocean processes is investigated, followed by the predictability of the acoustic field propagating through the ocean processes. The various measures of the acoustic field (proploss, coherence, etc.) one uses to quantify the ocean/acoustic interaction are considered, reflecting the needs of various acoustics applications. Which acoustics models are appropriate for describing propagation through particular oceanographic fields is also addressed. Fi-

\section{$3: 45$}

1pAO11. Shallow water acoustic channel monitoring with selective mode excitation. James C. Preisig and Josko A. Catipovic (Dept. of Appl. Ocean Phys. \& Eng., Woods Hole Oceanographic Inst., Woods Hole, MA 02543)

The shallow water environment is characterized by multiple processes that cover a wide range of temporal and spatial scales and that have a significant effect on the propagation of acoustic signals [G. V. Frisk, "Report on the Office of Naval Research Shallow Water Acoustics Workshop," 24-26 April 1991, WHOI-92-06]. The coupling between the modes of acoustic signals is potentially an effective means of monitoring these processes. Traditionally, a signal source is used to excite the acoustic medium. A receiver array is used to sort out the modes of the propagating signal and the coupling between particular modes is inferred from travel time differences. This approach provides relatively little control of the distribution of transmitted energy among the modes and adds uncertainty to the estimation of the coupling between particular modes. An alternative approach is proposed to use an array of sources to selectively excite modes of the medium. There are several advantages and challenges of using such an approach. First, this will improve the ability to estimate the actual coupling between different modes. Second, it is well known that propagation of and coupling between particular modes is most sensitive to environmental perturbations having particular spatial scales. [B. Comuelle and B. M. Howe, "High Spatial Resolution in Vertical Slice Ocean Acoustic Tomography," J. Geophys. Res. 92, No. C11 (15 October 1987)]. Therefore, by exciting particular modes and not exciting other modes, the emitted signal can be tuned to be most sensitive to environmental fluctuations on a particular spatial scale. A major challenge in this approach is the tuning of the source array to ensure that only the desired modes are excited.

\section{4:00}

1pAO12. A two-wave-number description of shallow water ocean acoustics. Robert M. Kennedy (Naval Undersea Warfare Ctr. Detachment, AUTEC, West Palm Beach, FL 33402-7517)

Wave-number spectrum is a useful measure of the spatial properties of the underwater acoustic field. A basic tenet of the wave-number spectrum approach is the spatial homogeneity of the cross-correlation (or cross-spectrum) function; a property that is frequently satisfied in deep water environments. Shallow water acoustic channels, on the other hand, are basically nonhomogeneous in the depth variable as a result of the addition of numerous normal modes or multipaths. Two-frequency spectrum have been used to analyze (temporal) nonstationary variables and to a lesser extent two-wave-number spectrum have found use investigating the nonhomogeneous noise in hydrophone array structures. For this paper, two-wave-number (vertical) spectrum were found for several canonical underwater acoustic waveguides. The physical significance of the results are identified by comparing them with similar spectrum from structural acoustic analysis. Particular attention is paid to the consequences of the nonhomogeneous structure to hydrophone array design and the channel parameters that emphasize the two-wavenumber character of the spectrum. The sensitivity of the results to intermodal correlation suggests a measurement approach for determining this basic component correlation.

\section{4:15-4:25 Break}

$4: 25-5: 15$

\section{PANEL DISCUSSION}

Panel Moderator: Peter H. Rogers

Panel Members: D. Weston, W. Carey, M. Buckingham, T. Ewart,F. Tappert, G. Frisk, J. Ewing, J. Austin, J. Zhou 


\title{
Session 1pEA
}

\section{Engineering Acoustics: Sound Intensity Measurement, Calibration, and Standards}

\author{
George S. K. Wong, Chair \\ National Research Council, Institute for National Measurement Standards, Ottawa, Ontario K1A OR6, Canada
}

Chair's Introduction-1:45

Invited Papers

1:50

1pEA1. Intensity measurement on time-varying signals. Gunnar Rasmussen (Brüel \& Kjær, 2850 Nærum, Denmark)

Intensity measurements are especially valuable when used for the detection of phenomena of a fast-varying nature. Sound pressure levels are greatly influenced by the character of the environment, the reverberation time, the location of acoustic centers, and other reactive conditions. The intensity data may be largely independent of these conditions especially when obtained in very short periods of time. Some standards require a wide frequency range, which often requires at least two spacings between the microphone acoustic centers and thus two measurements carried out with the same acoustical center for the probe. Many sources are not stationary and will therefore not offer the repeatability required for two measurements to be carried out with the required accuracy. Examples are vehicle passby, explosions, nonlinear structural vibration, reciprocating engines, and measurements during breakdown as well as run up and down. Techniques involving FFT technique may have serious shortcomings under such conditions. They will work extremely well during calibration and laboratory conditions. When there is a need for actual tests, possible errors may be difficult to assess. Spatial transformation will also require steady continuous signals. The response of transducers to very short duration signals, the influence of the transducer size and configuration, as well as ways of assuring a wide frequency range as required for standardized sound power measurement will be reported. The importance of the probe configuration for practical field measurements will be discussed.

1pEA2. Some recent developments in phase match of microphones. George S. K. Wong (Inst. for Natl. Measurement Standards, Natl. Res. Council, Ottawa, ON K1A 0R6, Canada)

Phase match data for condenser microphones obtained with a new coupler are discussed. The protective grids of the microphones have some influence on the phase match at frequencies above $1 \mathrm{kHz}$. The phase match theory is based on the exchange microphone method [J. Acoust. Soc. Am. 90, 1253-1255 (1991)], which is also capable of discerning the phase match between the amplifiers of the two measuring channels. The performance of the coupler is verified with direct electrical measurement of the phase difference between the two channels and by comparison with the corresponding measurements provided by the coupler. A comparison is made between the data obtained with the coupler and those acquired with the electrostatic actuator method. Since the electrostatic actuator method does not expose the microphone back cavity pressure equalizing vent to the same excitation, the phase match readings provided by the electrostatic actuator method are confirmed to be not valid, particularly at low frequencies.

$2: 30$

1pEA3. Progress on the development of an instrument standard for the measurement of sound intensity. G. Krishnappa (Inst. for Mech. Eng., Natl. Res. Council, Montreal Rd., Ottawa, ON K1A OR6, Canada)

ANSI and IEC working groups have been involved in the development of instrument standards for the measurement of sound intensity. The primary application of these standards is to instruments used for determining sound power of noise sources in accordance with the requirements of approved ANSI and ISO standards. The approach taken by both the working groups in the development of the documents is very similar with only minor variations, notably in the frequency range, specification of pressure residual intensity requirements, and testing devices. Requirements and performance verifications are specified for the complete instrument system comprising probe and processor, and separately for the probe and processor. Performance verification tests are written in terms of type of tests, periodic verification tests, and field checks. The paper will discuss in detail, the scope and purpose of the standard, instrument requirements, and methods for performance verifications.

1pEA4. Standing wave tube testing of sound intensity probes. R. W. Guy (Ctr. for Building Studies, Concordia Univ., 1455 de Maisonneuve Blyd. West, Montréal, PQ H3G 1M8, Canada)

To ensure correct measurement of sound intensity in highly reactive fields and lower frequencies $(\leqslant 400 \mathrm{~Hz})$ a standing wave field performance test has been specified in a draft standard (IEC 1043 Instruments for the measurements of sound-instruments which measure intensity with pairs of pressure sensing microphones, Draft Standard 1991). The purpose and requirements of the 
test are examined from theoretical and experimental perspectives, and suggestions for change are made. It is shown that differing tolerance limits are required if over and underestimate error cases are to be considered. A wider tolerance limit may be specified for the standing wave required by the test and fewer measurements need to be taken ( 2 in contrast to 15). [Work supported by the National Science and Engineering Research Council Canada_Individual Operating Grant.]

3:10

1pEA5. The standardization of sound power determination using sound intensity measurements: An analysis based on a computer simulation. Frédéric Laville and Jean Nicolas (Dept. of Mech. Eng., Sherbrooke Univ., Sherbrooke, PQ J1K 2R1, Canada)

The standardization of sound power determination using sound intensity measurements is a much more difficult task than in the case of pressure measurements. The difficulty comes from the fact that both the precision of each intensity measurement and of the resulting sound power depend strongly on the acoustic field. This effect has been analyzed with a computer simulation that has a broader scope than previous ones that were restricted to the evaluation of the sampling error for the source field only. Because the intensity estimated with two microphones is computed for sources represented by monopole arrays located inside as well as outside the measurement surface, the effects of instrumentation error and background noise field are included. The discrete point measurement standards proposed by ISO and ANSI are critically examined with the computer simulation. Among other results, it is shown how $F_{3}$ as defined in the ISO standard is a good indicator of the phase mismatch error but should not be used in conjunction with $F_{2}$ as suggested in the standard at this approach leads to variable error limits. [Work supported by IRSST.]

\section{Contributed Papers}

1pEA6. Field calibration and check-out of an intensity measurement system using a simplified probe and a reference sound source. Angelo J. Campanella (Campanella Associates \& ACCULAB, 3201 Ridgewood, Dr., Columbus, OH 43026)

There is need for a simple field check-out and calibration method for intensity measurement systems. Microphones, preamps, accompanying software, and data display constants can come in question after shipping, reassembly, and resetting. A simplified and standard method of exercising the total measurement system in a few minutes from microphone to display is presented. Microphones are mounted in the side-byside configuration described by Per Bruel [2nd ICA (Sept. 1985)]. The sound field around an ACCULAB RSS-101 [Inter-Noise 91, 961-964] reference sound source (RSS) is sweep-surveyed [Tachibana, InterNoise 91, 1041-1044] over the five sides of an enclosing 1-m cube. This intensity survey method tends to cancel external noise and reflections in a wide variety of environments (shops, offices, outdoors, etc.). Instrument software is then set to display sound power in octave bands that can be directly compared with the laboratory calibration values known for the RSS-101. Sound power agreement within 1 to $2 \mathrm{~dB}$ will be found A-weighted and in the $250-\mathrm{Hz}$ to $2-\mathrm{kHz}$ octave bands with a nominal 1 -in. (25-mm) microphone spacing. System performance in lower and higher frequency bands may be verified by repeating the test with appropriate microphone spacing.

\section{$3: 45$}

1pEA7. Fiber-optic interferometric and intensity microphones. Chonghua Zhou, S. V. Letcher (Dept. of Phys., Univ. of Rhode Island, Kingston, RI 02881), A. Shukla, and Raman Singh (Univ. of Rhode Island, Kingston, RI 02881)

Both interferometric and reflective-intensity fiber-optic microphones that exploit the fiber-optic advantages of electromagnetic-interference immunity, small size, and robustness are described. The interferometric microphone is a low-finesse Fabry-Perot sensor in which interference occurs between the light reflected from the end face of the fiber and that reflected from the membrane. The return signal is directed to a fast photodiode. The sensor has a flat frequency response from $50 \mathrm{~Hz}$ to 20 $\mathbf{k H z}$. The phase shift is directly proportional to the amplitude of the diaphragm displacement with a broad dynamic range. The sensitivity is $2 \mathrm{rad} / \mathrm{Pa}$ and the minimum detectable pressure is less than $0.001 \mathrm{~Pa}$ when the phase shift is measured at quadrature. The intensity-reflection microphone simply measures the change of the intensity of light that is recaptured by the fiber after reflection from the diaphragm. It has the advantage of low cost and ease of sound reproduction. In a dynamic range of more than $60 \mathrm{~dB}$, it is linear and has a flat frequency response from $50 \mathrm{~Hz}$ to $20 \mathrm{kHz}$. The minimum detectable pressure is $0.1 \mathrm{~Pa}$. [This work is supported, in part, by NSF Grant No. MSS-9101514.]

\section{4:00}

1pEA8. Underwater PVDF acoustic intensity probe. David Van Tol and W. Jack Hughes (Appl. Res. Lab., Penn State Univ., P.O. Box 30, State College, PA 16804)

The desired characteristics and properties of an underwater intensity probe are best achieved by constructing the sensor out of the polymer polyvinylidene fluoride (PVDF), which has the properties of high sensitivity, small thickness, and an impedance close to that of water. An array is being designed to measure the acoustic intensity in two dimensions and at several collinear points. Since the characteristic impedance of PVDF is close to that of water, the PVDF sensors are almost acoustically transparent. This helps to minimize the scattering and interference caused by the probe. The probe array is designed for use over a frequency range of $500 \mathrm{~Hz}$ to $5 \mathrm{kHz}$. Design data will be presented, and calibration measurements of magnitude and phase will show how well the probe performed. Some of the advantages, disadvantages, and construction problems with PVDF sensors will also be addressed. 
Session 1pNS

\author{
Noise and Architectural Acoustics: Wavelets I: Emphasis on Scattering and Statistical Signal Processing \\ David Lubman, Chair \\ GM Hughes Electronics, Building 676, MS N123B, P.O. Box 3310, Fullerton, California 92634
}

Chair's Introduction-1:30

Invited Papers

1:35

1pNS1. Phase space engineering for mid- and high-frequency submerged structural acousties: A wave is a wave is a wave, but what is a wavelet? L. B. Felsen (Dept. of Elec. Eng./Weber Res. Inst., Polytechnic Univ., Six MetroTech Center, Brooklyn, NY 11201)

Determining and interpreting the near and far zone mid-frequency acoustic scattering characteristics of submerged elastic structures in both the frequency and time domains requires wave-oriented spatially dependent pre-and post-signal processing. The most general framework for data analysis and synthesis is the eight-dimensional (space-time)-(wave-number-frequency) phase space that grants access by transforms and projections to various lower-dimensional subdomains that highlight distinct phenomenologies. In phase space engineering, "wavelets" are important basis elements for phenomenological and numerical parametrization, but there are two kinds: those usually proposed in favor of the wave aspect are smooth (analytic) compact propagators like beams or wavepackets, whereas those usually proposed in favor of the numerical processing aspect have bounded support and are generally nonsmooth. Thus, neither wavelet basis meets the demands of the other. Because scattering signatures from submerged structures have strong features driven by wave phenomenology, the processing must utilize space-wave-number portions of the phase space in addition to the conventional time frequency. Examples are shown to illustrate various wave-based parametrizations. The challenge is to construct a self-consistent hybrid scheme for phase space engineering that exploits the best of both types of wavelets. Then one can say: $a$ wavelet is a wavelet is a wavelet. [Work supported by ONR.]

2:05

1pNS2. Application of multiresolution decomposition to scattering. John J. McCoy (School of Eng., Catholic Univ. of America, Washington, DC 20064) and Ben Zion Steinberg (Tel-Aviv Univ., Tel-Aviv 69978, Israel)

The theory of multiresolution decomposition, using orthogonal wavelets, has received considerable attention as a tool for signal processing and more recently for developing efficient numerical algorithms for the solution of a class of integral equations. Relatively few studies have been reported of its use to investigate fundamental physics of the across-length-scale coupling that is a critical component for understanding the response of complex dynamical systems. In the context of the scattering of acoustic signals by a complex subscale heterogeneity in an elastic plate, the accommodation of the subscale heterogeneity in prediction models for the full-scale response, via a precisely defined operator, is demonstrated. This operator is seen to be invariant under changes in either the problem forcing or in the full-scale geometry of the plate system.

\title{
2;35
}

1pNS3. The multiscale structure of the passive scalar field in turbulent water jets. R. M. Everson (Ctr. for Fluid Mech., Brown Univ., Box 1966, Providence, RI 02912) and K. R. Sreenivasan (Mason Lab., Yale Univ., New Haven, CT)

The wavelet transform is applied to two-dimensional dye concentration data in axisymmetric turbulent jets at moderate Reynolds numbers. The transform permits an examination, at different scales, of the geometry of turbulent structures. Information about the number of structures at a given scale, their area and aspect ratio is obtained; long, stringy structures are observed at small scales in contrast to the short, rounded structures at large scales. The wavelet transform is also applied to temporally resolved sequences of laser-induced fluorescence images, which allows analysis of the evolution of structures at different scales, their interactions, and speeds. It is found that a significant part of the dynamics involves the merging of scales besides the usual splitting traditionally associated with cascading. Furthermore little coupling between structures at different scales is observed. A comparison with the vorticity field is made. 
1pNS4. Nonlinear and robust signal extraction based on wavelets. Andrew G. Bruce (Statistical Sciences, Inc., 1700 Westlake Ave. N., Seattle, WA 98109), David L. Donoho (Stanford Univ., Stanford, CA 94305), and R. Douglas Martin ${ }^{\text {) }}$ (Statistical Sciences, Inc., Seattle, WA 98109)

The wavelet transform is an important new tool in applied mathematics, engineering, and science. In a series of papers, Donoho and Johnstone (1992) develop a powerful theory based on wavelets for extracting nonsmooth signals from noisy data. Several nonlinear smoothing algorithms are presented that provide high performance for a wide range of spatially inhomogeneous signals. These algorithms are computationally very fast. However, like other methods based on the linear wavelet transform, these algorithms are very sensitive to outliers in the data. The effect of a large isolated observation on the wavelet transform is smeared through all wavelet scales. In this paper, outlier resistant wavelet transforms are developed. In these transforms, outliers and outlier patches are localized to just a few scales. Hence, it is easy to identify and remove the outliers. By using the outlier resistant wavelet transforms, the Donoho and Johnstone nonlinear signal extraction methods are improved upon. These algorithms prevent outliers from leaking into the extracted signal. Applications will be given for a variety of data sets, including ocean acoustic data

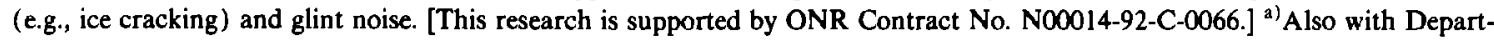
ment of Statistics, University of Washington, Seattle, WA 98195.

\section{3:45}

1pNS5. Application of wavelet analysis to machinery diagnosis. Gary R. Wilson, Mark D. Ladd, Russell D. Priebe, and Kevin W. Baugh (Appl. Res. Labs., Univ. of Texas at Austin, P.O. Box 8029, Austin, TX 78713-8029)

Conventional time-frequency spectral analysis, typically conducted with discrete Fourier transforms (DFTs), represents a signal as the sum of complex harmonic exponentials, and results in an analysis that has the same time and frequency resolution at all frequencies represented in the DFT. However, some processes are better represented as the sum of functions at different time scales rather than at different frequencies. Wavelet analysis provides such a representation, and under certain conditions can be interpreted as an analysis whose time and frequency resolutions change with frequency, as opposed to the constant time and frequency resolution of the DFT. Two different types of processes associated with rotating machinery, bearing vibrations, and periodic mechanical transients, are shown from physical considerations to have time scale properties that are appropriate for wavelet analysis. Measurements of bearing vibrations and mechanical transients were made and both Fourier and wavelet analyses were applied to each of the measurements to demonstrate their associated time-scale properties and the potential benefits of wavelet analysis for machinery diagnostics. [Work supported by ONR.]

\section{Contributed Papers}

\section{4:15}

1pNS6. Wavelet analysis of blade-vortex interaction noise. Wm. Constantine, C. Pezeshki, and M. Mosher ${ }^{2}$ (Dept. of Mech. and Mater. Eng., Washington State Univ., Pullman, WA 99164-2920)

Fourier analysis techniques for analyzing helicopter noise signals have been shown to be an adequate, yet inefficient tool for the design of helicopter noise control systems. Impulsive sources of noise, such as blade-vortex interaction (BVI) noise cannot be localized in time without collecting relatively large amounts of data at high sampling rates. Attempts to minimize the BVI phenomenon through helicopter blade control schemes in the Fourier domain are inherently inefficient since the control matrices must account for the large number of Fourier components needed to represent the signal. Time-frequency representations, such as the wavelet representation, offer an efficient alternative to these Fourier domain methods. Wavelet analysis has been performed on an ensemble of helicopter noise signals. An interactive wavelet program was designed using quadrature mirror filters developed by $\mathbf{S}$. Mallat. Using the bandpass representations of the signal, BVI noise was isolated and extracted from the overall helicopter noise signature. Future research will integrate the wavelet decomposition into an adaptive frequency domain controller for BVI noise minimization. ${ }^{\text {a) }}$ With NASA Ames Res. Ctr., MS T-42, Moffett Field, CA 94035.
1pNS7. The variable domain transform and detection and the simultaneous measurement of velocity and position of a target. $N$. Roosnek (R.B.R., Vlaskamp 82, 259 AC The Hague, The Netherlands)

It is known that for the detection of targets the cw pulse gives the best velocity resolution. The FM pulse gives a superior result for the position of the target. The latter, depending upon the form of the FM pulse, is biased by the velocity of the target. In the case of a large bandwidth pulse length product, the output of the pulse compression, the ambiguity function, depends upon the velocity and the form. The ambiguity function of the linear periodic FM pulse is invariant for the velocity. The other forms are sensitive for the velocity of the target. In this paper it will be shown that this property can be used nicely for the detection and measurement of the velocity and position of the target by means of the variable domain transform, which was presented in an earlier publication [N. Roosnek, J. Acoust. Soc. Am. 92, 3207-3212 (1992)]. The results of the application of this technique to classical FM and other FM pulse forms will be presented. 


\title{
Session 1pPA
}

\section{Physical Acoustics: General I}

\author{
Victor W. Sparrow, Chair \\ Pennsylvania State University, 157 Hammond Building, University Park, Pennsylvania 16802
}

\section{Contributed Papers}

1:15

1pPA1. Determination of texture coefficients in hexagonal polycrystalline aggregates with orthorhombic symmetry using ultrasonic waves propagating on one to three principal planes. P. J. Kielczynski, A. Moreau, and J. F. Bussière (Industrial Mater. Inst., Natl. Res. Council of Canada, 75 De Mortagne Blvd., Boucherville, PQ J4B 6Y4, Canada)

A method to characterize the texture of hexagonal polycrystalline aggregates with orthorhombic macroscopic symmetry is presented. Previous methods are extended to the analysis of waves propagating on three principal planes of the material. Five coefficients of the crystallographic orientation distribution function, $W_{200}, W_{220}, W_{400}, W_{420}$, and $W_{440}$, are determined from angular variations of the phase velocity of the four ultrasonic modes considered: the bulk longitudinal waves, Rayleigh surface waves, the fundamental symmetric plate mode (in the long-wavelength limit), and shear-horizontal mode. Analytical expressions for the texture coefficients are found for an arbitrary number of principal planes. Measurements are reported for a $\mathrm{Zr}-2.5 \mathrm{wt} \% \mathrm{Nb}$ pressure tube, an alloy used in CANDU nuclear reactors. The angular variations of the phase velocity of leaky Rayleigh waves and leaky surface skimming compressional waves are measured with a line-focus acoustic microscope. The results agreed with neutron scattering measurements.

\section{1:30}

1pPA2. A comparison of elastic constants of the quasicrystalline and cubic approximant phases of $\mathrm{AlCuLi}$, using resonant ultrasound spectroscopy. P. S. Spoor, M. J. McKenna, and J. D. Maynard (Dept. of Phys., Penn State Univ., University Park, PA 16802)

The anisotropy $\epsilon=\left|1-2 c_{44} /\left(c_{11}-c_{12}\right)\right|$ of a sample of quasicrystalline $\left(T_{2}\right.$-phase $)$ AlCuLi has been measured in our laboratory to be $0.0020 \pm 0.0004$; since a perfect quasicrystal should be isotropic, this could signify deviation from perfect icosahedral order. A comparison with the closely related $R$-phase cubic approximant, which is often present when macroscopic quasicrystals are grown, may provide a better basis for interpreting such measurements. Among the various methods of determining elastic properties, only resonant ultrasound spectroscopy, which uses the normal mode resonance frequencies to retreive the complete elastic tensor in one measurement, offers the necessary sensitivity to resolve such small anisotropies. Results on a recently received $\boldsymbol{R}$-phase sample, and on its neighboring icosahedral grains, will be compared with the results mentioned above. [Work supported by NSF Grant No. DMR-9000549 and by the Office of Naval Research.]

\section{$1: 45$}

1pPA3. Using piezoelectric film and resonant ultrasound for photoacoustic measurements of very low optical absorption in piezoelectric and dielectric crystals. M. J. McKenna, Wei-Li Lin, and J. D. Maynard (Dept. of Phys., Penn State Univ., University Park, PA 16802)
The photoacoustic effect is one of the most sensitive methods for measurements of the low optical absorption in glasses and crystals. Previously, a new photoacoustic technique has been developed, where a cw laser modulated at the acoustic resonant frequency of the sample generates an acoustic signal amplified by the quality factor of the resonance. With typical quality factors in the order of $10^{5}$, this technique is several orders of magnitude more sensitive than conventional pulsedlaser techniques. However, previous applications using noncontact capactive transducers required precise postitioning of the sample, transducers, and seperate sample supports. Recently, this difficulty has been reduced through the use of $9-\mu \mathrm{m}$-thick piezoelectric (PVDF) film transducers. By supporting the sample at the corners between the film transducers, the need for separate supports is eliminated, with only a small reduction in the quality factor of the resonance. In addition, a large improvement in the signal-to-noise ratio is observed. Recent measurements on large single crystals of calcium floride and quartz will be discussed. [Work supported by NSF Grant No. DMR-9000549 and by the Office of Naval Research.]

\section{2:00}

1pPA4. Focusing of fast transverse modes in silicon and slow transverse modes in zinc. Kwang Yul Kim and Wolfgang Sachse (Dept. of Theoret. and Appl. Mech., Thurston Hall, Cornell Univ., Ithaca, NY 14853)

This paper presents focusing of fast transverse (FT) modes in silicon and slow transverse (ST) modes in zinc, both observed at utrasonic frequencies. Using a (001)-oriented silicon disk, the FT modes are generated and detected by two tiny shear PZT transducers, both polarized in the [100] direction. One transducer fixed on the bottom surface acts as a source and the other serves as a detector scanning the top surface along the [100] direction across various points on the [010] axis at some distances away from the epicenter. The observed focusing pattern indicates strong concentration of FT modes on the narrow band about the (100) plane containing the source. For a (001)-oriented zinc crystal, strong focusing of ST or quasi-transverse (QT) modes toward the symmetry direction is observed: first in theoretical and experimental, epicentral Green's functions; second with very small PZT source and PZT detector; finally with focused pulsed laser excitation and small PZT detector. The focusing patterns observed both in silicon and in zinc are consistent with the results predicted by the theory of phonon focusing. [Work supported by ONR.]

\section{2:15}

1pPA5. Characterizing the performance of acoustic microscopes. Leonard J. Bond and U. W. Lee (Dept. of Mech. Eng., Univ. of Colorado, Boulder, CO 80309-0427)

The transfer functions for focused and defocused, coherent and confocal imaging systems developed in optics are applied to the equivalent ultrasonic imaging systems. Assuming that both of the two systems are free of attenuation and aberrations except defocus, the ideal complex amplitude line-scan signals across a step edge, with various degrees of defocus, are generated. The scalar magnitude of ideal complex image 
profiles are differentiated to get the unique theoretical IRF's and Fourier transformed to obtain the corresponding TF's. The acoustic system has been operated with a $50-\mathrm{MHz}$ lens-rod design focused transducer. The experimental single frequency and broadband image profiles of a step edge, with varying degree of defocus, are differentiated and Fourier transformed. The experimental IRF's and TF's are compared with the corresponding theoretical data. As the initial theoretical analysis is for the wave propagating in an ideal fluid, the effects of attenuation and aberrations are investigated to enable the experimental data to be compared with the theoretical data. The relation between broadband and single frequency acoustic images is described. A practical model for the detection limits for 2-D cracks using the response for a pair of step edges is proposed. The numerical calculations are compared with experimental data. Usually the strong contrast seen in the imaging of cracks is associated with the excitation of Rayleigh waves in surface. In this study, a similar sensitivity to detect cracks is achieved by the defocus effect of pure longitudinal waves, which can be clearly explained by theory.

\section{2:30}

1pPA6. Ultrasonic measurements of excess nonlinearity in polymers. Laszlo Adler and Peter B. Nagy (Dept. of Welding Eng., Ohio State Univ., Columbus, $\mathrm{OH} 43210$ )

To obtain nonlinearity parameters of solids one may use either harmonic generation of finite amplitude waves or the stress dependence of the sound velocity, the so-called acoustoelastic effect. In strongly viscoelastic materials, e.g., in polymers, both linear and nonlinear responses are significantly time-dependent and the quasistatic acoustoelastic effect is relatively much stronger than the dynamic harmonic generation effect. In this presentation, several modifications of the acoustoelastic method will be introduced in order to study the effect of excess nonlinearity in polymers due to fatiguing. An experimental system was developed where external bending load was used to degrade polymeric bars through fatigue and simultaneously monitor nonlinearity via the acoustoelastic effect. Due to the symmetry of the applied bending load, only the second-order nonlinearity (combination of second-, third-, and third-order elastic constants) was measured. It was demonstrated that for thin layers, such as adhesive bonds, a different acoustoelastic effect can be observed by measuring the stress dependence of the attenuation coefficient.

\section{2:45}

1pPA7. Time domain causality relations: Results for absorption obeying a power law. Thomas L. Szabo (Imaging Systems, Hewlett Packard, 3000 Minuteman Rd., Andover, MA 01810)

The Kramers-Kronig relations are used frequently to obtain velocity dispersion from attenuation frequency data and vice versa. For many cases of practical interest, attenuation is described by a power law equation, $\alpha(\omega)=\alpha_{0}|\omega|^{y}$, where $\alpha_{0}$ and $y$ are arbitrary real constants and $\omega$ is angular frequency. If $y \geqslant 1$, these relations fail because the PaleyWeiner theorem is no longer satisfied. Based on a time domain expression of causality, new time-causal relations, with the aid of generalized functions, provide a way of obtaining dispersion relations for the $y \geqslant 1$ cases. For $y<1$, the time-causal relations reduce to the KramersKronig relations. The new results for attenuation of the power law form indicate that dispersion is maximum for $y=1$, and it falls symmetrically to zero at $y=0$ and $y=2$. These results are in contrast to the approximate nearly local Kramers-Kronig theory [M. O'Donnell et al., J. Acoust. Soc. Am. 69, 696-701 (1981)], which predicts dispersion for values of $y=0$ to $y=2$. The two theories agree at $y=1$, but deviate elsewhere. Experimental data confirming the new theory will be given.

\section{3:15}

1pPA8. Causal time domain parabolic wave equations for power law absorption. Thomas L. Szabo (Imaging Systems, Hewlett Packard, 3000 Minuteman Rd., Andover, MA 01810)

The classic parabolic time domain wave equation describes acoustic propagation in a medium in which absorption is a quadratic function of frequency. For the general case of power law absorption, $\alpha(\omega)$ $=\alpha_{0}|\omega|^{y}$, where $\alpha_{0}$ and $y$ are arbitrary real positive constants and $\omega$ is angular frequency, generalized time domain parabolic wave equations are presented. The differential loss operator in the original classic parabolic equation is replaced by a single propagation convolution operator that accounts for both absorption and dispersion. These operators, based on new time causal relations, have different forms for $y$ as an even or odd integer or noninteger. The new equations are compared to those in the literature corresponding to the cases $y=0.5$ (acoustic duct), $y=1.0$ (medical and underwater applications), and $y=0$ or 2 (classic forms)

\section{3:30}

1pPA9. Acoustic pressure peak created at the impact of a missile on a gas-liquid interface: "The problem of the splash of the diver." Bernard Fromont, Michel Lagier (Thomson-Sintra ASM, 525, Route des Dolines, B.P. 38, 06561 Valbonne Cedex, France), and Didier Sornette (CNRS URA 190, Université de Nice-Sophia Antipolis, 06108 Nice Cedex 2, France)

A physical mechanism for the amplitude of the pressure peak created by the impact of a missile at a gas-liquid interface is proposed. The different factors affecting this acoustic pressure peak are discussed. They include the inclination of the missile trajectory, the existence of wavelets roughening the surface of the gas-liquid interface, and the finite compliance of the missile. The difference between the impact and a true shock is stressed: the missile impact indeed creates a true acoustic shock that however plays no role in the generation of the high-pressure peak.

\section{$3: 45$}

1pPA10. An approximate analysis for penetration of spaced foils. Michael El-Raheb (Dow Chemical Co., Central Res. Eng. Lab. Midland, MI 48674)

An approximate analysis for penetration of thin-spaced foils is described. When the projectile strikes the first foil, intense pressure ablates projectile and foil materials and a spherical shock forms propagating toward the next foil. As the spherically expanding shock reaches the second foil, it fails when shear stress along the perimeter of the footprint reaches ultimate shear stress. During this stage of impact, propagation has negligible effect on transfer of momentum. When stricken by material from foils above, each foil after the second deforms plastically into a conical shape ending with extensional failure. The cone's radius grows with time at a rate lower than the speed of propagation of shear waves in the foil's material. The effective wave front lags the actual front because of reversal in the direction of displacement close to the front. Since the mode of failure relies on maximum extensional strain, penetration will cease when speed of impact is lower than some critical speed that depends on maximum strain of the foil material. At this point, the circle of influence is expanding faster than transverse motion of the foil and maximum extensional strain in the cone of influence is never reached. It is found that weight efficiency of the shield rises inversely with foil thickness asymptotically as a result of mass entrained by propagation. The most efficient shield requires relatively thin layers of foil with material properties that maximize effective speed of propagation and maximum strain in the foil material. 
1pPA11. Oscillations of a deformed drop in an acoustic field. T. Shi and Robert E. Apfel (Dept. of Mech. Eng., P.O. Box 2159, Yale Univ., New Haven, CT 06520)

Because of the importance of acoustic levitation technology and no existing theory of oscillations of deformed drops in acoustic fields, a boundary integral technique, developed by Baker et al. [J. Fluid Med. 123, 477-501 (1982)], has been modified to study oscillations of axisymmetric liquid drops in air. The method consists of determining evolution equations for the position and velocity potential on the surface of the drop. The motion of the surface is determined from the potential by solving an integral equation. The potential is updated every time step by using the Bernoulli equation that depends on gravity, interfacial tension force, and acoustic radiation pressure. The acoustic field is determined for every time step by the Fourier transformation method developed by us. Given initial position and potential along the drop surface, the drop will oscillate around its equilibrium positions where it is statically deformed, which can be computed by introducing numerical damping. Some results on oscillations and frequencies of acoustically deformed drops will be presented. [Work supported by NASA through JPL Contract No. 958722.]

\section{4:15}

1pPA12. Thermoacoustics of traveling waves. Richard Raspet, Henry E. Bass, and John Kordomenos (Phys. Acoust. Res. Group, Univ. of Mississippi, University, MS 38677)

Standard thermoacoustic sources and refrigerators have the stack placed near one end in a standing wave tube. This selection of the stack position optimizes the thermoacoustic effect. For certain applications, however, the effect of a thermoacoustic stack on a traveling wave is of primary interest. Equations governing wave propagation through a thermoacoustic stack have been derived including equations for work flux. When the wave propagates in the direction of increasing temperature, the wave is amplified by an amount dependent upon the temperature gradient. Waves propagating in the opposite direction are attenuated. Counterpropagating waves in a standing wave tube encounter both. In this case, the results reduce to those developed earlier by Swift [J. Acoust. Soc. Am. 84, 1145-1180 (1988)] and others. [Work supported by ONR.]
1pPA13. Transient sound radiation from impulsively accelerated bodies. Sean F. Wu (Dept. of Mech. Eng., Wayne State Univ., Detroit, MI 48202)

This paper presents an integral formulation derived from the Kirchhoff integral theorem for predicting transient sound radiation from an impulsively accelerated body. The acoustic pressure is determined in an explicit form in the frequency domain, and subsequently converted to the time domain through Fourier transformations. This integral formulation can be extended to cases in which the body is subject to an arbitrarily time-dependent excitation. The transient acoustic pressure is shown to be expressible as a convolution integral of the impulse response function to the time history of the surface velocity of the body. Since there is no relationship between the integral formulations for the exterior and interior regions, solutions for the radiated acoustic pressure in the exterior region are unique, even at frequencies corresponding to the eigenfrequencies of the related interior boundary value problem. Analytical results based on this integral formulation agree perfectly with those obtained using other methods for transient sound radiation from an explosively expanding sphere, impulsively accelerated rigid sphere, impulsively accelerated baffled and unbaffled circular disks. [Work supported by ONR.]

4:45

1pPA14. Acoustic spiral wave field. Peter H. Ceperley and Alon Koren (Depts. of Physics and Elec. Eng., George Mason Univ., Fairfax, VA 22030)

While most low-amplitude acoustic waves in uniform media can be considered to travel straight from source to receiver, the energy flow and propagation directions in a spiral wave field change as the wave moves away from its source [P. H. Ceperley, Am. J. Phys. 60, 938-942 (1992)]. Thus, one would expect that directional microphones and acoustic imaging systems would give false information as to the location of the source in such a wave field. Two synchronized oscillators, four small speakers, and a horn were used to create a spiral wave in the laboratory. A table top robot arm and microphone were used to map out this sound field, which was then displayed in color using MathCad. The apparent propagation direction was measured using a directional microphone. [Work supported by ONR.]

MONDAY AFTERNOON, 17 MAY 1993

ADAM ROOM, 1:00 TO 5:05 P.M.

\title{
Session 1pSA
}

\author{
Structural Acoustics and Vibration: Intelligent Machine Acoustics \\ Aynur Ünal, Chair \\ Cogswell Polytechnical College, 10420 Bubb Road, Cupertino, California 95014
}

Chair's Introduction-1:00

Invited Papers

1:05

1pSA1. Monitoring, diagnosing, and actively controlling of complex systems using acoustic signatures and artificial neural networks. A. Ünal (Cogswell Polytech. College, 10420 Bubb Rd., Cupertino, CA 95014)

Artificial neural network-based technology enables us to monitor complex systems in real time with reasonable efficiency. Acoustic signatures and/or their transforms can be utilized for diagnosis with less false alarms by exploiting the robust feature extraction abilities of the artificial neural nets. Active controlling requires the instantaneous sensing and actuating abilities that cannot be obtained otherwise. Only the use of artificial neural nets enables us to annihilate the excessive vibrational fields in complex systems. Examples from real life will be given. 
1pSA2. Acoustic signatures: From natural to systems science. Robert D. Finch and Ben H. Jansen (College of Eng., Univ. of Houston, Houston, TX 77204-4792)

The interest in acoustic signatures began with the problem of detecting cracks in railroad wheels. When a wheel is struck with a hammer it produces a sound like that of a bell. If the wheel is cracked it sounds dissonant and muffled. By comparing sounds from the two members of a wheelset a measure of the difference in their mechanical properties is obtained. A fully automatic system was developed and installed on a Southern Pacific track in the 1980's. The story of this undertaking is an object lesson in systems science. Recently beams have been used as test objects in an attempt to resolve certain basic questions in the science of the acoustic monitoring method. These questions will be illustrated with results from a test fixture with various beams. The limitation of the vibration monitoring method is that other conditions, such as uncertainties in the geometry of the test object, its surface conditions, and loading can also affect the vibration response, and it is necessary to distinguish the effects due to harmful conditions from those due to harmless ones. The sensitivity of the method is thus determined by the need to make this distinction. [Work supported by NSF Grant No. MSS-9024224.]

\section{2:05}

1pSA3. Training and representation issues in machine diagnostic by neural networks. Samir I. Sayegh (Phys. Dept., Purdue Univ., Fort Wayne, IN 46805)

Neural networks have emerged as a viable and effective paradigm for pattern recognition and discrimination. A widely used algorithm, backpropagation, can be sensitive to the representation used for the input patterns to the network. This is particularly true in machine diagnostic. An application is presented to discrimination of good versus bad electric motors. Representations in terms of spectral coefficients, wavelets, and $\mathrm{KL}$ coefficients are contrasted.

1pSA4. Signal analysis techniques for acoustic monitoring. Pierre Y. Robin, Peeter M. Akerberg, Ben H. Jansen, and Robert D. Finch (College of Eng., Univ. of Houston, Houston, TX 77204-4793)

Impacting structures with a hammer and measuring the resonant frequency of the induced vibrations is a commonly used technique in acoustic monitoring. However, the transient and nonstationary characteristics of the vibrations limit the performance of classical spectral analysis. In this paper, a number of alternative techniques specifically designed to deal with the transient nature of impact-induced vibration signals are presented. Among the techniques discussed are the short-time Fourier transform (STFT), the discrete wavelet transform (DWT), and artificial neural networks (ANN). The results obtained with applying these techniques to the acoustic vibrations of intact and slotted beams are presented as an example. It was found with the STFT method, that a relationship exists between slot depth and decay rate. The DWT provided evidence that the decay undulates as a function of time, with a frequency that is related to the integrity of the beam. Early results with ANNs suggest that a net can be trained to differentiate between acoustic vibrations of intact and defective structures. [Work supported by NSF Grant No. MSS-9024224.]

\section{Contributed Papers}

3:05

1pSA5. The use of cluster methods in complex machine diagnostics. A. Ünal and A. Karakasoğlu (Cogswell Polytech. College, 10420 Bubb Rd., Cupertino, CA 95014)

Real-life examples will be given to assess the efficiency of the cluster techniques and artificial neural network-based methods in complex machine diagnostics.

1pSA6. An experimental investigation of the $\mathrm{AE}$ source location and magnitude on 2-D frame structures using intelligent signal processing. Rok Šribar and Wolfgang Sachse (Dept. of Theor. and Appl. Mech., Cornell Univ., Thurston Hall, Ithaca, NY 14853)

The results of experimental investigation of acoustic emission (AE) source characterization in 2-D frames is reported in this paper. Determination of the AE source location and magnitude from the displacement field is an inverse problem. It is known that humans have remarkable sensory abilities to undo a large number of demanding inverse problems with little effort. Here, nature is modeled by implementing intelligent signal processing algorithms to solve the problems of the $\mathrm{AE}$ source location and magnitude determination. The signal processing algorithms implemented are; FFNN trained by a modified backpropagation algorithm, and a linear system called an auto-associative processor (AAP). The standard feature of these algorithms is the use of a set of pre-processed, measured signals to form a system memory. The pre-processed signals used by the AAP algorithm are tutor augmented, while in the case of the FFNN the pre-processed signals are nonlinearly mapped to the training vectors provided by the tutor. The system memory is consequently utilized to process experimental signals to determine some of the AE source properties. [Work partially supported by NSF and ONR.]

\section{3:35}

1pSA7. Ultrasonic monitoring of tensile, fatigue, or creep specimens in situ. D. Hazony, G. Welsch (Case Western Reserve Univ.,

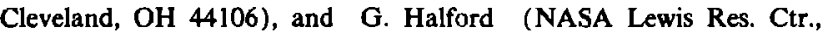
Cleveland, $\mathrm{OH}$ 44135)

A method is proposed for monitoring tensile, fatigue, or creep specimens while the testing is in progress. Two transducers, one transmitsone receives, are imbedded in both ends of the specimen. Sharp timelimited ultrasonic pulses permeate through the specimen from either end along the principle axis. Axial and radial strains may be monitored simultaneously by selectively tracking two of the received echoes. [see D. Hazony, "Edge effects in short pulse piezoelectric transducers," J. Acoust. Soc. Am. 86, 1230-1233 (1989), Sec. IV]. The method is especially useful at elevated temperatures, aggressive environments, relatively high fatigue-cycling frequencies, and a wide range of strains. Generation and growth of cracks may also be tracked and monitored. Data may be gathered from the beginning to the last few microseconds in the life of the specimen. Salient features of the tests will be discussed. Actual data, slides, and a video tape will be presented. [Work supported by TecSonics, Inc. of Twinsburg, Ohio.] 
1pSA8. A new technique for measuring structural dynamic response. Mei Q. Wu (6682 Ganymede Rd., Mississauga, ON L5N 6R1, Canada)

A modified cepstrum technique is introduced. It combines the cepstrum technique with an iteration procedure. The advantage of this technique is that it can determine the dynamic response of a structure from its vibration without any pre-knowledge of the excitation force. The restrictions of the technique are that it only applies to cases where the structural response is band limited in the frequency domain and the excitation force is short in time (comparing to the structural impulse response). When the structural response is not band limited, it can be determined in several limited frequency bands first and combined together later. In this paper, the theory of the modified cepstrum technique is reviewed, computer-simulated experiments are demonstrated, and an application example is presented. In the example, the magnitude of the structural dynamic response under $1400 \mathrm{~Hz}$ of a typewriter was determined by the modified cepstrum technique and compared to the dynamic response measured by conventional impulse excitation technique. The responses determined by the two techniques agreed well. [Work supported by IBM.]

\section{4:05}

1pSA9. Complex process controlling using wavelets. A. Ünal and F Ercal (University of Missouri-Rolla, Dept. of Comput. Sci., Rolla, MO 65401)

Complex process controlling requires a crucial reduction in the number of robust discriminitating features. The use of wavelet transformations with other artificial neural network-based methods will be given using real data.

1pSA10. Conditions of existing negative components in instantaneous frequency analysis. Jeung $T$. Kim and Byungduk Lim (Acoust. and Vib. Lab., Korea Res. Inst. of Standards and Sci., P.O. Box 3, Daedok Science Town, Daejon, Korea 305-606)

An instantaneous frequency analysis is a technique to examine a signature for the rotating machinery if the signal has several transitions within a cycle. This paper discusses the conditions of existing negative frequency components in the instantaneous frequency. By using a simple signal that consists of two frequency components, the instantaneous frequency analysis is conducted while the amplitude ratio between two frequency components has been changed. The calculation shows that depending on the amplitude ratio, the instantaneous frequencies have averaged, zero-valued, or negative components. It turns out that the negative-valued instantaneous frequencies, which have been regarded as the noise effect, are the consequence of the calculation process for the multisignal components. The criteria to show the condition of the negative values in instantaneous frequencies is given in terms of the relative amplitude ratio and the frequency difference. In this paper, a vibration signal monitored from a rotating machinery is also examined as an application example in order to show the existence of negative instantaneous frequencies components.

4:35

1pSA11. Meshing noise from timing chain drives. S. P. Liu, Sabih I. Hayek (Dept. of Eng. Sci. and Mech., Penn State Univ., University Park, PA 16802), K. W. Wang, and M. W. Trethewey (Penn State Univ., University Park, PA 16802)

Controlled experiments on a specially constructed timing chain drive test stand have shown that the most significant noise source in roller chain drives is from impacts between the chain links and the sprocket teeth during their meshing process. Despite its importance, a study that can analytically predict and experimentally verify the characteristics of meshing noise has yet to be performed. A method is presented that can quantify the meshing noise as a function of the critical system parameters, such as chain speed, tension, mass and shape of chain links, and sprocket geometry. A comprehensive model that integrates the chain transverse and longitudinal vibrations with the sprocket motion is developed. The approach also couples the local meshing impulse with the global system dynamic response of the vibrating chain. Parametric tests were carried out on a test stand in which a roller chain and up to three sprockets could be tested. Besides the chain speed and tension, the local vibration and the sound pressure levels are recorded. This fundamental investigation provides new insight into roller chain noise problems. [Work supported by General Motors Corp.]

\section{4:50}

1pSA12. Modeling of the vibration of a slotted beam under various conditions. XiuTing C. Man and Robert D. Finch (Dept. of Mech. Eng., Univ. of Houston, 4800 Calhoun Rd., Houston, TX 77204-4792)

An analytical solution for the vibration of a slotted beam with clamped-clamped end conditions was presented previously [J. Acoust. Soc. Am. 92, 2311 (A) (1992)]. The analytical solution for other classic end conditions, for example, free-free and pinned-pinned end conditions, is now presented. The theory is expanded to include damping as well. Solutions for general end conditions will also be presented. The effect of rotatory inertia, as in Timoshenko beam theory, is discussed quantitatively. Numerical results will be presented with some experimental results. [Work supported by NSF Grant No. MSS-9024224.] 


\title{
Session 1eID
}

\section{Tutorial on Noise Outdoors}

\author{
Allan D. Pierce, Chair \\ Pennsylvania State University, 157 Hammond Building, University Park, Pennsylvania 16802
}

\author{
Chair's Introduction-7:00 \\ Invited Paper
}

7:05

\begin{abstract}
1eID1. Noise outdoors. Tony F. W. Embleton ( 80 Sheardown Dr., Box 786, Nobleton, ON LOG 1N0, Canada)
Concerns about noise in the community date back to the dawn of recorded history, have continued through Roman times, the Middle Ages, and are still with us. Many of the principal wave-propagation mechanisms involved in the real atmosphere outdoors were understood at least qualitatively by the late 1800 's. Today, a good understanding of all the sound propagation phenomena is of considerable economic and social importance in connection with environmental impact studies. Reality is far more interesting than simple spherical spreading in the space above a hard, flat ground. Some grounds are hard like concrete or soft as snow. Corresponding reflection coefficients are usually less than unity and vary with angle. Grounds may not be flat, leading to shadow zones or alternatively multiple reflected paths. Wind convects sound waves, and gradients of wind or temperature refract waves either upward (temperature lapse) or downward (inversion). The atmosphere is rarely still and turbulence causes fluctuations in the acoustical effects. All these features are frequency dependent, and mutually interact. Measured sound levels outdoors owe as much to near-surface weather and to ground shape as to the obvious acoustical factors to source and receiver heights and their separation. Discussion will emphasize field measurements and simple physical interpretations.
\end{abstract}

\section{Session 2aAA}

\section{Architectural Acoustics and Musical Acoustics: New Auditorium Acoustics Measurements-Results and Comparisons II}

\author{
Michael Barron, Chair \\ School of Architecture and Building Engineering, University of Bath, Claverton Down, Bath BA2 7AY, United Kingdom
}

\section{Invited Papers}

8:30

2aAA1. Relationships among various room acoustic indices. 7-22-1, Minato, Tokyo, 106 Japan) and Yoshio Yamasaki

Hideki Tachibana (Inst. of Indust. Sci., Tokyo Univ., Roppongi Tokyo, 169 Japan)

(School of Sci. and Eng., Waseda Univ., Okubo 3-4-1, Shinjuku,

For the last 5 years, the authors have been making acoustical surveys of auditoria in the world at every opportunity. In these acoustical measurements, room impulse responses have been measured using a dodecahedral omnidirectional loudspeaker, monaural microphones, and a dummy head system (Neumann KU81i). In order to get accurate impulse responses with high $\mathbf{S} / \mathrm{N}$ ratio, the sweep-pulse method and synchronous averaging technique were adopted. From the monaural and binaural impulse responses measured in a lot of concert halls and theaters, such acoustic indices as $T_{60}$ (reverberation time), EDT (early decay time), $D_{50}$ (definition), $C_{80}$ (clarity), $T_{s}$ (center time), and IACC (inter-aural cross correlation) were obtained, and the relationships among them were statistically investigated. As a result, it has been found that such indices as $D_{50}, C_{80}$, and $T_{s}$ are highly correlated with each other and the correlations between IACC and other monaural indices are very low. The binaural impulse responses measured through the dummy head were convolved with dry music and speech signals by digital technique, and they are being used as the test signals for subjective experiments using a transaural reproduction system.

\section{8:55}

2aAA2. Relationships between objective measurements and perceptual interpretation: The need for considering spatial emission of sound sources. O. Warusfel, E. Kahle, and J. P. Jullien (IRCAM 31, rue St.-Merri, 75004 Paris, France)

The room acoustics laboratory at IRCAM has undertaken a series of objective and perceptual measurements in different concerts halls and opera houses throughout Europe. The measurements consisted in recording impulse responses at different seats, 
using a directive loudspeaker (at different locations on stage) oriented in various directions. The listening tests were undertaken during concerts with subjects seated at the measured locations. The subjects were asked to describe the room acoustic quality with the help of a structured questionnaire, based on the results of former psychoacoustic tests. The links between perception and the criteria based on the time distribution of sound energy, established in laboratory experiments, were confirmed and/or refined. Furthermore the results of this campaign emphasize dependencies of the perceptual factors on the spatial distribution of energy. Subjective description is strongly affected by orchestra size as well as location and directivity of the instruments. Thus a single location, directivity, and orientation of the source used for the measurements will not suffice for properly describing the perceived room acoustic quality. The analysis of the relations of subjective and objective data collected in that campaign, together with additional experiments, gives some hints as to how to define an appropriate measurement protocol.

2aAA3. Analysis of sound field on spatial information using a four-channel microphone system based on a regular tetrahedron peak point method. Katuaki Sekiguchi, Sho Kimura, and Toshiki Hanyuu (College of Sei. and Technol., Nihon Univ., 1-8 Kanda-Surugadai, Chiyodaku, Tokyo, Japan)

This paper describes the development of a sound field measurement method that is aimed at analyzing spatial information of the sound field in a room. This measurement system has four microphones and each of them is installed at the apex of a regular tetrahedron. With this method spatial information of an individual wave front is analyzed from the impulse response at four points that are completely synchronized. With the introduction of a deconvolution method, measuring accuracy for waveform analysis is greatly improved and the analysis becomes much faster. By using models for which the image source positions are already known, experimental studies have been conducted on the identification of sound source positions and frequency distortion by repeated reflections. Furthermore, virtual sound sources have been detected and the waveform in actual sound fields have been recomposed. The results of these experiments indicate that the measurements are accurate and the measurement method can be used effectively.

\section{9:45}

2aAA4. Recent experiences with binaural measurement techniques in the evaluation of concert hall acousties. J. Christopher Jaffe (Jaffe Holden Scarbrough Acoustics, 114A Washington St., Norwalk, CT 06854), Mahlon D. Burkhard (Sonic Perceptions, Norwalk, CT 06851), and Paul H. Scarbrough (Jaffe Holden Scarbrough Acoustics, Norwalk, CT 06854)

Binaural recording, measurement, and analysis techniques have been significantly improved over the past decade. The coupling of modern binaural recording heads with advanced computer-based signal processing and analysis equipment offers acoustical designers a valuable tool for use in both subjective and objective evaluation of concert hall acoustics. The authors will describe recent experiences in the use of binaural technology for such evaluations, focusing on specific examples of how the technology can be used to improve our understanding of the acoustical environment of these spaces. The specific halls that were studied include a 2300-seat multiple-use auditorium built in the late 1960's, an existing theatre that was converted into a 1900-seat symphony concert hall in the mid-1980's, and a 2700-seat symphony concert hall reconstructed in the mid-1970's.

\section{0:10}

2aAA5. A new physical measure for psychological evaluation of a sound field: Front/back energy ratio as a measure for envelopment. M. Morimoto (Environmental Acoust. Lab., Faculty of Eng., Kobe Univ., Rokko, Nada, Kobe, 657 Japan) and K. Iida (Kobe Univ., Kobe, 657 Japan and Matsushita Commun. Industrial Co., Ltd., Japan)

Broadening is one of the important characteristics for the psychological evaluation of a sound field. Several investigations indicated that broadening was comprised of two elemental senses, i.e., auditory source width (spaciousness) and envelopment [M. Morimoto et al, Proc. 13th ICA, Belgrade 2, 215-218 (1989); J. Acoust Soc. Jpn. 46, 449-457 (1990); and Hidaka et al., J. Acoust. Soc. Am. 92, 2469 (A) (1992)]. They inferred that the degree of interaural cross correlation of late reflections correlated with envelopment. This paper, however, shows the results of psychological experiments that envelopment is affected by the energy ratio of reflections coming from the front of the listener to those coming from the back of the listener, even if the degree of interaural cross correlation of the late reflections are equal. Namely, envelopment grows as the energy of the reflection coming from the back of the listener increases. This result suggests the need to measure the ratio which has never been measured.

10:35

2aAA6. The comparative evaluation of auditoriums-Theaters and concert halls. Paul S. Veneklasen (Paul S. Veneklasen Res. Foundation, 1711 16th St., Santa Monica, CA 90404)

A valiant enterprise is underway to make difficult physical measurements in concert halls and to permit comparisons of these measures among the halls. Early experience is proving most valuable. In this paper full scale measurements will be related to similar information that can be derived in more specific detail from measurements in small models. The data also hold promise of correlation with presentations in auditorium synthesis, which has over the last 25 years furnished design criteria for model studies leading to completed auditoriums. However, the missing link in an invaluable chain of design correlation is the ability to make reliable subjective comparisons and preferential judgments of sound in auditoriums. Suggestions are given for limitations and requirements for the ability to conduct these measurements. 
2aAA7. Spatial information measurements for investigating specular and diffuse sound fields in enclosures. A. Abdou and R. W. Guy (Ctr. for Building Studies (CBS), Concordia Univ., Montreal, PQ H3G 1M8, Canada)

Attempts to predict the sound quality in large rooms can be made at the design stage using scale or computer simulation models but they are usually based on the assumption that the sound field is diffuse. Quality is said to be measured in existing rooms by known room-acoustic indicators but most of them have been developed without regard to direction sensing. Measurements in existing halls show that the sound field is far from diffuse. Specular and diffuse surface reflections not only vary from one room to another but also from seat to seat in the same room. To quantify this phenomena, both spatial and temporal diffuseness has to be investigated. Measuring the directional characteristics of sound in existing halls provides the prospect of quantifying such distribution. This work describes the development of a PC-based system to measure the spatial information of sound fields in large rooms. The system utilizes sound intensity measurement from three microphone pairs arranged in Cartesian coordinates or one pair in three successive orientations to calculate intensity vectors. The sound field can then be visualized on an energy directional basis versus arrival time and hence analyzed in greater detail than hitherto possible. The potential for quantifying spatial specular and diffuse sound fields will be discussed and example results presented. Such results may lead to new acoustic indices with respect to direction dictated quality. [Work supported by NSERC, Canada, Individual Operating Grant OGPIN 013.]

\section{1:15}

2aAA8. Comparisons of $\mathrm{IACC}$ and LF measurements in halls. John S. Bradley (M-27, IRC, Natl. Res. Council, Montreal Rd., Ottawa, ON K1A OR6, Canada)

Lateral energy fractions, LF, and inter-aural cross correlations, IACC, have both been proposed as correlates of spaciousness in concert halls. Although, theoretically, related previous measurements have not shown a strong relationship between the two quantities. New measurement results from 80 locations in 10 different halls indicate varying relationships in each of the 6 octave bands from 125 to $4000 \mathrm{~Hz}$. At medium and higher frequencies both LF and IACC values vary among halls. At low frequencies only $\mathbf{L F}$ values vary significantly among halls. The two quantities are also shown to vary differently with position in halls. Statistical analyses of the data reveal other objective measures related to these two quantities.
2aAA9. Pilot study of simulated spaciousness. John S. Bradley (M-27, IRC, Natl. Res. Council, Ottawa, ON KIA OR6, Canada), G. A. Soulodre (McGill University, Montreal, PQ H3A 1E3, Canada), and N. Popplewell (Univ. of Manitoba, Winnipeg, MN R3T 2N2, Canada)

Spaciousness is said to include the sense that the sound in a concert hall is enveloping, that the apparent source width is increased, and the general feeling of being in a room or space. Strong early lateral reflections are generally accepted as essential for adequate spaciousness. The influence of other factors such as the level and the spectrum of the sound is not so well understood. This paper reports the results of initial subjective evaluations of simulated sound fields with varied early lateral reflections. Using paired comparison tests, judgments of the apparent source width were found to be well correlated with two types of objective measures (LF and IACC). As reported in earlier studies, louder sounds were judged to be more spacious and lower frequencies also led to greater apparent source width. [Work partially supported by the Natural Sciences and Engineering Research Council of Canada.]

\section{1:45}

2aAA10. Spaciousness judgments of binaurally reproduced sound fields. G. A. Soulodre (Graduate Program in Sound Recording, Faculty of Music, McGill Univ., Montreal, PQ H3A 1E3, Canada), John S. Bradley (Natl. Res. Council, Ottawa, ON KIA OR6, Canada), and Dale Stammen (McGill Univ., Montreal, PQ H3A 1E3, Canada)

Spaciousness is known to be a key subjective parameter in determining the acoustical quality of a concert hall. Spaciousness is described as the sense of being enveloped by the sound, or as an increase in the apparent width of the soundstage. Inter-aural cross correlations (IACC) and lateral energy fractions (LF) are two objective measures used to describe the relative spaciousness of concert halls. However, the two measures often do not correlate well with each other. In this study binaurally simulated sound fields were presented to listeners in paired comparison tests. Listeners were asked to identify the more spacious of the two sound fields. Binaural simulations of various North American concert halls were produced by convolving source material with impulse responses measured using a dummy head. The measured LF and IACC values are compared with the judged spaciousness. [Binaural impulse response data were obtained from a measurement tour partially supported by the Concert Hall Research Group.] 


\title{
Session $2 \mathrm{aAO}$
}

\section{Acoustical Oceanography and Underwater Acoustics: Ocean Acoustics in Shallow Water: What are the Issues? III}

\author{
Mohsen Badiey,* Cochair \\ University of Delaware, Graduate College of Marine Studies, Newark, Delaware 19716 \\ *Current: Office of Naval Research, Ocean Acoustics, Code 11250A, 800 North Quincy Street, Arlington, Virginia 22217 \\ Peter H. Rogers, Cochair \\ Georgia Institute of Technology, School of Mechanical Engineering, Atlanta, Georgia 30332
}

\section{Contributed Papers}

8:-00

2aAO1. Benchmark comparisons between an NRL, SCC high-frequency shallow water normal mode code and commonly used codes with more detailed explanations of the method. E. White and Michael F. Werby (Naval Res. Lab., Modeling and Simulation Branch, Code 7181, Stennis Space Center, MS 39529)

In the past, a new normal mode code developed at NRL, Stennis Space Center based on a new expansion method was discussed. The expansion functions are solutions of a related iso-velocity profile. The method is discussed in more detail and compared with old perturbation methods, the Galerkin method, and variational methods. Then benchmark comparisons with other codes are presented.

\section{8:15}

2aAO2. The split-step Padé solution. Michael D. Collins (Naval Res. Lab., Washington, DC 20375)

The parabolic equation (PE) method has been the most efficient range-dependent propagation model in ocean acoustics ever since the split-step Fourier solution was developed [F. D. Tappert, "Numerical solutions of a canonical nonlinear dispersive wave equation," SIAM Rev. 16, 140 (1974)]. Since the asymptotic accuracy of the split-step Fourier solution is restricted to leading order (a serious limitation for some problems), the PE method was extended to higher-order asymptotic accuracy using Padé approximations. Until recently, the higherorder PE has been solved using relatively inefficient finite-difference solutions, and the split-step Fourier solution has remained in widespread use despite the accuracy improvements. To improve efficiency without sacrificing accuracy, we apply Padé approximations to achieve higher-order accuracy in both the numerics and the asymptotics. The split-step Padé solution, which has been implemented for both fluid and elastic media, achieves both higher-order asymptotic accuracy and the efficiency of the split-step Fourier solution. This finite-difference solution is one to three orders of magnitude faster than other finitedifference solutions.

\section{$\mathbf{8 : 3 0}$}

2aAO3. New results for the elastic parabolic equation. Michael D. Collins (Naval Res. Lab., Washington, DC 20375)

New results are presented for the elastic parabolic equation (PE). Galerkin's method is applied to handle solid-solid interfaces efficiently. Rayleigh and Stoneley waves are handled with the elastic PE for the first time. Conversion formulas that are local in range are derived for obtaining quantities such as the horizontal displacement and the shear potential from the dependent variables of the nonstandard formulation of elasticity that is used with the elastic PE. The two-way elastic PE is implemented for problems involving realistic boundary and interface conditions and is applied to solve reverberation problems. The self- starter is generalized to problems involving a source in a solid layer. The energy-conserving $P E$ is generalized to elastic media. The elastic PE is applied to problems involving the propagation of an interface wave up a sloping bottom and the cutoff of modes and coupling into shear-wave beams in the sediment. The latter problem is analogous to an acoustic problem that was solved by Jensen and Kuperman ["Sound propagation in a wedge-shaped ocean with a penetrable bottom," J. Acoust. Soc. Am. 67, 1564-1566 (1980)].

$8: 45$

2aAO4. Spectral formalism of wave propagation in a range-dependent shallow-water waveguide. Dajun Tang (Woods Hole Oceanographic Inst., Woods Hole, MA 02543) and Yue-Ping Guo (MIT, Cambridge, MA 02139)

The study of acoustic wave propagation in a shallow-water environment invariably encounters the problem of range dependency, which usually comes in the forms of rough interfaces and medium inhomogeneity. For a range-independent, i.e., horizontally stratified problem where the wave equation can be solved using separation of variables, the well-established wave-number spectrum formulation has proved to be a powerful technique. When such a spectrum is known, the modal structure and its continuous component will completely determine the wave behavior in the waveguide. More importantly, knowledge of the spectral information can be used in inversion techniques to estimate environment parameters through acoustic probing. Here an approximate spectral formalism is developed for weakly range-dependent problems. More specifically, this approach is suitable for those cases where the change of environment is much slower than a horizontal wavelength. The formulation is based on an asymptotic expansion in which the lowest-order solution will result in the well-known adiabatic solution when the proper poles of the spectrum are evaluated. Since the field is expressed in the form of an approximate wave-number integral, a mode near its transition region where it changes from propagating to nompropagating in the water column can be handled properly. In addition, higher-order solutions can be expressed in a simple recursive manner. Some examples will be discussed to elucidate this approach.

\section{9:00}

2aAO5. Coupled modes in a waveguide with a range-dependent rigid basement. Gregory Gillette (The Johns Hopkins Univ., Appl. Phys. Lab., STA Group, Johns Hopkins Rd., Laurel, MD 20723)

Previous coupled mode approaches to acoustic propagation in ocean waveguides either (1) apply strictly to the case where interfaces other than the (free) ocean surface are range-independent, or else (2) they satisfy the boundary conditions at such interfaces approximately. In this paper coupled mode theory is extended to give a solution that exactly satisfies the boundary condition on a range-varying rigid basement. This is achieved by transforming the coordinate system so that at each range 
the basement coincides with a coordinate surface, and the partial separation of variables is construed with respect to the coordinate that is normal to that surface. Related work by other researchers is discussed, and the numerical implementation of the theory is described. Results for basements exhibiting both upsloping and downsloping regions are presented, and the exchange of radiated power between the modes is investigated for these cases.

\section{$9: 15$}

2aA06. Comparison of two numerical models for acoustic transmission loss in shallow-water waveguides. Steven G. Kargl and Raymond Lim (Coastal Systems Sta., Dahlgren Div., Naval Surface Warfare Ctr., Code 130B, Panama City, FL 32407-7001)

Transmission losses for the propagation of an acoustic wave field due to a point source in shallow water to some point along the watersediment interface are obtained from a fast-field algorithm (SAFARI) and an exact Green's function benchmark [R. Lim, J. Acoust. Soc. Am. 92, 1593-1611 (1992)]. The shallow water environment is modeled as a horizontally stratified medium where the layer of water is bounded above by a semi-infinite layer of air and below by a sediment consisting of a thin transition layer and a semi-infinite subbottom. The sediment layers can be viscous fluids and viscoelastic solids as well as poroelastic materials in the Green's function benchmark. The calculated transmission losses, that are to be discussed, correspond to a water depth of $12 \mathrm{~m}$ and a horizontal range that extends to $1 \mathrm{~km}$. A critical comparison of SAFARI against the exact benchmark suggests that extra care be exercised in the application of fast-field algorithms to shallow water problems, even at long ranges.

9:30

2aA07. On the measurement of environmental parameters with a volumetric array of hydrophones using the ambient acoustic field in an anisotropic, range-independent, shallow-water environment. George B. Smith (Naval Res. Lab., Code 7173, Stennis Space Center, MS 39529-5004), Charles L. Byrne (University of Lowell, Lowell, MA 01854), and Howard A. Chandler (Naval Res. Lab., Stennis Space Center, MS 39529-5004)

In a previous paper, a high-resolution estimator function that matched ambient acoustic field data from a vertical array of hydrophones to trial environments from a normal mode environmental model was used to estimate the parameters of the environment through which the acoustic energy passed. Shallow-water simulations were used to explore the response of the estimator as a function of several environmental parameters. The general conclusion was that the estimator produced unique and correct estimates for these parameters, but that the method probably would not extend, without modification, to volumetric arrays and anisotropic environments. In this work, it is shown that the inclusion of horizontal aperture (tilted line array) destroys the ability of this technique to distinguish the correct environment even when that environment is isotropic. Methods are explored to generalize the technique so that it works in anisotropic environments with volumetric arrays. [This work was supported by the Office of Naval Research, Program Element 61153N, with technical management provided by the Naval Research Laboratory, Stennis Space Center, MS.]

\section{$9: 45$}

2aAO8. Statistics of shipping noise vertical directionality in shallow water. Stephen K. Mitchell (Appl. Res. Labs., Univ. of Texas at Austin, Austin, TX 78712)

Even a cursory examination of ambient noise from a region of moderate or high shipping density shows that one of the most important features of noise in the frequency region from approximately 10 to 1000 $\mathrm{Hz}$ is its variability. A useful statistical summary of a noise time series $n(\theta, t)$, where $n(\theta, t)$ is the beam noise level from a vertical array steered in direction $\theta$ is the sample distribution function $D_{n}(L) . D_{n}(L)$ is computed from data as the fraction of time for which the noise $n(\theta, t)$ is less than or equal to $L$. A practical problem is to use known shipping densities for an area, together with a description of the acoustic propagation environment, to predict the distribution, $D_{n}(L)$. In this paper, an approach is presented for modeling the distribution function of vertical beam noise, and to demonstrate a comparison with experimental data from a shallow-water region. [This work is supported by the Office of Naval Research, Code 23, through Block RL3B.]

\section{0:00}

2aA09. Source localization based on coherent scattering from a rough seafloor in an uncertain underwater environment. George Haralabus, Dimitri Alexandrou, and Loren W. Nolte (Duke Univ., Dept. of Elec. Eng., Box 90291, Durham, NC 27708-0291)

In the present localization scenario, direct propagation from the source to the receiver is not permitted. The received field is comprised only by acoustic waves scattered from an infinitely hard seafloor and it is calculated using the Kirchhoff approximation. The performance of the optimum uncertain field processor is successfully tested in two mismatch situations, where either the location of the receiver array or the dimensions of bottom features are imperfectly known. The degradation of the localization performance due to discrepancies between the actual and the assumed scattering surface is demonstrated. This surface difference is expressed as a nondeterministic microroughness layer characterized by its root-mean-square height and its correlation length. Sensitivity study results are presented as a function of these two parameters. [Work supported by the Office of Naval Research, Code 1125GG, through contract No. ONR-N00014-93-I-0049 and Code 11250A, through contract No. ONR-N00014-91-J-1448.]

\section{$10: 15$}

2aAO10. Shallow-water broadband source localization in range and bearing by matched fields. David $P$. Knobles and Stephen $K$. Mitchell (Appl. Res. Labs., Univ. of Texas at Austin, P.O. Box 8029, Austin, TX 78712)

Data from a moving source received by three horizontally separated bottomed sensors in a shallow-water environment are analyzed using a broadband matched-field approach to source localization. The water depth was approximately $120 \mathrm{~m}$, and the low bottom loss in the area results in low spatial coherence of the broadband field. The modeled acoustic fields are constructed via a ray model with wave-like corrections. Localizations are made in the $x-y$ plane. The time evolution of the matched-field surfaces produced from the data agree well with simulated surfaces. Matched-field surfaces are generated successively in intervals of about $1.6 \mathrm{~s}$ in small search areas with a fine spatial grid to test the possibility of tracking a source. Results of the source tracking will be presented. The analysis also considers the effects of the sea floor and environmental mismatch of sea floor descriptions on localization in the context of spatial coherence. In addition the effect of varying the receiver separation on the ability to make a localization when a source is in the "broadside" region of the array is discussed. [This work is supported by the Office of Naval Research, Code 23, through Block RL3B.]

\section{0:30-10:45 Break}

\section{0:45}

2aA011. Performance stability of high-resolution matched-field processors to array-element location error in a shallow-water environment. Howard A. Chandler, George B. Smith, and C. Feuillade (Naval Res. Lab., Stennis Space Center, MS 39529-5004)

The effects of array-element location errors on the performance of four matched-field processing algorithms (Bartlett, maximum likelihood, sector focusing, and multiple constraint) have been investigated. The SNAP propagation model was used to generate the replica acoustic pressure field for a shallow-water channel with a depth variable soundspeed profile typical of a mid-latitude summer environment. It was also used to simulate a "detected" field due to an acoustic source in the 
presence of both uncorrelated and correlated (modal) noise. These were then correlated using the four algorithms for selected degrees of array deformation error. Results will be presented.

\section{1:00}

2aAO12. Vertical coherence of long-range reverberation in a Pekeris shallow-water waveguide. Ji-Xun Zhou, Xue-Zhen Zhang (School of Mech. Eng., Georgia Inst. of Technol., Atlanta, GA 30332), Ensheng Luo, and Dezhao Wang (Inst. of Acoust., Academia Sinica, Beijing 100080, People's Republic of China)

By using the averaged angular spectrum method for a shallow-water sound field [Zhou, Acta Acust. Sin. 5, 86-99 (1980)], an expression of vertical coherence of long-range reverberation in a Pekeris shallowwater waveguide is derived. The resulting vertical coherence coefficient depends on bottom reflection and bottom scattering at small grazing angles. In this talk, sea-going experiments and laboratory data analyses are presented. The measured vertical cross-correlation coefficient for long-range reverberation is given as a function of relevant parameters such as reverberation time, hydrophone separation, signal frequency. etc. It is found that the measurements agree with theoretical predictions very well. The results are encouraging for further research on the derivation of both small-angle bottom reflection and scattering from longrange reverberation in shallow water. [Work supported by ONR and the IAAS.]

\section{1:15}

2aA013. Adaptive multiuser receiver for shallow-water acoustic telemetry channels. Zoran Zvonar, David Brady (Dept. of Elec. and Comput. Eng., Northeastern Univ., Boston, MA 02115), and Josko A. Catipovic (Woods Hole Oceanographic Inst., Woods Hole, MA 02543)

Recent advances in coherent underwater communications technology are the backbone of an Acoustic Local Area Network (ALAN), which is designed for a real-time underwater telemetry with multiple sensors or unmanned underwater vehicles. In shallow-water areas ALAN is planned to cover $100-1000 \mathrm{~km}^{2}$ with nodes designed to communicate at $10 \mathrm{kbit} / \mathrm{s}$ at ranges of $3-4 \mathrm{~nm}$. The communication protocol is optimized for a shallow-water acoustic environment, minimizing retransmission from finite-energy transceivers and taking into account time-variant link reliability and long propagation time between the nodes. One of the key elements of this protocol is a multiuser receiver capable of simultaneously demodulating signals coming from several asynchronous cochannel modems. Unlike spread-spectrum systems the narrow-band transmission is used. The propagation conditions in shallow water are severe due to the highly dynamic multipath structures and the adaptive multiuser receiver performs joint multiple-access cancellation, equalization, phase tracking, and bit timing recovery. It consists of fractionally spaced feedforward section, coupled digital phaselocked loops, and feedback sections, which utilize previous decisions both from the desired and the interfering user. The adaptive algorithm is the combination of the recursive least-squares algorithm for filter tap weights and a second-order phase update for the carrier recovery loop.
Extensive testing has been carried out to confirm successful operation of the proposed receiver in a variety of propagation conditions using the experimental data provided by the Woods Hole Oceanographic Institution. The results indicate significant performance gain over the conventional receiver at the expense of a modest increase in complexity.

\section{1:30}

2aAO14. Comparisons and sensitivity analyses of numerical predictions of acoustic propagation in shallow-water and surface duct environments. P. Bilazarian, N. Kenig, D. Sutton (General Electric Co., Advanced Technol. Labs., ATL Bldg., Moorestown Corporate Ctr., Moorestown, NJ 08057), and M. Parish (General Electric Co., Moorestown, NJ 08057)

Comparisons of commonly used numerical models of acoustic propagation are performed over a wide frequency band in selected shallowwater environments. The models include range-independent propagation loss codes, such as Colossus II and Generic Sonar model (GSM), as well as range-dependent codes, such as SNAP and a wide-angle split-step parabolic equation (PE) model. For both range-independent and range-dependent shallow-water environments, SNAP model predictions typically provide close approximations to measured propagation loss data. Alternatively, large differences and model biases are generally prevalent in comparisons between measured data and Colossus II or PE predictions. Comparisons between the PE and GSM models are presented for sound propagation in surface duct environments. Results pertinent to the sensitivity of shallow-water propagation loss predictions to the amount of range dependence in environmental quantities, such as sound speed, bathymetry, and bottom-interaction parameters, are provided. Advanced 3-D visualizations are shown that illustrate the sensitivity of the optimum frequency of acoustic propagation in shallow water regions to variations in source-receiver geometries and environmental quantities.

\section{1:45}

2aA015. Simulated annealing and genetic algorithm in shear modulus inversion of shallow-water sediments. Altan Turgut (Dept. of Geosci., Univ. of Tulsa, 600 S. College Ave., Tulsa, OK 74104)

The shear modulus of the seabed is one of the most important geoacoustic parameters for modeling acoustic wave propagation in shallow water. A previously developed inversion method [Yamamoto and Torii, Geophys. J. R. Astron. Soc. 85, 413-431 (1986)] enables seabed shear modulus profiles to be determined by inverting measurements of gravity-wave induced sea-bed motion. It is a linearized inversion method that uses local derivative information to improve a starting model in an iterative fashion. Linearized inversions usually depend strongly on the starting model avoiding extensive exploration of model space and tend to be trapped around local minima. In this paper, two new global optimization methods, namely; simulated annealing and genetic algorithm, are introduced to avoid the limitations of the linearized inversion. Performance of these two methods has been tested by using both synthetic and real data and found to be highly efficient for the inversion of shear modulus profile of the seabed. 


\title{
Session 2aEA
}

\section{Engineering Acoustics: Application of Wave-vector Filters to Flow Noise Reduction}

\author{
Sung H. Ko, Chair \\ Naval Undersea Warfare Center Detachment, New London, Connecticut 06320
}

Chair's Introduction-8:45

Invited Papers

8:50

2aEA1. Approximate evaluation of the spectral density integral for a large planar array of rectangular sensors excited by turbulent flow. William Thompson, Jr. (Dept. of Eng. Sci. \& Mech. and Appl. Res. Lab., Penn State Univ., P.O. Box 30, State College, PA 16804) and Robert E. Montgomery (Naval Research Lab., Orlando, FL 32856-8337)

An approximate numerical procedure has been developed for rapidly evaluating the spectral density integral that predicts the output of a planar array of sensors excited by turbulent boundary layer pressure fluctuations. This procedure is particularly useful in cases where the transfer function factor of the integrand is not a simple function of the wave numbers in the flow and transverse directions. The procedure exploits the facts that the entire integrand is a separable function of these two wave numbers and, for large arrays, the array function factor of the integrand is a rapidly varying function of wave number, characterized by many similar-shaped lobes. In addition, a model for multilayered media is employed to provide the transfer function for boundary conditions that closely correspond to reality. Results generated by this procedure were compared to those from an exact evaluation of the integral that is possible if the transfer function is taken to be constant; there was agreement to within $0.2 \mathrm{~dB}$ or better over a broad frequency interval. Some results for a realistic transfer function are presented, such as the case of an elastomeric layer backed by an elastic plate with the sensors embedded at an arbitrary position within the layer. [Work supported by NAVSEASYSCOM 06UR.]

\section{9:15}

2aEA2. Fundamentals of outer decoupler design for flow noise reduction. Nathan C. Martin (Bolt Beranek and Newman, Inc., 70 Fawcett St., Cambridge, MA 02138)

Turbulent boundary-layer (TBL) wall-pressure fluctuations due to the flow of water over a sonar array produces significant levels of array self-noise. This flow noise background reduces the ability of the array to detect acoustic waves propagating from distant sources. These flow noise levels can be reduced by various methods including hydrophone area averaging and the separation of the array from the flow interface by structures generically referred to as outer decouplers. Examples of outer decouplers (O.D.) include sonar domes and elastomer-covered arrays. The fundamental objective of outer decoupler design is to provide a low pass filter in wave number $k$. Analytic modeling of the structural acoustic behavior of O.D. structures provides a basis for both understariding the wave-number filtering process and designing effective O.D.'s for specific applications. This paper reviews the basic structural acoustics of various $O$.D. classes to illustrate the differences in wave-number filtering performance at various frequencies and the application of these filter characteristics to array flow noise reduction. [Work supported by Office of Naval Research.]

9:40

2aEA3. Wave-vector filtering and flow noise reduction in finite structures. Y. F. Hwang (David Taylor Model Basin, Carderock Div., Naval Surface Warfare Ctr., Bethesda, MD 20084-5000)

It has been shown in the literature that the unwanted noise voltage output from a pressure sensing system due to the pressure fluctuations of a turbulent boundary layer can be minimized by exploiting the wave-number filtering action of finite hydrophones and hydrophone arrays, or by other wave-number filtering techniques. This paper discusses the similar effect in a finite structural panel subjected to the same excitation. However, in this case, the corresponding output is the effective modal force per unit area that excites the structural mode. The wave-number filtering characteristics of structural modes as a function of flow parameters, structural dimensions, mode order numbers, and boundary conditions are presented. The effective modal force per unit area is evaluated as the sum of contributions from three wave-number regions: the low-wave-number region, at and near the modal wave-number (within the major lobe) of a structural mode; the high-wave-number region, at and near the hydrodynamic coincident wave-number; and the intermediate wave-number region that lies between the above two regions. The relative contributions of the three wave-number regions are important criteria upon which further noise reduction strategies are devised. [Work supported by ONR.] 
2aEA4. Effect of various array structures on flow noise reduction. Sung H. Ko and Howard H. Schloemer (Naval Undersea Warfare Ctr. Detachment, New London, CT 06320)

Turbulent boundary layer pressure fluctuations can be reduced by filtering of its wave-number response with either a finite hydrophone or a hydrophone array, or by filtering the wave-number response through a layer of elastomer. In general practice, hydrophone arrays are embedded within a layer of elastomer to reduce the turbulent boundary layer pressure fluctuations and then mounted to an elastic structure. To understand the role of the mounting structure, this work will examine the performance of idealized and realistic array mounting configurations. Three configurations are modeled in this paper. The first one is a plane elastomer layer backed by a rigid surface. The second one is backed by an elastic plate with a finite thickness. Finally, the third one is backed by a layer of water. The front side of the elastomer layer is exposed to turbulent flow. The transfer functions for the three different configurations will be discussed. The results presented here are the numerically calculated noise reductions relative to the calculated noise level for a flush-mounted point hydrophone. In addition, an equivalent-plane-wave flow noise level is derived and compared among the three configurations to directly show any array performance similarities or differences.

\section{Contributed Papers}

\section{0:30}

2aEA5. Interaction of TBL-induced flexural waves, element spacing, and steering on the self-noise of hydrophone arrays. Paul $W$. Jameson and Richard N. Brown (BBN, 70 Fawcett St., Cambridge, MA 02138)

Most ship-mounted planar hydrophone array systems employ stiff structures for isolation from local noise. One type of structure is a plate ("window"), which isolates the hydrophones from high wave-number turbulence on the outer surface. Most arrays also use a heavy steel plate behind the array to achieve isolation from the inner surface. These plates support bending waves, which can be induced by TBL forces. Even though these waves are subsonic over the frequency region of interest, the noise stress can enter the array output through an aliasing lobe when the array is steered. This noise increase is frequency dependent, and increases with frequency after a critical value. This talk will indicate how this noise contaminates the array response and discuss the interplay of element spacing, steering, and frequency performance of the array.

\section{0:45}

2aEA6. Determination of electrical drives for a cylindrical wave-number calibration array. L. D. Luker (Naval Res. Lab., Underwater Sound Reference Detachment, P.O. Box 568337, Orlando, FL 32856-8337)

The "flow noise" produced by a turbulent boundary layer is generally characterized by pressure distributions that are transported along the surface at speeds much less than the sound speed in water and therefore have spatial wave numbers that are larger than the acoustic wave number. This paper describes several methods for computing the electrical drives for a prototype cylindrical wave-number calibrator (CWC) designed to generate a pressure field consisting of essentially a single nonacoustic wave number inside the free-flooded calibrator. This geometry is suitable for the evaluation of line acoustic sensors such as in towed sonar arrays. The prototype CWC consists of a cylindrical tube of PVDF with an electrode pattern along the tube length that allows the PVDF to be operated as 40 independent bands each of which can be driven at a specified amplitude and phase. The required drive voltages are calculated using the electroacoustic transfer matrix of the CWC at the desired drive frequency. This transfer matrix can be determined by actual measurements or by using a computer model of the CWC. Drives were computed using transfer matrices that were determined with several modeling methods including the acoustic-radiation program CHIEF and the finite-element program ATILA. Measured pressure fields inside the CWC are compared with desired and predicted fields at selected spatial wave numbers for drive frequencies of 800 and $1600 \mathrm{~Hz}$. These results are also compared with previous results produced using measured transfer matrices. [Work jointly supported by ONR and ONT.]

\section{1:00}

2aEA7. A planar eight-element flexural disk fiber optic acoustic sensor. D. A. Brown, G. B. Netzorg, B. White, D. Kapolka, ${ }^{\text {) }}$ David L. Gardner, and Steven L. Garrett (Phys. Dept., Naval Postgraduate School, Monterey, CA 93943)

Interferometric fiber optic flexural disk hydrophones made of aluminum, castable epoxy, and composite materials have previously been constructed and reported (D. Kapolka et al., J. Acoust. Soc. Am. 91, 2325(A) (1992)]. These hydrophones consist of a closed cylinder with fiber coils bonded to the sides of the two end caps in a push-pull fashion in order to increase acoustic sensitivity and allow for cancelation of acceleration induced signals. It is possible to sum in series the phase modulations induced in multiple transducers (e.g., flexural disks) using the two legs of the interferometer. This will enhance the spatial averaging capability of the sensor due to its increased size. The sensor selfnoise arising from flow-induced pressure fluctuations can then be reduced resulting in improved detectability. The fabrication and performance of a sensor comprising 8 flexural disks and 16 coils of optical fiber will be discussed. [Work supported by the Naval Sea Systems Command.] a ${ }^{\text {) }}$ T., USN.

\section{1:15}

2aEA8. Concerning the "displacement thickness theory" of boundary layer noise. Alan Powell (Dept. of Mech. Eng., Univ. of Houston, Houston, TX 77204-4792)

The streamlines just outside a boundary layer are pushed away from the wall by the displacement thickness $\delta^{*}$ as the flow slows down. Hydrodynamic fluctuations in $\delta^{*}$ have been considered to act as a compact monopole source distribution at the edge of the boundary layer, see Laufer et al. (1964), Lauchle (1981. 1989), Sornette and Lagier (1984, 1986). However, Rayleigh's (normal velocity) formula applied to a plane just outside the layer reduces to the $\delta^{*}$ theory only if the acoustic (sound-speed-dependent) contribution of the fluctuations there, due to the sound sources that must be within the layer, can be safely ignored. For a monopole source the $\delta^{*}$ theory yields the correct result, although the net strength must be zero (because of continuity), except for viscous dissipation effects. It also yields the correct results for tangential dipoles, pertinent to net wall shear stresses, and for tangential longitudinal quadrupoles. But the $\delta^{*}$ theory cannot represent normal longitudinal quadrupoles, an essential complementary component of plane boundary layer noise, Powell (1991): the extensive (noncompact) acoustic field contribution to the plane source distribution is then essential. 
2aEA9. Aerodynamic noise theory-An alternative monopole source term. Alan Powell (Dept. of Mech. Eng., Univ. of Houston, Houston, TX 77204-4792)

Lighthill's equation for the acoustic pressure perturbation may expressed as $\square^{2} p=\rho_{0}\left(\frac{1}{2} \xi^{2}-e_{i i}^{2}\right)$, where $\rho_{0}$ is the density of the incompressible inviscid noise generating flow, $\zeta$ is its vorticity, and $e_{i i}$ is the principal strain rates. In the hydrodynamic field $\left(\nabla^{2}\right.$ vice $\left.\square^{2}\right)$, pressure minima and maxima evidently always tend to occur in regions of vorticity squared and strain rates squared, repectively. For the far field, it is proved that the volume integral over the source region of the two monopole terms cancel exactly: the net monopole strength is always zero. Here the source region is not extensive as for the existing "simple source" theory. This is readily demonstrated in the limiting case, a steady vortex. This supports the gist of Ribner's 1964 intuitive sketch of turbulent flow eddies having central pressure minima with the maxima in the regions of collision: the notion of the dilatation in the center of a single vortex by itself being the source (Legendre, 1992) is evidently incomplete. Quadrupole radiation may be studied, in principle at least, by taking the second moment of the source strength, or by considering the acceleration of the source field explicitly, say, à la Lowson, 1965.
2aEA10. Improved integration methods for poles in transfer functions. Daniel Zwillinger and Paul W. Jameson (BBN, 70 Fawcett St., Cambridge, MA 02138)

When analyzing stress transmission in layered media as a function of in-plane wave number, the transfer function may exhibit pole-like behavior. These poles are associated with trace matching to bending waves in high stiffness layers. However, the exact interrelationship of multiple poles for the case of multiple various layers is not well understood. This condition presents difficulties to routines that integrate over the wave number. Hence, most users employ adaptive integration schemes that compensate for the poles by increasing the number of points at which the transfer function is evaluated. An improved approach, which involves singularity isolation, is being used in software being developed at BBN. This approach is to describe the pole in an analytical form and perform the integral analytically in the region in which the pole occurs. In this presentation, how to construct the analytic function for a single layer is illustrated, and the generalization to multiple layers is described. This approach leads to a reduction in computer processing time.

TUESDAY MORNING, 18 MAY 1993

QUEBEC SUITE, 9:00 A.M. TO 12:00 NOON

\title{
Session 2aNS
}

\section{Noise and Architectural Acoustics: Wavelets II: Emphasis on Perceptual and Biological Signal Processing}

\author{
John R. Bareham, Chair \\ Consultant, 20 Rogers Avenue, Marlborough, Massachusetts 01752
}

Chair's Introduction-9:00

Invited Papers

9.05

2aNS1. Application of wavelet transform to the perception of reproduced sound. Peter L. Schuck (Acoust. and Signal Processing, Inst. for Microstructural Sci., Natl. Res. Council, Ottawa, ON K1A OR6, Canada)

Various time frequency distributions including spectrograms, Wigner distributions and smoothings thereof, and more recently scalograms, have been used to analyze speech. Very little work has been presented on attempts to use these methods to study sound reproduction, that is, loudspeaker/listening room systems. The loudspeaker/room system alters the reproduced sound mainly through resonances, delayed resonances, and reflections. The wavelet transform offers another potential avenue to visualize loudspeaker/room interaction with the highly desirable property that it is "constant $Q$ " or constant percentage bandwidth as opposed to the other transforms that are constant bandwidth. Visual displays of the scalogram or squared modulus of the continuous wavelet transform are presented of resonances, delayed resonances, and echoes at, above, and below the threshold of audibility. In this way, the viability of the scalogram as a tool for the analysis of loudspeaker/room interactions is explored.

\subsection{5}

2aNS2. Acoustical applications of wavelets: Audio coding and sonar. Ahmed H. Tewfik (Dept. of Elec. Eng., Univ. of Minnesota, Minneapolis, MN 55455)

Wavelets are a family of basis functions for the space of finite energy signals. In a wavelet decomposition, a signal is expanded in terms of translates and dilates of a single function. Wavelets provide a flexible decomposition of signals. This flexibility is the foundation of a new generation of advanced low-bit-rate, high-quality audio coding procedures. By adaptively choosing the wavelet representations of consecutive segments of the audio signal one can optimally exploit the masking effect in human hearing. The performance of the resulting coding techniques surpasses that of known nonwavelet-based approaches. Another important property of wavelet decompositions is the fact that one can relate the structure of the wavelet coefficients of a given signal to the local time and frequency domain characteristics of the signal. In sonar processing, this fact has been exploited by a new class of robust direction of arrival estimation algorithms that can deal with background correlated noise of an unknown correlation structure. These algorithms separate signal from noise by analyzing the structure of the wavelet coefficients of the array outputs. 
2aNS3. Wavelet-like transforms in the auditory system of the bat. Tim Haresign, James A. Simmons, and Michael Ferragamo (Hunter Lab., Brown Univ., Providence, RI 02912)

Traditional views of neural processing of auditory signals in the nervous system have been shaped by conceptual models based on Fourier analysis-in the frequency domain for spectral selectivity or in the time domain for periodicity analysis. However, in recent years wavelet analysis has been proven to be a powerful new technique for signal processing. Evidence has been found for a wavelet-like representation of ultrasonic auditory signals in the inferior colliculus of the big brown bat, Eptesicus fuscus. The inferior colliculus is a laminar structure, with the neurons in each layer being sensitive to a specific, narrow frequency band. In response to a broadband stimulus the averaged activity of small groups of neurons exhibits a structured temporal pattern in its response. The temporal response pattern is dependent on the spectral-temporal structure of the input sound, and varies as a function of the frequency lamina from which the measurements are taken. Based on these results, it seems likely that information about ultrasonic signals is being segregated according to frequency and then transformed onto a different time scale within each frequency lamina as a wavelet-like burst, so that the time and phase relations within ultrasonic signals can be displayed by time and phase relations on a corresponding expanded physiological scale. [Work supported by ONR Grant No. N00014-89-J-3055, NIMH Grant Nos. MH-00521 and MH-19118, and NIH Grant No. DC-00511.]

\section{0:35-10:45 Break}

\section{Contributed Papers}

\section{0:45}

2aNS4. Improvement of the spatial Hankel transform method to determine the impedance of outdoor ground surfaces. C. Verhaegen, W. Lauriks, and A. Cops (Akoestiek en Warmtegeleiding, K. U. Leuven Celestijnenlaan 200 D, 3001 Leuven, Belgium)

The method of measuring the impedance of a ground surface based on the spatial Hankel transform, can be divided into three parts. First the pressure field is sampled on two horizontal lines near to the surface, from beneath a cylindrical symmetric sound source till infinity. Next a Hankel transform is performed on the data of each horizontal line. Finally the impedance of the surface for an incident angle ranging from 0 to $90 \mathrm{deg}$, is calculated. In practice, due to the finite maximum, measuring distance oscillations occur in the impedance curve as a function of angle of incidence. This effect can be avoided by using a spatial window before transforming the data. A Kaiser-Bessel window is selected to give the best results. A side effect of using a window is a shift from the real impedance values. Further improvement can be obtained by calculating more data points for a horizontal value larger than the maximum measuring distance. The method and its improvements will be illustrated with measurements on outdoor ground surfaces.

\section{1:00}

2aNS5. Comparison of STFT, Gabor, and wavelet transforms in transient vibration analysis of mechanical systems. Taner Önsay and Alan G. Haddow (Dept. of Mech. Eng., Michigan State Univ., East Lansing, MI 48824)

Short-time Fourier (STFT), Gabor, and wavelet transforms are applied to the analysis of vibration signals obtained from mechanical systems. Nonstationary vibration signals resulting from impacts, friction, and transient dynamic interactions are studied by employing the transforms to demonstrate the time-evolution of their spectra. In the application of the STFT, the time-window length and the overlap in window translation are varied and their effect on the resolution of the timefrequency information is discussed. Due to its optimal time-frequency localization characteristics, the Gaussian window used in Gabor transform is found to be particularly useful in the analysis of time-decaying sinusoidal signals, whereas for vibration signals having sharp variations the logarithmic zooming properties of wavelets result in better localization in the time-frequency domain. The use of different analyzing wavelets are considered for detection of transient vibrations and characterization of the associated dynamic behaviors. Based on applications and examples of synthetic signals, the advantages and the limitations of each transform technique are discussed and their conceptual similarities are emphasized.

\section{1:15}

2aNS6. Wavelet analysis of rhythm. Neil P. McAngus Todd (Dept. of Music, City Univ., Northampton Square, London EC1V OHB, England)

The application of wavelet analysis to rhythm is described. Rather than carry out the multiscale decomposition on the sound signal itself this method decomposes the sound energy flux [N. P. McAngus Todd, J. Acoust. Soc. Am. 92 (A), 2380 (1992)], This enables the analysis of rhythmic phenomena that have frequencies several orders of magnitude below pitch and are not represented in the sound signal. The decomposition is carried out by a logarithmically spaced Laguerre series approximation Gaussian filter bank. The analog design was discretized to a cascaded IIR structure using a bilinear transformation. This has the following advantages over the usual FIR approach: (a) plausibility of interpretation as a perceptual model since the ideal Gaussian is not physically realizable; (b) a smaller number of parameters; (c) smaller delay times than the Gaussian ideal; (d) avoidance of the need for down/up sampling. Two complementary structural components are obtained from the projection in the frequency-time plane of loci of zero crossings in the rate of change of energy: (a) segmentation structure, corresponding to positive second derivatives; (b) a stress structure, corresponding to negative second derivatives. A compact tree coding of rhythm is possible from points of convergence of the loci of zero crossings.

11:30-12;00

PANEL DISCUSSION

Panel Moderator: David Lubman 


\title{
Session 2aPA
}

\section{Physical Acoustics and Structural Acoustics and Vibration: Anderson Localization in Acoustics and Structural Vibration I}

\author{
Richard L. Weaver, Chair \\ Department of Theoretical and Applied Mechanics, University of Illinois, \\ Urbana, Illinois 61801
}

Chair's Introduction-8:25

Invited Papers

8:30

2aPA1. Localization and wave conversion phenomena in multi-coupled nearly periodic structural systems. Christophe Pierre, Matthew P. Castanier, and Wan J. Chen (3104 G. G. Brown, Dept. of Mech. Eng. and Appl. Mech., Univ. of Michigan, Ann Arbor, MI 48109-2125)

For disordered mono-coupled systems, wave localization causes an exponential spatial wave amplitude decay, with the associated decay constant known as the localization factor. In multi-coupled structural systems, with multiple wave types, wave conversions complicate the analysis of localization phenomena. In this work, Lyapunov exponents of the system wave transfer matrix are used to analyze localization in multi-coupled disordered systems. Lyapunov exponents are a measure of the exponential growth rate of wave vectors. In a mono-coupled system, the largest Lyapunov exponent is equivalent to the localization factor. Lyapunov exponents are calculated numerically and, for the case of weak disorder, approximated by perturbation techniques. Three examples are presented. First, a representative mono-coupled system is considered. The largest Lyapunov exponent is calculated and compared with localization factors found by Monte Carlo as well as perturbation methods. Then bi-coupled system, a multi-span beam resting on flexible supports, is examined. Lyapunov exponents are calculated and compared with the wave amplitude decay found in a single realization of the disordered system. Finally, a truss beam, which features four types of waves is examined, and the physical significance of the Lyapunov exponents is discussed.

9:00

2aPA2. Experimental observation of bending wave localization. George Cody, Ling Ye, Minyao Zhou, Ping Sheng (Exxon Res. \& Eng. Co., Rte. 22 East, Annadale, NJ 08801), and Andrew N. Norris (Rutgers University, Piscataway, NJ 08855)

Localization of bending waves has been observed for the first time for two-dimensional (2D) acoustic wave propagation in an inhomogeneous composite system consisting of a steel plate decorated with two sets of randomly attached Lucite blocks. A significant experimental feature of the localized modes is an exponentially decay of the mode intensity from their peaked centers, with a decay length that increases as $\left(f_{0}-f\right)^{-1}$ when the mode frequency $f$ approaches a quasimobility edge $f_{0}$. The minimum attenuation length is of the order of a block diagonal and is about $40 \%$ of the banding wave's wavelength. The experimental data, as well as results of finite-element calculations, identify the source of the localization phenomenon as strong scattering of the bending wave by shear and flexural resonances of the Lucite blocks. This result supports theoretical predictions that resonant scattering enhances localization [cf. Scattering and Localization of Classical Waves in Random Media, edited by P. Sheng (World Scientific, Singapore, 1990)]. Recent experiments on acoustic wave propagation in rough composite steel/refractory walls also exhibit exponential localization at frequencies corresponding to 3-D propagation. The experimental data suggest that a composite plate is a unique vehicle for the study of classical localization: at low frequencies, in 2-D and, at higher frequencies, in 3-D. The generic nature of the localization phenomenon suggests its application as a tunable attenuation mechanism for bending waves

\section{9:30}

2aPA3. Dynamic finite-element calculation of excitation modes in periodic and disordered Lucite-steel composite systems. Ping Sheng and Minyao Zhou (Exxon Res. and Eng. Co., 79 Rte. 22 East, Annadale, NJ 08801-0998)

Recent experiment by L. Ye et al. on Lucite-steel composite system [Phys. Rev. Lett. 69, 3080 (1992)] has demonstrated the localization of bending waves. This is particularly surprising on first sight because the huge acoustic impedance mismatch between Lucite and steel would seem to imply that Lucite should have very little effect on the steel. A dynamic finite-element approach was used to calculate the excitation modes in a 2-D periodic structure consisting of a steel plate docorated by a checkerboard pattern of Lucite blocks. The results show that the coupling between the steel plate bending wave and the flexural resonances of the Lucite blocks can drastically alter the dispersion relation of the bending wave, whereas the effect of periodicity is minimal Since the Lucite flexural resonance frequencies depend on the Lucite block height, its effect on the bending wave can be tuned in terms of the frequency as demonstrated experimentally by Ye et al. This talk will also report the results of calculations on a random 1-D Lucite-steel system. In particular, the localization characteristics will be emphasized. 
2aPA4. Fluid loading of irregular structures. David G. Crighton (Dept. of Appl. Math. \& Theor. Phys., Univ. of Cambridge. Cambridge CB3 9EW, United Kingdom)

An analytically and computationally tractable model for the response of complex fluid-loaded structures is that of a plane elastic plate or membrane subject to static fluid loading and single-frequency localized mechanical drive, and reinforced by a number of parallel "ribs" in regular, partially ordered, or random configurations. Coupling along the structure is provided by subsonic surface waves (equivalent to nearest-neighbor mechanical coupling) and by long-range acoustic/hydrodynamic fields. A variety of analytical and computational studies carried out by the Cambridge group will be presented, showing large effects of an isolated irregularity, Anderson localization in extensively irregular configurations under surface wave coupling, and delocalization (short circuiting) by weak long-range fields. [Work supported by ONR, Code 1132SM.]

\section{Contributed Papers}

\section{0:45}

2aPA5. Effects of rib complexity on plane wave localization phenomena. Douglas M. Photiadis (Naval Res. Lab., Code 7131, Washington, DC 20375-5000)

Spatial irregularity of an array of line masses has been previously predicted to give rise to Anderson localization of plane waves on a fluid-loaded plate. Here, the effects of additional rib complexity: nonvanishing moment of inertia, the ability to support vibrational waves along the rib, and the influence of some simple models of internal structure, are considered. It is shown that this system is nearly equivalent to a nearest-neighbor coupled line array where each site is now represented by two degrees of freedom, the effective force and moment exerted by each scatterer. In the absence of fluid-loading this immediately implies that any degree of irregularity produces Anderson localization. Simulation results illustrating typical localization effects in the presence of fluid-loading will be presented. Generally, more accurate modeling of the rib impedance is found to increase the rib scattering strength and hence increase localization phenomena.

\section{1:00}

2aPA6. The effect of periodically attached substructures on the excitation of submerged eylindrical shells. S.-H. Choi, J. D. Achenbach, and T. Igusa (Dept. of Civil Eng., Northwestern Univ., Evanston, IL 60208)

The presence of substructures in a cylindrical shell gives rise to dynamic interactions that influence the axial distribution of vibration along the shell. In this paper, Lagrange's equations are used to develop the equations governing the forced harmonic vibration of a submerged cylindrical shell with periodically attached internal substructures that is subjected to periodically placed loads. Modal expansions are used to describe the response of the shell and the fields in the fluid. First a shell without substructures but subjected to periodically placed ring loads is considered. The method of this paper is found to be nearly as accurate as a traveling-wave-based method for an infinite shell subjected to a single ring load. Two types of substructures are examined: circular panels with hinge connections and circular ribs with rigid connections. It is shown that the substructures confine the flexural motion to a region near the location of the applied forces. The localization effect decreases when the forcing frequency is close to the substrate's natural frequencies, and becomes more pronounced when within a periodic segment the ribs have a varying impedance. [Work supported by ONR.]

\section{1:15}

2aPA7. Band-edge localization and spatial textures of surface acoustic waves in weakly disordered 1-D superlattices. Jean-Pierre Desideri and Didier Somette (Lab. de Phys. de la Matière Condensée, CNRS URA 190, Faculté des Sciences, Parc Valrose, B.P. 70, 06108 Nice Cedex 02, France)

The extreme sensitivity of wave propagation on the presence of disorder near remnant geometric Bragg resonance (band edges) is exhibited experimentally for the first time using surface acoustic waves on very weakly corrugated solids. Anderson localization is identified by measuring the spatial structure of proper modes with an optical diffraction experiment and is found to occur most strongly precisely at the two borders of a remnant stop band. Exponentially decaying localized modes are decorated by a speckle-like wave pattern, which constitutes an observable fingerprint of the random systern. The amplitude of this spatial wave structure is found to exhibit a marked maximum at band edges thus providing a novel signature of localization, which used to probe localization in the interior of the remnant stop bands. 


\title{
Psychological and Physiological Acoustics: Psychological and Physiological Collaborations in Hearing I
}

\author{
William A. Yost, Chair \\ Parmly Hearing Institute, Loyola University Chicago, 6525 North Sheridan Road, Chicago, Illinois 60626
}

Chair's Introduction-8:00

Invited Papers

8:05

\begin{abstract}
2aPP1. Physiological correlates of the precedence effect: Implications for neural models. Tom C. T. Yin and Ruth Y. Litovsky (Dept. of Neurophysiol., Univ. of Wisconsin, Madison, WI 53706)

The precedence effect is an illusion whereby two similar sounds, delivered from different locations and in quick succession, are perceived as a single auditory event whose apparent location is dominated by the leading sound. Physiological correlates of the precedence effect have been looked for in the responses of single cells in the inferior colliculus (IC) of anesthetized cats. Studies were conducted in both dichotic and free-field conditions by simulating click pairs with variable interclick delays. Almost all cells show a form of echo suppression, whereby the response to a lagging click is suppressed to half-maximal value for interclick delays of 3-150 ms (mode of $29 \mathrm{~ms}$ ). This result dictates an important role for inhibition in the binaural circuits of the brainstem. It may also provide a resolution for the paradox in which Colburn's models of the medial superior olive (MSO) need no inhibition yet there is strong anatomical evidence for the presence of inhibitory synapses in both MSO and IC. [Work supported by NIH DCOO116 and NRSA DC00078.]
\end{abstract}

\section{8:40}

2aPP2. Modeling of precedence-effect behavior in single neurons and in human listeners. $H$. Steven Colburn and Hany Ibrahim (Dept. of Biomed. Eng., Boston Univ., 44 Cummington St., Boston, MA 02215)

Many of the physiological observations of Yin (reported in this session) are consistent with the precedence effect observed in human psychophysical data. In an attempt to understand the mechanisms involved in the generation of these observations, models for the observed neural interactions are being investigated. Since precedence-effect phenomena are fundamentally related to transient parts of stimuli, the models of these phenomena incorporate descriptions of responses to transient stimuli. In contrast to models for the observed binaural interaction in the medial superior olive (MSO) for sinusoidal stimuli, models for responses to click stimuli in the inferior colliculus (IC) must include inhibitory effects. These effects are long-lasting (tens of milliseconds) and asymmetric, consistent with earlier data from the IC with binaural clicks [L. H. Carney and T. C. T. Yin, J. Neurophys. 62, 144-161 (1989)]. The location of this inhibitory interaction is not clear since physiological measurements in lower levels of the brainstem (e.g., MSO) have not included the appropriate transient stimuli. The physiological responses from the IC will also be discussed in the context of recent psychophysical observations which suggest that the precedence effect may be a very central phenomenon. [Work supported by NIDCD.]

\section{9:15}

2aPP3. What determines the sound of a sinusoid? Roy D. Patterson (MRC Appl. Psychol. Unit, 15 Chaucer Rd., Cambridge CB2 2EF, United Kingdom)

"Damped sounds," constructed by repeating a short segment of a sinusoid with an exponential decay, were compared with "ramped sounds," constructed by reversing each damped sound in time. As the half-life of the decay function decreases through values less than the repetition period, the continuity of the sinusoid is disrupted and its distinctive sound quality fades away. However, these changes occur far sooner for the damped sound. Damped/ramped pairs with the same carrier frequency and repetition rate have identical power spectra, so simple spectral models predict no perceptual difference for these sounds. Excitation patterns generated with filterbanks and leaky integrators show that damped sounds have sharper spectral peaks than corresponding ramped sounds, so auditory models with simple leaky integrators predict the reverse of what is heard. A new model that employs strobed temporal integration to stabilize neural activity patterns suggests that ramped stimuli sound more sinusoidal because they produce more time intervals near the carrier period, although not in the carrier channel, and the perception is more continuous because the strobe threshold function is asymmetric in time.

\section{9:50-10:05 Break}

10:05

2aPP4. Neurophysiological correlates of the pitch of complex tones. Bertrand Delgutte (Eaton-Peabody Lab., Mass. Eye \& Ear Infirm., Boston, MA 02114, Res. Lab. of Electron., MIT, and Harvard-MIT Div. of Health Sci. \& Technol.), Peter A. Cariani (Mass. Eye \& Ear Infirm., Boston, MA, and Harvard-MIT Div. of Health Sci. \& Technol.), and Mark J. Tramo (Mass. Eye \& Ear Infirm., Boston, MA, and Harvard Med. School)

The pitch of complex tones plays an important role not only in speech and music, but also for perceptual segregation of sound sources. In order to study physiological correlates of these phenomena, autocorrelation histograms were recorded and computed 
from auditory-nerve fibers in anesthetized cats to estimate the aggregate interspike interval distribution for the entire auditory nerve. Results for many types of complex stimuli (including those used in classical pitch shift and pitch dominance experiments) suggest that the perceived pitch corresponds to the most frequent interspike interval in the auditory nerve. Also investigated was how interspike interval information might be used to separate simultaneously presented vowels. Psychophysical experiments show that both constituents in a pair of concurrent vowels are better identified when they have slight different pitches. These results show that concurrent vowels can usually be segregated from the information in the aggregate interspike interval distribution, and that peformance improves when the vowels have different pitches even though the method used does not rely on explicit pitch identification. [Work supported by NIH Grant Nos. DCO0119 and DCO0038.]

10:40

2aPP5. Detecting changes in spectral shape of complex auditory signals: Profile analysis. David M. Green (Psychoacoust. Lab., Psychol. Dept., Univ. of Florida, Gainesville, FL 32611-2065)

This is a review of current theories and recent experimental data on how human listeners detect changes in the spectrum of a complex sound. The effects of parameters such as the number of components in the complex, their density, bandwidth, and whether the components are harmonic or equally spaced in logarithmic frequency will be described. Some new data on how signal duration affects the ability to detect spectral change will also be reviewed. It is believed that three distinct sets of cues mediate the detection of changes in the spectral shape depending on the total bandwidth of the complex. For wide bandwidths, the primary cue is a simultaneous comparison of level over different frequency regions. For spectra that are one or two critical bands in width, the primary cue is a change in pitch. For spectra less than one critical band, the primary cue is a change in the temporal fluctuation or roughness of the sound related to the power spectrum of the envelope.

11:15

2aPP6. Nonlinearity of spectra processing in the dorsal cochlear nucleus (DCN). Eric D. Young, Israel Nelken (Johns Hopkins Univ., 720 Rutland Ave., Baltimore, MD 21205), and George A. Spirou (West Virginia Univ., Morgantown, WV 26506)

Most studies of the neural representation of complex stimuli assume a parallel filterband form of representation in which neural response is plotted against position on the basilar membrane (or best frequency, BF). Such a representation assumes that the neural response reflects the energy in the signal within the neuron's tuning curve and makes most sense if neural tuning properties are linear or only weakly nonlinear. By contrast, the principal cells of the DCN integrate energy across their response areas in a highly nonlinear fashion. These neurons give predominantly inhibitory responses to narrow-band stimuli and excitatory responses to broadband stimuli. Their responses to broadband stimuli cannot be predicted by a filtering model, in which the response equals the sum of narrow-band responses, weighted by the stimulus spectrum. Similarly, a narrow notch in a noise band centered on a unit's BF also gives an inhibitory response. This inhibitory response also cannot be predicted from narrow-band responses and may represent a specialization for detecting spectral notches produced by the directional filtering properties of the external ear. [Work supported by NIH, Keck Foundation, and Fulbright Foundation.]

TUESDAY MORNING, 18 MAY 1993

CANADIAN ROOM, 9:00 A.M. TO 12:00 NOON

\title{
Session 2aSP
}

\author{
Speech Communication: Speech Production, Cross-Language Research, and Synthesis (Poster Session) \\ Carol Y. Espy-Wilson, Chair \\ Electrical, Computer, and Systems Engineering Department, Boston University, 44 Cummington Street, Boston, \\ Massachusetts 02215
}

\section{Contributed Papers}

\begin{abstract}
All posters will be on display from 9:00 a.m. to 12:00 noon. To allow contributors an opportunity to see other posters, contributors of odd-numbered papers will be at their posters from 9:00 to 10:30 a.m. and contributors of even-numbered papers will be at their posters from 10:30 a.m. to 12:00 noon.
\end{abstract}

2aSP1. An MRI atudy of the physiological mechanism of FO lowering. Hiroyuki Hirai, Kiyoshi Honda (ATR Auditory and Visual Perception Res. Labs., 2-2 Hikaridai, Seikacho, Soraku-gun, Kyoto, 619-02 Japan), Ichiro Fujimoto, and Yasuhiro Shimada (Takanohara Central Hospital, 1-3-3 Ukyo, Nara City, 631 Japan)

The phenomena of $F 0$ lowering in utterances, widely observed in a word accent or near a phrase boundary, is not sufficiently explained by the relaxation of $F 0$ raising musculature, and the physiological mecha- nism for $F 0$ lowering has been a question to be examined. Morphological observation of laryngeal framework was performed in this study using the magnetic resonance imaging (MRI) during phonation. Midsagittal images near the larynx were taken in different $F 0$ levels in four male speakers. Relative positional changes among laryngeal components were measured to explore the chain of mechanisms that allows vocal fold shortening in low $F O$. In the results, vertical movements of the larynx were consistently observed to be correlated with $F 0$ levels. 
Movements of the hyoid bone and changes in laryngeal cavity size were also found. A rotation of the cricoid cartilage was always associated with laryngeal descent towards lower $F 0$. This rotation occurred to the direction of shortening the vocal folds, and its mechanism was confirmed by vertical sliding motion of the posterior plate of the cricoid cartilage along the physiological curvature of the cervical vertebrae. This mechanism can account for the well-known tendencies of laryngeal descent and strap muscle activity in $F 0$ lowering.

2aSP2. Nonlinearities in articulatory/acoustic relations and the evolution of the human vocal tract. $K$. Bretonnel Cohen (Dept. of Linguistics, Ohio State Univ., 1712 Neil Ave., Columbus, OH 43210)

The evolution of the modern human vocal tract is characterized by a progressive increase in the degree of flexion of the base of the skull. The acoustic consequences of this basicranial flexion were analyzed using a transmission line analog model and calculating variations in vocal tract transfer functions as the trailing edge of a constriction is moved anteriorly. The behavior of an unflexed tract was modeled by trading back and front cavity lengths. That of the modern human vocal tract was modeled similarly, except that after the distance of the trailing edge of the constriction tube from the larynx increased past the superior end of the pharynx, the cross-sectional area of the back cavity was increased (instead of its length) as front-cavity length decreased. The modern human vocal tract had a larger area of stability for the second formant frequency for [i]-like constrictions than that produced by the unflexed tract. It appears that the human vocal tract has evolved in the direction of increased size of quantal locations for vowel production.

2aSP3. The quasi-steady approximation, boundary-layer separation, and vibrations in the vocal tract. Richard S. McGowan (Haskins Labs., 270 Crown St., New Haven, CT 06511)

Studies of voice and other flow-induced vibratory phenomena in the vocal tract often rely on the quasi-steady approximation based on the smallness of the Strouhal number (a nondimensional frequency measure). However, the relevant parameter do decide the application of the quasi-steady approximation to boundary-layer separation in oscillatory flow situations is a function of both the Strouhal number and the Reynolds number. Boundary-layer separation is important because it helps to determine the pressure distribution on the vibrating solid surface. Thus, unsteadiness in separation could provide a mechanism for energy exchange between the solid and the air to account for self-sustained oscillation. Such a mechanism will be illustrated by a model for tonguetip trills. [Supported by NIH Grant Nos. DC-00121, DC-00865, and DC-01247 to Haskins Laboratories.]

2aSP4. Foreperiod effects on acoustic laryngeal reaction time. Ben C. Watson (Dept. of Otolaryngol., New York Medical College, Munger Bldg., \# 170, Valhalla, NY 10595)

In a simple reaction time task, foreperiod is the preparatory interval between presentation of a warning cue and a respond cue. Two studies examined effects of foreperiod duration, range, and distribution on laryngeal reaction time (LRT) values for production of the isolated vowel $/ a /$ The first study examined foreperiods presented in two series with different durations and ranges $(200-1000 \mathrm{~ms}$ and $700-2300 \mathrm{~ms})$. Each series had a rectangular distribution. The second study examined the same two foreperiod series presented in nonaging (equi-probable) distributions. Variable inter-stimulus intervals and subject instructions inhibited initiation of preparation prior to presentation of the warning cue. Laryngeal reaction time (LRT) values decreased as foreperiod increased in each series. LRT was longest after the shortest foreperiod in a series, regardless of its absolute length. The same absolute duration foreperiod (i.e., 700-1000 ms) yielded a longer LRT when it was embedded in a wider range of foreperiods. The quadratic relation between foreperiod duration and LRT [K. Izdebski, J. Speech Hear. Res. 23, $485-489(1978)$ ] was more pronounced when a series was presented as a nonaging distribution versus a rectangular distribution.

2aSP5. In vivo measurement of laryngeal mucosal wave speed in humans. Randall Wenokur, Gerald S. Berke, Bruce R. Gerratt, Jody Kreiman, and Ming Ye (Div. of Head/Neck Surgery, UCLA School of Medicine, CHS 62-132, Los Angeles, CA 90024, and VA Med. Ctr., West Los Angeles, CA 90073)

Measurement of laryngeal mucosal wave speed is potentially important for understanding laryngeal vibration and for guiding phonosurgery. However, measurement of wave speed requires knowledge of distance or phase angle on the vocal folds, and has not been possible in awake patients. A new method permitting such measurements in an office setting is described. This method can be applied using either a calibrated fiberoptic nasopharyngoscope or a calibrated laryngoscope to measure absolute distances in the larynx accurately, reliably, and noninvasively. Wave speeds were measured in one normal male speaker at varying levels of $F 0$ and intensity. Across conditions, measured mucosal wave speed ranged from 0.29 to $1.18 \mathrm{~m} / \mathrm{s}$. Wave speeds varied significantly with vocal intensity $(r=0.72)$, but not with $F 0(r=0.14)$. Theoretical implications of these findings will be discussed, along with clinical applications of the new techniques.

2aSP6. Jaw velocity events as correlates for the control of quantity contrast in Arabic. Mounir Jomaa and Christian Abry (Inst. de la Communication Parlée CNRS No. 368, Stendhal, BP 25, 38040 Grenoble, France)

Jaw vertical position of VCC vs VVC quantity contrasts in Arabic uttered at two speech rates were monitored. Events detected are: jaw lowering onset ( $\mathrm{VCO}$ ), and maximum velocity (MVV), jaw raising onset (CCO), and maximum velocity (MCV). Results show that at conversational rate, contrasts depend on differences in absolute timing along only one cycle ( $\mathrm{VCO}-\mathrm{VCO}$ ). At fast rate, relative timing values no longer carry quantity contrasts for two speakers (out of four) within this cycle, whereas the contrast is still maintained in the MVV-MVV cycle. Velocity profiles for the raising gesture show that speakers who lose contrasts in relative timing in the VCO-VCO cycle, are also speakers who lose them within this gesture at fast rate: in fact, they lose the absolute lead that geminates have over nongeminates in the acceleration phase of this gesture. Consequently, it appears that contrasts rely mainly on the timing of raising gestures that follow the vowel, thus hiding it faster when the consonant is a long one.

2aSP7. Proposal of a wavelet model for vocalic speech coarticulation. Robert C. Lange (Grad. Prog. in Acoust., Penn State Univ., 117 Appl. Sci. Bldg., University Park, PA 16802)

A recent development in the theory of wavelets is wavelet system characterization [R. K. Young, Wavelet Theory and its Applications (Kluwer, Boston, 1993)]. In wavelet system theory, the wavelet transform can be used to describe the time-frequency behavior of a transmission channel, by virtue of its ability to describe the time-frequency content of the system's input and output signals. The present research proposes a wavelet-system model of speech production; wherein, the system is the process of transformation from one speech state to another. The transmission channel generates an effected utterance from a control utterance. A speech production effect (such as voice quality, or the influence of the speaker) is thereby characterized in a time-frequency manner. The effect of CVC coarticulation is investigated in particular, using samples of real speech. In this case, an isolated vowel utterance (the control) is contrasted with an utterance of the same vowel imbedded within a $/ \mathrm{C}-\mathrm{C} /$ context (the effected). The channel thus describes (in terms of wavelets) the CVC coarticulation. [Work supported by Appl. Res. Lab.]

2aSP8. Inversion and control of an articulatory model of the vocal tract: Recovering articulatory gestures from sounds. Rafael Laboissiere (Inst. de la Commun. Parlee, 46 Ave. Felix Viallet, 38031 Grenoble Cedex, France)

To what extent are the basic concepts of robotics and control theory useful for the understanding of speech communication processes? In this paper, this question is addressed and a quantitative modeling framework is proposed, which has some bearings on classical concepts of feedforward and feedback control systems. The main feature of the model is the capability to solve the ill-posed inversion problem of determining the articulatory commands from speech goals specifications, whatever they are. A theoretical analysis shows that former models for motor control, 
namely the task-dynamics approach [Saltzman and Munhall (1989), Ecolog. Psychol. 1(4)] and the forward-inverse modeling [Jordan and Rumelhart (1992), Cognitive Sci.], are subcases of the present model. Simulations of well-known phenomena in speech, like compensation for bite-block perturbation and coarticulation of simple VCV sequences, can be successfully done in this framework. This modeling effort can feed the theoretical debate about invariance/variability in speech because it is a natural framework to address the following issues: (1) the nature of the control space used in programming speech gestures; (2) the suitable principles of control, defined as the choice of an architecture for the control and the constraints imposed on the control law to solve the inversion problem; and (3) the learning of an inverse model capable of generating suitable articulatory commands from a specification of the goals to be reached.

2aSP9. Syllable internal timing: Effects on vowel and consonant duration. Dawn $M$. Behne (Linguist. Inst., Univ. of Trondheim, N-7055 Dragvoll, Norway) and Lynne C. Nygaard (Speech Res. Lab., Dept. of Psychol., Indiana Univ., Bloomington, IN 47405)

The present study investigates the effects of vowel quality, postvocalic voicing, sentence position, focal stress, and speaking rate on the internal timing of syllable components. Previous findings [D. M. Behne and L. C. Nygaard, J. Acoust. Soc. Am. 90, 2254 (A) (1991); D. M. Behne and L. C. Nygaard, J. Acoust. Soc. Am. 91, 2340 (A) (1992)] have confirmed the combined effects of these factors on vowel duration and have shown that consonants can also be independently affected by sentence position, focal stress, and speaking rate. The goal of this paper is to characterize the internal timing of both component vowels and consonants when these factors affect syllable timing. Using the vowels $/ \mathrm{r}, 0, \mathrm{a}, \mathrm{x} /$, conversations were developed with $/ \mathrm{kVt} /$ and $/ \mathrm{kVd} /$ as target words. In each conversation a target word occurred in initial and final sentence position and was either focused or nonfocused by the discourse. Twelve subjects produced each conversation at three speaking rates. The results indicate that (1) speaking rate and focal stress have a global effect on syllable-internal timing, influencing both vowel and consonant durations, (2) phrase-final lengthening affects the nucleus and postvocalic consonant, and (3) postvocalic voicing and inherent vowel duration locally affect vowel duration, but have little effect on neighboring consonants. These findings verify that the factors being investigated can concurrently influence the syllable-internal timing, and suggest a hierarchy of effects on the internal-timing of a syllable, from speaking rate to inherent vowel duration.

2aSP10. Effects of speaking rate and stress on vowel durations and formant structures. Janet W. Stack (Dept. of Psychol., Univ. of South Florida, Tampa, FL 33620)

The effects of speaking rate and stress on the temporal and spectral quality of vowels in four adult male speakers were evaluated. Conversational style speech was used from which four vowels in two target words were analyzed. Target words were produced in two different sentence stress conditions. Vowel durations were measured, and formant values were obtained at the one-fourth, one-half, and three-fourths points of the syllables. Rapid rate tokens were consistently shorter in duration than normal rate. There was no differential duration shortening between stressed and unstressed words or vowels. Speakers were very consistent in their overall sentence compression, but word and vowel compressions showed nonsystematic individual differences. Target undershoot (any deviation greater than 1 Bark from the stressed normal rate condition) was found in only one speaker, for the first formant of one vowel. Formant movement from the one-fourth to the three-fourths point was not affected by rate or stress (determined as any change of more than $0.1 \mathrm{log}$ unit from the stressed normal rate condition) in any speaker. [Work supported by NIDCD.]

2aSP11. Context independence of F3 trajectories in American English $/ \mathbf{r} /$ 's. Carol Y. Espy-Wilson (Elec., Comput, and Systems Eng. Dept., Boston Univ., 44 Cummington St., Boston, MA 02215) and Suzanne Boyce (MIT, Cambridge, MA 02139)

In Boyce and Espy-Wilson [J. Acoust. Soc. Am. 89, 1916(A) (1991)] the coarticulatory effects of American English / $r$ / on surround- ing segments were reviewed, and data were presented to suggest that the articulatory gesture for $/ r /$ is relatively incompressible. Here, data are reported from a larger study that supports the previous claims. Comparing the trajectories through the intervocalic consonant of /wavam/ and /wawam/ with /waram/, we estimate the duration of the /r/ trajectory to be around $150 \mathrm{~ms}$. Further, the trajectory appears to be symmetrical. In addition to $/ \mathrm{r} / \mathrm{s}$ in clusters with labial consonants, we looked at $/ \mathrm{r} / \mathrm{s}$ following voiced stops $/ \mathrm{g} /, \mathrm{b} /$, and $/ \mathrm{d} /$. Given the commonly cited coarticulation hypothesis that (1) articulation will be initiated as soon as possible, and (2) that coarticulation will be more extensive when neighboring segments contain no incompatible gestures [W. Henke, unpublished Ph.D. dissertation, MIT (1966)], it was expected that $/ r /$ trajectories would start earlier in words with labial consonants. However, it was found instead that $/ r /$ trajectories remained the same for each consonant condition. This finding was aided by the fact that for each speaker in every condition at least one token was found where the full symmetrical $/ \mathrm{r} /$ trajectory could be observed. Results from 4 male and 4 female speakers of American English will be presented. Implications for coarticulation and speech recognition will be discussed.

2aSP12. Effects of frequency scale on inter-age comparisons of first and second formant transitions. Megan M. Hodge (Dept. of Speech Pathol. \& Audiol., Univ. of Alberta, 2-70 Corbett Hall, Edmonton, AB T6G 2G4, Canada) and Terrance M. Nearey (Univ. of Alberta, Edmonton, AB T6G 2E9, Canada)

Comparison of spectral measures from a wide age range of talkers, to draw inferences about inter-age differences in articulatory behavior, is confounded by the absolute resonant frequency differences that result from vocal tract size differences. Normalization procedures are required so that spectral differences due to vocal tract size differences are not misinterpreted as age differences in speech production behavior. Based on vowel (steady-state) data, the $\log \mathrm{Hz}$ scale has been favored over several other frequency scales that have been used to normalize spectra for analysis of production data [T. M. Nearey, Proc. Int. Conf. Spok. Lang. Process. 1, 583-586 (1992)]. The magnitudes of first and second formant transition extents of male talkers, who ranged from infancy to adulthood, were compared under three different normalization conditions ( $\log \mathrm{Hz}, \mathrm{ERB}$, and Bark scales). Each formant was measured in $\mathrm{Hz}$ at the onset and offset of the consonant-vowel transition in wideband spectrographic displays of the syllables $/ \mathrm{bar} / \mathrm{bi} / \mathrm{b}$, and $/ \mathrm{d} \mathrm{x} /$. These values were transformed to each scale and formant transition extents and rates were determined. The results are discussed relative to the potential of these scales to lead to artifactual differences in inferred inter-age articulatory differences.

2aSP13. Resistance of bilabials / $p, b /$ to anticipatory labial and mandibular coarticulation from vowel types $/ \mathrm{i}, \mathrm{a}, \mathrm{u} /$. Rudolph Sock and Anders Löfquist (Haskins Labs., 270 Crown St., New Haven, CT 06511-6695 and Inst. de la Commun. Parlée, Univ. Stendahl, BP 25 X, 38040 Grenoble, France)

The timing of lip and jaw movements and acoustic events was studied in one subject producing the six pseudowords /pipi,papa,pupu,bibi,baba,bubu/ at two speaking rates. Lip and jaw movements were recorded optoelectrically. Acoustic durations of bilabial closures served as a temporal base for analyzing consonantal resistance to vowel coarticulation. Within each temporal base, a phase was defined as the interval between peak raising or lowering velocity of an articulator and the acoustic onset of the following vowel. The proportion of time taken by each phase in the temporal base provides a measure of anticipatory coarticulation of the vowel in the consonant. Results suggest that the rounded vowel $/ w$ / has a higher coarticulatory influence on the labial consonant that the unrounded vowels. The voiced consonant $/ b /$ has less resistance to coarticulation than its unvoiced cognate. Both domains investigated show comparable phasing patterns, suggesting a general strategy of coarticulation for the successive consonants. Generally, all three articulators show similar gestural kinematic patterns. The results are discussed in terms of biomechanical constraints on speech production tightly coupled with the perceptual requirements 
of the linguistic code. [Work supported by the Fyssen Foundation and NIH.]

2aSP14. Effects of lexical status on children's and adults' perception of native and non-native vowels. A. C. Walley, James E. Flege, and L. A. Randazza (Depts. of Psychol. and Biocommun., Univ. of Alabama at Birmingham, Birmingham, AL 35294-1170)

Monolingual, English-speaking 5-year-olds, 9-year-olds, and adults heard stimuli from two "native," synthetic continua, in which the vowels ranged from English $/ \mathrm{I} /$ to $/ \mathrm{i} /$ in the context $/ \mathrm{b} \_\mathrm{b} /$ or $/ \mathrm{b} \ldots \mathrm{p} /$. Thus the endpoints of the first continuum constituted a word and a nonword ("bib" vs " "beeb"); the reverse held for the second continuum ("“bip" vs "beep"). Other subjects heard stimuli from two "foreign" continua, where the vowels ranged from English / I / to a foreign vowel $/ v /$ in the contexts described above. Thus the endpoints of the first continuum corresponded to a word and a nonword ("bib" vs " "bYb"); both endpoints of the second continuum corresponded to nonwords ("“bip" vs *"bYp"). After training on endpoints, subjects' identifications of the nine stimuli of a given continuum were examined to assess whether: children, like adults, exhibit a "lexical bias" effect for familiar vowels (from the "native" continua); vowel categories not bounded by another native vowel (as in the "foreign" continua) expand outward or become better defined with increasing age and/or lexical status.

2aSP15. Rhythm and compression in Caribbean Spanish. Henrietta J. Cedergren and Guillermo Toledo (Dept. de Linguist., Univ. du Québec à Montréal, C.P. 8888, Montréal, PQ H3C 3P8, Canada)

The goal of this research was the observation of temporal relations and degrees of compression in spontaneous speech. Recorded materials of casual speech from one female and one male speaker of a dialect of Panamanian Spanish were studied. Acoustic measurements were made by examining phonetic segments, syllables, and rhythmic groups embedded in intonational phrases through the use of digital spectrograms and oscillographic traces. Results indicate low degrees of temporal compression; rhythmic group duration shows significant linear correlation with rhythmic group size. In addition, rhythmic groups in intonational phrase preboundary position are subject to local deceleration, which affects both the penultimate stressed syllable and the following unstressed final syllable. Both speakers reveal a positive relation between the duration of the stressed and unstressed syllables. These findings suggest that casual speech style in this dialect has both syllable-timing and a locally bound deceleration. [Work supported by SSHRCC.]

2aSP16. Articulatory and intonationgl correlates of French focus. Gayle M. Ayers (Dept. of Linguist., Ohio State Univ., 1712 Neil Ave., Columbus, OH 43210-1298)

Jaw movement and $F 0$ patterns are investigated in a French corpus of neutral utterances and utterances with focus on the initial, medial, or final word. Initial-word length also varied between two or three syllables, and both natural and reiterant copies were produced. The maxima and minima of jaw displacement (jaw closure and opening), peak velocities, and $F 0$ peaks of the target words were measured. Jaw velocity and displacement were correlated identically in neutral and focus utterances. Displacement alone accounted for between $69 \%$ (disyllables) and $80 \%$ (trisyllables) of the velocity variation. Focused words tended to have a larger displacement on the first syllable. The observed variation in duration could not be accounted for by a displacementindependent variation in peak velocity. In neutral utterances $F 0$ peaks occurred during the last closing gesture of each nonfinal phrase, a pattern that can be represented phonologically by a demarcative phrasefinal $\mathrm{H}$ tone. In focused utterances, by contrast, $F 0$ peaks occurred during the first closing gesture of the focused word, although there was sometimes also a peak during some later gesture, as in the neutral utterances. The focus $\mathrm{H}$ tone is perhaps an accent tone of the English type. A possible correlation between jaw kinematics and the $F 0$ pattern is being investigated.

2aSP17. Effects of prosodic context on Taiwanese tones. Shu-hui Peng (Dept. of Linguist., Ohio State Univ., 1712 Neil Ave., Columbus, OH 43210-1298)
Duration and $F 0$ patterns for the high and high-falling tones of Taiwanese Amoy in different contexts were investigated in two experiments. The first examined the target tones in four prosodic positions: initial, medial, and final to a prosodic phrase, and utterance final. The nonutterance-final targets also varied in tonal context, being followed by high or mid tone. Words at the end of a prosodic phrase were substantially longer than in the other prosodic positions. The target tones were higher in frequency phrase-initially and phrase-medially than phrase- or utterance-finally. Tonal coarticulation occurred between the target tone and its following tone. When followed by high tone, the offset of the high target tone did not change, while that of the high-falling target tone increased. The second experiment investigated tonal coarticulation further by examining the high tone followed by five different tones in the middle and the end of a prosodic phrase. It is predicted that the tone height and the tone pattern of the target tone will vary depending on the following tone.

2aSP18. Interpreting complex articulations: The backward tongue movement in Ewe doubly articulated stops. Ian Maddieson (Phonetics Lab., Linguist. Dept., UCLA, Los Angeles CA 90024-1543), J. S. Perkell, M. L. Matthies, and Mario A. Svirsky (MIT, Cambridge, MA 02139)

In West African languages, $/ \mathrm{kp} /, / \mathrm{gb} /$ are described as plosives with simultaneous bilabial and velar closures. From auditory and acoustic evidence Maddieson and Ladefoged (1989) argued that the two articulations are slightly offset in time, with the velar leading the labial. This claim has now been directly tested. Two Ewe speakers provided data using the EMMA system (Perkell et al., 1992). Temporal asymmetry of the two articulations was found, as well as broad similarities to gestures in simple velar and labial stops. In labial velars there is more backward movement of the tongue dorsum than in plain velars; in particular, the tongue back retracts during the closed phase of the stop before releasing forwards. This retraction might result from (a) movements to create suction in the front cavity, (b) increased air pressure in the front cavity pushing the tongue backwards, (c) greater fortition of the velar contact resulting in more compression and displacement of the tongue, or (d) larynx lowering, creating suction in the pharyngeal cavity and pulling the tongue dorsum backwards. Comparison between labial velars and plain velars with respect to the height reached by the tongue back, coordination between movements of the tongue front and back, and voicing features in the acoustic signal suggest that, in these data, pharyngeal cavity expansion is the most likely explanation for retraction.

2aSP19. Phonetics of Jemez vowels. Alan Bell and Rebecca Heins (Dept. of Linguist., Univ. of Colorado, Boulder, CO 80309)

This paper presents a spectral and durational analysis of the vowels of Jemez, a Kiowa-Tanoan language spoken northwest of Albuquerque. The vowel system consists of six short vowels $/ i$ e $æ a \quad j$ i/ and six corresponding long vowels. For each long and short vowel, except /e/ and /ee/, there is a corresponding nasal vowel. The principal data for the analysis consists of repetitions of words containing each vowel pronounced by a single speaker in isolation and in sentences. Some data from another speaker and from other word contexts is also used. The vowels /e o i/ are realized at the same heights, substantially closer to /i/ than to $/ \mathfrak{x} \alpha /$. The vowels are monophthongs, except for $/ e /$ and /ee/, which have rising offglides. Long and short vowels do not differ significantly in quality. Nasal vowels are higher than corresponding oral vowels, contrary to cross-linguistic tendencies. Vowels are raised after the labialized consonants $/ \Phi^{w} k^{w} w /$ and the palatalized consonants $/ \int \mathbf{k}^{\mathrm{j}} \mathbf{k}^{\mathrm{jh}} \mathbf{k}^{\mathrm{j}^{\prime}} \mathrm{j} /$, but differences between the two contexts suggest that separate processes are involved. [Supported in part by a UROP grant from the University of Colorado.]

2aSP20. Phonetic observations on tone and stress in Mandarin Chinese. Corinne B. Moore (Dept. of Modern Lang. \& Linguist., Cornell Univ., Ithaca, NY 14853)

This study reports results of an experiment on Beijing Mandarin tones and stress. There are four lexical tones in Mandarin, plus a pitch pattern referred to as the "neutral tone," which occurs on atonic or 
unstressed syllables. The literature has been vague, however, about whether syllables that have undergone tone deletion as a result of destressing and underlyingly atonic syllables show the same surface pitch pattern. This experiment examined these lexical tones in stressed and unstressed contexts, and then compared unstressed tone patterns to that of the neutral tone. The experiment focused on duration and pitch range as effective correlates of stress. Unstressed syllables were found to be significantly shorter in duration than stressed syllables, corroborating previous studies. Pitch range was also significantly reduced in unstressed contexts in all cases except one. The data unexpectedly showed that changes in pitch range are a function of the pitch peak only; pitch valleys did not significantly change. Finally, the results provide evidence that unstressed syllables with underlying tones do not neutralize completely, but remain distinct from the neutral tone.

2aSP21. Preaspiration in Icelandic: Production and perception. Jörgen Pind (Inst. of Lexicography, Univ. of Iceland, Neshaga 16, 107 Reykjavík, Iceland)

Preaspiration in Icelandic occurs, e.g., when a vowel in a stressed syllable is followed orthographically by "pp", "tt", or "kk," and is commonly transcribed as [h] before a short stop. Stressed syllables in Icelandic thus present a three way contrast (involving stops) between word forms such as baka [ba:ğa], "to bake," (type V:C), bagga, [bag:a], "burden" (acc.sg.) (type VC:), and bakka, [bahg̊a], "to turn back" (type VhC). Durational measurements showed wide variability in segment durations depending on context. However, results also indicate that the ratio of the preaspiration to the vowel duration is reasonably constant and can be viewed as a higher-order invariant, comparable to the vowel/rhyme ratio in the quantity opposition [J. Pind, Phonetica 43, 116-139 (1986)]. Perception experiments showed that preaspiration can be cued by voice offset time, a speech cue analogous to voice onset time. The experiments further showed that durational relationships within the syllable rhyme influenced the perception of preaspiration to a great extent while factors outside the rhyme had a minor influence on the placement of the phoneme boundaries, indicating perceptual constancy.

2aSP22. VOT values of roiceless and voiced stop contrasts in Hindi and Kannada. H. S. Gopal (Dept. of Linguist., Univ. of California at Santa Barbara, Santa Barbara, CA 93106)

A number of languages from the subcontinent of India have a fourway voicing contrast-the aspirated voiceless, the unaspirated voiceless, the unaspirated voiced, and the breathy voiced stops (for example, [p", $\left.p, b, b^{\mathbf{l}}\right]$ ). It has been suggested that the primary difference between the unaspirated voiceless and the unaspirated voiced stop consonants (for example, [p] vs [b]) is one of voice onset time (VOT). This study investigated whether VOT adequately separates the unaspirated voiceless and the unaspirated voiced stop consonants in two languages, Hind and Kannada. Hindi is primarily a north Indian language and Kannada is primarily a South Indian language. Using four speakers, VOT values were measured for these two stop consonant series at four places of articulation-bilabial, dental, retroflex, and velar. The results will be discussed with reference to the adequacy of VOT as the primary acoustic feature distinguishing these two stop contrasts in the two languages.

2aSP23. Segmental duration changes due to variations in stress, vowel, place of articulation, and voicing of stop consonants in Greek. H. B. Kollia (City Univ. of New York, and Haskins Labs., 270 Crown St., New Haven, CT 06511)

Although Lisker and Abramson (1967) found no effect of the following vowel on the VOT of a stop consonant, Port and Rotunno (1979) found VOT to have greater values for voiceless stops followed by tense than by lax vowels. The purpose of the present study was to obtain a complete database on the VOT characteristics of voiced and voiceless initial stop consonants in Greek, and to examine the contextual effects on the VOT of the post-consonantal vowel, the stress pattern, and the distance of the stress from the initial stop consonant. The question here was whether the vowel effects found by Port and Rotunno for English would be seen in Greek, a language whose two stop categories have voicing lead and medium lag. Speakers read isolated disyllabic and trisyllabic words of four stress patterns. The utterance-initial stops $/ \mathrm{p}, \mathrm{t}, \mathrm{k}, \mathrm{b}, \mathrm{d}, \mathrm{g} /$ were followed by the five vowels of Greek, $/ \mathrm{a}, \varepsilon, \mathrm{i}, \mathrm{o}$, $u /$. Results indicated that both voicing lead and voicing lag increased for stops followed by higher than by lower vowels. Fourakis (1986) found that stress changes did not affect VOT of $/ p, t /$, only of $/ k /$. In this study, stress variations affected VOT for all stops. Furthermore, these effects were vowel dependent. [Work supported by NIH Grant Nos. DC-00121 and DC-00594 to Haskins Laboratories.]

2aSP24. Do women listen to men's voices with men's mouths in mind? Keith Johnson (Dept. of Biocommun., Univ. of Alabama, Birmingham, AL 35205), Peter Ladefoged, and Joyce McDonough (UCLA, Los Angeles, CA 90024-1543)

This paper reports the results of an acoustic study of vowels produced by Navajo speakers, which was supplemented by perception data using the method of adjustment. Analyses of vowels produced by male and female speakers showed these two groups distinguished long and short vowels differently. Male speakers had about the same formant values for both members of the long/short vowel pairs, while female speakers tended to distinguish long and short vowels by means of both a length and a vowel quality distinction. The perception data showed that when listening to a "male" synthetic voice all listeners (irrespective of sex) collapsed long and short vowels in the perceptual vowel space, selecting vowels that were similar to those in the male production data, but with more extreme formant values than those produced by the male speakers. One hypothesis is that Navajo women produce different long and short vowels, but when listening to a male speaker's vowels select as matches long and short vowels that do not differ significantly in quality. It is not known whether the men would listen to women's voices with women's mouths in mind.

2aSP25. Let your synthesizer breathe. D. H. Whalen (Haskins Labs., 270 Crown St., New Haven, CT 06511) and Charles E. Hoequist (BNR, Research Triangle Park, NC 27709)

Although synthesizers do not have any air flow, they are representing creatures that do. While it is quite common to have long stretches of speech without audible intake (especially with speakers trained in acting and broadcasting), synthesis might be somewhat less artificial with the intake of breath added. To see whether adding an audible intake would affect the perception of synthetic speech, breath intake (naturally produced) was introduced in a synthesis transcription task. Subjects received the breath-added stimuli on either the first half of the test or the second. The largest effect was a typical one of increased performance in the second half, due to increased experience with the synthesizer. However, those subjects who received the breath second had a much larger improvement than those who had it first, who were essentially the same in the second condition as the first. The results indicate that, as with almost anything that increases naturalness, allowing computers to breathe will improve the perception of synthetic speech. [Work supported by NIH Grant No. HD-01994.]

2aSP26. Spectral shape cues for vowels that predict the perception of multiple-tone steady-state stimuli. Stephen A. Zahorian, Zhong-Jiang Zhang, and Zaki B. Nossair (Dept. of Elec. and Comput. Eng., Old Dominion Univ., Norfolk, VA 23529)

In previous experiments for which multiple tone stimuli were synthesized such that either the formants or global spectral shape were matched to that of naturally spoken vowel tokens, it was found for both cases that vowel identity and quality was not well preserved [S. A. Zahorian and Z.-J. Zhong, J. Acoust. Soc. Am. 92, 2414-2415 (1992)]. In the present study several additional criteria were tested for selecting the amplitudes and frequencies of sinusoids with the objective that stimuli synthesized from these sinusoids would be perceived as most similar to original "target" vowel tokens. Of the methods investigated, vowel quality from stimuli synthesized from $N$ sinusoids was best preserved if these sinusoids match the $N$ largest peaks in the magnitude spectrum of the original vowels. Depending on the vowel, between 5 and 10 sinusoids are required such that the synthesized token is perceived as sounding nearly identical to the original token. A new metric for spectral shape that yields acoustically invariant cues to vowel perception in a manner 
consistent with the results of these experiments has been developed and will be presented. This new metric also predicts the number of sinusoids that are required to synthesize each vowel.

2aSP27. Articulatory underspecification as a concept for controlling a speech synthesizer. Bernd J. Kröger, Georg Heike, and Claudia Opgen-Rhein (Inst. Phonetik, Univ. Köln, Greinstr. 2, 5000 Köln 41, Germany)

A segmental model for generating articulator movements based on the principle of articulatory underspecification has been developed. This model is part of a computer-implemented speech production model controlling an articulatory speech synthesizer and capable of producing any utterance [B. J. Kröger, in Signal Processing VI: Theories and Applications, edited by J. Vandewalle et al. (Elsevier, New York, 1992), pp. 331-334]. Articulatory/phonatory control parameter time functions generated by this segmental model are compared to those generated by a gestural model. Qualitative comparison shows that the concept of segmental underspecification and the concept of cooccurrence (or overlap) of articulatory gestures result in similar articulator movements. [Work supported in part by DFG Grant No. He 434/21-1.]
2aSP28. Modified Klatt synthesizer (VSS-KS) and analyzer (INTANAKL) for control parameters. T. V. Ananthapadmanabha and H. N. Jayasimha (Voice and Speech Systems, Temple St., Malleswaram, Bangalore 560003 , India)

Klatt synthesizer (KLSYN88) is a successful hybrid formant filter model for speech synthesis [Klatt and Klatt, J. Acoust. Soc. Am. 87, 820-857 (1990)]. Several modifications are proposed to enhance the scope and quality of KLSYN88. For example, in VSS-KS, control parameters do not change abruptly; an impulse excitation is used for synthesis of stop consonants; aspirated stop consonants can be synthesized; microphonetic events with fine temporal resolution can be synthesized; parallel formant filter is correctly implemented; source-filter interaction (up to three formants) is implemented as formant frequency and bandwidth modulation rather than abrupt jumps; etc. INT ANAKL estimates many of the control parameters like formant frequency, bandwidth, level, voice source parameters, aspiration noise component, polezero component, etc. The estimated parameters are validated by comparing the short-time spectra of the original and synthesized speech and source waveforms. VSS-KS and INTANAKL are described in this paper.

TUESDAY MORNING, 18 MAY 1993

BANQUET ROOM, 7:55 A.M. TO 12:00 NOON

\title{
Session 2aUW
}

\section{Underwater Acoustics: Scattering and Reverberation}

\author{
John R. Preston, Chair \\ SACLANT Undersea Research Center, Viale San Bartolomeo 400, I-19100 La Spezia (SP) Italy
}

Chair's Introduction-7:55

Contributed Papers

8:00

2aUW1. Preliminary bistatic and monostatic scattering results from a steep basin feature in the Tyrrhenian Sea. J. R. Preston (SACLANT Undersea Res. Ctr. (SACLANTCEN), CMR 426, APO, AE 09613)

A feature scattering experiment was conducted by SACLANTCEN off the island of Palmarola in the Tyrrhenian Sea in March 1991. Estimates of this feature scattering are presented as a function of range, source type, and source depth. Area water depths ranged from 200 to greater than $3800 \mathrm{~m}$. The receivers were a horizontal array of 64 elements spaced at $0.5,1$, and $2 \mathrm{~m}$ and a vertical array with 32 elements and the same spacings. Source depths varied from 90 to $1800 \mathrm{~m}$ and receiver depths ranged from 90 to $150 \mathrm{~m}$. Depth charges were used as sources, as were low-frequency cw pulses and linear FM pulses of various pulse durations and bandwidths. Basin wide received reverberation levels and scattering strength values are also presented as functions of the estimated scatterer position on area bathymetry maps. Comparisons of shot and pulse data reverberation levels are presented. Estimates of monostatic bottom scattering strengths versus grazing angle are given using generic sonar model (GSM), PAREQ, and KRAKEN to interpret the data. Finally, comparisons of measured reverberation and modeled reverberation are presented.

\section{8:15}

2aUW2. Measurements of low-frequency, low-grazing-angle sea-surface scattering and feature strengths. Roger $C$. Gauss (Naval Res. Lab., Washington, DC 20375-5350) and Joseph M. Fialkowski (Planning Systems Inc., McLean, VA 22102)

Direct-path measurements of low-frequency $(200-1000 \mathrm{~Hz})$ and low-grazing-angle $(<10 \mathrm{deg}$ ) acoustic surface scattering were made in the Gulf of Alaska in April of 1990 and in March of 1992. Both narrowband (cw) and broadband (FM,PRN) transmissions were used to examine the strength of sea-surface backscatter as a function of frequency and environmental conditions. Results have revealed that in addition to a continuously distributed reverberation component, there are often strong spatially and temporally discrete scattering events. Scattering and feature strengths will be presented to quantify the relative contributions of these components to mean backscatter levels (such as were used in deriving the Ogden-Erskine scattering-strength curves), and to extend the scattering-strength results of Ogden and Erskine to lower grazing angles. Furthermore, the presented results have strong implications as to the types of bubble clouds that are responsible for each component (as will be discussed).

\section{$8: 30$}

2aUW3. Measurements of the spectral characteristics of low-frequency, low-grazing-angle surface reverberation. Roger $\mathrm{C}$. Gauss (Naval Res. Lab., Washington, DC 20375-5350) and Joseph M. Fialkowski (Planning Systems, Inc., McLean, VA 22102)

Direct-path measurements of low-frequency $(200-1000 \mathrm{~Hz})$ and low-grazing-angle ( $<10 \mathrm{deg}$ ) acoustic surface scattering were made in the Gulf of Alaska in April of 1990 and in March of 1992. Shortduration (0.6-2.4 s) $\mathrm{cw}$ and PRN waveforms were used to quantify the spectral character of surface/near-surface reverberation as a function of frequency and environmental conditions. Measures include spreading and peak-Doppler-shift statistics versus azimuth. Results over the range of wind speeds $(10-35 \mathrm{kns})$ have revealed a dominant zero-Doppler component and a weaker-than-expected dependence of spread on both sea conditions (wind speed and relative direction of the seas) and frequency. The results are consistent with sub-surface bubbles as the primary mechanism for surface reverberation when white caps are present, 
and give insight as to the nature of the bubble clouds that could give rise to the observed acoustic scattering.

\section{$8: 45$}

2aUW4. The impact of backscatter angular dependency on reverberation levels in shallow water under downward refracting conditions. Peter D. Herstein, Bernard F. Cole, Kerry E. Brown, Eugene M. Podeszwa, and David G. Browning (Naval Undersea Warfare Ctr.-New London Detachment, New London, CT 06320)

For downward refracting conditions in shallow water over a sandy bottom it was found that propagation loss was dependent on the source and receiver depth [P. D. Herstein et al., J. Acoust. Soc. Am. 92, 2302(A) (1992)]. The analysis is now extended to reverberation levels, considering the grazing angles corresponding to various source and receiver depths and both the first and second power sine of the angle dependencies of the backscattering. This is compared to a compilation of shallow water backscattering results.

\section{9:00}

2aUW5. Broadband bottom scattering measurements in shallow and deep water. Steven O. McConnell and John H. McCown (Areté Associates, P.O. Box 8050, La Jolla CA 92038)

Measurements using broadband signals with bandwidths up to 5 $\mathrm{kHz}$ were made in shallow and deep water off San Diego. At the shallow $(180-500 \mathrm{~m})$ location 1- to $6-\mathrm{kHz}$ coded signals were used for gathering simultaneous forward loss and backscattering data at grazing angles between $10^{\circ}$ and $40^{\circ}$. Time resolutions as small as $0.25 \mathrm{~ms}$ were achieved and from the forward loss measurements these high resolution data clearly revealed a medium scale $\left(\sim 10^{2} \mathrm{~m}\right)$ patchiness in the surficial sediment type alternating between sand and silt. The deep $(1800-\mathrm{m})$ water measurements were taken at a deep sea drilling project site using a combination of $1-$ to $5-\mathrm{kHz}$ coded pulses and $0.05-$ to $2 \mathrm{kHz}-$ bandpassed SUS charges. Fine scale subbottom layering with typical thicknesses of $1-10 \mathrm{~m}$ down to $130 \mathrm{~m}$ were delineated using these broadband source signals. The coded pulses yielded a better definition of this layered structure in the upper $40 \mathrm{~m}$, but were limited in depth of penetration because of increased attenuation at higher frequencies. [Work supported by ONR-AEAS.]

\section{9:15}

2aUW6. Analysis of low-frequency, broadband measured reverberation levels at Blake Escarpment. A. K. Kalra, J. K. Fulford, and J. A. Showalter (Naval Res. Lab., Stennis Space Center, MS 39529-5004)

Acoustic reverberation data were acquired at the Blake Escarpment using Naval Research Laboratory's (NRL's) deep towed acoustic/ geophysics system (DTAGS). DTAGS is similar to a seismic acquisition streamer system with its own omnidirectional source (LFM, from 250 to $650 \mathrm{~Hz}$ ) but consists of two collinear receiver arrays (an acoustic and a geophysics) and can be towed in deep waters (to $5000 \mathrm{~m}$ ). Although DTAGS acquires acoustic and geophysics data simultaneously, only the acoustic array data are analyzed here. The acoustic (field) data consist of backscattered reverberations and/or specular reflection events from all angles. These data are first transformed into $p$ - $t$ (ray-parameter versus time-intercept) domain and studied to retain the grazing angles (intermediate and high) of interest. These selected angles of interest are further analyzed to determine the reverberations versus frequency relationships, and also transformed back to space-time domain to determine the locations of the scattering patches that produced the reverberations. [Work supported by ONR, ARSRP Program.]

\section{9:30}

2aUW7. Limitations of the operator expansion method. Peter $J$. Kaczkowski and Eric I. Thorsos (Appl. Phys. Lab., Univ. of Washington, Seattle, WA 98105)
Preliminary studies of the operator expansion method applied to scattering from rough surfaces satisfying the Dirichlet boundary condition [P. J. Kaczkowski and E. I. Thorsos, J. Acoust. Soc. Am. 90, 2258 (A) (1991)] have indicated that this relatively new method [D. M. Milder, J. Acoust. Soc. Am. 89, 529-541 (1991)] has a broad range of validity. For moderate rms surface slopes, the method is accurate over almost all scattering angles, and represents a vast improvement over the Kirchhoff approximation and small perturbation methods. Further study of the operator expansion method has led to new insights that establish a link between the formal validity of the method and the validity of the Rayleigh hypothesis. While the Rayleigh hypothesis appears to place a strict limit on the operator expansion, numerical examples will be presented that illustrate that the accuracy of the scattering cross section computed by the operator expansion method degrades only gradually as the rms slope is increased beyond that limit. For scattering from surfaces rough in one dimension, the accuracy of the operator expansion solution is established through comparison with the solution to an integral equation. Studies of the convergence of the terms in the operator expansion series indicate how the convergence rate can be used to infer the accuracy of the solution at any given order. This property will be useful when applying the operator expansion method to scattering from surfaces rough in two dimensions, for which exact solutions are still very costly. [Work supported by ONR.]

\section{9:45}

2aUW8. Application of numerical grid generation to scattering from rough penetrable ocean bottoms. Kenneth E. Gilbert, Timothy J. Kulbago (Appl. Res. Lab. and the Grad. Prog. in Acoust., Penn State Univ., P.O. Box 30, State College, PA 16804), and Xiao Di (Univ. of Mississippi, University, MS 38677 and Natl. Res. Council, Ottawa, ON K1A 0R6, Canada)

Numerical grid generation methods have recently been applied with success to atmospheric sound propagation over irregular terrain [Di et al., J. Acoust. Soc. Am 92, 2431 (A) (1992)]. Here, the method was applied to scattering from rough ocean bottoms that allow sound to penetrate into the sediment. Considered are some simple geometries for which analytic results exist as well as more complex geometries that are treated numerically. Of interest are the relative contributions from surface scattering and volume scattering to the total acoustic backscatter. The ultimate objective of the investigation is to arrive at a simple, physically intuitive formulation that will aid in the interpretation of backscatter data from complex coastal environments. [Work supported by ONR.]

\section{0:00-10:15 Break}

\section{0:15}

2aUW9. Modeling of frequency broadening for sea surface reverberation. Eric I. Thorsos (Appl. Phys. Lab., Univ. of Washington, Seattle, WA 98105)

Even at low frequencies $(100-1000 \mathrm{~Hz})$ backscattering levels rise above rough interface scattering levels at higher sea states, most likely due to scattering from bubble clouds near the surface. Because the bubble clouds are convected in the orbital motion of the surface waves, frequency broadening will occur for backscattering of narrow-band signals. However, the moving rough surface leads to another effect. A scatterer below the moving surface is ensonified by the total field: incident plus the surface scattered field. Because the total field vanishes on the surface, the main scattering contribution comes from several meters below the rough surface at low frequencies. The magnitude of this total field will fluctuate with time as the surface waves move by the scatterer. Therefore, even if the scatterer is stationary the scattered field will have some frequency broadening, and this will be combined with the Doppler broadening due the orbital motion for a scatterer free to move. The possible importance of rough surface effects on frequency broadening has been examined using numerical simulations with a one-dimensional surface model. [Work supported by ONR.] 
sized steel and aluminum spheres will be presented. It will be demon-

2aUW10. Frequency and angle spreading for scattering from a rough ocean bottom. Diana F. McCammon and William Thompson, $\mathrm{J}_{r}$. (Appl. Res. Lab., Penn State Univ., P. O. Box 30, State College, PA 16804)

An analysis has been performed of the sound scattered from a Gaussian random rough ocean bottom when radiated from a time-harmonic point source and received by an omnidirectional receiver positioned at the same deep depth. Both source and receiver are moving, at low Mach number, in a plane parallel to the ocean's surface, with arbitrary independent velocities. The problem is idealized and analyzed as a linear system with stochastically varying components, viz., the propagation distances because of the reflections from the random rough bottom. An expression is developed for the power spectral density of the received signal as a function of the source amplitude function. This expression describes the dependence of the frequency shift of the received signal upon the source and receiver speeds and upon the azimuthal angles, grazing angles, and ranges from source to arbitrary scattering facet of the rough bottom to receiver. [Work supported by ONR/AEAS Program.]

\section{0:45}

2aUW11. Analysis of acoustic resonances in partial-wave space, and the nonresonant background. H. Überall (Phys. Dept., Catholic Univ. of America, Washington, DC 20064) and Michael F. Werby (NRL, Code 7181, Stennis Space Center, MS 39529)

The basic principle of the resonance scattering theory, or RST [Flax et al., J. Acoust. Soc. Am. 63, 723 (1978)] consists in separating the scattering amplitude for elastic objects into two parts: a nonresonant background (assumed that of rigid-body scattering for solid-metal and thick-shell objects, as shown above and earlier by Junger), and a series of resonance amplitudes that can be represented as in the Breit-Wigner nuclear scattering theory (cf. reference above). It is shown that this way of subdividing the scattering amplitude into two parts follows naturally from Hilbert-Schmidt theory. For not-so-thick shells, the correct background was given by Werby [original derivation published in Acoustic Resonence Scattering, edited by H. Überall (Gordon and Breach, New York, 1992)]. Subtraction of the appropriate background in partial wave space can be facilitated by representing the modal amplitudes (and resonances) as a function of frequency, or as done in a more recently introduced approach [Talmant et al., J. Acoust. Soc. Am. 86, 278 (1989)], by representing the total amplitude as a function of mode number. This furnishes an unambiguous classification scheme for all orders of resonances, and allows one to isolate fluid-borne waves as recently identified by Talmant $e t$ al.

\section{1:00}

2aUW12. Scattering of superpositions of localized waves from spheres. Des Power and Rod Donnelly (Faculty of Eng., Memorial Univ., St. John's, NF A1B 3X5, Canada)

Localized wave ( $\mathrm{LW}$ ) solutions of the homogeneous wave equation can represent either focused or extended pulses, depending on the choice of a free parameter present in the solution. One can form finite energy superpositions, with respect to this parameter, and one such superposition, known as a modified power spectrum (MPS) pulse, has been extensively studied [R. W. Ziolkowski, Phys. Rev. A 39, 20052032 (1989)]. In particular, the enhanced near-field LW propagation effect has been demonstrated by others, in the context of multi-timederivative transmit/receive systems $[R$. W. Ziolkowski and D. $K$. Lewis, J. Appl. Phys. 68, 6083-6086 (1990)]. The LW solutions are broadband, so it is perhaps to be expected that one could use this property, along with the enhanced focusing characteristic, to extract target parameters when scattering LW superpositions from objects. The acoustic MPS pulse backscattered spectra from a number of different strated that these spectra can be used to extract target size information. [Work supported by NSERC.]

\section{1:15}

2aUW13. Scattering from a rigid target in shallow water. Angie Sarkissian (Naval Res. Lab., Washington, DC 20375-5350)

An algorithm is developed to compute the field scattered from a rigid target placed in shallow water. The method of superposition is applied where the scattered field is approximated to be equivalent to the field produced by a large number of point sources placed on a surface inside the target. The source strengths are determined by minimizing the least-squares error of the normal velocity on the surface of the target. This algorithm is applicable even when the source or the receiver is close to the target as well as in the case where the target is close to the surface. Scattering computations are made for a cylinder with hemispherical endcaps in an isovelocity fluid with soft boundary conditions on top and rigid boundary conditions on the bottom. They are compared to the scattered field computed by using an algorithm discussed by Ingenito [F. Ingenito, J. Acoust. Soc. Am. 82, 2051-2059 (1987)] for the case when both the source and the receiver are distant from the target and when the target is distant from the surface. Results from both methods agree in the range of applicability of Ingenito's method.

\section{1:30}

2aUW14. Effects of ambient noise on the scattering from cylinders and spheres. Rebecca L. Honeycutt and Steven J. Johnson (Code 1945, Carderock Div., Naval Surface Warfare Ctr., Bethesda, MD 20084-5000)

The sound scattering due to an ambient noise field, approximated by a squared cosine function, is considered for infinite rigid and elastic cylinders and rigid spheres. For the cylinders, it is assumed that the acoustic wave front is parallel to the axis of the cylinder (normally incident). For this assumption, a closed-form expression for the scattered sound field-to-incident ambient noise field (signal-to-noise) ratio is obtained not only for the cosine squared directivity, but for any arbitrary directivity that can be expressed in terms of a Fourier series. For the sphere, it is assumed that the noise is circumferentially symmetric, which leads to a closed-form expression for the signal-to-noise ratio due to a cosine-squared directivity.

\section{1:45}

2aUW15. Acoustic characterization of a bubble injected into a fully developed turbulent flow field. Ali R. Kolaini, Ken Markewicz, and Veera Rajendran (Natl. Ctr. for Phys. Acoust., Univ. of Mississippi, Oxford, MS 38677)

An experiment was designed to investigate the characteristics of sound produced by the turbulent excitation of bubbles. By injecting a bubble into a fully developed turbulent flow, generated by a submerged axisymmetric horizontal water jet, the excitation of bubbles and shearinduced fission/fusion may occur. In the case of shear-induced bubble distortion by a turbulent jet, nonlinear coupling between volume pulsation and surface oscillations occur at exactly the same frequency as the radial breathing mode of the bubble. Particle tracking velocimetry (PTV) is used to characterize the turbulent flow field. Two high-speed Kodak Ekta-Pro video cameras are used to capture, simultaneously, several $180-\times 180-\times 10-\mathrm{mm}$ illuminated regions along the jet. An 8-W argon ion laser is utilized for illumination. The method provides not only a visualization of the various patterns and structures of the turbulent flow, but also yields quantitative instantaneous velocity data of the flow containing the bubble. The role of the turbulent flow characteristics in determining the acoustical bubble response is discussed. [Work supported by ONR.] 


\title{
Session 2pAA
}

\section{Architectural Acoustics and Musical Acoustics: New Auditorium Acoustics Measurements-Results and Comparisons III}

\author{
John S. Bradley, Chair \\ Institute for Research in Construction, National Research Council, Ottawa, Ontario K1A OR6, Canada
}

Invited Papers

1:30

2pAA1. Measurements informing the design process: Experiences in occupied hall analysis. Dana L. Kirkegaard (Kirkegaard \& Associates, 4910 Main St., Downers Grove, IL 60515)

In a performing arts facility, many design decisions take into account the influence that audience and performers will have in modifying the acoustics of the unoccupied or sparsely occupied hall. When correlating subjective experience to objective measures, it is very important to assess whether the phenomena being studied are dependent upon the presence of performers and/or audience, and to structure objective measurements accordingly. The impact of occupancy varies widely, and is dependent upon the location of important reflective surfaces. The musicians, music stands, and audience members can be obstacles to the direct sound and wall reflections. This impacts ensemble conditions onstage [Kirkegaard et al., "Concert Acoustics: The Performers' Perspective," J. Acoust. Soc. Am. Suppl. 1 84, S20 (1988)] as well as issues of clarity, imaging, and envelopment in the audience. In some notable cases, the character of the fixed absorption-seating predominantly-changes the spectrum and spatial quality of the reverberation with occupancy. Comparisons of occupied/unoccupied data will be used to illustrate the dependence of the early echo structure and reverberation upon occupancy and its relationship to the design process.

\section{1:55}

2pAA2. The Academy of Music, Philadelphia-Preliminary model studies of the potential for coupled volumes. A. Harold Marshall (Marshall Day Associates) and Dana L. Kirkegaard (Kirkegaard \& Associates, 4910 Main St., Downers Grove, IL 60515)

A 1:24 scale model of the occupied hall is being used to explore the feasibility of coupling additional volume to the Academy of Music in Philadelphia. Data from several schemes will be compared with respect to both listening position and source position. Model measurements are being carried out with the MIDAS test system. The model was calibrated to measurements made in the occupied hall.

2pAA3. Comparison of modern room acoustic measurements in auditoria: An overview of variables, data analysis, and objective criteria. Jerald R. Hyde (Consultant on Acoustics, 1335 Main St., St. Helena, CA 94574)

Over the last two decades, a set of "modern" room acoustic measures has been developed which utilizes the creation of an impulse response at various positions within a room. The idea has been to create a set of information for that space which facilitates correlation with subjective evaluation. Objective measures such as energy ratios, lateral energy fraction, and source strength have been reported frequently in the literature over the past decade, stimulating a concerted effort to standardize the methodology of both the measurements and the data analysis. The goal is to understand the effects of an almost endless set of variables on the data so that one will know which sets of information from different halls and measurement systems can be reasonably compared. This paper will report the results of different measurement systems data in the same auditorium as well as the difference in acoustical properties between the empty and occupied condition for the same space. An approach to how sets of data might be averaged will be explored along with a methodology for predicting full occupancy objective values from "empty" room data. Finally, a range of objective criteria for concert halls will be suggested.

2pAA4. The case for uniformity in the computer storage of room impulse response binary files. Richard $\mathrm{H}$. Campbell (Dept. of Elec. \& Comput. Eng., Worcester Polytech. Inst., 100 Institute Rd., Worcester, MA 01609)

The proliferation of computer-based acoustical data acquisition instruments, which store room impulse responses on magnetic media, suggests that some uniformity and interchangeability of these data would be beneficial. For example, a central, accessible public-domain library of room impulse responses would allow practitioners, researchers, and educators the opportunity of applying their respective post-processing techniques to any of these uniformly stored data from venues around the world. Suggestions are given for headers, data structures, and trailers based on current practice for two systems. An example of a data translation exercise from Ram-Soft-II to MLSSA is used to emphasize the need for a standard. 
2pAA5. Attenuation of sound passing over audience at grazing incidence. Eckard Mommertz (Inst. für Tech. Akust. RWTH Aachen, Templergraben 55, 5100 Aachen, Germany)

In halls with horizontally arranged seats the direct sound propagates nearly parallel to the audience, which causes a frequencydependent attenuation. Although the additional attenuation is remarkable not much experimental data are available in the literature so far; most measurements were carried out in empty halls. The present study examines the effects on sound passing over audience and unoccupied seats at grazing incidence. Measurements were carried out in two halls and for scale model seat rows, using maximum-length sequences in combination with the Hadamard transform. To improve the dynamic range the spectrum of the maximum-length sequences was digitally pre-emphasized in some cases. The investigation showed that the audience causes a considerable, almost constant attenuation in the range between $500 \mathrm{~Hz}$ and $1.5 \mathrm{kHz}$ with an exponential relationship between distance and sound preasure decrease. For higher frequencies the attenuation was dependent on the horizontal angle of incidence with regard to the seat rows. In some cases a significant decrease of sound pressure due to interference effects was observed. In the low-frequency range the well-known "seat-dip" effect occurred.

\section{Contributed Papers}

3:35

2pAA6. Full-wave modeling of the transmission of sound over theater seats: Near-field investigation. Dominique J. Chéenne, Robert D. Kubik, Robert C. Maher, and Ezekiel Bahar (Dept. of Elec. Eng., 209N WSEC, Univ. of Nebraska, Lincoln, NE 68588)

The full-wave theory [E. Bahar, J. Acoust. Soc. Am. 89, 19-26 (1991)] is applied to a computer simulation of sound transmission across a simplified model of theater seats. The acoustic response is derived for observation points above the seats for a range of incident angles and receiver heights. The results obtained from the model study are in good agreement with the experimental data recently obtained by J. S. Bradley [J. Acoust. Soc. Am. 90, 324-333 (1991)]. The full-wave solution accounts for the acoustic pressure diffusely scattered by the chairs as well as the zero-order field scattered by the finite floor and the direct wave from the source. It suggests that the "seat-dip effect" is mostly due to an interference phenomenon at the observation point between the direct field and the scattered field reflected off the floor. The model allows for a detailed analysis of the effect of scattered field by the chairs, both in front of, and behind the receiver. These results suggest the need for the use of high directionality microphones that can distinguish between the forward and backward scattered acoustic pressure during future experiments.

\subsection{0}

2pAA7. A comparison of objective measurements in concert halls and multi-purpose rooms. Loren Raia, Gary W. Siebein, Wei-hwa Chiang, Harold $w$. Doddington, and Wilhelm K. Schwab (Architecture Technol. Res. Ctr., Univ. of Florida, 231 ARCH, Gainesville, FL 32611-2004)

A series of monaural and binaural acoustical measurements were taken at multiple receiver locations from two source locations in four concert halls and in the concert configuration of four multi-purpose performing arts centers. Comparisons both within and among roorns were made to find similarities and differences in the measured acoustical properties of the two types of rooms. Specific investigations tried to relate how the architectural properties of the rooms affected the measured acoustical properties. [Work supported by the National Science Foundation and Concert Hall Research Group.]

\section{4:05}

2pAA8. An assessment of current acoustical measurements in auditoriums in relation to previously established guidelines derived from perceptual experiments in auditorium synthesis. John $\mathrm{J}$. LoVerde (Paul S. Veneklasen and Associates, 1711 Sixteenth St. Santa Monica, CA 90404)

Paul S. Veneklasen and Associates has used auditorium synthesis and acoustical modeling as a guide in the design of auditoria over the past 25 years [Paul S. Veneklasen, Int. Symposium on Arch. Acoustics,
21-42 (1974)]. Optimal settings for ratios of direct to envelopmental and direct to reverberant sound level were established based on perceptual data for a variety of program materials including classical music, music drama, speech, and pipe organ by experienced listeners including conductors, musicians, music critics, concert goers, and acousticians [ $P$. S. Veneklasen, Acoust. Soc. Am. 77th Meeting, Session P, 27-28 (1969)]. Current auditorium acoustic parameters were measured using impulse response techniques in the auditorium synthesis facility for a variety of direct, envelopmental, and reverberant sound level settings. The range of values for the various auditorium synthesis settings is shown for the measured acoustic parameters [source strength $(G)$, early decay time (EDT), early/late energy $(C)$, and lateral energy fraction (LF)]. Based on the guidance from auditorium synthesis, two additional parameters are introduced that should be considered for a more complete assessment of auditoria acceptability. These are the direct to envelopmental and direct to reverberant energy ratios.

\section{$4: 20$}

2pAA9. The control of reverberation. Paul $S$. Veneklasen and Mahabir Atwal (Paul S. Veneklasen Research Foundation, 1711 16th St., Santa Monica, CA 90404)

The variety of programs that a good modern auditorium should accommodate, requires that reverberation should be controllable if each program is to merit optimal acoustical environment. Guidance from auditorium synthesis suggests that, in contrast with the usual doctrine stressing reverberation time, the sound level of the reverberant sound relative to the direct sound is a dominant factor. Following on from the seminal work of Carl Eyring, this paper describes experiments and experience over the past 25 years that suggest a very practical way to achieve the necessary control.

\section{$4: 35$}

2pAA10. The French horn versus the concert hall. Daryl Caswell (Dept. of Mech. Eng., Univ. of Calgary, 2500 University Dr. N.W., Calgary AB T2N 1N4, Canada)

The acoustical problems presented by the French horn in concert halls and recording studios are a constant source of frustration for the acoustician. The unique performance practices employed by the horn player (hand in the bell, pointing the bell to the back of the hall) create confusing and seemingly contradictory acoustical demands. In addition, the nonscientific language of the performer tends to force the acoustician to disregard the very real concerns and knowledge of the musicians. For example, horn players want a diffuse and "mellow" sound but insist on acoustical conditions that allow clear and precise ensemble playing. This paper will describe a solution to the chronic problems experienced by the French horn section of the Calgary Philharmonic Orchestra in the new Jack Singer Concert Hall. This solution was possible because the researcher (a professional horn player and mechanical engineer) was able to translate the concerns of the horn players into scientifically acceptable terms and use this information to direct the project. 
diffusors. The theatre has a volume of $10500 \mathrm{~m}^{3}$, it is $29 \mathrm{~m}$ wide and the

2pAA11. Acoustic desigm of the new Princess of Wales Theatre. John P. M. O'Keefe (Aercoust. Eng. Ltd., 50 Ronson Dr., Suite 127, Rexdale, ON M9W 1B3, Canada)

The acoustic design of the new 2000-seat Princess of Wales Theatre in Toronto is based loosely on the neighboring Royal Alexandra Theatre. The new theatre is a traditional double balcony, horseshoe-shaped room, augmented by a modern interior design. Abstract sculptures by artist Frank Stella provide acoustic diffusion on the balcony facia. On the back walls, diffusion is provided by specially designed primitive root furthest seat is $30 \mathrm{~m}$ from the stage. Most materials in the theatre are either massive or stiff, to encourage acoustic warmth. The natural frequency of the balconies is approximately $6 \mathrm{~Hz}$, higher than normal rhythmic motion. At the Royal Alexandra the natural frequency is half this and the balconies are easily set into motion by patrons during rock musicals. A hybrid computer modeling routine combining the method of images and particle tracing was used to assist in the design. Measurements in the new theatre will be presented and will be compared to the Royal Alexandra and other similar venues.

TUESDAY AFTERNOON, 18 MAY 1993

SALON MACDONALD, 1:15 TO 4:30 P.M.

\title{
Session 2pEA
}

\section{Engineering Acoustics: Transducers, Sonar, Arrays, and Velocity}

\author{
Thomas R. Howarth, Chair \\ Naval Research Laboratory, Underwater Sound Reference Detachment, P.O. Box 568337, Orlando, Florida 32856-8337
}

\section{Contributed Papers}

$1: 15$

2pEA1. Deflections and vibrations of the unimorph flexural disk transducer. Daniel M. Warren, L. Dwynn Lafleur, and F. Douglas Shields (Natl. Ctr. for Phys. Acoust., Coliseum Rd., University, MS 38677)

Previously, a simple electroacoustic model of a transducer constructed of flexural mode piezoelectric disks [Warren et al., J. Acoust. Soc. Am. 91, 2325 (A) (1992)] and a derivation of the governing differential equations for the flexural disk as a layered piezoelectric plate [Warren et al., J. Acoust. Soc. Am. 92, 2292 (A) (1992)] have been presented. In the current work, solutions of the differential equations are sought for static deflections and dynamic vibrations, under a variety of boundary conditions. The technique used to treat laminated plates with lamina of differing radii, of which unimorphs are examples, is emphasized. The results are compared to the classic theories [S. Hanish, NRL Rep. 5259 (1959) and R. S. Woollett, USL Res. Rep. 490 (1960)] of which this work is an extension. [Work supported by ONR.]

2pEA2. Split-dual beam array transducer design and computer simulation. Dehua Huang, J. Lan, R. K. Jeffers, and S. G. Boucher (Airmar Technol. Corp., Meadowbrook Dr., Milford, NH 03055)

Both dual-beam and split-beam array transducers are widely employed in underwater sonar systems. The split-beam principle is used to find the position of individual targets, for target strength measurements and fish stock assessment. The dual-beam array transducer can transmit signals on one beam and receive the echoes with either one or two beams and has been widely used for target strength measurements and fish stock assessment. Here, a new array transducer has been developed, which combines the strengths of split-beam and dual-beam array transducers. The dual-beam mode is achieved by driving the linear array elements with Chebyshev weighting, while the split-beam mode is obtained by driving the quadripartite subarrays. Furthermore, this new array transducer can transmit signals in split-beam mode and receive the echoes in the dual beam mode, or vice versa. The configuration of the new array transducer will be presented. The beam patterns at different transmitting and receiving situations have been numerically simulated.
1:45

2pEA3. A finite element compatible bond graph modeling approach for transducers applied to the piezoelectric thickness vibrator. Won-Kyu Moon and Ilene J. Busch-Vishniac (Dept. of Mech. Eng., Univ. of Texas, Austin, TX 78712)

A bond graph modeling method suitable for transducers and compatible with finite element formulations is developed. This formulation leads to a new definition of the generalized displacements for a continuous system. The newly defined coordinates are easily interpreted physically and easily used in analysis of the system performance. The method is demonstrated for a piezoelectric thickness vibrator. Compared to the Mason equivalent circuit models, the bond graph modeling method presented offers the primary advantage of physical realizability. Compared to circuit models based on standard discrete electrical elements, the main advantage of the bond graph modeling method is a greater physical accuracy (which is the direct result of using multiport energic elements)

\section{2:00}

2pEA4. Effect of an underlying compliant layer on transducer performance. Rose M. Young (SFA, Inc., 1401 McCormick Dr., Landover, MD 20785) and R. D. Corsaro (Naval Research Laboratory, Code 7135, Washington, DC 20375-5350)

The performance of acoustic transducers (sensors and actuators) can be degraded or enhanced by the structure on which they are mounted. For various numerical structural acoustics studies, a simple computationally efficient model is required for estimating the change in transducer performance resulting from variations in the front and back face impedances. The approach used here is based on simple physical principles and intuition, which makes it well suited for incorporation in more-complicated structural models since it is conducive to direct extraction of physically meaningful forcing functions. As an example of its use, the performance of pressure sensors, velocity sensors, and acoustic drivers on an air-backed steel plate are estimated, and then the change in performance when a layer of compliant material is inserted between the transducer and the plate is calculated. Results are compared with a Mason-type equivalent circuit model.

2:15

2pEA5. Three-dimensional finite element model of a high-power, low-frequency ring-shell flextensional sonar transducer. Rogerio N. 


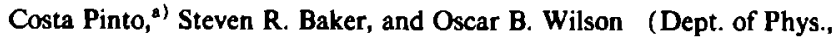
U.S. Naval Postgraduate School, Monterey, CA 93943)

A three-dimensional finite element model of a high-power, lowfrequency ring-shell flextensional transducer [Sparton of Canada, Ltd., model 34A0610] has been developed for use with the ATILA code [Decarpigny et al., J. Acoust. Soc. Am. 78, 1499-1507 (1985)]. A three-dimensional model is needed to represent nonaxisymmetric vibrations that can occur in a dense sonar array. The model was derived from a two-dimensional axisymmetric model developed by Blottman [J. B. Blottman, Naval Undersea Warfare Center, New London Laboratory, Technical Memorandum No. 89-1090 (1989)]. In extending Blottman's model to three dimensions, reasonable simplifications were made to minimize the number of elements required to faithfully represent the dynamical behavior of the transducer in the frequency range of interest. A description of the model and the results of in-air modal and in-water harmonic analyses will be presented. [Work sponsored in part by the Naval Postgraduate School and the Naval Undersea Warfare Center.]

a) Lieutenant Commander, Brazilian Navy.

\section{2:30-2:45 Break}

2pEA6. Abstract withdrawn.

2pEA7. Piezoelectric composites of the 1-3 type used as underwater sound sources. Wayne T. Reader and David F. Sauter (Vector Res. Co., Inc., Ste. 700, 2101 E. Jefferson St., Rockville, MD 20852)

Piezoelectric composites with 1-3 connectivity used in the thickness mode have traditionally used a relatively rigid polymer as the matrix encapsulant to force uniform surface motion. Finite element analyses are employed to show that these rigid polymers constrain the piezoelectric rod motion sufficiently to reduce rod displacements by $50 \%$ or more. The magnitude of the reduction depends upon the ceramic volume fraction, the rod aspect ratio, rod spacing, and of course the stiffness of the matrix. Further, it has been shown [W. A. Smith, ONR Tech Note (31 May 1991)] that the hydrostatic response, rather than the thickness mode alone, controls the performance of 1-3 composites used as underwater sound sources. It is shown in this paper that efficient lightweight underwater sound sources can be produced by: (1) using lightweight rigid cap plates and a very compliant matrix polymer to produce uniform surface motion comparable to the motion of the unconstrained rod, and (2) using rigid edge strips bonded to the cap plates to prevent edge motion, thereby effectively limiting the transmitting response to the thickness mode. [Work supported by ONR.]

\section{3:00}

2pEA8. Water depth and drive voltage dependence of the acoustic parameters of a barrel-stave flextensional projector. Dennis $F$. Jones (Defence Res. Establishment Atlantic, Dartmouth, NS B2Y 3Z7, Canada) and Mark B. Moffett (Naval Undersea Warfare Ctr., New London, CT 06320)

A low-frequency barrel-stave flextensional projector for sonar and general underwater acoustics applications has been built at the Defence Research Establishment Atlantic in Dartmouth, Nova Scotia. The projector was calibrated at the Seneca Lake sonar test facility at Dresden, NY, as a joint research venture with the Naval Undersea Warfare Center, New London, Connecticut. The transmitting voltage response (TVR), mechanical quality factor $\left(Q_{m}\right)$, electrical admittance, and electroacoustic efficiency $\left(\eta_{e a}\right)$ were measured for driving voltages up to $5.0 \mathrm{kV}$ rms at four depths: $30,61,91$, and $123 \mathrm{~m}$. At $5.0 \mathrm{kVrms}$, the resonance frequency $\left(f_{0}\right)$ increased from 780 to $840 \mathrm{~Hz}, Q_{m}$ decreased from 4.4 to 3.3 , and $\eta_{\mathrm{ca}}$ decreased from $87 \%$ to $71 \%$, as the depth was increased from 30 to $123 \mathrm{~m}$. The change in each acoustic parameter, averaged over the four depths of interest, was $-10 \mathrm{~Hz} / \mathrm{kV}$ rms for $f_{0}$, $+0.4 \mathrm{~dB} / \mathrm{kVrms}$ for the TVR at resonance, and $+0.1 / \mathrm{kVrms}$ for $Q_{m}$, as the driving voltage was increased from 0.1 to $5.0 \mathrm{kVrms}$. The dependence of the parameters on depth and driving voltage are discussed in terms of the interactions between the rubber boot and the staves. [Work sponsored in part by the U.S. Office of Naval Research.]

\section{3:15}

2pEA9. Electroacoustic eraluations of 1-3 piezocomposite rings. Thomas R. Howarth, Kurt M. Rittenmyer, and Robert Y. Ting (Naval Res. Lab., Underwater Sound Reference Detachment, P.O. Box 568337, Orlando, FL 32856-8337)

Two 1-3 piezocomposite rings have been recently electroacoustically evaluated for sonar application. The two rings have outer diameters of $345 \mathrm{~mm}, 101.6-\mathrm{mm}$ height and a wall thickness of $5 \mathrm{~mm}$. Both have a $5 \%$ Navy type II piezoceramic volume concentration loading. The host epoxy of one ring is soft while the other is hard. Both rings had 17.78 by $25.4 \mathrm{~mm}$ rectangular elements etched into a 3 by 3 element pattern in their outer electrode for direct in-water performance comparison with a previous design utilizing individual elements of the same dimensions [Howarth et al., J. Acoust. Soc. Am. 91, 2325-2326 (A) (1992)]. The in-water measurements include wide-band free-field voltage sensitivity (FFVS), transmitting voltage response (TVR), and radiation patterns in both the $X Y$ and $X Z$ planes. A laser Doppler vibrometry was used for in-air evaluation of the piezoelectric charge constant and surface displacement. Interelement coupling data will also be presented. [Work sponsored by the Office of Naval Research.]

\section{3:30}

2pEA10. Spacing effects in large-area hydrophone arrays. Richard N. Brown and E. F. Berkman (Systems and Technol. Div., Bolt Beranek and Newman, Inc., 70 Fawcett St., Cambridge, MA 02138)

It has long been known that large hydrophones filter out the highwave number, convective components of turbulent boundary layer (TBL) noise. A two-dimensional array constructed of $N \times M$ hydrophones might be expected to increase the filtering by a factor of $N \times M$, but, in practice, inter-hydrophone spaces ("gaps") diminish this benefit. An analysis, based on a wave-vector approach, has been done to study the effect of gaps in two-dimensional arrays. It is shown that the effect of gaps is to allow leakage into the array response function at wave numbers corresponding to aliasing lobes. This leakage can extend to moderately high wave numbers, although the details of the leakage are sensitive to the exact gap size. Numerical estimates of array self-noise due to flow noise are made using models of the TBL wall pressure 
wave-vector spectrum. It is shown that, at low values of $k_{0} L\left(k_{0}\right.$ is the acoustic wave number and $L$ is characteristic array length), the leakage from the convective and subsonic components of the TBL can be substantial, even for relatively small gaps.

\section{3:45}

2pEA11. Comparisons of two encapsulating methods on the acoustic performance of a passive cylindrical array. Alan $K$. Walden, Thomas R. Howarth, Mark L. Pecoraro, and Allan C. Tims (Naval Res. Lab., Underwater Sound Reference Detachment, Orlando, FL 32856)

The purpose of this study was to determine the effects of encapsulation methods on the acoustic performance of a passive, cylindrical, high gain, broadband (multi-octave) array. Both an oil-filled boot and a polyurethane coating were considered. The array consisted of 36 lead titanate elements mounted in a 3 by 12 matrix along 180 deg of the circumference of a steel cylinder. Original intentions were to rely on the oil-filled booting to ensure performance void of transverse mode coupling between neighboring elements. Measurements of the oil-filled array showed a "scalloping" effect in the radiation patterns as well as significant disturbances throughout the receiving response [Howarth et al., J. Acoust. Soc. Am. 91, 2325-2326 (A) (1992)]. This study took the same array and encapsulated it in a polyurethane elastomer. Experimental data indicate that many of the perturbations notes in the oilfilled configuration were considerably diminished after potting. Performance comparisons between the two encapsulating methods shall be presented and discussed. [Work supported by NSWS/Carderock Division.]

\section{4:00}

2pEA12. Optimized constant beam width arrays. Jefferson A. Harrell (Jet Propulsion Labs., 4800 Oak Grove Dr., Pasadena, CA 91109) and Elmer L. Hixson (Univ. of Texas, Austin, TX 78712)
It has been previously shown by the authors [JAES 34, 221 (April 1990); J. Acoust. Soc. Am. 91, 2326 (A) (1992)] that the main beamwidth of a loudspeaker array can be held constant over an octave by the superposition of two arrays by using suitable filter functions. The beam patterns are identical at the end (design) frequencies, however, deviations occur at frequencies between the extremes. The deviations are representative of unwanted acoustic energy if the deviation is positive when referenced to the desired pattern. While the array has some error at these design frequencies (for example, undesired side and backlobes), the total error energy integrated over the octave may be greater than that of other arrays that use element weights that allow more error at the design frequencies. Presented is an algorithm to minimize the deviations between design frequencies while minimizing the error energies, which is based on a Wiener adaptation. The results are compared to hand-generated guesses. The impact of linear and nonlinear cost functions is considered.

2pEA13. Speed of sound in pure water as a function of temperature. Nykolai Bilaniuk and George S. K. Wong (Inst. for Natl. Measurement Standards, Natl. Res. Council, Ottawa, ON K1A 0R6, Canada)

In view of the adoption of the International Temperature Scale of 1990 (ITS-90), which defines the International Celsius Temperatures, $t_{90}$, the dependence on temperature of the speed of sound in pure water is examined. Drawing on the experimental data published previously [V. A. Del Grosso and C. W. Mader, "Speed of Sound in Pure Water," J. Acoust. Soc. Am. 52, 1442-1446 (1972)], it is found that the change from the previous $t_{68}$ scale is significant. At $100^{\circ} \mathrm{C}$, the difference between the two scales $\left(t_{68}-t_{90}\right)$ is $0.026^{\circ} \mathrm{C}$, resulting in a difference of $0.022 \mathrm{~m} / \mathrm{s}$ for the speed of sound. The speed of sound is fitted to a new fifth-order polynomial applicable over the $t_{90}$ range $0-100^{\circ} \mathrm{C}$.

TUESDAY AFTERNOON, 18 MAY 1993

ADAM ROOM, 1:30 TO 4:45 P.M.

Session 2pMU

\title{
Musical Acoustics and Psychological and Physiological Acoustics: Auditory Organization in Music and Speech I: Theory, Phenomena, and Models
}

\author{
Punita G. Singh, Chair \\ Department of Psychology, McGill University, 1205 Doctor Penfield Avenue, Montreal, Quebec H3A 1B1, Canada
}

Chair's Introduction-1:30

Invited Papers

1:35

2pMU1. Auditory scene analysis: Theory and phenomena. Albert S. Bregman (Psychol. Dept., McGill Univ., 1205 Dr. Penfield Ave., Montreal, PQ H3A 1B1, Canada)

Many natural listening environments face the auditory system with a jumble of overlapping sound-producing events, from which it must recover separate descriptions of the individual sounds (auditory scene analysis). Its most basic method is "primitive" auditory grouping, a pre-attentive process that analyzes the incoming signal into components and then uses certain acoustic relations among them to link them into sets that later-acting recognition processes will prefer to treat as coming from separate events. Components (or features) will be linked when they exhibit relationships to one another that would be very improbable had they actually come from unrelated events. The evidence for grouping, derived from different relations, is allowed to compete and collaborate, in the linking of subsets of components or features. In a mixture, the listener tends to hear these subsets as distinct but overlapping sound sequences, each with its own properties. The perceived qualities that can be affected by this organizing of data include the melodic and rhythmic qualities of the perceived sounds, their pitches, timbres, numerosity, temporal order, and even their perceived spatial positions. 
2pMU2. Auditory grouping in lateralization, pitch, and vowel perception: A comparison of the effects of onset asynchrony and of mistuning. C. J. Darwin and Nicholas I. Hill (Lab. of Exptl. Psychol., Univ. of Sussex, Brighton BN1 9QG, UK)

This paper reviews the effect of two powerful cues to sound segregation, onset asynchrony and mistuning, in a number of different experimental paradigms. Although both cues substantially influence grouping in all three paradigms, there are quantitative differences across the paradigms. An onset asynchrony of a few tens of milliseconds is sufficient to allow listeners to hear out a single low-numbered harmonic from a complex and for it to be lateralized largely independently of the other harmonics. It is also enough to remove that harmonic from the calculation of vowel quality. But a substantially greater asynchrony (over 100 $\mathrm{ms}$ ) is needed in order to remove a single (mistuned) harmonic from a complex for the purpose of calculating its pitch. When a single low-numbered harmonic is mistuned, a mistuning of less than $1 \%$ is generally sufficient to allow it to be heard out. At about 3\% mistuning, the mistuned harmonic can be lateralized largely independently of the other harmonics, but will still make a full contribution to the pitch and the vowel quality of the complex. A substantially reduced contribution both to the vowel quality and to the pitch of the complex requires a larger mistuning-around $8 \%$. [Work supported by UK SERC \& MRC.]

2pMU3. Implicit assumptions in modeling higher level auditory processes. Richard E. Pastore (Ctr. for Cognitive and Psycholinguistic Sci., SUNY, Binghamton, NY 13902-6000)

There has been growing interest in the investigation of auditory stimulus processing at levels considered to be clearly beyond or above the limits imposed by the peripheral auditory system. Efforts to investigate such higher levels of processing of complex stimuli are nearly always based upon assumptions about perceptual and decision processes that limit the range of reasonably valid conclusions. Such assumptions are usually implicit and often not immediately recognized. To illustrate the critical role played by such implicit underlying assumptions, existing and new research on the perception of formant transitions in speech will be examined in terms of basic assumptions whose recognition can modify (and sometimes strengthen) conclusions about higher levels of perceptual processing. Discussion will focus on the implications of fundamental assumptions for the identification and demonstration of important principles of perceptual organizing (e.g., Gestalt, feature integration) and for testing hypotheses about alternative perceptual models, modes, or modules. [Research supported in part by NSF and AFOSR.]

3:05-3:15 Break

\section{Contributed Papers}

3:15

2pMU4. Perceptual organization of speech signals: Clues from studies of sinewave replicas of utterances. Robert E. Remez, Jennifer S. Pardo (Dept. of Psychol., Barnard College, 3009 Broadway, New York, NY 10027), and Philip E. Rubin (Haskins Labs., New Haven, CT 06511)

The perceptual organization of speech is a challenge for accounts proposing a two-stage model in which primitive auditory grouping is followed by schematic secondary processes. This difficulty stems from the diversity of acoustic constituents of speech signals, which is attributable in turn to the mechanics of vocal sound production. Primitive mechanisms that rely on similarity principles to group like elements therefore fail to parse the sound stream isomorphically to the sound sources. The insufficiency of primitive grouping mechanisms for organizing speech signals is often admitted, and knowledge-based latterly occurring processes are often invoked to explain the self-evident perceptual coherence of utterances. Studies with sinewave replicas of words and sentences show that phonetic perception occurs in conditions that thwart the application of primitive grouping mechanisms as they are presently understood. Psychophysical tests with sinewave words suggest that the perceptual organization of speech is distinct from other auditory organization. Because classic evidence and arguments discourage schema-based conceptualizations of phonetic processes, the two- stage model of organization appears to be implausible for speech. Perceptual organization of speech can better be rationalized by a phonetically specific facility that organizes and analyzes utterances. [Research supported by NIDCD and NICHD.]

\section{3:30}

2pMU5. Effects of discontinuous $F 3$ transitions on phonetic identification and fusion. Michael D. Hall and Richard E. Pastore (Ctr. for Cognitive and Psycholinguistic Sci., SUNY, Binghamton, NY 13902-6000)
According to general perceptual principles, figural goodness and feature integration of stimuli should reflect the continuity between components; in $\mathrm{CV}$ syllables this continuity might be between phonemedistinguishing glide (formant transition) and vowel formant. Two experiments examined continuity effects on identification and fusion for sinewave syllables based on common $\mathrm{CV}$ parameters. Experiment 1 provided a perceptual basis for equating isolated glides and stationary tones. Independent of glide direction, perceptual matches tended to be based upon temporally adjacent portions of the stimuli. Using matched stimuli, experiment 2 evaluated the relative contribution to phonetic identification of (1) frequency continuity of transition and formant and (2) transition direction. Subjects discriminated the original syllables from binaural and dichotic syllables containing either continuous or discontinuous $F 3$ transitions. Identification was influenced more by transition direction than by goodness of physical continuity. Furthermore, very different pattems of results were obtained for binaural conditions and dichotic conditions in which responses indicated fusion. The nature of information provided by transitions and operation of schema are discussed. [Work supported by NSF and AFOSR.]

\section{$3: 45$}

2pMU6. Effect of onset asynchrony and mistuning on the lateralization of a pure tone embedded in a harmonic complex. Nicholas I. Hill and C. J. Darwin (Lab. of Exptl. Psychol., Univ. of Sussex, Brighton BN1 9QG, UK)

The effect of onset asynchrony and mistuning on the binaural processing of multi-tone stimuli was investigated using a paradigm derived from that of Trahiotis and Stern [C. Trahiotis and R. M. Stern, J. Acoust. Soc. Am. 86, 1285-1293 (1989)]. A tonal complex comprising harmonics 2 to 8 of $100 \mathrm{~Hz}$ and presented with an IDT of $1.5 \mathrm{~ms}$ gave rise to a single image lateralized towards the ear receiving the leading signal. However, when the central $500-\mathrm{Hz}$ component was delayed by $40 \mathrm{~ms}$ relative to the flanking tones, it was heard out as a separate tone shifted towards the opposite side of the head. Similar effects were ob- 
served when the $500-\mathrm{Hz}$ component was mistuned from the flanking complex, with shifts of $\pm 3 \%$ being sufficient for the mistuned component to be lateralized in the vicinity of the mid-line. The results demonstrate that both onset asynchrony and mistuning influence which frequency components IDT information is integrated across. [Work supported by UK SERC and MRC.]

\section{$4: 00$}

2pMU7. Computer simulation of auditory stream segregation in sequential-tone sequences. Michael W. Beauvois (IRCAM, 31, rue St-Merri, F-75004 Paris, France) and Ray Meddis (Univ. of Technol., Loughborough LE11 3TU, UK)

A simple computer model is described that takes a novel approach to the problem of accounting for perceptual coherence among successive pure tones of changing frequency by using simple physiological principles that operate mainly at a peripheral, rather than a central level. Using a single set of parameter values, the model is able to reproduce a number of streaming phenomena found in the literature. These are: (1) the buildup of auditory streaming over time, (2) the temporal coherence and fission boundaries of human listeners, (3) the trill threshold, and (4) the stream-organization process exhibited by human listeners when presented with $\mathrm{ABC}$ tone sequences. Whereas streaming phenomena are generally accounted for in terms of an auditory scene-analysis process that works on the basis of Gestalt perceptual principles, the success of the model in reproducing experimental data obtained from humans justifies the potential value of a low-level analysis for explaining high-level psychological phenomena such as Gestalt auditory grouping, and suggests that some Gestalt auditory grouping may be the product of low-level auditory processing. [Work supported by SERC(UK) and CNRS(France).]

\section{$4: 15$}

2pMU8. A computer implementation of psychoacoustic grouping rules. Daniel P. W. Ellis (MIT Media Lab, Perceptual Computing Group, E15-368C, Cambridge, MA 02139)

Many of the rules employed by the human auditory system to fuse and segregate acoustic energy into separately perceived sources are described by Bregman [A. S. Bregman, Auditory Scene Analysis (MIT, Cambridge, MA, 1990)]. However, the precise application of these rules to anything but the most simple experimental stimuli is less well understood. This idealized model of low-level auditory processing [Ellis and
Vercoe, J. Acoust. Soc. Am. 91, 2334 (A) (1992)]; [Ellis, J. Acoust. Soc. Am. 92, 2376 (A) (1992)] is specifically designed to facilitate automatic grouping for real stimuli. This is achieved by representing sounds as sets of sinusoid tracks, the discrete time-frequency elements required by such rules. This system is discussed for grouping tracks, and it is shown that the results are obtained by applying simple rules for harmonicity, common fate, and proximity to some real sounds. The means by which ambiguity has been resolved between different types of rule, along with other issues arising from the model, will also be described.

2pMU9. Interactive computational auditory scene analysis: An environment for exploring auditory representations and groups. Malcolm Crawford, Martin Cooke, and Guy Brown (Dept. of Comput. Sci., Univ. of Sheffield, Regent Court, 211 Portobello St., Sheffield S1 4DP, England)

Computational modeling of auditory scene analysis (ASA) offers a new paradigm for experimentation. It permits a novel approach to the development of theories of grouping, and to the design of experimental stimuli. For example: (i) grouping algorithms can be implemented, and validated against experimental data; (ii) experimental data can be analyzed to suggest a possible representation in the auditory system, and to test conformance with expectations; (iii) computational implementation can expose deficiencies in current theory. Over the last 4 years, the Sheffield Auditory Group has developed a rich set of representations used for investigating computational ASA [G. J. Brown, "Computational Auditory Scene Analysis: A Representational Approach," Ph.D. thesis, University of Sheffield (1992); M. P. Cooke, Modelling Auditory Processing and Organisation (Cambridge U.P., Cambridge, UK, 1993)]. There representations include computational maps for onsets, offsets, frequency transitions, and periodicities, in addition to higher-level symbolic representations of acoustic components. Recently, an environment has been created that brings together this diverse collection into a uniform framework for display, resynthesis, and experimentation. The environment supports experimental investigation and allows the "debugging" of stimulus selection. Further, it acts as a canvas onto which the results of auditory grouping can be drawn. It also serves as a tutorial in this increasingly complex field. The practical application of these points is illustrated in a case study that maps the path from stimulus generation to grouping by listeners or machine.

TUESDAY AFTERNOON, 18 MAY 1993

QUEBEC SUITE, 2:00 TO 4:05 P.M.

\title{
Session 2pNS
}

\section{Noise: General}

\author{
George P. Wilson, Chair \\ Wilson, Ihrig and Associates, Inc., 5776 Broadway, Oakland, California 94618
}

Chair's Introduction-2:00

\section{Contributed Papers}

\section{2:05}

2pNS1. The generation of high-speed train noise from roughness. Paul J. Remington and Jonathon Webb (Bolt Beranek and Newman, Inc., 70 Fawcett St., Cambridge, MA 02138)

The forces generated in the contact area between railroad wheels and rails due to small scale roughness has been estimated using three analytical techniques: a simple average of the roughness, a point react- ing spring model, and a full elastic interaction model. Roughness data measured in a number of parallel tracks in the rolling direction on partially worn wheels and rails was used in these three models. The forces estimated by all three analytical techniques were remarkably similar in the range of wavelengths important for noise generation from high-speed (200-to $400-\mathrm{km} / \mathrm{h}$ ) trains. Wave-number decomposition of the roughness data has shown it to be bandlimited in the direction transverse to rolling, a result consistent with the similarity in the predictions of the three analytical techniques. The influence of wheel and 
rail profile and high roughness amplitudes at low wave number will be discussed.

\section{2:20}

2pNS2. Aerodynamic noise of high-speed ground vehicles. Bennett M. Brooks (Brooks Acoust. Corp., 27 Hartford Tumpike, Vernon, CT 06066)

A potentially serious problem for high-speed ground transportation vehicles is the aerodynamic self-noise generated simply by the vehicular movement through the air. This noise constitutes the floor to the total noise produced by the vehicle regardless of the method of propulsion. Several methods of calculating the sound pressure levels of this aerodynamic noise are developed based on the interaction of turbulent boundary layer flows with solid surfaces. These calculations are applied to a model for a high-speed train. Calculated noise levels compare favorably with the levels measured for unpowered vehicles operating at typical high speeds. [Work partially supported by United Technologies Corporation.]

2pNS3. Jet noise generation mechanism. Seungbae Lee and William C. Meecham (Dept. of Mech., Aerosp., and Nucl. Eng., Univ. of California, Los Angeles, CA 90024)

The breakdown process of the initial large-scale structures into substructures near the end of the potential core is examined and the production of aerosound as a result is discussed. The quadrupole-like directivity pattern of jet noise is numerically obtained by applying the large-eddy simulation (LES) technique for the fluid flow. The Lighthill analogy is used to obtain the acoustic far field. Most of the sound comes from a region near the end of the potential core. A discussion to explain this quasiaxisymmetric directivity pattern is given. The directivity maximum is obtained at about $45^{\circ}$ from the axis of the jet exhaust. This will be explained in terms of turbulent velocities. The convective effect on sources is introduced by ray tracing to calculate retarded times. The result is to obtain more radiation downstream, as is observed. The relation of this correction to the Lighthill theory will be discussed.

2pNS4. Development of efficient methods to reduce the noise level of dishwashers. Cüneyt Öztürk and Şemsettin Eksert (Arçelik A. S, Product Develop. Dept., Çayirova-41460, Istanbul, Turkey)

The aim of these studies was to keep the machine within the 50-dBA range to be competitive within the market. In order to achieve the ultimate goal of noise reduction, measurement methodology evolved on the basis of four steps. Source identification studies have led one to consider that the tub of the machine was driven to act as a loudspeaker because of permanent splashes of the sprayed waters and transmitted vibration of the motor housing, which was located over the tub. Studies have been focused around the elimination of high-frequency water noises and torsional noises of the motor that were dominant around the line frequency and double-line frequency and had a tendency to coincide with the natural frequency of the tub. Achievement of the proposed noise levels has led to studies of new treatment methods to keep the machine within the ranges of acceptable noise quality

\section{3:05}

2pNS5. Testing instruments that measure transient sounds. Robert W. Young (1696 Los Altos Rd., San Diego, CA 92109)

An unattended instrument for audible environmental noise must measure and report whatever and whenever sounds occur at the monitoring location. Test signals for steady sounds are here voltageequivalents of sound pressure levels; the signals are sine waves at onethird octave intervals, $10 \mathrm{~Hz}$ to $20 \mathrm{kHz}$. Test signals for responses to transient sounds are sound exposure levels of single-cycle sinebursts, 10 $\mathrm{Hz}$ to $20 \mathrm{kHz}$. For a wide-band system and $1 \mathrm{kHz}$, the sound exposure level of a single cycle relative to steady sound pressure level is theoret- ically $-10 \log (1000)=-30.0 \mathrm{~dB}$. With A-weighting, the relative sound exposure level is theoretically $-30.7 \mathrm{~dB}$; with CEL-493 integrating sound level meter No. 198107 the relative level was also $-30.7 \mathrm{~dB}$. At $32 \mathrm{~Hz}$ (more precisely $31.6228 \mathrm{~Hz}$ ), the theoretical relative A-weighted sound exposure level is $-10.6 \mathrm{~dB}$ [not $-10 \log 31.6228=$ -15.0 ]; with the CEL-493 the relative level was $-10.8 \mathrm{~dB}$. For a conventional sound level meter, at $1 \mathrm{kHz}$ the theoretical fast A-weighted sound level of a single cycle, minus steady sound pressure level, is $-21.8 \mathrm{~dB}$; for CEL-493 the difference was $-21.7 \mathrm{~dB}$. At $32 \mathrm{~Hz}$ the theoretical fast A-weighted sound level of the single cycle, minus steady level, is $-2.2 \mathrm{~dB}$; for CEL-493 the difference was also $-2.2 \mathrm{~dB}$.

\section{$3: 20$}

2pNS6. Vibroacoustical design for the connecting structure between a vibrating source and a lightweight envelopping structure. Nicolas Lessard, Frédéric Laville, and François Charron (Mech. Eng. Dept., Univ. de Sherbrooke, 2500 Boul. Université, Sherbrooke, PQ J1K 2Rl, Canada)

This research is on the design of the connecting structures between a vibrating source and a lightweight structure with application to ventilation devices with a $120-\mathrm{Hz}$ excitation by an electrical motor. The design method had two objectives: a low noise level and little variability in level (robust design). Three complementary approaches have been used to identify the various design, manufacturing, and assembling parameters that affect the low-frequency noise level. First, acoustical engineering judgment lead to basic design concepts. Two other approaches were then used to analyze the effect of these basics design concepts and various manufacturing and assembly parameters on the noise level. The first approach was a statistically designed experimentation to identify key parameters. The second approach was a finite element modelization including a sensitivity analysis of the quadratic velocity as a function of key parameters. The design approach applied to the case of an electrical motor in a box-like structure lead to the choice of a low-noise connecting structure as well as the identification of important manufacturing and assembly parameters to be controlled to ensure a low noise variability.

\section{3:35}

2pNS7. Micro-perforated sheet metal and its application to silencer design. Mei Q. Wu (6682 Ganymede Rd., Mississauga, ON L5N 6R1, Canada)

Micro-perforated sheet metal is used to make no-media silencers, i.e., silencers without fiberglass, mineral wool, or any kinds of fibrous filling materials. The advantages of no-media silencers are that they will not release small fibers to pollute the air and they will not be contaminated by the moisture and bacteria in the air. No-media silencers are extensively used in the air-handling system ducts of hospitals, pharmaceutical factories, and chemical laboratories. This paper summarizes the research work of developing a theory to predict the performance of no-media silencers. The theory is based on Maa's micro-perforation theory. Two modifications have been made to Maa's original theory so it can be applied to air duct silencer design. One of the modifications is to introduce the effect of grazing incident sound wave, and the other is to introduce the effect of air flow. Based on the modified theory, a computer program was developed to calculate the insertion loss and the pressure drop of a silencer. The calculated results were compared with test results. The comparison indicated a reasonably good agreement between the theoretical prediction and the test results. [Work supported by BVA Systems.]

$$
3: 50
$$

2pNS8. A new number-theoretic acoustical concrete masonry unit Peter D'Antonio (RPG Diffusor Systems, Inc., 651-C Commerce Dr., Upper Marlboro, MD 20772)

A new number-theoretic structural acoustic concrete masonry unit will be described. These new acoustical building blocks uniformly disperse reflections created by the flat surfaces of conventional absorbing 
block over a broad range of frequencies, while simultaneously providing extended broadband low-frequency absorption, load-bearing reinforced construction, fire-safety, high transmission loss, economy, and an aesthetic appearance. Random-incidence acoustical testing yielded an NRC of 0.85 for the unpainted block and 0.40 for the painted block, with $100 \%$ efficiency at $100 \mathrm{~Hz}$. The STC for painted block was 55 . Structural testing for both compressive strength and flexural mid-span deflection were in compliance with ASTM C90-90. A novel cavity de- sign, which provided extended low-frequency absorption will be described. The system consists of three blocks. The A and B blocks facing the noise source each contains a partial quadratic residue sequence and the rear mounting $\mathbf{C}$ block forms a structural cavity. The acoustical properties of the system address the needs of all musical facilities, movie theaters, auditoriums, school rehearsal rooms, and noise control applications including highway barriers, airports, power plant transformer substations, etc.

TUESDAY AFTERNOON, 18 MAY 1993

SALON RENAISSANCE, 12:55 TO 5:00 P.M.

\title{
Session 2pPA
}

\section{Physical Acoustics and Structural Acoustics and Vibration: Anderson Localization in Acoustics and Structural Vibration II}

\author{
Ping Sheng, Chair \\ Exxon Research and Engineering Company, 79 Route 22 East, Annandale, New Jersey 08801-0998
}

\author{
Chair's Introduction-12:55
}

Invited Papers

1:00

\begin{abstract}
2pPA1. Stop bands in periodic and random media. E. N. Economou (FORTH, P.O. Box 1527, 71110 Heraklio, Crete, Greece)
In periodic media, frequency regions may exist at which no bounded at infinity solution of the wave equation exists. In random systems frequency regions may appear at which all bounded solutions are localized, i.e., decay exponentially at infinity. Both regions give rise to stop bands. Such stop bands appear easily for acoustic waves in bubbly liquids. They are also predicted to exist for elastic waves in binary composites consisting of gold or lead spherical inclusions periodically placed in a $\mathrm{Si}, \mathrm{Be}_{0}$ or $\mathrm{SiO}_{2}$ (for gold inclusions only) matrix and occupying about $10 \%$ of the volume. The midfrequency $\omega_{g}$ of the stop band was found to satisfy the relation $\omega_{g}=y c_{i f} / R_{\dot{b}}$ where $c_{i l}$ is the longitudinal velocity in the inclusion material, $R_{i}$ is the radius of each inclusion, and $y$ is a number between 1 and 2 .
\end{abstract}

1:30

2pPA2. "Wave automaton" for wave propagation in the time domain. Didier Sornette, Patrick Sebbah, and Christian Vanneste (Lab. de Phys. de la Matière Condensée, CNRS URA 190, Univ. de Nice-Sophia Antipolis, Parc Valrose, 06108 Nice Cedex 2, France)

The new model introduced recently [Vanneste et al., Europhys. Lett. 17, 715 (1992)] for the dynamical propagation of waves in arbitrary heterogeneous media, which is efficient for calculations on large systems $(1024 \times 1024)$ over long times (several $10^{6}$ inverse band widths) will be discussed. Instead of starting from a wave equation or a Hamiltonian that needs to be discretized for numerical implementation, the model is defined by the set of $S$ matrices, one for each node, describing the interaction of the wave field with the scatterers. The different results are shown on wave packets in random media, which have been obtained using extensive numerical simulations on a parallel computer and these numerical results are compared with weak localization predictions. Finally, "wave automaton" is shown to be equivalent to a discretized version of the hyperbolic time-dependent wave and Klein-Gordon equations, when restricted to a suitable subclass of the control parameters, and the relationships between the two formulations of the wave propagation problem are made explicit. Compared to finite-difference versions of hyperbolic equations, the wave automaton is shown to be much more flexible for implementing arbitrary boundary conditions.

2pPA3. Anderson localization in the time domain: Numerical studhes of waves in two-dimenswonal disordered media. Richard L. Weaver (Dept. of Theor. and Appl. Mech., Univ. of Illinois, Urbana, IL 61801)

Experimental work in the time domain on Anderson localization and anomalous diffusion in dimensions greater than one is scant, being confined to some ultrasonic work by Weaver and, inasmuch as measurements of frequency-frequency intensity correlation functions imply measurements of time domain behavior, some microwave work by Genack and co-workers. Numerical work in the time domain is similarly poorly developed. No studies have been conducted with sufficient detail for determination of the effective transport behavior. This paper presents results obtained from new numerical experiments in an attempt to rectify this lack. A numerical model is introduced that allows the tracking of evolving wave energy density in a two-dimensional linear classical wave equation version of an Anderson model diagonally disordered Hamiltonian. The behavior of the evolving average wave energy density in effectively infinite large samples of this system (typically 300 sites by 100 sites) excited by tone burst line sources is presented and the results shown to collapse to an apparent single universal response function with interesting asymptotic character like $\exp \left(-r / \lambda-r^{2} / 4 \beta t\right)$ where $r$ is the distance from the source, $\lambda$ is the localization length, and $\beta$ is a 
constant not equal to the bare diffusivity. The effect of dissipation is also investigated, and, consistent with previous demonstrations, found to be trivial. [Work supported by NSF MSS-91-14360.]

\section{2:30-2:45 Break}

\section{$2: 45$}

2pPA4. Intensity correlation and the approach to localization. Azriel Z. Genack, Narciso Garcia, and Jiang Hong Li (Dept. of Phys., Queens College of the City University of New York, Flushing, NY 11367)

Because the conductance is the prime physical property of interest for electronics and also the universal scaling parameter of the electronic localization transition, considerable attention has focused upon the scaling of conductance. For classical waves, however, the localization transition can be followed not only by the scaling of the average of local or global quantities such as the intensity, transmission, or conductance, but also in a broad array of distributions and correlation functions, in which the proximity to the localization threshold is revealed, even in samples of a fixed size. Quantitative agreement is obtained between microwave measurements of spectral and spatial correlation in samples of randomly positioned polystyrene spheres and Langevin and macroscopic matrix calculations. The degree of correlation is shown to be the inverse of the dimensionless conductance even in the presence of absorption. The intensity distribution in this sample is also given in terms of the dimensionless conductance. Thus the approach to localization is revealed in the statistical character of propagation. The role of dielectric sphere resonances and the breakdown of the diffusion model is metallic mixtures will also be discussed. [Work supported by the NSF and the Petroleum Research Fund of the ACS.]

3:15

2pPA5. Pulses, nonlinearity, and Anderson localization. J. D. Maynard (Dept. of Phys., Penn State Univ., University Park, PA 16802)

It is now well known that disorder in one-dimensional systems results in normal modes that are exponentially localized (Anderson localization). The propagation of waves and pulses through a disordered system is dramatically affected by the localization, and there is some interesting exceptional behavior. The problem is made further interesting by the addition of nonlinearity. A basic question is whether or not nonlinearity destroys Anderson localization. Of all the references, [1] half predict that localization is destroyed, and half predict that it is not. Continuous wave and pulse experiments have been performed on nonlinear disordered acoustic systems and some interesting results have been obtained. An analysis of a nonlinear periodic system, which shows effects of basin crowding, will also be presented. [Work supported by NSF DMR 9000549 and ONR.] P. Devillard and B. Souillard, J. Stat. Phys. (1986); B. Doucot and R. Rammal, Europhys. Lett. (1987); C. Albanese and J. Frohlich, Commun. Math. Phys. (1988); Q. Li et al., Phys. Rev. B (1988); A. Soffer and M. I. Weinstein, Commun. Math. Phys.; R. Bourbonnais and R. Maynard, Phys. Rev. Lett. (1990); Yu. S. Kivshar et al. (1990); R. Knapp et al., Disorder and Nonlinearity, edited by A. R. Bishop, D. K. Campbell, and S. Pnevmatikos (1989).

\section{Contributed Papers}

\section{3:45}

2pPA6. Acoustic waves in bubbly liquids: (1) Can strong localization of waves be attained by internal resonances? (2) Acoustic wave propagation in one-dimensional stratified gas-liquid media: The different regimes. Didier Sornette (Lab. de Phys. de la Matiere Condensee, CNRS URA 190, Univ. de Nice-Sophia Antipolis, Parc Valrose, 06108 Nice Cedex, France and Lab. de Phys. Statist., CNRS and Univ. Paris VI and VII, Paris, France), J.-P. Bouchaud (Lab. Phys. Statist., Univ. Paris VI and VII, Paris, France), and $O$. Legrand (Univ. de Nice-Sophia Antipolis, Nice, France and X-RS, Parc-Club, Orsay, France)

An inhomogeneous medium with scatterers presenting internal resonances has been suggested to be an efficient system to localize classical waves. Within a mean-field approach describing the internal degrees of freedom of the scatterers and their strong interactions in the near field regime, the existence of two longitudinal modes associated with the scatterers and embedding medium degrees of freedom is found. Anderson localization is shown to be reached in a rather large frequency interval for the "slow" mode, above the single scatterer resonant frequency, whereas the "fast" mode is never localized. An experimental situation is discussed where the localization of the "slow" mode could be observed. This work appeared in J. Phys. I France 2, 1861-1867 (1992). Also considered is the problem of the propagation of acoustic waves in a one-dimensional bubbly liquid, either periodic or random. The response of the bubbly liquid to an incident wave in any range of concentrations, bubble size polydispersity, and frequency range has been computed exactly. Three main regimes are found, depending on the average value of the bubble radius $R$, average distance $d$ between bubbles, and wavelength $\lambda$ in the liquid: (1) "phonon" or "mass-spring" regime; (2) "bubble" regime; and (3) "stratified" regime. In the random systems, the Anderson localization length is computed both numerically and analytically in the limit of uncorrelated disorder, for various system realizations.

\section{4;00}

2pPA7. Nondestructive evaluations in multiple scattering media. Christian Vanneste (Lab. de Phys. de la Matière Condensée, CNRS URA 190, Univ. de Nice-Sophia Antipolis, Parc Valrose, 06108 Nice Cedex 2, France), Shechao Feng (Univ. of California, Los Angeles, CA 90024), and Didier Sornette (Univ. de Nice-Sophia Antipolis, Nice, France)

Due to its tendency to scramble the structural information, multiple scattering blurs the distinction between different materials containing different kinds and numbers of defects, or their configurations, beyond the scattering mean-free-path distance from the observation point, thereby rendering most random systems cloudy in appearance and opaque in the sense of structure delineation. However, it has recently been shown theoretically [Feng and Sornette, J. Acoust. Soc. Am. 90, 1742-1747 (1991)] that imaging of a new defect in a multiple scattering medium is in principle possible using speckle pattern correlations. Extensive numerical simulations and analytical calculations were performed to demonstrate the feasibility of detecting a new crack in a single given sample already containing many cracks using speckle pattern av- 
eraging technique at many different frequencies. Potential applications of this approach can be found in medical and industrial imaging needs in highly scattering systems.

$$
\text { 4:15 }
$$

2pPA8. Influence of sound-speed fluctuations and medium geometry on wave localization. Kavitha Chandra and Charles Thompson (Lab. for Advanced Comput., Univ. of Massachusetts, Lowell, MA 01854)

The influence of spatial geometry and material composition on acoustic radiation is considered. Numerical methods are presented that compute the scattered wave field for wavelengths between the Rayleigh scattering and geometrical optics regimes. The transition from extended to localized modes and their dependence on the wave number, average sound-speed fluctuations, and volume fraction of the scatterers is demonstrated. It is shown that the sound-speed contrast must exceed a minimum value for the localized modes to exist. A spatially varying eigenfunction decomposition of the intensity field is carried out to demonstrate this localization effect.

\section{$4: 30$}

2pPA9. Radiative transfer of ultrasound. Joseph A. Tumer and Richard L. Weaver (Dept. of Theor. and Appl. Mech., 104 S. Wright St., Univ. of Illinois, Urbana, IL 61801)

Multiple scattering of ultrasound in an elastic medium containing discrete random scatterers is modeled using radiative transfer theory. The uncorrelated phases of the scattered field allow one to write an energy balance equation for the spatially incoherent intensity. This ultrasonic radiative transfer equation contains single-scattering and propagation parameters that are calculated using the elastic wave equation.
Polarization effects are included through the introduction of an elastodynamic Stokes vector, which contains a longitudinal Stokes parameter and four shear Stokes parameters similar to the four Stokes parameters used in optical radiative transfer theory. The theory is applied to a statistically homogeneous, isotropic elastic half-space containing randomly distributed voids illuminated by a harmonic plane wave. Results on the angular dependence of backscattered intensity are presented. It is anticipated that this approach may be applicable to materials characterization through the study of the time, space, ultrasonic frequency, and angular dependence of multiply scattered ultrasound in elastic media with microstructure. [Work supported by NSF Grant No. MSS-9114360.]

\section{4:45}

2pPA10. Classical sound waves as a coherent superposition of phonons. Philip L. Marston (Dept. of Phys., Washington State Univ., Pullman, WA 99164)

It is generally recognized that single mode classical light waves can be represented as a particular superposition of photon occupation number states [R. J. Glauber, Phys. Rev. 131, 2766-2788 (1963)]. The analogous superposition of phonons is examined for a chain of equal masses coupled only through nearest-neighbor harmonic potentials. This relates phonons to classical normal modes. While the resulting coherent phonon state may be implicit in the work of others, it illustrates the quantum limitations on the normal mode amplitude $\xi$. The expectation value $(\xi)$ oscillates harmonically but the oscillating wave packet has a quantum spread of order $(\hbar / \omega M)^{1 / 2}$, where $M$ is the mass of the chain. This magnitude agrees with estimates by others on the quantum limitation on the detection of classical vibrations. [Work supported by ONR.]

TUESDAY AFTERNOON, 18 MAY 1993

CANADIAN ROOM, 1:00 TO 4:00 P.M.

\title{
Session 2pPP
}

\section{Psychological and Physiological Acoustics: Auditory Models, Temporal Patterns, Spatial Perception (Poster Session)}

\author{
Charles S. Watson, Chair \\ Department of Speech and Hearing Sciences, Indiana University, Bloomington, Indiana 47405
}

\section{Contributed Papers (Posters)}

\begin{abstract}
All posters will be on display from 1:00 to 4:00 p.m. To allow contributors an opportunity to see other posters, contributors of odd-numbered papers will be at their posters from 1:00 to 2:30 p.m. and contributors of even-numbered papers will be at their posters from 2:30 to 4:00 p.m.
\end{abstract}

2pPP1. Modeling and simulation of tympanograms. Lin Chen (Hear. Res. Labs., Dept. of Commun. Disord. and Sci., 215 Parker Hall, State Univ. of New York at Buffalo, Buffalo, NY 14214)

Tympanometry has become a standard evaluation procedure for children and adults in the audiological clinic. Because of the acoustical complexity of both the middle ear system and the testing device, there is a need to study tympanometry not only from an experimental but also a theoretical perspective to achieve a full understanding of the underlying mechanism. In the present study, a model has been developed for simulation of different type of tympanograms. In the model, the acoustic characteristics of the ear canal are described by a set of acoustic equations and those of the middle ear are described by the Zwislocki electric analog model. Model parameters corresponding to the ear canal length, the stiffiness of the middle ear, and the resistance of the cochlea are manipulated to simulate the effects of the applied pressure in the ear canal during a tympanometric procedure. Typical tympanograms such as $1 \mathrm{B1G}, 3 \mathrm{BIG}, 3 \mathrm{B3G}$, and $5 \mathrm{~B} 3 \mathrm{G}$ have been demonstrated with the model and are in good agreement with those actually recorded from the human ear. Some tympanograms seen under pathological and abnormal conditions are also simulated.

2pPP2. Integrated mathematical model of the ear. I. Free-field pressure to basilar membrane displacements. Joel Kalb (Army Res. Lab., Aberdeen Proving Ground, MD 21005-5425)

In order to provide insight into the processes operative in the ear especially at high sound pressure levels, an integrated mathematical model of the ear has been produced. The model's electroacoustic elements are conformal with the strucure of the ear, which promotes physical insight. For maximum utility the model is menued to allow parameter changes by the user. Mathematically, the model is equations of motion formulated as a system of coupled ordinary differential equa- 
tions, which allow both linear and nonlinear claculations in the time domain. The " $Q$ " of the basilar membrane is not constant and the range is selectable. The systems are linear, except at very high SPLs (130 $+\mathrm{dB})$ where the stapes becomes nonlinear. Input to the model is any 4096-point free-field pressure or pressure at the ear drum. Outputs are standardly numerical files of stapes displacements and basilar membrane displacements at any number of evenly spaced user-selected places. As a final output the model makes a "movie" of the basilar membrane displacements coordinated with the pressure history, which shows the effect of each part of the waveform.

2pPP3. Integrated mathematical model of the ear. II. Application and insights. G. Richard Price (Army Res. Lab., Aberdeen Proving Ground, MD 21005-5425)

The model, described by Kalb in the previous paper at this meeting, was initially designed to predict hazard from intense impulses [G. R. Price and J. T. Kalb, J. Acoust. Soc. Am. 90, 219-227 (1991)]. In this mode it correlates highly with auditory hazard from impulses and has provided a radically different approach to an impulse noise damage-risk criterion. A feature of major significance is the "movie" it produces showing basilar membrane displacement synchronized with temporal progression through the pressure history. This display allows visualization of intracochlear events, promoting insight into the ear's response to spectrally and temporally complex signals. In addition to research applications, the model has a potential pedagogical role. For example, if the basilar membrane is given a " $Q$ ' associated with active processes in the cochlea, then an additional range of demonstrations is possible. The "half-octave shift" for TTSs, the traveling wave, frequency localization, and the upward spread of masking can be shown. The model runs on a $\mathrm{PC}$ and with demonstrations is easily contained on a single floppy disk.

2pPP4. A mathematical model of hair cell transduction. Stefan Krol, Margaret F. Cheesman, and Donald G. Jamieson (Hear. Health Care Res. Unit, Univ. of Western Ontario, Elborn College, London, ON N6G $1 \mathrm{H} 1$, Canada)

A mathematical model is described for mechano-electro-chemical transduction in hair cells. The model relates the displacement of stereocilia to the intensity of firing of fibers attached to each hair cell through a cascade of four stages. The gating stage relates hair bundle displacement to the change in conductivity of the hair cell membrane. It is described by a mechanical version of the Hodgkin-Huxley model. The electric stage relates the change in conductivity to the receptor potential through a hair cell electrical network of the Davis type. The calcium kinetics stage relates free calcium ion $\left(\mathrm{Ca}^{2+}\right)$ concentration in hair cells to the receptor potential. It is assumed there are two states of calcium ions: free and bound (by buffers). The synoptic transmission stage relates free calcium concentration to the intensity of firing as described by the Hill equation. Using relevant parameters, the model simulates the behavior of low- and high-spontaneous firing rate fibers, and is being implemented as a component of a complete cochlear model. [Work supported by NSERC, URIF, and Unitron.]

2pPP5. Evaluation of a digital adaptive filter, noise-reduction scheme for normal and hearing-impaired listeners. Donald G. Jamieson (Hear. Health Care Res. Unit, Elborn College, Univ. of Western Ontario, London, ON N6G 1H1, Canada), Rob Brennan (Unitron Industries Ltd.), and Leonard E. Comelisse (Hear. Health Care Res. Unit)

A common complaint of hearing aid wearers is that they have difficulty when listening in a background of noise. The ability of a digital adaptive noise reduction filter to reduce background noise without degrading speech perception has been evaluated. Each of four normalhearing and six hearing-impaired subjects completed two behavioral tests of speech perception-the speech reception threshold (SRT) and the modified distinctive features differences test $[\mathrm{DFD}(\mathrm{m})]$-and one subjective measure of listener preference (paired comparisons). Each test was performed in three types of background noise presented at a level of $65 \mathrm{~dB}$ : white noise, low-pass filtered at $8.0 \mathrm{kHz}$; white noise, low-pass filtered at $1 \mathrm{kHz}$; and multitalker speech babble. For hearingimpaired subjects, the speech-plus-noise signal was filtered and ampli- fied to provide the NAL real-ear insertion gain. Results indicated that all subjects preferred the noise-reduction-processed speech-plus-noise signal over the unprocessed signal at positive signal to noise ratios. The effect of the adaptive digital filter on speech perception depended on hearing loss, noise type, and SNR. Feature analysis of DFD(m) responses indicated that processing had different effects on the error patterns for normal and hearing-impaired listeners.

2pPP6. ECoS: The experiment control system. Cathy Mandarino, Peter Bangarth, Ketan Ramji, and Donald G. Jamieson (Hear. Health Care Res. Unit, Dept. of Commun. Disord., Univ. of Western Ontario, London, ON N6G 1H1, Canada)

The experiment control system is a package of computer programs that facilitate the generation and conduct of identification and discrimination experiments using speech and other complex acoustical signals. The system permits microcomputers to be used to control experimental equipment, present signals to listeners, and record and analyze responses, without preparing individual computer programs for each experiment. The package contains three general programs. The GENER$A T E$ program is used to define experimental parameters via full-screen data entry forms. The core of the program is an entry form that allows one to specify four basic parts of an experimental trial: a warning signal, one or more stimulus presentations, the response( $s$ ) to be recorded, and any feedback to be provided after the response. Within each of these categories, a number of alternatives are permitted. The CONTROL program applies the parameters and data defined in GENERATE to run the experimental session. Typically, possible responses are displayed using high-resolution graphics on a computer video screen and subjects respond using a computer mouse. The $S C O R E$ program analyzes output from CONTROL and displays the results as response matrices with basic statistics such as means and standard deviations, as well as row and column sums. Output is provided as a screen display and printready file. ECOS is being made available to researchers as part of Version 4.0 of CSRE (the Canadian Speech Research Environment).

2pPP7. Encoding amplitude modulation in the cochlear nucleus: $A$ model. Christian Lorenzi (Inst. de Psychol., L.E.A.C.M., Univ. Lyon 2, 5 ave. Mendes-France, C.P. 11, 69676 Bron Cedex, France), Frederic Berthommier (TIMB-TIM3-IMAG, Univ. Joseph Fourier, 38700 Grenoble, France), and Nosrat Tirandaz (Univ. Lyon 2, France)

A physiological model of a post-cochlear mechanism responsible for enhancing the phase-locked activity of auditory neurons is proposed, when excited by sinusoidally amplitude-modulated (AM) tones. In the cochlear nuclei, chopper units respond to the summated low-frequency components of their peripheral inputs. This feature is due to the time constant of the excitatory postsynaptic potential. It is also the result of the specific time course of the cellular membrane potential during the refractory period. The physiologically plausible model is derived from Hodgkin-Huxley equations. AM tones and white noises were applied into the chopper model. In both experiments, the responses of the model showed a strong phase-locking for modulation frequencies between 100 and $300 \mathrm{~Hz}$. The mean gain of the chopper model suggests that chopper units enhance the phase-locked AM responses that they receive as inputs from auditory-nerve fibers. These results agree with earlier electrophysiological studies.

2pPP8, Differential synchronization rates of eighth nerve and midbrain fibers to AM tones and AM noise in bullfrogs (Rana Catesbeiana). S. Boatright-Horowitz and Andrea Megala Simmons (Dept. of Psychol., Brown University, Providence, RI 02912)

The American bullfrog (Rana catesbeiana) provides an excellent model for neuroethological studies in pitch perception. It is particularly useful in the development of an auditory model of temporal coding because of its sensitivity to periodicities in complex sounds. Using a dorsal surgical approach, recordings were made of the neural synchronization responses of 8 th cranial nerve fibers to $A M$ tones and $A M$ noise in the frequency range of 200 to $2500 \mathrm{~Hz}$ at different modulation depths, followed by single- and multi-unit recording in the midbrain (torus semicircularis) to the same stimuli. Analysis of synchronization rates of 
peripheral and central auditory sites elucidates the differences in neural temporal representation of the waveform periodicity for subjects ranging from recently post-metamorphic froglets to fully mature adults.

2pPP9. Involvement of medial olivocochlear system in detection in noise. Christophe Micheyl and Lionel Collet (Lab. Phys. Sens. Audition et Voix, URA CNRS 1447, Pavillon U. Hôpital E. Herriot, 3, Pl. d'Arsonval, 69437 Lyon Cedex, France)

In order to investigate the potent role of the efferents in perception in noise, medial olivocochlear feedback and signal-in-noise detection abilities were both examined in $\mathbf{3 0}$ normally hearing subjects. Medial olivocochlear feedback was assessed in terms of reduction in clickevoked otoacoustic emission amplitude under a contralateral acoustic stimulation consisting of a 30-dB SL broadband noise. Monaural detection thresholds of $\mathbf{1}$ - and $2-\mathbf{k H z}$ tones embedded in broadband noise were measured with and without the same contralateral stimulation sucessively. The finding of a correlation between the reduction in otoacoustic emission amplitude and the detection threshold of the $2-\mathrm{kHz}$ tone in the presence of the contralateral stimulation suggests that the detection abilities are partly related to the operation of the medial olivocochlear feedback. The results indicate moreover that in these subjects with the strongest medial olivocochlear feedback, the detection performance is improved by the addition of noise in the opposite ear whereas it decreases or remains constant in the other subjects.

2pPP10. Eye-closed positional and positioning nystagmus in an abnormal population-A comparison with two previous normative data studies. Yea-Wen Shiau, Bobert W. Keith, Laura W. Kretschmer, Myles Pensake, and Ernest M. Weiler (Dept. of Commun. Sci. and Disord., Univ. of Cincinnati, 332 Braunstein, ML \#379, Cincinnati, OH 45221-0379)

This retrospective study investigated the diagnostic power of two previous built sets of normative data (Barber and Wright, 1973; Schwallie, 1988) by examining eye-closed positional and positioning tests in 100 clinical subjects who had balance problems. The research questions were: (1) What is the prevalence of eye-closed positional and positioning nystagmus in an abnormal population? (2) Which normative data set is more useful in diagnosis? (3) Are the two sets of normative data useful in diagnosis? The findings were: (1) Essentially, the occurrence rate of pathologic nystagmus was higher in the abnormal population than normals. (2) Female subjects had more imbalance problems than males. The age range of female patients was 31 to 70 years, while most male subjects were between the age of 21 to 30 . (3) Both sets of normative data were found to be of diagnostic use; however, there was no evidence to show which is more useful. (4) Horizontal nystagmus data were more applicable in "peripheral" diagnostic categories while vertical data were applicable to both CNS and peripheral categories. It is suggested that eye-closed positional and positioning testr with both vertical and horizontal nystagmus deserve more emphasis in clinical situations.

2pPP11. A comparison of octave-band noise masking patterns obtained from normal-hearing and hearing-impaired listeners. Christine M. Rankovic and Patrick M. Zurek (MIT, Res. Lab. of Electron., Rm. 36-749, Cambridge, MA 02139)

In cases where spread of masking plays a role, interfering noise is more detrimental to speech reception for hearing-impaired than for normal-hearing listeners [e.g., Rankovic et al., J. Acoust. Soc. Am. 90, 2319 (A) (1991)]. To examine such effects, masking patterns for octave-band noises collected from 5 normal-hearing and 6 hearingimpaired subjects were compared. Maskers were centered on 0.5, 1.0, and $2.0 \mathrm{kHz}$ and varied in overall level from 70 to $95 \mathrm{~dB} \mathrm{SPL}$ in $5-\mathrm{dB}$ steps and masked thresholds were measured with 1/3-octave resolution. The hearing-impaired subjects' masking patterns were compared with the averaged patterns of the normal-hearing subjects and the following were found: (1) a low-frequency spread-of-masking (for both normal and impaired listeners) that is roughly constant for the masker levels used here; (2) generally shallower slopes on the high-frequency side of masking patterns for impaired listeners; and (3) for some impaired listeners, substantially greater masking within the masker band. These results will be discussed in relation to the design of hearing aids that modify the frequency-gain characteristic with the goal of reducing upward spread of masking, and will be compared with the maskingpattern equations for impaired listeners proposed by Ludvigsen [C. Ludvigsen, J. Acoust. Soc. Am. 78, 1271-1280 (1985)]. [Work supported by NIH.]

2pPP12. Time-frequency analyses of coherent frequency fluctuations among spontaneous otoacoustic emissions. Edward $M$. Burns (Speech and Hear. Sci., JG-15, Univ. of Washington, Seattle, WA 98195) and James W. Pitton (Univ. of Washington, Seattle, WA 98195)

Previously it has been shown [e.g., Keefe et aL, J. Acoust. Soc. Am. Suppl. 188, S17 (1990)] that in ears with multiple spontaneous otoacoustic emissions (SOAEs) there are often short-term amplitude fluctuations that are highly correlated (either positively or negatively) among SOAEs widely separated in frequency. In this report it is shown that in many such ears there are also coherent frequency fluctuations among widely spaced SOAEs. However, the demonstration of these small (a few tenths of a percent), short-term frequency fluctuations is difficult using the usual spectrogram-based analysis because of the inability of the spectrogram to accurately resolve frequency-modulated signals. To circumvent this drawback, advanced time-frequency representations (TFRs) have also been employed: e.g., the cone kernel TFR and the Wigner distribution. These time-frequency representations provide additional insights into the nature of the short-term frequency and amplitude fluctuations in SOAEs. [Work supported by NIDCD.]

2pPP13. Comparison of suppression across frequencies. Linda $\mathbf{M}$. Thibodeau and Marc A. Fagelson (Dept. of Speech Commun., Univ. of Texas at Austin, Austin, TX 78712)

Although much research has focused on the temporal, spectral, and intensity relationships between a masker and a suppressor, there has been little attention directed towards relative amounts of suppression in different frequency regions. The purpose of this experiment was to compare the magnitude of suppression at 500 and $2000 \mathrm{~Hz}$ in two forwardmasking experiments with seven normal-hearing persons. In the first experiment, the threshold for a $10-\mathrm{ms}$ probe was determined as the bandwidth of a $400-\mathrm{ms}$ masker increased from 0.05 to 1.6 times the probe frequency. Suppression was determined by observing a decrease in threshold when the masker bandwidth exceeded the estimated critical band. In the second experiment, the threshold for a $10-\mathrm{ms}$ probe was obtained with a $400-\mathrm{ms}$ tonal masker in two conditions: masker-alone and masker + suppressor. Suppressor frequencies ranged from 1.1 to 1.5 times the probe frequency. Suppression was indicated by a lower threshold in the masker + suppressor condition relative to that in the maskeralone condition. In both experiments, the magnitude of suppression was smaller for the $500-\mathrm{Hz}$ probe relative to the $2000-\mathrm{Hz}$ probe.

2pPP14. Detection of amplitude modulation with linear-frequency-modulated carriers. James W. Pitton (Dept. Elec. Eng. FT-10, Univ. of Washington, Seattle, WA 98195), Edward M. Burns, and Les E. Atlas (Univ. of Washington, Seattle, WA 98195)

Results are presented for detection of sinusoidal amplitude modulation (AM) superimposed upon linear-frequency-modulated, 120-ms tone bursts. Thresholds for detection of AM were obtained using an adaptive $2 \mathrm{IFC}$ procedure over a range of $\mathrm{AM}$ frequencies from 25 to $400 \mathrm{~Hz}$, and over a range of frequency modulation (FM) rates from 0 to $30 \mathrm{~Hz} / \mathrm{ms}$. The stimuli are a simplification of speech transitions, with both AM and FM, but consisting of only one signal component. These experiments explore the ability to detect modulations that are distributed across critical bands. For fast rates of FM, the stimuli will sweep through a critical band within one to two periods of the AM. A rapid frequency transition also broadens the spectrum of the signal, so that these stimuli do not possess the distinct sidebands of an amplitudemodulated, stationary tone. Thus, both temporal cues from periodicities within individual channels and place cues derived from the excitation pattern will weaken as the FM rate increases. Nonetheless, data indicate that listeners readily detect amplitude modulation for all modulation frequencies at the highest rates of FM. These data will be discussed in 
light of some current physiologically based models of auditory processing, wherein both within- and across-channel temporal information is utilized. [Work supported by the Virginia Merrill Bloedel Hearing Research Center, University of Washington.]

2pPP15. Temporal resolution of frequency-modulated signals. John P. Madden (Dept. of Speech and Hear., Cleveland State Univ., Cleveland, $\mathrm{OH} 44115$ )

Normal-hearing subjects were asked to discriminate between two sinusoidal signals. One, a 50 -ms glide, moved from its initial frequency over a linear path to its final frequency. The other, the step signal, was the same except that its trajectory followed a series of discrete steps in frequency. The smallest frequency increase per step (FIS) at which the step signal could be distinguished from the glide was determined. These FIS thresholds (FISTs) were obtained for signals with 2 to 11 steps at center frequencies of $0.250 .5,1.0,2.0,4.0$, and $6.0 \mathrm{kHz}$. At center frequencies from 0.5 to $2.0 \mathrm{kHz}$, the FIST was roughly constant for signals with up to about 8 steps. Beginning at about 9 steps, the FIST rose sharply, eventually reaching the limits of the possible FIS. It is inferred that beginning at this point, the duration of the individual steps (5-6 ms) limits discrimination. At center frequencies above $2000 \mathrm{~Hz}$, performance deteriorated markedly, unlike the results of gap detection studies. The implications of these results for mechanisms tracking FM signals are discussed. [Work supported by NIH.]

2pPP16. A difference limen for periodicity pitch perception related to spectral change. John W. Hawks and Christine L. Sapp (School of Speech Pathol. and Audiol., Kent State Univ., Kent, $\mathrm{OH}$ 44242)

Preliminary estimates to determine the relative amplitude relationship necessary for one spectral pattern to influence another in terms of the resulting periodicity pitch were made. The threshold of discrimination (DL) for a change in periodicity pitch was estimated for complex tones whereby either the even- or odd-order harmonic components were simultaneously decremented in amplitude. An adaptive up-down procedure employing a 2AFC task with two standards was utilized with minimally trained subjects. Stimuli were digitally constructed by summing sine waves spaced at $100-\mathrm{Hz}$ intervals. Three stimulus parameters were evaluated: (1) total number of components ( 5 or 6 ); (2) position of varied components (even or odd order); and (3) frequency range $(1400-1900 \mathrm{~Hz}$ or $2000-2500 \mathrm{~Hz}$ ). Difference limens obtained ranged from -8.2 to $-23.1 \mathrm{~dB}$ relative to the fixed component amplitude with an overall mean of $-13 \mathrm{~dB}$. The implications of these findings will be discussed.

2pPP17. Cross-modal enhancement of repetition detection for very long period recycling frozen noise. James A. Bashford, Jr., Bradley S. Brubaker, and Richard M. Warren (Dept. of Psychol., Univ. of Wisconsin-Milwaukee, Milwaukee, WI 5320I)

Guttman and Julesz [J. Acoust. Soc. Am. 35, 610 (1963)] reported that recycling of frozen noise segments can be readily detected for periods up to $1 \mathrm{~s}$ and can be detected with difficulty for periods of $2 \mathrm{~s}$. These experiments demonstrate that this limit can be exceeded with ease. It was found that a cross-modal cue (e.g., a light flash) presented synchronously with a randomly selected point in a 10-s recycled frozen noise allows listeners to tap accurately at the repetition rate when the recycled noise is subsequently presented without the cue-even following delays of $\mathbf{2 4} \mathrm{h}$ or more. Two mechanisms for the effect of cueing are suggested: (1) it provides an accurate time base for determining when repetition of salient events will occur; (2) cross-modality attentional effects highlight auditory events concurrent with the cue. Experiments distinguishing between these two mechanisms will be discussed along with other aspects of the effect. [Work supported by NIH.]

2pPP18. Properties of the structure of multi-tone sequential patterns that determine the difficulty of perceptually isolating single target components. Charles S. Watson, Gary R. Kidd, Aimée M. Surprenant, and Ward R. Drennan (Dept. of Speech and Hear. Sci., Indiana Univ., Bloomingion, IN 47405)
A method of adjustment was used to establish the importance of each of several structural properties of the context tones, in nine-tone sequences, in determining the perceptual isolability of target components. Successful "perceptual isolation" of a target tone was assumed to be achieved when frequency matches were as accurate as those achieved for tones presented in isolation, generally meaning less than $1 \%-2 \%$ for the 50-ms tones in these sequences. The context property that was found to primarily affect the frequency matches was the separation, in $\mathbf{H z}$, between the target tone and both the local and (to a lesser degree) the remote context tones. Other than its bandwidth, the form of the local pitch contour (the target tone plus the single tones immediately before and after it) had no clear effect on the ability to "hear out" the target tone, i.e., whether the local context was ascending, descending, concave up, or concave down. The contours of the remote context tones (first and last three in the patterns) likewise had no effect on performance. Performance ranged from $25 \%$ target tones isolated for the most difficult conditions to $90 \%$ for the easiest.

2pPP19. Temporally directed attention in the detection and discrimination of auditory pattern components. Gary R. Kidd (Dept. of Speech and Hear. Sci., Indiana Univ., Bloomington, IN 47405)

Thresholds for detection and frequency discrimination were determined for tones that occurred at unexpected temporal locations within 12-tone sequences. Expectancies were established by repeated presentations of a standard pattern on each trial. Temporal deviations were introduced in comparison patterns by advancing or delaying the onset of a single "target" tone while maintaining its serial position within the pattern. Rhythmic patterns consisting of 350 - and 150 -ms intertone intervals were used to allow for a large range of temporal displacements. Thresholds were determined for target tones that were advanced ("early" targets) or delayed ("late" targets) by various degrees. Thresholds for displaced targets were elevated with respect to nondisplaced targets for both detection and discrimination. However, for most listeners, there was little or no effect of temporal displacement on detection except when targets were advanced by $200 \mathrm{~ms}$ or more. Temporal deviations had more consistent effects on frequency discrimination for both early and late targets. The results are consistent with the view that, when presented with an auditory pattern, a listener's attention is dynamically allocated in time in response to the temporal structure of the pattern. [Work supported by NIH.]

2pPP20. Use of the psychophysical method of adjustment in tonal pattern discrimination. Charles S. Watson, Gary R. Kidd, Aimée M. Surprenant, and Ward R. Drennan (Dept. of Speech and Hear. Sci., Indiana Univ., Bloomington, IN 47405)

A difficulty in tonal-pattern research is that several thousand trials are typically required to approach asymptotic discrimination performance under minimal-uncertainty testing conditions. One solution to this problem is to use the method of adjustment to determine thresholds, rather than a forced-choice psychophysical method. In this study the extremely brief times that are required for a listener to achieve perceptual isolation for single components of a multi-tone patterns using the method of adjustment instead of a forced-choice method (minutes as opposed to hours) are demonstrated. A quantitative criterion for "perceptual isolation" reached when a frequency match is made that is as close to the standard as can be achieved when the standard and variable tones are both presented in isolation, rather than in pattern contexts. Not all adjustments are this accurate, however. The most useful distinction between difficult and easy adjustments is shown to be the percent of all the adjustments, for a given combination of target and context tones, that meet this perceptual-isolation criterion.

2pPP21. The effects of training method on frequency discrimination for individual components of complex tonal patterns. Robert $F$. Port, Catherine L. Rogers (Dept. of Linguist., Indiana Univ., Bloomington, IN 47405), Charles S. Watson, and Gary R. Kidd (Indiana Univ., Bloomington, IN 47405)

It has been assumed that subjects trained to detect increments in the frequency of all components of complex tonal patterns (broad focus) would be less accurate in detecting changes in a single target tone than 
subjects who have been trained to detect changes in only that component [e.g., Watson et al., J. Acoust. Soc. Am. 60, 1176-1186 (1976)]. In several experiments, using a number of 750 -ms ten-tone patterns, subjects were trained using one of three methods: in the first two, a S/2AFC procedure was used to train subjects to detect frequency increments in a specific target tone (group one) or to detect frequency increments that could occur in any of the ten components (group two), and in the third, subjects were trained only to identify the individual patterns. Subjects trained using these methods were tested on their ability to detect changes in various components of the patterns, including the target tone for the first group. In all of these experiments, only very slight differences in performance were found among the different groups. These results suggest that lengthy experience with a given pattern allows a listener to discriminate small differences in frequency in any of the individual components of that pattern, relatively independent of the nature of that experience.

2pPP22. Processing advantages for sequential pure tones related by small integer frequency ratios. E. Glenn Schellenberg (Dept. of Psychol., Cornell Univ., Ithaca, NY 14853) and Sandra E. Trehub (Univ. of Toronto, Erindale College, Mississauga, ON L5L 1C6, Canada)

The effect of frequency ratio complexity on the discrimination of changes to sequential tonal patterns was examined. Patterns consisted of two alternating pure tones (ABABA). Subjects heard a repeating background pattern (the standard pattern) presented in transposition (same frequency ratios between tones, different absolute frequencies), and were asked to identify when a contrasting pattern (different frequency ratios, different absolute frequencies) was substituted. Changes were significantly easier to identify when the component tones of the background pattern were related by small integer frequency ratios (3:2 or 4:3) and those of the contrasting pattern were related by more complex ratios (45:32) than when the situation was reversed. The effect did not extend to cases in which the relative differences in ratio complexity were reduced (3:2 vs $8: 5$ or $4: 3$ vs 5:4). A multiple regression analysis of the combined data sets showed that frequency ratio complexity of component tones in both the standard (background) and contrasting patterns made independent contributions in predicting these results. These findings cannot be explained by notions such as critical bandwidth. Moreover, the present test procedure may be a more sensitive measure of effects of tonal consonance and dissonance than others previously used.

2pPP23. An illusion based on dichotic fusion of harmonically related tones. Wenyi Huang. Michael D. Hall, and Richard E. Pastore (Psychoacoust. Lab., Ctr. for Cognitive and Psycholinguist. Sci., SUNY, Binghamton, NY 13902-6000)

In the octave illusion, dichotic pairs of octave-related tones are physically alternated across ears, with perception of only a single fused tone shifting both in pitch and from side-to-side in synchrony with the physical alternation. Huang et al [J. Acoust. Soc. Am. 91, 2374 (A) (1992)] demonstrated that dichotic fusion of octave stimuli is a primary factor in the occurrence of the illusion. Since the work of Helmholtz, it has ben known that fusion occurs most often for stimuli in an octave relationship and decreases in frequency for other specified relationships. Therefore it is conjectured that similar illusion patterns should be observed with specific frequency intervals other than octaves. Experiment 1 evaluated the incidence of dichotic fusion for different frequency integer ratio. Experiment 2 replicated parts of experiment 1 and determined the incidence of the illusion for some noninteger frequency ratios. Incidence of the illusion tended to be greater for stimuli that are known to more readily fuse. [Research supported by NSF and AFOSR.]

2pPP24. Buildup of echo suppression: Effects of echo delay and source position. Xuefeng Yang and D. Wesley Grantham (Vanderbilt Univ. Div. of Hear. and Speech Sci. and The Bill Wilkerson Ctr., 1114 19th Ave. S., Nashville, TN 37212)

When a subject in free field is presented with a test click and a simulated echo (originating from different positions on the horizontal plane), the degree of suppression of the echo increases with the number of clicks that precedes the test click [Freyman et al., J. Acoust. Soc.
Am. 90. 874-884 (1991)]. Two experiments were conducted to investigate aspects of this buildup of echo suppression. Echo suppression for a test click was quantified by measuring echo threshold (i.e., the echo delay for which subjects could just identify the direction of the echo) in various conditions in which the test click was preceded by a click train. In experiment 1, echo threshold was largest (the maximum buildup occurred) when the echo delay in the preceding click train was 10-20 ms. Experiment 2 investigated the effect of the spatial positions of the preceding train and its echo. For subjects who showed echo suppression buildup, it was maximal in those cases when the train's echo was in the same position as the test click's echo. Results will be discussed in terms of the spatial and temporal characteristics underlying the buildup of echo suppression. [Work supported by NIH.]

2pPP25. Discriminating the direction of auditory motion. Kourosh Saberi and Ervin R. Hafter (Dept. of Psychol., Univ. of California, Berkeley, CA 94720)

Dichotic click trains presented through headphones were used to simulate changes in the interaural delay produced by a source moving horizontally in space. The interaural time-difference (ITD) in each click was increased by a constant $\triangle I T D$ relative to the click immediately preceding it. Left motion was simulated with delays favoring the left ear and visa versa. The $d$ 's for direction measured in a single interval design were linearly related to the number of clicks in the train and, by definition, the extent of change in ITD from first click to last. In experiment II, the middle clicks were eliminated; leaving only the first and last clicks. Performance was essentially unchanged, suggesting that subjects relied on only the onset-offset position of the moving image in experiment I. To examine this hypothesis, experiment III presented a variety of conditions for the middle clicks. If motion detection were simply based on the onset and offset of the total stimulus, then the interaural configuration of the middle clicks should be inconsequential. It was not. These results are explained in terms peripheral adaptation and an integration time of about $65 \mathrm{~ms}$ [Work supported by NIH.]

2pPP26. Shifting auditory attention through space. Todd A. Mondor and Robert J. Zatorre (Dept. of Psychol., McGill Univ., 1205 Ave. Dr. Pentield, Montreal, PQ H3A 1B1, Canada and Montreal Neurological Inst.)

Auditory spatial attention was investigated by measuring the relation between azimuthal distance and the time necessary to shift attention. On each trial, listeners determined whether a pure or a complex tone had been presented. Either of these target tones could sound at any of five free-field locations arranged in a semi-circle in front of the listener. This arrangement required that subjects respond to targets presented up to $180 \mathrm{deg}$ from an initial attentional focus. Prior to the target tone, an attentional cue, consisting of a brief noise burst, was presented to the same position as the target. The time interval between the onset of this cue and the onset of the target tone was varied from 50 to 1400 ms to control the amount of time available to orient attention to the cued location. Results indicated that response time decreased as the opportunity to shift attention to the cued position increased. Thus, the cue was successful in effecting an attentional shift. However, performance was unaffected by the spatial extent of the attentional shift. Under these experimental conditions, therefore, the time required to move auditory spatial attention from one location to another appears to be independent of distance.

2pPP27. Localization and recall of the spatial position of sounds. Ingelise J. Loy and Robert J. Zatorre (Dept. of Psychol., McGill Univ., and Montreal Neurological Inst., 3801 University St., Montreal, PQ H3A 2B4, Canada)

Memory for the location of auditory stimuli was tested after delays of $1 \mathrm{~min}, 30 \mathrm{~min}$, or $24 \mathrm{~h}$. Eleven environmental sounds were presented to 22 subjects in each of 11 different free-field positions, spaced evenly in a semicircle around the subject in the horizontal plane. Initial localization acuity was recorded upon presentation of each stimulus. To test memory for location, target stimuli were presented over headphones, and subjects attempted to recall the original position. Memory for location was significantly less accurate than initial localization, even after 
1 min. Male subjects' accuracy decreased over time, but there was no effect of delay for female subjects. A large effect of stimulus position was also noted, with stimuli coming from central locations remembered better than those coming from the extremes, an effect not accounted for by azimuthal differences in localization accuracy. Analysis of the error distributions indicated that this pattern was largely due to the presence of right-left reversals. These reversals suggest the existence of two separate components to auditory spatial memory: side and azimuth, and that these two parts can be dissociated.

2pPP28. On the potential of fixed arrays for hearing aids. $R$. W. Stadler and W. M. Rabinowitz (Res. Lab. of Electron., MIT, Rm. 36-789, Cambridge, MA 02139)

Microphone arrays with fixed (time-invariant) weights are directed at enhancing a desired signal from one direction (straight ahead) while attenuating spatially distributed interference and reverberation. Using the theory of sensitivity-constrained optimal beamforming [Cox et al., IEEE Trans. Acoust. Speech Signal Process. ASSP-34, 393-398 (1986)], free-field arrays of head-sized extents were studied. The key parameters affecting array design and performance are the set of transfer functions from the target direction to each array microphone and the intermicrophone cross-spectral densities for isotropic noise. Design variables included the orientation of the array, the number and [as motivated by Soede, Ph.D. thesis, Delft Univ. of Technol. (1990)] the directionality of the microphones within the array, and the complexity and robustness of the required processing. For broadside orientation, a variety of arrays based on cardioid and hypercardioid microphones gave very similar performance. They can provide broadband intelligibilityweighted directivities (direct-target gain against isotropic noise) of 7-8 dB with easily implemented weights (simple scalars). For endfire orientation, as Soede recognized, similar directivities result with weights based on analog gains and pure time delays. However, with weightings that are chosen independently for each frequency, directivities up to $11 \mathrm{~dB}$ may be practical. [Work supported by NIH.]

2pPP29. Relative auditory distance perception of synthetic reflections and reverberation presented over headphones. William R. D'Angelo and Mark A. Ericson (AL/CFBA Bldg. 441, 2610 Seventh St., Wright Patterson AFB, OH 45433-7901)
Relative auditory distance perception of a direct path signal by itself, with synthetic reflection(s), and with reverberation presented over headphones was measured using a 2AFC task. The stimulus, either a 500 -ms pink noise burst or a 3-s phrase of male speech, was presented twice, first at a reference distance and second at an incremental distance from the reference. Reference distances included 5,14 , and $22 \mathrm{ft}$, and the incremental distances included multiples of $0.125,0.25$, and $0.5 \mathrm{ft}$, respectively. Stimuli pairs at each of the three distances were presented from four directions: front, back, left, and right. For each stimuli pair, the task of eight subjects was to indicate which of the two sounds appeared closer to himself/herself. The results of all locations indicated that the jnds for the direct path signal were $7 \%$, jnds for the reflections were around $6 \%$, and jnds for the reverberant sounds were about $12 \%$. All of the sounds appeared out-of-head, however, almost all of the sounds presented from behind were perceived to be in front. Side sounds appeared to be biased towards the front when presented at the further distances.

2pPP30. A comparison of maskers on spatially separated competing messages. Mark A. Ericson (AL/CFBA BIdg. 441, 2610 Seventh St., Wright Patterson AFB, OH 45433-7901)

The intelligibility of simultaneous speech signals from pairs of talkers was improved with spatial separation of talkers using synthetic means. The simultaneous speech signals of the talkers, male versus male, female versus female, and male versus female, were separated by $0,22,44,90,134$, and $180 \mathrm{deg}$. A simple left-right (180-deg) signal separation condition was also measured. Four listeners responded to both diotic and spatially separated presentations over headphones. The listeners were randomly prompted by a call sign to respond to one of the two talkers, providing an intelligibility baseline of $0 \mathrm{~dB}$ signal-to-noise ratio for each test phrase. Speech maskers used were either (1) pink noise or (2) a speech babble presented over headphones or, (3) pink noise presented by loudspeakers. In general, the female talkers were the least intelligible and the male versus female pair were the most intelligible. Spatial separations of $90 \mathrm{deg}$, or more, provided up to a $25 \%$ increase in intelligibility.

\title{
Speech Communication: Signal Processing and Auditory Front Ends for Speech Recognizers (Lecture and Poster Session)
}

\author{
B. H. Juang, Cochair \\ AT\&T Bell Laboratories, 600 Mountain Avenue, Murray Hill, New Jersey 07974 \\ Jont B. Allen, Cochair \\ AT\&T Bell Laboratories, 600 Mountain Avenue, Murray Hill, New Jersey 07974
}

\author{
Chair's Introduction-1:00 \\ Invited Papers
}

2pSP1. Signal analysis and representation for speech recognition. B. H. Juang (AT\&T Bell Labs., 600 Mountain Ave., Murray Hill, NJ 07974)

The acoustic front end that converts the signal waveform into a parsimonious representation is a critical compronent of a speech recognizer. Traditionally, this front end conversion process has relied on the short time spectral analysis framework in which power spectra of speech segments, on the order of $10 \mathrm{~ms}$, are successively and independently estimated, via all-pole modeling or bank of filters. In this paper, several key considerations in front end design are elaborated, ranging from the computational structure to the associated dissimilarity measures, in the context of prevalent short time spectral representations 
and the strengths as well as weaknesses of various speech analysis models are discussed, with an attempt to point out possible ways to improve the existing methods. It is further suggested how auditory modeling may help enhance the robustness of a speech recognizer by providing perceptually reliable measurements of speech.

2pSP2. How do humans process and recognize speech? Jont B. Allen (Acoustics Res. Dept., Rm. 2D553, AT\&T Bell Labs, Murray Hill, NJ 07974)

Until the performance of automatic speech recognition hardware surpasses human performance in accuracy and robustness, one stands to gain by understanding the basic principles behind how humans recognize speech. This problem has been studied extensively at Bell Labs between the years of 1920 and 1950 by Harvey Fletcher and his colleagues. The motivation for these studies was to quantify speech sounds in the telephone plant and to improve speech articulation and quality. To do this they studied the effects of filtering and noise on the average speech articulation errors for nonsense CVC syllables, words, and sentences. During WWII these studies were continued at Harvard, but under much more adverse conditions of filtering, noise, and distortion. These studies have recently been extended to include the effects of reverberation degradation due to changes in the modulation transfer function. Fletcher and his colleagues derived a linear speech information density function $D(\omega)$ for the CVC's and found a formula that accurately predicts the average CVC errors. The area under $D$ is called the articulation index. Many people are not familiar with Fletcher's derivation, which is very insightful. He then went on to find the relations between the errors for these nonsense speech sounds and words and sentences. This work has recently been reviewed and extended by Boothroyd. Taken as a whole, these studies can tell us a great deal about how humans process and recognize speech sounds.

I:45

2pSP3. Design of a feature-based speech recognizer aiming at integration of auditory processing, signal modeling, and phonological structure of speech. Li Deng (Dept. Elec. Eng., Univ. Waterloo, Waterloo, ON N2L 3G1, Canada and Spoken Language Systems Group, Lab. Comput. Sci., MIT, Cambridge, MA 02139)

The conventional design of speech recognizers tends to treat the "front end" (signal representation of speech) and the "back end" (lexical representation plus signal modeling and pattern matching) as separate modules. In the integrated framework being developed, signal representation is regarded as a process of "constrained optimization" with the objective function determined by the phonological structure (feature) of speech and by statistical representation of its acoustic/auditory correlates. The constraints are based on the significant properties known to be employed by the human auditory system. A preliminary version of a speech recognizer incorporating the above ideas will be presented where bundles of overlapping articulatory features are used as the basis for describing the phonological structure of speech. Several multi-valued features are assigned uniquely to each quasi-phonemic unit with "minimal" redundancy and "maximal" separability. Major contextual variations in speech are modeled as a natural result of overlapping the "intrinsic" values of one or more of these features across adjacent phonemic units. Knowledge from speech production and speech perception are utilized to limit the allowable feature overlaps. Linkage of this feature-based lexical representation to speech recognition is achieved by establishing a one-to-one mapping from a feature-overlap pattern to a directed graph where each node represents a unique composition of the features and is characterized by a time-series model. Given the explicit feature specification for each node in the overall speech space, a general set of acoustic/auditory measurements can be assigned specifically into the individual nodes based on the understanding of the acoustic/auditory correlates to the features.

2pSP4. Proper time-frequency distributions for speech. Les E. Atlas, James W. Pitton, and Patrick J. Loughlin (Dept. of Elec. Eng., FT-10, Univ. of Washington, Seattle, WA 98195)

The usual representations that are used for speech involve sliding windows across the signal in time and doing Fourier or other analysis on this windowed signal. However, this approach is inconsistent with the notion of a proper distribution. A proper distribution is defined to have the usual properties of a joint probability density function and it has been shown that proper time-frequency distributions exist for speech [Loughlin et al., Proc. ICASSP, V-125-V-128 (1992)]. The most obvious difference of these new distributions is the improved simultaneous resolution in time and frequency. However, this advantage may not be useful for speech recognition and some other advantages could be utilized. For example, the signal-dependent kernel of a proper distribution may be insensitive to changes in room acoustics and could also effectively normalize out differences in vocal tract length. Also, a proper distribution's representation of a periodically excited, time-varying resonator is quite different than that of a spectrogram's, and new features with greater pertinence for auditory modeling may be apparent. [Work supported by Boeing and the Washington Technology Center.]

\section{2:25}

2pSP5. Auditory front ends in speech recognition systems. Tatsuya Hirahara (NTT Basic Res. Labs., 3-9-11, Midori-cho, Musashino, Tokyo, 180 Japan)

The performances of several auditory front ends were evaluated in a phoneme recognition task using a VQ-HMM or an LVQ2 back end, and in a word recognition task using a DTW back end. The auditory front ends used in the experiments were different combinations of a fixed-Q cochlear filter (FQF), an adaptive-Q circuit (AQC) [T. Hirahara et al., Proc. ICASSP, $496-499$ (1989)], an inner-hair-cell model (IHC) and a lateral inhibition (LINH). Traditional DFT-based and LPC-based front ends were examined for comparison. In the VQ-HMM/LVQ2 phoneme recognition task, the AQC and the LINH improved the performance in both noise-free and noisy conditions. However, the best performance provided by an auditory front end was the same as that of the DFT front end. Unexpectedly, the IHC degraded performance in most cases. Hence, it was concluded that the auditory front ends provide little benefit. In the DTW word recognition task, the AQC improved the robustness for noise and 
speaker variation. The LINH greatly improved robustness for noise but degraded that for speaker variation. The best performance for noisy speech was obtained by the combination of FQF, AQC, and LINH, which outperformed traditional front ends thoroughly. Similarly, the best performance for multiple speakers was by the FQF in conjunction with the AQC. This combination completely outperformed other front ends. Hence, it was concluded that the auditory front end pays off. These results indicate that the difference in back ends and the task difficulty strongly affect the evaluation of the front ends.

2pSP6. A computational auditory model for real-world sounds of useful duration. Roy D. Patterson, John Holdsworth, and Michael Allerhand (MRC Appl. Psychol. Unit, 15 Chaucer Rd., Cambridge CB2 2EF, UK)

A psychoacoustic model of auditory processing has been developed to summarize peripheral processing and provide a convenient auditory simulator for research on real-world sounds like music and speech. Most of the processing is devoted to simulating the operation of the cochlea in a fairly traditional way, that is, with a bank of auditory filters and a bank of neural transduction units. The remainder is devoted to two novel processes: First, a form of quantized temporal integration introduced to account for the stablity of the auditory images produced by periodic sounds. Second, a spiral pitch processor that operates on the auditory image and measures the loudness, pitch, and strength of voicing in speech. The system is modular and offers displays of the auditory representation of the sound at each processing stage. Standard workstations process sentence length sounds promptly with larger databases processed overnight. The system is currently being used to assess vocal agitation in samples of normal and emotional speech.

\section{Contributed Papers (Posters)}

All posters will be on display and all authors will be at their posters from 3:05 to 4:05 p.m.

2pSP7. Multi-microphone cross-correlation based processing for robust speech recognition. Thomas $M$. Sullivan and Richard $\mathbf{M}$. Stern (Dept. of Elec. and Comput. Eng. and School of Comput. Sci., Carnegie Mellon Univ., Pittsburgh, PA 15213)

A new algorithm of signal processing for robust speech recognition using multiple microphones is described. The algorithm, loosely based on human binaural perception, consists of imposing time-aligning delays on the speech signals from each microphone and passing the delayed speech through a bank of bandpass filters and nonlinear rectifiers. The outputs of the nonlinear rectifiers within each frequency band are crosscorrelated, providing an estimate of the spectral profile of short-term energy in the speech signal that is resilient to the presence of off-axis noise sources. A cepstral representation of these energy estimates is used as the feature set for automatic speech recognition using the CMU SPHINX system. The multichannel cross-correlation-based algorithm was found to preserve the shape of vowel spectra in additive noise, and it provides better recognition accuracy than is obtained using equivalent single-channel processing with nonclosetalking microphones. The performance of this system was compared to that obtained using delayand-sum beamforming and conventional adaptive filtering approaches. Finally, some implications of these results for human binaural hearing will be commented on. [Work supported by Motorola and DARPA.]

2pSP8. Acoustic correlates of vocalic nuclei of syllables. James D. Miller, M. J. Gottfried, D. J. Meyer, and E. J. Frederick (Central Inst. for the Deaf, 818 S. Euclid, St. Louis, MO 63110)

Values for the fundamental frequency and $F 1, F 2$, and $F 3$ were obtained for a corpus of 1248 vocalic nuclei from CVCs ( 26 phonemically different vocalic nuclei $\times 4$ speakers $\times 2$ stress rate conditions $\times 3$ consonantal contexts $\times 2$ repetitions of each token) at 25 equally spaced times within each vocalic nucleus. This corpus included monopthongs $/ i, \mathbf{I}, \varepsilon, x, a, \wedge, \jmath, U, u /$, diphthongs /au, al, eI, oU, $\jmath /$, rhotic vowels /Ir, er, ar, or, $3 /$, and vowels followed by $/ t /$-/it, it, et, at, At, st, ut/. These were spoken in $/ b \_d /, / d \_d /$, and $/ g \_d /$ contexts. A standard back-propagation neural network with one hidden layer was trained to identify which of these 26 vocalic nuclei was spoken. Input data for the neural network was presented as (1) an auditory-perceptual space using $\left(x^{\prime} y^{\prime} z^{\prime}\right)$ or (2) other implementations of the fundamental frequency and the first three formants. Preliminary results indicate that the neural network is able to identify these nuclei on the basis of acoustic parameters alone. [Work supported by NIDCD.]
2pSP9. Multiple speaker localization using envelope time delays. Pierre Zakarauskas and Max S. Cynader (Dept. of Ophthalmol., Univ. of British Columbia, 2550 Willow St., Vancouver, BC V5Z 3N9, Canada)

It is well known that an array of $N$ sensors can discriminate between at most $N$-1 continuous sources. However, if the time series produced by the sources contain transients, this limit applies only to samples that are shorter than the duration of the transients. This principle has been applied to the problem of localizing two speakers by using only two microphones. Human speech contains a large number of more or less sharp transients. The time of arrival of the transients at each microphone is estimated by finding the local maxima in the envelope of the time series. The time series at each microphone is replaced with the value of the envelope at the local maxima location, and is set to zero everywhere else. The cross correlation of the two resulting time series presents peaks at the time delays corresponding to the correct location of speakers, whereas the cross correlation of the original time series had a single peak at the wrong time delay. It is shown that the difference is due to the nonlinearity of the operations performed on the time series.

2pSP10. Speaker-independent speech recognition using an auditory model front end incorporating the spectro-temporal masking effect. Kazuaki Obara, Kiyoaki Aikawa, ${ }^{a)}$ and Hideki Kawahara (ATR Human Information Processing Res. Labs., 2-2 Hikari-dai, Seika-cho, Soraku-gun, Kyoto, 619-02 Japan)

Speaker-independent speech recognition experiments using an auditory model front end with a spectro-temporal masking model demonstrated the improvement of the recognition performance and outperformed the auditory front ends without the masking model and the traditional LPC-based front ends. The auditory model front end composed of an adaptive $Q$ cochlear filter bank incorporating spectrotemporal masking has been proposed [J. Acoust. Soc. Am. 92, 2476 (A) (1992)]. The spectro-temporal masking model can enhance common phonetic features by eliminating the speaker-dependent spectral tilt that reflects individual source variation. It can also enhance the spectral dynamics that convey phonological information in speech signals. These advantages result in an effective new spectral parameter for representing speech models for speaker-independent speech recognition. Speakerindependent word and phoneme recognition experiments were carried out for Japanese word and phrase databases. The masked spectrum was calculated by subtracting the masking level from logarithmic power spectra extracted using a 64-channel adaptive $Q$ cochlear filter bank. The masking levels were calculated as the weighted sum of the 
smoothed preceding spectra. To cover the variability of the time sequences of the spectrum, multi-template DTW and hidden Markov model were used as the backend recognition mechanism. ${ }^{\text {a) Also at ATR }}$ Auditory and Visual Perception Res. Labs.

2pSP11. Fourier descriptors for time domain labeling of multi-speaker voiced/unvoiced stop consonants. Jack Green and Ben Pinkowski (Dept. of Comput. Sci., Western Michigan Univ., Kalamazoo, MI 49007)

Fourier descriptors (FD's), common shape features often used in computer image analysis, were used to describe time domain waveform shapes of speech signals. The signals examined were initial stop consonants contained in continuous speech samples of five speakers in the TIMIT corpus. The stops were extracted from a consonant/vowel (CV) combination, and information contained in the vowel portion of the utterances was not used. Data included with the corpus were used to segment the sounds. Sixty four FD's were calculated for each of the 58 samples used in the study. These FD's were used to label each stop as being either voiced or unvoiced. Preliminary results showed $61 \%$ of the stops were correctly labeled. Because time domain information requires less computational effort to obtain, a labeling could be obtained while frequency domain information is being calculated. The labeling could then be used to limit the search space in the frequency domain. Despite limitations of working in the time domain, it is possible that these results will approach $75 \%$. Present work is examining the use of neural networks as a method of increasing correct classification.

2pSP12. Evidence of word class effects for word recognition in sentences. Susan L. Goldman (MIT, Rm. 36-749, Cambridge, MA 02139) and Thomas D. Carrell (Northwestern Univ., Evanston, IL 60208)

Previous research has reported that because content words tend to be stressed and function words tend to be unstressed, word class (content-function word) perceptual differences are an artifact of stress differences [A. Cutler and D. Foss, Language Speech 20, 1-10 (1977)]. The present research provides evidence for the validity of word class differences for gated words in a sentence context. Subjects heard successively longer presentations of each target word. The first presentation included the sentence up to the target word onset. The second presentation included the same sentence context plus the first $50 \mathrm{~ms}$ of the target word. The duration of each successive presentation was $50 \mathrm{~ms}$ longer than the previous presentation. After each presentation, subjects were asked to identify the target word. The target words were content and function words balanced for stress. The results demonstrated that word class, independent of stress, significantly influenced the number of presentations required for identification. The results also showed that for the first presentation, subjects significantly chose words of the same word class as the target word, indicating that word class information is available before phonetic information. [Work supported by Northwestem University dissertation year grant.]

2pSP13. Phonetic classification of plosive voicing using computational modeling. Angela M. Darling, Stuart Rosen, Mark Huckvale, and Andrew Faulkner (Dept. of Phonet. \& Linguist., University College London, 4 Stephenson Way, London NW1 2HE, England)

One approach to investigating the roles of auditory and phonetic processing in specch perception tasks is to compare the performance of listeners with no phonetic processing capabilities (animals and machines) to those of human listeners. Here, a computational model of the peripheral auditory system was coupled to an artificial neural net pattern classifier in order to investigate the essential auditory and phonetic features that distinguish voiced and unvoiced plosives in initial position. The computational model and the human listeners were presented with three stimulus series, varying in voice onset time, representing English bilabials ("ba"-“pa"), alveolars ("da"-"ta"), and velars ("ga"-"ka"). The categorization functions obtained for the model and for human listeners will be compared with regard to their sharpness, and shift of phoneme boundary with place of articulation. [Work supported by the Joint Council Initiative in Cognitive Science/HCI (UK).]

2pSP14. Locating landmarks in utterances for speech recognition. Sharlene A. Liu (Res. Lab of Electron., Dept. of Elec. Eng. and Comput. Sci., MIT, Rm. 36-511, Cambridge, MA 02139)

Locating landmarks, or acoustically important points, in an utterance is the first step in a proposed method for feature-based speech recognition. The algorithm developed here is designed to locate landmarks caused by closures and releases of obstruent consonants (sounds produced with a pressure buildup behind a constriction) flanked by sonorants. Two characteristies of obstruents are: (1) voicing diminishes or stops completely at the onset and (2) noise is generated during the constricted interval (at the release in the case of stops and affricates). The algorithm thus monitors voicing changes by keeping track of lowfrequency signal energy and locates a landmark wherever a rapid change occurs. It also monitors higher frequencies for the presence of noise to aid in the detection of voiceless stop and affricate releases. With appropriate selection of time windows, smoothing intervals, and frequency bands, sonorant/obstruent boundaries for stops, fricatives, and affricates could be detected with only a few percent error. Semivowels and creaky voicing sometimes mistakenly cause a landmark to be detected, but more detailed analysis of the characteristics of these erroneous landmarks may overcome this problem. [Work supported by NSF.]

2pSP15. Speech recognition in noise: Comparing an auditory model with human performance. Georg $F$. Meyer and William $A$. Ainsworth (Dept. of Comput. Sci. and Dept. of Commun. and Neurosci., Keele Univ., Keele, Staffordshire ST5 5BG, UK)

A potential application for models of the auditory system is as the front end to speech recognition systems. The model discussed here consists of a physiologically plausible model of the cochlear nerve and of the major neuron types in the cochlear nucleus, the next stage of information processing in the auditory pathway. The recognition task for the machine and human observers was the identification of 100-ms-long plosive-vowel combinations in three noise conditions: clean, $3 \mathrm{~dB}$ and 0 $\mathrm{dB} \mathrm{S} / \mathrm{N}$ ratio. Human listeners were asked to identify the combination under a multiple forced choice regime while the auditory model was used as a front end to a hidden Markov model. Two types of noise were used: Continuous noise and gated noise coincident with the utterance. Physiological experiments show that continuous noise causes a substantial threshold shift while short duration coincident noise has no effect. Psychophysical experiments show that human recognition performance is also a function of the noise type. The performance of the auditory model with plausible threshold shifts is compared with human performance for both conditions.

2pSP16. A nonlinear filter bank model for the auditory periphery. Vikas Taliwal (Depts. of Biomed. Eng. and Math., Boston Univ.) and Laurel H. Carney (Dept. of Biomed. Eng., Boston Univ., 44 Cummington St., Boston, MA 02115)

The intrinsically nonlinear character of auditory processing has long been the bane of linear models for the auditory periphery. A previous computational model [Carney, J. Acoust. Soc. Am. 93, 401-417 (1993)] for single auditory nerve fibers contained a feedback mechanism to vary the bandwidth of a narrow-band gammatone filter as a function of sound pressure level. That model simulated the compressive nonlinearity, including aspects of temporal and average discharge rate characteristics of low-frequency auditory nerve fiber responses. The proposed model extends the previous work; its architecture is motivated by the most recent empirical evidence on outer hair cell (OHC) physiology. The nonlinear narrow-band gammatone filter is replaced with a broad second-order bandpass filter [G. von Békésy, J. Acoust. Soc. Am. 21, 245-254 (1949)] inside a feedback loop. The feedback path consists of a nonlinearity representing $\mathrm{OHC}$ transduction, followed by a lowpass filter for the cell membrane, and finally a nonlinearity representing OHC motility. A bank of such models allows representation of spatiotemporal discharge patterns across populations of auditory nerve fibers. [Work supported by The Whitaker Foundation and NIDCD.] 
Session 2pUW

\author{
Underwater Acoustics: Three-Dimensional Seismoacoustics
}

\author{
Jacques R. Chamuel, Chair \\ Sonoquest Advanced Ultrasonics Research. P.O. Box 153, Wellesley Hills, Massachusetts 02181
}

Chair's Introduction-12:55

Contributed Papers

1:00

2pUW1. New range-dependent antisymmetric edge waves along immersed curved elastic wedge with variable cross section. Jacques R. Chamuel (Sonoquest Advanced Ultrason. Res., P.O. Box 153, Wellesley Hills, MA 02181-5339)

Transient antisymmetric edge waves ( $100 \mathrm{kHz}-1 \mathrm{MHz}$ ) were propagated along the sharp edge of an immersed curved elastic wedge with variable cross section. Two basic geometrical configurations were investigated. The first was based on a truncated oblique solid cylinder ( $A$ $10-\mathrm{cm}$-diam aluminum cylinder obliquely cut at $22.5^{\circ}$ ) forming a convex wedge. The second utilized the intersection of an oblique borehole and a flat solid surface to develop a concave wedge. Aluminum and ice models were studied. The experimental results revealed that the velocity, amplitude, and number of existing antisymmetric edge modes continuously changed as the waves propagated along the range-dependent edge. The antisymmetric edge waves remained nearly nondispersive over a wide frequency range. As the apex angle increased, the number of edge modes decreased. The ratio of the flat side particle displacement to the adjacent curved side particle displacement increased rapidly as the edge apex angle varied between $90^{\circ}$ and $135^{\circ}$. Results from free and immersed wedge models were compared. The new findings contribute to the physical understanding of 3-D range-dependent fluid/solid seismoacoustic wave phenomena applicable to underwater acoustics, nondestructive testing, geophysics, acoustoelectronic devices, and propeller noise. [Work supported by ONR.]

\section{$1: 15$}

2pUW2. Three-dimensional ocean acoustic propagation: Beyond the wedge. Grant B. Deane (Marine Phys. Lab. 0238, Scripps Inst. of Oceanography, 9500 Gilman Dr., La Jolla, CA 92093-0238)

In recent years there has been an increasing interest in developing analytical solutions to various range-dependent, three-dimensional propagation problems. Calculation of the sound field from a point source in a homogeneous wedge is such a problem. However, although interesting, the wedge is not representative of the geometry of many shallow water channels. Over continental shelf and slope regions, the bottom is often faceted, that is to say, the channel can be represented as a "hybrid duct" constructed from two or more planar boundaries. The wedge (two planes) and a parallel-sided duct terminated by a wedge (three planes) are examples of hybrid ducts. $A$ technique has been developed for evaluating the field in a hybrid duct with perfectly reflecting boundaries. The new method is based on a novel extension of the method of source images, in which the concept of an image is abstracted into a set of generating sequences and a visibility criterion. A solution will be presented for the sound ficld in a duct terminated by a wedge as an example of this technique. The method is exact provided the diffracted component of the sound field is negligible. A description of how the source-image approach can be extended to hybrid ducts with penetrable boundaries will be given.
$1: 30$

2pUW3. Applications of a two-way, three-dimensional parabolic equation. Gregory J. Orris and Michael D. Collins (Naval Res. Lab., Washington, DC 20375)

A method was previously reported for solving three-dimensional propagation and backscattering problems involving a range-dependent environment that is separable in one of the horizontal Cartesian coordinates. With this approach, the two-dimensional parabolic equation (PE) is modified by a separation constant that appears in the wavenumber term. The self-starter has been modified to generate an accurate starting field for this problem. Large-scale problems can be solved efficiently when this approach is combined with the split-step Padé solution. This two-way PE is one of the few deterministic, three-dimensional reverberation models in existence and may be applied to problems involving a point source, a beamed array of sources, gradual range dependence, and scattering facets.

\section{$1: 45$}

2pUW4. Modeling global-scale three-dimensional sound propagation in the ocean with the parabolic equation method. Michael $D$. Collins, B. Edward McDonald, W. A. Kuperman (Naval Res. Lab., Washington, DC 20375), and Kevin D. Heaney (Planning Systems, Inc., McLean, VA 22102)

The parabolic equation (PE) method is efficient for solving rangedependent sound propagation problems, including relatively small-scale three-dimensional problems. Global-scale three-dimensional problems are well beyond current computer resources because the size of the computational grid increases with range. The adiabatic normal mode approximation, which ignores the effects of mode coupling, is applied to reduce problems of this class to a practical size. Each of the complex modal coefficients is a function of longitude and latitude and is determined by solving a two-dimensional PE. The wave number appearing in the $P E$, which is a function of longitude and latitude, corresponds to the modal eigenvalue. An interesting description of global-scale threedimensional propagation can be obtained by solving this twodimensional problem for a small number of modes. This approach is orders of magnitude faster than the three-dimensional PE even when all of the modes are considered. This model should be applicable to analyzing results from global-scale experiments such as the Perth to Bermuda transmissions [J. Acoust. Soc. Am. 71, 51-60 (1982)] and the Heard Island Feasibility Test [Baggeroer and Munk, Phys. Today 45(9), 22-30 (1992)].

\section{2:00}

2pUW5. Three-dimensional modeling of narrow-band pulse spreading using normal modes. Michael Wild and Robert Joyce (Naval Oceanogr. Office, Stennis Space Center, MS 39522)

Energy from a narrow-band pulse interacting with a time varying ocean surface will be spread into upper and lower sidebands. This can be modeled in a range-independent ocean environment using normal 
modes and a coupling scheme based on first-order perturbation theory. The structure of the scattered signal from the two-dimensional PiersonMoskowitz surface wave spectrum is presented. The Kraken normalmode model with environmental inputs from a shallow sea has been used, and a comparison is made with measured data.

\section{2:15}

2pUW6. Three-dimensional modeling of low-angle seismoacoustic backscatter. C. R. Bradley and R. A. Stephen (Dept. of Geol. and Geophys., Woods Hole Oceanographic Inst., Woods Hole, MA 02543)

Predictive modeling codes for seafloor backscatter (e.g., finite difference methods) frequently take advantage of coordinate system symmetries in order to reduce the amount of numerical computation required to obtain a solution. Line sources in models with twodimensional Cartesian coordinates $(x, z)$ are often computationally fastest but assume symmetry into and out of the sagittal plane. Twodimensional solutions in cylindrical coordinates $(r, z)$ give the correct spreading and waveforms for point sources but range-dependent features in the sagittal plane represent "rings" about the vertical axis through the source and are somewhat unrealistic. In both of these 2-D cases, propagation and scattering are only considered for $P-S V$ waves. In order to study the limitations of these 2-D approaches and to see if they are excluding any significant scattering mechanisms it is necessary to run some fully 3-D models. In addition to out-of-plane scattering of compressional body waves, vertically polarized shear body waves, and Stoneley (Scholte) waves, 3-D models include the new effects of $S H$ body waves and Love (interface) waves. In this paper backscatter coefficients for single facets on a homogeneous seafloor, for a canonically rough (Goff-Jordan) seafloor, and for volume heterogeneities beneath a flat seafioor are compared. For soft bottoms such as sediment the backscatter coefficients of these models are within $3 \mathrm{~dB}$. [Work supported by Office of Naval Research.]

2pUW7. Wave theory modeling of bi-static bottom reverberation in a stratified ocean. Henrik Schmidt (Dept. of Ocean Eng., MIT, Cambridge, MA 02139), Kevin LePage (BBN Systems and Technol., Cambridge, MA 02138), and Huaiyu Fan (MIT, Cambridge, MA 02139)

Recent results by Lepage and Schmidt [J. Acoust. Soc. Am. 92, 2467-2468 (A) (1992)] have shown that broadband representations of rough surface scattering scenarios may be obtained for layered acousticelastic media by using a computationally efficient, hybrid perturbation/ wave-number integration approach. These results are quite general for source characteristics and geometry but up until now the scattered field has only been obtained in the proximity of the scattering patch. Using a formulation similar to the one used by Schmidt and Glattetre for horizontal source arrays in a stratified ocean [J. Acoust. Soc. Am. 78, 2105-2114 (1985)], the two-dimensional Fourier representations of the scattering source strengths can be transformed into a cylindrical representation in terms of a Fourier series of Hankel transforms. The fact that the number of terms in the series is limited by the finite size of the scattering patch, and the fact that the kernels of the Hankel transforms are determined from a single, linear system of equations with multiple right-hand sides, makes this approach extremely efficient for simulation of long range bi-static reverberation. The seismoacoustic bottom reverberation in deep water is investigated for relevant bi-static scenarios, and the importance of elastic properties and the roughness characteristics is investigated. [Work supported by ONR.]

\section{2:45}

2pUW8. Three-dimensional scattering and wave-type conversion by delineated features in a stratified sea bed. Huaiyu Fan and Henrik Schmidt (Dept. of Ocean Eng., MIT, Cambridge, MA 02139)

The three-dimensional seattering from delineated features in an elastic sea bed, such as faults, small abyssal hills, and extended diapirs, is simulated numerically, and the seismoacoustic wave-type conversion introduced by such reverberant features is addressed. The ocean environment is assumed to be two dimensional: An infinitely long inhomogeneity is imbedded in or penetrating the bottom of an otherwise horizontally stratified ocean. Such reverberant features are assumed to be insonified by an obliquely incident acoustic beam. Due to the fundamental assumption of a two-dimensional environment, this threedimensional reverberation problem can be treated using Fourier synthesis of 2-D solutions. Here, the two-way solutions are obtained using a modified version of an existing, hybrid wave-number integrationboundary element (WI-BEM) approach [P. Gerstoft and $\mathrm{H}$. Schmidt, J. Acoust. Soc. Am. 89, 1629-1642 (1991)]. This WI-BEM approach inherently decomposes the total field into basic physical components, thus enabling direct identification of the different seismic wave types in the seattered field. The conversion into seismic interface (Scholte) waves and $S H$ waves in the bottom is investigated, and its importance for the bi-static reverberation is discussed. [Work supported by ONR.]

3:00

2pUW9. Stochastic analysis of a three-dimensional seismoacoustic field. Alexander H-D. Cheng (Dept. of Civil Eng., Univ. of Delaware, Newark, DE 19716), Mohsen Badiey, ${ }^{\text {a) }}$ and Indra Jaya (University of Delaware, Newark, DE 19716)

Based on geological information existing for a site on the New Jersey continental shelf [Badiey et al,, J. Acoust. Soc. Am. 90, 2372 (A) (1991)], a three-dimensional geoacoustic data set was generated using various widely accepted empirical relations. A statistical analysis is performed on the acoustic properties which reveals the random, inhomogeneous nature of seabed sediment. The statistical structure in both the horizontal and the vertical directions are quantified in terms of mean, standard deviation, and correlation length. The data set is then used in a theoretical modeling by both the deterministic and stochastic techniques. The deterministic technique utilizes an anisotropic, inhomogeneous, poroelastic model. The sensitivity of the various physical parameters to the seismoacoustic responses is examined. For stochastic analysis, the Monte Carlo technique is applied. Utilizing the statistical moments obtained from analyzing the field data, a large number of random realizations of seabed acoustic properties can be generated via a spectral technique in one or multi-dimension, and uni- or multi-variate conditions. The deterministic model is then applied to study the effect of the variability of the sediment properties on the acoustic wave field. a) Presently at Office of Naval Res., Ocean Acoustics, Code 11250A, 800 N. Quincy St., Arlington, VA 22217-5000. 


\title{
Session 3aEA
}

\section{Engineering Acoustics: Measurements and Techniques}

\author{
Victor Nedzelnitsky, Cochair \\ National Institute for Standards and Technology, Sound Building 233, Room A147, Gaithersburg, Maryland 20899-0001
}

Caroline Fu, Cochair

Boeing Computer Services, MS SF-AC, P.O. Box 24346, Seattle, Washington 98124

\section{Contributed Papers}

\section{8:30}

3aEA1. High-temperature fiber optic lever microphone incorporating a single fiber. Frank W. Cuomo (Dept. of Phys., Univ. of Rhode Island, Kingston, RI 02881), Trung D. Nguyen (Old Dominion Univ., Norfolk, VA 23508), and Allan J. Zuckerwar (NASA Langley Res. Ctr., Hampton, VA 23665)

The fiber optic lever microphone [Hu et al., J. Acoust. Soc. Am. 91, 3049-3056 (1992)], as described previously, contains a central transmitting fiber surrounded by six receiving fibers placed with the distal end in the vicinity of the reflecting microphone membrane. Recently, a new approach consisting of a single optical fiber connected to an optical fiber coupler has been implemented. This design provides increased optical throughput, ease of fabrication, improved stability with temperature cycling, and more repeatable sensitivity. The two embodiments will be compared with regard to their theoretical optical transfer functions and experimental performance with temperature cycling to $1000^{\circ} \mathrm{F}$.

\section{$8: 45$}

3aEA2. Finite baffle diffraction effects on second-order differential microphones. Michael A. Smith, Gary W. Elko, James E. West, and R. A. Kubli (Acoust. Res. Dept., AT\&T Bell Lab., Murray Hill, NJ 07974)

Second-order differential microphones provide an excellent means for obtaining a superdirectional response with frequency-independent directivity and small size. By subtracting the output of two closely spaced first-order differential dipole $[\cos (\theta)]$ microphones, a linear quadrupole is obtained whose directivity pattern is $\cos ^{2}(\theta)$. This particular directivity pattern is realized only when the microphones are in an acoustic free-field. When differential systems are placed on or near a finite surface, the effects of diffraction and scattering can significantly modify the directional response relative to the free-field conditions. Since most differential microphones in teleconferencing and business environments are placed on a desktop surface, it is important to understand the effects of diffraction on these systems. A second-order differential microphone was constructed that has been tested for directivity fluctuation in the presence of a large reflecting surface. These experimental results match predicted theoretical patterns for diffraction on a hard disk. Further experimentation with varying surface treatment and different reflector geometries have given valuable insight into the effects of diffraction and scattering on differential microphones placed on a finite reflecting surface.

\section{9:00}

3aEA3. A simple sensor for direct measurement of particle velocity in water. David L. Gardner (Naval Postgraduate School, Code PH/ Gd, Monterey, CA 93943), Thomas B. Gabrielson (Naval Air Warfare Center, Warminster, PA 18974), and Steven L. Garrett (Naval Postgraduate School, Monterey, CA 93943)

Normally, acoustic particle velocity is inferred from measurement of pressure or pressure gradient. In water, however, direct measurement of particle velocity is simple. A moving-coil sensor embedded in a neutrally buoyant package produces a voltage output directly proportional to the particle velocity in the surrounding fluid for frequencies above the resonance of the moving coil. Leslie et al. [J. Acoust. Soc. Am. 28, 711-715 (1956)] built such a sensor by mounting a moving-coil element inside a hollow brass sphere. They also presented the theory for its response with the assumption that the sphere was acoustically rigid. The device described in this work is considerably simpler to fabricate-the moving-coil element is cast into a small cylinder of syntactic foam to make the entire sensor neutrally buoyant-and all of the materials are readily available. In addition, the sensor is small and does not perturb the acoustic field as much as a rigid body would. This sensor can be used to measure acoustic particle velocity directly; it can be used in conjunction with a pressure hydrophone to distinguish between real and reactive power flows or to measure acoustic intensity; and, it can be used to demonstrate effectively the spatial variations of pressure and particle velocity in standing waves.

\section{9:15}

3aEA4. Acoustical holography with arbitrarily shaped sources in a cylindrical duct. Charles J. Konzelman (Dept. of Mech. Eng., Univ. of Victoria, P.O. Box 3055, Victoria, BC V8W 3P6, Canada)

Radiated sound-pressure measurements of a device designed to operate in the presence of a fluid flow are frequently conducted in wind or water tunnels in order to control flow conditions. However, due to environmental reverberation, these sound-pressure measurements may bear little resemblance to those obtained when the device operates in a free-field environment. In particular, it may be impossible to infer the far-field radiation pattern from such measurements. This study examines the possibility of using acoustical holography to determine the far-field radiation pattern in such cases. Numerical simulations are used to synthesize the pressure field radiated by an arbitrarily shaped source that has a prescribed normal velocity distribution and which operates in a rigid-walled cylindrical duct. Acoustical holography is then used to reconstruct the velocity distribution. Assuming that the source velocity does not change when the device is operated in a free-field environment, the reconstructed source velocity is used to determine the far-field radiation pattern. This far-field radiation pattern is compared with that obtained using the exact source velocity in order to assess the performance of the technique.

\section{9:30}

3aEA5. Improved laser interferometry for ultrasonic vibration measurements on diffuse surfaces. Peter B. Nagy and Laszlo Adler (Dept. of Welding Eng., Ohio State Univ., Columbus, OH 43210)

In spite of its obvious advantages over conventional contacts and immersion techniques, laser interferometry is not feasible in many acoustic measurements since its sensitivity is insufficient at ultrasonic frequencies. Part of the problem is that the maximum signal-to-noise 
ratio cannot be maintained on ordinary diffusely reflecting surfaces. Although these surfaces reflect a fair amount (5\%-50\%) of the incident laser light, this energy is randomly distributed among a large number of bright speckles. Unless the detector happens to see one of these bright speckles, the interferometer's noise level will be much higher than the optimum. In most ultrasonic measurements, when the vibrations to be detected are periodically excited by a transmitter, this adverse speckle effect can be almost completely eliminated by a very simple procedure. The conventional interferometer is mechanically modulated to assure the random occurrence of a few very bright speckles and to move the whole speckle pattern around at an appropriate speed. Random but frequent bright flashes detected from the surface of the specimen result. The bright periods are sufficiently long, to trigger the ultrasonic pulser and detect the transmitted signals before the flash subsides and close to maximum signal-to-noise ratio can be maintained everywhere on the surface of a diffuse object. Numerous examples are shown to demonstrate the significant improvement of the detection sensitivity by this novel approach.

\section{9:45}

3aEA6. Dual SAW sensor for the measurement of skin friction on structures exposed to turbulent fluid flow. Xiao Qi Bao, Vasundara V. Varadan, Vijay K. Varadan, and Wayne Pauley (Dept. of Eng. Sci. and Mech., Res. Ctr. for the Eng. of Electron. and Acoust. Mater., Penn State Univ., University Park, PA 16802)

A dual SAW sensor attached in cantilever fashion to a free-floating membrane flush mounted on a structure exposed to turbulent fluid flow are described. Shear stress on the membrane due to the flow conditions, deforms and bends the cantilever beam. The SAW devices are mounted such that one device is in compression and the other in tension. The cantilever arrangement magnifies the deformation of the SAW sensors by many orders of magnitude relative to the membrane. The SAW devices are attached to oscillator circuits and the change in frequency of the SAW oscillators due to the deformation can be accurately measured. The dual arrangement compensates changes in temperatures, electrical, and mechanical noise and other changes and the difference in frequency measured by a mixer is directly related to the shear stress on the structure. Experiments conducted in a wind tunnel are described. [Work supported by ONR.]

\section{0:00}

3aEA7. Acoustic calibrator for plethysmographic surface pressure sensors. Allan J. Zuckerwar (NASA Langley Res. Ctr., MS 238, Hampton, VA 23665)

Plethysmographic surface pressure sensors measure heart sounds, breathing sounds, blood pressure, blood flow sounds, fetal movement, and other sounds indicative of physiological conditions. An acoustic calibrator for such sensors should provide a specific acoustic impedance and permit a mounting arrangement resembling in-service conditions. The calibrator described here consists of a water-filled balloon, on which the test sensor is mounted at the equator. A hydrophone positioned in the center of the balloon serves as a nearly isotropic source at low frequencies. Since the excited sound field is not free but reverberant, a free-field calibration of the hydrophone as projector cannot be used. Rather, a rigidly supported reference sensor of known calibration is tightly coupled to the balloon surface to calibratc the amplitude of the local surface pressure (which is sensitive to mounting conditions) as a function of frequency. The calibration of an acoustically based fetal heart tone monitor will be presented as an example.

\section{0:15}

3aEA8. Integral method for modeling the sound field above a porous material. Celse K. Amédin, Alain Berry, and Yvan Champoux (G.A.U.S., Mech. Eng. Dept., Univ. de Sherbrooke, Sherbrooke, PQ J1K 2R1, Canada)

A new concept of a transmission technique for the characterization of porous material is presented. It is based on the accurate modeling of the sound field above the layer of material that rests on an horizontal infinite baffle. The sound field is generated by a waveguide mounted vertically under the baffle with its termination flush to the baffle. The waveguide is assumed to create a uniform distribution of particle velocity at the termination. The field in the material is expressed using a Green's function that accounts for the multiple reflections on the baffle and at the upper surface of the material. The field above the material is formulated in terms of the pressure gradient distribution over the upper surface. Starting with the boundary conditions at the upper surface (continuity of the sound pressure and normal particle velocity), the collocation method is used to solve for the pressure gradient at a mesh on this surface. This, in turn, allows one to calculate the sound pressure above the material and the transfer function between the volume velocity of the source and this sound pressure. Numerical results are presented, and the potential of this technique for the characterization of porous material is discussed.

10:30

3aEA9. Measurement of the real and imaginary piezoelectric, elastic, and dielectric constants for dispersive piezoelectric transducer materials. Stewart Sherrit, Harvey D. Wiederick, and Binu $\mathbf{K}$. Mukherjee (Phys. Dept., Royal Military College, Kingston, ON K7L 5LO, Canada)

The recent past has seen the introduction of many new types of piezoelectric materials with relatively high dissipation and low mechanical $Q$ (PVDF, ceramic-polymer composites). In order for designers to predict the performance of these materials in transducers, the imaginary components of the dielectric, elastic, and piezoelectric constants need to be known. This is typically done by analyzing the electrical impedance spectra and applying the methods described in the IEEE standard on piezoelectricity [IEEE Std. 176-1987] to calculate the relevant material constants. This paper describes new methods of analysis that expand on the IEEE standard and allow the experimenter to analyze materials with low mechanical $\boldsymbol{Q}$ and substantial piezoelectric loss factors. The paper also describes a technique to determine dispersion in the material constants from the experimental impedance or admittance spectra of the materials. [Work supported by DREA, DND-Canada.]

\section{0:45}

3aEA10. Effect of dynamical fluid compressibility and permeability on porous piezoelectric ceramics. $O$. Lacour, Michel Lagier (Thomson-Sintra, DASM, zone des Bouillides, Sophia-Antipolis, 06561 Valbonne, France), and Didier Sornette (Université de Nice, 06108 Nice Cedex 2, France)

For frequencies less than the Biot frequency, the ac piezoelectric properties of porous ceramics are studied in the presence of slightly compressive viscous fluids filling the pores inside the ceramics. Two regimes are found: (1) for $f$ smaller than a cross-over frequency $f_{c} \sim(\beta / \eta) L^{-2}$, where $L$ is the thickness of the sample and $\beta$ the fluid compressibility coefficient, the piezoelectric coefficients $d_{i j}$ are close to that of the dense nonporous ceramics weighted by the filling factor $(1-\phi)$; for $f>f_{a}$ the $d_{i j}$ are those of the empty porous ceramics as if the fluid was not present. These observations are explained in terms of a froquency-dependent penetration of the fluid pressure inside the porous ceramics as a result on the interplay between fluid compressibility and viscosity. The dependence of the hydrostatic piezoelectric coupling $d_{H}=d_{33}+d_{31}+d_{32}$ as a function of frequency observed experimentally is compared to an effective medium theory for the piezoelectric properties coupled to a permeability model. The experimental dependence of the cross-over frequency $f_{c}$ on sample sizes and on the fluid viscosity are in excellent agreement with this theory. The study shows that it is thus possible to obtain the desirable property of a high bydrostatic piezoelectric coefficient $d_{h}$, characteristic of light porous piezoelectric ceramics, and good mechanical properties under high applied pressure with the use of an internal fluid that allows one to equilibriate the pressure without destroying the piezoelectric properties of the porous ceramics. 
analysis of a finite amount of random data after acquisition are well

3aEA11. More on low peak-factor periodic functions. Logan $E$ Hargrove (Phys. Div.-Code 1112, Office of Naval Res., Arlington, VA 22217.5660 and Dept. of Mater. Sci. and Eng., Johns Hopkins Univ., Baltimore, MD 21218)

A low value of peak factor (PF), the ratio of the range to the rms value of a periodic function, is desirable for radar, sonar, communications, and test signals. A previous paper [L. E. Hargrove, J. Acoust. Soc. Am. 92, 2312 (1992)] described low PF functions constructed from $1<M<101$ harmonically related and equal-amplitude components with signs derived from spectra components of sinusoidally frequencymodulated sinusoids. In that paper, the flat and bandlimited spectra of the low PF periodic functions consisted of integer multiples $n, 1<n<M$, of a fundamental frequency $f_{1}$. In the present paper, similar low PF functions consisting of $M$ equal-amplitude components with frequencies which are higher multiples $n, 1<p<n<(p+M-1)$, of a frequency $f_{1}$ are described. A somewhat different set of values for PF obtain for the higher multiple cases. Some comparisons will be made with low PF periodic functions similarly derived from linearly frequency-modulated sinusoids. [Work performed in the ONR Scientific Officer Research Program.]

\section{1:15}

3aEA12. How much random data is enough? John C. Burgess (Dept. of Mech. Eng., Univ. of Hawaii, 2540 Dole St., Honolulu, HI 96822)

There is a relationship between the amount of data taken from a random signal and its statistical reliability. The usual assumptions are that the signal is stationary, white, and Gaussian. While procedures for known, little information appears to be available about planning before data acquisition. With the usual assumptions, an upper limit can be found for the amount of random data required to meet a specified statistical reliability. This can be done for both time and frequency domain applications.

11:30

3aEA13. A universal shading method-successive approximation method. J. Lan, M. J. Simoneau, R. K. Jeffers, and S. G. Boucher (Airmar Technol. Corp., 69 Meadowbrook Dr., Milford, NH 03055)

A completely new shading method, called the successive approximation method, is developed. The foundation of the method is the energy extreme theorem with a constraint condition: Construct a func-

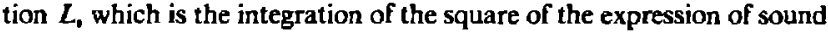
pressure multiplied by a control function, letting $L$ reach minimum will give a set of unknown numbers, therefore a sound pressure can be obtained for the specified control function, which is based on the expected function and the sound pressure obtained during the last iteration. After several iterations, the sound pressure will approach expected function. This method can be applied to any arrays, both in the far field and near field. A variety of patterns can be obtained by either amplitude shading, phase shading, or using unequally spaced arrays. Changing the constraint condition can alter the fitting way between the pattern to be shaded and the expected one. Several computed and experimental examples will be given, which includes: unequally spaced arrays; superdirectivity arrays; using unsymmetric amplitude shading to obtain fanshaped patterns with any low sidelobe level; using amplitude shading only to steer mainbeam.

WEDNESDAY MORNING, 19 MAY 1993

ADAM ROOM, 8:30 A.M. TO 12:00 NOON

\title{
Session 3aMU
}

\section{Musical Acousties and Psychological and Physiological Acoustics: Modulation and Auditory Grouping I}

\author{
John H. Grose, Chair \\ Division of Otolaryngology, Head and Neck Surgery, University of North Carolina, Chapel Hill, North Carolina
} 27599-7070

Chair's Introduction-8:30

Invited Papers

8:35

3aMU1. Modulation detection interference and sound source determination. William A. Yost and Stanley Sheft (Parmly Hearing Inst., Loyola Univ. of Chicago, 6255 N. Sheridan Rd., Chicago, IL 60626)

This paper will review work on modulation detection interference (MDI), especially in regard to its relationship to sound source determination. MDI represents the difficulty in processing temporal modulation of a probe stimulus when one or more spectrally remote maskers are similarly modulated. Some authors have argued that MDI is an example of cross-spectral processing and is a consequence of the auditory system using coherent modulation of spectrally disparate components as an aid in sound source determination. The evidence for and against these two arguments will be provided. Data from experiments involving complex modulation patterns and multiple maskers will be described along with those from previous work involving simple modulation of a single masker. Attempts to demonstrate that MDI is primarily a cross-spectral phenomenon and that it is related to sound source determination will be made. [Work funded by grants from the NIDCD, NSF, and the AFOSR.]

3aMU2. Identification of a single modulated component in a complex sound. Brian C. J. Moore (Dept. of Exp. Psychol., Univ. of Cambridge, Downing St., Cambridge CB2 3EB, England) and Sid P. Bacon (Arizona State Univ., Tempe, AZ 85287-1908)

In experiment 1, subjects were required to decide whether the frequency of a single-modulated carrier (with a suprathreshold modulation depth) in a complex sound was the same as or different from the frequency of a probe composed of a single-modulated 
carrier. They were able to perform well above chance, i.e., they could identify the modulated carrier. Performance was not greatly affected by whether the probe was presented before or after the complex or by the modulation depth used. Experiment 2 was similar to experiment 1, except that the level of each carrier in the complex was varied randomly from trial to trial. This produced only a very slight impairment of performance, indicating that short-term across-frequency differences in level were not used to identify the modulated component in experiment 1 . In both experiments 1 and 2, the identification of a 1000-Hz carrier was best when that carrier was part of a six-carrier harmonic complex, less good when it was part of a six-carrier inharmonic complex, and worst when it was part of a two-carrier complex. The results are interpreted in terms of perceptual grouping. [Work supported by MRC, UK, and NIH.]

9:25

3aMU3. Anomalies in the neural tonotopic-synchrony pattern as the basis for the segregation of tones. William Morris Hartmann (Dept. of Phys., Michigan State Univ., East Lansing, MI 48824)

It is possible to envision a comprehensive model for the segregation and integration of tones based upon synchrony and asynchrony of neural excitation patterns. Evidence for this model comes from experiments on mistuned harmonic matching, simultaneous and sequential octave tuning, and FM incoherence detection with harmonic and inharmonic tones. Such a model has the conceptual advantage of unifying temporal effects and mistuning effects upon segregation. A central remaining question in the formulation of this model is the role of tonotopic analysis. There is, in fact, tonal segregation attributable entirely to tonotopic oddity. More than that, there are tonotopic effects on the processing of synchrony anomalies. These can be seen in mistuned harmonic detection experiments with neighboring harmonics removed, or with added constant remote inharmonic confuser, or with several mistuned harmonics that are themselves either mutually synchronous or asynchronous. Such experiments have suggested a tuned-autocorrelator model with a bandwidth of about two critical bands. Tonotopically based synchrony models are, in turn, challenged by experiments on cross-channel interaction where excitation at intervening tonotopic sites has been deliberately squelched. [Work supported by the NIDCD, DC 00181.]

9:50-10:05 Break

10:05

3aMU4. Across-channel processing of amplitude modulation in cochlear hearing-impaired listeners. J. W. Hall, III and J. H. Grose (Div. Otolaryngol./Head \& Neck Surgery, Univ. North Carolina, Chapel Hill, NC 27599-7070)

Over the past few years, there have been several studies that have examined across-channel effects related to amplitude modulation (AM). Some of these effects, notably comodulation masking release (CMR), modulation detection interference (MDI), and monaural envelope correlation perception, have been hypothesized to be related to the analysis of signals in complex noise backgrounds, and to auditory grouping. Several studies of cochlear-impaired listeners using these paradigms have recently been completed. Cochlear-impaired listeners often report having difficulty processing signals in noisy backgrounds, and it is possible that the study of across-channel processes involving the coding of AM can shed light upon some components of this disability. Because physiological studies have indicated that neural/central abnotmalities can occur secondary to cochlear lesions, it is worthwhile to consider the effect of cochlear hearing loss on across-channel auditory processes. At this point, the extent to which cochlear-based losses in humans are associated with some degree of concomitant neural deficit is largely unknown. One of the specific goals of the research was to determine the peripheral versus central contributions to the hearing loss related deficits in the processing of across-channel AM cues. The results of the studies indicated that relatively mild acquired cochlear losses in adults are associated with effects that can be accounted for in terms of peripheral processing deficits. [Research supported by NIH NIDCD.]

\section{Contributed Papers}

10:30

3aMU5. Comodulation masking release for AM noise maskers. David A. Eddins and Beverly A. Wright (Psychoacoust. Lab., Psychol. Dept., Univ. of Florida, Gainesville, FL 32611)

Comodulation masking release (CMR) was measured for a puretone signal $(2500 \mathrm{~Hz})$ masked by five narrow-band ( $100 \mathrm{~Hz}$ wide) noises (2IFC, 3-down/1-up paradigm). Nose bands were centered at $1500,2000,2500,3000$, and $3500 \mathrm{~Hz}$ and had a spectrum level of $50 \mathrm{~dB}$ SPL. The stimulus duration was $500 \mathrm{~ms}$. Each band of noise was composed of 51 sinusoids. The noise envelopes were manipulated on two levels. On the first level, the phases and amplitudes of the 51 components were either the same (coherent) or different (incoherent) across the bands. On the second level, the bands were amplitude modulated with 10-Hz sinusoids and were either comodulated (same starting phase) or uncomodulated (phase shifted). Mean thresholds for three listeners were 69 and $63 \mathrm{~dB}$ SPL for incoherent and coherent noise, respectively, yielding a 6-dB CMR. For both incoherent and coherent noise, thresholds were lower in the presence of modulation. Thresholds were lower by about $3 \mathrm{~dB}$ for the uncomodulated noise and $21 \mathrm{~dB}$ for the comodulated noise. Thus a CMR occurred on the first level, incoherent minus coherent noise, and on the second level, uncomodulated minus comodulated noise. Combinations of the two treatments may augment or diminish the CMR. [Work supported by AFOSR and NIH.]

\section{0:45}

3aMU6. Effect of prior stimulation on modulation detection interference. Stanley Sheft and William A. Yost (Parmly Hearing Inst., Loyola Univ., 6525 N. Sheridan Rd., Chicago, IL 60626)

Thresholds were measured for detecting amplitude modulation of a 2049-Hz probe carrier in the presence of a two-tone masker complex ( 909 and $4186 \mathrm{~Hz}$ ). The probe and masker were modulated in-phase at $10 \mathrm{~Hz}$. The effect of prior stimulation on masked probe AM detection was evaluated by either preceding the probe with a 200-ms, 2049-Hz tone burst (precursor) or adding an unmodulated carrier fringe preceding the onset of probe modulation. Precursor and masker onsets were synchronous, with the interval between precursor offset and probe onset 
$(\Delta t)$ either 0,40 , or $160 \mathrm{~ms}$. The precursor was either unmodulated, modulated at $10 \mathrm{~Hz}$ in-phase with the masker, modulated at $10 \mathrm{~Hz}$ antiphasic to the masker, or modulated at $20 \mathrm{~Hz}$. With a $\Delta t$ of $160 \mathrm{~ms}$, precursor modulation characteristics had little effect. For $\Delta t$ 's of 0 and $40 \mathrm{~ms}$, modulation of the precursor lowered probe AM detection thresholds with performance generally best with $10-\mathrm{Hz}$ precursor AM. In the fringe conditions, the unmodulated probe carrier preceded the masker onset, with the probe- and masker-modulation onsets synchronous. Fringe duration was either 50,100 , or $200 \mathrm{~ms}$. The addition of the fringe improved performance only with the 50-ms fringe. Results will be discussed in terms of perceptual grouping of stimulus events. [Work supported by NSF.]

\section{1:00}

3aMU7. Monotic and dichotic modulation detection interference. Jane M. Opie and Sid P. Bacon (Psychoacoust. Lab., Dept. of Speech and Hear. Sci., Arizona State Univ., Tempe, AZ 85287-1908)

Monotic and dichotic modulation detection interference was measured for both practiced and naive subjects who listened to either all monotic or all dichotic conditions first. Subjects detected $10-\mathrm{Hz}$ amplitude modulation of a $1-\mathrm{kHz}$ target tone in the presence of a $4-\mathrm{kHz}$ masker that was either unmodulated or modulated from 2 to $80 \mathrm{~Hz}$ at a depth of $100 \%$. The target and masker were presented for $500 \mathrm{~ms}$ at a level of $60 \mathrm{~dB}$ SPL. Both practiced and naive subjects demonstrated less interference when the masker was presented to the ear opposite the target. On average, the maximum amount of interference was approximately $4 \mathrm{~dB}$ in the dichotic condition and approximately $7 \mathrm{~dB}$ in the monotic condition. In addition, the tuning for modulation detection was similar for the dichotic and monotic conditions, with the greatest interference observed for masker modulation frequencies that were the same as or similar to the target modulation frequency. [Work supported by NIH.]

\section{1:15}

3aMU8. Across-frequency interactions in an interaural correlation experiment. William S. Woods, Andrew R. Brughera, H. Steven Colburn, and Hany Ibrahim (Dept. of Biomed. Eng., Boston Univ., 44 Cummington St., Boston, MA 02215)

The work reported here investigated whether across-frequency comparisons can be made when the only relevant information is contained in binaural comparisons. Subjects were required to detect a difference in interaural correlation (IC) between a target band and surrounding fringe bands. Noise stimuli of 500-ms duration and 115-, 354-, 1000-, and $4000-\mathrm{Hz}$ bandwidth, centered on $500 \mathrm{~Hz}$, were used in an adaptive 2I,2AFC task. In each interval, the reference IC was roved between 0.7 and 1.0. In the target interval, the IC was additionally reduced in the 115- $\mathrm{Hz}$ target band centered on $500 \mathrm{~Hz}$. Results indicate that the threshold reduction-in-IC is lowest for the 115- and $4000-\mathrm{Hz}$ bandwidth conditions, and highest for the 354-Hz bandwidth condition, consistent with subjects' use of across-frequency comparisons of binaural information. For the 1000 - and $4000-\mathrm{Hz}$ conditions, subjects reported using the presence of a perceived narrow-band object in the target interval as the basis for decision. This basis was eliminated by delaying the onset of the 115- $\mathrm{Hz}$ target band $500 \mathrm{~ms}$ relative to the fringe band. Resulting thresholds were unchanged or smaller than in the corresponding synchronous condition, possibly due to the subjects' use of image-width cues.

\section{1:30}

3aMU9. The effect of amplitude comodulation on extracting sentences from noise: Evidence from a variety of contexts. Thomas D. Carrell (Commun. Sci. \& Disord., Northwestern Univ., Evanston, IL 60208-3570)

While signal-to-noise ratios range from about $50-90 \mathrm{~dB}$ in laboratory experiments, measurements of speech levels in most natural environments show much poorer listening conditions. For example, Teder [Hear. Instrum. 11, 32-33 (1990)] measured signal-to-noise ratios ranged from a high of $13 \mathrm{~dB}$ in a carpeted office to low of $1 \mathrm{~dB}$ in a 1986 Chevy Nova traveling at $55 \mathrm{mph}$. These numbers indicate an immense difference between speech signals presented to listeners in laboratory experiments as opposed to the real-world environment. Although speech intelligibility has been well studied in noise, there has been little study of the acoustic characteristics of speech that allow the message to be separated from the background noise. One characteristic of voiced speech that shows great promise in this regard is amplitude comodulation. In the present research, amplitude comodulated tone-analog sentences [Carrell and Opie, Percept. Psychophys. 52, 437-445 (1989)] were presented with simultaneous white noise and multispeaker babble at $0-, 5-$, and $10-\mathrm{dB}$ signal-to-noise ratios. It was found that the beneficial effect of amplitude comodulation was greater at the lower signalto-noise ratios.

\section{1:45}

3aMU10. Why do masker fluctuations as in interfering speech lower the speech-reception threshold? Joost M. Festen (Dept. Otolaryngol., Free Univ. Hospital, P.O. Box 7057, 1007 MB Amsterdam, The Netherlands)

Two experiments will be presented to explain the difference in speech-reception threshold (SRT) between conditions with a steadystate noise masker and an interfering voice. In experiment $I$, the possible role of comodulation masking release is investigated by manipulating the comodulation in the interfering voice by introduction of temporal shift among filter bands of various width. The spectral spread of masking from the manipulated interfering voice was controlled by interleaving mutually shifted speech bands with 1/3-octave bands of noise. Although comodulation in interfering speech appears to be very important for the low SRT, the contribution of across-frequency processing of masker fluctuations-commonly considered as the origin of CMR-is only $1.3 \mathrm{~dB}$. In experiment II, the level dependence of masking release with an interfering voice is investigated. The data confirm the hypothesis by Festen and Plomp (1990) that the release from masking with an interfering voice is limited by forward masking. It appears that up to about $55 \mathrm{dBA}$, the release from masking increases with level up to about $7 \mathrm{~dB}$. Above $55 \mathrm{dBA}$, the difference in SRT obtained with a noise masker or an interfering voice is constant due to the limited modulation depth of speech. 


\title{
Session 3aNS
}

\author{
Noise: Special Workshop on ASA's Role in Noise and its Control I \\ Robert M. Hoover, Cochair \\ Hoover and Keith, Inc., 11381 Meadowglen, Houston, Texas 77082 \\ T. James DuBois, Cochair \\ Southern California Edison, P. O. Box 800, Rosemead, California 91770
}

Invited Papers

8:30

3aNS1. Introduction to workshop goals. Bennett M. Brooks (Brooks Acoust. Corp., 27 Hartford Tnpk., Vernon, CT 06066), T. James DuBois (Southern California Edison, Rosemead, CA 91770), Kenneth M. Eldred (Ken Eldred Eng., East Boothbay, ME 04544), Robert M. Hoover (Hoover and Keith, Inc., Houston, TX 77082), Govindappa Krishnappa (Natl. Res. Council, Ottawa, ON K1A OR6, Canada), and Louis C. Sutherland (Consultant in Acoust., 27803 Longhill Dr., Ranchos Palos Verdes, CA 92074)

The goal of this workshop is to further explore the possible future roles of the Acoustical Society in noise and its control. The workshop will begin in the morning with an introductory keynote address with all participants to review the topics, objectives, and procedures for the day's discussion. The participants will then break out into small subgroups to define key issues and develop action plans. The entire group will reassemble to report, assess, and prioritize the proposed actions in each area.

8:40

3aNS2. A quiet, pleasant sounding environment-A goal for the ASA. Richard H. Lyon (MIT and RH Lyon Corp., Cambridge, MA 02139)

A quiet and tranquil environment may seem an unreachable goal, but this profession has a responsibility to assist in the striving for it. The ASA has the disciplinary strengths in engineering, standards, perceptual science, environmental control, and physiological and psychological health studies that are required for the effort. As an international scientific and engineering organization with strong constituencies in academia, industry, and government, the ASA has the credibility to provide the balanced leadership in education, technical developments, and policy analysis that is needed. The ASA needs to assemble and organize these resources to provide assistance to society at large in this complex area.

9:(10

Working Groups, Part 1

Working groups will be organized around the following areas of interest, as expressed by persons responding to the workshop announcement or at meeting registration. Working groups will develop presentations on problems, solutions, and recommended actions.

(1) ASA's role in Environmental Noise Control

(2) ASA's role in Industrial Noise Control

(3) ASA's role in Product Noise Control

(4) ASA's role in Prevention of Hearing Loss (occupational and nonoccupational)

(5) ASA's role with Government

(6) ASA's role in Education

Each working group discussion will focus on (1) what has the ASA done in the past, (2) what should be done now, and (3) how could the ASA accomplish this? 
Working group discussions will continue, focusing on (1) what has the ASA done in the past, (2) what should be done now, and (3) how could the ASA accomplish this? There may be some realignment of the participants in the groups.

\title{
Session 3aPA
}

\section{Physical Acoustics and Bioresponse to Vibration: Novel Applications of Ultrasound in Medicine I}

\author{
Ronald A. Roy, Cochair \\ Applied Physics Laboratory, University of Washington, Seattle, Washington 98105 \\ J. Brian Fowlkes, Cochair \\ Department of Radiology, University of Michigan, Ann Arbor, Michigan 48109-0553 \\ Chair's Introduction-8:00
}

Invited Papers

8:05

3aPA1. Everything you wanted to know about ultrasonic atherectomy. Howard Alliger (MedSonic, Inc., 1938 New Highway, Farmingdale, NY 11735)

An ultrasonic wire was developed that can be threaded through the blood vessels to disintegrate or liquefy occlusive material by cavitation. Using an entry point at the groin, the wire, varying in size from 0.018 to 0.032 in., is flexible enough to reach well below the knee as well as the coronary arteries. It is important to prevent transverse whipping motion in the long wire ( $2-4 \frac{1}{2} \mathrm{ft}$.), which reduces the needed linear motion, and tends to break the wire. Here, $110 \mathrm{~dB}$ of sound, mostly ultrasound, can be detected coming off the side of the wire at transverse vibration. There is only a small reduction in tip amplitude as the wire is bent. Most heating is at node points when the wire is straight, and at antinodes when the wire rubs against the surrounding catheter. To date, actual in vivo human tests have been more encouraging than in vivo bench top evaluations. There are several interesting reasons for this.

3aPA2. Acoustic radiation force and acoustical tweezers used in biomedical research. Junru Wu (Dept. of Phys., Univ. of Vermont, Burlington, VT 05405)

General analytical expressions for the acoustic radiation forces generated by focused and unfocused ultrasonic beams on a small compressible sphere [J. Wu and G. Du, J. Acoust. Soc. Am. 87, 997 (1990)] and a rigid cylinder [Wu et al., J. Acoust. Soc. Am. 87, 581 (1990)] are derived. A new tool for biomedical research, acoustic tweezers, is developed [J. Wu, J. Acoust. Soc. Am. 89, 2140 (1991)]. Several applications of acoustic radiation force at megahertz frequencies such as determination of the tensile strength of a single actin protein filament are discussed. [Work supported by NIH.]

9:05

3aPA3. Shear acoustic properties of soft biological tissues in medical diagnostics. Armen Sarvazyan ${ }^{a)}$ (Dept. of Chem., Rutgers Univ., New Brunswick, NJ 08903)

This report is a summary of the studies of shear acoustic properties of soft biological tissues performed by the author and his co-workers in the period from 1966 to the present. A number of techniques for measuring shear elastic moduli of soft tissue in a range of frequencies are described. Methods are considered based on the measurements of acoustic impedance, the shear and surface wave speeds, as well as of static moduli. Results of in vitro studies of animal and human tissues and of such model systems as various gels and rubbers are presented. In vivo studies were made on superficial tissues such as the skin and the nearest underlying tissues. It has been shown that surface acoustic waves can be successfully used in the diagnosis of various dermatitis and skin cancer as well as for monitoring of the efficacy of treatment. In response to the needs of the newly developing technique of ultrasonic elasticity imaging of the breast, systematic studies involving 162 excised samples of glandular, fatty, cancerous, and 
fibroadenoma tissues were performed and the ranges of variation of shear moduli were evaluated. ${ }^{\text {s)}}$ Permanent address: Inst. of Theoret. and Exp. Biophys., Russian Acad. Sci., Pushchino 142292, Russia.

9:35

3aPA4. Elastography: Technical considerations and preliminary clinical results. Jonathan Ophir, Ignacio Cespedes, Hari Ponnekanti, and Nabil Maklad (Univ. of Texas Med. School, Houston, TX 77030)

The elastography technique is based on computing localized strain levels in tissue, which are produced in response to a small axial compression, and which are detected by subjecting pre- and post-compression radiofrequency a-line pairs to crosscorrelation analysis. Additionally, the absolute stress field generated by the compressor is calculated from theory and from acoustic measurement of the applied stress. The local Young's moduli are estimated from the local axial stresses and strains, with reasonable sensitivity and resolution. Theoretical considerations and simulations were used to investigate the mechanical, acoustical, and signal processing tradeoffs which are encountered, and some of these have been studied experimentally in tissue mimicking phantoms and in tissues in vitro and in vivo. Some of the interesting results to date include improved signal-to-noise ratio in elasticity images (elastograms) as compared to sonograms, the possibility of imaging elasticity in certain acoustically shadowed areas, and the ability to observe certain structures that are invisible on sonograms. Several image artifacts have also been identified.

\section{0:05-10:20 Break}

\section{0:20}

3aPA5. Recent developments in high-frequency ultrasound imaging. F. S. Foster, G. R. Lockwood, L. K. Ryan, K. A. Harasiewicz, D. H. Tumbull (Dept. of Med. Biophys., Univ. of Toronto, Sunnybrook Health Sci. Ctr., Rm. S-130, 2075 Bayview Ave., Toronto, ON M4N 3M5, Canada), and C. J. Pavlin (Princess Margaret Hospital, Toronto, ON M4X 1K9, Canada)

Current medical diagnostic imaging methods are generally aimed at structures that are larger than $1 \mathrm{~mm}$. Nevertheless, there are numerous important clinical applications for sub-millimeter or microscopic resolution of superficial structures and structures accessible by endoscopic means. In this presentation, the development of high-frequency ( $40-100 \mathrm{MHz})$ ultrasound backscatter microscopy (UBM) will be described together with initial clinical and biological applications. UBM is essentially an extension of the powerful B-mode backscatter methods developed for clinical imaging in the 3- to 10-MHz frequency range. The development of new high sensitivity transducers in the 40 - to $100-\mathrm{MHz}$ range now permits visualization of tissue structures with resolution approaching $20 \mu \mathrm{m}$ and a maximum penetration of approximately $4 \mathrm{~mm}$. The design and characterization of ceramic and polymer devices for UBM applications will be discussed. Progress in the use of UBM to visualize the anterior segment of the eye will be reviewed and newer applications such as intravascular imaging, articular cartilage imaging and skin imaging will be examined.

\section{Contributed Papers}

\section{0:50}

3aPA6. Intra-arterial ultrasonic angioplasty: A feasibility study. Olivier K. Colliou and E. Carr Everbach (Dept. of Eng., Swarthmore College, Swarthmore, PA 19081-1397)

Current medical technologies for removing the arterial plaque of patients with cardiac disease include balloon angioplasty and laser ablation. In the first, balloons are introduced via catheter into the blocked artery and expanded to allow increased blood flow, but macroscopic plaque pieces can leave arterial walls and enter the circulatory system, causing brain embolism. In laser ablation, an optical fiber is passed into the artery and intense laser light is used to heat the plaque until micronsized particles are removed. Unfortunately, the ablation is not limited to the plaque, and punctures can occur in the healthy arterial walls once the plaque layer has thinned. A new technology, intra-arterial ultrasonic angioplasty, is being developed in which a wire, acting as an acoustic waveguide, is inserted into the artery and intense ultrasonic waves are used to ablate the plaque at the wire tip. Alternatively, tiny transducers can be inserted into the artery and excited to produce intense ultrasonic waves. Violent cavitation occurring at the plaque surface is believed to be responsible for the ablation, which occurs preferentially in the harder, stiffer plaque rather than in the softer, healthy vessel walls. A series of measurements an several of these acoustic devices and an anal ysis of the parameters that could be used to optimize their efficacy and safety are presented here.

\section{1:05}

3aPA7. Use of continuous wave ultrasound for measurement of the dynamic response of soft tissue to facilitate diagnosis of disease. Mardi C. Hastings and Lee E. Schroeder (Dept. of Mech. Eng., Ohio State Univ., 206 W. 18th Ave., Columbus, OH 43210)
A noninvasive, noncontact ultrasonic system for measurement of amplitudes of vibration of soft tissue has been developed for operation in air. The measurement method, based on phase modulation of low power, continuous wave ultrasound, was originally developed by Rogers and Hastings [U.S. Patent No. 4,819,643 (11 April 1989)] to measure the frequency response of auditory organs in fish underwater. In this study, the technique was used to measure the response of corneas of enucleated bovine eye globes to acoustic excitation at audible frequencies in air. Data were recorded at different levels of intraocular pressure (IOP) which is routinely clinically measured to sereen for glaucoma. A mathematical model for harmonic motion of the corneal shell was developed for correlation with empirical results. The stiffness and natural frequency of the thin corneal shell are functions of IOP; thus the data provide a noncontact measurement of this parameter. This ultrasonic measurement technique eliminates the problems associated with current contact tonometry methods which include maintaining sterility, abrading the corneal surface, and inherently altering the IOP. [Work supported by NSF Grant No. MSS 9058607 .]

\section{1:20}

3aPA8. Recent theoretical and experimental results with Albunex ${ }^{\circledR}$, a contrast agent for diagnostic ultrasonography. Charles $\mathrm{C}$. Church, Harold B. Levene, and James M. Keene (Molecular Biosystems, Inc., 10030 Barnes Canyon Rd., San Diego, CA 92121)

Albunex ${ }^{(\mathbb{D})}$ is an ultrasound contrast agent prepared by sonicating 5\% human serum albumin to produce stable, air-filled, albumin-coated microspheres. It is an effective ultrasound contrast agent for use during echocardiography and other ultrasound radiological procedures. Current research is directed toward understanding the physics of the inter- 
action of ultrasound with Albunex ${ }^{\oplus}$ in tissue. A theoretical model describing the dynamics of these bubbles has been developed by assuming a solid elastic shell. The shell (1) supports a strain that counters the effect of surface tension and thus stabilizes the bubble against dissolution; (2) increases the stiffness of the bubble and thus raises the resonance frequency of the bubble significantly; (3) increases the effective damping of the bubble and thus reduces the scattering cross section of the bubble markedly. Experimental measurements of speed of sound, attenuation coefficient, and backscatter coefficient have been made as functions of acoustic frequency and gas volume fraction. Comparisons between these measurements and theoretical predictions will be presented.

\section{1:35}

3aPA9. An investigation of mechanisms for drug-mediated cell damage using acoustic cavitation. R. J. Jeffers, R. Q. Feng, J. B. Fowlkes (Dept. of Radiol., Univ. of Michigan, Ann Arbor, MI
48109-0553), J. W. Hunt (University of Toronto, Toronto, ON, Canada), and C. A. Cain (Univ. of Michigan, Ann Arbor, MI 48109)

Several different research groups including this one have demonstrated that the application of ultrasound in the presence of certain anticancer agents will increase the in vitro and in vivo cytotoxicity of the drug significantly beyond the strictly additive effect of ultrasound alone. Recent in vitro experiments indicate that the addition of cavitation nuclei in the form of albumin-stabilized microbubbles can greatly enhance the cell killing potential of the combination treatment on HL-60 human promyelocytic leukemia cells. Several measurement techniques currently in use will be described for correlating cytotoxicity with cavitation activity in order to investigate the mechanism for cell damage. One of the more promising methods thus far has been time-integrated subharmonic emission versus cell lysis where the latter is measured by the amount of extracellular lactate dehydrogenase. The use of the subharmonic to indicate the amount and severity of cavitation accommodates variations in cavitation threshold and consistency of cavitation nuclei. [Work supported by USPHS Grant No. CA-55357.]

\title{
Session 3aPP
}

\section{Psychological and Physiological Acoustics: Cochlear Emissions, Applications, Implants}

\author{
Glenis R. Long, Chair \\ Department of Audiology and Speech Sciences, Purdue University, West Lafayette, Indiana 47907
}

\section{Contributed Papers}

\section{8:30}

3aPP1. Ontogeny of $2 f 1-f 2$ acoustic and cochlear microphonic distortion products. Ming Zhang (Dept. of Speech Pathol. and Audiol., Univ. of Iowa, 100 WJSHC, Iowa City, IA 52242) and Paul J. Abbas (Univ. of Iowa, 127 WJSHC, Iowa City, IA 52242)

The development of the acoustic distortion product (ADP) and cochlear microphonic distortion product (CMDP) $2 f 1-f 2$ was studied in pigmented rats, beginning 10 days after birth (p10). Both the ADP and CMDP were measured as a function of stimulus frequency (1.04 to $10.24 \mathrm{kHz}$ with an $f 2 / f 1$ ratio of 1.25 ) and level (20 to $80 \mathrm{~dB} \mathrm{SPL}$ ). The onset of the ADP occurred first at $f 2=5.12 \mathrm{kHz}$ (p11-13), then at higher and lower frequencies. The amplitude of the ADP also matured first at $f 2=5.12 \mathrm{kHz}(\mathrm{p} 17-18)$. This result is similar to the earlier work [S. J. Norton et al., Hear. Res. 51, 73-92 (1991); C. M. Henley et al., Hear. Res. 43, 141-148 (1989); M. Lenoir and J.-L. Puel, Hear Res. 29, 265-271 (1987)]. The onset and maturation of the DMDP was similar to ADP's, except at lower frequencies where the CMDP developed somewhat earlier. There is a strong correlation in amplitudes of the ADPs and CMDPs across ages, consistent with the hypothesis that the ADP and CM DP may be linked to the same process [D. T. Kemp and A. M. Brown, Hear. Res. 13, 39-46 (1984)].

\section{$8: 45$}

3aPP2. Comparison of cubic distortion product otoacoustic emissions generated by the interaction of a spontaneous emission and an external tone and by the interaction of two external tones. Glenis $R$. Long, Chuang Sun (Dept. of Audiol. and Speech Sci., Purdue Univ., West Lafayette, IN 47907), and Carrick L. Talmadge (Purdue Univ., West Lafayette, IN 47907)

DPOAEs generated by the interaction of one external tone and one spontaneous otoacoustic emission (SOAE) are maximal when the external stimulus begins to suppress the level of the emission. Consequently, investigations of the $f 2 / f 1$ and $L 2 / L 1$ ratios producing max- imum distortion are limited. DPOAEs generated in this way were systematically compared to DPOAEs generated by two external tones. First, another external tone with the same frequency as the SOAE was inserted into the ear canal to entrain the spontaneous emission and maintain a known signal at the frequency of the spontaneous emission. Second, DPOAEs generated by two external tones with same frequency and level ranges as above were measured in ears with no spontaneous emissions in the frequency range examined. The pattern of maximum DPOAEs are interpreted in terms of the excitation patterns of external tones and SOAEs on the basilar membrane.

\section{9:00}

3aPP3. Correlation of distortion product otoacoustic emissions (DPOAE) with hearing loss produced in a "toughening" exposure. Donald Henderson and Malini Subramaniam (Hear. Res. Labs., State Univ. of New York at Buffalo, 215 Parker Hall, Buffalo, NY 14214)

The relation between distortion product otoacoustic emissions (DPOAE) and hearing loss was studied using a "toughening" exposure. The subjects were monaural chinchillas [ $N=5$ ]; DPOAE were measured using a Virtual 330 and hearing was estimated using evoked potentials recorded from a chronic electrode in the colliculus. Hearing thresholds and DPOAE were measured before any exposure began, then after each 6-h exposure of $0.5 \mathrm{kHz} \mathrm{OB}$ at $95 \mathrm{~dB}$ SPL for 10 days and, finally, 5 days after the last exposure. Over the 10 days of exposure, the daily threshold shifts became progressively less. Initially, the DPOAE were depressed below the noise of the DPOAE system, but by days 8 to 10 , the subjects had recovered to pre-exposure levels even though there were still significant shifts in hearing thresholds. Five days postexposure, hearing levels were normal and DPOAE were normal or enhanced. Results will be discussed in terms of "toughening" induced by noise exposure. [Work supported by NIH 1 R01 DCol237-01A11.]

\section{9:15}

3aPP4. Human cochlear traveling wave delays in normal and impaired ears. Barry P. Kimberley, Anya Lee (Dept. of Surgery, Univ. 
of Calgary, 3330 Hospital Dr. NW, Calgary, AB T2N 4N1, Canada), David Brown, and Jos Eggermont (Univ. of Calgary, AB T2N 4N1, Canada)

Previous work using electrocochleographic measures [J. J. Eggermont, J. Acoust. Soc. Am. 65, 463-470 (1979)] suggested that human cochlear traveling wave velocities were often increased in ears with cochlear loss. A new method to measure cochlear delays using DPE phase responses has been developed [Kimberley et al., J. Acoust. Soc. Am. (submitted 1992)]. This method relies on more purely cochlear responses in comparison with previous techniques. The method is also rapid and noninvasive. Cochlear traveling wave delays were estimated using the phase responses of distortion product emissions associated with $f 2$ frequencies ranging from $10 \mathrm{kHz}$ to $700 \mathrm{~Hz}$. Frequency-specific delay comparisons were made between two groups, namely 21 young adults (42 ears) with normal hearing and 20 adults (40 ears) with mild/moderate high-frequency hearing losses (30-50 dB HL) at two or more audiometric frequencies. The effect of frequency-specific cochlear hearing loss on traveling wave delays are analyzed and compared with the results previously obtained by Eggermont using electrocochleographic techniques. [Work supported by the Alberta Heritage Foundation for Medical Research.]

\section{9:30}

3aPP5. Effects of click polarity on ABR parameters in the older population. Vishakha W. Rawool (Commun. Disord. \& Special Educ., Bloomsburg Univ., Bloomsburg, PA 17815)

The auditory brain-stem response (ABR) is known to be useful for detection of acoustic tumors and the onset of symptoms for acoustic tumors is usually between $\mathbf{3 0}$ and 50 years of age. Hence, this study was designed to evaluate the effects of stimulus polarity in the older population at intensity levels that are used in obtaining ABRs for clinical diagnosis. Nine subjects between the age of 40-69 years participated in the study. Rarefaction and condensation clicks were delivered at 70 and $80 \mathrm{~dB} \mathrm{nHL}$. The latencies were significantly earlier for rarefaction clicks for components II and V $(p<0.05)$. The interpeak interval I-II was significantly shorter for rarefaction clicks than for condensation clicks $(p<0.03)$. The amplitudes of the IV-V complex did not change with stimulus polarity at $80 \mathrm{~dB} \mathrm{nHL}$, but at $70 \mathrm{~dB} \mathrm{nHL}$ larger amplitudes were observed with rarefaction clicks yielding a significant polarityintensity interaction $(p<0.04)$. The ratio of the IV-V complex with wave I was larger for rarefaction clicks at both the stimulus levels. These results will be discussed in reference to clinical diagnosis and auditory physiology.

\section{9:45-10:00 Break}

\section{0:00}

3aPP6. Liquid transmission of sound directly into the cochleg. Samuel Gilman (Sam Gilman Assoc., P. O. Box 25176, W. Los Angeles, CA 90025) and J. Phil Mobley (House Ear Inst., Los Angeles, CA 90057)

An implantable hearing prosthesis is being investigated based on the conduction of sound through a liquid-filled tube directly from an electronics module into the cochlea, thus by-passing the external and middle ears completely. Hearing could be provided up to the functioning limits of the cochlea regardless of pathological or functional conditions of the external or middle ear. The system also has wide frequency response with low distortion at high sound levels. Scale model acoustic tests (described in an accompanying paper) confirmed the calculated performance. The band output is linear with applied transducer voltage up to more than $140 \mathrm{~dB} \mathrm{SPL}$, is flat $( \pm 5 \mathrm{~dB})$ from 150 to $6000 \mathrm{~Hz}$, rising $10 \mathrm{~dB}$ to $10 \mathrm{kHz}$ and shows no standing waves. Transmission losses are essentially zero over the entire frequency range so that a maximum efficiency is obtainable. Biocompatible transmission liquid and tubing sizes and material are selected to match the specific acoustic impedance of the cochlea at the interface and couple most efficiently to the transducer.

\section{0:15}

3aPP7: Performance of a liquid-filled audio transmission line. $\mathrm{J}$. Phil Mobley (House Ear Inst., 2100 W. Third St., Los Angeles, CA 90057) and Samuel Gilman (Sam Gilman Assoc., W. Los Angeles, CA 90025)

The acoustic characteristics of a liquid-filled transmission line have been investigated over the range of $32 \mathrm{~Hz}$ to $12.5 \mathrm{kHz}$. As described in the accompanying paper "Liquid transmission of sound directly into the cochlea," this method has been investigated to the transmission of audio sound through a scale model of the system. Measurements of dynamic range, frequency response, and distortion were made on an acoustic coupling system consisting of a cylindrical piezoelecric transducer (operated in a radial mode) coupled with a liquid-filled transmission line. When compared with similar methods of transmitting sound into the cochlear such as electromagnetic transducers, mechanical vibrators driving the ossicular chain, and bone conduction transducers, the liquid transmission method shows superior performance. Signal-to-noise ratios range up to $60 \mathrm{~dB}$, frequency responses were flat $( \pm 5 \mathrm{~dB})$ over 150 - to $6000-\mathrm{Hz}$ range, and distortion measured as low as $0.2 \%$ using a $1-\mathrm{kHz}$ fundamental and ten harmonics. These measurements were made at SPL levels exceeding $140 \mathrm{~dB}$ using an identical piezoelectric transducer as a receiver terminated with simulated cochlear load.

10:30

3aPP8. The quadratic property of the human auditory sensation induced by focused amplitude-modulated ultrasound. Shengke Zeng and Richard B. Beard (Biomed. Eng. and Sci. Inst., Drexel Univ., Philadelphia, PA 19104)

Three modes of low-intensity $\left(\leqslant 100 \mathrm{~mW} / \mathrm{cm}^{2}\right)$ monotone amplitude-modulated ( $A M$ ) ultrasound are focused on the region of the human cochlear and the human auditory response patterns are observed. The AM ultrasonic signal is defined as $f(t) \cos \omega t$, where $f(t)$ is the monotone modulation signal and $\omega$ is the angular frequency of the ultrasonic carrier. The three modes of $f(t) \cos \omega t$ are: (1) carriersuppressed AM mode, $\cos \Omega t \cos \omega t$; (2) square-rooted AM mode, (1 $+\cos \Omega t)^{1 / 2} \cos \omega t$; (3) standard AM mode, $(1+\cos \Omega t) \cos \omega t$, where $\Omega$ is the angular frequency of the monotone. They are applied in sequence to stimulate the subject. The pattern of auditory sensation of the subject to the ultrasonic stimulations follows the square of the modulation signal, $f^{2}(t)$, which are: (1) $\cos ^{2} \Omega t$; (2) $\left[(1+\cos \Omega t)^{1 / 2}\right]^{2}$; (3) $(1+\cos \Omega t)^{2}$. The subject senses the pure tone of $2 \Omega$ for the first mode of the ultrasonic stimulation, the pure tone of $\Omega$ for the second mode of the ultrasonic stimulation, and the mixed tones of $\Omega$ with relatively weaker intensity of $2 \Omega$ for the third mode of the ultrasonic stimulation. The response patterns reveal that the AM ultrasound induced human auditory sensation has the property of $f^{2}(t)$. This quadratic property is similar to the parametric demodulation of the focused AM ultrasound in water which has the form of $d / d t\left[f^{2}(t)\right],(B \gg 1)$ [Novikov, Nonlinear Undenwater Acoustics (Acoustical Society of America, Woodbury, NY, 1987), pp. 86-95]. [Work partially supported by Electro-Stim Corp.]

\section{0:45}

3aPP9. The effect of the square-root preconditioning on amplitude-modulated ultrasonic hearing. Shengke Zeng and Richard B. Beard (Biomed. Eng. and Sci. Inst., Drexel Univ., Philadelphia, PA 19104)

The discovery of the quadratic property of the human auditory sensation induced by the focused amplitude-modulated (AM) ultrasound ( Zeng and Beard, "The quadratic property of the human auditory sensation induced by focused amplitude-modulated ultrasound," preceding abstract, this meeting) suggests the need for preconditioning of the AM ultrasound stimuli. The preconditioning can cancel the harmonic distortion which is caused by the quadratic effect in ultrasonic hearing. This quadratic harmonic distortion slopes from about $-3 \mathrm{~dB}$ at $250 \mathrm{~Hz}$ (modulation frequency) to $-11 \mathrm{~dB}$ at $2 \mathrm{kHz}$ (modulation 
frequency). The preconditioned AM ultrasound is in the form of $\sqrt{\left|f(t)_{\min }\right|+f(t)} \cos \omega t$, where $f(t)$ is the modulation signal and $\omega$ the angular frequency of the carrier. The non-negative of $\left|f(t)_{\min }\right|$ $+f(t)$ guarantees that its square root can always be obtained. The auditory perception to the preconditioned AM ultrasound is in the form of the square of the modulation signal, $\left|f(t)_{\min }\right|+f(t)$, which eliminates the harmonic distortion. In auditory perception test, the ultrasound which is modulated by the preconditioned and unconditioned consonant-vowel-consonant (CVC) syllables is used to stimulate the human cochlea. The subject's preconditioned ultrasonic intensity thresholds for CVC syllable recognition are lower than the unconditioned ultrasonic intensity thresholds for the same CVC syllable recog. nition. [Work partially supported by Electro-Stim Corp.]

\section{1:00}

3aPP10. Forward masking patterns and pitch estimation for bipolar and remote ground stimulation in a subject implanted with a Cochlear Pty. Limited "20+2" cochlear implant. Lawrence T. Cohen, Graeme M. Clark (Dept. of Otolaryngol., Univ. of Melbourne, Parkville 3052, Australia), and Lesley Whitford (Univ. of Melboume, Parkville 3052, Australia)

A new electrode array, recently developed by Cochlear Pty. Limited, enables "remote ground" as well as standard bipolar stimulation. To compare the patterns of electrical stimulation for these two operating modes, forward masking and pitch estimation investigations were performed with a single patient. The masking patterns were studied using a bipolar +1 or a remote ground masker, followed by a bipolar +1 probe. For maskers on apical and middle electrodes, masking patterns were more apical for bipolar +1 than for remote ground maskers, while for maskers on a basal electrode, masking was similar for the two masker modes. For a bipolar +1 masker on a middle electrode, masking was skewed strongly toward the apical end of the array. In the apical half of the array, while remote ground pitch reduced regularly with movement apically, bipolar +1 pitch dropped abruptly to a constant low value for the nine most apical electrodes and was lower than remote ground pitch for each electrode. The results show that remote ground stimulation may provide a different, and at least for this patient a better, range of pitch percepts, as well as other benefits such as reduced current requirement. [Work supported by NIH.]

\section{1:15}

3aPP11. Temporal pitch coding for cochlear implantees: The effects of carrier rate and amplitude modulation of pulsatile electrical stimuli. Colette M. McKay, Hugh J. McDermott, and Graeme M. Clark (Univ. of Melbourne and Australian Bionic Ear and Hear. Res. Inst., 384-388 Albert St., East Melbourne 3002, Australia)

The pitch evoked by sinusoidally amplitude-modulated current pulse trains on single electrodes was studied. Modulation frequencies of 100,150 , and $200 \mathrm{~Hz}$, together with carrier pulse rates from 200 to 1200 $\mathrm{Hz}$ were studied. The results showed that, in general, the pitch of the stimulation was close to but higher than that of a nonmodulated pulse train of rate equal to the modulation frequency. This general rule did not hold, however, whenever the carrier rate was both less than four times and not a multiple of the modulation frequency. Modulation depth was also found to significantly affect the pitch when less than approximately $7 \%$ of the current. In this region, the pitch rose uniformly with reduction in modulation depth and became equivalent to that of the carrier rate near the modulation detection threshold. The results of this investigation have relevance to speech processing schemes utilizing pulsatile stimulation, in which modulations in the processor output at the fundamental frequency of speech may provide a voice pitch percept to implantees. [Work supported by NIH.]

\title{
Session 3aSA
}

\section{Structural Acoustics and Vibration: Plates and Shells}

\author{
Courtney B. Burroughs, Cochair \\ Applied Research Laboratory, Pennsylvania State University, P.O. Box 30, State College, Pennsylvania 16801
}

\author{
Jean Nicolas, Cochair \\ Department of Mechanical Engineering, Université de Sherbrooke, 2500 Boul. Université, Sherbrooke, Quebec J1K 2RI, \\ Canada
}

\section{Contributed Papers}

\section{8:15}

3aSA1. Free vibration of a rotating annular plate including the effect of bending, membrane, shear deformation, and rotatory inertia. André Côté, Noureddine Atalla, and Jean Nicolas (Dept. of Mech. Eng., Univ. de Sherbrooke, 2500 Boul. Université, Sherbrooke PQ J1K 2R1, Canada)

Free response of an annular plate constrained at the inner radius is investigated using a variational approach. In the method used here, special attention has been taken to include all possible effects in linear theory: pure bending, Mindlin's shear deformation, rotatory inertia, and membrane effect. Membrane effect and bending (pure bending plus shear deformation) are then shown to be independent, thus allowing a separated evaluation of the natural frequencies. The influence of rotation is also investigated for both membrane and bending movement. This general problem is solved using the Rayleigh-Ritz method including symmetric and antisymmetric terms in the polynomials. Special cases where symmetric and antisymmetric natural frequencies are not equivalent can then be shown. The use of polynomials also produced stable results for a future acoustic study. Finally, the critical speed of rotation is determined for a nonrotating observer. Results are presented for every case. [Work supported by N.S.E.R.C. and I.R.S.S.T.]

\section{8:30}

3aSA2. Mean flow effects on the vibroacoustic behapior of baffled plates in heary fluids. Franck Sgard and Noureddine Atalla (G.A.U.S, Génie Mécanique, Univ. of Sherbrooke, Sherbrooke, PQ J1K 2R1, Canada)

The originality of the present paper lies on the development of a formulation accounting for mean flow effects in the vibroacoustic model of a baffled plate. The importance of those effects on the vibrational behavior and stability of a baffled plate with arbitrary boundary conditions, as well as its acoustic radiation pattern, is assessed. The analysis is based on a finite-element method for the calculation of the plate 
transverse vibrations and the use of the extended Kirchhoff's integral equation to account for fluid loading with mean flow. A boundaryelement method is used to compute the acoustic radiation impedance. The formulation shows explicitly the effects of mean flow in terms of added mass, stiffness, and radiation damping. Furthermore, the added stiffness is shown to be responsible for the instabilities that occur as the flow speed increases. Details of the formulation as well as its numerical implementation are exposed and results showing the effect of the mean flow on the different vibroacoustic indicators (mean square velocity, radiated acoustic power, modal radiation efficiencies) are presented.

\section{8:45}

3aSA3. The role of acoustic coupling on the radiation from a vibrating source. Lucio Maestrello (NASA Langley Res. Ctr., MS 463, Hampton, VA 23681-0001), Abdelkader Frendi (Analytical Services and Mater., Inc., Hampton, VA 23369), and Alvin Bayliss (Northwestern Univ., Evanston, IL 60201)

The response of an acoustically excited flexible surface together with the resulting sound radiation was considered. A model was employed that accounts for the full coupling between the surrounding fluid and the vibrating surface in three dimensions as well as two dimensions. The model couples the nonlinear Euler equations with an equation describing the nonlinear vibration of the plate. Different regimes of plate vibration and acoustic radiation were described, including linear, nonlinear, quasiperiodic, and apparently chaotic dynamics. It was found that, in the linear regime, the effect of the fluid/structure coupling is not significant. In the nonlinear regime, the effect of the coupling is to significantly reduce the harmonic content in both the plate vibration and the acoustic field. Furthermore, the nonlinear radiation exhibits a relative increase in harmonic content and an overall reduction in level as the acoustic propagation distance increases.

\section{9:00}

3aSA4. Acoustic radiation of a nonbaffled vibrating plate with arbitrary boundary conditions in light fluids. Carol Gauthier, Noureddine Atalla, and Jean Nicolas (G.A.U.S., Mech. Eng. Dept., Univ. de Sherbrooke, Sherbrooke, PQ J1K 2R1, Canada)

A study of the acoustic radiation in light fluids of a nonbaffled vibrating plate with arbitrary boundary conditions is conducted. $\mathbf{A}$ new approach using integral formulations combine with polynomial interpolations (Ritz method) is proposed to compute both the vibrations and the acoustic radiation of the plate. The formulation used to compute the acoustic radiated power is expressed in terms of jump of pressure through the plate. Simulations using different boundary conditions have been conducted in both cases, with baffled and with nonbaffled plate Comparison between both cases reveals the considerable effect of baffles on the modal and nonmodal radiation efficiencies in the lower frequencies. Comparisons are also made between cases with different boundary conditions (clamped-clamped-clamped-clamped, free-free-free-free, and simply supported-SS-SS-SS). Details of the formulations along with their numerical implementations are described and the results showing the effects of a baffle on the acoustic radiation are presented. Finally, the comparisons between the various cases are commented as a conclusion of the study.

\subsection{5}

3aSA5. Dispersive waves in fluid-loaded, multilayer, composite plates with lossy material. M. Kim and Y. F. Hwang (David Taylor Model Basin, Carderock Div., Naval Surface Warfare Ctr., Bethesda, MD 20084-5000)

One of the outstanding features of the multilayer composite plate is that it can be tailored for a specific structural-acoustic application by intelligently arranging the various layers of elastic and viscoelastic materials. In this study, the multilayer composite plate under consideration possesses a layer of viscoelastic material with high material loss factor for attenuating the propagating waves. For a fluid-loaded case, certain propagating modes dissipate their energy through radiation into surrounding fluid medium, and decreases in amplitude in the direction of propagation even if no material loss factor is assumed. Study of the wave dispersion of this composite plate indicates that the dispersion characteristics and the mode shape of the propagating wave are affected by the amount of damping in the viscoelastic material. The reasons for the aforementioned effect are discussed. Numerical examples are presented for various plate configurations with the viscoelastic layer given a range of material loss factor. [Work supported by ONR.]

\section{9:30}

3aSA6. Spatial attenuation of bending waves in the infinite plates coupled by a viscous damping layer. Jongmin Kang (Dept. of Mech. Eng. and Inst. for Manufacturing Res., Wayne State Univ., Detroit, MI 48202) and Adnan Akay (Carnegie Mellon Univ., Pittsburgh, PA 15213)

Plate vibrations can be reduced by a viscous damping layer between a primary excited plate and a secondary plate, of which frequency responses are considered by Ingard and Akay [J. Vib., Struct. Rel. Design 108, 178-184 (1987)]. For a free vibration, complex wave numbers are determined from a dispersion relation, where the imaginary part is related with the attenuation due to the damping layer. The steady state responses both in the plates and fluid layer are calculated when the primary plate is under a time harmonic line-driven force. Energy and power flow of a complex wave number are also considered. The complex wave number in a vibrating plate increases as the thickness of the damping layer decreases, such that the attenuation is greater and the period in space is shorter, and the waves in a damping layer become unstable. For a given thickness of a damping layer, as the exciting frequency is higher, the damping effects are weaker, since the boundary layer thickness becomes thinner. When a secondary plate of a same material is attached to attenuate the vibration of a primary plate, the secondary plate must be thicker than the primary plate to achieve an efficient vibration reduction. The bending waves in the primary plate are attenuated very fast, and become identical with the bending waves in the. secondary plate at far field.

\section{9:45}

3aSA7. A new model for predicting the sound transmission properties of orthotropic plates with general boundary conditions. Roland Woodcock and Jean Nicolas (G.A.U.S., Mech. Eng. Dept., Univ. de Sherbrooke, Sherbrooke, PQ J1K 2R1, Canada)

Actually, the structures used in many industrial areas are of particular type: they generally consist of an assemblage of different materials (multilayer structures) and they exhibit anisotropic properties. Real structures are of finite size and their properties are influenced by boundary conditions. The studies on the sound transmission through structures have been limited to the case of simply supported boundary conditions or clamped ones. The present work is intended to extend these different aspects. A new general model is developed for the characterization of multilayer systems consisting of orthotropic plates with general boundary conditions. This modeling concerns the transmission properties of oblique incident acoustic waves or diffuse field. The aim of the study is the development of tools allowing (1) the understanding of the different mechanisms involved in the transmission process, (2) studies on the influence of different parameters such as boundary conditions, the effect of structures size, and orthotropic properties. This model will allow the simulation of real conditions. A variational approach is used with a Ritz basis for the expansion of the flexural displacement of the structure. The different vibroacoustic parameters are determined from the coefficients in the basis. The transmission coefficient requires the calculation of either the radiation impedances, or the radiated power in the far field. An important discussion will be presented concerning this question. Among the areas applications of this model, one can mention aeronautics involved by structures of composite type, architectural acoustics, shipbuilding, etc. 
This paper treats the nonaxisymmetric motion of a thin elastic

3aSA8. An analytic model for the vibrations of rectangular shells of variable curvature and thickness. Masahiko Okajima and Courtney B. Burroughs (Grad. Prog. in Acoust., Penn State Univ., P.O. Bax 30, University Park, PA 16804)

A flexible and powerful model is developed for analyzing the vibration of a rectangular platform shell whose curvature and thickness are arbitrary. The shape of the shell can have almost any conceivable representation, since the curvature and thickness are represented by bicubic polynomials of the centerline arc length. The vibration model includes the effects of shear deformation, rotary inertia, and centerline extension. The equations of motion are solved by an alternative form of the Rayleigh-Ritz method. The resulting integral formulas for the stiffness and mass matrix elements are evaluated by a set of simple computer routines that do symbolic manipulations of algebra and calculus. Predictions of the natural frequency for the several shell geometries are compared to published data, with good agreement. A parameter study shows the dependence of the resonance frequencies and mode shapes on the curvature and the thickness of the shell.

\section{0:15-10:30 Break}

\section{$10: 30$}

3aSA9. Dispersion predictions for waves along a cylindrical shell immersed in fluid. Martin G. Manley (Grad. Prog. in Acoust., Penn State Univ., P.O. Box 30, State College, PA 16804)

The behavior of guided flexural waves of an infinite, elastic, circular cylinder immersed in fluid was considered previously [M. Manley, J. Acoust. Soc. Am. 92, 2387 (1992)]. The fluid is of lower density than the solid. The asymptotically exact dispersion relation derived previously is applied to determine dominant terms for dispersion of axial waves in the vicinity of the ring frequency. The effect of fluid loading on the cutoff frequency for the longitudinal mode is examined. [Supported by the PSU Applied Research Laboratory Exploratory and Foundational Research Program.]

\section{$10: 45$}

3aSA10. Vibrations and acoustic radiation of a simply supported cylindrical shell under circumferentially moving loads: Modeling and experiments. Raymond Panneton, Frédéric Laville, and Alain Berry (G.A.U.S., Univ. of Sherbrooke, Sherbrooke, PQ J1K 2R1, Canada)

The vibrational and acoustical behaviors of a simply supported cylindrical shell, immersed in a light fluid and excited with circumferentially moving radial loads are considered in the frequency domain. The equation of motion is developed using an energy method. To solve the problem, the shell displacements and boundary pressures are expanded in a series of in vacuo shell modes. The circumferentially moving loads induce a displacement with spatial and temporal dependences of the form $e^{j N_{\varphi}}$ and $e^{-j N \Omega t}$, where $N$ is an integer and $\Omega$ is the rotational speed of the loads. For this type of dependence, the equation of motion shows critical speeds for which the modal amplitudes are maximum. Also, a modal analysis is conducted to describe the general behavior of the shell, in the frequency domain, in terms of quadratic velocity, radiated sound power, and radiation efficiency under moving loads. This analysis shows that high levels of quadratic velocity and radiated sound power occur for the critical speeds of rotation. In order to validate this modeling, numerical results are compared with previous published works and laboratory experiments. This paper is the continuation of the paper presented at 124th ASA Meeting [J. Acoust. Soc. Am. 92, 2387 (A) (1992)].

\section{1:00}

3aSA11. Thin elastic spherical shells: the nonaxisymmetric motion. Jin-Meng Ho (SFA, Inc., 1401 McCormick Dr., Landover, MD 20785 and Naval Res. Lab., Washington, DC 20375-5000) spherical shell; it is motivated by the problem of a finite cylindrical shell with hemispheric shell endcaps. Here, the spherical shell is subject to intrinsic azimuthal deflection, as opposed to the well-documented case where the vibration is rendered axisymmetric by the special choice of coordinates. The kinematics of deformation is discussed, based on the thin shell assumption referred to in the literature as the Kirchhoff hypothesis. This leads to the expressions of the total kinetic and potential energies and external work in terms of the displacements of the middle shell surface; application of Hamilton's principle then yields the desired dynamical equations of nonaxisymmetric motion. It is found, as two special cases, that the spherical shell undergoing free vibrations supports shear waves, in addition to compressional and flexural waves; but that for fluid loading, the former decouples from the acoustic wave while the latter observe the same dispersion relations as in the axisymmetry case.

\section{1:15}

3aSA12. Time-domain fluid-solid interaction and the proliferation of response frequencies. Janet B. Jones-Oliveira (LLNL, L-84, P.O. Box 808, Livermore, CA 94551) and Leo P. Harten (Paradigm Assoc., Inc., Cambridge, MA 02138)

Exact modal solutions for the shell displacements and fluid pressure field resulting from the transient loading of a submerged, spherical shell are well-known [H. Huang, J. Acoust. Soc. Am. 45, 661-670 (1969)] Comparisons of the shell deformation time histories with experimental data have been published previously [J. B. Jones-Oliveira and P. J. Wender, 55th Shock \& Vib. Bull., Suppl. 3, 59-76 (1986)]. Herein, the analysis of the unloaded versus fluid-loaded frequencies reveals the effect of the fluid on the structual response to be more complex than simple damping and/or added mass. The fluid introduces a proliferation of frequencies in the structural response which is independent of the loading. The additional frequencies, which are introduced as a function of the expansion mode number via the fluid coupling, are associated with additional roots of an ever increasing higher-order system. The relative influence of the low, intermediate, and high frequencies are investigated. It is shown that while the proliferant frequencies are strongly damped and contribute little to the late time behavior in the far field, they can dominate the early time behavior. [This work was performed under the auspices of the U. S. Department of Energy by Lawrence Livermore National Laboratory under contract No. W-7405Eng-48.]

\section{1:30}

3aSA13. Radiation and propagation properties of elastic waves on conical shells. Y. P. Guo (Dept. of Ocean Eng., Rm. 5-204, MIT, Cambridge, MA 02139)

This talk discusses the radiation and propagation properties of elastic waves on conical shells by making use of a previously developed theory [Y. P. Guo, J. Acoust. Soc. Am. 92, 2388 (A) (1992)]. The three types of dominant waves in the mid-frequency domain, namely, the compressional, shear, and flexural waves are examined. It is shown that when propagating toward the apex of the cone, shear waves are turned back at caustics but compressional waves are cut off only where they have become subsonic. The radiation losses of these waves are also discussed. It is found that compressional waves radiate much more efficiently than shear waves on a cone, indicating that much less compressional wave energy would be reflected by a cone-shaped endcap. This is indeed what was observed in these scattering experiments.

\section{1:45}

3aSA14. Vibrations of core walls with connecting beams. Ö. Sümer, G. Așkar, and M. C. Dökmeci (Civil Eng. Dept., Boğaziçi Univ., 80815, Bebek, Istanbul, Turkey)

Vibrations of a singly symmetric core wall structure coupled with connecting bearns is studied using Vlasov's theory. Due to the presence 
of connecting beams, shear transfer occurs between the open edges of the core wall. The connecting beams are modeled as a continuum media with equivalent stiffness property. The results include warping effects and shearing deformations in the plane of the wall. Formulations for displacements and rotations at the top of the structure are given [cf. Ö. Sümer and G. Aşkar, Thin-Walled Struct. 14, 193-208 (1992)]. Results are compared with those of open core walls without connecting bearns. [Work supported by TÜBITAK.]

WEDNESDAY MORNING, 19 MAY 1993

BALLROOM, 8:00 TO 11:45 A.M.

\title{
Session 3aSP
}

\section{Speech Communication: Applications in Speech Science}

\author{
Donald G. Jamieson, Chair \\ Department of Communicative Disorders, Elborn College, University of Western Ontario, London, \\ Ontario N6G 1H1, Canada
}

Contributed Papers

8:00

3aSP1. An actuarial approach to cochlear implant candidacy in deaf children. I: Development of test instruments. Orna Eran and Arthur Boothroyd (Grad. School, City Univ. of New York, 33 W. 42 St., New York, NY 10036)

Cochlear implants are being used extensively in the habilitation and rehabilitation of profoundly and totally deaf children. It is generally accepted that an implant can provide significant access to acoustic speech information to children who cannot obtain this information through more conventional devices. Appropriate decisions regarding implant candidacy can be difficult in this population, however, because many of the children who are potential implantees are too young to perform reliably on tests that measure speech perception capacity. The purpose of this presentation is to describe two tests of speech perception assessment that are used with young deaf children. THRIFT is a threeinterval, forced-choice oddity procedure. The results depend on the adequacy of sensory data rather than on cognitive and linguistic status. IMSPAC is an imitative test for children as young as 3 years. In older subjects, scores obtained on IMSPAC correlate with those obtained on the THRIFT. The presentation will include data on applicability, reliability, validity, and efficiency of both tests. [Work supported by NIH Grant No. 2PO1DC00178.]

\section{8:15}

3aSP2. An actuarial approach to cochlear implant candidacy in deaf children. II: Comparison of implants and hearing aids. Arthur Boothroyd and Orna Eran (Grad. School, City Univ. of New York, 33 W. 42 St., New York, NY 10036)

The perception of speech pattern contrasts, via hearing aids, was measured in approximately 100 hearing-impaired children with a wide range of hearing losses. A regression function for performance on hearing loss were generated. Using identical tests, data were collected from approximately 60 profoundly deaf children who had been fitted with cochlear implants. Using the regression function obtained from the hearing aid users, the implantees' speech perception scores were converted to "equivalent hearing loss." The cumulative distribution of equivalent hearing loss was used to estimate the probability that a child with a given hearing loss would be expected to perform better with a hearing aid than with an implant. The data suggest that the hearing loss should be in excess of $105 \mathrm{~dB}$ before the odds in favor of superior performance with an implant are acceptably high. A factor analysis of the implantees data suggests four relatively independent factors that were labeled: perception, age, experience, and age-at-onset. [Work supported by NIH Grant No. 2PO1DC00178.]
8:30

3aSP3. Single- versus multichannel vibrotactile supplements to intonation and stress by normal-hearing and hearing-impaired adults. Lynne E. Bernstein, Edward T. Auer (Ctr. for Auditory and Speech Sci., Gallaudet Univ., 800 Florida Ave., N.E., Washington, DC 20002), David C. Coulter (Coulter Assoc., Fairfax, VA 22031), Paula E. Tucker (Gallaudet Univ., Washington, DC 20002), and Marilyn E. Demorest (Univ. of Maryland Baltimore County, Catonsville, MD 21228-5398)

The possible benefit of a wearable, single-channel versus eightchannel tactile aid for conveying voice fundamental frequency (F0) was estimated in three experiments. Severely or profoundly hearingimpaired ( $\mathrm{HI}$ ) and normal-hearing ( $\mathrm{NH}$ ) adults identified position of stressed words and rising versus falling intonation in sentences previously recorded for this purpose by Bernstein et al. [J. Acoust. Soc. Am. 85, 397-405 (1989)]. In experiment 1, NH subjects performed the identification task in counter-balanced visual-alone (VA) and visual-tactile (VT) conditions. Both tactile configurations conveyed intonation but neither conveyed stress. In experiment 2, NH subjects performed the task tactile alone. Both stress and intonation were conveyed. In experiment 3, pre- and post-lingually HI subjects demonstrated effects of the aid for identification of intonation but not of stress. As in the previous study (Bernstein et al, 1989), visual stress was highly accurate in all VA conditions and tactile information shown to be present in experiment 2 did not improve identification in VT conditions.

\section{8:45}

3aSP4. Automated derivation of phonemic spectral distribution in speech output of nonlinear hearing aids. Faye Erickson (Lexington Ctr., 30th Ave. and 75th St., Jackson Heights, NY 11347), Eddy Yeung, and Arthur Boothroyd (City Univ. of New York, New York, NY 10036)

Interactions occur between the temporal characteristics of nonlinear processing schemes and the temporal and spectral properties of speech. Uncertainties in the nature and extent of these interactions make it difficult to predict hearing aid performance with speech input. An option is empirical measurement, but the collection of spectral data on a sample of phonemic segments can be prohibitively time consuming. This paper describes progress toward the development of an automated process. Input is derived from digitized samples of connected speech in which the temporal locations of segments of interest are already known. Recordings of speech output are, themselves, digitized. Using a known onset marker, the segments of interest are automatically extracted, subjected to FFT transformation, and integrated over a moving 1/3-oct window. The intensities and frequencies of key spectral points from each spectrum are displayed on a graph of intensity versus frequency for 
comparison with the input data. [Work supported by NIDRR Grant No. H133E80019.]

\section{$9: 00$}

3aSP5. Estimated hearing handicap (using the AMA method and a self-evaluation questionnaire) versus reduction in speech intelligibility in quiet and noise. Michael J. Nilsson (House Ear Inst., 2100 West Third St., Los Angeles, CA 90057), Donna Felker, Allen Senne (House Ear Clinic, Los Angeles, CA 90057), and Sigfrid D. Soli (House Ear Inst., Los Angeles, CA 90057)

Three methods of estimating hearing handicap were compared in subjects with bilateral sensorineural losses. Sentence speech-reception thresholds (sSRT) measured in quiet and $65 \mathrm{~dB}(\mathrm{~A})$ noise were compared to the American Medical Association (AMA) method, based on pure-tone thresholds above $25 \mathrm{~dB} \mathrm{HL}$, and to a self-evaluation of handicap (Hearing Handicap Inventory for Adults, HHIA). The AMA correlated highest with sSRTs in quiet $(r=0.92)$, followed by SSRTs in noise $(r=0.81)$, and directional hearing in noise $(r=0.84)$. The HHIA was confounded by age effects, and increasing age decreasing perceived handicap. Thresholds were also measured with noise at a sensation level (SL) $25 \mathrm{~dB}$ above sSRTs to partially compensate for audibility differences among subjects. In this noise condition, the AMA correlated with sSRTs in noise $(r=0.76)$ and directional hearing in noise $(r=0.58)$, though the directional hearing is better predicted by pure-tone thresholds at $3 \mathrm{kHz}(r=0.64)$. Stepwise regression analysis suggests handicap continues below 0\% AMA handicap in the quiet and 25-dB SL noise conditions.

\section{9:15}

3aSP6. Speech changes during exacerbation in multiple sclerosis. J. Anthony Seikel, Charles L. Madison, David G. Drumright, Marilyn Russell (Univ. Prog. in Commun. Disord., Washington State Univ. at Spokane, W. 601 First Ave., Spokane, WA 992040399), N. Roger Cooke, and Charles E. Brondos (Neurology Associates, Spokane, WA 99204)

Multiple sclerosis (MS) is a demyelinating disease with unpredictable course and variable periods of symptom exacerbation and remission. This study identified characteristics of speech in MS that signaled increased disease activity of exacerbation. Speech of eight MS and eight control subjects was recorded at 5-week intervals for a year. Stimuli were CVCs in the carrier "Say again." Durations measured included vowel in "say," initial stop consonant VOT $(/ p, t, k, b, d, g /)$, target vowel $(/ \mathrm{i}, \mathrm{a} /)$, and post-vocalic closure preceding final stops $(/ \mathrm{t}, \mathrm{d} /)$. A number of speech segments were longer for MS than controls. Data from sessions were aligned by date of exacerbation of individual MS subjects and analyzed to determine changes in speech occurring before, during, and after exacerbation. Exacerbations revealed striking reductions in duration for vowels, closure durations, and overall sentence duration, and increases in voiceless VOT duration.

\section{9:30}

3aSP7. Perception of supraperiodic voices. Jody Kreiman, Bruce R. Gerratt, Kristin Precoda, a) and Gerald S. Berke (VA Med. Ctr., West Los Angeles, Audiol. \& Speech Pathol. (126), Wilshire \& Sawtelle Blvds., Los Angeles, CA 90073 and Div. of Head/Neck Surgery, UCLA School of Medicine, Los Angeles, CA 90024)

The study of voice quality is built largely upon the assumption of vocal (quasi-)periodicity. Supraperiodic phonation types challenge traditional concepts of vocal periodicity, and thus are of theoretic and practical importance, especially when measuring or describing pathologic vocal function. Unfortunately, the literature describing such phonation is confusing. It has been argued [Gerratt et al., J. Acoust. Soc. Am. Suppl. 183 , S66 (1988)] that the traditional term "diplophonia" has been applied to two distinct phonation types. In one (true diplophonia), the two vocal folds vibrate at different rates, producing a waveform resembling a high-frequency wave modulated by a lower frequency envelope. In the second phonation type ("bicyclicity"), a pattern of two cycles repeats; cycles within the pattern differ in period and/or amplitude, giving a big-small-big-small appearance to the waveform. To investigate the perceptual characteristics of these phonation types, sets of male and fernale voices were constructed that included equal numbers of diplophonic, bicyclic, and noisy voices. Expert listeners judged the dissimilarity of pairs of these voices. Multidimensional scaling analyses confirmed that bicyclicity and diplophonia are easily distinguished from each other and from rough or breathy voices. Psychoacoustic studies examining factors underlying the nature of the bicyclic and diplophonic percepts will also be described. Implications of findings for periodicitydependent models of laryngeal function will be discussed. ${ }^{\text {a) }}$ Also at Bell-Northern Res., Verdun, PQ H3E 1H6, Canada.

\section{$9: 45$}

3aSP8. A comparison of voice analysis systems for perturbation measurement. Steven Bielamowicz, Jody Kreiman, Bruce R. Gerratt, Marc S. Dauer, and Gerald S. Berke (Div. of Head and Neck Surgery, UCLA School of Medicine, CHS 62-132, Los Angeles, CA 90024 and VA Med. Ctr., West Los Angeles, CA 90073)

Dysphonic voices are commonly analyzed using automated digital voice analysis software. However, reliability and validity of acoustic measures obtained from these programs has been questioned, especially in the assessment of dysphonic voices. This study evaluated the agreement among various commercially available speech analysis programs and a handmarked voice analysis system. Sustained vowels of 25 male and 25 female speakers with mild to severe dysphonia were digitized and jitter, shimmer, and harmonics-to-noise ratio were analyzed using CSpeech, Computerized Speech Laboratory (CSL), and SoundScope. The effect of dysphonic severity on the measures provided by these four programs was also assessed. The clinical utility and theoretical limitations of automated, perturbation-based measures will be discussed, especially with regard to the indeterminant nature of $F 0$ in some types of dyhsphonia.

\section{0:00}

3aSP9. A comparison of frequency spectra of normal and hoarse voices. Amando García, José Romero, and Francisco Cervera (Dept. of Appl. Phys., Univ. of Valencia, Ave. Dr. Moliner, 46100, Burjassot, Spain)

In the framework of source research for developing an auxiliary tool for voice rehabilitation, the differences between normal and hoarse voices have been analyzed. Five women with normal voices (control group) and five hoarse women (experimental group) have been selected as study subjects. Frequency spectra from laboratory voice recordings of the five Spanish vowels for all subjects have been obtained with a real time analyzer. The frequencies of fundamental pitch $(F 0)$ and the first four formants $(F 1, F 2, F 3$, and $F 4)$ are similar for both groups of voices. However, this demonstrates that there is a loss of harmonics in people with hoarse voices. The main intensities (spectrum envelopes) for experimental group subjects are always lower than that for control group subjects. The differences between the two groups are specially located for the frequency range of $1000-3000 \mathrm{~Hz}$ (about $20 \mathrm{~dB}$ ).

\section{0:15}

3aSP10. Nasometric evaluation of velopharyngeal function in nonliterate subjects. Ian R. A. MacKay (Dept. of Linguist., Univ. of Ottawa, Ottawa, ON K1N 6N5, Canada) and Ann W. Kummer (Children's Hospital Med. Ctr., Pavilion Bldg., Cincinnati, OH 45229-2899)

The Kay Elemetrics nasometer measures nasalance, a parameter of speech that reflects the proportion of total acoustic energy that is emitted nasally, making it possible to infer velopharyngeal (VP) function noninvasively. Nasometric evaluation is potentially widely applicable in the clinical assessment of suspected VF impairment. For clinical use, a patient's mean nasalance on a passage of known phonetic composition 
must be compared to age-appropriate population norms. Most potential clinical subjects are young; many are preliterate. Passages for which norms have been established (Zoo, Rainbow, Nasal Sentences) are syntactically, semantically, and lexically complex, phonetically heterogeneous, phonologically mature, and long. Individual child subjects' nasalance scores, if obtained at all, are therefore likely to be contaminated by artifacts created through hesitation noises, filled pauses, phonetic deviance from normed target, age differences, and measurement errors induced by coaching procedures. Differences in phonetic content between normed and actual utterances are almost inevitable; they lead to uninterpretable results. This study reports a technique for obtaining clinically useful nasalance scores from young, preliterate subjects, even those evidencing phonological deficits or noncompliant behavior. Large- $n$ norms for preschool and primary children are presented.

\section{0:30}

3aSP11. Developmental phonological impairment: An acoustic description of improved production. Kim A. Wilcox, Sherrill R. Morris (Dept. of Speech-Lang.-Hear.: Sci. and Disord., Univ. of Kansas, Lawrence, KS 66045), and Kathleen A. Siren (St. John's Univ., Jamaica, NY 11439)

Although misarticulation of speech is one of the most common communication problems of young children, there is little available information concerning the phonetic form of young children's errors. This paper reports on a longitudinal analysis of the acoustic properties of speech samples from two phonologically impaired children. Both children were enrolled in remediation programs that focused on the correct production of stops and fricatives. Bimonthly samples were taken from each child over a period of several months coinciding with significant improvement in perceived phonetic accuracy. Both durational and spectral aspects of the children's speech were monitored throughout the period. The relationships between changes in specific acoustic properties and correct perception as well as interspeaker differences are discussed.

\section{$10: 45$}

3aSP12. Effects of alterations in auditory feedback on stuttering frequency during fast and normal speech rates. Joseph Kalinowski, Joy Armson, and Andrew Stuart (Dalhousie Univ., 5599 Fenwick St., Halifax, NS B3H 1R2, Canada)

That stuttering is ameliorated under altered auditory feedback conditions (e.g., masking and delayed auditory feedback) has been known for over $\mathbf{4 0}$ years. While this effect was initially attributed to auditory factors, more recent explanations have suggested that a change in speech production, specifically slowed speed rate is responsible. The purpose of this study was to determine if stutterers could demonstrate fluency enhancement under various altered auditory feedback conditions at normal and fast speech rates. Using these rates, stutterers read eight 300-syllable passages under nonaltered auditory feedback (NAF), delayed auditory feedback (DAF), frequency-altered feedback (FAF), and a combination of DAF and FAF (DAF + FAF). Results showed that stutterers significantly reduced stuttering under all altered auditory conditions at both speech rates. These results indicate that a slowed speech rate is not necessary to achieve fluency enhancement under altered auditory conditions. Because sensory and motor events are inseparable components within the speech process, it is proposed that reduction in stuttering occurs as a result of alterations to both auditory feedback and speech production. [Work supported by NIH-DC-00201 awarded to Haskins Laboratories.]

\section{1:00}

3aSP13. Perception of coarticulatory cues in the speech of profoundly hearing-impaired and normally hearing children. Shari $R$. Baum (SHCD, McGill Univ., 1266 Pine Ave. W., Montreal, PQ H3G
1A8, Canada) and Robin S. Waldstein (CUNY Graduate Ctr., New York, NY 10036)

Two experiments investigated the perception of coarticulatory cues in the speech of normally hearing (NH) and profoundly hearingimpaired (HI) children. To examine anticipatory coarticulation, five repetitions of the syllables [ši šu ti tu ki ku] produced by nine NH and nine HI children were edited to include only the aperiodic consonantal portion. To explore perseveratory coarticulation, comparable segments were excised from the syllables [iš uš it ut ik uk]. Ten listeners were asked to identify the missing vowel. Results revealed that, for anticipatory coarticulation, listeners were able to identify the absent vowel with better-than-chance accuracy for all stimuli but the HI speakers' [š] tokens. For perseveratory coarticulation, identification accuracy was worse overall, but still significantly above chance for the NH stimuli; for the HI children's productions, only [k] tokens yielded better-thanchance vowel identification. Listener's identification of NH speakers' tokens was significantly better than that of HI speakers' productions for both anticipatory and perseveratory contexts. Results are discussed in relation to the nature of speech production in the $\mathrm{HI}$ and the role of contextual cues in speech perception.

\section{1:15}

3aSP14. Processing of stop-vowel syllables by normal and language-impaired children. Rachel E. Stark (Audiol. and Speech Sci., Purdue Univ., West Lafayette, IN 47907) and John M. Heinz (Kennedy-Krieger Inst., Baltimore, MD 21205)

It has been claimed that language-learning disordered children have impaired rapid-rate auditory processing. One manifestation is the failure to identify stop-vowel syllables; another less fine-grained discrimination of stop-vowel syllables. In the present study, 20 language-impaired (LI) and 20 normal (LN) children ( 6 to 10 years) attempted to identify $/ \mathrm{ba} /-/ \mathrm{da} /$ syllables within an adaptive paradigm. When they failed to do so, they were given a change/no change discrimination task with these stimuli. Formant transition duration (FTD) ranged from 80 to 40 ms. The syllable pairs had either five or two formants. As in previous studies, LI children failed the identification task, even for syllables of 80-ms FTD, significantly more often than LN. They were able to discriminate the syllables. Number of formants was not a significant variable. A subgroup of LI and LN children were subsequently asked to produce the test syllables. In both groups, children unable to produce the syllables had difficulty in identify them. The reverse was not true. Perception difficulties were not predictive of production errors. [Work supported by NIH.]

\section{1:30}

3aSP15. Reading disability: A deficit in rate of auditory processing or in phonetic coding? Maria Mody and Michael Studdert-Kennedy (Haskins Labs., 270 Crown St., New Haven, CT 06511)

The study investigates a general hypothesis regarding the bases of certain reading disabilities: poor readers suffer from an impaired rate of auditory processing [P. Tallal, Brain Lang. 9, 182-198 (1980)], such that they are unable to discriminate or detect the order of a pair of brief complex tones at short interstimulus intervals. Such children also had difficulty with two synthetic speech sounds, $/ \mathrm{ba} /$ and $/ \mathrm{da} /$, on analogous tasks, which was similarly attributed to a deficit in the processing of rapid acoustic changes, such as those which occur in formant transitions signaling place of articulation in stop consonants. The present study, in establishing the significantly lower performance by poor readers, compared to good readers, with $/ \mathrm{ba} /$ and $/ \mathrm{da} /$, at short interstimulus intervals $(100,50$, and $10 \mathrm{~ms})$, however, failed to show any significant difference between the groups when the acoustic-phonetic contrast of the stimulus pair to be discriminated was increased, as with $/ \mathrm{ba} /-/ \mathrm{sa} /$ or $/ \mathrm{da} /-/ \mathrm{Ja} /$. Further, that the good and poor readers here, did not differ significantly on discrimination at these short interstimulus intervals, with acoustically similar nonspeech sinewave analogs of $/ \mathrm{ba} /$ and $/ \mathrm{da} /$, appears to implicate the role of phonetic processing. 


\title{
Session 3aUW
}

\section{Underwater Acoustics: Acoustic Imaging}

\author{
J. Robert Fricke, Chair \\ Massachusetts Institute of Technology, Department of Ocean Engineering, Room 5-218, 77 Massachusetts Avenue, \\ Cambridge, Massachusetts 02139
}

Chair's Introduction-7:55

Invited Papers

$8: 00$

\begin{abstract}
3aUW1. Application of sidescan sonar to geological sea floor mapping. David C. Twichell and Thomas F. O'Brien (US Geological Survey, Woods Hole, MA 02543)

Digital sidescan-sonar images collected during a systematic mapping of the deep water part of the US continental margins, show a more complex near-surface geology than anticipated from previous mapping programs. For example, reconnaissance mapping with the GLORIA system (6.5-kHz operating frequency) in the Gulf of Mexico revealed channels extending hundreds of kilometers across the basin floor and discontinuous high-backscatter deposits in areas previously inferred to be laterally uniform deposits. Subsequent studies over part of the same area using the 27- to 30-kHz SeaMARC system permitted a detailed mapping of smaller channels and the high-backscatter deposits around their ends while cores permitted a link between seafloor geology and the imagery. Sandy and silty beds ranging from $10-118 \mathrm{~cm}$ thick occur in the areas of high backscatter and muds from areas of low backscatter. The imagery and cores in some cases permit mapping the extent and geometry of individual deposits. The deposits are a series of laterally discontinuous sand and silt lenses with abrupt edges rather than a continuous sheet of sand as previously was inferred for this area. This complex geology needs to be incorporated in efforts to understand the interaction of acoustic signals with the seabed.
\end{abstract}

\section{$8: 25$}

3aUW2. Swath acoustic imaging of the seafloor. Christian de Moustier and Clyde Nishimura (Naval Res. Lab., Code 7420, 4555 Overlook Blvd., Washington, DC 20375-5350)

Recent developments in bathymetric sidescan sonar systems and in multibeam echosounders have allowed these systems to produce coregistered bathymetry and sidescanned acoustic images of the seafloor in near real time. As a result, in addition to producing geometrically correct images with much less ambiguous information than conventional sidescanned seafloor images, the effects of the local relief and beam patterns on the magnitude of the backscattered signals can be readily compensated to bring out the angular dependence of acoustic backscatter across the swath. By isolating regions of homogeneous tonal appearance in the resulting image, a first-order assessment of the spatial variability in the composition of the bottom can be made. In such areas of the image, where the seafloor is presumably uniform in composition, the angular dependence of acoustic backscatter can be used to infer statistical terrain characteristics such as surface roughness at smaller spatial scales than obtainable with the bathymetry alone. The deterministic approach of shape-from-shading described in a companion presentation by $\mathrm{C}$. Nishimura (same session) can potentially lead to greater bathymetric resolution. Examples are provided with data from multibeam and bathymetric sidescan sonar systems. [Work supported by ONR.]

\section{$8: 50$}

3aUW3. Microbathymetry from Shape From Shading of sidescan acoustic imagery. Clyde Nishimura and Christian de Moustier (Naval Res. Lab., Code 7420, 4555 Overlook Blvd., Washington, DC 20375-5350)

Shape From Shading (SFS) is a methodology for extracting the topography of a surface given the acoustic (or electromagnetic) backscattered imagery. As a sidescan image is of generally finer spatial resolution than multibeam bathymetry at the same frequency, there is some promise that SFS can provide information on the microtopography of an area. However, several key assumptions need to be made in order to apply SFS techniques on sidescan data; foremost of these assumptions is that of homogeneity of the backscattering properties of the seafloor across the imaged area. The sidescan sonar device can also introduce ambiguities in the SFS method if a flat-bottom assumption is used to position the pixels and/or the far-field transmit beam pattern is poorly known. The results obtained from SFS can be readily compared to acoustic data collected from new bathymetric sidescan systems that produce coregistered bathymetry and sidescan images (companion presentation by C. de Moustier, same session). [Work supported by ONR.]

\section{9:15}

3aUW4. Neural computing techniques for acoustic image processing. Mark Dzwonczyk (Sensor and Commun. Electron. Div., C. S. Draper Lab., 555 Technology Square, Cambridge, MA 02139)

Computational architectures modeled after the biological nervous systems, so-called neural networks, have been shown to excel at pattern classification problems where the input data set is large and corrupted by noise and the solution formulation is 
not well defined. This paper discusses the application of neural computing techniques to a target detection problem with side-scan sonar data. A brief review of the fundamental concepts of feed-forward neural networks is first presented. Image segmentation and classification with a large analog electronic neural network are then described. It is shown that such neural computing approaches can be expected to be more robust than conventional statistical classifiers and are far better suited for deployed, real-time implementation of the system architecture.

\section{9:40}

3aUW5. Acoustic imaging with a bottom-mounted sonar. Darrell R. Jackson (Appl. Phys. Lab., College of Ocean and Fishery Sci., Univ. of Washington, Seattle, WA 98105)

Phase-coherent processing of data from a bottom-mounted, circularly scanning sonar provides a view of the time evolution of the seafloor that is unlike that obtained with conventional sonar. These techniques were developed under the ONR STRESS program, and will be illustrated using data from the STRESS experiment as well as from a later experiment. Ping-ping correlation is used to provide images of cumulative change in the bottom as well as images of current rate of activity. Correlation between echo signals received on the upper and lower halves of the receiving array provides images of fine-scale bathymetry. Time series of all these image types will be presented as false-color movies. Means of extracting quantitative measures from the images will be discussed.

10:05

3aUW6. Shape reconstruction from high-frequency sonar data for robotic tasks. John J. Leonard, Chryssostomos Chryssostomidis, and Bradley A. Moran (Sea Grant College Prog., MTT, E38-300, 292 Main St., Cambridge, MA 02139)

This talk will describe an approach to the recovery of geometric object shape descriptions from high-frequency sonar data for robotic applications. The goal is to recover explicit geometric surface descriptions for man-made objects, by fusing the geometric constraints of multiple sonar returns obtained by a moving autonomous underwater vehicle (AUV). Automated sensor data interpretation is made difficult by the pervasive problem of data association-uncertainty in the origins of measurements-in addition to the uncertainty in the values of measurements (noise) that is present in more traditional estimation and control problems. This problem is particularly severe when using sonar, for it is impossible to determine the geometry (e.g., sphere, cylinder, plane, corner, or spurious multiple reflection) that produced a single time-of-flight sonar return in isolation. One must first group measurements from different sensors and/or different sensing locations that have a common origin, to characterize the reflecting surface geometry. State estimation or surface fitting techniques can then be performed on correctly classified sets of measurements to cormplete the shape recovery process. Experimental results will be presented for two applications: (1) land robot map building and localization using the $50-\mathrm{kHz}$ Polaroid ultrasonic ranging system, and (2) underwater scene reconstruction using a 1.25-MHz mechanically scanned profiling sonar.

$10: 30$

3aUW7. Three-dimensional acoustic tracking of zooplankton. Jules S. Jaffe (Marine Phys. Lab., Univ. of California at San Diego, San Diego, CA 92093-0238)

As part of a National Science Foundation initiative in Global Ecology (GLOBEC) our group in acoustic imaging in the Marine Physical Laboratory, Scripps Institution of Oceanography, U.C.S.D., has been operating a multibeam acoustic irnaging system whose purpose is to track small animals (zooplankton) in the water column in three dimensions. The system has been constructed to operate at a frequency of $420 \mathrm{kHz}$ and consists of a set of side-scan sonarlike transducers. Together, the eight transmitters and eight receivers allow imaging of an $8 \times 8$ image. Lateral resolution is $2 \times 2 \mathrm{deg}$ and the range resolution of the system is on the order of centimeters. The system produces frames (3-D images) at a rate of approximately 1 frame per second. To date, the system has been used to image the trajectories of small fish and test targets in a test tank environment. Animated movies, derived from the data have been fabricated which clearly show the animal's movements. Quantitative analysis of the systems's performance has confirmed the three-dimensional locations of the animals that are produced from the data.

\section{0:55-11:15 Break}

\section{Contributed Papers}

\section{1:15}

3aUw8. Inverting, imaging, and mapping ocean-basin reverberation onto high-resolution bathymetry. Nicholas C. Makris and Jonathan M. Berkson (Naval Res. Lab., Washington, DC 20375)

The objective of this paper is to determine the relationship between low-frequency ocean-basin reverberation and bathymetry. Long-range reverberation data acquired on the western flank of the Mid-Atlantic Ridge (MAR) are mapped onto high-resolution bathymetry. Since reverberation was measured via horizontally towed line array, there is a "right-left" ambiguity about the array's axis and a decrease in resolution for off broadside beams and more distant returns. These ambiguities make an objective correlation between reverberation and bathymetry difficult. Therefore, an optimization procedure has been applied for eliminating the right-left ambiguity and maximizing resolution by simultaneously inverting data taken with differing array locations and orientations. Combined with range-dependent propigation modeling, the images show that distant reverberation is significant when the intermediate propagation path is free from bottom interaction. Since the experiment site is primarily bottom-limited, such paths are not abun- 
dant, and a sparse azimuthal distribution of strong returns at convergence zone intervals is often found. In general, strong returns coincide with prominent bathymetric features, such as abyssal hillsides. However, such correlations must be made judiciously because the azimuthal resolution of distant returns is often on the order of the width and separation between ridges in the MAR's continuously rugged bathymetry.

\section{1:30}

3aUW9. Acoustic daylight: Design and performance of a multi-beam imaging system. John R. Potter and Michael J. Buckingham (MPL 0238, Scripps Inst. of Oceanogr., University of California at San Diego, 9500 Gilman Dr., La Jolla, CA 92093-0238)

A multibeam "Acoustic Daylight" [Buckingham et al., Nature 356 (1992)] system is being developed that is capable of forming real-time images at $30-\mathrm{Hz}$ frame rate with 127 simultaneous beams. Objects at $10-$ to 200 -m range will be imaged, primarily employing acoustic information over $8-70 \mathrm{kHz}$. The raw data rate of such a system is over 25 Mbyte/s, which poses serious beamforming, real-time imaging, and data storage problems. The solutions we have developed include: an acoustic lens in the form of a spherical reflector with multi-element sensor (which achieves beamforming geometrically rather than through phasing); frequency-estimator preprocessing; and an economic 40 MFlop DSP engine. The color and intensity of each pixel of the final image will be determined by the spectral shading and intensity of the received signal in each beam. The limitations of the design are explored in terms of their impact on expected performance. Image simulations have been computed based on a numerical sum of a closed-analytic expression for acoustic energy scattering from a submerged body. The illuminating noise field is chosen to represent a shallow-water waveguide. Several objects are chosen for image synthesis, corresponding to obvious applications of interest. [Work supported by the Office of Naval Research.]

\section{1:45}

3aUW10. Detection of buried landmines by imaging with surface waves. Peter A. Krumhansl, Bill G. Watters, and David A. Sachs (BBN STC, 70 Fawcett St., Cambridge, MA 02138)

A technique has been developed to search an area of soil for buried landmines from a remote position. A two-dimensional synthetic aperture array of seismic sources and receivers is laid down adjacent to the search area. Broadband seismic sources generate Rayleigh surface waves that illuminate a search area out to a radius of $6 \mathrm{~m}$ and a depth of about $0.5 \mathrm{~m}$. An array of vertical geophones receives backscattered signals that are recorded digitally. Unconsolidated soils have high rates of attenuation so an array with up to $21-\mathrm{dB}$ of gain is used. A delay-and-sum near-field beamformer computes a $60 \times 80$ pixel image of the region's scattering strength. The search area image displays the location and character of landmine echoes along with clutter. A large amount of field data has been collected at several sights. The mine echoes have been detected at ranges up to $5 \mathrm{~m}(\mathrm{Pd}=0.8)$. Landmines have been distinguished from rocks and holes, and false alarms due to system artifacts are being reduced. [Work supported by U.S. Army BRDEC.]

\section{2:00}

3aUW11. Imaging of ocean subbottom structure using swath mapping data: Feasibility studies. Mrinal K. Sen, Faruq A. Akbar, Paul L. Stoffa (Inst. for Geophys., Univ. of Texas at Austin, 8701 N. MoPac Expwy., Austin, TX 78759), and Joseph A. Gettrust (NRL-Stennis Space Center, MS 39529)

With the aim of resolving small scale geologic features within upper $100 \mathrm{~m}$ of sediments, NRL-SSC has developed a high-resolution swath mapping device based on SeaMARC sidescan sonar technology but with additional parametric $500-\mathrm{Hz}, 1-\mathbf{k H z}$, and $2-\mathbf{k H z}$ source capabilityMethods have been developed for modeling and imaging of such data in order to address the problem of image resolution. A modeling method that uses an clastic Kirchhoff-Helmholtz (KH) formulation rapidly calculates the arrival time, and amplitude of scattered signal. KH synthetics have been compared with elastic finite difference results. Synthetic phase data are computed by ray tracing for a given subsurface model. Also, a Kirchhof migration code has been developed that uses both the travel time and angle for imaging conditions. The imaging method has been tested on synthetic data with various degree of random noise added so as to investigate how the signal-to-noise ratio affects the images. These methods will be applied to real data collected by NRL. Like any imaging method, we require an estimate of subsurface velocity. This is being done by using several different possible velocity models and comparing the imaging results. [Work supported by ONR.]

\section{2:15}

3aUW12. Reverberation imaging of large scale under ice keel distrlbution. T. C. Yang (Naval Res. Lab., Washington, DC 20375) and T. Yates (Vector Res. Co., 2101 E. Jefferson St., Rockville, MD 20852)

Low-frequency reverberation data received on a large aperture planar array of hydrophones are used to estimate the under-ice scattering strength over a basin wide area. The result is interpreted as a large scale under-ice keel distribution which, unlike the ice surface ridge distribution, has not been measured over a wide area. The reverberation data were collected during the CEAREX 89 experiment using explosive sources. Scatterer localization was performed for each time segment. The range-bearing ambiguity distributions at depth near the surface was range gated according to estimated two-way travel times, and combined to form the ambiguity surface for a large area. Ice keel distribution is obtained by removing the two-way transmission loss to and from the surface scatterers. This image is compared with that obtained using conventional beamforming techniques. 


\title{
Meeting of Accredited Standards Committee S2 on Mechanical Shock and Vibration
}

to be held jointly with the

\section{U.S. Technical Advisory Group (TAG) Meeting for ISO/TC 108 Mechanical.Vibration and Shock}

\author{
S. I. Hayek, Chairman S2 \\ Applied Research Laboratory, Penn State University, P.O. Box 30, State College, Pennsyluania 16801 \\ D. F. Muster, Chairman \\ U.S. Technical Advisory Group (TAG) for ISO/TC 108 \\ 4615 O'Meara Drive, Houston, Texas 77035
}

\begin{abstract}
Standards Committee S2 on Mechanical Shock and Vibration. Working group chairs will present reports of their recent progress on writing and processing various shock and vibration standards. There will be a report on the interface of S2 activities with those of ISO/TC 108 (the Technical Advisory Group for ISO/TC 108 consists of members of S2, S3, and other persons not necessarily members of those committees) including a report on the current activities of ISO/TC 108, and its most recent meeting, which took place in London, United Kingdom, from 22 March to 2 April 1993.
\end{abstract}

\author{
Session 3pAA \\ Architectural Acoustics: Sound Transmission and Reverberation \\ Richard H. Talaske, Chair \\ Talaske-Joiner Group, 137 North Oak Park Avenue, Oak Park, Illinois 60301
}

\section{Contributed Papers}

$\mathbf{1 : 0 0}$

3pAA1. Performance transmission loss and the "high low" rating method for predicting and measuring sound resistance of walls, ceilings, and floors. Tom Paige (Vibron Ltd., 1720 Meyerside Dr., Mississauga, ON L5T 1A3, Canada)

This method uses a two-number rating system as opposed to the single-number rating used in the STC method. The advantage of using two numbers is to distinguish between the speech- and low-frequency sound transmission loss performance. This makes the rating useful for applications involving music sources or mechanical system noise, in addition to speech-frequency applications to which the STC method is limited. The two numbers correspond to the speech-frequency performance transmission loss (SPTL) and the low-frequency performance transmission loss (LPTL). As explained in this paper, SPTL and LPTL predictions and measurements are a function of the source spectrum, the receiving-room noise criteria and the sound transmission loss data. When applied to common wall, floor, and ceiling constructions, SPTL and LPTL predictions correlate very well with measurements in the 500- and 125-Hz octave bands, respectively, thus simplifying field testing for performance verification.

$$
\text { 1:15 }
$$

3pAA2. Design and commissioning of a new floor sound transmission facility. R. E. Halliwell, J. D. Quirt, and A. C. C. Warnock (IRC,
Natl. Res. Council, M27 Montreal Rd., Ottawa, ON K1A OR6, Canada)

The pair of reverberation room that have been used at National Research Council Canada for about 30 years, are deemed too small by current ASTM standards. A new pair of rooms that incorporate some interesting ideas to satisfy the requirements and recommendations in both ISO and ASTM standards has just been constructed. One vertical cross section through each room is a five-sided polyhedron. The test specimen is at one of the sides. This design allows the room volumes to be large (about $175 \mathrm{~cm}^{3}$ each), the specimen to be a normal size, and yet to be one complete surface of the room. The niche between the two room is minimal. Both rooms are resiliently supported as is the floor support. The upper room is of a lightweight construction and the lower is of poured concrete. Floors are constructed on wheeled, heavy concrete frames that can be moved in or out of the slot between the rooms. A crane outside the room allows other test frames with floors already constructed to be inserted into the specimen opening. Some details of the design will be presented as well as sound transmission results from reference specimens from all three pairs of rooms at NRC. These differ quite markedly in size and results for receiving rooms ranging from $65-250 \mathrm{~cm}^{3}$ can be compared. A small amount of a detailed examination of the sound fields in the rooms based on measured and calculated data will be presented. 
formed on four windows installed in field conditions ranging from high absorption with no flanking to very large reverberant spaces with flank-

3pAA3. Inter-laboratory comparisons of low-frequency sound transmission: I. Conventional and intensity measurements. $M$. Vorländer (Phys. Tech. Bundesanstalt, Bundesallee 100, Postfach 3345, W-3300 Braunschweig, Germany) and A. C. C. Warnock (IRC, Natl. Res. Council, Ottawa, ON K1A 0R6, Canada)

Sound transmission through nominally identical steel plates was measured in two laboratories; NRC, Canada and PTB, Braunschweig in Germany. The two laboratories differ markedly in construction; one has rooms with volumes 65 and $250 \mathrm{~m}^{3}$ and the other volumes of 54 and 69 $\mathrm{m}^{3}$. The specimens measured $1.5 \times 1.25 \mathrm{~m}$ and were mounted in window test frames that satisfied the recommendations of ISO 140 . The measurement frequency range was extended to $50 \mathrm{~Hz}$ in each laboratory. As well as conventional 1/3-octave band measurements, sound reduction index was measured using sound intensity techniques. Measurements were made in both directions, that is, each room served in turn as the source room and then the receiving room. Similar behavior was seen in each laboratory; conventional measurements into the smaller room tended to give higher values of sound reduction index. When the mean values of sound reduction index for each laboratory for both directions of measurement are compared, agreement is good but there are still differences at low frequencies that need explanation. This paper presents the measured data. Possible reasons for the differences are discussed in the companion paper.

\section{1:45}

3pAA4, Inter-laboratory comparisons of low-frequency sound transmission: II. Narrow-band measurements and computer simulations. A. C. C. Warnock (IRC, Natl. Res. Council, M27 Montreal Rd., Ottawa, ON K1A OR6, Canada) and M. Vorländer (Phys. Tech. Bundesanstalt, W-3300 Braunschweig, Germany)

Sound transmission through nominally identical $1.5 \times 1.25-\mathrm{m}$ steel plates was measured in two laboratories; NRC, Canada and PTB, Braunschweig in Germany. This sound paper presents results from a more detailed examination of the behavior of the sound fields at low frequencies in the laboratories. The long-term aim of the work is to understand reasons for poor reproducibility of low-frequency sound reduction measurements in laboratories. Narrow-band sound transmission measurements were made between the two pairs of rooms at PTB and NRC. In each case, the same procedure was used; loudspeakers were fed with a pseudorandorn sequence and the impulse response was calculated using a Hadamard transform. The two sound transmission suites were also simulated using FEM and BEM programs. Comparisons between the measured and calculated results will be presented and discussed.

3pAA5. Noise isolation measurements of partitions and windows under highly absorptive and flanking conditions in the field. Angelo J. Campanella (Campanella Assoc., 3201 Ridgewood Dr., Columbus, OH 43026)

Installed partitions, windows, and doors are often not situated ideally for sound isolation performance testing in terms of ASTM E 90 laboratory sound transmission loss (TL) and STC values. Test room quality specified in the ASTM E 336 and E 966 field methods may preclude TL measurement due to high absorption, small size, or flanking. Methods of Buckingham [NBS SP 20, 193-219 (S-506)], London [NBS RP 26, 419-453 (RP 1338) (1941)], and Donato [J. Acoust. Soc. Am. 40, 1-3 (1966)] were examined for field tests. Buckingham proposed the room space average sound-level method now used in ASTM E 90, ASTM E 336, and E 966. Donato proposed that a term of value $1 / 8$ be added within the $10 \log (\mathrm{S} / \mathrm{A})$ absorption normalization term to account for direct sound. London proposed sound-pressure level measurements (SPL) flush to the specimen receiving room surface for field tests. Flush SPL can be normalized with receiver room space average SPL or absorption via RT60 to determine TL. Tests were recently pering. The flush SPL method combined with RT60 normalization provided the most credible results for comparison with laboratory STC, and also offered immunity to flanking. Data and comparative results will be presented.

\section{2:15}

3pAA6. Flanking transmission in wood-frame constructions caused by simple construction faults. T. R. T. Nightingale (Bldg. M-27, Acoust. Lab., Inst. for Res. in Construct., Natl. Res. Council, Ottawa, ON K1A 0R6, Canada)

Flanking transmission can seriously degrade the performance of building elements in the field. In cases of very poor construction, the actual field transmission loss may bear very little resemblance to the laboratory data. A facility has been constructed to examine the effects of flanking transmission in wood-framed construction. Measured sound insulation data are presented for partitions with potentially common construction faults. The faults considered are rank ordered as to their severity. Standard airborne and impact tests, surface velocity and intensity measurements are also assessed for their effectiveness as measurement tools.

3pAA7. Review of collected data for the experimental checking of a new reverberation time formula. Domenico Stanzial (Natl. Res. Council of Italy, Cemoter Inst., Via Canal Bianco 28, I-44044 Cassana, Ferrara, Italy), E. Carletti (Cemoter. Inst. Ferrara, Italy), G. Brambilla (Natl. Res. Council of Italy, Inst. of Acoust. “O. M. Corbino"), and P. Fausti (Ferrara University)

The experimental checking of a new reverberation time equation proposed by D. Stanzial in a recent paper [D. Stanzial, "A heuristic approach to reverberation time in acoustics, based on energy transfer," submitted to J. Acoust. Soc. Am. (1992)] has been carried out in rooms of different size and different acoustic absorption characteristics. Although not rigorously true in a general acoustic field situation, the experimental checking of the new formula has been accomplished "in a standard way" using the measurement of the $T_{60}$ reverberation time directly obtained as the decay time of the squared acoustic pressure. On the right side of the reverberation time formula, $T_{60}=\gamma\{-0.166[1 / \ln (1$ $-\alpha)]+0.012[\ln (\alpha) / \operatorname{nn}(1-\alpha)]-0.012\}$, the local acoustic absorption coefficient $\alpha$ has been determined, for each testing room, from active and reactive sound intensity measurements. $A$ review of the collected data as well as a first analysis of the experimental checking are reported here.

\section{2:45}

3pAA8. Role of the absorption distribution and generalization of Sabine's reverberation law in chaotic rooms: Geometrical and wave theory. Fabrice Mortessagne, O. Legrand, and Didier Sornette (Lab. de Phys. de la Matière Condensée, CNRS URA 190, Faculté des Sci., Parc Valrose, B.P. 70, 06108 Nice Cedex 02, France)

The theoretical validity of Sabine's law of reverberation in the true geometrical acoustic limit (TGAL) with mirror reflection relies solely on the conditions that almost all rays are chaotic and that absorption is "sufficiently weak," as demonstrated by Joyce [J. Acoust. Soc. Am. 58, $643(1975)$ ]. It is shown here that exponential decay law may still hold in the TGAL for realistic large absorptions, depending on the spatial distribution of the absorption within the chaotic room. This dependence stems from the role of special ray trajectories that are analyzed. It is also demonstrated theoretically and numerically that Sabine's reverberation time is modified at large absorptions due to fluctuations in the number of encounters with the absorbing walls. This novel mechanism applies to many physical examples where a system decays from an initial metastable state with a generic exponential decay law, be it thermally activated or by quantum tunneling. A partial account of this work appeared 
in Europhys. Lett. 20, 287-293 (1992). Finally, a wave theory is given of Sabine's law for room acoustics relying on ergodic properties of the eigenmodes. Besides the global problem of reverberation, wave acoustics in ergodic rooms offer a true richness of phenomena that can be tackled with the help of tools developed in "Quantrum Chaos." A preliminary account of this work appeared in Lect. Notes Phys. 392, 267 (1991).

\section{3;00}

3pAA9. Performance of a large new anechoic chamber at the Canadian Bureau of Radiation and Medical Devices. S. H. P. Bly (Bureau of Radiat. and Med. Devices, 775 Brookfield Rd., Ottawa, ON K1A 1C1, Canada), A. D. Lightstone (Valcoustics Canada Ltd., Toronto), T. G. Hewlings, and J-L. Lourador (Resonance TJL, Montreal)

The large new anechoic chamber is to be used for health effects research and standards development for noise, and for other acoustics research not feasible elsewhere in Canada. The chamber's free-field performance was quantified using measured deviations from the $1 / R$ law for the variation of sound pressure with distance, $R$, from a point source in a free field. In measurements at $\mathbf{4 6}$ locations throughout the chamber, deviations were typically less than $1 \mathrm{~dB}$ for frequencies ranging from the design cutoff of $50 \mathrm{~Hz}$ to $20 \mathrm{kHz}$. The largest deviation was $1.9 \mathrm{~dB}$. Impulse response analysis was also used to examine sound reflections. Background noise was found to be less than the design criteria used, which ranged from $22 \mathrm{~dB}$ at $50 \mathrm{~Hz}$ to $4 \mathrm{~dB}$ at and above $1600 \mathrm{~Hz}$. Comparisons will be made to the few other chambers in the world which are of a similar size.

\section{3:15}

3pAA10. Recent data on audience and chair absorption. Leo $L$. Beranek ( 975 Memorial Dr., Ste. 804, Cambridge, MA 02138)

In 1960 (J. Acoust. Soc. Am. 32, 661-670) and 1969 (J. Acoust. Soc. Am. 45, 13-19), the author published a statistical study of audience and seat absorption based on data taken in a large number of concert halls and opera houses. This paper presents some more recent sound absorption data taken in reverberation chambers and in concert halls which shed light on the influence of seat construction on audience absorption.

WEDNESDAY AFTERNOOON, 19 MAY 1993

BALLROOM, 2:00 TO 3:25 P.M.

\title{
Session 3pID
}

\section{Committee on Tutorials: Hot Topics in Acoustics}

\author{
Allan D. Pierce, Chair \\ Graduate Program in Acoustics, Department of Mechanical Engineering, The Pennsyluania State University, \\ University Park, Pennsyluania 16802
}

This is the first of a new series of special "Hot Topics" sessions, planned so that there will be one such session at each successive serniannual meeting of the Society. At each session three or four of the technical committees and specialty groups will each present a tutorial talk on one or more topics that happen to be of especial current interest. Such talks will be directed to persons within the broader acoustical community and are intended to help individuals in the audience to become familiar with issues and achievements that are in areas not immediately perceived as lying within their areas of personal dominant interest.

\author{
Chair's Introduction-2:00
}

\section{Invited Papers}

2:05

3pID1. Hot topics in structural acoustics and vibration. Albert J. Tucker (Office of Naval Res., Arlington, VA 22217)

Acoustic holography has allowed the theoretical concept of identifying and separating radiating supersonic surface waves (identified by low wave numbers) from nonradiating subsonic surface waves (high wave numbers) to be verified experimentally. Frequency-wave-number filtering, which embodies the idea of spatial and temporal filtering, may hold the key for assessing advances in active noise control as well as for developing innovative concepts for damping treatments for passively controlling radiation. For implementation of active control, spatial anti-aliasing filters for sensors and shaped structural actuators (which prevent actuation of high wave numbers) may provide a path to stable active control technology for complex structures. The physical understanding that supersonic radiating waves are membrane waves of structures and fast compressional or shear waves of elastic bodies can lead to new ideas for incorporating damping mechanisms within structures. To extend the concept of spatial-temporal filtering to arbitrary bodies, further analytical, experimental, and signal processing efforts will be required.

3pID2. Hot topics in speech communication. Terrance M. Nearey (Dept. of Linguistics, Univ. of Alberta, Edmonton, AB T6G 2E7, Canada), Richard S. McGowan (Haskins Labs., New Haven, CT 06511), and Juergen Schroeter (AT\&T Bell Labs., Murray Hill, NJ 07974-0636)

In speech perception, the issues emphasized are related to long-term representation of speech units, language-specific differences in speech perception by infants and adults, and some new results from cochlear implant and other hearing research relating to the auditory coding of speech cues. New results on the question of prototypes for speech units and how speaker and contextual variability affect listeners' behavior are reviewed. Next, there is a survey of recent findings on the effect of language background on infants' and adults' perception of speech. Finally, results from perceptual studies of cochlear implant patients involving 
systematically designed stimulus sets are summarized. In speech production, focus is on the applications of instrumentation, on numerical simulation, and on sensory feedback. There is discussion of measuring instruments that are beginning to provide data on motor compensation and coproduction, and sophisticated numerical simulations that are being developed to answer questions about vibratory mechanisms, air flow, and their relation to sound output. Finally, there is a discussion on the roles of receptor and auditory feedback on articulatory coordination and timing. In speech processing, stress is placed on the progress made in auditory representations, in computational models of language, and in statistical methods for text-to-speech synthesis. In auditory-based representations, 100- to 200-ms speech segments may serve as a basis for distance measures for speech coding that reflect perceptual acceptability of acoustic distortions, and as speech-recognition features that capture dynamic aspects of speech production. In spoken-language processing, the gap between stochastic models of speech (e.g., HMM's and more traditional models of language structure (syntax) and content (semantics) is being bridged. Finally, text-to-speech synthesis is moving, albeit slowly, from hand-tuned to automatically trained systems.

3pID3. Hot topics in underwater acoustics. Arthur B. Baggeroer (Dept. of Ocean Eng., Rm. 5-204, MIT, Cambridge, MA 02139)

There have been two major changes that have modulated the temperature of topics in underwater, or ocean, acoustics since the last "hot topics" session. First, close coupling among the acoustics, the ocean environment and its processes, numerical models, and the signal processing continues to lead to new methods for learning about the ocean and to improved performance of acoustic systems. Second, in response to the changing demands upon future sonars, there has been much more attention to both active systems and shallow water environments. Several "hot" (at least warm) topics will be discussed: adaptive focusing systems including phase conjugation, self-cohering arrays, and matched-field processing/tomography; global acoustics and ocean climate monitoring; high resolution bathymetric mapping; reverberation; seismoacoustics of interface waves; shallow water acoustics; ambient noise and acoustic daylight; and high data rate acoustic telemetry. (The order is not an indication of relative temperature.)

3pID4. Hot topics in physiological acoustics: The adaptive cochlea. Jozef J. Zwislocki (Inst. for Sensory Res., Syracuse Univ., Syracuse, NY 13244-5290)

There are several areas of hot topics in physiological acoustics. However, from the point of view of acoustics, the cochlea that both transmits sound and transduces it into electrochemical events that initiate the digitized neural code must be the closest to an acoustician's heart. The cochlea has been a hot topic since the discovery in the late 60 's that a live cochlea acted in a different way from a passive post-mortem one. Subsequent discoveries revealed that it was able to emit sound and that its mechanoelectrical transducers of one type, the outer hair cells, were bidirectional and also acted as electromechanical transducers, providing in this way a feedback loop. Now it has been found that neither the cochlear transfer functions nor its input-output functions are invariant, but that they change with the intensity and duration of sound. These nonlinear dynamics suggest explanations for the nonlinear growth of loudness with sound intensity and for the shift between the frequency of a damaging sound and the frequency of maximum hearing loss. They also show that the location of maximum cochlear excitation cannot be a code for subjective pitch.

WEDNESDAY AFTERNOON, 19 MAY 1993

ADAM ROOM, 1:00 TO 3:35 P.M.

\title{
Session 3pMU
}

\section{Musical Acoustics and Psychological and Physiological Acoustics: Modulation and Auditory Grouping II}

\author{
Albert S. Bregman, Chair \\ Department of Psychology, McGill University, 1205 Dr. Penfield Avenue, Montreal, Quebec H3A 1B1, Canada
}

Chair's Introduction-1:00

\section{Invited Papers}

1:05

3pMU1. Is there an across-frequency mechanism sensitive to FM coherence? Robert P. Carlyon (Exptl. Psychol., Sussex Univ., Brighton BN1 9QG, England)

Much recent research has focused on one's ability to perform simultaneous comparisons between different regions of a sound's spectrum. In particular, it has been suggested that the patterns of modulation at the outputs of different "auditory filters" can be compared, and that this ability can be used to group together the commonly varying components of a single source. The case for an across-frequency mechanism that can compare patterns of amplitude modulation (AM) is well established, but that for a corresponding mechanism for frequency modulation (FM) is not. When attempting to identify such a mechanism, it is important to control for the fact that frequency-modulating one component of a harmonic complex out of phase ("incoherently") from the rest causes it to become mistuned, and that listeners might detect this mistuning rather than the incoherence per se. This 
presentation reviews existing evidence that (i) for pairs of inharmonically related tones, listeners cannot discriminate coherent from incoherent FM, and (ii) for harmonic sounds, sensitivity to FM incoherence can be predicted from that to the resulting mistuning. New experiments are described that show that recent contradictory evidence, which has been interpreted as revealing an across-frequency mechanism sensitive to FM incoherence, can be accounted for by the existence of within-channel cues.

1:30

3pMU2. Hearing melodies in continuous frequency modulations. Laurent Demany and Kenneth I. McAnally (Lab. de Psychoacoust., Univ. Bordeaux 2, 146 rue Léo-Saignat, F-33076 Bordeaux, France)

A $1-\mathrm{kHz}$ carrier was frequency modulated by the exponential of periodic time functions corresponding to the sum of a few sinusoids (e.g., $\sin \omega t+\sin 3 \omega t$ ). The modulations were symmetric on the dimensions of time and $\log$ frequency $[y(t)=y(k-t)$ $\left.=-y\left(k^{\prime}-t\right)\right]$. Each had three local maxima and three local minima per cycle. The peak-to-peak frequency excursion was typically 0.5 oct. At low rates of modulation, the stimuli were heard as complex glissandi. However, for modulation rates around $1.5 \mathrm{~Hz}$, the dominant percept was a melodic motif comprising the pitches of the local maxima. Generally, the local minima were not heard as auditory "events" (pitch singularities). A similar perceptual asymmetry was not observed for sequences of discrete tones corresponding to the local maxima and minima of the continuous modulations. Additional experiments were performed to determine the frequency difference limens for maxima and minima of continuous frequency modulations. The carriers were modulated by the exponential of one cycle of a cosine function, starting at phase $\pi$ or phase 0 . For maxima and minima around $1 \mathrm{kHz}$, frequency shifts of maxima were markedly better detected than frequency shifts of minima. These results are in contrast to those predicted by consideration of the upward spread of masking.

$1: 55$

3pMU3. Auditory image formation using a CMR paradigm: The role of AM, FM, and harmonicity. J. H. Grose (Div. Otolaryngol./Head \& Neck Surgery, Univ. North Carolina at Chapel Hill, Chapel Hill, NC 27599-7070)

When a pair of comodulated narrow bands of noise is inserted into a larger set of independently comodulated noisebands, the amount of comodulation masking release (CMR) measured for the larger set is reduced. This interference effect can be mitigated to some extent by presenting further bands of noise to the contralateral ear which are comodulated with the intervening pair of bands. One interpretation of this release from interference is that the additional contralateral bands serve to draw the intervening pair of bands into a separate auditory image and that this more distinct segregation of the two sets of comodulated bands allows the auditory system to better perform a CMR on the original comodulated set. The purpose of this study was to apply the paradigm to situations where the commonality between the intervening pair of noisebands and the contralateral bands was some combination of three variables: AM (correlated, uncorrelated), FM (present, absent), and spectral spacing (harmonic, inharmonic). Results indicated that the release from interference occurred most often when coherent AM featured in the commonality, although a slight effect of coherent FM was also observed. [Work supported by the NIDCD R01-DC01507.]

\section{Contributed Papers}

2:20

3pMU4. The effects of deviation and phase disparity of frequency and amplitude modulation on musical blend. Pamela J. Goad (Systematic Musicology, School of Music DN-10, Univ. of Washington, Seattle, WA 98195)

Two experiments tested the effects of deviation and phase disparity of frequency and amplitude modulation on an aspect of musical blend referred to as fusion. The experimental procedure was a two-alternative forced choice design. The stimuli were two simultaneously presented complex tones in a musical interval of a major sixth. The spectral envelopes of the complex tones were similar to oboe tones at fundamental frequencies of 312 and $523 \mathrm{~Hz}$. These tones were modulated by a sinusoid at a rate of $5 \mathrm{~Hz}$. Frequency and amplitude deviations representative of musical instrument vibratos were constructed with peak frequency deviation varying by $0 \%$ (unmodulated), $1.0 \%$, and $2.2 \%$. Amplitude modulation indices were subjectively matched to the tone quality of the frequency modulation deviations. Also, the modulation of one tone relative to the other tone in a stimulus was varied by $0,45,90$, and $180 \mathrm{deg}$. Both tones of a stimulus had the same deviation but they could differ in phase of modulators. Results show subjects were attuned to deviation where greater deviations decreased the amount of fusion. However, subject variability showed two other decision strategies occurring; a combination of deviation and phase disparity, and phase disparity alone.

\section{2:35}

3pMU5. Effect of frequency transposition on discrimination of amplitude patterns. Annie H. Takeuchi and Louis D. Braida (Res. Lab. of Electron., Rm. 36-747, MIT, Boston, MA 02139)
Sheft and Yost [J. Acoust. Soc. Am. 91, 2333 (1992)] reported that frequency transposition impaired discrimination of amplitude modulation patterns. They suggested that our failure to supplement speechreading effectively with amplitude envelopes derived from a particular frequency band of speech and carried at a lower center frequency [Grant et al., Q. J. Exp. Psychol. 43A, 621-645 (1991)] might reflect a general auditory limitation on the ability to compare amplitude modulations across carrier frequencies. This study examined the effect of systematic practice on performance in an $A X Y$ three-interval amplitudemodulation discrimination task in which all signals were narrow-band noises with bandwidths of $50 \mathrm{~Hz}$ and durations of $400 \mathrm{~ms}$. The difference in center frequency between the $A$ stimulus and the $X$ and $Y$ stimuli increased in intervals of $10 \%$ to more than an octave. With practice, the decline in discriminability was substantially lessened and some subjects showed almost no decline. This result suggests that even if perception of amplitude modulation is initially dependent on carrier frequency, listeners can leam to compare amplitude modulation patterns across large frequency differences. [Work supported by NIH.]

\section{2:50}

3pMU6. Dichotic beats of mistuned consonances. M. Patrick Feeney and Edward M. Burns (Dept. of Speech and Hear. Sci., JG-15, Univ. of Washington, Seattle, WA 98195)

Temporal envelope perception, aural harmonics, and combination tones have all been proposed as mechanisms to explain the beats of mistuned consonances (BMC). This study used dichotic presentation of mistuned octaves to explore a between-channel temporal explanation. A 2I-2AFC procedure was employed to determine subjects' ability to detect BMC. Masking in dichotically complementary frequency regions was used to eliminate effects caused by aural harmonics and acoustic 
crossover. Subjects were able to detect dichotic BMC under these conditions. Performance deteriorated with an increase in fundamental frequency as expected from previous studies on monotic BMC and on binaural beats. Envelopes of pairs of beating sinusoids were used to further explore the temporal basis of BMC. Subjects were able to detect mistuned consonant envelopes for both monaural and dichotic presentation. A third experiment examined the detection of the "sweep-tone" pitch effect using the same stimuli. The results of these experiments will be discussed in terms of within- and between-channel phase perception. [Research supported by the Virginia Merrill-Bloedel Hearing Research Center, University of Washington.]

\section{3:05}

3pMU7. The contribution of onset, offiset, and comodulation to the grouping of noise bands. Christian Kaernbach (Inst. für Neuroinformatik, Univ. Bochum, Postfach 1021 48, 4630 Bochum, Germany)

Auditory grouping uses two difference kinds of cues; "vertical" curs act on small temporal sections and are based on similarily principles (frequency, harmonicity, onset/offset, modulation), whereas "horizontal" (sequential) cues depend on the history of the stimulus. The present study addresses the relative importance of several vertical grouping cues. Twenty naive subjects were presented with auditory scenes of one to five elements. The elements were noise bands with a width of $50 \mathrm{~Hz}$ and a duration of 0.12 to $0.36 \mathrm{~s}$. The center frequencies, the onsets and the offsets were randomized; in half of the scenes the noise bands were comodulated. The subjects were asked how many events they had heard. The number of perceived auditory events was on the average smaller than the number of presented noise bands. This could be shown to depend mostly on onset synchrony, to a slightly smaller degree on offset synchrony, and only very little on comodulation.

3pMU8. Temporal asynchrony and within-signal frequency discrimination for complex tones. Irwin Pollack (Mental Health Res. Inst., Univ. of Michigan, Ann Arbor, MI 48109-0720)

Within-signal frequency discrimination for mixtures of two complex tones of different fundamental frequency ("modulation detection") was examined under conditions of temporal asynchrony of the individual complex tones. Over a wide range of temporal conditions, the accuracy of discrimination of fundamental frequency: is primarily determined by the temporal overlap of the two complex tones; is independent of the duration of the leading complex tone preceding the mixture; and, is negatively related to the duration of the lagging complex tone following the mixture. The latter result suggests a form of retroactive interference ('backward masking') upon the processing of within-signal frequency differences for mixtures of complex tones.

WEDNESDAY AFTERNOON, 19 MAY 1993

QUEBEC SUITE, 1:30 TO 3:30 P.M.

\title{
Session 3pNS
}

\section{Noise: Special Workshop on ASA's Role in Noise and its Control II}

\author{
Robert M. Hoover, Cochair \\ Hoover and Keith, Inc., 11381 Meadowglen, Houston, Texas 77082 \\ T. James DuBois, Cochair \\ Southern California Edison, P. O. Box 800, Rosemead, California 91770
}

1:30

Working Groups, Part 3

\begin{abstract}
Working groups will have been organized around the following areas of interest, as expressed by persons responding to the workshop announcement or at meeting registration. Working groups will develop presentations on problems, solutions, and recommended actions.
\end{abstract}

(1) ASA's role in Environmental Noise Control

(2) ASA's role in Industrial Noise Control

(3) ASA's role in Product Noise Control

(4) ASA's role in Prevention of Hearing Loss (occupational and non-occupational)

(5) ASA's role with Government

(6) ASA's role in Education

Working group discussions from the morning session will continue, focusing on (1) what has the ASA done in the past, (2) what should be done now, and (3) how could the ASA accomplish this?

3pNS1. Final group presentation. Bennett M. Brooks (Brooks Acoustics Corp., 27 Hartford Turnpike, Vernon, CT 06066), T. James DuBois (Southern California Edison, Rosemead, CA 91770), Kenneth M. Eldred (Ken Eldred Engineering, East Boothbay, ME 04544), Robert M. Hoover (Hoover and Keith, Inc., Houston, TX 77082), Govindappa Krishnappa (Natl. Res. Council, Ottawa, ON KIA OR6, Canada), and Louis C. Sutherland (Consultant in Acoustics, Ranchos Palos Verdes, CA 92074)

This final plenary session of the Workshop will include presentations by the moderators/recorders of all the working groups on their findings. The final set of recommendations will be presented to the meeting of the Technical Committee on Noise, to be held on Thursday evening, 20 May 1993 for further action. 


\title{
Session 3pPA
}

\section{Physical Acoustics and Bioresponse to Vibration: Novel Applications of Ultrasound in Medicine II}

\author{
J. Brian Fowlkes, Cochair \\ Department of Radiology, University of Michigan, Ann Arbor, Michigan 48109-0553 \\ Ronald A. Roy, Cochair \\ Applied Physics Laboratory, University of Washington, Seattle, Washington 98105
}

Chair's Introduction-1:25

Contributed Papers

\section{1:30}

3pPA1. Ultrasound enhanced generation of hydroxyl radicals from anticancer drugs: Adramycin and Mitomycin C. Darayash Tata, Tom R. Tritton (Dept. of Pharmacology, Univ. of Vermont, Burlington, VT 05405), and Junru Wu (Univ. of Vermont, Burlington, VT 05405)

It has been reported that $\mathrm{OH}$ production in anticancer drug Adramycin and Mitomycin $\mathbf{C}$ suspensions is dramatically enhanced by applying $0.5-\mathrm{MHz} \mathrm{cw}$ ultrasound (US). Time course studies were performed to characterize the $\mathrm{OH}$ generation with and without US exposures. The results show that to a first approximation $[\mathrm{OH}]$ can be expressed as superposition of a rapid exponentially growing term and a slow linear varying term. The experiment indicated that the first term is due to US. In view of these observations it is concluded that US activated some shortly lived $\mathrm{OH}$ generating pathways.

\section{$1: 45$}

3pPA2. An alternative to the mechanical index as a means of assessing the safety of exposures to diagnostic ultrasound. Charles $\mathrm{C}$. Church (Molecular Biosystems, Inc., 10030 Barnes Canyon Rd., San Diego, CA 92121)

The mechanical index (MI) is a means of providing the users of diagnostic ultrasound equipment with a real-time, on screen display of the potential for a given exposure to produce tissue damage by mechanical means. As originally conceived, MI was based on a calculation of the lowest rarefactional pressure $P_{n}$ needed to produce the growth and subsequent collapse of an optimally sized, pre-existing gas bubble such that the peak temperature attained upon collapse was at least $5000 \mathrm{~K}$. While the assumption that stabilized microbubbles exist within the body may be necessary when assessing the safety of medical exposures to ultrasound, that assumption is unproven. If it is assumed that there are no pre-existing, stabilized gas bubbles in tissue, it is possible to calculate a "maximum threshold" rarefactional pressure amplitude $P_{\mathrm{rm}}$, above which cavitation is highly probable. By combining the theory for spontaneous homogeneous nucleation and the rate of cavitation observed during clinical lithotripsy procedures, an estimate of this (frequencyindependent) threshold may be obtained. Assuming that an "acceptable" cavitation rate in tissue is $10^{-20}$ events $/ \mathrm{m}^{3} / \mathrm{s}$ and that an additional clinical safety factor of 2 is desirable, it is possible to show that $P_{\mathrm{rmt}}=3 \mathrm{MPa}$. For comparison, at a frequency of $5 \mathrm{MHz}$, if $\mathrm{MI}=0.4$, then $P_{r}=0.9 \mathrm{MPa}$; if $\mathrm{MI}=1.0, P_{r}=2.2 \mathrm{MPa}$. [Work supported by NIH.]

3pPA3. The role of acoustic radiation force in contrast enhancement techniques using bubble-based ultrasound contrast agents. J. B. Fowlkes, E. A. Gardner, J. A. Ivey, and P. L. Carson (Dept. of Radiol., Univ. of Michigan, Ann Arbor, MI 48109-0553)
The movement of bubbles in an acoustic field has recently been used to manipulate bubbles for the purpose of contrast enhancement in medical applications. Some ultrasound contrast agents consist of stabilized microbubbles which, when exposed to an acoustic field, can be collected at specific points in space and may be moved by translating the field. One application for this type of bubble manipulation is the collection of bubbles within the urinary tract for urinary reflux diagnosis. Experiments conducted on sacrificed dogs indicate that bubbles can be moved in a controlled fashion within the bladder using external acoustic fields. In addition, acoustic radiation force could be used to overcome hydrodynamic forces in flowing blood. Bubbles could be pushed to a vessel wall to collect contrast and when the sound field was turned off, a bolus of contrast agent would reenter the flow for measurement of tissue perfusion. In preliminary experiments, a bolus of bubbles was created in blood flowing through dialysis tubing. This paper will review these experimental findings and present an examination of the mechanisms involved in each application. [Work supported in part by USPHS Grant No. DK-42290.]

\section{2:15}

3pPA4. A novel acoustical approach to biomedical thermodynamics. Armen Sarvazyan, ${ }^{\text {) }}$ Tigran Chalikian, Kenneth J. Breslauer (Dept. of Chemistry, Rutgers Univ., New Brunswick, NJ 08903), Theodor Funck (Max-Planck-Inst. for Biophys. Chem., Germany), and Vladimir Belonenko (VNIIGAZ, Moscow, Russia)

The principal thermodynamic quantities that describe biomolecular structures and interactions $\left(\Delta G^{\circ}, \Delta H^{\circ}, \Delta S^{\circ}, \Delta C_{p}\right.$ ) traditionally have been obtained indirectly by measuring the temperature dependence of some equilibrium property. A more direct approach for obtaining the relevant thermodynamic data is based on calorimetric techniques. Significantly, these thermodynamic parameters also can be derived by measuring the speed of sound $U$ over a range of pressures and temperatures, since $U$ is a function of the second derivative of the free energy over pressure. This talk will describe a novel approach that employs acoustical measurements for determining energetics of biologically relevant molecules and their interactions with small ligands (e.g., drugbiopolymer interactions). Preliminary results will be presented that illustrate how acoustic measurements allow for a thermodynamic characterization of model biomolecular systems. Advantages and complementary features of the acoustical versus calorimetric approaches for obtaining the requisite thermodynamic data will be discussed. ${ }^{\text {a)}}$ Permanent address: Inst. of Biophys., Russian Acad. Sci.

3pPA5. Feasibility of a mechanical waveguide to control the ultrasonic field in pulsed Doppler blood flow measurement. James $J$. Finneran and Mardi C. Hastings (Dept. of Mech. Eng., Ohio State Univ., 206 W. 18th Ave., Columbus, OH 43210) 
The location and size of the sample volume is of considerable importance in pulsed Doppler blood flow detection and velocity measurement. This study examines the feasibility of using a mechanical waveguide to control the ultrasonic field (and thus the sample volume size) of a flat disk transducer operated in pulse-echo mode. The waveguide controls the axial location of the natural focus and the lateral beam width at the natural focus. Experimental measurements are compared to theoretical results derived from a transient field model of the pulse-echo system. Results confirm that the waveguides offer a potentially useful and inexpensive means of altering the pulse-echo field of a flat disk transducer. [Work supported by NSF Grant No. MSS 9058607.]

\section{$2: 45$}

3pPA6. Lithotripter shock wave propagation through layered media. Joseph Jankovsky, P. Ted Christopher, and E. Carr Everbach (Dept. of Eng., Swarthmore College, Swarthmore, PA 19081-1397)

Recent advances in the computation of shock wave front propagation [P. T. Christopher and K. J. Parker, J. Acoust. Soc. Am. 90, 488-499 (1991)] have allowed the prediction of spark-gap lithotripter waveforms both on and off-axis for a variety of geometries and conditions. Since in both urinary and biliary lithotripsy, shock pulses must propagate through layered human tissues (e.g., fat, muscle, and other soft tissues) it is especially important to understand how waveforms evolve in layered media. Comparisons of computational predictions and experimental results are presented for a laboratory lithotripter of our own construction whose axis of symmetry is vertical. Measurements of waveforms were made using a novel PVDF membrane hydrophone [E. C. Everbach, J. Acoust. Soc. Am. Suppl. 1 87, S128 (1990)] immersed in oil-water layers horizontally stratified by buoyancy forces. The comparisons show sufficient agreement to allow the possibility of optimizing lithotripter reflector geometries so that waveforms could be tailored to accommodate tissue configurations of individual patients.

\section{3:00}

3pPA7. Temporal response of an elastic sphere kidney stone and gallstone model to pulsatile plane wave excitation. Robert $C$. Waag and Timothy J. Case (Dept. of Elec. Eng., Univ. of Rochester, Rochester, NY 14627)

An elastic sphere has been employed to model kidney stones and gallstones. Temporal harmonic amplitudes of the stress tensor components in the sphere were expressed in a spherical coordinate system using acoustic displacements found from an orthogonal function series with coefficients determined by boundary conditions. Corresponding temporal responses were then obtained via Fourier transformation and principal stresses as well as stress combinations proposed by von Misses and Tresca for the onset of inelastic behavior were calculated. Crosssectional and three-dimensional displays of these stresses in the sphere at sequential instants of time shows the temporal growth and reverberant decay of the stress field resulting from an incident pulsatile plane wave. The model may be employed to study the influence of the incident waveform shape on the distribution of induced stress in kidney stones and gallstones.

WEDNESDAY AFTERNOON, 19 MAY 1993

BANQUET ROOM, 1:00 TO 3:45 P.M.

\title{
Session 3pPP
}

\section{Psychological and Physiological Acoustics: Binaural Processing and Sound Localization}

\author{
R. H. Dye, Jr., Chair \\ Parmly Hearing Institute, Loyola University, 6525 North Sheridan Road, Chicago, Illinois 60626
}

Contributed Papers

1:00

3pPP1. Binaural masking and lateralization of click trains with alternating interaural time delays. Uma Balakrishnan and Richard L. Freyman (Dept. of Commun. Disord., 6 Amold House, Univ. of Massachusetts, Amherst, MA 01003)

Broadband click trains were used to investigate the effect of onset and ongoing interaural time delays (ITDs) on lateralization and binaural masking. Within a train, successive click pairs had ITDs that were either identical or altemated between two values. The interclick interval was $2 \mathrm{~ms}$ and the total duration of each train was $250 \mathrm{~ms}$. Perceived intracranial position of the signals was estimated using an acoustic pointer. Detection thresholds for the same trains were obtained in the presence of a continuous white noise masker with a fixed interaural delay. It was found that lateralization generally followed the ITD of the very first click pair in the train. Masked thresholds were dependent upon the proximity of one or both signal ITDs to the masker ITD, but were independent of the perceived lateral position of the signal relative to that of the masker. These results suggest that while the onset ITD may dominate lateralization for click trains, it has no influence on masked binaural detection of the same signals. [Work supported by NIDCD Grant No. DC01625.]
1:15

3pPP2. The relative contributions of targets and distractors in laterality judgments. R. H. Dye, Jr. (Parmly Hearing Inst., Loyola Univ., 6525 N. Sheridan Rd., Chicago, IL 60626)

A stimulus classification paradigm that allows the estimation of the relative contributions of target and distractor components of judgments of perceived laterality was used to assess the effect of frequency separation $(\Delta f)$. A $753-\mathrm{Hz}$ tone was the target and the distractor was 553 , $603,653,703,728,778,803,853,903$, or $953 \mathrm{~Hz}$. The duration of the tones, which were gated simultaneously, we either 25 or $100 \mathrm{~ms}$. Each block of 100 trials presented the target and the distractor at ten different interaural delays, with each combination presented once. Preceding the test interval was a diotic presentation of the target. Subjects indicated whether the target component appeared to the left or right of the intracranial midline. Left-right judgments were plotted as a joint function of the target and distractor delays. The slope of the best-fitting linear boundary between left and right responses was used to estimate the relative weights given to the target and the distractor. The target weights were greater for 100 -ms durations and decreased as $\Delta f$ fell below $100 \mathrm{~Hz}$. For all conditions, left-right judgments were partially dependent on distractor delay. [Work supported by NIH.] 
angles and stimulus bandwidth will be presented, and implications for

3pPP3. Effects of different samples of reproducible noise on detection of a 20-Hz-wide interaural phase shift centered on $500 \mathrm{~Hz}$. A. N. Grange and William A. Yost (Parmly Hearing Inst., Loyola Univ., 6525 N. Sheridan Rd., Chicago, IL 60626)

Subjects' thresholds were first determined for detection of an interaural phase shift of a $20-\mathrm{Hz}$ band of equal-amplitude wideband noise. The narrow-band target was centered on $500 \mathrm{~Hz}$, and generated a dichotic, Huggins-type pitch. A diotic band of either $100,125,150$, or 900 $\mathrm{Hz}$ symmetrically surrounded the target band. Interaural phases for frequency components outside of the diotic band were drawn randomly from a rectangular distribution. After thresholds were determined via a tracking procedure, 20 independent samples were generated for each condition at each subject's threshold. A two-interval forced choice task was used in which each interyal was comprised of a 250-ms forward fringe followed by a $250-\mathrm{ms}$ observation period. Sixty trials were presented in a block with each sample presented three times. Order of presentation was randomized across runs and each sample was presented a total of 96 times. $P(C)$ ranged from chance $(50 \%)$ to over 90\% across samples having identical interaural parameters. In conditions where subjects' thresholds were similar, allowing the same set of samples to be used for two or more subjects, there was a strong intersubject correspondence with respect to $P(C)$. Possible sources of these sample-specific effects will be discussed. [Work supported by NIH.]

\section{1:45}

3pPP4. Detection of a target signal in localized versus nonlocalized noise. Kim Abouchacra, Tomasz Letowski (Dept. of Commun. Disord., Penn State Univ., 5 Moore Bldg., University Park, PA 16802), and Joel Kalb (U.S. Army Res. Lab., Aberdeen Proving Ground, MD 21005-5425)

Detection thresholds were measured for a spectrally complex target signal presented in either localized or nonlocalized speech-spectrum noise (65 dBA). Thresholds were obtained for 50 normal hearing subjects, in 40 different combinations of azimuth of the target and noise sources. In general, the results showed that the detectability of the target signal in localized noise was better than in nonlocalized noise. In the conditions where the target signal was presented in localized noise, the findings revealed that (a) poorest thresholds occurred when the target and noise were coincident; (b) thresholds improve as the amount of spatial separation between the target and noise source increases; and (c) best performance occurred when either the target or noise source was off of the median plane and at least a $45^{\circ}$ azimuth separation existed between the sources. Improvements in detection thresholds amounted to as much as $15-18 \mathrm{~dB}$, depending on the condition.

\section{2:00}

3pPP5. Evaluation of a linear interpolation scheme for approximating HRTFs. Abhijit Kulkami and H. Steven Colburn (Dept. of Biomed. Eng., Boston Univ., 44 Cummington St., Boston, MA 02215)

As part of a study of virtual acoustical environments, the ability of subjects to distinguish interpolated HRTFs from empirical HRTFs in the horizontal plane was investigated. Each interpolated HRTF was obtained by averaging empirical HRTFs from two positions chosen symmetrically about the location of interest. Empirical HRTFs were measured from the KEMAR mannequin in anechoic space. Subjects were tested using a 4I, 2AFC paradigm for several angles of interpolation using lowpass noise stimuli. Theoretical analysis predicted a useful lateralization cue for large interpolation angles. For a $2.2-\mathrm{kHz}$ lowpass stimulus, subjects reported the use of such a cue and performed at $96 \%$ correct (averaged across four subjects) for a 30-deg angle. For a 10-deg angle, subjects reported no lateralization cue and performed near chance ( $53 \%$ correct), until they discovered a spectral cue increasing performance significantly ( $69 \%$ correct). Monaural performance was comparable to binaural performance, consistent with predictions for the use of spectral information. Results for combinations of interpolation both basic and applied contexts will be discussed. [Work supported by NIDCD.]

$$
\text { 2:15-2:30 Break }
$$

3pPP6. Monaural echo suppression: Detectability of a delayed signal based on vowel formant structure. Xiaofen Chen and Marion $F$. Cohen (Dept. of Commun. Sci., U-85, Univ. of Connecticut, Storrs, CT 06269)

In order for echo suppression to occur, the ear must be convinced that the echo makes a reasonable acoustic match with the direct sound. It is plausible that the potential acoustic cues used to perform this task are similar to those used for auditory grouping, and include spectral location of vowel formants. An experiment was performed to investigate the role of vowel formant structure in monaural echo suppression. In a 2IFC adaptive procedure, the detectability of a masked signal, consisting of the second formants of two synthetic vowels, presented successively, was measured. The masker consisted of the first, third, and fourth formants of the same two vowels, repeated, so that it had four altemating segments. The signal either preceded the second segment of the masker, or was delayed relative to that segment. When the vowel order of the signal matched that of the second and third segments of the masker, the delayed signal was less detectable than when the vowel order was reversed in the signal. Likewise, when the vowel order of the signal matched that of the masker, the delayed signal was less detectable than the leading signal. These data suggest that the auditory system is able to group vowel formants based on expected spectral location and use that for echo suppression. [Work supported by AFOSR.]

\section{2:45}

3pPP7. Minimum audible angle for clicks with simulated echoes: Effects of aximuth and standard. Ruth Y. Litovsky (Dept. of Neurophysiol., 273 Med. Sci. Bldg., Univ. of Wisconsin, Madison, WI 53706) and Neil A. Macmillan (Univ. of Massachusetts, Amherst, MA 01003)

Minimum audible angle (MAA) was measured in free field with a center position at $0^{\circ}$, and $25^{\circ}$ or $50^{\circ}$ on the right. Stimuli were 6 -ms-wide band noise bursts presented as single bursts or noise pairs under precedence-effect conditions. In the single-noise condition, one burst was presented from the right or left. In precedence lead discrimination, a leading sound was presented from the left or right speaker and a lagging sound from a speaker at center. In precedence lag discrimination, the lagging sound was on the right or left and the leading sound was at center. The test stimulus was either presented in isolation or was preceded by a single standard noise at center. MAAs were lowest for the single condition, slightly higher for lead discrimination, and much higher for lag discrimination. Presence of a standard yielded a benefit in lag discrimination, especially at $0^{\circ}$. Thresholds at $50^{\circ}$ were significantly higher than at $0^{\circ}$ and $25^{\circ}$, especially in lag discrimination. The data can be described by a model in which judgments are based on apparent azimuth of the fused stimulus, and the lagging sound in a pair perceptually "pulls" the image along the azimuth dimension. [Work supported by NSF and NIH.]

\section{3:00}

3pPP8. Auditory spatial resolution in the horizontal plane as a function of reference angle: Microstructure of the azimuth function. David W. Chandler, ${ }^{\text {a) }}$ D. Wesley Grantham (Vanderbilt Univ. Div. of Hear. and Speech Sci. and The Bill Wilkerson Ctr., 1114 19th Ave. S., Nashville, TN 37212), and Marjorie R. Leek (Walter Reed Army Med. Ctr., Washington, DC 20307-5001)

Auditory spatial resolution in the horizontal plane was measured in five normal-hearing subjects by determining the minimum audible angle (MAA) for brief $1.0-\mathrm{kHz}$ tones. Using a left-right discrimination paradigm, MAAs were determined for 22 reference azimuths ranging from 
$-62^{\circ}$ to $+63^{\circ}$. The gross pattern of the mean data was similar to that reported previously for more sparsely sampled azimuth functions: spatial resolution tended to be best when the reference sound was near midline, becoming worse as the reference moved toward the periphery. However, there were remarkable asymmetries and nonmonotonicities in individual subject's MAA versus azimuth functions, and these nonmonotonic features were highly subject-dependent. Although such individual differences may be due partly to differences among subjects in head and pinna shape, it is possible that the highly individual and idiosyncratic MAA functions also reflect subject-dependent nonmonotonicities in the underlying interaural time difference (ITD) discrimination functions as reference ITD is varied in fine increments. [Work supported by NIH and by the U.S. Army.] [) Current address: ENT/ Audiology (HSHJ-SET-A), Madigan Army Med. Ctr., Tacoma, WA 98431-5374.

3:15

3pPP9. Horizontal and vertical MAAs for a wide range of sound source locations. Adelbert W. Bronkhorst (TNO Inst. for Perception, P.O. Box 23, 3769 ZG Soesterberg, The Netherlands)

Minimum audible angles (MAA) in both the horizontal and the vertical directional were measured for sound source locations distributed over virtually the complete sphere around the listener. Stimuli were noise bursts with either a fixed (flat) spectrum, or a random spectrum with 1/3-octave levels drawn from a uniform distribution with a 20-dB range. It was found that, in general, horizontal MAAs are smallest for sound sources in or near the median plane and vertical MAAs are smallest for sources around $90^{\circ}$ azimuth. The results for the randomspectrum stimuli show some unexpected asymmetries. Though listeners were almost unable to localize sources in the median plane with elevations in excess of $30^{\circ}$ (vertical MAAs were then $60^{\circ}$ or more), they attained moderate MAAs for lower elevations. The MAA for frontal presentation was even similar to the result obtained with fixed-spectrum noise. Results furthermore show that horizontal MAAs for sources at $90^{\circ}$ azimuth are much larger for positive elevations than for negative elevations. These findings indicate that localization acuity is optimal for sound sources around or below the horizontal plane.

\section{$\mathbf{3 . 3 0}$}

3pPP10. Use of auralization to accomplish small elevation shifts of a loudspeaker in the median plane. David L. Bowen (RH Lyon Corp., 691 Concord Ave., Cambridge, MA 02138) and Jens Blauert (Ruhr-Universität Bochum, Bochum, Germany)

The use of frequency domain equalization to change the perceived elevation of a loudspeaker over a fairly small range in the frontal plane $\left( \pm 20^{\circ}\right)$ will be described. Because of the application (the center channel speaker in home video theater), several constraints were in effect. Among these were (1) the effect must be applicable over a broad range of people, and over a fairly broad listening area, (2) the equalization applied must not introduce an objectionable amount of "coloration" into the sound, and (3) the resulting circuit must be feasible to implement into a consumer product. The procedure used was to first determine equalizations from ratios of head-related transfer functions measured on an artificial head and on human subjects. Salient features were then determined and implemented in hardware for listening tests, where further refinements took place. Despite a common assertion that there is little directional dependence in the median plane below $4-5 \mathrm{kHz}$, listening tests indicated a more convincing effect took place when several features below $4 \mathrm{kHz}$ were included in the equalizations. It was also found effective to include a feature at $12.5 \mathrm{kHz}$, even if source material was limited to male speech. The results of demonstrating a $20^{\circ}$ to $0^{\circ}$ "delevator" to 125 groups of 12 people will also be discussed. [Work supported by Thomson Consumer Electronics.]

WEDNESDAY AFTERNOON, 19 MAY 1993

DRAWING ROOM, 1:30 TO 3:30 P.M.

\title{
Session 3pSA
}

\section{Structural Acoustics and Vibration: Active Control and Structural Transducers}

\author{
Mauro Pierucci, Chair \\ Department of Aerospace and Engineering Mechanics, San Diego State University, San Diego, California 92182
}

\section{Contributed Papers}

1:30

3pSA1. Asymmetric actuation and sensing of a beam using piezoelectric materials. François Charette, Guy Plantier, Catherine Guigou, and Alain Berry (G.A.U.S., Mech. Eng. Dept., Univ. de Sherbrooke, Sherbrooke, PQ J1K 2R1, Canada)

The purpose of the present work is the modeling of a beam actuated by a ceramic piezoelectric on the top with a PVDF piezoelectric on the opposite side acting as a sensor. The two piezoelectric are different in types, may be of arbitrary length and can be positioned independently along the beam. Since there is only one actuator, the asymmetric excitation induces transverse and axial displacement in the beam. This asymmetric actuation contrast with the symmetric actuation normally considered in the literature. Symmetric actuation implies no axial displacement. Here, both piezos are considered perfectly bonded to the beam and the Bernoulli-Euler hypothesis is used for the displacement field. The variational approach with Hamilton's principle is put to use in the theoretical model. Hamilton's principle states that the definite time integral of the Lagrangian shall be stationary. This formulation, which is energy based, allows one to consider any boundary conditions and to take into account the dynamic effects of both piezos on the beam response. The theoretical model gives the axial and transverse displacements, the strains, the mean quadratic velocity of the beam, and the voltage of the PVDF piezo sensor as a function of the voltage given to the actuating ceramic piezo. Experimental results are compared to the theoretical model for the case of the beam with free-ends boundary conditions.

\section{$1: 45$}

3pSA2. Active control of finite beam volume velocity using shaped PVDF sensors. Catherine Guigou and Alain Berry (G.A.U.S., Mech. Eng. Dept., Univ. de Sherbrooke, Sherbrooke, PQ JIK 2RI, Canada)

Active control of volume velocity using shaped PVDF sensors is 
analytically studied for finite beam with different boundary conditions. Controlling the volume velocity of a finite beam is equivalent of controlling the far-field pressure above the center of the baffled beam. The shape of the PVDF sensor is determined in such a way that the output signal of the sensor is directly proportional to the volume velocity. A general expression of the PVDF sensor shape is obtained and is shown to depend only on the beam characteristics (material properties and boundary conditions). However, the shape of the sensor is independent of the type and frequency of excitation. This type of shaped PVDF sensors detects only contributions from the odd modes, which are the only ones without a nonzero volume velocity and also the most effective radiators. When a simply supported beam is subjected to a harmonic input force, the output signal of the shaped PVDF sensor is minimized by a control point force. The far-field radiated power, the mean-square velocity, and the radiation efficiency before and after the control are extensively studied. The results demonstrate that such shaped PVDF sensors are effective to control the sound radiation from the most efficient radiating flexural modes.

\section{2:00}

3pSA3. Curved piezo-actuator models for vibration control of cylindrical shells. Venkata R. Sonti and James D. Jones (1077 Ray W. Herrick Labs, School of Mech. Eng., Purdue Univ., West Lafayette, IN 47097-1077)

An analytical model of curved piezo-actuators (for active noise and vibration control of cylindrical shells) is developed using Love's theory of thin shells. It is found that a curved uniformly polarized piezoactuator of standard size exerts five types of effective forces. The amplitude variation of these effective forces as a function of the actuator thickness shows that some of the forces attain an optimum value for a specific thickness. Also, single-sided and double-sided actuator configurations are examined. The double-sided case includes actuators driven in-phase and out-of-phase. It is found that under some of the various configurations considered above different sets of forces reinforce or cancel each other. The model is developed under the assumption that the actuator size is small in comparison to the cylindrical shell and thus it does not cause any appreciable change in the overall mass or stiffness of the system. The validity of the assumption is tested by evaluating the response of the shell to the actuator in two ways, one neglecting and the other including the mass and stiffness of the actuator.

\section{2:15}

3pSA4. Active control of nonlinear vibration of a panel structure. Lucio Maestrello (NASA Langley Res. Ctr., MS 463, Hampton, VA 23681-0001)

Experimental results show that one can convert the nonlinear periodic motion of a panel structure to a linear one by controlling the system about the fundamental orbit through a time-dependent, phaseshift forced perturbation. An aircraft fuselage panel structure is forced at resonance by plane acoustic waves. Once the nonlinear response is triggered, the oscillation evolves through a sequence of subharmonic and harmonic bifurcations. The radiated acoustic pressure also contains harmonics whose level increases with distance relative to the fundamental. Control is achieved by actively forcing the panel either with a piezoceramic element or with an electrical shaker. Results show that the energy is transferred from the fundamental to the harmonics and vice versa as the response changes from linear to nonlinear and back to the linear state. An analytical model using a control linearization technique of Duffing's equation with periodic forcing is discussed.

\section{2:30}

3pSA5. Modeling the sensor/actuator response of a finite disk transducer flush mounted on a plate subject to fluid loading. Vasundara V. Varadan, Jaehwan Kim, and Vijay K. Varadan (Res.
Ctr. for the Eng. of Electron. and Acoust. Mater., Dept. of Eng. Sci. and Mech., Penn State Univ., University Park, PA 16802)

It is important to model the sensor and actuator function of a finite disk-shaped piezoelectric transducer in the environment that it will function in. This will involve taking into account the structure on which it is mounted, modeled here as a flat, solid, elastic plate and fluid loading to which the structure may be subject to. In addition, material damping in the plate and the transducer must be taken into account. The sensor response involves computation of the voltage induced in the transducer by an incident acoustic wave in the fluid or a force to which the plate may be subjected to. The actuator response involved computation of the acoustic field radiated into the fluid when the transducer is subject to a known voltage. A hybrid numerical method involving finite element modeling in the plate and transducer region and a plane wave representation in the fluid region will be used. [Work supported by ONR.]

\section{$2: 45$}

3pSA6. Active control of radiated noise and vibrations from an automobile fuel tank. Vasundara V. Varadan, Suk-Yoon Hong, and Vijay K. Varadan (Res. Ctr. for the Eng. of Electron. and Acoust. Mater., Dept. of Eng. Sci. and Mech., Penn State Univ., University Park, PA 16802)

The use of a co-located piezoelectric, disk-shaped sensor/actuator is described for controlling several modes of vibration of an actual automobile fuel tank that is excited by a shaker. The shaker is attached to the tank where the fuel pump would normally be attached. Single mode control as well as multi-mode vibration control was achieved for both the empty as well as partially filled tank. The radiated noise caused by the structural vibration can also be controlled. Experimental results and short video presentation of the experiment will be made.

\section{3:00}

3pSA7. Active systems for attenuation of noise. O. L. Angevine (Angevine Acoust. Consultants, Inc., 1021 Maple St., P.O. Box 725, East Aurora, NY 14170) and M. J. M. Jessel ${ }^{\text {) }}$ (Retired from Ctr. Natl. de la Récherche Sci., Marseille Cedex 09, France)

Both theoretical studies and experience with active systems for noise reduction disclose constraints necessary for successful systems. These include the following. (1) Topological fitness: A net of antisources suitable to the primary noise source. (2) Density of cancellers: Quite different resuits are produced by dense than by sparse arrays. (3) Directivity: Different results are achieved by directional cancellers than by nondirectional ones. (4) Causality: Cancellation requires repetitive patterns of the noise to be attenuated, or the ability to sample the noise to be canceled earlier than the production of the antinoise by the time interval needed for processing the data. Some consequences of these constraints are discussed. 'Present address: L'Aragon F-13008, Marseille, France.

\section{3:15}

3pSA8. Active noise control simulations in a parallelepipedic cavity. Serge Brunet, Alain Berry, and Jean Nicolas (G.A.U.S., Dept. of Mech. Eng., Univ. de Sherbrooke, Sherbrooke, PQ J1K 2R1, Canada)

Over the last few years, much attention has been focused on the development of active noise control. This development has been spurred by the available low-cost computing power, improvements in actuator technology, and the development of effective control algorithms. Many applications exist for the control of a noise field within complex enclosures such as aircraft, helicopter, and automobile cabins. In order to understand the basic underlying working principles, an analytical model based on a modal approach was developed to simulate the acoustic response of a parallelepipedic cavity (consisting of a flexible simply 
supported plane structure mounted on a rigid cavity) resulting from the application of acoustic or mechanical excitations on the flexible plane structure. The controllability of the noise field was then investigated with the use of acoustic point sources within the cavity as secondary noise sources and piezoelectric devices on the flexible plane structure as counter vibration sources. The presentation shall consist of a short summary of the analytical model development, results and comparisons for some of the tested control schemes.

WEDNESDAY AFTERNOON, 19 MAY 1993

CANADIAN ROOM, 1:00 TO 3:45 P.M.

Session 3pSP

\title{
Speech Communication: Speech Processing Issues in Analysis and Modeling
}

\author{
Janet C. Rutledge, Chair \\ Department of Electrical Engineering and Computer Science, Northwestern University, Evanston, Illinois 60208-3118
}

\section{Contributed Papers}

1:00

3pSP1. Evaluation of objective vowel classifiers. James D. Miller, F. E. Kramer, D. J. Meyer, S. Lee, and R. M. Uchanski (Central Inst. for the Deaf, 818 S. Euclid, St. Louis, MO 63110)

Four classifiers were examined for their ability to identify nine monophthongal American English vowels. The classifiers, (1) Bayesian, (2) a standard back-propagation neural net with one hidden-layer, (3) a modified ellipse method, and (4) an automatic region-drawing method, operated on two-dimensional vowel representations. Additionally, three diferent types of two-dimensional data were evaluated; (a) ( $\log F 1, \log F 2$ ), (b) (norm $\log F 1$, norm $\log F 2$ ) normalized by a sensory reference [Miller, J. Acoust. Soc. Am. 85, 2114-2134 (1989)], and (c) $\left(x^{\prime}, y^{\prime}\right)$ of the auditory-perceptual space [Miller, J. Acoust. Soc. Am. 85, 2114-2134 (1989)]. Our corpus of 2304 vowels spoken in a CVC context by male and female talkers includes stress and speakingrate variations [Fourakis, J. Acoust. Soc. Am. 90, 1816-1827 (1991)]. For each vowel utternace and each data type, a single two-dimensional data point is computed from the vocalic segment selected by Fourakis. Separate training (75\% of the data) and testing (25\%) subsets of the corpus were used in a jacknife procedure. In general, all the classifiers except the ellipse method performed similarly, and obtained the highest scores using $\left(x^{\prime}, y^{\prime}\right)$ data. However, unless training and testing of the classifiers is restricted to vowels that are relatively steady-state, identification scores are less than ideal $(<90 \%)$. [Work supported by NIDCD.]

\section{1:15}

3pSP2. Evaluation of speaker normalization methods for vowel recognition using neural network and nearest-neighbor classifiers. Gail A. Carpenter and Krishna K. Govindarajan (Cognitive and Neural Systems Dept., Boston Univ., 111 Cummington St., Boston, MA 02215)

Intrinsic and extrinsic speaker normalization methods were compared using a neural network (fuzzy ARTMAP) and $L_{1}$ and $L_{2}$ $K$-nearest neighbor (K-NN) categorizers trained and tested on disjoint sets of speakers of the Peterson-Barney vowel database. Intrinsic methods included one nonscaled, four psychophysical scales (bark, bark with end correction, mel, ERB), and three log scales, each tested on four combinations of $F_{0}, F_{1}, F_{2}, F_{3}$. Extrinsic methods included four speaker adaptation schemes each combined with the 32 intrinsic methods: centroid subtraction across all frequencies (CS), centroid subtraction for each frequency (CSi), linear scale (LS), and linear transformation (LT). ARTMAP and K-NN showed similar trends, with K-NN performing better, but requiring about ten times as much memory. Among intrinsic methods, ARTMAP and K-NN performed optimally using all the differences between bark scaled $F_{i}$ (BDA). The ordering of performance for the extrinsic methods were LT, CSi, LS, and CS. For all extrinsic methods, ARTMAP performed best using BDA; K-NN chose psychophysical measures for all except CSi. [Work supported by BP, DARPA, NSF, AFOSR, ONR.]

3pSP3. Modeling vowel identification by listeners: $A$ comparison of cepstrum-based and auditory models at reduced signal-to-noise ratios. D. D. Paschall and Peter F. Assmann (Human Devel. and Commun. Sci., Univ. of Texas at Dallas, Box 830688, GR4.1, Richardson, TX 75083)

This study compared the abilities of two computational models to predict the identification responses of listeners to vowels presented in quiet and with pink-noise maskers at signal-to-noise ratios (SNRs) of $-6,-3,0,+3$, and $+6 \mathrm{~dB}$. One model used a representation derived from cepstral analysis. The other used a representation derived from a model of peripheral auditory analysis which (i) filtered the stimulus with a bank of bandpass filters; (ii) compressed the filtered waveforms using a simulation of hair cell transduction; (iii) computed the shortterm autocorrelation function of the compressed, filtered waveform in each channel; and (iv) summed the autocorrelation functions across filter channels to generate a pooled autocorrelation function (PACF). Classification was performed by discriminant function analysis, using noise-free tokens as input to the classifier during the training phase. Performance using the cepstrum-based model was better than human listeners in quiet, but deteriorated rapidly with the audition of background noise. In contrast, the PACF-based model performed at levels similar to listeners in quiet, and mirrored their gradual decline in accuracy with decreasing SNR.

\section{$1: 45$}

3pSP4. Estimation of articulatory-to-acoustic mapping using input and output measurements. Masaaki Honda and Tokihiko Kaburagi (Information Sci. Res. Lab., NTT Basic Res. Labs., 3-9-11 Midori-cho, Musashino-shi, Tokyo, 180 Japan)

An articulatory-to-acoustic mapping was estimated from real speech data and articulatory data measured by a magnetic position sensing device. The mapping function consisted of an acoustic model of the vocal tract and a multi-layered neural network to map articulatory positions on the midsagittal plane into the vocal tract log area function. Input variables to the network included eight positions on the tongue, lower and upper lip, jaw, and velum as well as two unknown inputs that locally control the log area function below and around the larynx. The weighting variables of the network and the unknown inputs were iteratively trained by the gradient method so as to minimize the cepstral distance between the pre-emphasized input speech spectrum and the 
synthesized spectrum. When a database consisting of 27 sentences was used to examine the mapping accuracy for a three-layered neural network configuration with 112 hidden nodes, the spectral error of the mapping was $1.3 \mathrm{~dB}$ for vowel data and $1.8 \mathrm{~dB}$ for sentence data. The mapping accuracy was fair enough for producing natural sounding speech from real articulatory movements.

\section{2:00}

3pSP5. Linear autoregressive modeling of unvoiced speech. Gernot Kubin and Bishnu S. Atal (AT\&T Bell Labs., Rm. 2D-461, 600 Mountain Ave., Murray Hill, NJ 07974)

Recent interest in nonlinear prediction of speech has brought up the need to assess the performance limitations of linear speech models. While nonlinearity is essential in the sound production mechanisms this need not be reflected in a speech signal model. A linear model may produce the same perceptual effect for a human listener as the nonlinear one. The validity of a linear autoregressive model has been tested for the unvoiced segments of speech. In this experiment, a linear prediction residual is first computed from the speech signal. For unvoiced segments, the residual is replaced by white Gaussian noise with its energy matched every $5 \mathrm{~ms}$. The residual is left unmodified for voiced segments. The linear prediction synthesis filter is excited with the composite residual signal to reconstruct speech. The resulting speech quality has been evaluated in a formal MOS test where 32 listeners rated speech material from 12 speakers uttering 3 sentences each. The present approach yielded an MOS of 4.0. In the same test, 16-bit linear PCM obtained a score of 4.1. In conclusion, a linear model with random excitation is sufficient to reproduce unvoiced speech with very high perceptual quality. A demonstration tape will be played during the presentation.

\section{2:15}

3pSP6. Automatic classification of labial and alveolar stop consonants. Stephanie Zierten and Carol Y. Espy-Wilson (Elec. Comput. and Systems Eng. Dept., Boston Univ., Boston, MA 02215)

This study investigates the automatic classification of labial and alveolar stop consonants. The algorithm implemented was proposed by Lahiri et al. [J. Acoust. Soc. Am. 76, 391-404 (1984)], and uses relative and dynamic measures relating the spectral shape at the burst with that of the onset of the following vowel. The purpose of the measure is to capture that (1) for labials, the difference in energy between the burst release spectrum and the vowel spectrum is either less at low frequencies than at high frequencies or the difference is compatible in both frequency ranges and (2) for alveolars, the difference in energy between the burst release spectrum and the vowel onset spectrum is less at high frequencies than at low frequencies. To make these distinctions, the algorithm draws slope lines on the burst and the vowel onset spectra based on $F 2$ and $F 4$ and computes a ratio of the difference (in $\mathrm{dB}$ ) of the slope lines at 3500 and $1500 \mathrm{~Hz}$. In the automatic procedure, $F 2$ and $F 4$ are picked from LPC spectra based on the covariance method. Preliminary results obtained from word-initial stops consonants occurring in monosyllabic words show that the algorithm does classify correctly many of the stop consonants. However, an analysis of the errors suggests the need for refinements. In particular, the alveolars classified as labials had less energy than the vowel between 0 and $4000 \mathrm{~Hz}$ (falling slope line), but more energy than the vowels at frequencies higher than $4000 \mathrm{~Hz}$. In the case of labials, the energy difference at low frequencies was not less than that at high frequencies, but, the labials did have comparable or less energy to that of the vowel at frequencies above 4000 $\mathrm{Hz}$. The refinements suggested by these findings will be made and results will be presented.

\section{$2: 30$}

3pSP7. An LVQ2-trained connected-phoneme hidden Markov model for automatic phonetic segmentation and labeling. Y. Chun Kuo and Janet C. Rutledge (Dept. of Elec. Eng. and Comput. Sci., Northwestern Univ., Evanston, IL 60208-3118)
A connected-phoneme hidden Markov model (HMM) is proposed to perform automatic segmentation and labeling. Individual phonetic models are first created by a left-to-right HMM. The large connectedphoneme HMM is formed by grouping all these phonetic models together. Therefore, each state of this big HMM uniquely represents an English phoneme. The large connected-phoneme HMM is not trained by the Viterbi algorithm since the most probable state sequence dose not necessarily yield the correct segmentation and labeling. Learning vector quantization (LVQ2) is used to train the connected-phoneme HMM such that the phoneme confusions can be reduced. The proposed algorithm has two potential advantages over the existing speech recognition schemes. (1) With the aid of the unique representation of each state of the big HMM, more insight into speech characteristics can be gained, which is essential for the improvement of speech recognizers. Errors caused by insertion, deletion, and substitution can be properly analyzed and adjusted. (2) The computation load for the LVQ2 training is considerably less than the Viterbi training. Therefore, the LVQ2 training is also more suitable for a limited speech data base

\section{$2: 45$}

3pSP8. A comparison of synchronous and asynchronous analysis of speech. Grant J. Jensen and William J. Strong (Dept. of Phys. and Astron., Brigham Young Univ., Provo, UT 84602)

Speech having a high signal-to-noise ratio and digitized at $16 \mathrm{kHz}$ was used for the comparison. An "ideal" synchronous analysis was obtained by handmarking the speech events-voicing periods, plosive bursts, and so on. An asynchronous analysis was obtained from $25-\mathrm{ms}$ Hamming windowed segments of speech stepped at 7.5-ms intervals. In both cases the speech sample-event or windowed segment-was Fourier transformed and displayed in a "trispectral" space. The synchronous analysis produced erratic event-to-event spectra for short voicing periods. The asynchronous analysis produced erratic segment-tosegment spectra for long voicing periods. It also tended to smear shortduration, transitional features. Various examples of trispectral plots obtained from the two methods will be shown for comparison.

\section{3:00}

3pSP9. Strident-feature extraction in English fricatives. Nabil $\mathrm{N}$. Bitar, Armen Y. Balian, and Vinay Chandra (Elec. Comput. and Systems Eng. Dept., Boston Univ., Boston, MA 02215)

An automatic method was developed to classify fricatives as strident/nonstrident. The method is based on relative energy measures in the frequency bands $(2-4 \mathrm{kHz}, 4-6 \mathrm{kHz}$, and $6-8 \mathrm{kHz})$. Preliminary results based on 1176 fricative sounds extracted from sentences in the TIMIT database showed that most of the fricatives / $\check{\mathbf{s}} \check{\mathrm{s}} \mathrm{z} /$ were classified as strident and most of the fricatives $/ f \vee \theta \delta /$ were classified as nonstrident. An analysis of the remaining fricatives showed that their acoustic realizations differed substantially from their canonical form. The typically strident fricatives did appear to be "weak" and the typically nonstrident fricatives did appear to be "strong." Factors influencing the change in the manifestation of these fricatives are phonetic context and stress. A detailed analysis of the observed variability will be presented.

\section{$3: 15$}

3pSP10. Methods for separating adjacent sounds with the same manner of articulation. Armen Y. Balian and Nabil N. Bitar (Elec. Comput. and Systems Eng. Dept., Boston Univ., Boston, MA 02215)

A difficult problem in automated time alignment is the separation of neighboring sounds with the same manner of articulation, such as two adjacent vowels or two adjacent fricatives. Usually, a broad classifier will find only one region that contains both sounds. In this study, the automatic detection of acoustic events that separate such sequences is investigated. For example, an algorithm based on abrupt changes in the energy between 2000 and $4000 \mathrm{~Hz}$ separates $/ \mathrm{v} / \mathrm{s}$ and $/ \check{s} / \mathrm{s}$, which are adjacent to $/ \partial /$ 's or $/ \mathrm{s} /$ 's. Preliminary results based on 38 cases of 
adjacent fricatives taken from the TIMIT database show that $68 \%$ of the automatically placed boundaries agreed with the labeled boundaries. Of the remaining 12 cases, $67 \%$ were considered to be as good as, or even better than the TIMIT labeling. The fricative separation algorithm is presently being refined and tested across a larger database containing other similar sounds. Methods for separating adjacent stops and adjacent vowels will also be presented.

\section{3:30}

3pSP11. Alternative procedures for spectral estimation. Issam Kheirallah and Donald G. Jamieson (Hear. Health Care Res. Unit, Univ. of Western Ontario, Elborn College, London, ON N6G 1H1, Canada)

This paper compares four alternative procedures for estimating the acoustical characteristics of speech and other complex signals: the fast
Fourier transform (FFT), two autoregressive techniques (the autocorrelation and modified covariance methods), and the Cone-Kernel method. All four techniques are now widely available to researchers in Version 4.0 of the CSRE (Canadian Speech Research Environment) software system. FFT spectral estimates are characterized by many known tradeoffs: good frequency resolution is obtained at the expense of good time resolution, and vice versa. Autoregressive methods overcome some of the inherent limitations of the FFT method, but still use a quasi-stationary approach to analyze nonstationary signals such as speech. The Cone-Kernel method offers the opportunity for both good frequency and good time resolution, and cancels many of the cross terms that have made it difficult to interpret previous approaches based on the Wigner distribution. Examples of applying the various analysis procedures to signals will be shown, including situations where the Cone-Kernel approach facilitates the interpretation of certain details of the speech signal.

WEDNESDAY AFTERNOON, 19 MAY 1993

CANADIAN MUSEUM OF CIVILIZATION, 4:30 TO 5:30 P.M.

\title{
Plenary Session and Awards Ceremony
}

\author{
Herman Medwin, Chair \\ President, Acoustical Society of America
}

Presentation of Awards

R. Bruce Lindsay Award to Michael D. Collins

\section{Gold Medal to David T. Blackstock}

The Museum will be kept open exclusively for the Society until 7:30 p.m. so that members can visit the exhibits. Members are also invited to view the 20-minute film "Momentum," which presents spectacular views of Canada projected at 48 frames per second in the new high definition IMAX HD technique.

Shuttle bus service will operate continuously between the Chateau Laurier and the Museum. The first bus will leave the Chateau Laurier from Mackenzie. 


\title{
Session 4aAA
}

\section{Architectural Acoustics and Engineering Acoustics: Acoustic Measurement Techniques}

\author{
Gary W. Elko, Cochair \\ AT\&T Bell Laboratories, Murray Hill, New Jersey 07974-2070 \\ Timothy J. Foulkes, Cochair \\ Cavanaugh Tocci Associates, 327 F Boston Post Road, Sudbury, Massachusetts 01776
}

Invited Papers

8:30

4aAA1. Measurement and in situ calculation of room acoustic parameters. Torben Jacobsen (Bruel \& Kjaer Instruments, 121 Keeler Dr., Ridgefield, CT 06877)

Room acoustic parameters should describe the subjective evaluation of a room. The most important parameter for evaluation of acoustic comfort in a room is the reverberation time, but additional parameters are necessary for evaluation and design of concert halls and auditoria. Beside the reverberation time and EDT (early decay time) it is possible from the integrated impulse response to calculate acoustical parameters such as "early to late sound index" (clarity), "early energy fraction" (deutlichkeit), "center time," and "total sound level." These measurements and calculations are today possible to perform in situ by a sound level meter with application modules connected to a sound source and a lap-top computer; traditionally these measurements required a much bigger measurement system. Therefore, by means of this easy portable instrumentation, the results may be inspected in the field and additional measurements can be done if necessary. During the presentation, the above acoustical parameters are mentioned and measurement results, obtained by instrumentation from Bruel \& Kjaer, from a European concert hall and from a hall in the USA are described.

\section{8:55}

4aAA2. Improved acoustical measurements with MLSSA. Douglas D. Rife (DRA Labs., 24 Halifax Ct., Sterling, VA 20165)

Two fundamental properties of MLS techniques, namely, phase randomization and energy consenation are exploited by MLSSA to achieve improved acoustical measurements. These include (1) the conversion of nonstationary interfering noise as well as weak nonlinear distortion into stationary noise and, as a consequence, (2) the possibility of correcting, as a post-processing operation, systematic errors in measured Schroeder decay plots whether due to interfering noise or transducer nonlinearities, (3) one-shot STI and RASTI measurements that properly account for contemporaneous background noise including nonstationary noise and, (4) the possibility of characterizing transducer nonlinear distortion by means of incoherency measurements that exclude, or at least attenuate, the contribution of the background noise. This last possibility is in contrast to traditional dualchannel coherence measurements that unavoidably lump together the effects of nonlinearity and noise. All of these results are demonstrated theoretically as well as experimentally using actual MLSSA measurements.

$$
\text { 9:20 }
$$

4aAA3. Measurements and analysis with SYSid. Sunil Puria (Ariel Corp., 433 River Rd., Highland Park, NJ 08904 and Res. Lab. Electron., MIT, Cambridge, MA 02139), Jont B. Allen, Gary W. Elko (AT\&T Bell Labs., Murray Hill, NJ 07974), and Patricia S. Jeng (CUNY, New York, NY 10036)

To make measurements a person sometimes has the need for many different test instruments on ones bench. The philosophy behind SYSid (SYStem identification) is to integrate many commonly used test instruments into a single yet accurate and easy to use system. The SYSid software package runs on a DOS platform with the Ariel DSP-16+installed. The theory of operation in SYSid is simple. SYSid excites the system being measured with a stimulus and synchronously averages the measured response of the system. The stimulus can be a chirp, single tone, or multiple tones, MLS, or it can be user-defined (i.e., speech, pink noise, octave-band noise, etc.). It is important to synchronously average a systern response in order to obtain accurate phase information. Synchronous averaging also allows one to measure a signal that is below the system noise floor. SYSid then uses FFT techniques to deconvolve the stimulus from the measured response and to further analyze the data. From this basic mode of operation SYSid can perform many types of analyses including phase responses, group delay, impulse response, Hilbert envelope, reverse energy time curve, RT60, waterfall displays, electrical impedance, etc. In addition to these linear measurements, SYSid also provides the capabilities to make distortion measurements due to nonlinearities in the system. These include harmonic distortion, intermodulation distortion, THD $+\mathrm{N}$, input-output functions, and spectral contamination.

\section{9:45}

4aAA4. Development of a multi-beam array microphone for multi-channel pickup of sound fields and its applications to room acoustics. Ryou Ikezawa and Takashi Nishi (Auditory Sci. \& Acoust. Res. Div., NHK Sci. \& Tech. Res. Labs., 1-10-11, Kinuta, Setagaya-ku, Tokyo, Japan)

A new multi-beam array microphone (MAM for short) has been developed. The MAM is composed of four concentric rings, each of which has 12 microphones elements, and a common center microphone. The MAM synthesizes the output phase of each 
microphone by compensating for the path differences. It has 12 main beams that point in 12 different directions at intervals of 30 deg in the horizontal plane. These main beams are sharp over a wide frequency range. By utilizing these characteristics, the MAM can be applied to many fields such as precise acoustic measurement of sound fields, automatic talker locations, and beam steering. When reproducing the original sound field by this system, 12 loudspeakers arranged in a circle around a central point are connected to the corresponding 12 outputs of the MAM. A new acoustical parameter based on spatial correlation of the sound field (correlation between the 12 MAM outputs) is proposed by utilizing the MAM.

\section{0:10}

4aAA5. Directional microphones in computer simulated and real rooms. David A. Berkley and James E. West (Acoust. Res. Dept., AT\&T Bell Labs., Murray Hill, NJ 07974)

Directional microphones are being used increasingly for teleconferencing in a variety of environmental conditions such as varying size and design of the rooms in which they function. However, little is known about the subjective effects of varying environments on the hypothetical improvements produced by these complex microphone systems. It is possible, but quite difficult and expensive, to directly test any proposed microphone system design in a variety of actual or experimental rooms. An alternative approach is use of computer simulation, both of the microphone system and the room environment. A complex microphone system can be modeled as a collection of point microphones distributed in space and summed with appropriate time delays. Established room simulation modeling methods were used. Combining both models together, with appropriate attention to spatial and time resolution of the microphone model, allows calculation of the impulse response of the complete structure, in an arbitrary room environment. The resulting impulse response can be used as a filter for speech. Results of such simulations are presented and compared with real room results.

\section{0:35-10:45 Break}

\section{Contributed Papers}

\section{0:45}

4aAA6. A new in situ method to measure the directivity index. Gary W. Elko (Acoust. Res. Dept., AT\&T Bell Labs., Murray Hill, NJ 07974)

The directivity index is a considerably useful measure in quantifying the directional properties of electroacoustic transducers. For microphones used in reverberant environments, the directivity index gives a rough estimate of the gain in signal-to-noise ratio. The measurement of the directivity index of a transducer is complicated by the need to measure over a spherical surface that is in the far field of the transducer. This talk will present a new method for measuring the directivity index in a diffuse reverberant environment. The method uses the frequency response spectral variance to estimate the distance from a point source to a point receiver in a reverberant field. If the source-to-receiver distance, reverberation time, room surface area, and volume are known, the directivity factor can be calculated explicitly from the spectra variance. The method and its limitations will be discussed in the talk.

\section{1:00}

4aAA7. Finding the direction of a sound source using a vector sound-intensity probe. Robert Hickling, Wei Wei (Natl. Ctr. for Phys. Acoust., Univ. of Mississippi, University, MS 38677), and Richard Raspet (Univ. of Mississippi, University, MS 38677)

Good spatial resolution of a sound source can be achieved with the four closely spaced transducers in a vector sound-intensity probe. In contrast, a receiving array, generally requires an extensive distribution of transducer elements. Experimental results are presented that demonstrate the ability of a vector probe to determine the direction of a sound source in water, both without and with noise interference. Measurements of the sound-intensity vector, or sound-power flux, were performed using the cross-spectral relation. Spectral subtraction and averaging over the frequency range of a source, were found to be effective tools for determining the direction of the source when there is interference and background noise.

\section{1:15}

4aAA8. Minimally intrusive occupied hall measurement techniques. Jont B. Allen (AT\&T Bell Labs., Murray Hill, NJ 07974), Dana L.
Kirkegaard (Kirkegaard \& Associates, 4910 Main St., Downers Grove, IL 60515), and Sunil Puria (MIT, Cambridge, MA 02139)

With high-powered portable computers and digital signal processing boards it is possible to make high-quality, broadband impulse response measurements that are minimally intrusive to audience and performers. In January 1992 the authors designed a stimulus for measurements at Symphony Hall, Boston that permitted stimulus levels near or below the audience's masked threshold. Synchronous averaging of the response increased the signal-to-noise ratio and minimized inaccuracies due to random audience and performer movement. Subsequent measurement sessions in Davies Symphony Hall, San Francisco and the Academy of Music, Philadelphia provided the opportunity to fine-tune the stimulus type, number of averages, and various source characteristics, including: spectrum, level, directionality, and placement relative to intrusion upon audience and performers. Source directionality and placement were selected to optimize convolution with recordings of solo violin. Typically, measurements are made at levels above the masked threshold following a brief announcement. There are interesting exceptions when occupied hall measurements can only be made at levels near or below the masked threshold. In either case, occupied hall measurements must be designed to minimize the intrusion upon the concert setting, particularly when working with major symphony orchestras.

\section{1:30}

4aAA9. DISC project: Auralization of virtual environments and the Drrectional Scattering Coefficient. Peter D'Antonio (RPG Diffusor Systems, Inc., 651-C Commerce Dr., Upper Marlboro, MD 20772)

Accurate prediction of a room's impulse response requires the appropriate directional scattering coefficients, as well as computational algorithms, which utilize these data in modeling programs. The directional scattering coefficients define how sound interacts with the various absorbing, reflecting, diffusing, and diffracting surfaces in a room, as a function of the incident direction and frequency, sample size, mounting, and observation direction. Unfortunately, these data and algorithms do not exist. The acoustics community is now preparing to meet the challenge of augmenting random-incidence absorption coefficients and establishing a database of $D /$ rectional Scattering Coefficients (DISC) for all commonly used architectural materials, in a new basic research initiative called the DISC project. The goals of the DISC project are as follows. (1) Design and implement computer-controlled automatic test- 
ing instrumentation and facilities to experimentally map the fullspectrum backscattered hemisphere for all commonly used architectural materials for all angles of incidence and various sample sizes and mountings. (2) Develop a geometrical convolution algorithm that can be used with the image model to utilize directional information and provide $n$-bounce impulse responses, which agree with experimental measurements within accepted error limits, as judged by auralization test. (3) Conduct subjective perception auralization studies with headphones and in an acoustically optimized loudspeaker listening environment.

\section{1:45}

4aAA10. A rapid method for measuring tube attenuation. $W$. T. Chu (Acoust. Lab., Inst. for Res. in Construction, Natl. Res. Council, Ottawa, ON K1A OR6, Canada)

It has long been recognized that the acoustic impedance is the chief characteristic of the performance of acoustical materials. The impedance tube provides an easy way of measuring this characteristic at normal incidence. It is also known that the effect of tube attenuation must be included in the impedance tube analysis for accurate measurements. Although theoretical prediction can be deduced from the well-known Helmholtz-Kirchhoff formulation, disagreements with experimental results (up to 15\%) have been reported. Previous results relied on locating and measuring the pressures as a number of maxima and minima along the standing wave pattern inside the tube. The method was te- dious. A more rapid method for measuring the tube attenuation is possible based on the recently proposed three microphone method for impedance tube measurements [W. T. Chu, J. Acoust. Soc. Am. 92, 2315(A) (1992)]. The procedure will be presented together with results from both computer simulations and actual experiments.

\section{2:00}

4aAA11. Maximum length sequence based testing of hearing aids. Todd Schneider and Donald G. Jamieson (Hear. Health Care Res. Unit, Univ. of Western Ontario, London, ON N6G 1H1, Canada)

An automated dual-channel maximum-length sequence (MLS) test system for the electroacoustic characterization of hearing aids has been developed. This test system applies a speech-shaped MLS acoustically to a hearing aid and measures the electro-acoustic frequency response. This method provides results that compare favorably to those obtained using the method employed by Kates [J. M. Kates, J. Rehab. Res. Develop. 27, 255-278 (1990)] and the ANSI standard method [ANSI S3.42 (1992)]. Test results show that MLS-based testing is significantly faster than noise-based testing. Two signal-biased MLS-based testing methods have also been developed. These methods apply a bias signal to force the hearing aid into a mode of operation where the frequency response, with adaptive or subtractive bias signal cancellation, is measured using a low-level, speech-shaped MLS. This method has proved valuable for the characterization of automatic signal processing hearing aids. [Work supported by ORTC and OMH.]

\title{
Session 4aAB
}

\section{Animal Bioacoustics: Marine Bioacoustics}

\author{
Ann E. Bowles, Chair \\ Hubbs-Sea World Research Institute, 1700 South Shores Road, San Diego, California 92109
}

\section{Contributed Papers}

\section{9:00}

4aAB1. Observations of humpback whale songs in Australian waters. Douglas H. Cato (Maritime Operations Div., Mater. Res. Lab., Defence Sci. and Technol. Organisation, P.O. Box 44, Pyrmont, NSW, 2009, Australia)

Songs of humpback whales have been recorded in Australian waters for more than 10 years, and show features that are broadly similar (though different in detail) to the complex and stereotyped songs observed in the northern hemisphere. Observations have been made along the migration paths that follow the east and west coasts of the continent. Recordings at similar times but separated by thousands of kilometers along a particular coast are similar, but the song off the east coast does not appear to be related to that off the west coast. Both the characteristics of the sounds and the song pattern have changed progressively, but the rate of change has varied substantially from year to year. On the east coast the song has been observed to degenerate from its usual complex pattern to one with little structure, during a year of rapid change, and then return with time to its more usual form.

\section{9:20}

4aAB2. Bioacoustic transient detection by image convolution. David K. Mellinger and Christopher W. Clark (Bioacoust. Res. Program, Cornell Univ., 159 Sapsucker Woods Rd., Ithaca, NY 14882)

A method is presented for detecting bioacoustic transient signals. The method detects a common feature of animal calls, frequency sweeps, or combinations of frequency sweeps over time, by convolving a spectrogram image with a two-dimensional kernel designed to find lines in images. This kernel has positive (excitatory) regions to achieve strong convolution operator response on desired frequency sweeps, and negative (inhibitory) regions to reject noise and interfering sounds. Design parameters control permissible variation in the sweeps. This technique is useful in screening long recordings for desired call types and perhaps for identifying individuals. For screening, it is compared with two other approaches on recordings of Bowhead Whale calis in a noisy Arctic environment: a hidden Markov model and a matched filter. This method performs better than both others on the Bowhead data, perhaps well enough for practical use. It also detects perfectly 15- to 20-Hz calls of unknown origin, possibly blue whales, in a moderately noisy environment. For identifying individuals, tests of spotted dolphin "signature whistles" are underway; it is hoped that similar bottlenose dolphin whistles will soon be tested.

\section{9:40}

4aAB3. Transmission beam pattern of a false killer whale. Whitlow W. L. Au, Jeffrey L. Pawloski, ${ }^{\text {) }}$ Ted W. Cranford, Robert C. Gisner, and Paul E. Nachtigall (Naval Command Control and Surveillance Ctr., P.O. Box 997, Kailua, HI 96734)

The echolocation transmission beam pattern of a false killer whale (Pseudorca crassidens) was measured in the vertical and horizonta] planes. A vertical array of seven broadband miniature hydrophones was used to measure the beam pattern in the vertical plane and a horizontal 
array of the same hydrophones was used in the horizontal plane. The measurements were performed in the open waters of Kaneohe Bay, Oahu, Hawaii, with the whale performing a discrimination task. The width of the beams in both planes were similar to those of the Atlantic bottlenose dolphin (Tursiops truncatus), and broader than those of the beluga whale (Delphinapterus leucas). The major axis of the vertical beam was directed slightly downwards between $0^{\circ}$ and $-5^{\circ}$, in contrast to the +5 to $10^{\circ}$ for Tursiops and Delphinapterus. The beam in the horizontal plane was directed forward. Differences in the fatty structure of the melon of Pseudorca, Tursiops, and Delphinopterus could explain differences in the elevation angle of the vertical beam axis. ${ }^{\text {a) }}$ At SAIC, Kailua, HI 96734.

\section{0:00}

4aAB4. Living sounds from the sea. Helen M. Walkinshaw (Box 72, Peapack, NJ 07977)

In mid-1951, with initial support by ONR, Bell Telephone Laboratories began an exploratory program of undersea acoustics research that lasted some 40 years. As a result, a diverse collection of in situ recordings of marine life slowly accrued from chance encounters during other planned experiments. Most have been identified as marine mammalsvarious species of whales and porpoise-a few remain unknown even now. The precise mechanism for producing certain very low frequency sounds ( $20 \mathrm{~Hz}$ and below) also remains unclear. The original recordings were made on several bottomed and off-bottom suspensions of hydrophones with effective bandwidths of from $5-400 \mathrm{~Hz}$ and $10-1000$ Hz. Their installation depths ranged from 220 to below 2000 fathoms, cable-connected to shore, enabling continuous monitoring of the under- sea environment. Four samples are presented. Locale and circumstances of their acquisition are described, as are geographic scope and observed seasonal occurences. Copies of the original recordings are available for study.

\section{0:20}

4aAB5. Measurement and analysis of the two-peak resonance of fish swimbladders. Wen-xu Zhou and Peter H. Rogers (School of Mech. Eng., Georgia Inst. of Technol., Atlanta, GA 30332)

The object of this research is to study the frequency response of the two-chambered goldfish swimbladder. An ultrasonic system was devised to measure swimbladder frequency response in vivo in a noninvasive, nonintrusive manner. Measurements were also made for oscars which have a single-chambered swimbladder. Unlike the oscar, two peaks often appear in the response curves of goldfish. To explain this two-peak resonance, an experimental model and an analytical model have been developed. The experimental model employs two gas bubbles to simulate the swimbladder. The experimental results show that the interaction between the two chambers of the goldfish swimbladder causes the two-peak phenomenon. The analytical model treats an oscillating system with two degrees of freedom. The parameters for the analytical model are related to the physical parameters of the system by theory. From comparison of the experimental and theoretical models, it is found that the damped-spring-mass system with two degrees of freedom reproduces what has been observed in the two-bubble experiment and does a reasonable job of modeling the two-chambered goldfish swimbladder.

\title{
Session 4aBV
}

\section{Bioresponse to Vibration and Psychological and Physiological Acoustics: Effects of Aging on Hearing and Touch}

\author{
Ronald T. Verrillo, Chair \\ Institute for Sensory Research, Syracuse University, Syracuse, New York 13244-5290
}

Chair's Introduction-8:15

Invited Papers

8:20

4aBV1. Presbyacusis: An overview. John H. Mills (Dept. of Otolaryngol. and Commun. Sci., Medical Univ. of South Carolina, 171 Ashley Ave., Charleston, SC 29425)

Presbyacusis is defined operationally as age-related hearing loss. It is a common problem and will become even more prevalent in the future. Age-related histopathological changes have been discovered at nearly every location in the aging auditory system from the external ear to the cochlea to the auditory brainstem and temporal lobe. Even for a given person, histopathology can be observed at multiple sites. Moreover, for individual human subjects it remains difficult, if not impossible, to separate age-related hearing loss from hearing loss caused by exposure to noise and other ototoxic agents (sociocusis), or from hearing loss caused by other disease processes (nosoacusis). Most of the research on presbyacusis can be placed into three categories: cross-sectional epidemiologic (audiometric surveys) studies; clinical studies with the emphasis on histopathology of the cochlea, psychophysics, and speech perception; and most recently, there have been a number of efforts to develop an animal model (mouse, rat, gerbil) of the aging human ear. Highlights from these research efforts will be presented with a focus on evoked potentials and masking in aging human and animal subjects. [Work supported by NIH DCOO422.]

\section{8:50}

4aBV2. The pathophysiology of presbyacusis: Does the aging cochlea need a jump start? Richard A. Schmiedt (Dept. of Otolaryngol. Med., Univ. of South Carolina, 171 Ashley Ave., Charleston, SC 29425-2242)

The Mongolian gerbil is used as an animal model to study the pathophysiology underlying presbyacusis. Gerbils are raised from birth in a quiet vivarium or are exposed to a long-duration wideband noise $(0.5-4.0 \mathrm{kHz}$ for $1-2$ years at $85 \mathrm{~dB}$ SPL) at some time during their life. Quiet-aged ears have thresholds that are increased somewhat, especially above $4 \mathrm{kHz}$, but nonlin- 
earities such as otoacoustic emissions and two-tone suppression are present and are largely unaffected by the aging process. Morphologically, hair cells in quiet-aged animals are highly conserved, whereas the lateral wall system degenerates, resulting in a decreased endocochlear potential (EP). Current injection to bolster the EP in these quiet-aged ears can enhance the response to low- and high-level stimuli. Noise-aged ears, while also showing increased thresholds, can be differentiated from their quietaged counterparts by a lack of nonlinear phenomena. Two-tone suppression is absent as are otoacoustic emissions. Thus, aging in quiet or in noise can have very different outcomes with regard to overall peripheral function, even when the respective audibility curves are similar. [Work supported by NIH POI DC00422.]

4aBV3. When feeling is failing: The effects of aging on the sense of touch. Ronald T. Verrillo, George A. Gesheider, Stanley J. Bolanowski, and Kathryn E. Hoffman (Inst. for Sensory Res., Syracuse Univ., Syracuse, NY 13244-5290)

The vast majority of reports in the literature concerning the psychophysical characteristics of touch is based on studies of college-aged persons. If the skin is to be utilized as a surrogate channel of communication for the profoundly deaf, it is essential that one know how aging affects the sense of vibrotaction. Life-span studies are rare indeed although there is a recent intensified interest in the sensory systems of elderly persons. Being reported are the results of a research program showing changes, from childhood to the elderly, of the threshold of detectability, suprathreshold responses, gap detection, and temporal and spatial summation. The Pacinian system shows a general decline with age and "recruitment" in the growth of sensation magnitude. Changes are also noted in gap detection and in the capacity to summate over time and space. [Work supported by NIDCD-DC01243.]

\section{9:50-10:00 Break}

\section{Contributed Papers}

10:00

4aBV4. Human infants' sensitivity to interaural level differences. Daniel H. Ashmead, D. Wesley Grantham, Walter Murphy, and Anne Marie Tharpe (Div. of Hear. and Speech Sci., Vanderbilt Univ., and Bill Wilkerson Ctr., 114 19th Ave. S., Nashville, TN 37212)

Infants' sensitivity to interaural differences was assessed using a lateralization paradigm. Infants aged 5 to 6 months heard speech sounds with interaural level or time differences (ILD, ITD), while an observer made forced choice judgments about the side of the louder (or leading) sound. In experiment $1(N=10)$, the stimuli were $10 \mathrm{~dB}$ ILD, $100 \mathrm{~dB}$ ILD (monaural), and $400 \mu \mathrm{s}$ ITD. Performance was well above chance $(70 \%, 74 \%$, and $78 \%$ correct), indicating appropriate responses to "easy" interaural differences. In experiment $2(N=16)$, a group threshold for ILD sensitivity was determined, using ILDs of 2,5, 8, and $100 \mathrm{~dB}$ (as well as the 10 and $100 \mathrm{~dB}$ ILD trials from experiment 1). The threshold estimate was $6.6 \mathrm{~dB}$, far higher than adult thresholds of 0.5 to $1 \mathrm{~dB}$. These findings, together with previous work on infants' free field sound localization, suggest that ILDs are not a strong basis for sound localization in this age range. [Work supported by NIH.]

\section{0:15}

4aBV5. Gap detection in young and old adults. Bruce $A$. Schneider (Dept. of Psychol., Erindale Campus, Univ. of Toronto, Mississauga, ON L5L 1C6, Canada) and Margaret Kathleen Pichora-Fuller (Univ. of British Columbia, Vancouver, BC V6T 1Z3, Canada)

Threshold for detecting a gap between two Gaussian-modulated, $2-\mathrm{kHz}$ tones $(\sigma=0.5 \mathrm{~ms}$ ) were determined in young and old listeners. The gap detection thresholds of old subjects were more variable and about twice as large as those obtained from young subjects. Moreover, gap detection thresholds were not correlated with audiometric thresholds in either group. Estimates of the width of the temporal window of young subjects, based on the detection of a gap between two tone pips, were smaller than those typically obtained when a relatively long duration pure tone is interrupted [Moore et al., J. Acoust. Soc. Am. 85, 1266-1275 (1989)]. Because the amount of time it takes to recover from an adapting stimulus is likely to affect gap detection thresholds, smaller estimates of temporal window size would be expected in this paradigm if the amount of adaptation produced by the first tone pip was negligible. The larger gap detection thresholds of old subjects indicates that they may have larger temporal windows than young subjects.
10:30

4aBV6. Neural processing of temporal gaps in the infertor colliculus of the awake young and old CBA mouse. Joseph P. Walton, Robert D. Frisina, and William O'Neill (Otolaryngol. Div. and Dept. of Physiol., Univ. Rochester School of Medicine, Rochester, NY 14642-8629)

The ability to detect brief silent intervals or gaps in sound forms one important property of temporal resolution. Psychoacoustical evidence suggests such fundamental processing correlates with more complex perceptions, such as speech perception in noise. Moreover, studies have also implicated deficits in temporal resolution as a possible cause for the difficulties observed in presbycusis. The current study compared neuronal response properties and minimal gap thresholds (MGT) in the young (2-4 month) and old ( $>24$ month) CBA mouse model of presbycusis. The following measures were obtained from over 40 single units in each age group; intensity functions for 100 -ms tone and noise bursts, spontaneous rate, Q10 bandwidth, and gap functions. Neural correlates of gap detection were studied by imbedding a silent gap (widths from 0.5 to $20 \mathrm{~ms}$ ) between two noise bursts presented at $30 \mathrm{~dB}$ above unit threshold. MGT was quantified by comparing spike counts in multiple time windows before, during, and after the gap. Comparison between young and old response patterns indicate: (1) PST histograms displayed numerous patterns including; onset, onset-sustained, on-off, primarylike, buildup, and off responses, (2) a wide range of spontaneous rates were encountered from 0 to 60 spikes/s, (3) MGT varied with response type regardless of age, and (4) the lowest MGTs (1-2 ms) observed were found both in young and old animals, but the frequency of occurrence was lower in the older animals. [Work supported by NIH-NIA P01 AG09524-01Al.]

\section{0:45}

4aBV7. Sound localization in noise with hearing protectors: The effect of aging. Sharon M. Abel and Valerie H. T. Hay (Samuel Lunenfeld Res. Inst., Mount Sinai Hosp., 600 University Ave., Toronto, ON M5G 1X5, Canada)

Sound localization ability was assessed in two groups of 24 normalhearing subjects, aged $20-35$ and $40-60$ years. The test was conducted in a semi-reverberant chamber, using an array of six speakers, surrounding the subject at azimuth angles $60^{\circ}$ apart, at a distance of $1 \mathrm{~m}$. Three variables were investigated: the wearing of hearing protectors (unoccluded, conventional E-A-R plug and E-A-R muff, and Bilsom 2392 
stereophonic muff with limited amplification), background (quiet vs 65 dB SPL white noise), and stimulus frequency ( 500 vs $4000 \mathrm{~Hz}$ ). For each of the 16 listening conditions, one block of 120 trials was presented. Within a block, the test sound, an 80-dB SPL 300-ms one-third octave noise band, was randomly presented from each speaker on 20 trials. The subject judged the direction using a hand-held response box with an array of microswitches configured like the speaker array. Analysis of the overall and conditional proportions of correct response and the correct and incorrect response times is currently underway. Focii of interest are the effects of (1) stereophonic amplification on accuracy and (2) aging on decision reaction time.

\section{$11 \cdot 00$}

4aBV8. A comparison of complex vibrotactile pattern perception on the OPTACON by young and old observers. Roger W. Cholewiak and Amy A. Collins (Dept. of Psychol., Green Hall, Princeton Univ., Princeton, NJ 08544-1010)

College-aged and, more recently, senior observers are being surveyed for the ability to identify and recognize complex vibrotactile patterns. These patterns are presented to the left index fingertip on the 144-pin vibratory array from the OPTACON, a reading machine for the blind, in a "static" mode in which all elements that spatially define the pattern are turned on for its 26-ms duration. Pattem complexity ranges from simple line segments, identified only by number, to the 26 letters of the alphabet. The ability of these observers to discriminate between two simple patterns is first measured. They are then trained to identify these patterns, and are tested in a masking situation. Observers are then trained to identify a larger set of abstract patterns, and finally the alphabet. In addition, two measures of basic sensitivity are taken on both groups: threshold at ten different frequencies $(10-400 \mathrm{~Hz})$ of sinusoidal vibration, and the growth of magnitude for suprathreshold levels of four of these frequencies. Comparisons will be discussed. [Work supported by NIDCD.]

\section{1:15}

4aBV9. Age-related changes in mechanoreceptor-specific vibrotactile thresholds for normal hands. A. J. Brammer and J. E. Piercy (Inst. for Microstructural Sci., Natl. Res. Council, Ottawa, ON K1A OR6, Canada), S. Nohara, H. Nakamura (Kanazawa Univ., Kanazawa, Japan), and P. L. Auger (Ctr. Hospitalier de l'Unjv. Laval, Ste-Foy, PQ, Canada)

Mechanoreceptor-specific vibrotactile perception thresholds have been measured at the fingertips using the tactometer [A. J. Brammer and J. E. Piercy, in Proc. UK Informal Group Meeting on Human Response to Vibration, Buxton (September 1991)]. Thresholds were obtained from the left and right hands of healthy male white-collar workers, aged from 25 to 73 years, without signs, symptoms, or history of neurological disease, or of exposure to vibration. Groups were formed from: (1) oriental professionals (mean age $34 \pm 5.9$ years, $N=24$ ); (2) occidental professionals (mean age $40 \pm 5.0$ years, $N=18$ ); and (3) oriental workers (mean age $62 \pm 5.6$ years, $N=24$ ). A preliminary analysis of the data suggests that there is littie effect of age on thresholds mediated by the SAI and FAI receptors. Thresholds mediated by the FAII receptors decreased in sensitivity at a average rate of $2.6 \mathrm{~dB}$ per decade increase in age. There are also a tendency for the SAI and FAI receptor thresholds in groups 1 and 3 to be more sensitive than those in group 2.

\section{1:30}

4aBV10. Age-related changes in mechanoreceptor-specific vibrotactile thresholds with exposure to hand-transmitted vibration. G. Coutu-Wakulczyk (Ctr. for Res. on Gerontology and Geriatrics, Univ. of Sherbrooke, 1036, Belvédère South, Sherbrooke, PQ J1H 4C4, Canada), A. J. Brammer, and J. E. Piercy (Natl. Res. Council, Ottawa, Canada)

Mechanoreceptor-specific vibrotactile thresholds have been determined at the fingertip of the third finger (both hands) of manual workers using the tactometer. Age-related changes in thresholds were examined in $\mathbf{8 2}$ hands exposed to vibration, which were classified according to the Stockholm Workshop Stages of the hand-arm vibration syndrome (HAVS), and 38 unexposed hands. The age of subjects ranged from 19 to 54 years, and the exposure to vibration ranged from 2 to 27 years. Thresholds from asymptomatic, unexposed persons were employed to separate the influence of aging from that due to vibration exposure. A preliminary analysis of the data suggests that age does not influence the development of either the sensorineural symptoms or vascular signs of HAVS, a finding of consequence to the management of workers' activities in the workplace.

\section{1:45}

4aBV11. Wheel chair vibration and bioresponse criteria. Robert D. Collier (Dept. of Mech. Eng., Tufts Univ., Medford, MA 02155)

Wheel chairs are being used increasingly in outdoor environments including paved and unpaved roads and sidewalks with widely varying roughness. Wheel chair design and development for active users is a lively field in which weight, stability, control, and comfort are important design criteria. An ongoing research project at Tufts includes baseline laboratory and field vibration measurements of both conventional and new prototype wheel chairs and associated bioresponse. A test track includes a range of roughness conditions. Representative results show: (1) wheel hub vibration levels on the order of $1-3 \mathrm{~g}(0-200 \mathrm{~Hz})$ with impulse waveforms of 5-10 ms occurring several times a second; (2) frequency response functions of a standard wheel chair structure contain several low-frequency resonances below $100 \mathrm{~Hz}$ that are lightly damped; (3) cushions provide comfort but limited low-frequency vibration and shock isolation. A review of models of whole body vibration together with an analysis of wheel chair dynamics provide guidance for vibration isolation/stability design criteria for nonstationary impulsive forces. An isolator that can be installed on an existing chair has been designed and evaluated. 


\title{
Session 4aMU
}

\section{Musical Acoustics and Psychological and Physiological Acoustics: Auditory Organization in Music and Speech II: Melody and Rhythm}

\author{
William M. Hartmann, Chair \\ Department of Physics, Michigan State University, East Lansing, Michigan 48824
}

Chair's Introduction—8:30

Invited Papers

8:35

4aMU1. Auditory organization in music: Let the (defunct) composer speak. Pierre L. Divenyi (Speech and Hear. Res., V.A. Med. Ctr., Martinez, CA 94553)

Auditory organization of music as the stimulus must reflect to a large degree the organization and the structure of "musical building blocks"-elements consisting of notes and simultaneous clusters of notes, as well as patterns built from successive elements-intended by the composer. One aspect of this organization is the internal cohesion of successive elements, i.e., the formation of a melodic structure or an auditory stream [A. S. Bregmann, Auditory Scene Analysis (MIT, Cambridge, 1991)]. Experimental and theoretical foundations of auditory stream cohesion and stream segregation, developed during the last two decades, make it possible to derive rules that are both solidly anchored in psychoacoustic reality and that, at the same time, are well-integrable with music theory (see the following paper by David Huron in this session). However, much can be learned from observing musical compositions to see how the composer makes the elements come together to form melodic entities, or to break up into separately audible voices (streams). While, for the most part, compositional practice confirms predications of psychoacoustic-auditory stream theory, not infrequently, the results are contrary to expectations and, sometimes, to common sense. Excerpts from pieces throughout Western music history will be shown and demonstrated in an effort to illustrate usual as well as unusual examples of melodic organization. [Work supported by the National Institutes of Health and the Department of Veterans Affairs.]

9:05

4aMU2. A derivation of the rules of voice-leading from perceptual principles. David Huron (Conrad Grebel College, Univ. of Waterloo, Waterloo, ON N2L 3G6, Canada)

The tradition rules of voice-leading in Western music are elucidated using empirically established perceptual principles. Six core principles are shown to account for the majority of traditional rules of voice-leading given in music theory texts. The pertinent principles include the principle of dissonant spread, the principle of tonal fusion, the pitch proximity principle, and the pitch comodulation principle. These principles are treated as axioms in a formal system from which the traditional rules of voice-leading are derived. All of the major rules of voice-leading are derived-including such arcane rules as avoid doubling the leading-tone, and when approaching an octave by similar motion, ensure that one part moves by diatonic step. In the process of the derivation, several novel rules arise that are not found in treatises on voice-leading. For these novel rules, computer-based score analysis show that composers do indeed write in a manner consistent with these additional rules.

\section{Contributed Papers}

\section{9:35}

4aMU3. The dynamics of musical structure formation: Strange attractors, orbit formation, and the topology of musical structure. Donald Casadonte (Dept. of Music, Ohio State Univ., 1899 College Rd., Columbus, OH 43210)

Since the time of the ancient Greeks music theorists have dreamed of a "musical space" in which musical events reside. In this paper the complete phase space portrait of a piece of music, Bach's Two Part Invention Na 4 in D Minor is reconstructed using techniques of dynamical systems theory. The space consists of a central strange attractor surrounded by wandering orbits that continually "revisit the attractor as the musical structure develops. This simple example is taken as a paradigm for more general types of music structure, in which stable melodic lines are topologically represented as orbitally stable subsets in phase space (most commonly as strange attractors), and the transitional or nonmelodic sections are topologically unstable or wandering subsets. Musical structure is generated as the musical "trajectories" contained in the different simultaneous musical lines of a composition interact via the stable and unstable regions of the musical phase space structure ( $\omega$-limit structure) defined by the composer. This $\omega$-limit hypothesis is illustrated with several classical music examples. The implications of time-evolutionary pictures of the unfolding of musical structure and the topological process involved in the composer-shaping of melodic contours are discussed.

\section{9:50}

4aMU4. The effects of training and predictability on the discrimination of sequences of tones with various frequency ranges. Kathy Barsz, Maya Jariwala, Jeffrey E. Peter, Melissa A. Scism, Larissa A. Barnauskas, Shannon M. King, and Margaret $M$. McCarthy (Dept. of Psychol., SUNY, Geneseo, NY 14454)

Listeners discriminated sequences: two different higher frequency tones alternated with two different lower frequency tones, each $200 \mathrm{~ms}$. Sequences began at a random point and repeated without pause. Predictability was manipulated by holding the standard sequence constant 
(PREDICTABLE) or by varying it (UNPREDICTABLE). The frequency range between the higher and lower tones was two octaves (WIDE) or a minor second (NARROW). Medium range sequences (a minor third) were presented in two contexts: with the wide range sequences (MED/W) or with the narrow range sequences (MED/N). Sequences differed only in the relationship between the higher and lower tones. Fifty listeners each discriminated the sequences in one condition (MED/N or MED/W in either a PREDICTABLE or UNPREDICTABLE block); ten returned for two extra sessions (practice). Discrimination of the wide range sequences was at chance and only improved with practice when the block was predictable, although listeners still had a difficult time. Performance on this task was affected by frequency range; performance on the narrow range sequences benefited from practice and improved predictability There was a clear practice effect for the medium sequences, but only when the block was predictable and they were presented with the narrow range sequences.

\section{0:05}

4aMU5. Spectro-temporal factors in the perceptual segregation of tonal sequences. Punita G. Singh and Albert S. Bregman (Dept. of Psychol., McGill Univ., Montreal, PQ H3A 1B1, Canada)

Spectral factors such as differences in harmonic content are powerful cues in the organization of tonal sequences. Temporal factors such as rise time, however, have been shown to be poor cues [W. M. Hartmann and D. Johnson, Mus. Perc. 9(2), 155-184 (1991)]. The relative salience of these factors was investigated further using complex tones sequenced in a repeating ABA-"gallop" format, under conditions in which tones $\mathbf{A}$ and $\mathbf{B}$ had the same, or different harmonic content and/or temporal envelope shape. The $F 0$ difference between $A$ and $B$ was initially $0 \mathrm{~Hz}$, but increased over the course of a trial, until terminated by a listener, indicating perceptual segregation of the input sequence into sub-sequences comprising $\mathbf{A}$ and $\mathbf{B}$ tones, respectively. The $F 0$ difference required to reach this segregation threshold provided a measure of the efficacy of stimulus features of $A$ and $B$ as cues for sequential organization. Sequences combining differences in spectral content and temporal envelope shape required the smallest $F 0$ change for segregation. Sequences of tones with the same harmonic structure and temporal envelope required the largest $F 0$ changes, while the other conditions fell in the middle of this range. Results will be discussed in terms of implications for sequential organization as well as for the perception of timbre. [Work supported by NSERC, Canada.]

\section{0:20-10:35 Break}

\section{0:35}

4aMU6. Perceptual organization of temporal patterns in music. Annie H. Takeuchi (Res. Lab. of Electron., Rm. 36-747, MIT, Cambridge, MA 02139)

The temporal structure of music is based on meter, a hierarchical structure in which each level consists of periodic alternation of strong and weak pulses. This experiment examined the extent to which listeners have internalized metrical hierarchies and use them to perceptually organize temporal patterns. Musically experienced subjects rated how well probe tones that varied in temporal location and in loudness (accent level) fit into simple metrical contexts. Whereas Palmer and Krumhansl [J. Exp. Psychol: Human Perc. \& Perf. 16, 728-741 (1990)] reported that listeners rated such probe tones on the basis of metrical location (whether they occurred on accented beats, unaccented beats, or between beats), in this experiment, the accent level of tones interacted with metrical location in determining probe tone ratings. Accented probe tones were judged better fitting when they occurred on accented beats than on unaccented beats or between beats. Unaccented probe tones showed the reverse pattern. Most subjects organized the patterns according to a three-level hierarchy for triple meter and a four-level hieracrchy for quadruple meter. [Work supported by NSF.]
4aMU7. Rhythm and musical tuning joined in the minds of infants. Michael P. Lynch (Dept. of Audiol. \& Speech Sci., 1353 Heavilon Hall, Purdue Univ., West Lafayette, IN 47907-1353)

Perceptual grouping in music occurs in the context of tuning relations of musical scales, but perceptual relations between rhythm and tuning have received limited study. Because evidence suggests infants perceive rhythm, an important question is whether tuning interacts in the developing mind with other factors contributing to perceptual grouping. Six- and 12-month-old infants detected elongations of silence intervals in 6-note stimuli consisting of two 3-note triads from either the Western major (often heard), Western augmented (seldom heard), or Javanese pelog (culturally foreign) scales. The 2 triads in each stimulus were 1 octave apart, with either the first triad an octave above the second triad, or the reverse. Therefore, the latter but not the former stimuli conformed to the Gestalt principle of good continuation. Elongations of silence intervals were after either the third (between-group) or fourth (within-group) note. Six-month-olds and 12-month-olds performed reliably better than chance only in the testing conditions involving good continuation, and the 12-month-olds' performance was reliably best in the condition involving also within-group silence elongation and the Western major triad. These findings suggest that good continuation and infants' perceptual knowledge of tuning characteristics interacted in their perception of auditory groupings. [Work supported by NICHD Grant No. HD28527.]

\section{1:05}

4aMU8. Segmentation and stress in the rhythmic structure of music and speech: A wavelet model. Neil P. McAngus Todd (Dept. of Music, City Univ., Northampton Square, London EC1V OHB, England)

The rhythm of a piece of music or a poem can be described by hierarchical tree structure. A new application of wavelet analyses to rhythm in music and speech is described whose result is a hierarchical structure [N. P. McAngus Todd, J. Acoust. Soc. Am. 92, 2380 (A) (1992)]. This structure has two complementary components that are obtained from the projection of loci, in the frequency-time plane, of zero crossings of the slope of the energy flux surface in the energy-time plane: (a) a segmentation structure, corresponding to positive second derivatives at the zero crossings; (b) a stress structure, corresponding to negative second derivatives at the zero crossings. The value of the stress is given by the second derivative, which has a "Mexican hat" impulse response. These structures resemble the grouping/metrical structures of the theory of F. Lerdahl and R. Jackendoff [A Generative Theory of Tonal Music (MIT, Cambridge, MA, 1983)]. Example analyses of musical and speech performance are demonstrated for this method and evidence is provided that the resultant structures have a reasonable perceptual basis. The possibility of using this technique for the synthesis of rhythm using a cascade structure is discussed.

\section{1:20}

4aMU9. Detection of regular and irregular repeated tones in noise: Rhythm helps only if rhythm is detected. C. R. Hetherington, ${ }^{2)} \mathrm{C}$. D. Creelman, D. T. Stuss, ${ }^{\mathrm{a}}$ and M. Schmuckler (Dept. of Psychol., Univ. of Toronto, Toronto, ON M5S 1A1, Canada)

Individual thresholds for $1024-\mathrm{Hz}, 200-\mathrm{ms}$ tones in noise (PEST, 80\% 2IFC) were established in ten listeners, and then tones at this level were presented in a rhythmic pattern or in an irregular temporal pattern with the same average spacing and density of tones. Blocks comprising 200 tones were alternated randomly between the two conditions. A questionnaire administered at the conclusion of the session showed that half the subjects had become aware of the rhythmic pattern. Subjects were separated into two groups for data analysis; those who were aware and those who were unaware of the rhythmic pattern. In a subsequent session, detection threholds were again determined, and the subjects did a five-category rating detection. The rating data showed approximately 
the detectability predicted by the PEST procedure. No significant difference in this task was found between individuals from the aware the unaware groups. Relative to the detectability established with PEST $\left(d^{\prime}=1.19\right)$, rhythmic presentation yielded greatly enhanced detectability measures in listeners who were aware of the rhythm. Subjects who were unaware of the rhythm showed depressed detectability, both for rhythmic and arhythmic presentation. ")Also at Rotman Res. Inst. of the Baycrest Ctr., 3560 Bathurst Str., North York, ON M6A 2El, Canada.

\section{1:35}

4aMU10. Functional activation of right temporal and occipital cortex in processing tonal melodies. Robert J. Zatorre, Alan C. Evans, and Ernst Meyer (Montreal Neurolog. Inst., McGill Univ., Montreal, PQ H3A 2B4, Canada)

Cerebral blood flow changes were measured to examine the cortical basis for perceptual analysis of musical information. Twelve normal right-handed volunteers underwent positron emission tomography (PET) scanning using the bolus water technique while listening to two types of stimuli: (1) unfamiliar tonal melodies, and (2) sequences of noise bursts, acoustically matched to the melodies for duration, intensity, and onset/offset characteristics. Subtracting the latter from the former PET image provides information regarding melodic processing beyond that associated with nonspecific auditory stimulation. Anatomical localization was provided by superimposing the average PET subtraction image on the average magnetic resonance image for the subjects tested. Results indicated significant increases in blood flow while listening to melodies in the right associative auditory cortex, suggesting that specialized neural systems in the right temporal lobe participate in higher-order perceptual analysis. In addition, regions of significant activity were observed in the right occipital lobe, within areas classically described as associative visual cortex. This result may implicate these regions in hitherto unexplored aspects of auditory processings, or may possibly reflect engagement of visuospatial processing mechanisms in perception of melodies.

THURSDAY MORNING, 20 MAY 1993

SALON RENAISSANCE, 8:25 A.M. TO 12:15 P.M.

\title{
Session 4aPA
}

\section{Physical Acoustics: Acoustics in Space}

Robert E. Apfel, Chair

Department of Mechanical Engineering, Yale University, P.O. Box 2159, Yale Station, New Haven, Connecticut 06520

\author{
Chair's Introduction-8:25
}

Invited Papers

8:30

4aPA1. Microgravity investigations in the physics of fluids using acoustic techniques. Eugene H. Trinh (Jet Propulsion Lab., MS 183-401, California Inst. of Technol., Pasadena, CA 91109)

The Earth gravitational field strongly affects the motion of extended liquid surfaces, it establishes unavoidable density gradients, and it leads to the segregation of immiscible fluids having different density. Accessing a low gravity environment introduces the opportunity to investigate the effects of low-magnitude capillary and thermocapillary forces in systems such as twoand three-dimensional free-liquid surfaces (drops, bubbles, and floating zones) and stable multi-phase samples. Acoustic methods have already been shown to be effective in allowing the contactless positioning and manipulation of drops and bubbles in low gravity, as the other presentations in this session demonstrate. This successful implementation of acoustics to space-based reseanch is currently represented by the Drop Physics Module flight facility for microgravity experiments. There is, however, additional opportunity for the application of advanced quantitative acoustic measurement techniques for the determination of the properties of fluid phases or for the management of fluids in low gravity. Experiments in microgravity require a variety of novel supporting capabilities, and acoustics could provide some viable initial approaches. On the other hand, the observation of strictly acoustic effects such as streaming, cavitation, and radiation pressure in the reduced gravity environment might also provide new clues useful for the understanding of these phenomena. [Work supported by NASA.]

\section{9:00}

4aPA2. Thermogeoustic life sciences refrigerator. Steven L. Garrett, D. Felipe Gaitan, David K. Perkins (Dept. of Phys. and Space Systems Academic Group, Naval Postgraduate School, Code PH/Gx, Monterey, CA 93943), and D. Andrew Helseth (Actran Systems, Orlando, FL)

This thermoacoustic refrigerator is being built to preserve biological samples (blood, urine, etc.) during Space Shuttle operations. It employs two high-power electrodynamic drivers capable of generating $60 \mathrm{~W}$ of acoustic power each, two "stacks," and four internal heat exchangers in order to produce $700 \mathrm{BTU} / \mathrm{h}(205 \mathrm{~W})$ of cooling in the refrigerator mode at $4^{\circ} \mathrm{C}$ and 400 $\mathrm{BTU} / \mathrm{h}(117 \mathrm{~W})$ of cooling in the freezer mode at $-22^{\circ} \mathrm{C}$. The working fluid is a 2-MPa mixture of He and Xe gas. Both hot and cold heat exchangers use circulating fluids to remove heat from the insulated sample enclosure and exhaust enthalpy from the engine. This talk will describe the acoustical design and electronic control systems that permit long-term autonomous operation while on orbit. Except for the fact that the entire systems operates from a 28- $V_{d c}$ power supply, it is not unlike a typical small household refrigerator. [Work supported by NASA-Life Sciences Division and managed by General Electric Government Systems.] 
4aPA3. Materials characterization of surfaces of water drops with surfactants by acoustic means: The need for a microgravity environment. Robert E. Apfel, R. Glynn Holt, Yuren Tian, Xiaoyu Zheng, and Shi Tao (Yale Univ., New Haven, CT 06520-2159)

The goal of this laboratory's ground-based and microgravity research is to determine the rheological properties of liquid drops of single or multiple components in the presence or absence of surface active materials by exciting drops into their quadrupole resonance and observing their free decay. The resulting data coupled with appropriate theory should enable one to understand better the physics of the underlying phenomena, providing a better foundation than earlier empirical resuits could. The space environment makes an idealized geometry available (spherical drops) so that theory and experiment can be properly compared, and allows a "clean" environment, namely, an environment in which no solid surfaces come in contact with the drops during the test period. Moreover, by considering the oscillations of intentionally deformed drops in microgravity, a baseline is established for interpreting surface characterization experiments done on the ground by this and other groups. Results of work at Yale and in the first United States Microgravity Laboratory (USML 1, summer 1992) will be presented. [Work supported by NASA through JPL Contract No. 958722.]

\section{0:00}

4aPA4. Bubble dynamics in low gravity and related experiments on Earth. Philip L. Marston (Dept. of Phys., Washington State Univ., Pullman, WA 99164-2814) and Eugene H. Trinh (Jet Propulsion Lab., Pasadena, CA 91109)

A dual-frequency acoustic levitator containing water was developed for studying bubble and drop dynamics in low gravity. It was flown on USML-1 where it was used in NASA's Glovebox facility. High-frequency (21- or 63-kHz) ultrasonic waves were modulated by low frequencies to excite shape oscillations on bubbles and oil drops ultrasonically trapped in the water. Bubble diameters were typically close to $1 \mathrm{~cm}$ and the low gravity alters biases present in related experiments in $1 \mathrm{~g}$. Other observations concerned the agglomeration or coalescence of bubbles in response to acoustic Bjerknes forces, the response of bubbles to surfactant injection, oscillations of bubbles coated by oil, and ultrasonic cavitation. Observations suggest that shape oscillations tend to shift the coated bubble away from the oil-water interface of the coating. Supporting experiments in 1-g extending earlier results [Asaki et al., J. Acoust. Soc. Am. 93, 706-713 (1993)] will also be examined. [Work supported by NASA and by ONR.]

\section{0:30-10;45 Break}

\section{0:45}

4aPA5. Acoustics for space applications: A progress review. Taylor G. Wang and A. V. Anilkumar (Ctr. for Microgravity Res. and Applications, Vanderbilt University, Nashville, TN 37235)

A wide spectrum of containerless science experiments can be performed in a microgravity environment by employing the levitation and manipulation capabilities of acoustics. Acoustic radiation forces can be used to position, rotate, oscillate, and deform liquid samples; and thereby help perform controlled experiments. These aspects have been successfully demonstrated in space with the Drop Dynamics Module (DDM, May 1985), and the more recent Drop Physics Module (DPM, June 1992). The mastering of these noninvasive manipulation techniques opens the way for future drop physics and containerless materials processing experiments. In the latter context, the acoustic method is useful in the control of any molten material, regardless of it being conducting, or charged, or not.

\section{Contributed Papers}

11:15

4aPA6. Undercooling and solidification of acoustically levitated molten drops. Kenichi Ohsaka and Eugene H. Trinh (Jet Propulsion Lab., California Inst. of Technol., M/S 183-401, 4800 Oak Grove Dr., Pasadena, CA 91109)

Undercooling of a liquid prior to the nucleation of a solid is necessary for producing novel microstructures including metastable phases that are not formed by conventional solidification processes. Because containerless techniques such as acoustic levitation are capable of achieving a high undercooling level of liquids, they are under consideration as possible techniques for material processing on Earth and in space. For an application of acoustic levitation on Earth, however, there is a strong concern that ultrasound may prematurely initiate the nucleation of the solid and prevent further undercooling in experiments designed for the study of solidification in the deeply undercooled liquid. An experiment using a model material with a single axis levitation system shows an effect of ultrasound as the occasional nucleation of the solid at low undercooling levels. This premature nucleation may be explained by thermodynamic considerations which predict an increase in effective undercooling of the liquid upon the collapse of cavities. [Work supported by NASA.]

\section{1:30}

4aPA7. Drops in space: Spacelab experiments on surfactant-bearing drops. R. Glynn Holt, Yuren Tian, Xiaoyu Zheng, and Robert E. Apfel (Dept. of Mech. Eng., Yale Univ., P.O. Box 2159 Yale Station, New Haven, CT 06520)

Utilizing a dedicated acoustic positioning apparatus called the Drop Physics Module (DPM) aboard the First United States Microgravity Laboratory (USML-1) mission, single surfactant-bearing drops were excited into their quadrupole resonance and observed during free decay. Surface shear and dilatational viscosity and Gibb's elasticity are inferred from the frequency and damping measurements using a modification of a previous theory [H.-L. Lu and R. E. Apfel, J. Fluid Mech. 222, 587 (1991)]. Measurements were performed for various combinations of surfactant type, concentration, and drop size. Results from groundbased experiments in ultrasonic fields will also be presented and compared. [Work supported by NASA through JPL Contract No. 958722.]

\section{1:45}

4aPA8. Experiments and analysis of shape oscillations of bubbles levitated in water on the Earth. Thomas J. Asaki and Philip L. Marston (Phys. Dept., Washington State Univ., Pullman, WA 99164-2814) 
Levitation and excitation of shape oscillations for bubbles in water were demonstrated for the size range 1.6 to $12-\mathrm{mm}$ diameter [T. J. Asaki, P. L. Marston, and E. H. Trinh, J. Acoust. Soc. Am. 93, 706-713 (1993)]. Novel methods of experimentation and analysis in the current research should complement experiments carried out in microgravity. Current research concerns the understanding of equilibriuim shape effects on steady-state and freely decaying shape oscillations. These oscillations were studied with an optical extinction method. The measurements probe the surface rheology of the bubble. The experimental method has been shown to be applicable to saline solution as well as to degassed but otherwise untreated sea water. [Research supported by ONR and DOE GAANN fellowship.]

\section{2:00}

4aPA9. The effect of compressibility on acoustical streaming near rigid boundaries. Quan Qi (Dept. of Theor. and Appl. Mech., Univ. of Illinois at Urbana-Champaign, Urbana, IL 61801)
The effect of compressibility on acoustic streaming near rigid boundaries is discussed for plane traveling, as well as, for plane standing waves. The investigation is motivated by the fact that neglecting compressibility in the study of acoustic streaming near a rigid boundary lead to an incomplete description of the streaming velocity field both inside and outside the boundary layer. It is shown that an improved description may be obtained by including the effect of compressibility in the analysis of flow field in the viscous-acoustic boundary layer. The resulting acoustical streaming velocity outside the boundary layer is shown to depend not only on the amplitude of the primary wave, but also on the Prandtl number and the ratio of the specific heat of the fluid medium. Calculations based on typical numerical data suggest that the streaming velocity outside the boundary layer is larger than the classical results obtained with the incompressible fluid assumption. Relevance to the on-going study of ultrasonic cleaning will also be briefly mentioned. [Work supported by the Hunt Fellowship.]

THURSDAY MORNING, 20 MAY 1993

CANADIAN ROOM, 8:00 TO 11:45 A.M.

\title{
Session 4aPP
}

\author{
Psychological and Physiological Acoustics: Signal Detection, Loudness, Noise \\ Robert D. Sorkin, Chair \\ Department of Psychology, University of Florida, Gainesville, Florida 32611
}

\section{Contributed Papers}

\section{8:00}

4aPP1. Comparison of adaptive procedures for obtaining psychophysical thresholds using computer simulation. Monica $L$. Hawley and H. Steven Colburn (Dept. of Biomed. Eng., Boston Univ., 44 Cummington St., Boston, MA 02215)

In order to compare the efficacy and efficiency of various adaptive psychophysical procedures, a program that simulates these procedures has been developed and used to compare several adaptive methods for three experiments: binaural detection, interaural intensity discrimination, and interaural time discrimination. The simulation program takes as input a psychometric function, which specifies the probability of a correct response for any value of the adapting variable. A threshold is generated by choosing the next value of the variable according to the rules of the procedure being simulated, including the rules for stopping and calculating the threshold estimate. The software generates distributions of thresholds and statistical analyses are performed, such as bias and variance of the obtained distribution. The program has been applied to psychometric functions appropriate for normal-hearing and hearingimpaired listeners for each of the three experiments noted above, and has compared a number of adaptive procedures including the Transformed Up-Down (such as 2-down-1-up), PEST, BUDTIF, and Weighted Up-Down methods. [Work supported by NIDCD and Dept. of Education.]

8:15

4aPP2. A threshold estimate in a yes-no task using a maximum likelihood procedure. David M. Green (Psychoacoust. Lab., Psychol. Dept., Univ. of Florida, Gainesville, FL 3261 1-2065)

A maximum-likelihood procedure for estimating threshold in a yes-no task is presented. The absolute thresholds at six audiometric frequencies for a group of normal-hearing listeners were measured using this procedure. The average standard deviation of the threshold estimate is less than $5 \mathrm{~dB}$ with only 12 trials per threshold estimate. The total time taken to measure the audiogram was less than $3 \mathrm{~min}$. The estimate of false-alarm rate is poor and strongly biased, but can be considerably improved by a simple modification that increases the testing time about 25\%. [Research supported by National Science Foundation and National Institutes of Health.]

\section{$8: 30$}

4aPP3. Psychoacoustic models of group signal detection. R. D. Sorkin and Huanping Dai (Dept. of Psychol., Univ. of Florida, Gainesville, FL 32611)

A number of auditory scientists have proposed models for the multichannel signal detection task: an observer must detect a signal that has components in several partially correlated channels (spectral, spatial, or temporal). This task has been reframed as a group detection problem: an array of observers must detect a signal by combining the observations of the individual detectors. It is shown how the performance of the ideal group detection system depends on the number of detectors, the correlation among detector observations, and the distribution of individual sensitivities. Also considered are some cases where the group decision is based on the binary outputs of the individual detectors. The results are applicable to a variety of different types of group detection systems. [Work supported by AFOSR.]

$$
8: 45
$$

4aPP4. Detection of unexpected tones in noise: The effect of signal duration. Beverly A. Wright and Huanping Dai (Psychoacoust. Lab., Dept. of Psychol., Univ. of Florida, Gainesville, FL 32611)

The effect of signal duration on the detectability of unexpected tones masked by a continuous wideband noise was assessed using a probesignal method. This method encourages the listener to attend to a target frequency by presenting the signal most often at that frequency, and only occasionally at other unexpected probe frequencies. The attention contour (percent correct as a function of probe frequency) was considerably broader with 5 -ms than with 295 -ms signals. However, auditory filter shapes measured using the notched-noise technique were very sim- 
ilar for those two signal durations, indicating that the results obtained in the attention experiments do not simply reflect peripheral frequency selectivity. Further supporting this interpretation, probe tones having the same frequency but a different duration from the target were poorly detected. It is proposed that the subject listens through a time-frequency window whose location and shape in the time-frequency plane is determined by the duration and frequency of the target. [Work supported by the NIDCD and NIH.]

\section{9:00}

4aPP5. Distribution discrimination of signal duration. Daniel $L$. Weber and Stephen P. Sanger (Dept. of Psychol., Wright State Univ., Dayton, $\mathrm{OH} 45435$ )

This experiment examined a listener's ability to judge signal duration in the context of a 2IFC, distribution discrimination procedure. A "standard" distribution for the duration of a $1-\mathrm{kHz}$ sinusoid had a mean of $10,30,100,200$, or $500 \mathrm{~ms}$. Comparison distributions had means $1.05,1.1,1.2$, and 1.4 times that of the standard. Both were normal distributions with standard deviations equal to the difference in the means. (All conditions thus yield a $d^{\prime}$ of 1.0 for an ideal observer making a single observation. Obtained $d$ 's vary as the separation of the means changes with respect to the duration jnd.) In each observation interval, listeners were presented with $1,2,3,4,5,6,8,12$, or 16 different samples (a fixed number within a block of trials) from one of the two distributions and had to identify which interval was drawn from the comparison (longer mean) distribution. The observed increase in $d^{\prime}$ with up to six presentations is well described by the observationintegration model [D. M. Green, J. Acoust. Soc. Am. 30, 904-911 (1958)] in all conditions. Our listeners generally fail to show improvement beyond 8 presentations, so the partitioned-variance model [D. E. Robinson and B. G. Berg (1986)] described by Lutfi [R. A. Lutfi, J. Acoust. Soc. Am. 86, 934-944 (1989)] provides a better fit to the entire set of data. [This research was supported by a grant from AFOSR through WPAFB AL/CFBA.]

\section{9:15}

4aPP6. Pure-tone thresholds of 1-month-old infants. Lynne A. Werner and Lisa Rickard Mancl (Dept. Speech and Hear. Sci., Univ. of Washington, WJ-10, Seattle, WA 98195)

Thresholds for the detection of tones at $0.5,1$, and $4 \mathrm{kHz}$ were estimated for 1-month-old infants using insert earphones. On each trial, the infant was presented with either 8 repetitions of a tone burst or with no sound for an 8-s period.Based on the infant's response, an observer, blind to trial type, gave a confidence rating at the end of each trial. Correct infant responses were reinforced. The level and frequency of the tone were fixed within a session; several sessions, at different levels, were completed for each subject. The confidence ratings were used to calculate $p(A)$. Performance for a session was taken as the highest $p(A)$ achieved in a block of 8 trials. Thresholds were calculated as the level at which $p(A)=0.83$, where 0.83 just exceeds chance for 8 trials. Thresholds were calculated for infants who achieved $p(A)>0.83$ in at least one session and who had monotonic psychometric functions. The results were in close agreement with previous studies and suggest that I-monthold infants are more sensitive at lower frequencies. The current procedure uses fewer trials to estimate a threshold and allows thresholds to be estimated for a higher proportion of the infants tested. [Work supported by NIH.]

\section{9:30}

4aPP7. The internal signal-to-noise ratio in hearing-impaired listeners. Mariorie R. Leek, W. Van Summers, and Mary T. Cord (Army Audiol. \& Speech Ctr., Walter Reed Army Med. Ctr., Washington, DC 20307-5001)

The reduced frequency resolution often demonstrated by listeners with sensorineural hearing impairment might have at least two related effects on their perception of speech in background noise. The internal representation of the peaks and valleys in the speech spectrum become smeared, resulting in less precise frequency analysis than can be achieved by normal-hearing persons. In addition, when the speech is embedded in noise, poorer frequency resolution will, in theory, result in a greater-than-normal decrease in the signal-to-noise ratio at the outputs of the auditory filters. This poorer-than-normal internal $\mathrm{S} / \mathrm{N}$ might account for the common observation that noise is more degrading to speech understanding by hearing-impaired listeners than by normalhearing listeners. To assess the validity of this notion, both normalhearing and hearing-impaired subjects participated in an identification experiment using highly simplified vowel-like harmonic complexes presented in quiet and in three levels of broadband noise. After equating individual performance in terms of percent correct identification in quiet, trading relationships were established between amount of spectral contrast in the stimuli (level differences between peaks and valleys in the spectra) and levels of the background noise. When the effect of spectral smearing introduced by reduced frequency analysis was compensated by increasing the input spectral contrast, the effects of additional background noise were similar for normal-hearing and hearingimpaired groups of subjects. Results from individual listeners will be discussed in light of the degree of impaired frequency resolution as determined by auditory filter shape measurements. [Work supported by NIH.]

\section{9:45}

4aPP8. The growth of loudness of brief sounds. Mary Florentine (Commun. Res. Lab., Dept. of Speech-Language Pathol. and Audiol., 133FR, Northeastern Univ., Boston, MA 02115), Soren Buus (Northeastern Univ., Boston, MA 02115), and Torben Poulsen (Tech. Univ. of Denmark, DK-2800 Lyngby, Denmark)

The purpose of this paper is to review the literature on temporal integration of loudness and to derive the form of the loudness function for brief stimuli from existing data. The review of 19 studies reveals that the level difference between equally loud sounds of different durations varies with overall level. The variation is orderly across durations. For $1-\mathbf{k H z}$ tones, the level differences between equally loud 5- and $200-\mathrm{ms}$ stimuli appears to vary nonmonotonically with level. The available data indicate that it ranges from about $17 \mathrm{~dB}$ at low sensation levels to about $10 \mathrm{~dB}$ around $95 \mathrm{~dB}$ SL. For complex sounds, the level difference between equally loud 5- and 200-ms stimuli appears to decrease linearly from about $17 \mathrm{~dB}$ at low sensation levels to about $10 \mathrm{~dB}$ around $80 \mathrm{~dB}$ SL. The loudness functions derived for 5-ms stimuli are steeper than those for 200 -ms stimuli. For the $1-\mathbf{k H z}$ tones, the exponent of the best-fitting power function increases from 0.30 for 200 -ms tones to 0.33 for 5-ms tones. [Work supported by NIH-NIDCD and The Technical University of Denmark.]

\section{0:00-10:15 Break}

\section{0:15}

4aPP9. Predicting intensity discrimination from power-law modifications of the loudness function. William S. Hellman (Dept. of Phys., Boston Univ., Boston, MA 02215) and Rhona P. Hellman (Dept. of Psychol., Northeastern Univ., Boston, MA 02115)

Several modifications of Stevens' power law have been introduced to account for the steepening of the loudness function below about $40 \mathrm{~dB}$ SPL. The relation between intensity discrimination $(\Delta I / I)$ and loudness is determined for three proposed modifications: (a) $L_{\mathrm{s}}=K(I$ $\left.-I_{0}\right)^{n}$, (b) $L_{s}=K\left(I^{n}-I_{0}^{n}\right)$, and (c) $L_{s}=k\left(\left[I+c I_{0}\right]^{n}-\left[c I_{0}\right]^{n}\right)$, where $I_{0}$ is the sound intensity at threshold. Below 15 dB SPL Eqs. (a) and (b) gencrale a rapidly decreasing function for intensity discrimination, whereas the function derived from Eq. (c) rises with a slope of -1 in $\log$ - $\log$ coordinates. The slope of the measured intensity-discrimination function is compatible with results predicted for pure tones from $\mathbf{E q}$. (c) but not with those predicted from Eqs. (a) and (b). [Work supported in part by NIH.] 


\section{0:30}

4aPP10. Loudness adaptation for high-frequency tones. Andrzej Miskiewicz, Rhona P. Hellman, Carol H. Meiselman, and Bertram Scharf (Northeastern Univ., 360 Huntington Ave., Boston, MA 02115)

Loudness adaptation was measured for pure tones at $4,12,14,16$, and $17 \mathrm{kHz}$. Loudness adaptation refers to the decline in the loudness of a continuous sound as a function of exposure time. More than 100 students judged - by the method of successive magnitude estimationthe loudness of the tones over a 6-min exposure time. Earlier investigations for frequencies up to $4 \mathrm{kHz}$ had shown that loudness adapts only at sensation levels up to approximately $30 \mathrm{~dB}$ [B. Scharf, Hear. Res. Theory, 1-56 (1983)]. The current measurements show that at $12 \mathrm{kHz}$ and above, loudness adapts at sensation levels above $30 \mathrm{~dB}$ as well as below. At high frequencies, loudness adaptation becomes greater as frequency increases up to $16 \mathrm{kHz}$; at $17 \mathrm{kHz}$ adaptation is nearly the same as at $16 \mathrm{kHz}$. The marked loudness adaptation of steady tones at very high frequencies and relatively high sensation levels is ascribed to a restricted spread of excitation in the auditory system resulting from the steep rise of the threshold curve at the upper bound of hearing. [Work supported by NIH.]

\section{0:45}

4aPP11. Loudness adaptation in listeners with high frequency impairment. Jennifer L. Janson, Emest M. Weiler, David E. Sandman, Yea-Wen Shiau (ML \#379, Dept. of Commun. Sci. \& Disord., Univ. of Cincinnati, Cincinnati, $\mathrm{OH}$ 45221), Todd Nelson, Joel Warm (University of Cincinnati), and Eleanor Stromberg (Cincinnati Speech \& Hear. Ctr., Cincinnati, $\mathrm{OH}$ )

High-frequency impairment was found to have a significant effect on ipsilateral loudness magnitude adaptation in complex interactions [Korman et al, J. Acoust. Soc. Am. Suppl. 1 81, S76 (1987)]. The present study used simple comparisons of 12 listeners with high-frequency hearing loss, age matched with 12 having no loss. All were tested at $1 \mathrm{kHz}$ for adaptation at 40 and $60 \mathrm{~dB}$ HTL. [See Weiler et al., Brit. J. Audiol. $15,204-205$ (1981).] The hearing loss group showed significantly more adaptation at $40 \mathrm{~dB}$ than at 60 , while normals showed the same amount. Based on the two studies, it appears that high-frequency hearing loss does have some effect on the loudness function at $1 \mathrm{kHz}$ where hearing appears to be normal.

\section{1:00}

4aPP12. Equal sensation functions in bilateral sensorineural-impaired hearing. Rhona P. Hellman and Carol H. Meiselman (Auditory Perception Lab., Northeastem Univ., 360 Huntington Ave., Boston, MA 02115)

Equal sensation functions were generated for individuals and groups with hearing losses ranging from 39 to $73 \mathrm{~dB}$ at the stimulus frequency. The functions were obtained directly from conventional loudness matching, and indirectly from magnitude estimation, magnitude production, and cross-modality matching. The results of the direct and indirect loudness-level determinations lie within a relatively narrow band. On the average, the intrasubject variability across procedures ranges from 4 to $6 \mathrm{~dB}$. The corresponding intersubject variability has a mean value of $4.6 \mathrm{~dB}$. Moreover, cross-modality matches obtained for 36 listeners exhibit temporal stability for intersession intervals as long as 5 years. Loudness functions constructed from the data are better described by Zwislocki's [Handbook of Mathematical Psychology, 3-97 (1965)] proposed loudness equation than by either of the more widely applied power-law modifications. [Work supported by the Rehab R\&D Service of the VA and by NIH.]

\section{1:15}

4aPP13. Enhancement of cochlear signal cell and $\mathrm{CM}$ responses after exposure to an intense tone or noise. Bradley W. Pietras and Jozef J. Zwislocki (Inst. for Sensory Res., Syracuse Univ., Syracuse, NY 13244-5290)

The phenomenon of decreased cochlear sensitivity after exposure to intense noise is well known. It predominantly affects low levels of stimulation and, when not too severe, it usually exhibits sensitivity recruitment at higher levels. By contrast, in Mongolian gerbils, enhanced receptor potentials of outer hair cells have been found, as studied through intracellular recording from Hensen's cells. According to previous studies, alternating potentials of the latter faithfully reflect those of the former. The enhancement affected mainly high levels of stimulation, and was practically absent in low level responses. It did not seem to affect the shapes of Hensen's cells' transfer functions. It was present at the cochlear location of $2 \mathbf{k H z}$ BF after exposure to either a $1-\mathrm{kHz}$ tone or a broadband noise, both at $100 \mathrm{~dB}$ SPL. It increased over exposure durations as long as 60 to $90 \mathrm{~min}$ and decreased slowly after exposure termination. The enhancernent did not appear to be correlated with fluctuations in Hensen's cell resting potentials or the endolymphatic potential. It was weak when measured in CM. [Work supported by NIDCD.]

\section{1:30}

4aPP14. Changes in distributions of fodrin and F-actin in the cochlea after noise exposure as revealed by confocal microscopy. Vlasta Spongr, Giuseppe Attanasio, and Donald Henderson (Hear. Res. Labs., State Univ. of New York at Buffalo, 215 Parker Hall, Buffalo, NY 14214)

Changes in distributions of two cytoskeleton proteins, $240 \mathrm{kD}$ alpha fodrin and filamentous actin, were studied in chinchillas exposed to 125 dB SPL impact noise and sacrificed immediately or $24 \mathrm{~h}$ after the exposure. To preserve the physiological integrity of the organ of Corti as much as possible, the surface preparations were studied using confocal fluorescence microscopy by serial, noninvasive optical sectioning. Immediately after noise exposure the damaged OHCs showed staining at the cuticular plates for both proteins, which varied in intensity. However, the lateral surfaces of the hair cells were not labeled. After $24 \mathrm{~h}$, scars have replaced the missing cells and intense labeling of F-actin was seen in the Deiter's cells bodies and phalangeal processes. The results will be discussed in terms of role of fodrin and F-actin in the pathology induced by noise exposure. [Work supported by NIDCD 1R01DC01237-O1A1.] 


\title{
Session 4aSA
}

\section{Structural Acoustics and Vibration: Structural Intensity}

\author{
Joseph M. Cuschieri, Chair \\ Department of Ocean Acoustics, Florida Atlantic University, Center for Acoustics and Vibration, Boca Raton, \\ Florida 33431
}

Chair's Introduction-8:30

Invited Papers

8:35

4aSA1. Power flow analysis for structural finite element models. Stephen A. Hambric (Comput. Mech. Div., Code 1282, Carderock Div., NSWC, Bethesda, MD 20084-5000)

Research on calculating mechanical power flow quantities (steady-state structural intensities and power dissipation) in finite element models from dynamic forces and velocities output by the NASTRAN computer program is summarized. Mechanical power formulations for the axial, torsional, and bending motion of beams and the membrane and bending motion of plates are presented. The formulations have been implemented in the program McPOW (Mechanical POWer) [S. A. Hambric, J. Vib. Acoust. 112, 542-549 (1990)], which functions as a post-processor to NASTRAN. Results are shown from a study comparing experimental and finite element flexural power measurements for a point excited straight beam with compliant end mounts. The good agreement of the experimental and numerical power spectra serve to validate the finite element mechanical power method. Also, animations of the power flow response of a cantilever plate model over a frequency range encompassing several low- to mid-order flexural and membrane modes were performed on graphics computers and recorded on videotape, and will be presented to show the benefit of visualization techniques in understanding mechanical power analyses.

\section{9:05}

4aSA2. A modal power balance approximation for point connected flexural beams. Peter Kasper, Paul Bremner, and Paris Constantine (Vibro-Acoustic Sci. Lid., Level 5, 15 Orion Rd., Lane Cove, NSW 2066, Australia)

In recent years, the application of power flow techniques to the analysis of beams and plates has led to an increased understanding of how noise is transmitted through structures. When applied in terms of mode-to-mode transmission, power flow evaluation has promise in modeling dynamic response at frequencies for which statistical energy analysis is not appropriate. Modal power flow calculations can, however, be computationally prohibitive. This paper introduces a modal power balance approach by which the computational effort can be reduced. The approach involves formulating the mode-to-mode power exchanged between two beams in terms of a set of modal power balance equations. These equations are analogous to the sub-system power balance equations of statistical energy analysis. By introducing suitable approximations, the equations can be integrated over frequency and length to express power flow relationships in terms of band-limited, modal mean-square values. The approach is applied to the configuration of two force and moment coupled flexural beams. The results are compared with a classical modal solution.

4aSA3. Identification of structural power flow for multiple correlated sources. Peter Wagstaff, Mei Jin (Univ. of Compiègne, B.P. 649, 60206 Compiègne, France), and Boudjema Bouizem (Acovib. Sarl. Centre de Transfert, Compiègne, France)

Structural intensity measurements may be used to identify the direction of propagation of vibrations in single and multiple source situations. In the latter case standard techniques do not permit the individual contribution of each source to be identified. Previous publications have presented the results of experiments carried out at Compiègne using selective intensity techniques which are based on multiple input multiple output models for partially correlated inputs. These techniques break down when the inputs are highly correlated. In order to solve this problem and to simplify the measurement of the reference signals for the inputs a method has been developed to estimate the structural intensity due to each source based on the use of the frequency response function matrix associated with specific points on the structure. The inputs to the structure are initially identified using a pseudo-inverse of the measured data and the intensity due to each input may then be estimated using the original data and eliminating the terms due to the effects of the other inputs. Results are presented for a simple structure with two inputs.

\section{0:05-10:20 Break}

\section{$10 ; 20$}

4aSA4. Laser vibrometer methods for measuring far-fleld structural intensity. Timothy E. McDevitt (Appl. Res. Lab., Penn State Univ., P. O. Box 30, State College, PA 16804)

There is a need to develop measurement techniques that can quickly and accurately provide structural intensity data over an entire surface without interfering with the structural response. To this end, methods have been developed to measure the flexural 
intensity in a reverberant beam by using scanning laser vibrometry; kinetics and standing wave methods have been employed with a single scanning vibrometer while a finite difference method has been employed with a two-channel scanning vibrometer. Experimental results for the first two methods, which each provide a single value of intensity on the beam, are compared with experimental results for the finite difference method, which was used to provide values of intensity at various positions along the beam. Similarly, experimental data on intensity in longitudinal waves along a rod are also presented for the vibrometer(s) set in the differential Doppler mode. Results indicate that flexural intensity is measured very consistently by the three methods and that longitudinal intensity measurements are feasible if the longitudinal motions, induced by bending waves, are taken into account. [Work supported by ARL Penn State.]

10:50

4aSA5. Active vibration control using structural intensity. Scott D. Sommerfeldt (Appl. Res. Lab. and Grad. Prog. in Acoust., Penn State Univ., P. O. Box 30, State College, PA 16804), Sabih I. Hayek, and Allan E. Schwenk (Penn State Univ., State College, PA)

The subject of structural intensity has been investigated by a number of researchers over the last few years, as a means of gaining an increased understanding of the energy in the structural field. In the area of active control, structural intensity is also of interest for applications that involve energy propagation in the structure. For such cases, controlling the structural intensity, as opposed to the structural vibration, has the potential of providing more effective control of the energy in the structural field. Initial results obtained by implementing an active control system based on structural intensity are presented. The results indicate the potential of obtaining improved control of the structural field, particularly for the case where the sensors are positioned in the near field of the actuators. Some of the issues involved with implementing a control system based on structural intensity are also discussed.

\title{
Contributed Paper
}

11:20

4aSA6. Vibro-acoustlc power flow in plate-fluid layer systems. Taner Önsay (Dept. of Mech. Eng., Michigan State Univ., East Lansing, MI 48824)

Attenuation of flexural vibrations by viscous compressible fluid layers is studied by using a power flow analysis. A fluid layer trapped between vibrating surfaces may increase low-frequency damping and cause significant changes in the dynamic behavior of a coupled plate. A prototype system consisting of a clamped plate and a coupled finite air layer is analyzed by using a transfer matrix method. Spectral and spatial distributions of power flow are obtained at different layer thicknesses. Particular attention is given to various dynamic regimes of the fluid layer. Significance of the viscous shear forces and compressibility during the dissipative-to-stiffness transition of the fluid layer are emphasized. At very small layer thicknesses, the increased flow resistance caused by viscous shear forces resulted in local stiffening of the fluid layer which blocked the transmission of the vibrational power. Contributions from three different power flow components, shear, bending and acoustic, are discussed in reference to the dynamic interactions of flexural waves with the fluid layer. The results obtained for different system configurations are briefly reviewed.

THURSDAY MORNING, 20 MAY 1993

BALLROOM, 8:00 A.M. TO 12:00 NOON

\section{Session 4aSP}

\section{Speech Communication: Developmental and Nonphonetic Factors in Speech Communication}

\author{
Peter C. Gordon, Chair \\ Department of Psychology, Harvard University, 33 Kirkland Street, Cambridge, Massachusetts 02138
}

\section{Contributed Papers}

8:00

4aSP1. Speech perception as a talker-contingent process. Lynne $C$. Nygaard, Mitchell S. Sommers, and David B. Pisoni (Speech Res. Lab., Dept. of Psychol., Indiana Univ., Bloomington, IN 47405)

The present study reports an experiment designed to investigate the nature of perceptual adaptation and memory representation for spoken words produced by familiar and unfamiliar talkers. To determine how familiarity with a talker's voice affects perception of spoken words, two groups of subjects were trained to recognize the names of ten voices (five male; five female) over a 9-day training period. One group of subjects then identified words presented at four signal-to-noise ratios that were produced by the same set of talkers that they had learned to recognize during training. Control subjects identified the same words at the same signal-to-noise ratios but the words were produced by a set of new talkers that these subjects had not heard during training. The results showed that the ability to explicitly identify a talker's voice im- proved intelligibility of novel words produced by the same talkers. Subjects who heard familiar voices in the word intelligibility task were better at identifying novel words in noise than control subjects who heard unfamiliar voices. The results suggest that speech perception may be a talker-contingent process whereby familiarity with aspects of the talker's vocal source facilitates the subsequent phonetic analysis of the acoustic signal. [Work supported by NIH grant to Indiana University.]

\section{$8: 15$}

4aSP2. The effects of speaking rate and stimulus variability on the perception of spoken words by young and elderly subjects. Mitchell S. Sommers and Larry E. Humes (Depts. of Psychol. and Speech and Hear. Sci., Indiana Univ., Bloomington, IN 47405)

This study cornpared spoken word recognition in young and elderly normal-hearing listeners as a function of speaking rate and stimulus 
variability. Monosyllabic words were presented in quiet at one of three speaking rates (fast, medium, or slow). In one condition, all stimulus items were presented at the same speaking rate while in a second condition speaking rate was varied from trial to trial. In control conditions, items were presented at either fixed or varying overall amplitudes. For young listeners, spoken word identification did not differ as a function of speaking rate. Older subjects, in contrast, demonstrated similar identification scores for slow- and medium-rate items but had significantly reduced identification performance for words presented at fast speaking rates. Identification accuracy was also reduced for older listeners in the mixed-as opposed to single-rate condition but only for those items presented at the fast speaking rate. Young listeners were not affected by variability due to speaking rate and neither group showed reduced identification as a result of variability in overall amplitude. Implications for speech perception in older listeners are discussed in terms of current views about perceptual normalization for different sources of variability. [Work supported by NIH.]

\section{8:30}

4aSP3. Talker differences and perceptual normalization. James $S$. Magnuson and Howard C. Nusbaum (Dept. of Psychol., Univ. of Chicago, 5848 S. University Ave., Chicago, IL 60637)

Recognition performance for speech is generally worse for utterances produced by a mix of several talkers compared to utterances produced by a single talker. This performance impairment can be attributed to those aspects of talker normalization used to determine the vocal characteristics of the talker each time the talker changes. The present study investigated the size and nature of talker differences that may affect normalization. Spoken words were generated by a text-tospeech system for matched pairs of synthetically defined talkers. All but two of these pairs differed only in average fundamental frequency. One remaining pair of talkers differed in perceived gender but both talkers had the same average pitch; the other pair differed in both gender and pitch. Response times in a speeded word recognition task were compared for blocks of stimuli produced by a single talker and blocks of stimuli produced by a mix of one of the pairs of talkers. The results are important for understanding how listeners use pitch differences between talkers during talker normalization.

\section{$8: 45$}

4aSP4. Attentional effects on phonetic encoding of acoustic cues. Peter C. Gordon (Dept. of Psychol., Harvard Univ., 33 Kirkland St., Cambridge, MA 02138) and Elizabeth J. Pyatt (Harvard Univ., Cambridge, MA 02138)

The role of attention in the phonetic encoding of acoustic cues was studied by examining the effect of a nonspeech distractor task on listeners' classification of the speech sounds $/ \mathrm{ra} /$ and $/ \mathrm{a} /$. Consistent with previous results [Gordon et al., Cog. Psychol. 25 (1993)] it was found that the impact of a relatively strong cue ( $F 3$ transition) on phonetic classification was reduced when listeners could not devote full attention to the speech sound. In contrast with previous results, the impact of a relatively weak cue (an aperiodic burst during the formant transition) was also reduced when full attention was not possible. This result conflicts with models in which the importance of attention in phonetic encoding is greater for acoustic cues whose phonetic importance is very great. It suggests that the role of attention in phonetic encoding depends on specific characteristics of acoustic cues.

\section{9:00}

4aSP5. Changing the deployment of attention to phonetic structure. Alison K. Baldwin and Howard C. Nusbaum (Dept. of Psychol., Univ. of Chicago, 5848 S. University Ave., Chicago, IL 60637)

Previous research on perceptual learning of synthetic speech and non-native phonetic contrasts suggests that listeners shift the focus of their attention from one set of acoustic cues to another, although this hypothesis has never been tested directly. The present study was carried out to determine whether training could alter perception of stopconsonant place of articulation of shifting attention from a transition cue to the spectral shape of the release burst. CV syllables were synthesized with transitions specifying one place of articulation and release burst shape specifying another. One group of subjects was given feedback consistent with the category specified by the transitions; the other group was given feedback consistent with the bursts. Although subjects initially tended to respond based on transition cues, feedback was effective for both groups. Furthermore, for subjects who showed learning, attentional changes also generalized to the same phonetic categories in new vowel contexts. The implications of these results for theories of speech perception and perceptual learning will be discussed.

\section{9:15}

4aSP6. The abstractness of lexical representations. James $T$. Myers (Lang. Percept. Lab., Dept. of Psychol., SUNY at Buffalo, Buffalo, NY 14260)

This study attempted to address a fundamental question concerning lexical representations, namely, whether they are encoded in an abstract phonemic form, where only information that is lexically distinctive is recorded, or more concretely. To this end a same-difference task was given to native speakers of American English. Subjects found that the allophone of $/ \mathrm{t} /$ that occurs before $/ \mathrm{r} /$, as in "truck," more similar to the distinct phoneme found in "chuck" than to the allophone of $/ t /$ found in "tuck." By contrast, subjects found the allophone of $/ t /$ that occurs after $/ \mathrm{s} /$, as in "stuck," more similar to the allophone of $/ \mathrm{t} /$ that occurs in "tuck" than the acoustically similar but distinct phoneme found in "duck." It is hypothesized that while the second segment of words like "stuck" is truly an allophone of $/ t /$, the first segment of words like "truck" is in fact the same phoneme as in "chuck." The forms being compared are thus represented phonemically in both cases. [Work supported by NIDCD.]

\section{9:30}

4aSP7. On the perceptual integrality of duration and amplitude cues to stress. Alice Turk and James R. Sawusch (Dept. of Psychol., Park Hall, SUNY at Buffalo, Buffalo, NY 14260)

Traditionally, amplitude, duration and $F 0$ have been proposed as independent cues to stress [D. Fry, J. Acoust. Soc. Am. 27, 765-768 (1955)]. However, there is psychoacoustic evidence that the perception of loudness depends on duration [B. Scharf, "Loudness," in Handbook of Perception, edited by E. C. Carterette and M. P. Friedman (Academic, New York, 1978), Vol. 4, pp. 187-242]. Beckman proposed the intensity integral as a more appropriate cue to stress than either amplitude or duration alone [M. Beckman, Stress and Non-stress Accent (Foris, 1986)]. The present study tested the perceptual integrality of duration and amplitude using Garner's paradigm [W. R. Garner, The Processing of Information and Structure (Erlbaum, Hillsdale, NJ, 1974)]. Preliminary results suggest that duration and amplitude are integral. Further, the integrality is asymmetric: Variation in duration interfered with responding to amplitude to a greater extent than amplitude variation interfered with responding to duration. These findings suggest that both duration and energy (the integral of amplitude over duration) may serve as auditory cues to stress. [Work supported by NIDCD Grant Nos. R01-DC00291 and T32-DC00036 to SUNY at Buffalo.]

\section{9:45}

4aSP8. The distinctiveness of word onsets. David W. Gow, Jr., and Peter C. Gordon (Dept. of Psychol., Harvard Univ., Cambridge, MA 02138)

Acoustic measurements examined the distinctiveness and salience of word onsets and matched syllables that occurred in non-word-initial position. Three naive speakers rehearsed and read phonemically identical one- and two-word sequences such as "cartel" and "car tell" under three conditions: citation form, read slowly in sentenial context, or read quickly in sentenial context. Analyses examining the timing of segments 
and syllables, and the degree of vowel reduction under each condition showed that word-initial syllables were longer, and showed less vowel reduction than their non-word-initial counterparts. These results suggest that word onsets differ acoustically from nonword onsets in a manner that may enhance their perceptual salience. These results are discussed in relationship to the Good Start model of lexical access and lexical segmentation. [Work supported by AFOSR.]

\section{0:00-10:15 Break}

\section{0:15}

4aSP9. Perceptual identification of phonologically flapped words. Jan Charles-Luce, Elvira Ragonese, and Betina Lewin (Dept. of Commun. Disord. and Sci., SUNY at Buffalo, Buffalo, NY 14260)

Native English speakers originally produced bisyllabic words containing intervocalic alveolar stops, e.g., writer and rider. These words were embedded in both semantically neutral and semantically biasing passages. In addition, both neutral and biasing passages were produced in two conditions. In one condition, a listener was present in the recording room with the speaker. In the other condition, the speaker was alone in the recording room. The production results showed that flapping did not occur in the listener-present condition but that it did in the listener-absent condition. The present investigation examined whether these production results are functional for the listener. All production tokens were excised from the original passages and presented to subjects auditorily for identification. Subjects were presented with pairs of stimuli, e.g., writer and rider. On each trial subjects heard one member of the pair. They were to circle the word that they thought they heard in a response booklet. Percent correct identification results show that subjects were best at correctly identifying the tokens when they had been produced originally in the listener-present, neutral passage and worst at identification in the listener-absent, bias passage. [Work supported by NIH.]

\section{0:30}

4aSP10. Further evidence for activation of sublexical units in spoken word recognition. Emily A. Lyons and Paul A. Luce (Lang. Percept. Lab., Dept. of Psychol., SUNY at Buffalo, Buffalo, NY 14260)

At the previous meeting of the Society, data from an auditory lexical decision experiment were presented that demonstrated that subjects are faster to respond to bisyllabic words in which the first syllable is a word (e.g., KIDNEY) than to bisyllabic words in which the first syllable is a nonword (e.g., CHIMNEY). These findings suggest that the activation of component lexical items embedded in longer words has demonstrable effects on recognition time. Data from a single word shadowing experiment will be presented that replicate the previous findings from auditory lexical decision. These results provide further evidence for activation of sublexical units in spoken word recognition and demonstrate that sublexical activation has facilatory-not inhibitory-effects on recognition time. [Work supported by NIH Grant No. DC-00879-01 to the State Univ. of New York at Buffalo.]

\section{0:45}

4aSP11. The relation between age of $\mathrm{L2}$ learning (AOL) and degree of perceived global foreign accent. James E. Flege, Murray J. Munro (Dept of Biocommun., VH 503, Univ. of Alabama at Birmingham, Birmingham, AL 35294), and Ian R. A. MacKay (Univ. of Ottawa, Ottawa, ON K1N 6N5, Canada)

Degree of accent was assessed in five English sentences spoken by 243 Italians who immigrated to Canada between the ages of two and 22 years (an average of $12 \mathrm{Ss}$ in each of $21 \mathrm{AOL}$ subgroups). The Ss' average age was 44 years (range: 25-56); their average length of residence in Canada was 31 years (range: 14-44). All Ss responded to a language-background questionnaire (LBQ) that provided information regarding age or arrival and length of residence in Canada, frequency of $L 1$ and L2 use, self-estimated L1 and L2 pronunciation and comprehension abilities, strength of concern for pronunciation, imitation/ musical ability, and motivation to learn English. The sentences were rated for degree of accent using our standard technique by ten native English Canadians. The results are presented of a multiple regression analyses relating the various $L B Q$ variables to the foreign accent scores, and report on tests of two hypotheses: (1) foreign accents first emerge at an AOL of about 7 years; and (2) intra-subject and inter-subject variability in $\mathbf{L 2}$ pronunciation accuracy increases systematically as a function of AOL beyond the age at which foreign accents first emerge. [Work supported by NIH.]

\section{1:00}

4aSP12. Age-related differences in the discrimination of two-formant transitions. Robert Allen Fox, Fauzia Chaudry, and Lida Wall (Div. of Speech and Hear. Sci., Ohio State Univ., 110 Pressey Hall, 1070 Carmack Rd., Columbus, OH 43210-1002)

In the past 3 years age-related differences in the ability to process rapidly changing acoustic information (e.g., formant transitions) in making phonetic decisions (e.g., identification of consonants and/or vowels) have been investigated. The data obtained in these studies have been consistent with the claim that older adults as well as children show a decreased ability to process dynamic acoustic information, compared to that of young (college-aged) adults. The present study examines age-related differences in the discrimination of several synthesized twoformant transition and steady-state continua. Two continua represented a [w1]-[wz]-[wx] continuum (differing in terms of the duration of the transition) and the last set of stimuli represented a [ba]-[da] continuum. Three groups of listeners (children 9-12 yrs, young adults 19-25 yrs, and older adults 58-71 yrs) were required to discriminate between two stimulus tokens using a version of Levitt's up-down adaptive procedure. Significant differences were found between the age groups with the lowest jnd's found in the young adults. However, phonological considerations (e.g., changing location of phoneme boundaries across stimulus types) also had a significant effect on jnd's. These data will be discussed with regard to possible explanations of developmental differences (in the case of children) and aging differences (in the case of older adults). [Work supported, in part, by a grant from the National Institute on Aging No. SR01 AG08353-04.]

\section{1:15}

4aSP13. Sonority contrasts dominate young infants' vowel perception. Francisco Lacerda (Inst. of Linguist., Stockholm Univ., S-106 91 Stockholm, Sweden)

Three groups of normal Swedish infants were tested on their ability to discriminate equal (in Bark) sonority (high/low) and chromacity (front/back) vowel contrasts. The infants were $2-3,6-7$, and 10-11 months old, living in monolingual Swedish environments. The 2-3 month olds were tested using the high-amplitude sucking technique. The older infants were tested with the head-turn procedure. One of the vowel pairs provided a $[a] /[\wedge]$ sonority contrast, that was conveyed only by a 1.8-Bark difference in $F 1$. The other pair was a [a]/[a] chromacity contrast, in which the vowels differed also by 1.8 Bark but in this case along $F 2$. In spite of the procedural differences, the results show that the three groups of subjects could detect the sonority contrast but that no reliable discrimination could be inferred for the corresponding chromaticity contrast. [Work supported by The Bank of Sweden Tercentenary Foundation, Grant No. 90-0150.]

\section{1:30}

4aSP14. Allophonic constraints and segmentation in infant speech perception. Elizabeth A. Hohne and Peter W. Jusczyk (Dept. of Psychol., Park Hall, SUNY at Buffalo, Buffalo, NY 14260)

The role of allophonic constraints in speech perception was studied with 2-month olds and adults. English word and nonword stimuli were selected to have the same phonemic content, but different allophonic structure. In particular, some of the stimuli contained an allophone of the phoneme category / $t$ / that is used in signaling a boundary between 
two words (e.g., the $/ t /$ in "night rate"), whereas others contained an allophone appropriate to the middle of a word (e.g., the $/ t$ / in "nitrate"). Two-month old infants discriminated allophones corresponding to different word and nonword boundary locations. When vowel duration and other prosodic variables surrounding the allophones were equated, infants continued to discriminate them. These results suggest that the basis for discrimination is not simply prosodic in nature. The adult data, measured by a same/different discrimination task, differed from the infant data in the patterning of responses. There is some indication the adult data can be explained in terms of lexical effects. [Work supported by NICHD.]

\section{1:45}

4aSP15. Do infants remember voices? Peter W. Jusczyk, Elizabeth A. Hohne, Ann Marie Jusczyk, and Nancy J. Redanz (Dept. of Psychol., Park Hall, SUNY at Buffalo, Buffalo, NY 14260)
Recent research on infants' representation of speech typically focuses on linguistic features such as phonetic and prosodic cues. However, speech also carries information about things such as emotional tone and talker identity. To what extent do infants extract and remember such information from the speech signal? The present study asks whether infants preserve information about talker identity when they encode information from speech. Infants 7.5 to 8.25 months old were exposed to recordings of stories read by the same talker for 10 days in a 2-week time period. Two weeks later, the infants were tested in a head turn preference procedure. The stimuli were lists of words from the stories. Half of the lists were produced by the original talker; the other half by a novel talker. The infants listened significantly longer to the familiar voice suggesting that they developed a representation of that voice. An ongoing study is investigating whether infants actually listen longer to familiar words from the stories or to novel items of the same phonetic complexity and lexical frequency. [Work supported by NICHD.]

THURSDAY MORNING, 20 MAY 1993

BANQUET ROOM, 7:55 A.M. TO 12:15 P.M.

Session 4aUW

\title{
Underwater Acoustics: Signal Processing
}

\author{
G. L. D'Spain, Chair \\ Marine Physical Laboratory, Scripps Institution of Oceanography, San Diego, California 92152-6400
}

Chair's Introduction-7:55

\section{Contributed Papers}

\section{8:00}

4aUW1. Passive localization and classification at low signal-to-noise via Bayesian inversion. Nolan R. Davis and Richard Pitre (Code 7144, Naval Res. Lab., Washington, DC 20375-5000)

The Bayesian inversion approach [R. Pitre and N. R. Davis, J. Acoust. Soc. Am. 92, 2418 (1992)] is employed to examine integration time dependence of a posteriori source location distributions and to perform spectral estimation. Model calculations are presented for low signal-to-noise conditions.

\section{8:15}

4aUW2. Holographic array processing in the presence of ambient noise. Azmi A. Al-Kurd and Robert P. Porter (Appl. Phys. Lab., Univ. of Washington, 1013 NE 40th St., Seattle, WA 98105)

A holographic array processing (HAP) algorithm is presented for source localization in a nonhomogeneous medium. Conventional array processing algorithms, such as matched field processing, require precise knowledge of the medium between the source and the receiving array. The holographic array processing method relaxes this stiff requirement and calibrates the integrated effect of the medium between a reference (virtual) array near the source, and the receiving array. Using conjugation and averaging techniques at the receiving array an image of the source wave front at the location of the virtual array is reconstructed without explicit knowledge of the characteristics of the medium. The target signal is measured at the receiving array while a reference source is deployed in the vicinity of the target and allowed to transmit at incremental depths. In this paper it is assumed that the ocean is to be horizontally stratified and range dependent. The target's signal is observed at the receiving array in the presence of additive, spatially correlated ambient noise. In the reconstruction step the volume of the ocean in the vicinity of the target is scanned. Both the maximum likelihood method (MLM) and the conventional Bartlett processor are used to estimate the reconstructed field near the target by comparing the virtual image of the wave front with a weighting function that depends on the replica field from the test source. In a noise-free situation, the magnitude of the reconstructed field is greatest at the location of the target. The detection criterion of the noisy signal is based on maximizing the intensity of the reconstructed field as a function of range and depth.

\section{8:30}

4aUW3. Holographic array processing using truncated arrays. Azmi A. Al-Kurd and Robert P. Porter (Appl. Phys. Lab., Univ. of Washington, 1013 NE 40th St., Seattle, WA 98105)

Imperfect knowledge of the salient characteristics of the propagation medium limits the performance of acoustic array processors at long ranges in the ocean. Holographic and phase conjugation techniques can be used to diminish the range integrated effect of the medium and reconstruct the wave front in the vicinity of a scatterer or other signal source. Then, using a backpropagation technique, which focuses sound at the position of the unknown source, the location of the source can be determined. In deep water, the idealistic situation of having a receiving array and reference source that span the water column is prohibitively complex. In this paper the effect of truncating both the receiving and virtual arrays on the performance of the holographic array processing algorithm is presented. The analysis was carried out using normal mode theory, and the simulation for a range-dependent ocean was performed using a wide angle PE (parabolic equation) code, FEPE. It is shown that the holographic array processing algorithm is more sensitive to the length of the virtual array than to the length of the receiving array.

\section{$8: 45$}

4aUW4. Broadband localization and deconvolution. T. C. Yang (Naval Res. Lab., Washington, DC 20375)

An algorithm is presented for detecting and localizing a passive 
broadband signal in an underwater acoustic waveguide. The processor estimates the modal spectrum of a broadband signal for source localization; as a consequence, the source spectrum and signature (time domain waveform) are estimated. (Broadband modal decomposition is the equivalent of time domain deconvolution for source signature extraction.) In addition to the usual lofargram, a frequency-depth plot is proposed to display a broadband signal. Source localization is conducted in the frequency domain by summing the (range and depth) ambiguity functions over the signal bandwidth. Sidelobes are suppressed in broadband processing if the signal has sufficient bandwidth. Coherent summation of the ambiguity functions yields the expected timebandwidth gain if source signature is known (the extended matched filter processing). Numerical simulations are shown for an Arctic waveguide. The processor works for transient as well as continuous broadband signals.

4aUW5. A normal-mode based approach for localization of transient signals. S. M. Jesus (UCEH-University of Algarve, PT-8000 Faro, Portugal)

Normal-mode modeling is a well-accepted representation for acoustic signals propagating in a number of environmental conditions. Detection of the spatial normal-mode structure makes possible signal localization enhancement against the noise that has no spatial structure. A simple algebraic argument allows one to separate the vector subspace that contains the signal from the vector subspace that contains the noise and to obtain a narrow-band estimate of the source location [S. M. Jesus, Signal Process. 28, 117-122 (1992)]. This subspace splitting algorithm has been extended for localizing broadband transient signals assuming that the signal only has a normal-mode structure. It is shown with synthetic data that the proposed broadband algorithm outperforms both the generalized minimum variance and the conventional processors. As an example, this processor has been used to localize short transient pulses collected in a 120 -m depth shallow water area with a 62-m aperture vertical array. The experimental results show that stable and accurate localizations could be obtained during long time intervals. This shows that the sound field, received over a given frequency band, is relatively stable over time and is in agreement with the predictions given by a standard normal-mode propagation model.

\section{9:15}

4aUW6. Detection of transients using the nonstationary bispectrum. Martin L. Barlett, Kevin W. Baugh, and Gary R. Wilson (Appl. Res. Lab., Univ. of Texas at Austin, P. O. Box 8029, Austin, TX 78713-8029)

An algorithm based on the nonstationary bispectrum is proposed for detection of sampled finite duration signals. The detection algorithm uses a combination of coherent and incoherent smoothing in the frequency domain to produce a test statistic which is suitable for display as a spectrogram. A model transient waveform imbedded in Gaussian noise is used to evaluate the detection performance of the proposed detector. Detectors based on the spectral correlation and power spectrum are used as metrics to evaluate the relative effectiveness of the proposed detector. Performance is investigated both as a function of signal-to-noise ratio for a fixed transient duration and processing length and as a function of transient duration/processing length mismatch at a fixed signal-to-noise ratio. The benefits and limitations of the proposed test statistic based on the nonstationary bispectrum relative to the other detectors investigated will be noted. [Work supported under contract with Space and Naval Warfare Systems Command.]

\section{9:30}

4aUW7. Model-based matched-filter processing of Doppler-shifted signals in a time dispersive ocean environment. J.-P. Hermand (SACLANT Undersea Res. Ctr., Viale Bartolomeo, 400, I-19138 La
Spezia, Italy) and W. I. Roderick (Naval Undersea Warfare Ctr. Div., Newport, RI 02841-5047)

Dispersive multipath propagation in an ocean medium distorts wideband linear frequency-modulated (LFM) transmit1ed signals. As a result, the performance of correlation receivers is degraded if the receiver does not account for the Doppler of the incoming signal or the multipath (energy splitting) in the medium. In this study, results are presented that demonstrate that the performance of a conventional correlation can be improved if the reference (replica) channel is compensated in both Doppler and time dispersion. The model-based matched filter is generated by correlating the received signal with a reference channel that consists of the transmitted signal convolved with the impulse response of the medium and Doppler compensated. The channel impulse responses were predicted with a broadband propagation model using environmental (sound velocity) data. The data were collected during a February 1990 experiment conducted in deep water in an area west of Sardinia. The acoustic data set consisted of linear frequency-modulated signals, with a time-bandwidth product of 4000 , transmitted from a moving source and received on a towed array. Comparison with conventional processing shows improvement (about $3 \mathrm{~dB}$ ) in peak output signal-to-noise ratio for the propagation conditions encountered in the experiment. [Work supported by ONR.]

\section{9:45}

4aUW8. Target tracking using matched-field processing. Michael J. Wilmut (Royal Roads Military College, FMO Victoria, BC VOS 1BO, Canada). John M. Ozard, and Bryan Woods (Defence Res. Establishment Pacific, FMO, Victoria, BC VOS 1B0, Canada)

The objective of this paper is to illustrate the use of matched-field processing (MFP) for tracking low signal-to-noise ratio targets moving linearly and at constant speed. The input to the tracker consists of the positions and power of the largest peaks on the MFP ambiguity surface. These largest peaks usually include the match at or near the source position even at low signal-to-noise ratio. An exhaustive search for the best matching track over all possible target tracks (that is allowing varying speed and heading) is beyond the scope of today's computers for any realistic search region. In this paper, an efficient algorithm is described based on examining the average Bartlett statistic along a set of linear tracks that connect only the largest peaks. This set was restricted to the physically possible tracks to further reduce the number to be examined. Examples of the ambiguity surfaces and the probability of examining the true track are given. The algorithm performance is a function of the scenario, signal-to-noise ratio, number of ambiguity surfaces, and number of peaks examined on each surface. It is shown that if the true target track is one of those examined its Bartlett statistic is almost certainly maximum. This efficient tracking requires only modest computing beyond that required to generate the ambiguity surfaces.

\section{0:00}

4aUW9. Synthetic aperture processing of a moving $\mathrm{Cw}$ sound source in a range-dependent underwater environment. Thomas $\mathbf{N}$. Lawrence and Nancy R. Bedford (Appl. Res. Lab., Univ. of Texas at Austin, P. O. Box 8029, Austin, TX 78713)

Synthetic aperture processing is a method of extracting horizontal wave numbers from recordings on one hydrophone. Such an approach has been previously suggested; most recently by Collins et al. [J. Acoust. Soc. Am. 92, 2366(A) (1992)], who proposed single hydrophone matched-field processing. A similar method was used by Frisk et al. [J. Acoust. Soc. Am. 86, 1928 (1989)] to derive bottom properties from data. The experimental method requires that a source (or receiver) move at a constant depth and a constant velocity with respect to a fixed receiver (or source). The moving source thus sweeps out a synthetic aperture with range, and array element recordings are processed by means of a Fourier transform of complex pressure with range, yielding the wave-number spectrum (or modal eigenvalues) over the chosen aperture. A series of overlapping apertures over the source track shows the evolution of the wave-number spectrum with the change in 
source-receiver range. Using a vertical array, estimations can be made for mode depth functions over the aperture of the array, mode attenuation, and mode eigenvalues with source range, i.e., those parameters needed for matched-field processing. Comparisons will be made to lowfrequency acoustic data from a range-dependent environment. Experimental results will be compared with parameters obtained from an adiabatic normal mode model.

\section{0:15-10:30 Break}

\section{0:30}

4aUW10. Sound-speed profiles from vertical array data. David $H$ Berman (Dept. of Phys. and Astron., Univ. of Iowa, Iowa City, IA 52242)

Provided mode amplitudes are uncorrelated with one another, it is possible to extract sound-speed information from the covariance of the pressure field along a vertical array [D. H. Berman and S. N. Wolf, J. Acoust. Soc. Am. 91, 2447(A) (1992)]. Conditions under which modes are in fact uncorrelated will be discussed here. In addition, a method for correcting effects of array tilt will be presented. The main difficulty of the extraction scheme is that second derivatives of the covariance are required. Various attempts at smoothing data, including fitting the covariance matrix to Pekeris normal modes will be described. The method will be applied to covariance matrices assembled from data gathered near San Diego in 1985. [Work supported by U. S. Naval Research Laboratory.]

\section{$10: 45$}

4aUW11. Self-cohering conditionally constrained minimum variance matched-field processing in a random ocean environment. Jeffrey $L$. Krolik (Dept. of Elec. Eng., Duke Univ., Box 90291, Durham, NC 27708-0291)

In recent work, minimum variance (MV) beamforming with soundspeed perturbation constraints (MV-SPC) [J. L. Krolik, J. Acoust. Soc. Am. 92, 1408-1419 (1992)] was proposed as a means of improving the robustness of $\mathrm{MV}$ matched-field source localization methods to random sound-speed profile inhomogeneities in the ocean channel. In this paper, an extension of the approach is developed that uses wave-front measurements from a source-of-opportunity to substantially improve MVSPC beamformer performance. The proposed method consists of MVSPC beamforming with constraints derived from the statistics of hypothesized point-source wave-front replica vectors conditioned on the measurement of a source-of-opportunity wave front at a particular point in time and/or space. Using a priori knowledge of the space-time correlation of sound-speed inhomogeneities, optimal prediction of the conditional signal wave-front correlation matrix is used to compute more precise MV-SPC beamformer constraints. The method also includes an accurate procedure for estimating a source-of-opportunity wave front in a multiple source environment. [Work supported by ONR.]

\section{1:00}

4aUW12. Further comments on heamforming with acoustic measurements at a single point in the ocean. G. L. D'Spain and W. S. Hodgkiss (Marine Phys. Lab., Scripps Inst. of Oceanogr., San Diego, CA 92152-6400)

Previously, the idea of using multi-component acoustic measurements at a single point in the ocean, rather than spatially distributed measurements of pressure, to obtain information on the spatial dependence of the ocean acoustic field was introduced [G. L. D'Spain and $\mathbf{W}$. S. Hodgkiss, J. Acoust. Soc. Am. 91, 2364(A) (1992)]. The basis for this single-point measurement approach is a Taylor series expansion of the pressure field. The purpose of this presentation is to further extend these ideas, including the use of adaptive time-varying array weights to perform correlated noise cancellation and the application of Capon's minimum variance method to measurements in a second order Taylor series expansion. In addition, ways of processing the data collected by a line array of multicomponent sensors in order to eliminate spatial aliasing when the interelement spacing is somewhat greater than the halfwavelength criterion will be presented. Finally, the application of highresolution beamforming techniques with actual multi-component (hydrophone and geophone) data for the determination of the azimuthal directionality of the underwater sound field below $5 \mathrm{~Hz}$ in several experiments will be discussed. [Work supported by ONR and ONT.]

\section{1:15}

4aUW13. Optimal linear array configuration and weighting through simulated annealing. Pierre Zakarauskas and Michael V. Greening ${ }^{\text {) }}$ (Defence Res. Establishment Pacific, FMO, Victoria, BC VOS 1BO, Canada)

This paper reports on the application of the optimization technique called simulated annealing to the problem of finding the best array configuration for a conventional beamformer. The question the optimization process has to answer is: "For a given maximum array length and number of sensors, what is the placement and weighting of the elements that will give the narrowest mainlobe while keeping the sidelobes below a given level $S$." It is emphasized that the technique is fairly general, and that different optimization criteria could easily be substituted. During the search, a cost $C$ is calculated for each randomly generated array configuration and set of weights. The cost increases with the width of the mainlobe, and is proportional to how much the sidelobes are above $S$. When the search is complete, the array configuration may or may not occupy the whole length that was accorded to it, and the sidelobes are all at or below $S$. By keeping the part of the cost function that does not change and using table look-up for the transcendental functions, the search can be made quite efficient, taking only on the order of minutes of CPU time on a VAX 9000 computer for 14 receivers. ${ }^{\text {) }}$ Present address: Datavision Computing Services Ltd., 1545 Pandora Ave., Ste. 203, Victoria, BC V8R 6R1, Canada.

\section{1:30}

4aUW14. Beamforming towed array data when knowledge of the hydrophone positions is imperfect. Brian G. Ferguson (Maritime Operations Div., Defence Sci. and Technol. Org., P. O. Box 44, Pyrmont 2009, Australia)

Information on the various sound sources that contribute energy to the underwater acoustic environment is often obtained by processing the data from a thin flexible line array of hydrophones towed behind a vessel. The acoustic data from the array are processed digitally in the frequency and spatial domains. The process of spatial filtering, which is commonly referred to as beamforming, assumes that the horizontal line array is straight with the transverse displacements of the hydrophones being zero with respect to the array axis. Using real acoustic data, this paper shows the effects on conventional and adaptive beamformers when these transverse displacements are finite and the array shape is nonlinear. Furthermore, signal processing techniques are presented that significantly reduce the influence of these effects on the performance of the beamformers. By processing the array's acoustic data when a planewave acoustic source is present, these techniques estimate the horizontal and transverse displacements of the hydrophones for subsequent input to the spatial filters.

\section{1:45}

4aUW15. A generalized beamformer based on a spatial bispectrum. Edmund J. Sullivan (Code 103, Naval Undersea Warfare Center, Newport, RI 02841) and Geoffrey S. Edelson (Univ. of Rhode Island, Kingston, RI 02881)

The bispectrum is the expected value of the third-order cumulant of the data sequence of a signal in either space or time. Much analysis based on the bispectrum has already been carried out in the time domain, where information regarding the stationarity, linearity, and Gaussianity of the signal series can be analyzed. In this paper, it is shown that these concepts can be directly applied to the spatial domain, resulting in 
a generalization of the standard $k$ - $\omega$ beamformer. It is shown that the ability of the bispectrum to indicate nonstationarity in the data series allows this generalized beamformer to account for nonplanar aspects of the incoming signal wave front, thereby allowing for source localization in which bearing and range estimation evolve in a self-consistent framework. Thus bearing estimation and waye front curvature ranging appear as special cases. It is also shown that the technique is applicable to the analysis of more complicated propagation scenarios such as normal mode propagation.

12:00

4aUW16. Signal parameter estimation via transfer matrix analysis. Woon Hyun Cho (Acoust. Simulation Lab., Naval R\&D Ctr., Jinhae P. O. Box 18, Kyungnam, Korea)
A new algorithm is developed for estimating the delay times and spectra in mixed wave fields. The algorithm is applicable to a spatial and temporal data window in which more than two events are present. In implementing the method it is assumed that data are recorded at equispaced hydrophones located in a spatial window in which the delay times are constant. Application of a least-squares criterion reduces the mathematics to an eigenvalue problem. The eigenvalues are complex, and their magnitude determines the frequency-dependent SNR. The phase is a linear function of frequency with a slope that determines the delay time over unit hydrophone spacing. The input data consist of the cross-power spectra between subarrays that contain the same numbers of elements and are shifted by zero or one hydrophone separation. Examples illustrate the application of the algorithm to synthetic data.

\title{
Meeting of Accredited Standards Committee S12 on Noise
}

to be held jointly with the

\section{U.S. Technical Advisory Group for ISO/TC 43/SC1 Noise and ISO/TC 94/SC12 Hearing Protection}

\author{
D. L. Johnson, Chairman S12 \\ EG\&G Special Projects, Albuquerque Operations, Albuquerque, New Mexico 87119-9024
}

\author{
H. E. von Gierke, Chairman, respective U.S. Technical Advisory Groups (TAGs) for ISO/TC 43/SC1 \\ and ISO/TC $94 / \mathrm{SC} 12$ \\ 1325 Meadow Lane, Yellow Springs, Ohio 45387
}

\begin{abstract}
Standards Committee S12 on Noise. Working group chairs will report on their progress for the production of noise standards. The interaction with ISO/TC 43/SC1 and ISO/TC 94/SCl2 activities will also be discussed, with reference to the international standards under preparation. The Chair of the respective U.S. Technical Advisory Groups (H. E. von Gierke) will report on current activities of these International Technical Subcommittees under ISO and preparation for the next meeting of ISO/TC 43/SC1, scheduled to take place in Oslo, Norway from 31 May to 4 June 1993.
\end{abstract}

THURSDAY AFTERNOON, 20 MAY 1993

TUDOR ROOM, 1:00 TO 5:00 P.M.

\author{
Session 4pAB \\ Animal Bioacoustics: Effects of Man-Made Noise on Animals \\ Ann E. Bowles, Chair \\ Hubbs-Sea World Research Institute, 1700 South Shores Road, San Diego, California 92109 \\ Chair's Introduction-1:00 \\ Invited Papers
}

1:05

4pAB1. Effect of intense tone stimulation on the anatomy of the peripheral auditory system in bony fishes. Mardi C. Hastings (Dept. of Mech. Eng., Ohio State Unjv., 206 W. 18th Ave., Columbus, OH 43210)

Results of current and previous studies by M. C. Hastings et al. [J. Acoust. Soc. Am. Suppl. 1 79, S80 (1986); J. Acoust. Soc. Am. Suppl. 1 80, S75 (1986); and J. Acoust. Soc. Am. Suppl. 1 81, S7 (1987)] and P. S. Enger [Hearing and Sound Communication in Fishes (Springer-Verlag, New York, 1981), pp. 243-255] to determine the effect of intense tone stimulation on the anatomy of the peripheral auditory system in bony fishes are summarized. In these studies different species of fish were exposed to pure tones at frequencies between 200 and $1000 \mathrm{~Hz}$ at different sound pressure levels for $1 \mathrm{to} 4 \mathrm{~h}$. The sensory epithelia in the saccule and legena were then examined using scanning electron microscopy to assess damage to hair cells. These experimental 
evaluations indicated (1) the possibility of a gross place-type mechanism for frequency discrimination and (2) a possible breakdown of the Weberian apparatus in goldfish (Carassius auratus) at extremely high sound pressure levels because the primary site of hair cell damage switched from the saccule to lagena. A mathematical model for the dynamic motion of the swimbladder and Weberian ossicles has been developed based on the work of R. McN. Alexander [J. Exp. Biol. 38, 747-757 (1961)], which includes viscoelastic properties of the tissue. The model supports previous experimental observations and indicates that motion is no longer transmitted to the saccule when the system is excited by sufficiently high sound pressure levels. [Work supported in part by ONR and NIH.]

4pAB2. Hearing recovery in neonatal chicks after exposure to intense pure tones. James C. Saunders, Henry J. Adler, and Daryl E. Doan (Dept. of Otorhinolaryngol. Head and Neck Surgery, Univ. of Pennsylvania, Philadelphia, PA 19104)

Neonatal chicks exposed to a 120-dB SPL pure tone at $0.9 \mathrm{kHz}$ for 48 to $200 \mathrm{~h}$ exhibited a severe loss in auditory function. The growth and recovery of hearing loss was traced with a variety of peripheral evoked-potential and single-cell recordings. The growth of threshold shift and the loss of frequency selectivity reached asymptotic levels after $40 \mathrm{~h}$ and then remained constant to $200 \mathrm{~h}$. At 0 days of recovery the magnitude of threshold shift in the most affected frequencies was $55-60 \mathrm{~dB}$ and tuning curves showed a $50 \%$ loss in selectivity. Nearly complete recovery of sensitivity and selectivity was noted 12-15 days post exposure. Severe recruitment was also seen in functions relating sound level to evoked-potential amplitude or single cell rate-intensity functions. The abnormal coding of intensity returned to normal within 3 days. The loss in function was associated with significant cochlear damage, and recovery was associated with considerable cochlear repair. The repaired cochlea, however, does not have a normal appearance. It remains to be explained how auditory function can completely recover in a cochlea with severe permanent structural defects. [Work supported by the NIDCD, PLHRF, and NOAR.]

\section{$1: 45$}

4pAB3. Effects of simulated aircraft noise on heart rate and behavior of desert ungulates. Paul R. Krausman (School of Renewable Natural Resources, Univ. of Arizona, Tucson, AZ 85721), Mark C. Wallace, Mara E. Weisenberger, Donald W. DeYoung, and O. E. Maughan (Univ. Arizona, Tucson, AZ)

The effects of simulated low-altitude jet aircraft noise on the behavior and physiology of 6 captive desert mule deer (Odocoileus hemionus crooki) and 5 mountain sheep (Ovis canadensis mexicana) were evaluated. Heart rate and behavior in relation to ambient temperature, number of simulated overflights/day, and noise levels [range $=92-112$ decibels $(\mathrm{dB}$ )] that the animals were exposed to were measured. Heart rates during simulated overflights ( $n=112 /$ treatments/season) were compared to data collected prior to and following treatment periods. Differences between heart rates for animals, noise levels, and number of overflights between seasons were documented. All animals became habituated to sounds of low-altitude aircraft. Although heart rates increased during overflights they returned to resting rates in $<2 \mathrm{~min}$.

4pAB4. Effects of aircraft noise on time-activity budgets of wintering black ducks. John T. Conomy (Zoology Dept., North Carolina State Univ., Raleigh, NC 27695), Jaime Collazo, and W. James Fleming (North Carolina Cooperative Fish and Wildlife Research Unit, North Carolina State Univ., Raleigh, NC 27695)

The primary goal of this study was to determine if the time-activity budget (TAB) of wintering black ducks (Anas rubripes) was significantly altered by military aircraft noise at the U. S. Marine Corps target range in Piney Island, North Carolina. Sound levels were measured concurrently with behavioral observations. Over a sampling period of 81 days, exceedances $>80 \mathrm{~dB}$ occurred on 289 occasions, the mean duration of exceedances was $5.09 \mathrm{~s}$, and the mean sound pressure was $85.7 \mathrm{~dB}$. Black ducks spent between $0.2 \%$ and $0.5 \%$ of their time reacting to aircraft. Correspondingly, the energetic costs of these reactions were low. TABs of black ducks in the high noise environment of Piney Island were within the expected range of those in low noise environments based on published literature. In a follow-up study, captive black ducks were subjected to simulated jet noise at levels approximately those recorded in the field. Measured levels of reactions to noise stimuli indicated that ducks habituated within 1 day. These results suggest that low reaction levels recorded in the field reflect the species' habituation capabilities to some kinds of disturbance. [Work supported by USMC and USAF.]

\section{2:25-2:45 Break}

\section{Contributed Papers}

\section{2:45}

4pAB5. Experimental radar-activated hazing system. Richard A. Weber and Barbara A. Filas (Knight Piesold and Co., 1600 Stout St., Ste. 800, Denver, CO 80202)

Migrating birds often use mine tailing facilities during migration, especially in arid areas throughout the west. Process chemicals in tailing facilities can adversely affect birds that use the facility as a resting and feeding location. Traditional audio hazing techniques have been ineffective because birds become habituated to the devices' continuous and regular sound production. This project involved development and testing of a radar-activated bird deterrent system to haze birds away from tailing facilities. The system was tested at a mine tailing facility in northeastern Nevada. It was designed to provide radar coverage over a tailings facility and to automatically activate several audio hazing devices when a bird entered a specified zone. The theory behind such a system is that hazing is much more effective while birds are still in flight. Audio hazing devices activated included a stereo system, fire alarm, propane cannons, and a device designed and built by Knight Piesold to launch firecracker-like cartridges. Data were collected during the fall of 
1990 and spring of 1991 . The data collected shows that the system has promise as a method to haze migratory birds away from the tailings facility.

\section{3:00}

4pAB6. Effects of simulated aircraft noise on hearing, food detection, and predator avoidance behavior of the kit fox, Vulpes macrotis. Ann E. Bowles and Jon K. Francine (Hubbs-Sea World Res. Inst., 1700 South Shores Rd., San Diego, CA)

Four kit foxes were captured south of Gila Bond, Arizona, in an area not overflown by aircraft. Hearing thresholds were measured by startle inhibition with a San Diego Instruments Startle Recording System. Shaped 200 -ms tone bursts ranging from $100 \mathrm{~Hz}$ to $40 \mathrm{kHz}$ in octave steps were delivered using a step-up, step-down procedure. Startle responses were elicited by a 40 -psi air puff $30 \mathrm{~ms}$ in duration following $100 \mathrm{~ms}$ after the tone burst and startle intensity and latency were measured. Threshold at best frequency lay between -10 and $-15 \mathrm{~dB}$ re: $20 \mu \mathrm{Pa}$ at $2-4 \mathrm{kHz}$ and declined rapidly below $1 \mathrm{kHz}$ and above 20 kHz. Foxes were trained to identify simulated prey and predator noise at the minimum level required for detection. Latancies to respond were measured in the absence and presence of simulated aircraft noise, simulated by recordings of F-4 fighter aircraft played for 40 min at $96 \mathrm{~dB}$ re: $20 \mu \mathrm{Pa}$ with onset rate of $25 \mathrm{~dB} / \mathrm{s}$. Foxes were also exposed to aircraft noise for $3 \mathrm{~h}$ while asleep during the day. Results available to date indicate that foxes can detect test signals and respond in the presence of aircraft noise; latencies to response are altered during tests but not after; and no changes in activity are detected during daytime exposures.

\section{$3: 15$}

4pAB7. Marine turtle auditory behavior. S. Moein, M. Lenhardt, D Barnard, J. Keinath, and J. Musick (Virginia Inst. of Marine Sci., College of William and Mary, Gloucester Point, VA 23062)

Auditory capacities of the Atlantic Loggerhead (Caretta c. caretta) were assessed to develop a sound repelling system to be used in dangerous areas. To estimate sensitivity, auditory brain-stem responses (ABRs) were recorded in ten animals to clicks and tones $(250,500$, 750 , and $1000 \mathrm{~Hz}$ ). Animals, removed from water, had electrodes implanted subcutaneously. Stimuli were presented to eardrum with a vibrator. Consistent responses occurred within the first $10 \mathrm{~ms}$ after stimulation, not unlike that recorded in nonmarine forms. ABR waveforms increase in latency with stimulus attenuation. Prior to ABR recording, sound-induced head movements were noted in most, but not all animals. Sound-induced swimming was also observed. A flight response within the artificial environment of a small tank may not predict natural behavior, hence animals will be placed in a saltwater pen and the experiments repeated with a sound source away from the animal. Finally animals fitted with transmitters will be released in the lower Chesapeake Bay. After settling into a selected area, they will be approached by a moving sound projector to determine if this sound induces flight.

4pAB8. A method for measuring wildlife noise exposure in the field. B. Andrew Kugler and David S. Barber (BBN Systems and Technologies, A Division of Bolt Beranek and Newman, Inc., 21120 Van Owen St., Canoga Park, CA 91316)
The effects of aircraft noise on wildlife have received much attention from the environmental community, especially when associated with low altitude aircraft overflights. In the past, much of the research on this subject used surrogate information to describe noise events in field studies. For example, noise exposure was often measured at an observer location, far removed from the animal under study, or inferred from the presence of aircraft flyovers. This paper describes the development and application of an animal noise monitor (or ANM) that can be mounted on a collar and worn by a subject animal over extended periods of time. The approach allows for measurement of noise exposure of the animal whose behavior is being observed, without external interference other than initial capture. The device is capable of recognizing aircraft noise events, calculating a variety of acoustic descriptors associated with these events, and retaining the information in memory for up to 6 months until the ANM is recovered from the field. The ANM, in addition to time- and date-stamping of each event, also contains a monitor that can measure and record the activity of an animal during and immediately after a noise intrusion. The ANM is capable of withstanding harsh environments and operates over a wide temperature range. This instrumentation has been used recently on a caribou noise effects study in Alaska.

\section{$3: 45$}

4pAB9. Sonic boom/animal stress project report on elk, antelope, and Rocky Mountain bighorn sheep. Thomas D. Bunch and Gar W. Workman (Depts. of Animal, Dairy, and Veterinary Sci. and Fisheries and Wildlife, Utah State Univ., Logan, UT)

The animal/noise project was initiated as a result of a proposed extension of the Air Force Gandy supersonic range (MOA) in westem Utah and eastern Nevada. The research was conducted at the Utah State University Green Canyon Ecology Center, and at Gold Hill, in the desert of northwestern Utah. The experimental animals included elk, antelope, and Rocky Mountain bighorn sheep. These animals were instrumented with heart rate and body temperature transmitters, which were surgically implanted in the animals. The animals were released in large enclosures, and in some cases were released to the wild for disturbance tests. This was done to determine effects of various disturbances on heart rate and to establish a baseline physiologic database of normal heart rate and body temperature. The animals were subjected to various types of disturbances, including people on foot, motorcycles, fourwheeled vehicles, fixed wing aircraft, helicopters, and F-16 jet aircraft flown subsonic and supersonic, etc. These projects indicated that animals habituated to most disturbance factors in a short period of time. The exceptions included people on foot who entered the research enclosures where the animals were kept; fixed wing aircraft at low levels of flight; and helicopter flights at low elevations near the animal enclosures. The animals habituated to subsonic and supersonic jet overflights after about four passes over the animals. This habituation seemed to be permanent, as these same animals did not respond when tested at a later date.

\section{4:00-4:05 Break}

\section{4:05-5:00}

PANEL DISCUSSION: Is there a need for standards to protect animals from man-made noise?

Panelists: Ann E. Bowles, Frank T. Aubreg, W. Cummings 
Session 4pAO

\title{
Acoustical Oceanography: Acoustical Determination of Ocean Parameters and Processes
}

\author{
Peter H. Dahl, Chair \\ Applied Physics Laboratory, University of Washington, Seattle, Washington 98105
}

Contributed Papers

$1 ; 00$

4pAO1. Breaking waves and bubble plumes. Peter H. Dahl and Andrew T. Jessup (Appl. Phys. Lab., College of Ocean and Fishery Sci., Univ. of Washington, Seattle, WA 98105)

A large part of our knowledge of oceanic bubbles originates from acoustic measurements, for example, boundary layer stability [Thorpe, Philos. Trans. R. Soc. London Ser. A 304, 155-210 (1982)] and Langmuir structure [Zedel and Farmer, J. Geophys. Res. 96 (C5), 88898900 (1991)] are known to influence bubble field properties. What remains elusive are details of the transient properties of the bubble field, such as the spatial and temporal scale of bubble plumes due to breaking waves. During January 1992 acoustic measurements of bubble plumes from breaking waves were made from the R/P FLIP operating $\mathbf{4 0 0} \mathrm{nmi}$. Off the coast of California. Acoustic measurements were made from two sonars, a four-beam system $(20-50 \mathrm{kHz})$ and a single-beam system $(240 \mathrm{kHz})$, placed at the end of a $12-\mathrm{m}$ subsurface boom attached to FLIP's hull at $28.5-\mathrm{m}$ depth. The subsurface boom was specifically designed for this application, allowing the sonars to look up at the ocean surface from a stabilized platform. To document breaking wave activity over the acoustic system, a video camera was mounted on FLIP's aft boom and trained upon the acoustic footprint on the ocean surface. Surface waves, CTD, current, and meteorological measurements were also made. Space-time images of acoustic volume scattering clearly show bubble plume growth and decay. Details of the spatial and temporal scales of transient bubble plume and breaking wave event combinations are presented. In one case characterized by $U_{10}=10 \mathrm{~ms}$ and $H_{1 / 3}=2.3 \mathrm{~m}$, newly generated bubble plumes were observed to reach a maximum depth of approximately $4 \mathrm{~m}$ in about $60 \mathrm{~s}$ after wave breaking occurred. [Work supported by ONR Code 124.]

\section{1:15}

4pAO2. Statistical characteristics of bistatic surface scatter. Peter D. Neumann (Grad. Prog. in Acoust., Penn State Univ., P.O. Box 30, University Park, PA 16804) and R. Lee Culver (Penn State Univ., University Park, PA 16804)

The statistical characteristics of the envelopes of bistatic surface reverberation data taken in January 1992 are compared against the Rayleigh distribution. Agreement with some slight deviations from Rayleigh behavior was found in the statistical characteristics of the data for all available wind speeds ( 1.4 to $7.2 \mathrm{~m} / \mathrm{s}$ ) at frequencies from 20 to $40 \mathrm{kHz}$. Additionally, the data were divided in time according to the dominant surface scattering mechanism, surface roughness or bubble layer, and the statistical characteristics of each of these portions examined for Rayleigh behavior. This type of analysis allows for an increased amount of data to be used as a result of using data not only from all of the transmitted pulses but also from a number of different times in the time series instead of at a single time as was done in the first part of the analysis. The statistical characteristics of these portions of the time series were also found to be Rayleigh with only slight deviations in the tails of the distribution. [Work supported by ONT.]
$1: 30$

4pAO3. Coherent and incoherent scattering from oceanic bubbles. Kausik Sarkar and Andrea Prosperetti (Dept. of Mech. Eng., Johns Hopkins Univ., Baltimore, MD 21218)

In recent years, an effective medium theory has been applied to model the contribution of bubbles for low-frequency oceanic backscattering [Prosperetti et al. and Sarkar and Prosperetti, both submitted to J. Acoust. Soc. Am.] This approach differs from the more traditional one used to account for the effect of bubbles at higher frequencies, in which bubbles are treated as individual scatterers. Here, the relationship between these two apparently different approaches is clarified and a unified theory is presented. In this way, a better understanding of the rationale and limitations for the older theory is achieved. Applications to scattering and bubble counting are described. [Work supported by ONR.]

\section{$1: 45$}

4pA04. Resonance frequency and damping constant of a spherical gas-vapor bubble in an infinite medium. Yi Mao, Lawrence $A$. Crum, and Ronald A. Roy (Appl. Phys. Lab., Univ. of Washington, Seattle, WA 98105)

The resonance frequency $f_{0}$ and damping constant $\delta$ for free volume oscillations of a spherical bubble in an infinite medium are derived from the linearized, fundamental equations of fluid dynamics. There are three possible modes (acoustic, thermal, and mass diffusion) of wave propagation that satisfy the governing equations for the gas-vapor mixture inside the bubble. Outside it, only the acoustic and thermal diffusion modes need consideration because gas diffusion across the bubble surface into the liquid appears negligible for free oscillations. The parameters $f_{0}$ and $\delta$ are values such that the boundary conditions can be satisfied. The dependence of $f_{0}$ and $\delta$ on bubble size, ambient pressure, and temperature is examined. When a bubble contains a high concentration of gas, the values of $f_{0}$ and $\delta$ agree with the results of Devin [J. Acoust. Soc. Am. 31, 1654-1667 (1959)]. When the concentration of vapor is high, it is found that there exists only one resonance frequency for free oscillations [compare the results of R. D. Finch and E. A. Neppiras, J. Acoust. Soc. Am. 53, 1402-1410 (1973)]. [Work supported by ONR.]

2:00

4pAO5. Direct measurements of the sound speed in the oceanic surface bubbly layer. Eric Lamarre (R. M. Parsons Lab., MIT, Cambridge, MA 02139) and W. Kendall Melville (Scripps Inst. of Oceanogr., La Jolla, CA 92093-0213)

It is well known that wave breaking events create a bubbly layer a few meters thick below the water surface. The most important characteristic of this shallow channel is its sound-speed profile. Results are presented from two recent field experiments in which the sound-speed profile was directly measured in the first few meters below the surface for frequencies in the range $5-40 \mathrm{kHz}$. Preliminary results in moderate sea states (wind speeds less than $10 \mathrm{~m} / \mathrm{s}$ ) indicate that significant departures from the sound speed in bubble-free water occur in a shallow 
$[O(1 \mathrm{~m})]$ layer and are intermittent in time. Two dominant time scales are present. A fast time scale associated with the surface waves, which advect the bubble clouds horizontally with respect to the buoy; and a slow time scale $O(100 \mathrm{~s})$. Sound-speed reductions up to $150 \mathrm{~m} / \mathrm{s}$ have been observed at 50-cm depth with 10-min means well above the 5 to 15 $\mathrm{m} / \mathrm{s}$ previously cited in the literature. Other results including ambient sound, acoustic attenuation, and upward looking sonar measurements of the bubble clouds will be discussed. [Work supported by ONR.]

\section{2:15}

4pAO6. Sensitivity of modal amplitudes to warm surface mixed layers in the Greenland Sea. Rich Pawlowicz (Dept. of Appl. Ocean Phys. and Eng., Woods Hole Oceanogr. Inst., Woods Hole, MA 02543), Guoliang Jin,, James F. Lynch (Woods Hole Oceanogr. Inst., Woods Hole, MA), and Peter Worcester (Scripps Inst. of Oceanogr., La Jolla, CA)

Six near-surface acoustic transceivers were moored in the Greenland Sea in an array approximately $200 \mathrm{~km}$ in diameter from October 1988 to August 1989. Transmissions with a center frequency of $250 \mathrm{~Hz}$ and a bandwidth of $100 \mathrm{~Hz}$ were made at 4-h intervals over most of the array, resulting in a multipath structure of early ray arrivals and later nearsurface modal arrivals. During February and March the almost isothermal temperature profile resulted in an arrival pattern with the later arriving lower-order modes have greater amplitude. However, from mid-April onwards the pattern changed dramatically as amplitudes of the lowest-order mode became far smaller, and slightly earlier arriving modes became somewhat louder. At the same time, net surface fluxes changed sign, so one expects the formation of a shallow warm surface mixed layer (although the somewhat sparse historical data suggests that warm surface mixed layers are not seen for at least another month) One explanation for the amplitude changes is that modal coupling is induced by horizontal variations in the depth of this warm layer. ${ }^{\text {a) }} \mathrm{On}$ leave from Shanghai Acoustics Laboratory.

\section{2:30-2:45 Break}

\section{2:45}

4pAO7. Low-frequency sound source for various acoustical oceanography and seafloor exploration applications. Dimitri $M$. Donskoy (Davidson Lab., Stevens Inst. of Technol., Castle Point on the Hudson, Hoboken, NJ 07030)

A new design of a low-frequency sound source has been developed and tested. The oscillation of a rigid body and an arrangement for converting a dipole oscillation of the rigid body to a monopole pulsation are used in this radiator. The oscillation of the rigid body provides large displacement of a radiating surface without deformation of the material. Monopole pulsation of the entire source provides much higher acoustic radiation than dipole oscillation of a small (as compared with sound wavelength) body. The vibromotive force is generated by oscillation of a mass inside the rigid body. To prove the concept, a small version of the source has been built. It has a diameter of $0.32 \mathrm{~m}$, a frequency of 35-150 Hz, and an acoustic power output of $25 \mathrm{~W}$ on a $80-\mathrm{Hz}$ frequency. The full scale source will be capable of radiating different kinds of signals (cw, FM, or pulse) in the frequency range of tens to hundreds of $\mathrm{Hz}$ with a source level over $200 \mathrm{~dB}$ re: $1 \mu \mathrm{Pa}$ and efficiency of approximately $70 \%$. A high source level in the low-frequency range can be achieved due to the lack of limitation in the displacement of the radiating surface. The source has a potential for high reliability and relatively low cost due to very simple construction, the use of highty reliable and inexpensive mass-manufactured units (like electric motors), and very small material deformation.

\section{3:00}

4pAO8. ATOC-New Zealand receiver site survey and acoustic test. R. W. Bannister, K. M. Guthrie, J. S. Kay (Defence Sci. Establishment, Devonport, Auckland, New Zealand), G. E. J. Bold, M. D. Johns, S. M. Tan, and C. T. Tindle (Univ. of Auckland, Auckland, New Zealand)

Possible New Zealand receiver sites for the ATOC experiment have been investigated. A suitable site requires a water depth of $2000 \mathrm{~m}$ at a distance of $\mathbf{3 0} \mathrm{nm}$ or less from the coast and an unobstructed acoustic path to California. A detailed site surveyed and an acoustic trial were conducted in September 1992. In the acoustic trial a series of $1.8-\mathrm{kg}$ SUS charges was detonated off the California coast. Good signals were received in New Zealand at a distance of $10360 \mathrm{~km}$.

\section{3:15}

4pAO9. Enhancement of hydrodynamic flow noise by the regulation of air bubbles in a turbulent water jet. Murray $S$. Korman (Dept. of Phys., U.S. Naval Academy, Annapolis, MD 21402) and Lawrence A. Crum (Univ. of Washington, Seattle, WA 98105)

An experiment is performed to show that the near-field hydrodynamic flow noise (generated by a submerged water jet) is enhanced when the turbulent flow is modified to become a two-phase flow containing air bubbles. Pressure spectra, in the band 5-5000 Hz, are measured using a digital spectral analyzer from signals generated by a hydrophone placed at the axial and radial position ( $Z=4 D$ and $R=4 D$ ) from the nozzle exit diameter, $D=0.635 \mathrm{~cm}$. An amplification factor, $G=\int p_{\text {two-phase }}^{2} d f / \int p_{\text {single-phase }}^{2} d f$, is measured as a function of the gross void fraction $\beta$ of the air bubbles. Here, we measure $\beta$ to be the ratio of gas volume flow rate (through the bubblemaker located at the nozzle entrance) to the water volume flow rate, $Q_{g} / Q_{w}$. The amplification $G$ is measured as a function of $\beta$ by keeping $Q_{w}$ constant (the nozzle velocity $U=13.8 \mathrm{~m} / \mathrm{s})$ and varying $Q_{s}$. Results show that $G \sim \beta^{2}$ and $G \sim 20$ at $\beta=0.0065$. The mean-squared pressure fluctuation $p^{2} \equiv \int p_{\text {two-phase }}^{2} d f$ is measured as a function of $U$ by keeping $Q_{g}$ fixed and varying $Q_{u r}$ Results show that $p^{2} \sim U^{m}$, where $m=3.6,4.3$, and 4.7 for $Q_{8}=50,86$, and 136 sccm, respectively. [Work supported by NCPA.]

\section{$3: 30$}

4pAO10. Use of the vector Bragg wave-number scattering condition to measure turbulence. Louis Goodman, Diane Szargowicz (Naval Undersea Warfare Ctr., Newport, RI 02841), John Oeschger, S. V. Letcher (Univ. of Rhode Island, Kingston, RI 02881), and Peter Hebda (Americom Corp.)

Acoustic scattering that satisfies the far-field Bom approximation leads to a simple linear relationship between the scattered pressure field and the scattering field, namely the two are Fourier transform pairs. A set of laboratory experiments have been devised to exploit this relationship for the case of scattering from a turbulent field. Data are taken in a multiple scattering angle configuration such that the Bragg wavenumber vector associated with each scattering angle pair is in the same direction. Use of broad bandwidth source and receiver transducers (250 to $750 \mathrm{kHz}$ ) then allows a three-dimensional fully resolved wavenumber spectra of the turbulent field to be estimated. Results are presented for scattering from a turbulent buoyant plume. The data can also be used to examine the validity of the fundamental assumption of the far-field Born approximation. 


\title{
Session 4pMU
}

\author{
Musical Acoustics: Analysis of the Sound and Structure of Musical Instruments
}

\author{
Uwe J. Hansen, Chair \\ Department of Physics, Indiana State University, Terre Haute, Indiana 47809
}

Chair's Introduction-1:30

Contributed Papers

1:35

\begin{abstract}
4pMU1. Dynamic mechanical properties of violin wood and the tone qualities of violins. I: Audiofrequency complex shear compliance, modulus, and shear sound velocity and attenuation measurements. Edwin R. Fitzgerald (Johns Hopkins Univ., 3400 N. Charles St., Baltimore, MD 21218), Carleen M. Hutchins, and Morton A. Hutchins (Catgut Acoust. Soc., Montclair, NJ 07042)
\end{abstract}

Values of complex shear compliance $\left(J^{*}=J^{\prime}-i J^{\prime \prime}\right)$, modulus, $\left(G^{*}\right.$ $\left.=1 / J^{*}\right)$, and shear sound velocity and attenuation have been measured over a continuous frequency range from 10 to $10000 \mathrm{~Hz}$ for samples of spruce and maple wood used in the construction of six violins. Measurements were made with an automated electromagnetic transducer system in which samples are sheared while clamped between stainless steel blocks. [E. R. Fitzgerald, Proc. Am. Chem. Soc. Div. Polymeric Mater. Sci. 60, 573-578 (1989); E. R. Fitzgerald, U.S. Patent 5,081,870 (1992)]. Four wood samples were measured for each finished violin, viz., samples of spruce and maple with and cross grain. As reported earlier, values of the shear parameters vary with frequency and grain orientation, but also with moisture content and the clamping force on the sample faces while they are vibrated in shear [E. R. Fitzgerald, J. Acoust. Soc. Am. 89, 1927(A) (1991)]. The details of the mechanical spectra of the six sets of samples vary, but, in general, several sharp, microstructural compliance modes are superimposed on broad retardation spectra [E. R. Fitzgerald, J. Acoust. Soc. Am. 33, 1305-1314 (1961)]. Comparisons among the six sample sets are made for the same clamping force at $20^{\circ} \mathrm{C}$ and $52 \%$ R.H.

$1 ; 50$

4pMU2. Dynamic mechanical properties of violin wood and the tone qualities of violins. II: Relation of dynamic mechanical properties to the plate tuning of violins. Carleen $M$. Hutchins, Morton A. Hutchins (Catgut Acoust. Soc., 112 Essex Ave., Montclair, NJ 07042), and Edwin R. Fitzgerald (Johns Hopkins Univ., Baltimore, MD 21218)

Desirable mechanical properties of density, vibrational damping. Young's modulus, velocity of sound, and the ratio of velocity to density for spruce tops and maple backs of violins have been fairly well established [I. Barducci and G. Pasqualini, Nuovo Cimento 5 (5), 416-466 (1948); J. C. Schelleng, J. Acoust. Soc. Am. 35, 326-338 (1963); F. Rocaboy and V. Bucur, Catgut Acoust. Soc. J. 1 (6) (Ser. II), 21-28 (1990)]. However the longitudinal to cross grain ratios of Young's modulus and the shear modulus, as well as their relationships in both spruce and maple have not been studied extensively. This work is concerned with the extent to which these ratios can vary and still make possible the optimum plate tuning of modes \#1, \#2, and \#5 in violin top and back free plates. From a 1975 shipment of commercially available German spruce and maple, ten sets of violin top and back flitches were built into 10 normal violins. The plate tunings of six sets of these will be charted and discussed in relation to their mechanical properties.
2:05

4pMU3. Dynamic mechanical properties of violin wood and the tone qualities of violins. III: Relation of dynamic mechanical properties to the tone and playing qualities of violins. Carleen M. Hutchins (Catgut Acoust. Soc., 112 Essex Ave., Montclair, NJ 07042)

Six violins constructed from the wood tested by E. R. Fitzgerald and M. A. Hutchins, and the plates tuned by C. M. Hutchins as reported in the previous two papers, have been played by several competent performers whose comments will be discussed in relation to plate tunings and final tone and playing qualities. The six violins will be demonstrated by members of the Ottawa Symphony.

2:20

4pMU4. The monopole cello. Paul S. Veneklasen (Paul S. Veneklasen Res. Found., 1711 16th St., Santa Monica, CA 90404)

The conventional cello is one of the orchestral instruments whose sound is usually poorly projected from an orchestra, especially in its lower register. One of several reasons for this, in common with others of the string family, is that the foot of the bridge is connected to both top plate and back by a sound post constraining these points to vibrate in phase. As a result, in the lower frequency range, the instrument radiates as a bi-polar sound source. If the top plate and back can be coupled so as to vibrate in apposing phase, then the instrument would radiate as a mono-pole source with a potential substantial increase in sound output. This paper describes an exploratory attempt to induce such operation.

$$
\text { 2;35 }
$$

4pMU5. Experimental determination and theoretical prediction for the frequency compromise in bowed string and wind instruments. Xavier Boutillon and Charles-Auguste Faure (Lab. d'Acoust. Musicale, Boîte Courrier 161, 4 place Jussieu, 75252 Paris Cedex 05, France)

In musical instruments, eigenfrequencies of resonators (strings, pipes) are generally not strictly harmonic. When the oscillation is sustained by means of an external nonlinearity (bow,reed) the harmonic frequencies of the vibration cannot fully coincide with these eigenfrequencies. The notion of reactive power balance brings a new insight on the frequency compromise for a given spectrum. On the violin, flattening of the pitch when the bow pressure increases may be explained and quantitatively predicted by this sumrule [X. Boutillon, J. Acoust. Soc. Am. 90, 754-763 (1991)]. Measurements of the frequency compromise (without and with flattening) will be presented on a bowed string and compared with theoretical predictions. Experimental results shed light on one aspect of the rosin friction curve between the bow and the string. The same approach is relevant on wind instruments and might give information on the reed dynamical properties. [Work supported by CNRS and French Ministry of Culture.] 
3:05

4pMU6. Measurement of flow rate past a clarinet reed. Fang-Chu Chen and Gabriel Weinreich (Randall Lab. of Phys., Univ. of Michigan, Arn Arbor, MI 48109-1120)

The relation of the air flow through a clarinet mouthpiece to the pressure difference across it and the size of the read opening has been investigated by connecting the mouthpiece to an air reservoir that is pumped to a partial vacuum and allowed to fill through the mouthpiece. The pressure in the reservoir is measured ten times per second until atmospheric pressure is restored. In principle, the rate of change of pressure determines the rate of air flow, so that the desired relationship is obtained. One difficulty comes from an ignorance of the reservoir air temperature, since completely adiabatic conditions cannot be assumed. Another is that the rate of change of pressure is sometimes too large to assure internal equilibrium of the reservoir gas. There is a plan to eliminate the first difficulty by adding an independent temperature sensor, and to alleviate the second by using a larger reservoir. Preliminary measurements, including comparison with results in the literature, will be presented. [Work supported by NSF.]

\section{3:20}

4pMU7. Root locus equations for the vibrating diaphragm model of jet-pipe action. S. A. Elder (Phys. Dept., U.S. Naval Academy, Annapolis, MD 21402)

The vibrating diaphragm model of jet-pipe action described in an earlier paper [S. A. Elder, J. Acoust. Soc. Am. 96, 2411 (A) (1992)] has been extended by the development of root locus equations for laminar and turbulent half-jet resonators, which allow solution for the shear wave propagation constant at resonance, and estimation of the oscillation amplitude. The "force-drive" term in the pipetone system is shown to be associated with the inertia of the separated shear layer, with power being delivered to sustain the oscillation whenever the inertial force has a component in phase with sound particle velocity in the pipe mouth. Turbulent boundary layers thus produce corresponding larger driving force, for the same frequency and jet speed, since they tend to be thicker and so possess larger inertia. [Work partially supported by U.S. Naval Academy Research Council.]

\section{3:35}

4pMU8. Acoustics of a Chinese hand-exercise ball. Edward $R$. Mansell and Thomas D. Rossing (Phys. Dept., Northern Illinois Univ., DeKalb, IL 60115)

Pairs of steel balls, 3-4 cm in diameter, have been used in China as hand exercisers since the time of the Ming dynasty. Often a sounding device, consisting of a coil spring and a small steel ball, is placed inside the balls to produce a rather delicate tinkling sound as the balls are moved in circles in the hand. It is claimed that the balls can produce sounds "which are like the whistling of a dragon and singing of a phoenix." The sound spectrum shows many strong partials up to 10 kHz or higher. The most identifiable pitch of $\mathrm{G}_{\mathbf{g}}$ results from a partial at $773 \mathrm{~Hz}$

4pMU9. Scaling of bells. H. John Sathoff (Phys. Dept., Bradley Univ., Peoria, IL 61635) and Thomas D. Rossing (Northern Illinois Univ., DeKalb, IL 60115)

The fundamental frequency of a bell has roughly the same dependence on thickness and diameter as flexural vibrations in a circular plate, and thus in designing a bell chime or ensemble of bells, it would be possible, in principle, to scale all dimensions proportional to $1 / f$. Tonal considerations often dictate otherwise, however, and in practice different scaling laws are found in ensembles of large and small carillon bells, church bells, handbells, and ancient Chinese bells that have been studied.
4pMU10. Acoustics of bass handbells. Thomas D. Rossing and Edward R. Mansell (Phys. Dept., Northern Illinois Univ., DeKalb, IL 60115)

Handbells tuned to frequencies below $100 \mathrm{~Hz}$ radiate inefficiently, since their fundamentals lie several octaves below the coincidence frequency for bending waves. In order to obtain a higher radiation efficiency and thereby enhance the sound of bass handbells, Malmark has created a new bell design using aluminum rather than bronze. The acoustical properties of two $G_{1}$ bass handbells, one of alurninum and one of bronze, are compared. The aluminum bell radiates an enhanced fundamental, as expected, and a much stronger third harmonic as well. Both bells radiate a strong sixth harmonic, which is attributed to parametric radiation from the $(3,0)$ mode, which is tuned to three times the fundamental frequency [T. D. Rossing and H. J. Sathoff, J. Acoust. Soc. Am. 68, 1600 (1980)]. The aluminum bell not only gives an enhanced bass tone, but it is considerably lighter and easier to handle than the bronze bell.

\section{$4: 20$}

4pMU11. Acousties of Choirchimes ${ }^{\mathrm{m}}$. Gregory $H$. Canfield and Thomas D. Rossing (Phys. Dept., Northern Illinois Univ., DeKalb, IL 60115)

Tuning-fork resonators, useful in acoustics lecture demonstrations [T. D. Rossing, J. Acoust. Soc. Am. 92, 2401 (1992)], can vibrate in a wide variety of modes. The Malmark Choirchime ${ }^{\mathrm{TM}}$, consisting of a closed-end tuning-fork resonator with an attached handbell clapper, has become a popular musical instrument. The modes of vibration of two Choirchimes $^{\mathrm{TM}}$, tuned to $\mathbf{A}_{3}$ and $\mathbf{A}_{4}$, are described. The observed modes can be classified into five categories: parallel bending, symmetric bending, out-of-plane bending, parallel torsional, and symmetric torsional. The lowest symmetric bending mode radiates a strong fundamental, which is greatly enhanced by the resonance of the tube, resulting in a sound spectrum in which the fundamental dominates (the next partial is generally $20 \mathrm{~dB}$ or more below the level of the fundamental).

\section{$4: 35$}

4pMU12. Increasing the depth of field in holographic interferometry. Dell O. Fystrom (Phys. Dept., Univ. of Wisconsin, LaCrosse, WI 54601) and Thomas D. Rassing (Northern Illinois Univ., DeKalb, IL 60115)

Holographic interferometry is widely used to determine the modes of vibration in a wide variety of objects, such as musical instruments. Use of two or more object beams is a convenient and inexpensive way to increase the depth of field (or coherence volume) in holographic interferometry. This technique was used, for example, to make time-average holograms of the inside and outside surfaces of a vibrating drum shell simultaneously.

4pMU13. Developing a MIDI pitch detector. Youngmoo Kim and E. Carr Everbach (Dept. of Eng., Swarthmore College, Swarthmore, PA 19081-1397)

Recent interest in automated pitch detection [J. Acoust. Soc. Am. 92, 2428-2430 (A) (1992)] has led to develop a device whose purpose is to transcribe the musical lines of single-voice instruments (e.g., flute, trumpet) in a MIDI format using Apple Macintosh ${ }^{\mathbf{T}}$ computers and software. In contrast with other devices requiring rapid digital sampling of the audio signal and extensive computational and digital signal processing resources, this is an essentially analog electronic device based upon a phase locked loop and matched filter that strips off the higher harmonics of the instruments' waveform. The signal that results is nearly sinusoidal, allowing the use of the Macintosh's own modest built-in digital sampling capabilities for transcription of the musical line played on the instrument into a MIDI format. 


\title{
Session 4pPA
}

\author{
Physical Acoustics: Nonlinear Acoustics
}

\author{
R. Glynn Holt, Chair \\ Department of Mechanical Engineering, Yale University, New Haven, Connecticut 06520
}

\section{Contributed Papers}

$1 \cdot 30$

4pPA1. An experimental investigation of the nonlinear interaction of noncollinear sound beams. Ronald A. Roy (Appl. Phys. Lab., Univ. of Washington, Seattle, WA 98105) and Junru Wu (Univ. of Vermont, Burlington, VT 05405)

The nonlinear interaction between two pulsed, Gaussian ultrasonic beams propagating through degassed and filtered water was studied. One beam had a center frequency of $3.5 \mathrm{MHz}$ and a peak pressure amplitude (in the interaction region) of $450 \mathrm{kPa}$. The other possessed a center frequency and peak pressure amplitude given by $3.0 \mathrm{MHz}$ and $320 \mathrm{kPa}$, respectively. With the axes of the beams intersecting perpendicularly in the near-field, no sum-frequency scattered pressure amplitude greater than $0.03 \mathrm{~Pa}$ was detected outside the interaction region. The insertion of a brass sphere into the center of the interaction region resulted in the generation of two partially collinear scattered beams, and a sum-frequency scattered signal level of $0.9 \mathrm{~Pa}$ was subsequently detected at a $45 \pm 0.5^{\circ}$ scattering angle. Experiments with collinear beams generated using a single projector driven by two electrically summed tone bursts (center frequencies $=3.0$ and $3.5 \mathrm{MHz}$ ) indicate the production of sum and difference frequencies in quantitative agreement with Westervelt's theory for the parametric end-fire array [J. Acoust. Soc. Am. 32, 934A (1960)]. [Work supported by APL IR Funds.]

\section{1:45}

4pPA2. Acoustic nonlinearity parameter inversion from harmonic intergetion. T. J. Plona, S. Kostek, C. V. Kimball, and R. D'Angelo (Schlumberger-Doll Res., Ridgefield, CT 06877-4108)

This paper is concerned with the measurement of the acoustic nonlinearity parameter of fluids $(\beta=1+B / 2 A)$ using harmonic interactions of finite amplitude acoustic waves. The data consist of the on-axis pressure field emanating from a planar circular transducer, measured by a small probe. The source is excited by either a single frequency signal $(f)$ or by a composite signal of two frequencies $\left(f_{1}\right.$ and $\left.f_{2}\right)$. The attenuation of the fluid is measured independently by a standard linear wave propagation technique. The model for predicting the finite amplitude pressures is the Kuznetsov-Zabolotskaya-Khokhlov equation (KZK), which has been used extensively to model nonlinear acoustic beam fields. By taking the pressure measurements just beyond the Rayleigh distance, and providing that the sound pressure level is not too high such that the measurements are taken within one shock formation distance, a quasilinear approximation to the KZK equation is used, which is sufficiently accurate to represent the first-order interactions ( $2 f, f_{1}+f_{2}, f_{1}-f_{2}$, etc.) and computationally efficient, thus rendering the one-parameter inversion problem manageable. The accuracy of the inversion technique will be discussed for data consisting of both secondharmonic and difference frequency signals. Results for various fluids will be shown and discussed.

2:00

4pPA3. Absorption of sound by noise in one dimension. Bruce Denardo, Andrés Larraza, Anthony A. Atchley, and Steven Dorff (Phys. Dept., Code PH/De, Naval Postgraduate School, Monterey, CA 93943)
A special case of a prediction by O. Rudenko and A. Chirkin [Sov. Phys. JETP 40, 945-949 (1975)] is that a small-amplitude monofrequency sound wave will decay as a Gaussian as a result of interaction with finite-amplitude noise, if the waves are all moving in one direction. An experiment to verify this will be discussed. The apparatus includes a 21-m-long, 7-segment, thick-walled aluminum tube, with two highintensity compression drivers coupled to one end and an absorber at the other end. A simplified perturbative treatment of the problem, as well as possible applications, will be presented. The importance of this system as one in far-off equilibrium [A. Larraza, to be submitted to Phys. Rev. Lett.] will also be discussed. [Work supported by NPS Direct-Funding Program and ONR.]

\section{2:15}

4pPA4. Viscous effects in the acoustic levitation of gas bubbles. Darren L. Hitt and Andrea Prosperetti (Dept. of Mech. Eng., Johns Hopkins Univ., Baltimore, MD 21218)

Previous experimental work on the dynamics of acoustically levitated gas bubbles [Crum and Prosperetti, J. Acoust. Soc. Am. 73, 121127 (1983)] has shown a discrepancy between the theoretical and measured values of the levitation number, $L e=\rho g /|\nabla \rho|$, in the nonlinear regime of oscillation. In an attempt to reconcile these differences, the existing inviscid theory is extended to include the effects of viscous drag forces in the dynamic equilibrium of the bubble. [Work supported by NSF.]

4pPA5. Sonoluminescence spectra. Anthony A. Atchley, J. T. Carlson, D. Felipe Gaitan, S. D. Lewia, X. K. Maruyama (Phys. Dept., Naval Postgraduate School, Monterey, CA 93943), M. E. Lowry, M. J. Moran, and D. R. Sweider (Lawrence Livermore Natl. Lab., Livermore, CA 94550)

The spectra of picosecond sonoluminescence were measured with two separate instruments (a spectrometer/CCD detector system and a scanning monochrometer/PMT detector system) for bubbles levitated in water and in glycerin/water mixtures. Results of measurements made with the scanning monochrometer/PMT detector system, which required 10 to $15 \mathrm{~min}$ to acquire a complete spectrum, agree favorably with those published previously by Hiller et al. [Phys. Rev. Lett. 69, 1182 (1992)]. However, measurements made with the spectrometer/ CCD detector system, which required approximately $30 \mathrm{~s}$, indicate that the spectrum continually changes over this time scale, with no apparent changes in the input parameters. [This work was supported in part by the Defense Nuclear Agency and the Office of Naval Research. Portions of this work were performed under the auspices of the U.S. Department of Energy by the Lawrence Livermore National Laboratory under Contract number W-7405-ENG-48.]

\section{2:45}

4pPA6. Temperature distribution and heat transfer from the pulsating bubble in ultrasonic field. H. Yang, J. C. Ryu, H. Kwak (Mech. Eng. Dept., Chung-Ang Univ., Seoul 156-756, Korea), and J. Hong (Ohsan Tech. College, Ohsan 447-749, Korea) 
Gas bubble behavior immersed in liquid under a high-intensity ultrasonic field was studied by using a self-consistent model. In this model, the temperature distribution of the gas inside the bubble was obtained by solving analytically the continuity and energy equations for the gas. Also heat transfer between the gas inside the bubble and the surrounding liquid was considered by solving the mass, momentum, and energy equation for the liquid. With this model, the polytropic approximation is no longer required to calculate the internal pressure of the oscillating bubble under ultrasonic field. Under the periodic ultrasonic field with an amplitude of $110 \mathrm{kPa}$ and a frequency of $20 \mathrm{kHz}$, the temperature of air at the bubble center with equilibrium radius of $20 \mu \mathrm{m}$ reached as high as $5000 \mathrm{~K}$ at the time of bubble collapse. The heat flux from the bubble to the surrounding fluid by conduction is above 10 $\mathrm{MW} / \mathrm{m}^{2}$ at this stage. The pressure inside the bubble at this stage is about 10 bar, which is much less than that obtained by a previous model. Calculation results support the hot spot theory, which states that the phenomenon of sonoluminescence is due to chemiluminescence by the high temperature of gas inside the bubble.

\section{3:00}

4pPA7. The radial oscillations of a gas bubble in a surfactant solution. Yuren Tian, Xiaoyu Zheng, R. Glynn Holt, and Robert E. Apfel (Ctr. for Ultrason. and Sonics, Yale Univ., 2159 Yale Station, New Haven, CT 06520)

In a surfactant solution, a gas bubble can be covered by an adsorbed film of surfactant. When the bubble is excited into radial oscillations by a sound field, the surface concentration of the surfactant varies with the surface area and the mass interchange between the surface and the bulk liquid. As a result, the surface tension becomes a function of the bubble radius. Previous theoretical work on this subject [R. E. Glazman, J. Acoust. Soc. Am. 74, 980-986 (1983)] will be commented on and the equations governing the radial oscillations for this kind of gas bubble will be discussed. By numerically solving the equation under different initial bubble sizes, sound pressures, and driving frequencies, one can determine the effects of variable surface tension, surface viscosity, and surface mass transfer on the motions of the gas bubble. Thresholds of transient cavitation will also be estimated by including these effects. [Work supported by NASA through JPL, Contract No. 958722.]

\section{3:15}

4pPA8. The acoustic interaction forces between two fluid spheres in an acoustic field. Xiaoyu Zheng and Robert E. Apfel (Ctr. for Sonics and Ultrasonies, P. O. Box 2159, Yale Univ., New Haven, CT 06520)

An object in a sound field will scatter the incident wave and experience the so-called acoustic radiation force, which is a nonlinear time average effect associated with the incident wave. When two objects are present, the total acoustic radiation force on either object will include the interaction portions due to the other's scattered field of the primary wave. The acoustic interaction force between two fluid spheres in a plane wave field is investigated by calculating the force on one sphere, which is assumed to be small compared to the center distance between them. Using the method of Yosioka [K. Yosioka and Y. Kawasima, Acustica 5, 167-173 (1955)], a general formula for the radiation force on one sphere is derived that shows dependence on the resonant modes of the other. It is noted that in the radiation force expression there are cross terms involving the primary plane wave and scattered wave. For two small bubbles, this result is the same as the Bjerknes force expression that is obtained by others under the approximation of very long wavelength. Calculations are also done for the case of a standing wave field. [Work supported by NASA through JPL Contract No. 958722.]
3:30-3:45 Break

\section{3:45}

4pPA9. Ultrasonic four-wave mixing mediated by a suspension of microspheres in water: Comparison between two scattering theories. Harry J. Simpson (Naval Res. Lab., Code 7136, 4555 Overlook Ave. SW, Washington, DC 20375) and Philip L. Marston (Dept. of Phys., Washington State Univ., Pullman, WA 99164-2814)

Two ultrasonic pump waves are used to produce a grating in a suspension of 25- $\mu \mathrm{m}$-diam latex particles. A higher frequency ultrasonic wave is used to probe the established grating to produce ultrasonic Bragg scattering. The scattering depends strongly on the pump waves and is an unusual class of nonlinearity. A previously summarized model of the interaction [H. J. Simpson and P. L. Marston, J. Acoust. Soc. Am. 90, 2244 (A) (1991) and 91, 2351 (A) (1992)] uses an Epstein layer approximation and effective medium approximation. This previous analysis is compared with a Fourier series approximation of the grating and the resulting predicted scattered wave fields. The Fourier series expansion is only valid when the Bragg condition is satisfied, thus giving no information off the Bragg peak. The two models match at higher pump pressures and predict similar onset pressures, but differ slightly in the medium pressure region. Both results are compared to experimentally measured scattering amplitudes for a range of probe frequencies and pump pressures. [Work supported by ONR.]

\section{4:00}

4pPA10. Rayleigh wave nonlinearity. Mark F. Hamilton, Yuri A. Il'insky, and Evgenia A. Zabolotskaya (Dept. of Mech. Eng., Univ. of Texas at Austin, Austin, TX 78712-1063)

The elastic nonlinearity in Rayleigh waves is investigated theoretically. Studies have revealed that nonlinearity in Rayleigh waves involves nonlocal as well as local effects [Parker and Talbot, J. Elast. 15, 389 (1985)]. Finite amplitude effects on sound in fluids are entirely local, and the effects on different wavelets are therefore independent. In contrast, the effects of nonlocal Rayleigh wave nonlinearity on a given wavelet are influenced by the properties of the entire wave. A timedomain evolution equation for Rayleigh wave propagation on the surface of an isotropic solid reveals the existence of nonlocal nonlinearity [Zabolotskaya, J. Acoust. Soc. Am. 91, 2569-2575 (1992)]. In the present study, the nonlinear operator in the evolution equation is investigated numerically and analytically. Numerical results show the dependence of the nonlinearity on the global properties of the waveform. An explicit analytical expression derived for the nonlinear operator permits the local and nonlocal contributions to be easily distinguished. Local versus nonlocal effects on waveform distortion are discussed. [Work supported by the David and Lucile Packard Foundation and by ONR.]

\section{4:15}

4pPA11. Development of an algorithm for evaluating finite amplitude waves resulting from arbitrary periodic excitation. Jerry $\mathbf{H}$. Ginsberg (School of Mech. Eng., Georgia Inst. of Technol., Atlanta, GA 30332)

Without incorporation of weak shock theory or an explicit description of dissipation, a numerical evaluation of the distortion of finite amplitude planar waves generated by an arbitrary period excitation is extremely limited in its domain of validity. Prior deseriptions of such waves using weak shock theory have apparently been restricted to the case of initially harmonic signals. The present study describes a computational algorithm combining the small signal version of Earnshaw's solution with weak shock theory implemented in the form of the "equal area rule." The major steps in this algorithm are: (1) development of a nominal waveform at a specified location based on equal increments in the phase variable, (2) identification of (possibly multiple) shocks and the corresponding instants at which the equal area rule is satisfied, (3) 
coalescence of overlapping shocks associated with each of the harmonics contained in the initial signal, and (4) reconstruction of the shocked waveform using equal time increments in order to permit evaluations using FFT's. The presentation will describe the algorithm, and use a variety of test problems to illustrate the various manifestations of shocks.

4pPA12. Burgers' equation generalized for absorption obeying a power law. Thomas L. Szabo (Imaging Systems, Hewlett Packard, 3000 Minuteman Rd., Andover, MA 01810)

Burgers' equation applies to finite-amplitude waves propagating in a medium with absorption that has a quadratic frequency dependence. Numerical solutions of a modified Burgers' equation have been obtained in the frequency domain for other types of losses; however, a complete set of time domain nonlinear equations corresponding to power law attenuation has not been available. Power law attenuation is defined by the equation, $\alpha(\omega)=\alpha_{0}|\omega|^{y}$, where $\alpha_{0}$ and $y$ are arbitrary real constants, and $\omega$ is angular frequency. Blackstock [J. Acoust. Soc. Am. 77, 2050-2053 (1985)] has suggested that Burgers' equation could be generalized if an appropriate operator $L$ could be found such that the equation could become $p_{z}-L^{*} p=B p p_{\pi}$ where $p$ is pressure, $B$ is a constant, $\tau$ is delayed time, and the subscripts denote derivative operations. The $L$ operators have been derived based on a new causality principle and parabolic wave equations for power law loss. The resulting time domain equations extend Burgers' approach to finite amplitude propagation in media with arbitrary power law absorption.

4:45

4pPA13. On the instability of one-dimensional acoustic standing waves. Zinoviy A. Goldberg (1990 Ford Dr., \#1206, Cleveland, OH 44106)

The paper theoretically considers the instability of acoustical standing waves in a nondispersive medium in a plane-parallel layer excited at the border of the layer or by a distributed force. The instability is caused by the nonlinear resonance interaction that sets in motion the parametric temporal amplification of the spontaneously formed standing waves. Discussion will focus on the results of the deviation of the frequencies of the parametrically excited subharmonic waves from the natural frequencies of the layer. This phenomenon was noted by a number of investigators [L. Adler and M. A. Breazeale, J. Acoust. Soc. Am. 48, 10771083 (1970); N.-C. Yen, J. Acoust. Soc. Am. 57, 1357-1362 (1975); Z. A. Goldberg, in Problems of Nonlinear Acoustics, XIth Int. Symp. on Nonlinear Acoustics (in Russian), Part I (SO AN USSR, Novosibirsk, 1987), pp. 154-158]. It is shown that this phenomenon is not connected with the borders of the layer but it is peculiar to waves running in opposite directions that replaced the standing wave. The cause of these results is the instability of the standing waves to waves with deviated frequencies.

\title{
Session 4pPP
}

\section{Psychological and Physiological Acoustics: Complex Sound Processing}

\author{
Virginia M. Richards, Chair \\ Department of Psychology, University of Pennsylvania, 3815 Walnut Street, Philadelphia, Pennsylvania 19104
}

\section{Contributed Papers}

\section{1:15}

4pPP1. Pitch, masking, and Ohm's law for hearing. Jerry Malzan (14 Vermont Ave., Toronto, ON M6G 1X7, Canada)

Ohm's law for hearing asserts that psychological phenomenon of pitch can be identified with the physical phenomenon of frequency. More precisely, the assertion is that perceived pitches are precisely those appearing in the Fourier spectrum of a periodic vibration having all frequencies in the audible range. Experiment shows, however, that both parts of this if and only if statement are false. Pitches may arise without a corresponding frequency (the "pitch" problem) and pitches may be absent when there is a corresponding frequency (the "masking" problem). Here an attempt is made to deal with these problems simultaneously, using only the fact that the ear is a short-time analyzer, rather than the long-time analyzer that Ohm's law for hearing would require. The model used is quite simple, requiring only one parameter, and is in reasonable accord with experimental results. Residue pitch is computed in the time domain, while other pitches are computed in the frequency domain with the possibility, however, of masking. [Work supported by NSERC.]

\section{$1: 30$}

4pPP2. Offset dominance in pitch perception. Shigeaki Aoki (NTT Human Interface Labs., 1-2356, Take Yokosuka-shi, Kanagawa 238-03, Japan) and T. Houtgast (TNO Inst. for Perception, Soesterberg, The Netherlands)
Possible effects of onset or offset dominance for pitch information are investigated with the same experimental approach, which clearly showed the familiar onset dominance in the perception of binaural cues [Houtgast and Aoki, J. Acoust. Soc. Am. Suppl. 1 87, S64 (1990)]. A brief stimulus, with a duration of $5,10,20$, or $40 \mathrm{~ms}$, is subdivided in two parts with durations $T_{1}$ and $T_{2}$, each part consisting of a sinusoidal signal of either 1 or $2 \mathrm{kHz}$. The measuring paradigm aims at assessing the critical ratio $T_{1} / T_{2}$ for which both parts contribute equally to the overall sensation of pitch for these brief stimuli. This critical ratio is found to be greater than one, indicating a dominance of the pitch information presented in the later part of the stimulus. The data can be summarized in a weighting function: the perceptual weight of the pitch information as a function of time before signal offset. This weighting function shows a peak of enhanced weight for the last few milliseconds just before stimulus offset, in contrast with the enhanced weight for the first few milliseconds following stimulus onset as found previously in case of binaural cues.

\section{$1: 45$}

4pPP3. Serial position effects in temporal pattern discrimination. Toktam Sadralodabai, Robert D. Sorkin, and DeMaris A. Montgomery (Dept. of Psychol., Univ. of Florida, Gainesville, FL 32611)

Observers were presented with two successive 8-tone sequences; the task was to discriminate between the temporal patterns defined by the inter-tone intervals in each sequence (average inter-tone interval $=50$ 
ms, tone duration $=25 \mathrm{~ms}$, tone frequency $=1000$ or $2500 \mathrm{~Hz}$ ). The observers had to indicate whether the two patterns on each trial were the same or different. A conditional-on-a-single-stimulus (COSS) technique [B. C. Berg, J. Acoust. Soc. Am. 86, 1743-1746 (1991)] was used to evaluate the salience of each temporal position. The probability of responding DIFFERENT is computed given the magnitude of $\left|t_{1, i}-t_{2, i}\right|$, the absolute difference between the inter-tone intervals at each serial position $(i=1,2, \ldots, 7)$. The temporal information in the first and last positions had the greatest influence on the observer's response. These results have implications for models of temporal pattern discrimination. [Work supported by AFOSR.]

2:00

4pPP4. Spectro-temporal enhancement measured by loudness matching. Deborah A. Fantini (Dept. of Psychol., Essex Univ., Colchester CO4 3SQ, UK), Quentin Summerfield, and Alan R. Palmer (MRC Inst. of Hear. Res., University Park, Nottingham NG7 2RD, UK)

If one member of a series of equal-amplitude harmonics starts a few hundred milliseconds after the others, it may be "enhanced" and stand out perceptually. Viemeister and Bacon [J. Acoust. Soc. Am. 71, 15021507 (1982)] demonstrated that components produce more forward masking when enhanced than when present in a harmonic series without enhancement. Thus, enhancement appears to involve an increase in the internal level of a component. The present study was conducted to determine whether this result is also found in a loudness-matching task. Stimuli were derived from a harmonic series with a fundamental of 200 Hz. A 500-ms adaptor consisted of the series without the 1.8-, 2.0-, and 2.2-kHz components. A 100 -ms standard included the $2-\mathrm{kHz}$ component. Subjects heard the standard in the left ear followed by an identical 100 -ms sound (the "comparison") in the right ear. They matched the loudness first of the background components and then of the $2-\mathrm{kHz}$ component between the comparison and the standard. Preceding the standard by the adaptor reduced the loudness of the background components by $2 \mathrm{~dB}$ and raised the loudness of the 2-kHz component by 5 to $15 \mathrm{~dB}$ relative to the background components. Thus, loudness matching, like forward masking, demonstrates that enhancement increases the internal level of a component, but shows also that the levels of the background components are reduced.

\section{$2: 15$}

4pPP5. Detecting asynchrony of a single component with synchronous and asynchronous standards. Jan Zera and David M. Green (Psychoacoust. Lab., Psychol. Dept., Univ. of Florida, Gainesville, FL 32611)

Detecting asynchrony in either the temporal onset or offset of individual components in a multicomponent complex was investigated. A synchronous standard was constructed with all components having the same duration and starting and stopping simultaneously. The asynchronous standard was constructed by linearly delaying successive components of a complex, either at onset or at offet. The signal was created by altering the temporal position of a single component of the complex, either at onset or offset, relative to its temporal position in the synchronous or asynchronous standard. Thresholds were measured at onset or offiset both for harmonic signals and for complexes-in which the components were logarithmically spaced in frequency. For the onset condition in either the harmonic or logarithmic complex, the thresholds for detecting the asynchrony of a single component were greatly increased when the standard was asynchronous as opposed to synchronous. For the offiset condition, the thresholds were not greatly affected by whether the standard was synchronous or asynchronous. [Research supported by NIH, AFOSR, and a Fulbright fellowship.]

\section{2:30}

4pPP6. Decision rules in spectral-shape discrimination with and without signal uncertainty. Huanping Dai (Psychoacoust. Lab. Psychol. Dept., Univ. of Florida, Gainesville, FL 32611-2065)
In typical profile-detection tasks there is little difference, about $3 \mathrm{~dB}$ in signal-to-standard ratio, between signal threshold when the signal frequency is fixed (signal known) and when the signal frequency is randomly selected on each trial (signal unknown). For an ideal observer, this difference should be $8 \mathrm{~dB}$ when using 21 components. One hypothesis about the discrepancy between the human and ideal observer is that, for the human observer, the central noise (e.g., fluctuations of the decision criterion) is much greater than the peripheral noise. This assumption can account for both the slope of the psychornetric function obtained in conditions when the signal frequency is known and the relatively small threshold difference between the signal-known and signal-unknown conditions. [Work supported by NIH.]

\section{2:45-3:00 Break}

4pPP7. Stimulus-oriented model for the discrimination of Gaussian noise samples. Martin E. Rickert and Donald E. Robinson (Dept. of Psychol., Indiana Univ., Bloomington, IN 47405)

Consider a two-interval same-different task in which listeners are asked to discriminate between trials on which a sample of noise is presented twice and trials on which two different samples are presented. Previous research has used this method to determine the effects of a wide range of stimulus conditions including temporal location of an uncorrelated segment, duration, bandwidth, correlation, and overall level [T. E. Hanna, Percept. Psychophys. 36, $409-416$ (1984); S. F. Fallon and D. E. Robinson, J. Acoust. Soc. Am. 92, 2630-2635 (1992)]. The data indicate that discriminability is best when changes occur at the end of the sample and that the ratio of the duration of the target segment to the total duration is independent of overall duration. In this paper, a stimulus-oriented model for discriminating samples of Gaussian noise is developed. The model is a derivative of the equalization-cancellation theory of binaural masking level differences [N. I. Durlach, J. Acoust. Soc. Am. 35, 1206-1218 (1963)] and of Jeffress' models of monaural masking [L. A. Jeffress, J. Acoust. Soc. Am. 44, 187-203 (1968)]. This approach begins with a cancellation stage in which the waveforms from the two intervals are jittered in amplitude of differenced. Then, the power of the difference waveform is approximated in a manner similar to Jeffress' leaky integration. Predictions of the model will be compared to the data of Fallon et al. [Work supported by AFOSR.]

\section{3:15}

4pPP8. Profile analysis in the spectro-temporal domain. T. Houtgast and Adelbert W. Bronkhorst (TNO Inst. for Perception, P. O. Box 23, 3769 ZG Soesterberg, The Netherlands)

In this study on energy profile discrimination, the basic sound consists of five Gaussian-shaped tone pulses (wavelets). The choice of the timing and the frequencies of the individual wavelets is such that they are always arranged in a linear array in the $\log (f)-t$ spectrogram, with the orientation of the array as an important parameter (horizontal, vertical or any oblique orientation). An additional parameter of these five-wavelets arrays is the mutual separation between the wavelets. For a given orientation and separation, the reference sound contains all five wavelets with equal energy, while in the target sound the (relative) energy of the middle wavelet is reduced: The just-detectable decrement of the middle wavelet is determined with a standard adaptive 2AFC procedure, including a roving-level paradigm. This form of profile analysis with the present five-wavelets arrays shows that (1) the performance is better with a purely temporal pattern (horizontal array) than with a purely spectral pattern (vertical array), and (2) for some specific oblique orientations of the array the performance is extremely poor, with just-detectable decrements of the middle wavelet of the order of 20 dB. 
4pPP9. Discrimination of broadband, multi-component, common-envelope signals. J. N. Ananthraraman, Ashok K. Krishnamurthy (Dept. of Elec. Eng., Ohio State Univ., Columbus, OH 43210), and Lawrence L. Feth (Ohio State Univ., Columbus, $\mathrm{OH} 43210$ )

The EWAIF model was based on the listener's ability to discriminate between narrow bandwidth, two-component complex tones. These tones shared a common envelope as described by Voelcker [Proc. IEEE 54 (1966)]. Later, the EWAIF model was applied to multi-component signals such as those used in profile analysis. Those signals were neither narrow bandwidth nor common envelope signals; however, the EWAIF model predicted a constant difference in pitch for just-detectable increments to the center component of otherwise flat-spectrum signals. Recently, Versfeld and Houtsma [Q. J. Exptl. Psychol. 43 (1991)] have applied to EWAIF model to a number of periodic signals, some of which are broadband. They report the failure of the model to predict the discriminability of these signals. Versfeld and Houtsma did not ensure that their test signals were common envelope pairs. Thus, the addition of envelope differences to their signals may have given their listeners additional cues upon which they could base discrimination. Signal pairs have been defined that are similar to those used by Versfeld and Houtsma, but that have common envelope properties. Recently the EWAIF model have been reformulated into what is better called an intensityweighted average of instantaneous frequency (IWAIF) model. In this presentation, the relationship between EWAIF and IWAIF model predictions for discrimination of relatively simple, periodic signals are demonstrated and then the IWAIF model is applied to the discrimination of broadband complex signals. [Work supported by AFOSR.]

\section{$3: 45$}

4pPP10. Short-term IWAIF model for frequency discrimination. Ashok K. Krishnamurthy and Lawrence L. Feth (Dept. of Elec. Eng. \& Div. of Speech and Hear. Sci., Ohio State Univ., Columbus, OH 43210)

The intensity/envelope weighted average of instantaneous frequency (IWAIF/EWAIF) has been used to model listener perfornance in complex signal discrimination [Feth, J. Acoust. Soc. Am. Suppl. 188, S48 (1990)]. Previous work with this model has used the entire signal duration for the IWAIF computation. However there exist pairs of signals such as the STEP and GLIDE signals used by Madden and Feth [Feth et al., J. Acoust. Soc. Am. Suppl. 1 86, S122 (1989); Madden and Feth, J. Speech Hear. Res. 35, 436-442 (1992)] that have the same long-term IWAIF values, but are nonetheless readily discriminable by listeners. The addition of a short-term running IWAIF computation extends the application of the IWAIF model to such dynamic signals. Also described is an ideal detector for the short-term IWAIF model that assumes that independent, white, Gaussian internal noise is added after the IWAIF computation. The noise variance is derived from frequency DL (FDL) data. It is shown that this detector is able to predict listener performance in the STEP versus GLIDE discrimination task. Applica- tions of the short-term IWAIF model in deriving frequency modulation DL (FMDL) from the FDL are also presented. [Work supported by AFOSR.]

4:00

4pPP11. Intermediate detection of changes in modulation and power spectra. Virginia M. Richards and Mark Ian Sanderson (Dept. of Psychol., 3815 Walnut St., Univ. of Pennsylvania, Philadelphia, PA 19104)

In an effort to examine the shift from modulation-based discrimination for narrow-band complex stimuli to across-frequency spectral comparisons for wideband complex stimuli, the mutual discriminability of 11 stimuli was determined. The five "base" stimuli were the sum of three tones. By altering the relative phases of the tones, modulation depths ranging from maximum (SAM) to minimum (QFM) were generated using five equal log steps. The central frequency was $1000 \mathrm{~Hz}$, and modulation rates ranged from 20 to $240 \mathrm{~Hz}$. Three additional stimuli had envelopes identical to three of the base stimuli, but had periodic fine structures. Three other stimuli had fine structures identical to three of the base stimuli, but no envelope modulation. For observers tested thus far, where the modulation rate was $20 \mathrm{~Hz}$, discriminability is well described as being based on changes in modulation depth. Where the modulation rate was $240 \mathrm{~Hz}$, the discrimination appears to depend on changes in long term power spectra. For intermediate modulation rates, the data do not appear to reflect a gradual change from modulationbased detection to power spectrum-based detection, but the intervening cues appear not to be well defined by our stimuli.

\section{$4: 15$}

4pPP12. Critical bands for envelope cues. Bruce G. Berg, Curt Southworth, and Matthew Turner (Dept. of Cognitive Sci., Univ. of California, Irvine, CA 92717)

Previous studies show that temporal envelopes can be used to discriminate complex sounds. This study estimates the effective bandwidth over which the auditory system extracts an envelope. Listeners discrim. inate a stimulus consisting of $n$, equal-intensity tones centered at 1000 $\mathrm{Hz}$, from a stimulus with an intensity increment of the $1000-\mathrm{Hz}$ tone. A roving level procedure is used to degrade absolute intensity cues, while variation of the digital-to-analog conversion rate degrades pitch cues. Theoretically, only envelope cues are preserved. Across conditions, tones are equally spaced with separations of $10,20,40$, or $80 \mathrm{~Hz}$. Within conditions, the number of tones varies from 3 to 41 . Thresholds increase as the number of components increases until a certain bandwidth is reached, after which a "Fletcher-type" breakpoint is observed with thresholds either decreasing or remaining constant. For individuals, the bandwidth at the breakpoint is roughly the same across conditions. Sizable individual differences are found, ranging from a factor of 1.5 to 4 greater than critical bandwidths estimated from tone-in-noise detection tasks. A model based on the power spectrum of the envelope is evaluated. [Work supported by ONR.] 


\title{
Session 4pSA
}

\author{
Structural Acoustics and Vibration: Numerical Methods and Experimental Techniques
}

\author{
Jerry H. Ginsberg, Cochair \\ School of Mechanical Engineering, Georgia Institute of Technology, Atlanta, Georgia 30332 \\ Wayne T. Reader, Cochair \\ Vector Research Company, Inc., 6903 Rockledge Drive, Suite 1200, Bethesda, Maryland 28817
}

Contributed Papers

1:30

4pSA1. External system modeling: An approach to minimizing vibroacoustic model sizes for design evaluation. B. K. Gardner and J. S. Bolton (1077 Ray W. Herrick Lab., School of Mech. Eng., Purdue Univ., West Lafayette, IN 47097-1077)

Numerical vibroacoustical models of dynamic systems can easily become so large that they are impractical when used to analyze alternative system designs. Hence, when evaluating designs for single subcomponents of a large system, the analysis is often limited either to that sub-component combined with idealized boundary conditions, or to the sub-component and a small portion of the rest of the system (i.e., the exterior system). In this paper a technique, based on the receptance method, is presented that allows a model of the complete system to be replaced by a complete model of the sub-component combined with a minimal model of the exterior system. Thus the in situ dynamics of a particular sub-component may be evaluated without having to solve the entire system. This approach allows sub-component designs to be assessed quickly so that the merits of many potential designs may be evaluated. The assumptions required by this approach are: steady-state conditions, linearity, and that the forces acting on the external system are unaffected by changes in the sub-component. The external system model may be obtained either numerically or experimentally. The proposed approach is demonstrated here analytically using a system of beam-like elements.

\section{1:45}

4pSA2. Analysis of vibration transmission through joints using component modes. Jerry E. Farstad and Rajendra Singh (Dept. of Mech. Eng., Ohio State Univ., 206 W. 18th Ave., Columbus, OH 43210)

In many cases, the most practical approach for reducing noise in built-up machine assemblies is to reduce the vibration transmitted through joints to the machine housing as much as possible. In this paper, a new analysis framework for estimating the vibration transmitted among machine components joined at discrete locations in vibrating assemblies is presented. The method is based on a recently developed modal synthesis approach. Natural frequencies and modes of the disassembled components subject to specific boundary conditions applied at the joint locations are used to compute the natural frequencies and modes of the assembly from a variational formulation. Lagrange multipliers are used to enforce the constraints that motions of components at joint locations be identical. The modal properties of the assembly are then used to compute its forced response. Since the Lagrange multipliers used to enforce the constraints represent the interfacial joint forces, evaluating these from the forced response provides an efficient way of computing the dynamic power transmission among components, which is probably the best measure of transmitted vibration. In addition to theory, a simple example of an assembly of two lumped parameter systems is included.
2:00

4pSA3. Estimation of dynamic forces via modal decomposition of operational deflection shape measurements. David C. Warwick (Hull Struetures and Acoust. Branch, Carderock Div., Naval Surface Warfare Ctr., Bethesda, MD 20084) and John J. Gilheany (Catholic Univ. of America, Washington, DC 20064)

The results of modal analysis are used to derive a set of modal filters that are applied to operational deflection shape measurements to estimate modal contributions and physical forces for structures subject to multiple, correlated and uncorrelated, external excitations. Singular value decomposition is applied to measured cross spectral density functions to decompose response measurements into contributions associated with each independent external excitation. Generalized and physical forces, coherent to each independent source, are determined from estimated modal parameters. The approach is demonstrated for single and multiple, correlated and uncorrelated, external excitations.

\section{2:15}

4pSA4. A simple test of the modal strain energy method. J. Gregory McDaniel (BBN Systems and Technol. Corp., 10 Moulton St., Cambridge, MA 02138) and Jerry H. Ginsberg (Georgia Inst. of Technol., Atlanta, GA 30332)

Nashif et al. [Vibration Damping (Wiley, New York, 1985)] present a "modal strain energy method" (MSEM) that has seen increased use in the finite element community to estimate modal loss factors in elastic-viscoelastic structures. The method enables one to predict dynamic response without explicitly accounting for hysteretic damping in the free vibration analysis. Despite its wide usage, there does not seem to have been prior formal analyses of its validity. The presentation will report such a test of MSEM predictions for a two-degree-of-freedom system in which the two masses are connected by dissipative and elastic elements. The evaluations explore the dependence of MSEM on basic parameters characterizing stiffness, inertia, and damping. The main conclusion is that MSEM is very accurate if dissipation within the viscoelastic substructure is sufficiently low. Raising either the inertia or stiffness of this part of the system seems to enhance the accuracy of MSEM for a fixed value of its damping factor.

\section{2:30}

4pSA5. Quantum chaos methods applied to high-frequency plate vilurations. O. Legrand (Lab. de Phys. de la Matière Condensée, CNRS URA 190, Faculté des Sci., Parc Valrose, B.P. 70, 06108 Nice Cedex 02, France and X-RS, Parc-Club, 91893 Orsay, France), C. Schmit (Div. of Phys. Theor., IPN, Orsay, France and X-RS, Pare-Club, Orsay, France), and D. Sornette (Lab. de Phys. de la Metière Consensée, CNRS URA 190, Nice, France and X-RS Parc-Club, Orsay, France)

The first investigation of the quasiclassical regime of thin plate vibrations in the high-frequency regime is presented. Using an efficient 
numerical scheme to compute many eigenfrequencies of a stadiumshaped plate, it is found that the distribution of level spacings and the spectrum rigidity follow the GOE (Gaussian orthogonal ensemble) random matrix statistics. An asymptotic equivalence between the spectrum of a clamped plate and that of a membrane with a mixed boundary condition is derived. Also, examples of eigenmodes that present scars are present, and their quantization is given. [This work has been published in Europhys. Lett. 18, 101-106 (1992).]

\section{2:45}

4pSA6. Sound pressure field into reverberant chamber containing structures excited acoustically. Hugues Nélisse, Alain Berry, F. Charron, and Jean Nicolas (G.A.U.S., Mech. Eng. Dept., Univ. de Sherbrooke, Sherbrooke, PQ JIK 2R1, Canada)

A method has been developed to obtain the sound pressure field for all points in a large reverberant chamber whether or not it contains a flexible structure excited acoustically by a so-called diffuse field. The approach is based on an integral equation method for the pressure in the chamber. The Helmholtz-Kirchhoff theorem is expressed using a pressure jump function across the surface of the structure [see, for instance, D. Ouellet et al., J. Acoust. Soc. Am. 89, 2131-2139 (1991)]. A modal expansion over modes of rigid cavity and in-vacuo modes of the structure is used. It leads to a set of linear coupled equations reflecting the existence of a fluid-structure interaction. Numerical resolution of the set of equations allows one to obtain physical quantities of interest, such as the distribution of pressure on each side of the panel and consequently the jump of sound pressure across the structure. Convergence of the solution, as well as its frequency range of validity, will be discussed.

\section{3:00-3:15 Break}

\section{3:15}

4pSA7. Structural acoustic modeling using a new high accuracy acoustic infinite element. David S. Burnett (Rm. 1A-254, AT\&T Bell Labs., Whippany, NJ 07981)

This new 3-D time-harmonic acoustic infinite element uses a truncated form of the exact asymptotic series solution for general scattered and/or radiated fields, providing an accurate approximation all the way to infinity. The infinite element and a companion fluid-structure coupling element have symmetric matrices that fit seamlessly into any structural finite element code, providing a structural acoustic capability as versatile and powerful as the structural code itself. Most important, this approach is several orders of magnitude faster, for the same accuracy, than the traditional and almost universally used boundary element method (BEM) for the acoustics, i.e., the surface Helmholtz integral equation, which the author also developed and used for several years. The reason is that the bandwidth of the coupled system equations is much smaller for infinite elements (being a domain method) than for the BEM. Experience with both methods has revealed that the severe computational inefficiency of the BEM limits practical problems to low frequencies and simple structures, whereas this infinite element approach can handle the full range of frequencies and structural complexities that are encountered in purely structural analyses, at little additional cost.

\section{3:30}

4pSA8. Numerical predictions of acoustic radiation by unbaffled, finite plates from non-intrusive velocity measurements. Todd $L$. Harbold and Courtney B. Burroughs (Appl. Res. Lab., Penn State Univ., P.O. Box 30, State College, PA 16801)

The spatial distribution of the amplitude and phase of the velocity of vibrating rectangular flat plates is measured using a scanning laser Doppler vibrometer. These measurements are conducted in a nonanechoic environment with the plate in air and water, driven by a single shaker at frequencies of resonance and nonresonance. Using free-field Green's functions and fast Fourier transform (FFT) algorithms, the free-field acoustic radiated field for the unbaffled plate is determined. In order to compute the acoustic radiation, the velocity field must be specified in the plane of the plate, both on and off the surface of the plate. Therefore, an iterative process is used to determine the velocity field of of the plate surface. Descriptions of the plate vibration measurements and algorithms used to compute the free-field acoustic radiated field are presented and discussed. Examples of results of measurements conducted at and off resonance are given.

3:45

4pSA9. Simultaneous measurements of in-plane and out-of-plane surface displacements of shell vibrations at several points by an array of miniature laser probe heads. Ming Yang. Yves H. Berthelot, and Jacek Jarzynski (School of Mech. Eng., Georgia Inst. of Technol., Atlanta, GA 30332)

Experiments are being conducted to measure directly by laser Doppler interferometry the in-plane and out-of-plane components of the surface motion of a vibrating shell simultaneously at several surface points. The proposed design requires three Bragg cells to generate three coherent optical beams with different carrier frequencies. Three $1 \times 4$ fiber optic connectors are used to transmit each beam to four surface points. At each point, both the out-of-plane component and one of the in-plane components of the displacement vector are measured. The light scattered by the vibrating surface is Doppler shifted and collected into a large core multimode fiber situated close to the surface and terminated by a photodiode. The signals are acquired and digitized for direct phase demodulation on a computer by means of Hilbert transforms. [Work supported by ONR.]

\section{4:00}

4pSA10. Direct measurements of axial and circumferential in-plane surface displacements of shell vibrations with an automated scanning laser Doppler interferometer. Hyun-Gwon Kil and Jacek Jarzynski (School of Mech. Eng., Georgia Inst. of Technol., Atlanta, GA 30332)

The surface displacement of a vibrating shell has been directly measured by laser interferometry. The system is designed to measure the two in-plane components of the surface displacement: the axial and the circumferential components. The system is completely automated for scanning around the cylindrical shell both circumferentially and axially, for focusing at each point until the detected signal is stable and sufficiently above the noise level, and for recording and transferring the data to a computer. The data are analyzed in the frequency-wave-number domain with fast Fourier transforms and Prony decomposition to separate the extensional waves (fast waves, low wave numbers) from the flexural waves (slow waves, high wave numbers). The shell under consideration is $0.94 \mathrm{~m}$ long and $1.6 \mathrm{~mm}$ thick. It is freely suspended in air and it is excited radially by a shaker at various frequencies up to twice the ring frequency which is about $10 \mathrm{kHz}$. The optical probe head is very compact (about 4 by $4 \mathrm{~cm}$ ) and it is designed to take full advantage of optical fiber technology. [Work supported by ONR.]

\section{4:15}

4pSA11. Experimental investigation of vibration excitation for structural sound radiation study. Dany Couture, Yvan Champoux, and Claude Lesage (G.A.U.S., Mech. Eng. Dept., Univ. de Sherbrooke, Sherbrooke, PQ JIK 2RI, Canada)

Over the last decade, several models have been proposed for modeling the sound radiation of vibrating structures. These analytical or numerical models are frequently considered for simple or semi-complex structures such as plates and shells. They were sometimes validated through simple experiments and the theories were often judged valid when only trends between theory and experimental results were obtained. The discrepancies were almost systematically associated with the limitations of the experimental set up. In order to use experimental results as a basis to challenge or validate the theories, accurate results must be obtained. The precise measurement of the structure excitation 
is deemed necessary. Laterally flexible rods are often used between the shaker and the structure to avoid the introduction of unwanted excitations or the inadvertent modification of the structure. A load cell that measures moments and longitudinal force has been developed and was used to study the influence of different excitation configurations. Alignment must be taken into account. The use of flexible rods will be examined and useful guidelines will be established.

\section{$4: 30$}

4pSA12. Mode localization phenomenon analysls on infinite ribbed plates. Ten-Bin Juang, Anna L. Pate, and Alison B. Flatau (Dept. of Aerosp. Eng. and Eng. Mech., Iowa State Univ., Ames, IA 50011)

An analysis of the mode localization phenomenon is demonstrated for disordered structural systems of infinite ribbed plates in air and in water. While for infinite ordered ribbed plates there exist frequency passbands for which vibrations propagate extensively throughout the structure, the introduction of disorder disables the propagation of vibrations to arbitrarily large distances and confines vibrations within a region close to the excitation force. Based upon the mathematical model built in a paper to be published, inertance transfer functions and vibrational responses due to line force excitation are implemented to investigate the passband and stopband characteristics and the localization phenomenon of multiple-rib plates in air and in water. Two facts are confirmed. First, water provides an energy path that reduces the decoupling effect between the bays of ribbed plates observed in the presence of air and consequently, diminishes the localization phenomenon. Second, passbands can merge at certain frequencies, as determined by rib size and dynamic properties, but have nothing to do with the number of ribs or the transmission span. For completeness, acoustic responses are presented for some specific cases of the structural system as well.

THURSDAY AFTERNOON, 20 MAY 1993

CANADIAN ROOM, 1:00 TO 5:00 P.M.

\title{
Session 4pSP
}

\section{Speech Communication: Consonant Perception and Other Topies in Speech Communication (Poster Session)}

\author{
Margaret F. Cheesman, Chair \\ Department of Communicative Disorders, University of Western Ontario, London, Ontario N6G 1H1, Canada
}

\section{Contributed Papers}

\begin{abstract}
All posters will be on display from 1:00 to 5:00 p.m. To allow contributors an opportunity to see other pasters, contributors of odd-numbered papers will be at their posters from 1:00 to 3:00 p.m. and contributors of even-numbered papers will be at their posters from 3:00 to 5:00 p.m.
\end{abstract}

4pSP1. An exploration of phoneme structure and models of classification for place of articulation. Xiaofeng $\mathrm{Li}^{\mathrm{a}}$ and Jennifer $\mathrm{L}$. Cho (Ctr. for Cognitive and Psycholinguistic Sci., Binghamton Univ., Binghamton, NY 13902-6000)

Speech perception research has typically investigated phoneme categories and specified trading relation effects in terms of limits defined along single dimensions. However, phoneme categories vary along a number of significant physical dimensions, with stimuli distributed across the multidimensional perceptual space often differing in goodness of category membership. The current study used a set of synthetic $\mathrm{CV}$ syllables based on F2-and F3-onset frequencies to examine the internal structure of several phonetic categories within the same vowel context. Experiment 1 used a free classification task to establish the perceptual structure of categories differing in place of articulation. Experiments 2, 3 , and 4, respectively, related response times of a speeded classification task with category goodness ratings and a multidimensional phonetic perceptual space based upon similarity ratings. The multidimensional space was quite distinct compared to a prior space obtained for a different vowel context. Results are discussed in terms of prototype and exemplar models of phonetic classification. [Research supported in part by grants from NSF and AFOSR to R. E. Pastore, and NIH.] ${ }^{\text {a) Now at }}$ Indiana University.

4pSP2. Trading relations; discrete versus continuous perception. Richard Wright (Dept. of Linguistics, UCLA, Los Angeles, CA 90025-1543)

The results of experiments on trading relations have been interpreted as evidence for theories that speech perception is discrete, such as the motor theory [e.g., Libermann and Mattingly, 1985]; however, the experimental stimuli have not tested the predictions of theories of con- tinuous perception [e.g., the FLMP, Massaro, 1987]. The present study addressed this issue using a pair of experiments that use a "slit-split" stimulus continuum varying in $/ \mathrm{p} /$ burst amplitude, rather than formant transitions, along the spectral dimension and varying in preburst silence duration along the temporal dimension. Experiment 1 was a 21AX task in which subjects responded "same" or "different" to ten blocks of stimuli that were presented in pairs with a $300 \mathrm{~ms}$ ISI and a 1000-ms inter-block interval. Experiment 2 was a forced choice identification task in which subjects responded "slit" or "split" to ten blocks of stimuli that were presented singly with a $1000 \mathrm{~ms}$ ISI. A trading relation occurred between the amplitude of the burst and the duration of the silence. This finding is incompatible with a theory in which a burst is absent or present.

4pSP3. Perceptual weight of acoustic cues for voicing in French [t, d]: Population differences. Thomas R. Sawallis (IASCP, 63 Dauer, Univ. of Florida, Gainesville, FL 32611)

An experiment is presented concerning perception of edited consonant length, voscing amplitude, and burst +aspiration amplitude in a corpus of natural tokens of French intervocalic [t, d]. The editing is designed using the statistical distributions of those cues as previously measured in the selfsame corpus. This design largely preserves the natural variation of the nontarget cues, and is an advantage over the traditional use of synthesis or prototype based series. Then, mathematical tools borrowed from signal detection theory are used on the perceptual test results to derive sensitivity measurements for the cues. Measurements of this type can be compared autonomously, without trading relationship-style equivalences, across cues, environments, languages, etc., and this property makes them amenable to use not only in speech perception, but also in formal phonology and general linguistics. In this paper, they are used to explore differential use of cues of listener groups 
based on native language (French versus other native languages) and on linguistic training ("naives" versus phoneticians, linguists, language teachers, etc.).

4pSP4. An acoustic analysis of the effects of cue deletions on the perception of stop consonants. Roberto Guadagno and Donald G. Jamieson (Hear. Health Care Res. Unit, Elborn College, Univ. of Western Ontario, London, ON N6G 1H1, Canada)

Cue deletion was used to examine the contribution of various acoustic cues to the identification of the English stop consonants $(/ \mathrm{b} /, / d /$, $/ \mathrm{g} /, / \mathrm{p} /, / \mathrm{t} /, / \mathrm{k} /)$. Stimuli were based on tokens spoken by four talkers (two men and two women) in a syllable-medial context $(/ \mathrm{A}$ Cil $/$ ). Cues within five regions centered on the burst were examined: (1) the transition from the preceding vowel into consonant closure, (2) the closure interval, (3) the consonant release, (4) the initial transition from release to the following vowel, and (5) the final transition to the vowel. Each region either remained at the full normal amplitude or was attenuated fully (i.e., effectively replaced by silence). Four listeners who had normal hearing identified the 768 signals constructed from each of the possible combinations of attenuated and full-amplitude regions (i.e., 32 combinations $\times 6$ stimuli $\times 4$ speakers). Analyses of these identification responses demonstrates the relative importance of each region, and of each combination of regions, for consonant identification. Acoustic measures explain some aspects of the confusion errors which occurred.

4pSP5. Selective adaptation of context-conditioned voiceless fricatives. Margaret F. Cheesman and Kristina G. Greenwood (Dept. of Commun. Disord., Univ. of Western Ontario, London, ON N6G 1H1, Canada)

Two fricative-vowel continua were synthesized. One ranged from $/ \mathrm{si} /$ to $/ \mathrm{ji} /$ and the other from / su/ to / $\mathrm{u} /$. Listeners' identification of the consonant portion of the syllables, as either "s" or "sh," depended on both the frequency of the frication noise and on the vowel quality. Selective adaptation of these continua was investigated in three experiments. In experiment 1 , the endpoints of the continua were used as adaptors, and the identification boundary shifted towards the adapting stimulus. In experiment 2 , ambiguous frication noises (that were identified as $/ \mathrm{s} /$ before $/ \mathrm{u} /$ and $/ \mathrm{j} /$ before the $/ \mathrm{i} /$ ) adapted the identification boundary in opposite directions, depending on which of the two vowels followed the noise and, therefore, on the perceptual identity of the consonant. In experiment 3 , the isolated $/ i /$ and $/ u$ / vowels were demonstrated to be ineffective as adaptors. The selective adaptation effects observed in these experiments were not determined by the acoustical information in the consonant or the vowel alone, but rather the contextconditioned percept of the fricative. [Work supported by NSERC.]

4pSP6. Multidimensional scaling of the native language prototype effect. Paul Iverson and Patricia K. Kuhl (Dept. of Speech and Hear. Sci., WJ-10, Univ. of Washington, Seattle, WA 98195)

Previous experiments with human adults and infants have demonstrated that variants of the vowel $/ i$ / are more difficult to discriminate from a prototypic $/ i /$ than from a nonprototypic $/ i /$, suggesting that prototypes assimilate neighboring stimuli [P. K. Kuhl, Percept. Psychophys. 50, 93-107 (1991)]. A series of experiments examined this influence of prototypes using multidimensional scaling. Adults discriminated pairs of $/ \mathrm{i} /$ tokens and RTs were measured. Multidimensional scaling was used to place the tokens in a Euclidian space where pairs of tokens with long RTs were close in the space and pairs with short RTs were far apart. Tokens were more closely spaced near the prototypic $/ i /$, demonstrating that prototypes assimilate proximate regions of the vowel space. This effect was stronger with long ISIs $(2500 \mathrm{~ms})$ than with short ISIs ( 25 and $250 \mathrm{~ms}$ ), suggesting that vowel prototypes distort memory for individual tokens. [Work supported by NIH.]

4pSP7. Training Japanese listeners to identify English $/ r /$ and $/ 1 /: A$ replication and extension. David B. Pisoni, Scott E. Lively (Speech Res. Lab., Dept. of Psychol., Indiana Univ., Bloomington, IN 47405), Reiko A. Yamada, Yoh'ichi Tohkura (ATR Human Information Processing Res. Labs., Kyoto, Japan), and Tsuneo Yamada (Osaka Univ., Osaka, Japan)
Monolingual native speakers of Japanese were trained to identify English $/ \mathrm{r} /$ and $N$ using a modified version of Logan, Lively, and Pisoni's [J. S. Logan et al., J. Acoust. Soc. Am. 89, 874-886 (1991)] high variability training procedure. Both the talker's voice and the phonetic environment were varied during training. Subjects improved in their ability to identify $/ \mathrm{r} /$ and $/ \mathrm{l} /$ from the pre-test to the post-test and during training. Generalization accuracy depended on the voice of the talker producing the $/ r /-\Lambda /$ contrasts: Subjects were significantly more accurate when words were produced by a familiar talker than when they were produced by an unfamiliar talker. Three months after the conclusion of training, subjects were given the post-test and the tests of generalization again. Surprisingly, accuracy decreased only slightly on each test, even though no training or exposure to $/ \mathrm{r} /$ and $/ /$ occurred during the 3-month interval. These results demonstrate that the high variability training paradigm is effective in modifying nonnative listeners' phonetic perception and that these changes are extremely robust over time. [Work supported by ATR.]

4pSP8. Effect of extended training on $/ r /$ and $/ /$ identification by native speakers of Japanese. Reiko A. Yamada (ATR Human Information Processing Res. Labs., Kyoto, 619-02 Japan)

It has been reported (1) that being exposed to the American English (AE) speaking environment in adulthood has less of an effect on the perception of $\mathrm{AE} / \mathrm{r} /$ and $/ /$ sounds for native speakers of Japanese than experience at younger ages, and (2) that laboratory training of those sounds on adult-Japanese has little effect. Recently, however, Logan et al. [J. S. Logan et al., J. Acoust. Soc. Am. 89, 874-886 (1991)] and Lively et al. [J. Acoust. Soc. Am. (1993)] showed that identification training with natural tokens produced by multiple talkers was effective in improving Japanese listeners ability to identify $/ r /$ and $/ 1 /$. In the present paper, the effect of extended training was examined. Subjects were adult native speakers of Japanese with no experience in living abroad. The training procedure was identical to Lively et al.'s procedure, except that subjects were trained for $\mathbf{4 5}$ sessions and were given two midterm tests. The results showed that (1) most of the subjects, even those whose score was around $60 \%$ in the pre-test, improved to more than $85 \%$ correct in the post-test, (2) some of the subjects kept improving throughout the 45 training sessions, while the others met asymptote with less training, and (3) the training effect in all the subjects generalized to the identification of new words produced by both familiar and unfamiliar talkers. The results are discussed in the context of recent theories of perceptual learning and development of phonological categories. [Work supported by ATR.]

4pSP9. Vowel continuity and perception of $/ \mathrm{ba-wa/}$. Andrew J. Lotto (Dept. of Psychol., Univ. of Wisconsin, Madison, WI 53706), Kerry Green (Univ. of Arizona, Tucson, AZ 85721), and Keith R. Kluender (Univ. of Wisconsin, Madison, WI 53706)

When $\mathrm{CV}$ syllables have longer vowels following a stop $/ b /$ or glide $/ w /$, the duration of transition necessary to perceive a stimulus as $/ w /$ is longer as well. Perceived continuity/duration of following vowels was manipulated by exploiting the phenomenon of auditory induction. All syllables were $315 \mathrm{~ms}$ in duration, and transition duration was varied from $15-65 \mathrm{~ms}$ for four series of syllables. After the first $100 \mathrm{~ms}$ of each syllable (transition + vowel), broadband noise either replaced or was added to the second $100 \mathrm{~ms}$ of syllable. Noise was either the same intensity as or $12 \mathrm{~dB}$ greater than the intensity of the vowel. Results from a signal detection task indicated that listeners could reliably report when the signal was deleted and replaced by the less intense noise; however, they could not reliably report whether the vowel was deleted when the noise was more intense $(+12 \mathrm{~dB})$. Listeners were asked to label these four series of stimuli as beginning with $/ b /$ or $/ w /$, and there was no effect of either the presence or absence of the vowel or of noise intensity. Even when subjects's phenomenal experience was one of intact continuous syllables $(+12-\mathrm{dB}$ noise with vowel present or absent, or equal intensity noise with vowel present), subjects' labeling boundaries were virtually identical to those for syllables perceived as interrupted (equal intensity noise with vowel absent) or for syllables of only 100-ms total duration. Theoretical implications and results from 
follow-up experiments will be reported. [Work supported by NIDCD Grant No. DC-00719 and NSF Grant No. DBS-9258482.]

4pSP10. A developmental study of the perception of onset spectra for stop consonants in different vowel environments. Ralph $\mathrm{N}$. Ohde, Katarina Haley, and Houri K. Voperian (Div. of Hear. and Speech Sci., Box 552, Sta. 17, Vanderbilt Univ. School of Medicine, Nashville, TN 37232)

The purpose of this study was to assess the perceptual role of integration and dynamic time-varying spectral cues for stop consonants in children and adults. Stop-consonant syllables were synthesized in the context of two vowels, $/ i, a /$. These syllables were synthesized with and without bursts, and contained formant motions and/or formant onset frequencies appropriate for each stop-consonant place of articulation. In addition, syllable duration varied between 10 to $46 \mathrm{~ms}$. Children at five age levels, $4,5,7,9$, and 11 , and a control group of adults were trained to identify the consonant as either $/ b /, / d /$, or $/ g /$. Preliminary findings indicate that dynamic time-varying spectral changes are not essential for accurate identification of stop consonants across all contexts. The results also show that the integration of noise bursts and formant onset frequencies, and syllable duration improve identification of alveolar and velar stops, particularly for children. The findings will be discussed relative to: (1) the differences between children and adults in processing integration and dynamic time-varying spectral cues, and (2) the developmental role of stimulus duration in the perception of stop consonants. [Work supported by NIH, DCOO464.]

4pSP11. A preliminary test of prototype theory for a [ba]-to-[da] continuum. Joan E. Sussman (Dept. of Commun. Disord. and Sci., State Univ. of New York at Buffalo, Buffalo, NY 14260)

Ten listeners participated in identification, overt prototype [A. Samuel, Percept. Psychophys. 82, 307-314 (1982)] and sejective adaptation tasks for a 15-item continuum. Stimuli varied linearly by 90 - and 178-Hz steps of formant frequency transition onsets for the second and third resonances, respectively. The prototype was defined as the "best example of ba" with 'best' explained per Samuel [1982, p. 309]. Results showed significant intra- and inter-subject variability for prototype choice. Each subject consistently chose his/her prototype on average $39 \%$ of the time, ranging from $20 \%-53 \%$. Subjects chose stimuli $2-8$ as prototypes, with the highest number ( 3 ) choosing stimulus 3 along the continuum. Further, contrary to Samuel's (1982) results for a [ga]-[ka] continuum, the greatest selective adaptation shift was obtained for the most extreme [ba] adaptor (average shift of $8.7 \%$ ), with the prototype (average shift of $6.5 \%$ ) and boundary adaptors (average $5.3 \%$ shift) resulting in smaller shifts. Results including reaction time data, will be related to prototype theory for consonant categories.

4pSP12. Acoustic correlates of phonetic prototypes: Velar stops. Katharine Davis and Patricia K. Kuhl (Dept. of Speech and Hear. Sci., WJ-10, Univ. of Washington, Seattle, WA 98195)

Studies on vowels by Kuhl and her colleagues have shown that certain stimuli in a vowel category are rates as "better exemplars" (prototypes) than others. The present study extends this work to consonants. Ten adult speakers of American English recorded 20 different words with initial velar stops before the vowel $/ æ /$. These 200 tokens were digitized and edited to include only two pitch periods of the following vowel in addition to the initial consonant. The resulting stimuli were presented to 8 adult subjects who were asked to rate their "representativeness" as members of the voiced or voiceless category ( $1=$ poor, $7=$ excellent). Acoustic parameters of the stimuli were correlated with subjects' ratings to determine which variables (including voice onset time, burst duration, burst locus, etc.) had the most effect on subjects' goodness judgments. Initial results [K. Davis and P. K. Kuhl, Proc. ICSLP, 495-498 (1992)] showed that VOT was strongly correlated with perceived category goodness. Now additional acoustic cues have been included and multiple regression analyses have been used which show effects of VOT, aspiration amplitude, and fundamental frequency of the following vowel in certain cases. The present study also investi- gates the response of Hindi speakers to the identical stimuli when judging goodness according to Hindi voicing categories.

4pSP13. Training native English speakers to identify Hindi dental and retroflex consonants. John S. Pruitt (Dept. of Psychol., Univ. of South Florida, Tampa, FL 33620)

Training listeners to perceive consonantal contrasts that do not $\alpha$ cur in their native language has proved to be difficult. Cross-language training studies usually produce about $10 \%$ improvement in performance. This improvement has not transferred to related material in different linguistic contexts. The present research had four aims: (1) to investigate the difficulty experienced by American-English listeners in identifying Hindi dental and retroflex stop consonants in different voicing conditions, (2) to test a new, computer-based, interactive training method, (3) to examine transfer of training to new voicing conditions, to a new vowel context, and to the voice of a new speaker, and (4) to test the hypothesis that increasing stimulus variability (in this case, training with one versus two voicing conditions) increases transfer of training. Subjects had differential difficulty identifying dental versus retroflex consonants produced in different voicing conditions. Further, this relative difficulty was affected by which speaker produced the contrasts and, to a lesser extent, by the vowel context. The computer-based training improved subjects' consonant identification. However, this improvement showed little transfer to new stimuli. Finally, increasing stimulus variability during training did not affect transfer of training. [Work supported by NIDCD.]

4pSP14. Cue interaction in an intervocalic voiceless affricate/fricative contrast. Stuart Rosen, Angela M. Darling, Andrew Faulkner, and Mark Huckvale (Dept. of Phonet. \& Linguistics, Univ. College London, 4 Stephenson Way, London NW1 2HE, England)

The distinction between intervocalic voiceless affricates and fricatives is a useful one for studies of cue interaction in speech for two main reasons. First, there are at least three temporal cues that are known to interact in this contrast (the duration of the silence preceding the frication, the rise time of the frication, and the duration of the frication). Second, there have been a number of claims that auditory processes may play a crucial role both in the perception of some of the individual cues (in particular, rise time), and in accounting for the interaction of some cues (in particular, silence duration and rise time). New empirical data is presented from a multifactorial identification experiment (involving the simultaneous variation of silence duration, frication rise time and frication duration) and the extent to which various theories of affricate/ fricative perception can account for the observed results is examined. [Work supported by the Joint Council Initiative in Cognitive Science/ HCI (UK).]

4pSP15. Individual variability in the use of acoustic cues to place and voicing contrasts. Valerie L. Hazan and Shi Bo (Dept. of Phonet. and Linguistics, Univ. College London, 4 Stephenson Way, London NWI 2HE, UK)

At the 123rd Meeting of the Society, initial results were reported of a study on individual variability in the perception of word and sentence material in synthetic and degraded natural speech conditions. The same group of $\mathbf{5 0}$ listeners, homogeneous in terms of a set of basic listener characteristics, were also tested on a series of identification tests. These included a $/ g /-/ d /$ place contrast and $/ g /-/ \mathrm{k} /$ voicing contrast presented in three vocalic contexts $(/ \mathrm{i} /, / \mathrm{ei} /, / \mathrm{x} /)$. Each contrast was presented in various full- and reduced-cue conditions, in order to evaluate the relative weighting given by listeners to different acoustic cues. For the place contrast, perceptual weighting given to the burst and formant transition cues varied widely according to individual listeners and vocalic context. However, for the voicing contrast, much more homogeneous results were obtained. No strong relation was found between listeners' performance on reduced-cue identification tests for place and voicing contrasts and intelligibility scores for VCV utterances and sentence material. [Work supported by SERC.] 
4pSP16. The perception and processing of foreign-accented speech: Contributions of various phonetic parameters. Harriet S. Magen (Dept. of Cognitive and Linguistic Sci., Brown Univ., Providence, RI 02912)

This research investigates the relative importance of various parameters contributing to the perception and processing of foreign accented speech. A native Spanish speaker of fluent heavily English read phrases designed to elicit prosodic and segmental deviations from American English. Parameters investigated were: prosodic (phrasal intonation and lexical stress); vowel segmental (vowel epenthesis, tense-laxness, reduction); consonant segmental [VOT, final /s/ deletion, fricative voicing $(/ \mathbf{z}-\mathrm{s} /)$, manner $(\bar{s}-\tilde{c})]$. English-speaking listeners rated extent of foreign accent of the Spanish phrases as actually produced and as edited by computer. Results showed that listeners are sensitive to manipulations, even those involving a brief portion (some as short as 60 ms) of a 3-s phrase, if the manipulation involves a phonological rather than subhonemic category. Results of a word monitoring experiment on the same stimuli will be discussed in terms of the contribution to perception and processing of subphonemic versus phonological and segmental versus prosodic parameters. [Work supported by NIH.]

4pSP17. Coarticulatory information in word recognition: A phonetic priming study. Robert Rossi (Dept. of Modern Languages and Linguistics, Cornell Univ., Ithaca, NY 14853), Martha Burton (Penn State Univ., University Park, PA 16802), Allard Jongman, and Joan Sereno (Comell Univ., Ithaca, NY 14853)

A series of experiments was conducted to investigate whether subphonemic coarticulatory information affects spoken word recognition. A phonetic priming paradigm was used in which isolated fricatives served as primes for target words beginning with a fricative. In particular, prime fricative segments ([s] $]_{i}$ and $\left.[s]_{u}\right)$, representing [s] excised from the vowels [i] and [u], respectively, were used as primes for the targets [si] and [su]. Acoustically similar prime conditions (prime and target fricatives had similar coarticulatory information) were contrasted to acoustically dissimilar prime conditions (prime and target fricatives had contrasting coarticulatory information). Preliminary results show that there was no difference in identification between targets that were preceded by similar as compared to dissimilar primes. Interestingly, however, target identification was speeded when preceded by an identical prime-target match, that is when the prime and target fricatives were the exact same tokens. Results will be discussed in terms of the role of subphonemic information in word recognition.

4pSP18. Methods for testing vowel perception in the field. Peter Ladefoged (Phonet. Lab., Linguistics Dept., UCLA, Los Angeles, CA 90024-1543) and Keith Johnson (Univ. of Alabama, Birmingham, AL 35205)

Based on procedures developed by Johnson et al. [J. Acoust. Soc. Am. 91, 2387(A) (1992)] programs have been written for Macintosh and MS-DOS portable computers for experiments on the perception of vowel quality in the field. In one version, vowels determined by 15 different values of $F 1$ and 22 values of $F 2$, with $F 3$ determined by algorithm, were digitized and stored. In the basic experiment listeners try to match one of these stimuli to a selection of their own vowels. Listeners can hear any of the stored stimuli by clicking with a mouse on a square in a $22 \times 15$ matrix. In other experiments, listeners estimate the distance between pairs of stimuli, or select the better match to one of their own vowels from pairs of vowels that are algorithmically varied from trial to trial in accordance with the previously selected better match. A later version of these programs, not yet tested in the field, generates vowels in real time. The advantage of this system is that the fundamental frequency, the vowel duration, and the third formant can be manipulated by the subject. This is important when matching vowels in languages in which tone or length or rhotacization is contrastive.

4pSP19. The perception of speech gestures. Aimée M. Surprenant (Dept. of Speech \& Hear. Sci., Indiana Univ., Bloomington, IN 47405), Louis Goldstein (Yale University and Haskins Labs., New Haven, CT), and Ian Neath (Purdue Univ., West Lafayette, IN)
An experiment was conducted to evaluate the hypothesis that speakers perceive the intended gestures of the talker, not merely the sound that is carried to their ears. This is a central assumption of the revised motor theory of speech perception [Liberman and Mattingly, Cognition 21, 1-36 (1985)]. Or relevance to this hypothesis is how listeners perceive instances of consonant gestures that show extreme overlap in casual speech and that may, therefore, lack the expected correlates in the acoustic signal (so-called "hidden" gestures). To test this, utterances that showed varying degrees of actual gestural overlap were selected from an $x$-ray microbearn study investigating the production of consonant sequences. These were presented to listeners in a phoneme monitoring task. Results show that as the temporal distance between centers of overlapping gestures decreases, detection also decreases. That is, as the target gesture becomes more and more hidden by the subsequent gesture, subjects report hearing it less and less. This result is qualified by the finding that the magnitude of the gesture also decreases when a gesture is hidden. This research is a first step towards using natural utterances with known articulatory properties to investigate theories of speech perception.

$4 p S P 20$. The effects of clear speech on crassmodal speech perception. J. -P. Gagné (École d'orthophonic et d'audiologie, Univ. de Montréal, C.P. 6128, Montréal, PQ H3C 3J7, Canada), Carol A. Querengesser, and Paula Folkeard (University of Westem Ontario, London, ON, Canada)

Six talkers were videotaped while they spoke a list of sentences twice: first while using conversational speech and then while using clear speech. The recorded stimuli were randomized (across talkers and type of utterances) and presented to three groups of subjects. Each group observed the stimuli under one of three experimental conditions: audioonly (A); visual-only (V); or, audio-visually (AV). For the two conditions in which the auditory signal was provided, a broadband noise was used to degrade the signal. The percent correct keyword recognition scores obtained from the subjects were used to determine the speech intelligibility of individual talkers. For each talker two speech intelligibility scores were obtained (in each sensory modality): one each for the tokens of conversational and clear speech. The results indicated that, in all sensory modalities, the talkers were more intelligible when they produced clear speech than when they used conversational speech.

4pSP21. Alternative scoring systems for the modified distinctive features difference test $[D F D(m)]$. Penny A. C. Anderson and Donald G. Jamieson (Hear. Health Care Res. Unit, Elborn College, Univ. of Western Ontario, London, ON N6G 1H1, Canada)

The modified distinctive features difference test [Cheesman et al., Proc. Int. Conf. on Spoken Lang. Processing (1992)] measures the ability to identify the 21 dominant English consonants in an /ACil / context. The test contains 84 stimuli ( 21 sounds, each spoken by four speakers of Central Canadian English). Target sounds are normally presented in a noise background. The possibility that the sensitivity of the test increases when listener's DFD(m) confusion errors are scored was examined in terms of phonetic features rather than in the more traditional, whole-itern terms. Several alternative scoring approaches were examined: the classical place, manner, and voice distinction and those systems used by Chomsky and Halle (1968), Miller and Nicely (1955), and Feeney and Franks (1982). Scoring using a phonetic feature system does offer certain advantages over whole-item scoring. [Work supported by NSERC and the Ontario Ministry of Health.]

4pSP22. The vanished Lombard effect. William Weiss (Univ. of Ottawa, Ottawa, ON K1N 6N5, Canada)

It is well known that the presence of noise produces an increase in vocal levels [E. Lombard, Ann. Maladies Oreille Larynx 37, 101-119 (1911); H. Lane and B. Tranel, J. Speech Hear. Res. 14, 677-709 (1971)]. Pick et al. [J. Acoust. Soc. Am. 85, 894-900 (1989)] suggested that through training the effect could either be enhanced or reduced but not completely eliminated. It is possible, nevertheless, that people with voice training react differently to that effect [W. D. Ward and $\mathbf{E}$. $\mathbf{M}$. Burns, J. Res. Singing 1 (2), 24-44 (1978)]. Two groups, one with voice training $(n=23)$ and one without voice training $(n=23)$ were 
recorded with and without masking noise. It was found that $39 \%$ of the masked speech samples of the trained group failed to produce the Lombard sign whereas only $9 \%$ of the untrained $(n=23)$ did not increase vocal levels under masking noise. Voice training was significantly related to cases where the Lombard effect was absent. This contradicts Pick et al.'s conclusion that the Lombard sign is always present in masking noise.

4pSP23. Listener performance under variations in signal content. Martin Schultz (Commun. Disord. and Sci., Southern Illinois Univ., Carbondale, IL 62901) and Tan Kah (Southern Illinois Univ., Carbondale, IL 62901)

Signal detection theory holds that performance can be modeled under conditions of physical specification of signals and systems, with no attention given to signal informational content. By contrast, Shannon [Bell Syst. Tech. J. 30, 50-64 (1951)] proposed that performance is open to coding influences and, therefore, may vary with signal content. This study examined performance of subjects for three-element sequences wherein the content varied systematically from words, to nonword, vocal system outputs, to speech-patterned noises. Signals formed a twenty sequences closed set, randomly selected and delivered with equal probabilities. Subjects were trained prior to experimental runs to a $90 \%$ criterion but probably not to asymptotic performance, using trial-by-trial feedback. All signal elements were represented by multiple tokens and all signals were of equal power and duration. Training was accomplished in the "clear," signal/noise ratio was an experimental variable and both training and experimental conditions were self-paced. Subjects were paid for performance throughout. Results are discussed from several theoretical perspectives.

4pSP24. Distribution of sound levels for consonants and vowels within individual frequency bands. Christopher $\mathrm{W}$. Turner (Dept. of Commun. Sci. and Disord., Syracuse Univ., 805 S. Crouse Ave., Syracuse, NY 13244)

While it has been long known that vowels are, in general, more intense than consonants in naturally spoken speech, this statement is based upon the overall sound levels of the phonemes. The purpose of the present study was to determine the sound levels of consonants and vowels within individual frequency bands, with potential application to the provision of consonant audibility to hearing-impaired patients via amplification. The NST speech materials were digitized and subjected to $1 / 3$ octave-band analysis in contiguous 23 -ms samples. The samples were then classified as vowels or consonants, based upon waveform and spectral analysis. A large majority of the samples were vowels, reflecting their greater duration. The long-term consonant spectrum was flat, in contrast to the more familiar low-pass shape of the traditional speech spectrum. In the higher-frequency bands, consonant levels were in many cases more intense than the vowels. [Work supported by NIDCD Grant No. 00377.]

4pSP25. On the correlation among neighboring energy pixels in the speech spectrogram. T. Houtgast (TNO Inst. for Perception, P.O. Box 23, 3769 ZG Soesterberg, The Netherlands)

The energy at any location $f_{t_{i}}$ in the speech spectrogram can be derived by convolution of the speech signal with appropriate Gaussianshaped cosine and sine waves. This leads to the so-called pixel energy, with an integration window centered at $f t_{i}$ and Gaussian-shaped in both frequency and time. Analysis of the correlation for pairs of pixels at a given mutual distance in the spectrogram provides a parametric representation of speech, which shows interesting characteristics. One such representation is the spectral correlation matrix: the correlation for pairs of energy pixels drawn at the same instance at two different frequencies $f_{i}$ and $f_{j}$, with $f_{i}$ and $f_{j}$ chosen from a range of values at appropriate intervals along the frequency scale. It is found that, for a given talker, this spectral correlation matrix stabilizes after analyzing a speech token of about 10 to $15 \mathrm{~s}$ : Its features are then typical of speech as such, and do not depend on the actual content of the speech token. Details of such spectral correlation matrices appear to be characteristic of the talker.

4pSP26. Density of voicing information in purely temporal consonant identification. Richard J. Baker and Stuart Rosen (Dept. of Phonet. \& Linguistics, Univ. College London, 4 Stephenson Way, London NW1 2HE, England)

Speech waveforms containing purely temporal information (i.e., flat spectra and instantaneous amplitudes identical to natural speech, obtained by randomly changing the polarity of consecutive samples) contain quasiperiodic fluctuations in amplitude coincidental with voice periods. The temporal complexity of such waveforms can be reduced by half-wave rectifying the speech prior to removal of the spectral information. This study addresses the question of whether such simplification of the waveform improves the transfer of voicing information in a VCV context in normal hearing adults. The results of consonant identification experiments will be presented in which (a) the two polarities of half-wave rectification are compared to the unrectified condition (equivalent to full-wave rectification) and (b) the waveforms are lowpass filtered at the upper extreme of the fundamental frequency distribution of the speaker, prior to full-wave or half-wave rectification and removal of spectral information. Such filtering removes much of the quasi-periodicity arising from voicing in the full-wave rectified condition. [Work supported by the MRC UK.]

4pSP27. CSRE: A speech research environment. Donald G. Jamieson (Dept. of Commun. Disord., Elborn College, Univ. of Western Ontario, London, ON N6G 1H1, Canada), Ketan Ramji (UWO), Issam Kheirallah (UWO), and Terrance M. Nearey (University of Alberta)

CSRE (The Canadian Speech Research Environment) is a comprehensive, microcomputer-based system designed to support speech research using IBM/AT-compatible micro-computers. CSRE provides a powerful, low-cost facility in support of speech research, using massproduced and widely available hardware. Functions include speech capture, editing, and replay; several alternative spectral analysis procedures, with color and surface/3-D displays; parameter extraction/ tracking and tools to automate measurement and support data logging; alternative pitch-extraction systems; parametric speech (KLATT80) and nonspeech acoustic synthesis, with a variety of supporting productivity tools; and a comprehensive experiment generator/controller, to support behavioral testing using a variety of common testing protocols. The CSRE project is nonprofit, and relies on the cooperation of researchers at a number of institution. Version 3.0 of CSRE has been used since 1989 by researchers in 12 countries. Version 4.0 offers a wider range of functions, runs faster, uses higher resolution displays, and supports additional hardware systems, including digital signal processing boards. 
Session 4pUW

Underwater Acoustics: Ocean Seismoacoustics

\author{
David J. Thomson, Chair \\ Defence Research Establishment Pacific, FMO, Victoria, British Columbia VOS 1BO, Canada
}

Chair's Introduction-1:25

Contributed Papers

4pUW1. Low-frequency bottom scattering strengths for a shallow water site in the Gulf of Mexico. Peter G. Cable (BBN Systems and Technol, Union Sta., New London, CT 06320-6147), Theo Kooij, and Mike Steele (BBN Systems and Technol., Arlington, VA 22209)

Acoustic transmission and reverberation measurements from $25 \mathrm{~Hz}$ to $1 \mathrm{kHz}$ were made in 100 fathom water on the West Florida Shelf during Area Characterization Test I (ACT I) in September 1992. Explosive charges were detonated at $90-\mathrm{m}$ depth and reverberation data were received on collocated vertical and horizontal hydrophone line arrays, each array having 25 hydrophones in nested sub-apertures designed for 100,200 , and $400 \mathrm{~Hz}$. Near-monostatic source-to-receiver separation $(2 \mathrm{~km})$ and bistatic separations of 10 to $40 \mathrm{~km}$ in $10-\mathrm{km}$ increments were employed in the test. From the reverberation data and the transmission loss measurements total monostatic and bistatic bottom scattering strengths have be determined and compared. Also, from the vertical array data monostatic scattering strengths versus scattering angle have been determined. The scattering strengths from ACT I have been compared with determinations from other shallow water measurements having similar bottoms. In addition, these results have been compared with angle and frequency dependencies for different scattering models. [Work supported by the DARPA Adverse Environments Program.]

\section{$1: 45$}

4pUW2. On the acoustic probing of the sea bottom in shallow water from near-field data. Marc Lambert and Dominique Lesselier (Lab. des Signaux et Systèmes CNRS-ESE, 91192 Gif-sur-Yvette Cedex, France)

The probing of the acoustic profiles of the seabed in shallow water from the sound pressure observed on a line array in presence of a cylindrical source, is dealt with. The seabed is modeled as a depthinhomogeneous fluid sediment overlying an homogeneous fluid subbottom. As is well known, the propagation is characterized by the depthdependent Green's function in the horizontal wave-number (spectral) domain, which is a function of the plane wave reflection coefficient of the seabed, with extension to wave numbers larger than the one of water. Conversely, seabed parameters are sought by analyzing features of the Green's function, such as location and magnitude of peaks with then measurements needed in the far field. Here, using receivers near the source, where the "continuum" part of the field is preponderant, directly provides an estimate of the reflection coefficient (at least at low wave numbers). This allows the application of solution methods of the 1-D inverse scattering problem, with limitations due to the discrepancy between estimate and true coefficient. Corresponding issues: retrieving the reflection coefficient, probing the parameters profiles, are investigated and illustrated by examples taken in various source, array, and seabed configurations.
4pUW3. The effect of frequency-dependent bottom reflectivity on transmission loss in shallow water over a sandy bottom. J. Matthew Tattersall, Dmitry Chizhik, Bernard F. Cole (Naval Undersea Warfare Ctr., New London CT 06320), and F. R. DiNapoli (SAIC, New London, CT 06320)

The effect of frequency dependence of bottom reflectivity on acoustic transmission in shallow water over a sandy bottom is investigated using transmission loss data from measurements conducted at a wide range of frequencies. Biot's theory of acoustic wave propagation in fluidsaturated porous media is applied to prediction of bottom reflectivity for a sea bottom consisting of medium grain size sand. For a homogeneous half-space the theory predicts a frequency-dependent bottom reflectivity in contrast to the fluid or solid bottom models. Transmission loss predicted using the Biot model of the sediment shows agreement with measurements at all frequencies considered, which ranged from 100 to $8000 \mathrm{~Hz}$. The fluid model, which assumed a dispersionless velocity and an attenuation that is proportional to frequency, has performed poorly at the low frequency of $100 \mathrm{~Hz}$. [Work supported by Naval Sea Systems Command 06UR1.]

\section{2:15}

4pUW4. A simple ray model of plane-wave reflection from a thin sediment layer. David M. F. Chapman (Defence Res. Establishment Atlantic, P.O. Box 1012, Dartmouth, NS B2Y 3Z7, Canada) and N. Ross Chapman (Defence Research Establishment Pacific, FMO Victoria, BC VoS 1BO, Canada)

Plane-wave reflection from a thin, elastic solid sediment layer over a hard basement is modeled by a simple ray model that includes only two ray families: a family of compressional waves that transit the sediment layer any number of times before returning to the water; and a similar family that includes doubly converted compressional waves. (The latter are compressional waves that are converted to shear waves at the basement, make one or more round-trip transits of the sediment layer, and are converted back into compressional waves at the basement.) The theory is applied to homogenous sediment layers with low shear speeds, and it provides valuable insight into the physical processes governing reflection from several seabed environments of interest. The coherent sum of all the rays belonging to these families leads to a closed-form expression for the overall reflection coefficient that includes several characteristic features also simulated by full wave-theoretical numerical models, such as bottom-loss resonances related to sediment shear waves and large losses associated with the excitation of interface waves at the sediment/basement boundary. Calculated examples are provided.

\section{2:30}

4pUW5. Approximate methods for shallow water acoustics with solid sea beds. Z. Y. Zhang and C. T. Tindle (Physics Dept., Univ. of Auckland, Auckland, New Zealand)

The reflection properties of representative shallow water solid sea 
beds can be reproduced by several simple approximations. An "equivalent fluid" with real parameters can be found provided the shear wave speed in the bottom is less than about $600 \mathrm{~m} / \mathrm{s}$. An equivalent fluid with complex density is useful if the shear wave speed is less than about 1000 $\mathrm{m} / \mathrm{s}$. A perfectly reflecting boundary placed at a "complex effective depth" gives a very simple approximation, again for shear speeds up to $1000 \mathrm{~m} / \mathrm{s}$. The acoustic field at long ranges is determined by reflection at low grazing angles and is reproduced well by all three approximations. In all cases algebraic expressions are given for the various parameters. If the complex effective depth is matched to each mode by iteration it provides a rapid means of finding exact normal mode eigenvalues for both the trapped and leaky modes. All normal modes are leaky for a soft solid sea bed and have complex parameters even when there is no attenuation.

\section{2:45}

4pUW6. Finite difference modeling of geoacoustic interaction at anelastic seaftoors. R. A. Stephen and S. A. Swift (Woods Hole Oceanogr. Inst., Woods Hole, MA 02543)

A major problem in understanding sound propagation in the seafloor is to distinguish between the loss of energy due to intrinsic attenuation (anelasticity) and the loss of energy due to scattering from intermediate scale heterogeneities and bottom roughness. Energy lost to intrinsic attenuation (heat) disappears entirely from the system. Energy lost to scattering is conserved in the system and can effect observations as incoherent noise (time spread, angle spread) and/or mode converted waves. Dougherty and Stephen $(1988,1991)$ showed that the finite difference synthetic seismogram method can be applied to the seafloor scattering problem. Recently, this two-dimensional finite difference code has been extended to include intrinsic attenuation using the formulation of Day and Minster (1984). The formulation assumes a stress-strain relation for which $Q$ is independent of frequency over a specified bandwidth. For each node on a two-dimensional grid arbitrary values of compressional and shear $Q$ can be specified in addition to the usual values of compressional and shear velocity and density. Fluids are represented by setting the shear modulus to zero. $Q$ s computed from the output time series are in good agreement with the input values for homogeneous media. Now by forward modeling the trade-off between scattering and intrinsic attenuation in seafloor models with both surface roughness and volume heterogeneities can be studied. Results can be compared with field observations from vertical seismic profiles (VSP's) in the seafloor and marine refractions experiments.

\section{3:00}

4pUW7. Tube waves, seismic waves, and effective sources. $R$. Burridge, S. Kostek (Schlumberger-Doll Res., Ridgefield, CT 06877-4108), and A. L. Kurkjian (Schlumberger Cambridge Res., Cambridge CB3 OEL, England)

The radiation of waves from a monopole source in a fluid-filled borehole into an elastic medium is considered. A simple asymptotic analysis, based on the smallness of the ratio of the borehole radius to the wavelength, reveals the interaction between tube waves and seismic waves. The pressure field in a tube wave acts as a secondary source of seismic waves and conversely an incoming seismic wave excites a tube wave. The asymptotic analysis leads to a characterization of these sources in terms of the solution to two-dimensional elastostatic problems. These may be solved exactly when the borehole has an elliptical cross section even in an anisotropic formation. Also the borehole need not be straight provided that its radius of curvature is large compared with a wavelength. The problem of two boreholes, one containing a source and the other a receiver (crosswell tomography geometry) is analyzed and an explicit expression for the received signal is derived.
3:15-3:30 Break

\section{3:30}

4pUW8. The influence of bottom geoacoustics on the dispersive behavior of Scholte interface waves. Hassan B. Ali and L. Dale Bibee (Naval Res. Lab., Stennis Space Center, MS 39529-5004)

Scholte seismic interface waves can be an important component of very-low-frequency (VLF) acoustic propagation, particularly in shallow-water environments. The propagation characteristics of these seismic waves, including their presence in the water column, are dependent on bottom geoacoustics (particularly shear speeds) and the types of layers overlying the basement level. This work examines the results of recent NRL/Stennis Space Center measurements and compares them with numerical predictions based on the SAFARI model. Scholte waves measured off the Oregon Margin (1991) are shown to have strikingly different characteristics from those obtained off the San Diego coast (1992). In particular, those obtained off the San Diego coast are characterized by strongly dispersed Scholte waves in the $1-$ to $5-\mathrm{Hz}$ band, whereas those measured off the Oregon Margin contain mostly lower frequencies $(1-2 \mathrm{~Hz})$ and display less clearly defined dispersive behavior. The differences in the two data sets are attributed to the significantly diverse bottom/subbottom geoacoustics at the two test sites. [Work supported by The Office of Naval Research under the NRL/SSC Interface Waves Project.]

$3: 45$

4pUW9. Simulation of the transmitted pressure field inside a water-saturated porous (sand) sediment. A. C. Kibblewhite and C. Y. Wu (Dept. of Phys., Univ. of Auckland, Auckland, New Zealand)

As part of a study of the effects of porosity on reflectivity the influence of an underlying solid basement on the reflection from the interface between the water and a porous sedimentary structure has been examined analytically and numerical calculations made for various versions of this model. This paper presents the results of two applications of the codes developed. In the first the transmitted pressure field inside a sand sediment, resulting from a source above the water-sediment interface, is calculated and compared with that measured in a recent experiment which appears to demonstrate the Biot slow wave [Boyle and Chotiros, J. Acoust. Soc. Am. 91, 2615-2619 (1992)]. The simulation appears to confirm the demonstration of the slow wave in the tank experiment but suggests it is identified with a different feature in the experimental contour plot. In the second example signatures of the vertical component of the seabed displacement are calculated at various ranges from a very low pressure source, in a simulation of the potential use of both the shear and slow wave in the study of the geoacoustic properties of nearbottom sediments. [Work supplied by ONR.]

\section{4:00}

4pUW10. Solution of wave scatter problems in poroelastic media using exact analytical and boundary integral equation methods. Chris Zimmerman (Appl. Res. Lab.) and M. Stern (Univ. of Texas at Austin, Austin, TX 78712)

Methods are developed for obtaining solutions to the harmonic form of the equations governing wave propagation and wave scatter in a poroelastic medium. An analytical solution in the form of an infinite series is obtained for the problem of scatter of an incident plane compression wave by a sphere. The incident wave may be composed of any linear combination of Biot "fast" and "slow" waves, and the sphere may be fixed and rigid, may be composed of elastic solid, or it may be a fluid-filled cavity. A desingularized set of boundary integral equations is developed for the harmonic problem, and these are incorporated into a three-dimensional boundary element computer program. Results obtained from this boundary element program are compared to results obtained using the series solution discussed above. The forbidden frequency problem is shown to be inherently absent from the poroelastic problem. 
4pUW11. An empirical model for acoustic backscatter from trapped gas bubbles in sediment. Frank A. Boyle and Nicholas P. Chotiros (Appl. Res. Labs., Univ. of Texas at Austin, Austin, TX 78713-8029)

Recent experimental backscatter data suggest a volume scattering mechanism involving resonant trapped gas bubbles [N. P. Chotiros and F. A. Boyle, J. Acoust. Soc. Am. 89, 1852(A) (1991)]. A preliminary model for shallow grazing angle high-frequency acoustic backscatter from sediment is developed. The model begins with an estimation of grain and pore size distributions based on analyses of existing measurements taken at sea. The bubble size distribution is assumed to follow the pore size distribution. This bubble size distribution is then combined with an existing model for resonant backscatter from bubbles [Clay and Medwin, Acoustical Oceanography: Principles and Applications] to arrive at a model for backscatter from sediments. The model is then compared with existing experimental data.
4pUW12. Reflection and propagation loss over porous sediments. Nicholas P. Chotiros and M. Stern (Appl. Res. Labs., Univ. of Texas at Austin, P.O. Box 8029, Austin, TX 78713-8029)

A full wave acoustic propagation model in a stratified viscoelastic medium [H. Schmidt and F. B. Jensen, J. Acoust. Soc. Am. 77, 813-825 (1985)] was combined with Biot's theory for propagation and reflection in a porous medium [Stern et al., J. Acoust. Soc. Am. 77, 1781-1788 (1985)] to produce a model of acoustic propagation in a stratified porous medium. Sample results were compared with a normal mode model [T. Yamamoto, J. Acoust. Soc. Am. 73, 1587-1596 (1983)]. The model was extended to account for the effect of gas bubbles in the pore fluid [J. A. Hawkins and A. Bedford, J. Acoust. Soc. Am. Suppl. 1 88, S131 (1990)]. Significant differences between viscoelastic, poroelastic, and gassy poroelastic models were investigated. [Work supported by ONR.]

\author{
Meeting of Accredited Standards Committee S3 on Bioacoustics
}

to be held jointly with the

\title{
U.S. Technical Advisory Group (TAG) Meetings for ISO/TC 43 Acoustics, IEC/TC 29 Electroacoustics, and ISO/TC 108/SC 4 Human Exposure to Mechanical Vibration and Shock
}

\author{
J. D. Royster, Chair S3 \\ 4706 Connell Drive, Raleigh, North Carolina 27612 \\ H. E. von Gierke, Chair \\ U.S. Technical Advisory Group (TAG) for ISO/TC 43 and ISO/TC 108/SC 4 \\ 1325 Meadow Lane, Yellow Springs, Ohio 45387 \\ V. Nedzelnitsky \\ U.S. Technical Advisor for IEC/TC 29
}

National Institute of Standards and Technology (NIST), Building 233, Room A149, Gaithersburg, Maryland 20899

\begin{abstract}
Standards Committee $\mathbf{S 3}$ on Bioacoustics. The current status of standards under preparation will be discussed. In addition to those topics of interest, including hearing conservation, noise, dosimeters, hearing aids, etc., consideration will be given to new standards which might be needed over the next few years. Open discussion of committee reports is encouraged.

The international activities in ISO/TC 43 Acoustics, and IEC/TC 29 Electroacoustics, and ISO/TC 108/SC 4 Human Exposure to Mechanical Vibration and Shock, will also be discussed. The chairs of the U.S. Technical Advisory Groups for ISO/TC 43 (H. E. von Gierke), and IEC/TC 29 (V. Nedzelnitsky), will report on current activities of these Technical Committees and Subcommittees. Reports will be given on preparations for the next meetings of ISO/TC 43 (in Oslo, Norway, from 31 May to 4 June 1993), and of IEC/TC 29 (in Oslo, Norway, from 24 to 28 May 1993). A report will be given on the ISO/TC 108/SC 4 meeting held in London, United Kingdom, from 29 March to 1 April 1993.
\end{abstract}




\title{
Meeting of Accredited Standards Committee S1 on Acoustics
}

to be held jointly with the

\section{U.S. Technical Advisory Group Meetings for ISO/TC 43 Acoustics and IEC/TC 29 Electroacoustics}

\author{
G. S. K. Wong, Chair S1
}

Institute for National Measurement Standards (INMS), National Research Council, Ottawa, Ontario KIA OR6, Canada

H. E. von Gierke, Chair

U.S. Technical Advisory Group (TAG) for ISO/TC 43

1325 Meadow Lane, Yellow Springs, Ohio 45387

V. Nedzelnitsky

U.S. Technical Advisor for IEC/TC 2,

National Institute of Standards and Technology (NIST), Building 233, Room A149, Gaithersburg, Maryland 20899

Standards Committee S1 on Acoustics. Working group chairs will report on their preparation of standards on methods of measurement and testing, and terminology, in physical acoustics, electroacoustics, sonics, ultrasonics, and underwater sound. Work in progress includes measurement of noise sources, noise dosimeters, integrating sound-level meters, and revision and extension of sound level meter specifications. Open discussion of committee reports is encouraged.

The international activities in ISO/TC 43 Acoustics, and IEC/TC 29 Electroacoustics, will also be discussed. The chairs of the respective U.S. Technical Advisory Groups for ISO/TC 43 (H. E. von Gierke), and IEC/TC 29 (V. Nedzelnitsky), will report on current activities of these Technical Committees. Reports will be given on preparations for the next meetings of ISO/TC 43 (in Oslo, Norway, from 31 May-4 June 1993), and of IEC/TC 29 (in Oslo, Norway, from 24-28 May 1993).

\section{Session $5 \mathrm{aAO}$}

\section{Acoustical Oceanography: Determination of Environmental Parameters by Matched-Field Processing}

Alexandra I. Tolstoy, Chair

Naval Research Laboratory, Code 5120, 4555 Overlook Avenue, S. W., Washington, DC 20375

\author{
Chair's Introduction-7:55 \\ Invited Papers
}

8:00

5aAO1. A review of initial matehed-field inversion experiments. Orest Diachok (SACLANT Undersea Res. Ctr., Viale San Bartolomeo 400, La Spezia 1938, Italy)

The first successful demonstration of the feasibility of localizing a source in the ocean with matched-field processing was in the Arctic Ocean (Fizell and Wales). This environment/site was chosen because of the essential range independence of its sound-speed structure and minimal bottom reflectivity. Replica fields were generated with Kutschale's well-tested, precise but computationally exhaustive FFP code. Soon thereafter Porter demonstrated that the precision of his much faster normal mode code equaled FFP, permitting computations of large numbers of replica fields and rapid "testing" of numerous environmental hypotheses," i.e., matched-field inversion. The first inversions were of under-ice reflectivity and phase versus grazing angle (Livingston and Diachok). Other inversions through matched-field processing soon followed: ocean sound-speed profile (Kerengeleu and Diachok; and Goncharov and Voronovich), and bottom geoacoustic parameters (Fizell and Diachok; Chapman and McKirdy; and Collins, Kristensen et ah).

\section{8:20}

5aAO2. Reducing instability through dimensionality reduction, with application to matched-field processing. Charles $L$. Byrne (Dept. of Math., Univ. of Massachusetts at Lowell, 1 University Ave., Lowell, MA 01854)

High-resolution estimators of source parameters, such as Capon's maximum-likelihood (ML) method, become unstable in the presence of nonwhite ambient noise and can degrade rapidly when the data includes perturbations due to model mismatch, finite 
averaging, system phase errors and the like. To combat the instability, some have suggested the use of multiple mainlobe constraints or derivative constraints (Vural, Steele). Stability through beamspace processing was proposed by Gray and was suggested as a way of avoiding nonwhite noise by Bienvenu and Kopp; dimensionality reduction (DR) is an extension of this idea. In matched-field processing (MFP), DR can be introduced through mode-space processing (Yang) or through "reduced ML" (Byrne). When the number of propagating modes approximates the number of phones more dramatic improvement over ML can be achieved through "sector-focusing" (SF) (Byrne and Steele). The basic idea of SF is to project the data vectors onto a subspace of lower dimension. Application of SF to MFP is considered by Frichter et al. [J. Acoust. Soc. Am. 88, 2843-2851 (1990)].

8:40

5aAO3. An application of matched-field processing to the determination of under-ice reflection coefficients and other parameters using long-range, low-frequency data. E. Livingston (Naval Res. Lab., Washington, DC 20375-5000)

Average low-frequency under-ice reflection coefficients were estimated from long-range Arctic data (FRAM IV) by matchedfield processing techniques (Livingston and Diachok, 1989). The method is a straightforward application of optimization using multiple thresholds over a finite set of parameter values, and will be described and discussed with regard to its advantages, disadvantages, and appropriate problem settings. The performance of this method in problems of determining sound-speed structure, optimum replica mode number, and several other types of parameters will be analyzed. In particular, the possible application of the method to the determination of bottom reflection coefficients is considered.

5aA04. Matched-mode processing (MMP) vertical slice tomography. E. C. Shang and Y. Y. Wang (CIRES, Univ. of Colorado/NOAA/Wave Propagation Lab., 325 Broadway, Boulder, CO 80303)

For a practical global scale ocean acoustic thermometry system, information given by vertical slice tomography might be the most fundamental outcome because 3-D mapping is difficult in such a case. Usually, vertical slice modal tomography gives only the "range-averaged" information. Moreover, the "range-averaged" information of sound-speed profile or temperature profile has bias due to the nonlinearity of acoustic travel time. In this talk, the bias has been discussed and the possibility of estimation range-dependent information by using a "differential" MMP vertical slice tomography scheme is investigated. Some numerical simulations will be presented. [Work supported by DARPA and NOAA.]

\section{9:20}

5aA05. The evaluation of acoustic parameters in shallow-water matched-field processing. C. Feuillade (Naval Res. Lab., Stennis Space Center, MS 39529-5004)

The principal application of matched-field processing (MFP) over the last several years has been the determination of acoustic source information, primarily location parameters. In shallow water, where the channel characteristically promotes strong boundary interactions, the successful implementation of MFP methods requires accurate environmental information to accurately model the acoustic field. When the environmental data used to calculate the replica field are incomplete or inaccurate (leading to data "mismatch") MFP performance can be seriously impaired. While it is clear that errors in all of the contingent acoustic parameters will affect MFP, much work has been devoted to establishing a scale of relative importance for these quantities, so that appropriate effort can be expended to obtain reliable values for the most important ones and to determine the effects that inaccuracies in them will have. In this paper, a review is presented of some of the numerical and experimental studies that have been performed to understand the data mismatch issue; and also a discussion of how this has motivated and directed the search for "robust" MFP methods that provide stable performance even when the environment is not known well will be presented. One particular class of estimators, involving "dimensionality reduction" eigenvector techniques, attempt to overcome this problem by isolating the effects of mismatch in the vector space outside of that which is processed. This talk describes how this approach may be adapted to do the inverse problem: to use MFP to provide data about the acoustic environment. [This work was supported by the Naval Research Laboratory.]

\section{9:40}

5aA06. Estimation of seismoacoustic properties of the ocean bottom by matched-field inversion. N. Ross Chapman and $M$. L. McKirdy (Defence Res. Establishment Pacific, FMO Victoria, BC VOS 1B0; Canada)

Matched-field processing of acoustic field data provides a straightforward but powerful method for estimating the elastic properties of the ocean bottom. The success of the inversion depends in part on having an efficient algorithm for searching the multidimensional parameter space of ocean bottom models. This paper considers the use of simulated annealing as a search algorithm for matched-field inversion (MFI), and presents results of inversions for data obtained from the PACIFIC ECHO experiments. A method is described for adaptively guiding the annealing process by adding a feedback loop that assesses the significance of the matched-field correlations for each parameter. The search is guided to regions of the parameter space associated with above-average correlations. Simulations for a vertical line array (VLA) are presented to demonstrate the limits on performance of MFI with respect to uncertainties in experimental geometry, and low signal-to-noise ratio. The method is applied to VLA data from thin-sediment PACIFIC ECHO environments where the age of the upper oceanic crust varied from 0.1 to 66 million years. The MFI results indicate an age dependence of the compressional and shear wave speeds of the basalt, and the values are significantly lower than those obtained for thickly sedimented crust. 
5aAO7. Inversion for bottom sound speed and bathymetry in a 3-D shallow-water Arctic environment. John M. Ozard, Trevor W. Dawson, and S. E. Dosso (Defence Res. Establishment Pacific, FMO Victoria, BC VOS 1BO, Canada)

Propagation modeling and matched-field processing (MFP) require a good knowledge of ocean-bottom properties on a grid of points covering the search region. These parameters can be difficult to measure in range-dependent Arctic shallow waters. An effective environmental model based on MFP inversion can be easier to obtain than extensive geophysical measurements and more effective for MFP if the same propagation model is used both for inversion and MFP. An even more effective model for MFP is obtained if inversion is carried out for several consecutive source positions. In developing MFP-based inversion for this scenario, recovering the bathymetry alone was investigated first, then both sound speed and bathymetry, for sources at a number of known ranges. After these successful inversions, inversion for recovering the unknown position of one source and the bathymetry was investigated. Both of these cases employed two-dimensional modeling but it is recognized that significant changes in the predicted field can occur when the third dimension is taken into account. Examples of seamounts and realistic shelf slope environments were modeled in three dimensions and the effect of inverting in two dimensions for bottom properties in the shelf slope case was investigated.

10:20-10:30 Break

\section{Contributed Papers}

\section{0:30}

5aA08. Parameter estimation bounds on estimating geophysical parameters using matched-field tomography. Arthur B. Baggeroer and Henrik Schmidt (Depart. of Ocean Eng., Rm. 5-204, MIT, Cambridge, MA 02139)

Matched-field tomography uses full wave modeling to infer geophysical parameters of the propagation medium. As such, it is fundamentally a problem in estimation theory. The signal processing literature has long used bounding methods to establish performance limitations and identify coupling among estimates of a set of parameters. The most extensive of these is the Cramer-Rao bound, which has been applied to the source localization problem for matched-field processing. The Cramer-Rao, as well as other bounds for analyzing the sidelobe, or ambiguity, problem can be applied to matched-field tomography. These will be derived and applied to problems in identifying the sound-speed profile and elastic properties of the seafloor.

\section{0:45}

5aAO9. Global inversion using genetic algorithms. P. Gerstoft (Saclant Undersea Res. Ctr., La Spezia, Italy)

An inversion of sound fields for determining unknown environmental parameters can be separated into four parts: (1) discretization of the environment and discretization or transformation of the data, (2) efficient and accurate forward modeling, (3) efficient optimization procedures, and (4) uncertainty analysis. While much work had been done on the first two, much less has been done on the latter two, especially for object functions with several local minima. Global optimization methods accept the multiple minima and try to find the global minimum, without doing an exhaustive search. These methods are based on directed Monte Carlo search, and two promising methods are simulated annealing and genetic algorithms (GA). These have been compared and it has been found that GA's often are superior. The global methods are time demanding and in order to speed up the convergence, gradient steps can be taken during the iteration process. The examples here will be based on a horizontally stratified environment where all the material and geometrical parameters can be taken as unknown parameters. The solution is presented in terms of a pasteriari probability function describing the parameters. From this the most likely model parameters can be found and their uncertainty and importance can be assessed.

\section{1:00}

5aAO10. Matched-field inversion for range-dependent bottom properties and source location. S. E. Dosso and John M. Ozard
(Defence Res. Establishment Pacific, FMO Victoria, BC VOS 1B0, Canada)

Ocean-bottom properties such as bathymetry and bottom sound speed can be difficult to measure directly in ice-covered Arctic waters, particularly if they vary with range. However, inversion procedures hold much promise for determining range-dependent bottom properties from acoustic measurements. The inversion is formulated as an optimization problem by assuming a discrete model of unknown parameters and a bounded search space for each parameter. The optimization then involves finding the set of parameter values which minimizes the mismatch between the measured and modeled acoustic fields. Two practical problems are considered. The first problem involves determining rangedependent bathymetry and bottom sound speed using a number of sources at known ranges. The second problem involves source localization when available knowledge of the bathymetry is inadequate. In this case, the inversion is carried out for source range and depth as well as bathymetry. Although the bathymetry cannot be determined uniquely in this manner, an effective bathymetry model can be computed which allows accurate source localization. This latter case is essentially an extension of the method of "focalization" [M. D. Collins and W. A. Kuperman, J. Acoust. Soc. Am. 90, 1410-1422 (1991)] to bottom parameters.

\section{1:15}

5aAO11. Using circulation models to improve ocean imaging (and vice versa). W. A. Kuperman, C. Shen (Naval Res. Lab., Washington, DC 20375), and M. B. Porter (New Jersey Inst. of Technol., Newark, NJ 07102)

Circulation models predict the ocean weather (temperature), which in turn determines the manner in which sound propagates. The ocean models, like their atmospheric counterparts, tend to drift away from reality as they are marched forward in time. In order to prevent this, tomographically constructed images of the ocean are assimilated into the circulation model. Furthermore, flow constraints provided by the ocean circulation model are used to exclude unrealistic structures and thereby enhance the quality of the tomographic imaging. The algorithmic issues of linking acoustic and ocean circulation models in this fashion, and the benefits of doing so, are discussed.

\section{1:30}

5aAO12. Demonstration of the large and small sector limits for large vertical array sector focused matched-field processing in a deep water environment. George B. Smith (Naval Res. Lab., Code 7173, Stennis Space Center, MS 39529-5004) and Dane Morris (Neptune Sciences, Inc., Slidell, LA 70458) 
Sector focused matched-field processing is a technique that has been developed to stabilize high-resolution estimators against random phase errors and environmental mismatch. This technique produces satisfactory high-resolution matched-field localization over a wider latitude of environments than does conventional high-resolution techniques. Its implementation requires the selection of several parameters such as sector size, shape and dimensionality. In this work, general guidance is given for the use of this technique by establishing the properties of the small and large sector limits. Specifically, it is demonstrated that in the small sector limit, sector focusing behaves like replica correlator processing (robust and low resolution). Whereas, in the large sector limit, sector focusing behaves like reduced maximum-likelihood processing (high resolution, but not as robust). The demonstration is accomplished by plotting signal gain degradation versus three sediment sound-speed parameters. These plots show a small hot spot at the correct environment immersed in very cool surroundings for the conventional highresolution estimator (mvdr). Whereas, for sector focusing, the plots show a rather wide swath of acceptable environments. In most cases, the technique peaks on the correct environment. [This work was supported by the Office of Naval Research, with technical management provided by the Naval Research Laboratory, Stennis Space Center, MS.]

\section{1:45}

5aAO13. Application of neural and statistical classifiers to the problem of seafloor characterization. Zoi-Heleni Michalopoulou and Dimitri Alexandrou (Duke Univ., Dept. of Elec. Eng., Box 90291, Durham, NC 27708-0291)

Simulated seafloor backscatter is obtained by employing the Kirchhoff approximation and the statistical properties of bottom reverberation. Such data are presented to multilayer perceptrons for training and testing, aiming at the development of a neural processor that discriminates among signal returns coming from seafloors with different roughness parameters. Experiments on the same data sets are performed with optimum Bayesian classifiers as well; a comparison of the results indicates suboptimum performance for the perceptrons. The same procedure is followed with real data collected by the bathymetric system Sea Beam over Horizon Guyot and Magellan Rise. In this case, the perceptron performance is comparable to that of the Bayesian classifier, which is no longer optimum, since no prior knowledge of the probability distribution parameters is available. In addition, self-organizing maps have been applied to both synthetic and real data sets and resulted in a successful separation of the output space into distinct regions corre- sponding to the existing classes. [Work supported by the Office of Naval Research, Code 1125GG, through Contract No. N00014-93-I-0049.]

\section{2:00}

5aAO14. Application of a maximum-likelihood processor on acoustic backscatter for the estimation of the seafloor roughness parameters. Zoi-Heleni Michalopoulou and Dimitri Alexandrou (Duke Univ., Dept. of Elec. Eng., Box 90291, Durham, NC 27708-0291)

The objective of this work is seafloor characterization using a method that takes advantage of the statistical properties of bottom reverberation and employs maximum-likelihood estimation to obtain estimates for the seaftoor parameters. The Helmholtz-Kirchhoff model for backscatter is here used to link the angular dependence of backscattering strength to the correlation properties of the seafloor roughness. The suggested estimation procedure is applied on simulated backscatter data and on real data recorded over Horizon Guyot and Magellan Rise by the bathymetric system Sea Beam. The real data estimation results are compared to curve fitting estimates acquired by the same data sets presented by Moustier and Alexandrou [J. Acoust. Soc. Am. 90, 522531 (1991)]. [Work supported by the Office of Naval Research, Code $1125 \mathrm{GG}$, through Contract No. N00014-93-I-0049.]

\section{2:15}

5aAO15. Waveform analysis of seafloor Scholte waves. Guust Nolet (Dept. of Geolog. and Geophys. Sci., Princeton Univ., Princeton, NJ) and LeRoy M. Dorman (Scripps Inst. of Oceanog., UCSD, La Jolla, CA 92093-0215)

A new method of nonlinear waveform inversion to model Scholte waves has been tested. Synthetic displacements were computed for an explosive source at the ocean bottom for frequencies up to about $2.5 \mathrm{~Hz}$. At the distance range of interest $(<1 \mathrm{~km})$ only the first few modes of the Scholte waves are present, with most energy in the fundamental mode. Linear perturbation theory is used in our scheme to predict changes in wave number produced by changes in the model. This theory degenerates at those frequencies where modes can escape the sediments and where the phase velocity changes suddenly from approximately 0.1 to $1.5 \mathrm{~km} / \mathrm{s}$. The step length for wave-number changes were limited to deal with this problem. This did not noticeably degrade convergence. The method was tested on observed waveforms from an experiment at Deep Sea Drilling Project site 469 . This site is at $3.8-\mathrm{km}$ water depth just off the Patton Escarpment, about $300 \mathrm{~km}$ west of San Diego. The seismic velocities there are quite uniform laterally. The surficial shear velocity is about $30 \mathrm{~m} / \mathrm{s}$. [Work supported by ONR.]

\title{
Session 5aMU
}

\section{Musical Acoustics and Psychological and Physiological Acoustics: Auditory Organization in Music and Speech III: Tonality, Pitch, Timbre, and Voices}

\author{
Richard E. Pastore, Chair \\ Psychology Department, State University of New York, Binghamton, New York 13902-6000
}

Chair's Introduction $-8: 30$

\section{Invited Paper}

\section{8:35}

5aMU1. Sensitivity to duration and frequency of occurrence of tones in music. Lola L. Cuddy (Dept. of Psychol., Queen's Univ. at Kingston, ON K7L 3N6, Canada)

Understanding music in the Western idiom includes perceptual sensitivity to the tone relationships and hierarchy of stabilities contained in the tonal-harmonic system. These features of the tonal-harmonic system are correlated with statistical properties of 
tonal music. For example, the frequency with which each tone is sounded has been shown to be closely related to its relative prominence in the tonal hierarchy. Listeners may be sensitive to such regularities, and, it has been argued, may use this information to develop, or elaborate, a sense of tonality for the music. This experimental work has pursued the issue of sensitivity to regularities in music by examining listener's sensitivity to duration and frequency of occurrence in tone sequences for which construction rules deviated from the rules of Western tonality. Sensitivity will be described both for abstract sequences of pure tones and for musical materials provided by contemporary composers. It is contrasted with an alternative perceptual strategythat of assimilation to the tonal hierarchy. [Work supported by NSERC.]

\section{Contributed Papers}

\section{9:05}

5aMU2. A parallel learning model of musical pitch perception. Bernice Laden (School of Music, Univ. of Washington, DN-10, Seattle, WA 98195)

Musical pitch perception involves the ability to extract and internalize a template that represents harmonic information. Template extraction is important to other perceptual processes such as melody recognition and chord classification, although these involve extraction of intervallic information. In order to explore how templates might be learned, a parallel learning algorithm was developed. This algorithm is based on the notion that a stimulus does not need to be physically present for a response to be learned. An artificial neural network was trained with this algorithm to identify the pitch of a tone complex. The network had 116 input units (log frequency) and 88 output units (pitch). After training, it correctly identified the pitch of 88 tone complex prototypes. It was tested with a variety of incomplete patterns as well as dyads and triads. All test patterns were correctly identified, although output strength dropped when simultaneous tone complexes were presented. The results indicate the parallel learning algorithm may be superior to other learning algorithms in terms of both accuracy and speed.

\section{9:20}

5aMU3. Similarity judgments of fifth-spaced tones and dyads thereof. Richard Parncutt (Faculty of Music, McGill Univ., 555 Sherbrooke St. W., Montreal, PQ H3A 1E3, Canada)

A fifth-spaced tone (FST) is a complex tone whose partials are equally spaced in log frequency at intervals of a musical fifth (seven semitones, frequency ratio $\sim 2: 3$ ) across a broad frequency band (like Shephard tones, but with fifths instead of octaves). The perception of single FSTs (monads), and dyads of FSTs spanning the intervals M2 (two semitones, frequency ratio $\sim 8: 9$ ) and $\mathrm{m} 3$ (three semitones, 5:6), was investigated by asking ten musicians and ten nonmusicians to rate the similarity of all pairwise combinations of monad, M2 dyad, and $\mathrm{m} 3$ dyad. Sequential intervals ranged from 0 to 6 semitones (i.e., all possible intervals in the chromatic scale). Results were compared with predictions according to the theories of virtual pitch [Terhardt et al., J. Acoust. Soc. Am. 71, 679-688 (1982)] and pitch commonality [R. Parncutt, Harmany: A Psychoacoustical Approach (Springer-Verlag, New York, 1989)]. The salience of virtual pitches by comparison to spectral pitches in monads and dyads of FSTs was found to be consistently lower than predicted by the models. [Work supported by NSERC Canada.]

\subsection{5}

5aMU4. Stimulus properties critical to normalization of instrument timbre. Jennifer L. Cho, Michael D. Hall, and Richard E. Pastore (Ctr. for Cognitive and Psycholinguistic Sci., Binghamton Univ., Binghamton, NY 13902-6000)

The perceptual system appears to engage in active, time-consuming processes called normalization that maintain perceptual constancy by adjusting for source differences [e.g., Mullennix and Pisoni, J. Acoust. Soc. Am. 85, 365-378 (1989)]. Prior music research [Cho et al, J. Acoust. Soc. Am. 89, 1988(A) (1991)] demonstrated normalization for irrelevant timbre variability in chord identification. The present investigation identified important global components of instrument timbre that may be differentially subject to normalization. Using intact and physically manipulated natural stimuli, experiment 1 used similarity scaling to directly assess contributions of previously identified temporal and spectral properties of timbre. Contributions of attack and upper harmonics were evaluated. Results indicated that similarity was based primarily on the upper harmonics, with little contribution from attack functions. The relevance of these stimulus properties to normalization was evaluated in experiment 2 using an $\mathrm{AX}$ chord discrimination task. Normalization, as indicated by RT, was inversely related to similarity. Information present in higher harmonics appears to be the major contributor to instrument timbre and thus is most relevant to normalization. [Research supported by NSF and AFOSR.]

\section{9:50}

5aMU5. Multidimensional scaling of synthetic musical timbre: Perception of spectral and temporal characteristics. Séverine Samson (Hôpital de la Salpétrière, Paris, France), Robert J. Zatorre (Montreal Neurolog. Inst., 3801 Univ. St., Montreal, PQ H3A 2B4, Canada), and James O. Ramsay (McGill Univ., Montréal, PQ H3A 1B1, Canada)

The perceptual correlates of the acoustic parameters involved in musical timbre have not yet been clearly established. To investigate this issue, perception of single tones and melodies were examined using nine hybrid synthetic sounds, derived from crossing three levels of spectral and temporal changes (number of harmonics and rise time, respectively). Multidimensional scaling analysis was carried out on the pairwise dissimilarity judgments of 15 subjects, using an 8-point rating scale. The configuration resulting from the analysis was best fit by a three-dimensional model, but the first two were most important, and accounted for $44 \%$ and $38 \%$ of the variance, respectively. The perceptual space derived from the analysis indicates that these two orthogonal dimensions corresponded closely to the spectral and temporal differences inherent to the stimuli. Similar results were obtained with melodies as with single tones. Thus, it appears that even musically unselected subjects are sensitive to spectral and temporal information in a tone, and are able to use them independently in making perceptual judgments of musical timbre.

\section{0:05}

5aMU6. Abstraction of musical tonality. Willi R. Steinke, Lola L. Cuddy, and Ronald R. Holden (Dept. of Psychol., Queen's Univ. at Kingston, ON K7L 3N6, Canada)

Data were collected from 100 subjects for a series of music tests and standardized tests of intelligence and cognitive abstraction. Music tests focused on abstraction of tonality. Three tests employed the probe-tone technique [C. L. Krumbans] and E. J. Kessler, Psych. Rev. 89, 334-368 (1982)] for both harmonic and melodic materials. Four other tests collecting tonality ratings with melodic sequences and chord progressions were constructed for this study. The subject sample comprised the following ranges: age (18-40 years); general education (7-22 years); music education (none to professional). Results confirmed the reliability of the probe-tone technique. Results for all music tests were significantly intercorrelated and significantly correlated with music theoretic 
predictions. Amount of music training, but not level of general intellectual functioning or level of general education, was implicated in the subject's responses to music tests. [Work supported by NSERC.]

\section{0:20-10:35 Break}

\section{$10: 35$}

5aMU7, Early musical acculturation. Michael P. Lynch (Dept. of Audiol. \& Speech Sci., 1353 Heavilon Hall, Purdue Univ., West Lafayette, IN 47907-1353)

Humans can universally, under typical circumstances, appreciate music. This appreciation is presumably dependent on the ability to process musical structure, but it is not clear how this processing develops. The present study was designed to contribute toward clarification of this issue. Western infants were tested in detection of mistunings (increase of $3.2 \%$ in frequency of a randomly selected melody note) in melodies based on either native or nonnative musical scales. Infants repeatedly heard the well-tuned versions of the melodies and learned to turn their heads toward the sound source when they heard a mistuning. If infants, like adults, have developed schematic knowledge of musical tuning, then their performance in this task would be expected to be better in testing conditions involving native scales than in conditions involving nonnative scales. Although infants at 6,9 , and 12 months of age all performed the task with reliably better than chance success, statistically better native than nonnative mistuning detection was evident only at 12 months of age. This finding suggests that perceptual reorganization for musical tuning occurs in an age range that is similar to that observed for speech perception in infants. [Work supported by NICHD Grant No. HD28527.]

\section{0:50}

5aMU8. Perception of the pitch of speech and of the pitch of music by children. Eduardo Castro-Sierra (Lab. of Psychoacoust., Hospital Infantil de México, Dr. Márquez No. 162, 06720 México D.F., Mexico)

The perception of fundamental changes within word (Mandarin and Cantonese) and phrase (English) samples was compared to the perception of fundamental frequency changes between the members of pairs of steady complex tones or of tonal intervals between the members of pairs of small tunes composed of similar tones in 5- to 12-year-old monolingual speakers of these languages. Some of these children had had training in Western instruments for from 1 to 5 years. A certain correlation was found between the perception of tonal intervals of major and minor second magnitude between notes in the pairs of tunes and the perception of similar intervals within the English phrase samples among native speakers of this language. This correlation was increased in subjects with a musical background. In contrast, there was a lack of correlation between the perception of frequency changes between isolated tones and the perception either of frequency changes within Mandarin or Cantonese word samples or of frequency changes at the end of English phrase samples.

\section{1:05}

5aMU9. Segregation of voiced and whispered concurrent vowels in English and Japanese. Andrew P. Lea and Minoru Tsuzaki (ATR Human Information Process. Res. Labs., 2-2 Hikaridai, Seicha-cho, Kyoto, 619-02 Japan)

This paper presents new evidence that listeners can use the difference between voiced and whispered speech to segregate both voiced and whispered members of concurrent vowels consisting of voiced/ whispered pairings. Previous experiments performed by Lea and Summerfield [Acoust. Soc. Jpn. Tech. Rep. H-92-31] required English listeners to identify the members of pairs of steady-state synthetic English vowels, called concurrent vowels. Accuracy of identification was higher when one vowel was voiced and the other whispered compared to control conditions. Surprisingly, the improvement in accuracy was restricted to the whispered member of the voiced/whispered pair, thus suggesting that the whispered vowel was segregated, but not the voiced vowel. Lea and Summerfield's study was replicated in this paper using Japanese vowels and listeners, however, the results suggest that both the voiced and whispered members of the voiced/whispered pair were segregated. These incompatible results either indicate a difference in segregational ability between English and Japanese listeners, or a difference in procedure between the experiments. Correspondingly, further experiments were performed using Lea and Summerfield's English vowels and English and Japanese listeners which show that the incompatible results are mainly due to the different methods of matching the levels of the voiced and whispered vowels between the two experiments. Thus the evidence suggests that the Japanese results indicate the true segregational ability of listeners.

\section{1:20}

5aMU10. The apparent duration of the restored speech segments. Minoru Tsuzaki, Hiroaki Kato, and Masako Tanaka (ATR Human Information Process. Labs., 2-2 Hikaridai, Seika-cho, Soraku-gun, Kyoto, 619-02 Japan)

To investigate how the auditory mechanism restores the noisereplaced speech segments, the apparent durations for the perceptually restored speech were measured. The results were obtained that supports the hypothesis that the subjects restore the "missed" part of speech based on the internal template whose temporal structure corresponds to the "original" sample. The threshold of duration discrimination was measured using an up-and-down two-interval task. In one interval, a "standard" stimulus was presented that had the temporal structure of the natural sample. In the other interval, a "comparison" was presented in which the third mora was either lengthened or shortened. There were four conditions in a two-factorial design. The first factor was the noise replacement in the comparison, depending on whether the part of the modified segment in the comparison was replaced by a noise. The second factor was the noise replacement in the standard. In the lengthening direction, only the noise replacement in the comparison resulted in the increment of the discrimination threshold irrespective of the noise replacement in the standard. This suggests that only the noise replacement in the modified part is effective in the perceptual judgment of the durations.

\section{1:35}

5aMU11. Reaction times to phonemic restoration. Shigeaki Amano (NTT Basic Res. Labs., 3-9-11 Midori-cho, Musashino, Tokyo, 180 Japan)

To investigate how acoustic information is integrated into phonemic restoration, reaction times to noise-replaced plosives in a vowelconsonant-vowel (VCV) sequence were measured. There were (a) a naturally spoken VCV, (b) a VCV made by concatenating the independently spoken $\mathrm{V}$ and $\mathrm{CV}$, and (c) a VCV made by cross splicing the initial $\mathrm{V}$ and the following $\mathrm{CV}$. In a naturally spoken VCV, the consonant information at the end of the initial $V$ matches that at the beginning of the following $\mathrm{CV}$. In an independently spoken VCV, however, there is no relationship between the consonant information at these locations, and in a cross-spliced VCV, they conflict. When the noisereplaced plosive was perceptually restored to the original plosive, reaction times to the plosive in the naturally spoken VCV was shortest. The next shortest reaction times were to the plosive in the independently spoken stimuli, and the longest times were to that in the cross-spliced VCV. This order was reversed, however, when the noise-replaced plosive was perceptually restored to the nonoriginal plosive. These results show that the integration of acoustic information at the end of the initial $\mathrm{V}$ is primary and faster than the integration of information at the beginning of the following $\mathrm{CV}$.

\section{1:50}

5aMU12. Neural network supporting auditory-vocal integration in singing. David W. Perry, Bessie Alivisatos, Alan C. Evans, Ernst 
Meyer, Michael Petrides, and Robert J. Zatorre (Dept. of Neuropsychol., McGill Univ., 3801 University St., Montréal, PQ H3G 2B4, Canada)

Although much is known about the auditory nervous system, in both man and nonhuman primates, and about the neural substrates for vocal production, particularly in nonhuman primates, relatively little is known about how these two systems are integrated in vocal behavior. The present study used positron emission tomography with magnetic resonance imaging to investigate regional changes in blood flow during the performance of a rudimentary singing task by 12 healthy righthanded volunteers. When activation during the repetetive singing of a single target pitch was compared to that during passive auditory stim- ulation with complex tones in the same frequency range, a widely distributed network of activated brain regions was observed. Some regions of activation were plausibly explained by the auditory stimulation of self-vocalization (right medial geniculate body and right primary auditory cortex), while others were clearly related to the act of vocalization (periaqueductal grey, subcallosal anterior cingulate gyrus, supplementary motor area, cerebellum and primary motor face region, bilaterally). Finally, the neuroanatomical connections of the mid-dorsal insula, activated bilaterally, and of the dorsomedial thalamus are suggestive of a particular role in auditory-vocal integration. It is proposed that auditory-vocal integration, both in singing and in speech, involves complex neural interconnections between many of the above regions.

FRIDAY MORNING, 21 MAY 1993

QUEBEC SUITE, 8:30 TO 11:30 A.M.

\title{
Session 5aNS
}

\section{Noise: Developments and Current Issues in Measurement of Noise Exposure}

\author{
John J. Earshen, Chair \\ Angevine Acoustical Consultants, Inc., 1021 Maple Street, P.O. Box 725, Aurora, New York 14052-0725
}

Chair's Introduction-8:30

Invited Papers

8:35

5aNS1. Update on modern noise dosimeters. Robert Krug (Cirrus Res., Inc., 6423 w. North Ave., Wauwatosa, WI 53213)

Noise dosimeters have been undergoing a quiet revolution in recent years. They have progressed from the simple single reading dosimeter, into a multifunction general purpose noise measuring terminal. Dosimeters are available that can measure noise dose with two or three separate criterion levels as well as separate channels for peak, A-, and C-weighting. Time histories are stored and retrieved with durations as short as every $1 / 16 \mathrm{~s}$ for several hours. Exceedance levels are available along with time-weighted average, equivalent sound level per day, and many additional features. Readout is possible with front panel displays, printer interfaces, and computer storage. While functions have increased greatly, size and weight have been reduced. Electromagnetic susceptibility problems still exist but are being addressed in the current generation of dosimeters.

\section{9:00}

5aNS2. Testing the response of personal noise exposure meters (noise dosimeters) to impulse-like signals using a laboratory reference noise dosimeter and a theoretical model (after ANSI S1.25-1978). David J. Evans (Acoust., Mass \& Vib. Group, NIST, Bldg. 233, Room A149, Gaithersburg, MD 20899-0001)

A laboratory reference noise dosimeter consisting of a measuring amplifier and instrument controller was assembled and characterized. A theoretical expression was obtained for the A-weighted, rms-detected output of a noise dosimeter assuming an input signal equal to that of a tone-burst sequence containing bursts comprised of an integral number of cycles of a sine wave. The output of the theoretical detector was numerically integrated to obtain percentage criterion exposure or time-weighted equivalent level. Equivalent levels were measured and calculated for each sequence in a set of tone-burst sequences with burst durations of 1 to 1000 cycles and crest factors of 4.8 to $30 \mathrm{~dB}$. The agreement between equivalent levels obtained using the reference dosimeter and theoretical calculations was typically $+0.1 \mathrm{~dB}$. The results of these measurements and calculations were then used as a basis of comparison for testing the response of commercially available noise dosimeters to a subset of the tone-burst sequences. [Work supported by OSHA.]

5aNS3. What OSHA is doing to minimize the effect of radio interference on acoustical instruments. Raymond F. Feldman (U.S. Dept. of Labor, OSHA Cincinnati Lab., USPO Bldg., Room 108, 5th \& Walnut St., Cincinnati, OH 45202)

Acoustical instruments are more susceptible to radio interference than many users find acceptable. Degraded performance caused by electromagnetic fields is called electromagnetic susceptibility (EMS). In the past, performance standards for acoustical instruments, and all industrial hygiene instruments in general, have not adequately addressed this problem. EMS deficiencies can range from subtle deviations to gross errors in sound measurements. It has even been reported, that EMS has caused audiometers to malfunction and blast the ears of patients with sound levels of 80 and $90 \mathrm{dBA}$. The OSHA Cincinnati Laboratory has been working to reduce EMS effects on sound instruments that are used by OSHA and MSHA. Manufacturers are being persuaded to meet EMS performance specifications for instruments which these agencies purchase. These requirements assure the instruments will operate properly in industrial and mining environments. In 1992, ASA established an ANSI/ASA working group to write an EMS standard for acoustical instruments. 


\section{Contributed Papers}

10:00

5aNS4. The importance of memory when predicting annoyance on the basis of equal energy. George A. Luz (US Army Environmental Hygiene Agency, Aberdeen Proving Ground, MD 21010-5422)

In 1981, CHABA published a report showing the annoyance for a 24-h day-night average sound level from sonic booms/blasts (measured with C) to increase at a faster rate than that from transportation noise (measured with A). In 1979, Luz and Lewis presented a theoretical argument that this difference was due to a difference in the loudness functions for the different noise sources. In 1992, Schomer et al. provided experimental proof for these differing loudness functions. A radical implication of Schomer et al.'s demonstration is that the equal energy rule is inappropriate for the assessment of heavy weapons noise and other types of explosions. A more conservative approach is that the equal energy rule is a description of human memory for the annoyance of noise. Using examples from past studies, an attempt is made to define the limits over which memory can be expected to conform to the equal energy rule.

\section{0:15}

5aNS5. Design of a bearing test probe using active acoustic noise attenuation. Boaz Rafaely and Miriam Furst (Dept. of Elec. Eng. Syst., Faculty of Eng., Tel-Aviv Univ., Tel-Aviv 69978, Israel)

Most hearing tests today are done in soundproof rooms. A method is proposed for conducting tests in a regular room while using a hearing test probe with active acoustic noise attenuation. The system consists of passive and active attenuation parts. The passive attenuation is achieved by a foam plug placed in the ear canal. The active attenuation is achieved by using two mini-microphones, a receiver and a DSP board. The first microphone is placed at the ear canal entrance and receives the acoustic noise. It then transfers the noise to the DSP board (with the TMS320, placed in a PC), which by using an adaptive algorithm transmits the antinoise signal to the receiver placed in the ear canal. The second microphone is placed near the receiver and produces the error signal to the adaptive process. The system was analyzed on a larger scale acoustic model and a 20 - to $40-\mathrm{dB}$ noise attenuation was achieved for tones up to $4 \mathrm{kHz}$ (not including the plug passive attenuation). Theoretical analysis of the acoustic filter transfer function (from the outer microphone to the inner microphone) was done using computer simulation, and showed good agreement with measurement. Implementation of the system for broadband noise attenuation will be discussed. Preliminary results have shown that the characteristics and configuration of the acoustic probe and the DSP controller specifications have to be considered.

\section{0:30}

5aNS6. Measuring attitudes toward co-workers affected by hearing loss in noisy industry. R. Hétu, L. Getty, L. Philibert, and $J$. Beaudry (Groupe d'Acoustique, Université de Montréal, Montréal, PQ H3C 3J7, Canada)

Previous studies have shown that the attitudes of hearing workers toward their hearing-impaired colleagues strongly contribute to the handicap associated with occupational hearing loss (OHL). In order to characterize such attitudes, a questionnaire has been designed. In phase I, a preliminary version of 45 items was equally divided into measures of beliefs, attitudes and reported behaviors toward co-workers affected by OHL. Answers from 176 workers from a metal products plant allowed to identify discriminative items that belonged to an appropriate factorial structure. In phase II, answers from 125 miners to a second version of the questionnaire, comprising 32 items, permitted further refinements. The final version was tested with 283 workers from three metal products plants, with a response rate of $86 \%$. Answers on the scale measuring beliefs showed that noise-exposed workers did not know many of the consequences of OHL. Attitudes toward hearing impaired co-workers were generally favorable, but this finding appeared to reflect a strong social desirability bias. Reported behaviors indicated that a majority of workers are not inclined to adopt conducts that favor communication with victims of OHL. These findings offer useful clues for developing an awareness raising program on the consequences of OHL. [Work supported by IRSST.]

\section{0:45}

5aNS7. Effects of high-intensity impulse noise on the hearing of humans wearing hearing protection. James $\mathrm{H}$. Patterson, Jr. (US Army Aeromed. Res. Lab., P.O. Box 577, Fort Rucker, AL 36362-5292) and Daniel L. Johnson (EG \& G Special Projects, Albuquerque, NM 87119-9024)

Current exposure limits for high-intensity impulse noise are based on very limited amounts of data. This study was undertaken to provide new data on the effects of a variety of impulse noises on humans exposed while wearing hearing protection. The impulses used in this study were produced by detonation of explosive material. Two types of impulses were used. One had an A duration of approximately $3.0 \mathrm{~ms}$, while the other had an A duration of approximately $0.8 \mathrm{~ms}$. Exposures were presented at one impulse per minute. The results of the long duration impulse have been presented previously. This presentation focuses on the short duration impulse. Sixty-five volunteers were exposed on successive days to six impulses with intensities ranging from 178 to $196 \mathrm{~dB}$ SPL for the shorter impulse. The number of impulses then was increased from 6 to 100 at the tolerable level. The results indicate that minimal hearing protection is adequate for 6 of the 0.8 impulses up to $196 \mathrm{~dB}$ SPL. It was inadequate for 12 or more impulses at 193 or for 50 or more impulses at $190 \mathrm{~dB}$ SPL. Protection is adequate for 50 impulses at 187 . Hearing protectors which provide more attenuation were adequate for all conditions used in this study.

\section{1:00}

5aNS8. Real-ear attenuation provided by noise-reducing earphone enclosures. Tom Frank and Amy C. Ritter (Dept. of Commun. Disord., Penn State Univ., 5-A Moore Bldg., University Park, PA 16802)

Real-ear attenuation at threshold values were obtained for Audiocup, AudioMate, Auraldome II, and Madsen ME-70 noise-reducing earphone enclosures (N-REEs) housing a TDH-type earphone mounted in supra-aural cushion using ten subjects following the procedures specified in ANSI S12.6-1984. Overall, the attenuation provided by each N-REE increased as frequency increased and was about $8 \mathrm{~dB}$ from 125 to $250 \mathrm{~Hz}, 21 \mathrm{~dB}$ from $500 \mathrm{to} 1000 \mathrm{~Hz}$, and $35 \mathrm{~dB}$ from 2000 to $8000 \mathrm{~Hz}$. The Audiocup provided the least $(3 \mathrm{~dB})$ and the AudioMate, the most $(16 \mathrm{~dB})$ attenuation from 125 to $250 \mathrm{~Hz}$. The Auraldome II provided the least ( $18 \mathrm{~dB}$ ) and the AudioMate provided the most $(25 \mathrm{~dB})$ attenuation from 500 to $1000 \mathrm{~Hz}$. Each N-REE provided similar attenuation ( $35 \mathrm{~dB}$ ) from 2000 to $8000 \mathrm{~Hz}$. Compared with the attenuation values for a TDH-type earphone in a supra-aural cushion (re: ANSI S3.1-1991), each N-REE provided significantly more attenuation except for the Audiocup, Auraldome II, and Madsen ME-70 at $125 \mathrm{~Hz}$ and the Audiocup at $250 \mathrm{~Hz}$. Implications for hearing testing using N-REEs as a means to reduce high levels of ambient noise will be discussed. 
$8000-\mathrm{Hz}, 500-$ to $8000-\mathrm{Hz}$ test frequency ranges, respectively; however,

5aNS9. Ambient noise levels in audiometric test rooms. Tom Frank and Dennis L. Williams (Dept. of Commun. Disord., Penn State Univ., 5-A Moore Bldg., University Park, PA 16802)

Octave-band ambient noise levels measured in 136 diagnostic and 202 industrial audiometric test rooms were compared with the maximum permissible ambient noise levels (MPANLs) specified in ANSI S3.1-1991. The mean octave-band ambient noise levels for the diagnostic rooms were less than the ears covered but higher than the ears not covered MPANLs for each test frequency range specified in ANSI S3.11991. Specifically, $68(50 \%), 69(51 \%)$, and $112(82 \%)$ of the diagnostic rooms met the ANSI ears covered MPANLs for the 125- to only $14(11 \%)$ for the $125-$ to $8000-\mathrm{Hz}$ and $250-$ to $8000-\mathrm{Hz}$ test frequency ranges and $39(29 \%)$ for the 500 - to $8000-\mathrm{Hz}$ test range met the ANSI ears not covered MPANLs. The mean octave-band noise levels for industrial rooms were lower than the ANSI ears covered MPANLs for the $500-$ to $8000-\mathrm{Hz}$ test frequency range except at 250 and $500 \mathrm{~Hz}$ where the levels exceeded the ANSI MPANLs by 2.4 and $6.4 \mathrm{~dB}$, respectively. For the industrial rooms, $66(33 \%)$ met the ANSI ears covered MPANLs for the $500-$ to $8000-\mathrm{Hz}$ test range. Overall, both the diagnostic and industrial test rooms most often failed to meet the ANSI MPANLs at 250 and $500 \mathrm{~Hz}$. Implications for diagnostic and industrial hearing testing will be discussed.

FRIDAY MORNING, 21 MAY 1993

SALON RENAISSANCE, 8:30 TO 11:45 A.M.

\title{
Session 5aPA
}

\section{Physical Acoustics: Outdoor Sound Propagation}

\author{
Carl K. Frederickson, Chair \\ University of Mississippi, University, Mississippi 38677
}

Contributed Papers

8:30

5aPA1. On the placement of microphones for outdoor measurements. Scott Morgan (Dept. of Phys., Southeastern Louisiana Univ., P.O. Box 878 SLU, Hammond, LA 70402), Henry E. Bass, Richard Raspet, Majorie Klugerman, and Stephen Wood (Univ. of Mississippi, University, MS 38677)

Acoustic measurements outdoors typically involve microphones located either on the surface or on stands which hold the microphone approximately $1.0 \mathrm{~m}$ above the ground. Arguments given for placing microphones on the surface include lower wind noise and ease of installation. Quite often microphones on the surface are mounted above a large solid sheet. In this talk, the effects of wind noise and ground impedance on the optimum design of measurement systems for different frequency and source position are considered.

\section{$8: 45$}

5aPA2. Micrometeorologically forced intermittency in atmospherically propagated signals. Scott D. Hansen (Grad. Prog. in Acoust., Penn State Univ., Appl. Sci. Bldg., University Park, PA 16804) and Dennis W. Thomson (Penn State Univ., University Park, PA 16802)

Line of sight, continuous acoustic signal propagation experiments were conducted as a part of the Joint acoustic Propagation experiment (JAPE) performed during July and August of 1991 at White Sands Missile Range. One of the purposes of JAPE was to examine the effects of the micrometerological phenomena on low-frequency sound. During one experiment, pure tones of 80,200 , and $500-\mathrm{Hz}$ were propagated simultaneously for a period of $1 \mathrm{~h}$ over distances up to $1000 \mathrm{~m}$. Towerbased measurements included both microphones and turbulence sensors spaced logarithmically to a height of $32 \mathrm{~m}$. Frequency, height-, and range-dependent statistics of the recorded acoustic transmission loss (TL) are interpreted in terms of the ambient surface and boundry layer micrometeorological processes. In addition, nonlinear attractor-based parameterizations of signal variability are evaluated.
5aPA3. Micrometeorological effects on line-of-sight acoustic propagation. Edward R. Maniet, Jr. (Grad. Prog. in Acoust., Penn State Univ., Appl. Sci. Bldg., University Park, PA 16802)

Experimental studies investigated the effects of atmospheric surfacelayer convective turbulence on the amplitude fluctuations of line-ofsight sound propagation. Several pure tones between 25 and $125 \mathrm{~Hz}$ were continuously monitored at a distance of $758 \mathrm{~m}$ for periods of 4 to 6 days by two microphones at heights varying between 0 and $8 \mathrm{~m}$. Simultaneous surface-layer micrometeorological measurements were made at the same site. Statistics of the acoustic transmission loss (TL) were analyzed along with the sensible heat flux $(Q)$, a measure of the strength of convectively driven turbulence. It was observed that only the lowest studied frequency, $27.6 \mathrm{~Hz}$, showed any dependence on the value of the sensible heat flux. The average standard deviation of the TL for the $27.6 \mathrm{~Hz}$ tone was twice as great under strongly convective conditions than under conditions with weak or no convection. It is suggested that turbulent static pressure fluctuations associated with surface-layer thermal plumes were responsible for the increased TL variability at $\mathbf{2 7 . 6}$ Hz.

\section{9:15}

5aPA4. Prediction of the sound-speed profiles and the index of turbulence associated with various meteorological conditions. A. L'Espérance (G.A.U.S., Mech. Eng. Dept., Univ. de Sherbrooke, Sherbrooke, PQ J1K 2R1, Canada) and Y. Gabillet (Ctr. Sci. et Tech. du Bâtiment de Grenoble, 38400 Saint-Martin d'Hères, France)

Sound propagation over the ground is greatly influenced by the sound-speed profiles and the atmospheric turbulence, and there exist a number of models taking these effects into account. However, for practical outdoor sound propagation studies, it appears to be unrealistic to measure the effective sound-speed profiles (SSP) and turbulence index $\left\langle\mu^{2}\right\rangle$ to obtain acoustical predictions. For a complete prediction model, these parameters should therefore be estimated from general atmospheric conditions. To fulfill the needs of acoustical prediction models, a meteorological model was developed to estimate SSP and $\left\langle\mu^{2}\right\rangle$. This model is based on estimations of the roughness length and the Monin- 
Obukhov length, which define the shape of the wind-speed profiles, temperature profiles, and the index of turbulence [Panofski and Dutton, Atmospheric Turbulence (Wiley, New York, 1984)]. The roughness length is estimated according to the surface of the site, and the MoninObukhov length using an empirical relation proposed by Golder [Bound. Layer. Meteorol. 3, 47-58 (1972)]. To verify the precision and the limitation of this model, the theoretical SSP and $\left\langle\mu^{2}\right\rangle$ were compared to experimental results for various meteorological conditions. These comparisons show the capability and the limitation of this meteorological model, which may limit the accuracy of a complete acoustical prediction scheme.

9:30

5aPA5. A fast phase-screen method for sound propagation through a turbulent atmosphere. Xiao Di (Natl. Ctr. for Phys. Acoust., Univ. of Mississippi, University, MS 38677 and Inst. for Microstruct. Sci., Natl. Res. Council, Ottawa, ON K1A 0R6, Canada), Kenneth E. Gilbert, and Lucy J. Ameling (Penn State Univ., State College, PA 16804)

An "integrated-turbulence" phase screen method has been developed to efficiently treat sound propagation through turbulence having both small-scale and large-scale structure [X. Di and K. E. Gilbert, J. Acoust. Soc. Am. 92, 2404 (A) (1992)]. In this paper, the method is used with two different parabolic equation (PE) algorithms: a PE based on a Fourier transform, split-step range step and a PE based on a Crank-Nicolson range step. It is shown that the phase-screen approach allows one to take advantage of the much longer range step possible with the Fourier method and thereby speed up the calculation by a factor of approximately 100 relative to the Crank-Nicolson result. Consequently, with the phase-screen method and the Fourier PE, it is practical to directly compute the statistics of the fluctuations in the acoustic field. Results are shown from such calculations and comparisons are made with available experimental results.

\section{9:45}

5aPA6. A numerical study of sound propagation through a turbulent atmosphere. Daniel Juve, Philippe Blanc-Benon, and Patrick Chevret (Dept. of Acoust., Ecole Centrale de Lyon, 36 Av. Guy de Collongue, 69131 Ecully Cedex, France)

A numerical simulation of the effect of turbulent conditions on the propagation of acoustic waves in the atmosphere has been developed. The turbulence is represented as a set of realizations of a random field generated by a limited number of random Fourier modes [Karweit et al., J. Acoust. Soc. Am. 89, 52-62 (1991)]. Through each individual realization, the acoustic pressure field is computed in the parabolic approximation. Ensemble averaging is thus performed to obtain the statistical properties of the field: mean level, standard deviation of the fluctuations, probability density functions, etc. Results will be given in particular for upward refracting conditions, when turbulence diffracts sound into the deterministic shadow zone.

\section{0:00}

5aPA7. Sound propagation through atmospheric turbulence: Multifractal phase fluctuations. R. H. Mellen and G. Siling (Marine Sci. Inst., Univ. of Connecticut, Groton, CT 06340)

Measurements of phase fluctuations in sound propagation through turbulent air show frequency spectra that approximate the Kolmogorov $\beta=5 / 3$ scaling law over several decades. This suggests a fractal-like process for which the apparent fractal dimension would be $D=(5-\beta) /$ $2 \approx 5 / 3$. However, most natural phenomena are found to be multifractal rather than monofractal. Multifractal theory treats, not only first and second moments of the process, but also the relation between moments (including nonintegral). For monofractals, this relation is simply a linear function of the moment number and a codimension $0<C_{1}<1$. The degree of multifractality $0<\alpha<2$ is determined from systematic deviations from linearity. The parameters $\alpha, \beta$, and $C_{1}$ are all indicators of the nature of the nonlinear energy cascade. Analysis of experimental data obtained at $5 \mathrm{kHz}$, range $\approx 4 \mathrm{~m}$ and wind speed $\approx 12 \mathrm{~m} / \mathrm{s}$ is reported here. Results indicate that phase fluctuations are "hard multifractal" ( $\alpha>1)$, which is comparable to measures of velocity fluctuations in atmospheric turbulence.

\section{0:15-10:30 Break}

10:30

5aPA8. On the aging of sonic booms. Kenneth J. Plotkin (Wyle Labs., 2001 Jefferson Davis Hgwy., Ste. 701, Arlington, VA 22202)

Sonic boom minimization exploits the shaping of nonasymptotic "mid-field" signatures of large aircraft [F. E. McLean, NASA TN D-2877 (1965)]. This is assisted by the "freezing" effect of downward propagation in the real atmosphere [W. D. Hayes and H. L. Runyan, Jr., J. Acoust. Soc. Am. 51, 695-701 (1971) ]. Cleveland and Blackstock [J. Acoust. Soc. Am. 92, 2331 (A) (1992)] have recently shown that true freezing occurs for flight altitudes in excess of $80000 \mathrm{ft}$. Recent studies of boom minimization, however, show greater success at much lower altitudes, and that shaping is increasingly more difficult at higher flight altitudes. Parametric analysis of the age parameter with regard to altitude and distance shows that successful minimized booms are not close to asymptotic freezing, and that "freezing" is not necessarily the best perspective. There is concern that increasing flight altitude as fuel load decreases might put a low-boom aircraft design into an off-design condition of higher boom. It is shown that, for realistic flight profiles, the increased age parameter is more than offset by reduced weight, so that sonic boom loudness will decrease throughout supersonic cruise.

\section{$10: 45$}

5aPA9. Noise production by turbulent flow over an unscreened measurement microphone. Scott Morgan (Dept. of Phys., Southeastern Louisiana Univ., P.O. Box 878 SLU, Hammond, LA 70402) and Richard Raspet (Univ. of Mississippi, University, MS 38677)

In previous work, it has been shown that the wind noise in an unscreened microphone placed in a low-speed turbulent flow is caused by the turbulence intrinsic in the flow [S. Morgan and R. Raspet, J. Acoust. Soc. Am. 92, 1180-1183 (1992)]. The theory of pressure fluctuations in a turbulent flow as related to wind noise in an unscreened measurement microphone is discussed in this paper. A Poisson's equation for fluctuation pressure in terms of mean and fluctuation velocity derivatives with respect to spatial dimensions results from the theory. This implies that the mean and fluctuation velocities must be known at every point in space in order to solve for the fluctuation pressure at a given point; however, through comparison of theory with experimental data, it is shown that the fluctuations in the boundary layer near the microphone where velocity gradients are very large provide the major source of wind noise. An approximation method for estimating the contribution of this "local interaction" is also discussed.

\section{1:00}

5aPA10. Acoustic impedance of porous pavements: Comparison between a microstructural and a phenomenological model. Michel C. Bérengier (L.C.P.C., Ctr. de Nantes, BP 19, 44340 Bouguenais, France), Jean-François Hamet (I.N.R.E.T.S., 69676 Bron Cedex, France), Gilles A. Daigle, and Michael R. Stinson (Inst. for Microstruct. Sci., Natl. Res. Council, Ottawa, ON K1A 0R6, Canada)

For the last 6 years, new porous road structures (drainage asphalt or pervious macadam) have been studied in several countries, particularly in Europe. In addition to their drainage qualities (i.e., elimination of splash and spray), these porous structures lead to reduced noise levels from traffic. To study further their acoustical performance, measurements of propagation over real road surfaces have been made, for grazing and oblique incidence conditions. The experimental results are compared to theoretical predictions based on two different models for the surface impedance: One model is a microstructural model that requires 
airflow resistivity, porosity, tortuosity, and shape factor; the other is a phenomenological approach that has been extended to include energy dissipation through thermal conductivity effects. Additionally, these impedance models are compared to direct measurements of the acoustical properties, e.g., absorption coefficient and impedance.

\section{1:15}

5aPA11. Determination of the properties of air-filled porous media using level difference and probe microphone measurements. Carl $\mathrm{K}$. Frederickson, James M. Sabatier, and Richard Raspet (Univ. of Mississippi, University, MS 38677)

Level difference measurements have been used to determine three properties of air filled porous media; porosity, tortuosity, and flow resistivity [Soil Sci. Soc. Am. J. 54, 658-672 (1990)]. These can be used to find the characteristic acoustic impedance of a porous medium. It can be shown that the level difference measurement alone does not contain enough information to uniquely determine more than two of these properties. A second measurement is needed to determine one of the properties. A probe microphone can be used to determine either the flow resistivity or the tortuosity; either of which can then be used in the level difference calculation to determine the other two properties. An experiment has been performed to determine the viability of this combined technique for characterizing the ground. Probe microphone measurements were made in a container of $0.526-\mathrm{mm}$ glass beads. This same size of bead was then used in level difference measurements. As the beads are spherical the porosity is known. Values of flow resistivity and tortuosity obtained from both the level differences and probe microphone measurements agree fairly well. [Work supported by the US Department of Agriculture.]

\section{1:30}

5aPA12. A stochastic, relaxation model for sound propagation in porous media. D. Keith Wilson (Dept. of Appl. Ocean Phys. and Eng., Woods Hole Oceanogr. Inst., Woods Hole, MA 02543)

By assuming that the averaged acoustic fluid velocity and temperature fields satisfy simple relaxational diffusion equations, a new model for sound propagation in porous media has been developed. The resulting model agrees quite well with the exact solutions for uniform circular or triangular pores, despite not being based upon any specific assumptions regarding the pore geometry. Other benefits of the new model include simpler equations (no Bessel or Kelvin functions are used) and the need for fewer parameters. Extension of the model to fractal pore surfaces will also be discussed.

FRIDAY MORNING, 21 MAY 1993

ADAM ROOM, 8:00 A.M. TO 12:15 P.M.

\title{
Session 5aPP
}

\section{Psychological and Physiological Acoustics: Psychological and Physiological Collaborations in Hearing II}

\author{
Richard R. Fay, Chair \\ Parmly Hearing Institute, Loyola University of Chicago, 6525 North Sheridan Road, Chicago, Illinois 60626
}

\section{Contributed Papers}

8:00

5aPP1. Three-process model of auditory-nerve fiber tuning curve shapes. Eric Javel (Div. of Otolaryngol., Duke Univ. Med. Ctr., Durham, NC 27710)

Cat auditory-nerve fiber tuning curves were fitted by a three-process model consisting of an acoustic resonance (for tuning curve "tails"), a skewed function formed by subtracting two exponentials (for basilar membrane resonances), and a Gaussian function (for the active gain responsible for sharp tuning). Thresholds were taken as the sum of pressures provided by the two resonances, multiplicatively enhanced in a spatially dependent manner by the active gain. Fits were generally excellent. "Tails" possessed center frequencies (1-3 kHz) and minima (60-80 dB SPL) that varied from animal to animal and depended on fiber CF. Basilar membrane resonances possessed average minima of 30-50 dB SPL in low-threshold fibers, tuning that increased in sharpness with CF or threshold, and center frequencies that were typically lower (for CFs $>1 \mathrm{kHz}$ ) or higher (for CFs $<1 \mathrm{kHz}$ ) than the center frequencies of the associated active gains. Average active gain magnitude in low-threshold fibers increased smoothly from $<10 \mathrm{~dB}$ in low-CF fibers to $>40 \mathrm{~dB}$ in high-CF fibers, and was reduced or absent in most high-threshold fibers. The average spatial extent of the active gain was remarkably constant at $1 \mathrm{~mm}$ regardless of gain magnitude, fiber CF, or threshold sensitivity. [Work supported by NIDCD.]

\section{8:15}

5aPP2. Characterizing auditory filter nonlinearity at $2 \mathrm{kHz}$. Stuart Rosen and Richard J. Baker (Dept. of Phon. \& Linguist., Univ. College London, 4 Stephenson Way, London NW1 2HE, England)

Although it is well known that psychoacoustically measured audi- tory filters broaden with increases in level, it is not yet clear what aspect of the acoustic stimulus controls this nonlinearity-that is, whether the change in bandwidth is controlled primarily by the level of the probe or masker. Therefore, a new method has been developed for fitting filter shapes to notched-noise data in which filter parameters depend explicitly on signal level (probe or masker). By applying this technique to notched-noise data in which both fixed-probe and fixed-masker paradigms have been used at a range of levels, it was possible to show that models in which filter parameters depend on probe level fit the data considerably better than models in which filter parameters depend upon masker level. Furthermore, it was possible to describe the nonlinear changes in auditory filter bandwidth at $2 \mathrm{kHz}$ with only five parameters, allowing the implementation of reasonably realistic computational nonlinear auditory filters whose shape depends on their output. [Work supported primarily by the Medical Research Council (UK), with important contributions from the Hearing Research Trust.]

\section{$\mathbf{8 : 3 0}$}

5aPP3. A comparison of peripheral and "attentional" auditory filters. Donna L. Neff, Theresa M. Dethlefs, Walt Jesteadt (Boys Town Natl. Res. Hosp., 555 N. 30th St., Omaha, NE 68131), and Robert A. Lutfi (Univ. of Wisconsin, Madison, WI 53706)

The relation between peripheral and "attentional" filters was explored in simultaneous masking for eight normal-hearing listeners. Thresholds for a sinusoidal signal at $1000 \mathrm{~Hz}$ were measured as a spectral gap in a masker was progressively widened. For peripheral filters, the masker was a notched noise and a one-parameter ROEX model was used to describe filter properties. For "attentional" filters, the maskers were multicomponent complexes of two or ten components of random frequency content, and the ROEX model was again applied. Indices of 
peripheral filter width did not distinguish listeners who showed large versus small effects of masker uncertainty in the "attentional" filter conditions. Indices of peripheral processing efficiency, however, were poorer for listeners showing large uncertainty effects, as were measures of both the width and processing efficiency of presumed "attentional" filters. An alternative analysis of the "attentional" filter data will examine whether component entropy or relative variance $[R$. A. Lutf, J. Acoust. Soc. Am. (to be published)] can account for the decline in masking observed as the spectral gap in the masker is increased. [Work supported by AFOSR and NIDCD.]

\section{$8: 45$}

5aPP4. Masking can explain the effects of notched noise on forward-masked intensity discrimination. Fan-Gang Zeng (House Ear Inst., 2100 W. Third St., Los Angeles, CA 90057)

Plack and Viemeister [J. Acoust. Soc. Am. 92, 1902-1910 (1992)] investigated the role of "off-frequency" listening in a nonmonotonic intensity discrimination function under forward masking, in which a midlevel hump was originally reported for 25 -ms sinusoidal standards presented $100 \mathrm{~ms}$ after an intense masker [Zeng et al., Hear. Res. 55, 223-230 (1991)]. Plack and Viemeister used a notched noise to prevent off-frequency listening and found that the notched noise removed or severely reduced the midlevel hump. A problem with the use of a notched noise in their experiment is that the level of the notched noise increases as a function of the standard level, introducing on-frequency masking in the form of elevated thresholds. They measured intensity discrimination at sensation levels between 10 and $14 \mathrm{~dB}$, independent of the standard level. In the present study, the experiment of Plack and Viemeister was replicated with a narrow-band $(800-1200 \mathrm{~Hz})$ noise presented simultaneously with the sinusoidal standards to produce threshold elevations similar to the equivalent notched noise conditions. Presumably, the narrow-band noise does not prevent off-frequency listening, but severely reduces the midlevel hump in a fashion similar to the notched noise. This result suggests that the effects of notched noise on forward-masked intensity discrimination are mainly due to the onfrequency masking. The possible role of excitatory and suppressive masking at the auditory nerve level will be discussed. [Work supported by NIH.]

\section{9:00}

5aPP5. Diminished response to acoustic transients in aged rodents. J. Ison, P. Bowen, J. Barlow, M. Taylor (Dept. of Psychol., Univ. of Rochester, Rochester, NY 14627), and Joseph P. Walton (Univ. of Rochester, Rochester, NY 14627)

The startle reflex to an intense noise burst $(S 2)$ is depressed if weak transients $(S 1)$ precede $S 2$. Noise pips and gaps in noise carriers preceded $S 2$ at various lead times in young and old CBA/J mice. Old mice differed from young in three ways: (1) reflex amplitudes were lower; (2) prepulse thresholds for inhibition were raised, especially for high frequency pips; and (3) inhibition appeared at later lead times. Inhibition thresholds, but not its slowed development, were remedied with a stronger $S 1$. Comparable effects were seen in the F344 rat. Single units in inferior colliculus in the mice showed that the aged IC had lost sensitivity to weak pulses. Minimal gap thresholds (1-2 ms) did not vary with age, but the younger animals had more units with lower gap thresholds. The reduced behavioral thresholds of the weak $S 1$ likely result from the presbycusislike sensitivity changes. The slow onset time of inhibition may reflect the reduced size of the population of transientsensitive units. [Work supported by NIH Grant No. AG09524 and the RICHS.]

\section{9:15}

5aPP6. Temporal pattern perception in the goldfish: Methods and models. Richard R. Fay (Dept. Psychol. and Parmly Hear. Inst., Loyola Univ. of Chicago, 6525 N. Sheridan Rd., Chicago, IL 60626)

How goldfish perceive temporal patterns of acoustic clicks was in- vestigated using a stimulus generalization paradigm with classical respiratory conditioning. Fish were conditioned to suppress respiration to trains of high-pass-filtered clicks with periodic inter click intervals (ICI). Five groups of animals were conditioned using ICIs between 4 and $25 \mathrm{~ms}$, and then tested for response to eight novel ICIs between 4 and $25 \mathrm{~ms}$. Response magnitude to novel ICIs (generalization) is a monotonic decreasing function of the difference between the conditioning and test ICIs. In further experiments, test stimuli consisted of temporally jittered periodic ICIs, and various pseudorandom ICI distributions. A model predicting the magnitude of generalization to these patterns was developed assuming that generalization is proportional to the number of ICIs common to conditioning and testing stimuli, and that the internal representation of ICIs has a variance predicted from discrimination data. Evidence was obtained that ICIs are analyzed, but that ICI representation is not necessarily congruent with that for pure tones of the same period. [Work supported by a Center Grant from the NIH NIDCD.]

9:30

5aPP7. Effect of stimulus intensity on the pitch of a complex tone's spectral edge. Adrianus J. M. Houtsma, Armin Kohlrausch, Peter A. Kef (Inst. for Percept. Res./IPO, P. O. Box 513, 5600 MB Eindhoven, The Netherlands), and Johannes W. Horst (University Hospital, 9700 RB Groningen, The Netherlands)

Six subjects made pitch matches between a fixed-intensity sinusoidal comparison tone and a complex test tone with harmonics 1 through 20 in zero-phase and fundamentals between 46 and $54 \mathrm{~Hz}$ at sensation levels between 10 and $70 \mathrm{~dB}$. Tone and intertone gap durations were 500 ms. All subjects performed six matches at all five fundamental frequency values and at seven intensities. As control, subjects also matched pitches between pure tones around $1000 \mathrm{~Hz}$. Mean matches to complex tones were found to depend on intensity, at a subject-averaged rate of $0.12 \% \mathrm{~dB}$, always going from a pitch below the edge frequency at low sensation level to a pitch above the edge frequency at high sensation level. This is consistent with evidence from cochlear nerve data by Evans [Philos. Trans. R. Soc. London Ser. B 336, 381 (1992)]. Standard deviations of complex-tone matches were typically only a factor two larger than those of pitch matches between pure tones, even at the lowest sensation levels. This is not well understood in terms of cochlear nerve data which suggest that edge pitch should fade away at sensation levels below 30-40 dB [Horst et al., J. Acoust. Soc. Am. 78, 1898-1901 (1985)].

9:45

5aPP8. Edge pitches of complex tones obtained at high frequencies. Armin Kohlrausch, Adrianus J. M. Houtsma, and Sebastiaan J. Kloos (Inst. for Percept. Res./IPO, P. O. Box 513, 5600 MB Eindhoven, The Netherlands)

The upper edge pitch of harmonic complex tones was matched by adjusting the frequency of a sinusoidal comparison tone. Complexes with upper-edge frequencies between 2 and $8 \mathrm{kHz}$ and fundamental frequencies of 50,100 , and $200 \mathrm{~Hz}$ were synthesized by adding all harmonics with equal amplitudes and with zero phases. Tones and intertone gaps had durations of $500 \mathrm{~ms}$. Stimuli were presented diotically through Etymotic ER-2 insert earphones at a sound-pressure level of 60 dB. Five subjects performed ten matches for each complex, from which the standard deviation (s.d.) was calculated and expressed as percentage of the edge frequency. The s.d. generally increased with the edge frequency and with the fundamental frequency. For $50-\mathrm{Hz}$ fundamental, the s.d. was only a factor two larger than in a sine-on-sine match, and four of the five subjects could perceive the edge pitch up to $8 \mathrm{kHz}$. For higher fundamental frequencies, more and more subjects lost the pitch percept at high edge frequencies, and for $200 \mathrm{~Hz}$ only one subject consistently matched the edge pitch at $8 \mathrm{kHz}$. The results emphasize the role of temporal processes in the perception of edge pitch as well as the importance of phase locking at the frequency of the edge component. 


\section{0:00-10:15 Break}

\section{0:15}

5aPP9. A computer model of the physiological basis of the extraction of pitch from tone complexes. Ray Meddis and Michael J. Hewitt (Dept. of Human Sci., Univ. of Technol., Loughborough LE11 3TU, UK)

A low pitch sensation can be heard when a mid-frequency (e.g., $500-4000 \mathrm{~Hz}$ ) carrier is amplitude modulated at a rate between 100 and $400 \mathrm{~Hz}$. The output of multipolar neurons in the ventral cochlear nucleus are known to reflect strongly modulation of the acoustic stimulus and even to amplify it. Other cells in the inferior colliculus respond by increasing their rate of firing when a rate of amplitude modulation, critical to that cell, is applied to a stimulating tone. The system to be described consists of a model of the auditory periphery that simulates auditory-nerve fiber input, arrays of multipolar point-neuron models that receive this input, and arrays of inferior colliculus unit models that receive input from the multipolar cells. Individual model units behave similarly to physiological units. Arrays of IC model units also show many properties associated with psychophysical studies of pitch including the ability to extract ambiguous pitch from inharmonic tone complexes.

\section{0:30}

5aPP10. Phase sensitivity in psychoacoustical and physiological experiments. S. Vranić-Sowers (Dept. of Elec. Eng., and Inst. for Syst. Res., Univ. of Maryland, College Park, MD 20742), H. Versnel, and S. A. Shamma (Univ. of Maryland, College Park, MD 20742)

The physiological and psychophysical experiments in vision $[R$. $L$. De Valois and K. K. De Valois, Spatial Vision (Oxford, New York, 1990)] indicate the existence of cortical cells that are tuned to a particular frequency of a sine wave grating. In present psychoacoustical experiments, listeners' sensitivity to changes in phase of a sinusoidal ripple (in frequency domain, 41 components) was measured for different ripple frequencies and phases. The results were interpreted on the basis of responses to the same stimuli recorded from units of the primary auditory cortex $(\mathbf{A I})$ in the anaesthetized ferret. Since phase sensitivity is associated with the asymmetry (or, ratio of excitatory and inhibitory portions) of cell's response area, which varies along the isofrequency axis [Shamma et al., "Organization of response area of ferret primary auditory cortex," J. Neurophysiol. (in press, 1993)], similar variations are expected for the phase sensitivity. The phase detection for any arbitrary stimuli (seen as a Fourier series) might be predicted from the corresponding results in single-ripple frequency sounds. Prediction for the spectral peak stimuli described in [Vranić-Sowers and Shamma, "The representation of spectral profiles in the auditory system," $J$. Acoust. Soc. Am. (submitted, 1992)], is discussed. [Work supported in part by AFOSR and NRL.]

\section{0:45}

5aPP11. Modulation-following responses and peripheral neural adaptation. Robert L. Smith, Chris Passaglia, and My Nguyen (Inst. for Sensory Res., Dept. of Bioeng. and Neurosci., Syracuse Univ., Syracuse, NY 13244-5290)

In response to tones that are amplitude modulated by sinusoids, a voltage at the modulation frequency can be recorded from the round window of anesthetized gerbils. This "modulation following response" (MFR) displays a number of properties that suggest that it is predominantly composed of summated auditory-nerve responses and may provide a convenient monitor of peripheral neural adaptation. The MFR is a maximum at tone onset and then decays or adapts towards a sustained value that is maintained throughout the duration of the stimulus. At sufficiently low-stimulus intensity levels, the MFR can be reduced by simultaneous or forward masking in a frequency-selective manner sim- ilar to the compound action potential (CAP) at tone onset. The MFR is generally eliminated, along with the CAP, by the application of Tetrodotoxin at the round window. The MFR also exhibited a bandpass "modulation transfer function" and a complex dependence on intensity level that differ from single-unit response characteristics, and appear to reflect the spatiotemporal summation producing compound responses at the round window. [Work supported by NSF.]

\section{1:00}

5aPP12. Cochlear nucleus neural mechanisms preserve complexsound feature encoding in the presence of background noise. Robert D. Frisina, Joseph P. Walton, and Kenneth J. Karcich (Otolaryngol. Div., Univ. of Rochester School of Medicine, Rochester, NY 14642-8629)

Rapid sound amplitude changes-amplitude modulation (AM)are important features of communication sounds like speech, and play a role in other perceptual phenomena including sound localization and pitch perception. Some cochlear nucleus $(\mathrm{CN})$ neurons preferentially amplify AM information, relative to their auditory-nerve (AN) inputs [Frisina et al., Hear. Res. 44, 99-122 (1990)]. An important question from scientific and clinical perspectives is: How does the remarkable ability of some $\mathrm{CN}$ neurons to process $\mathrm{AM}$ change when stimuli are presented in more realistic acoustic environments containing background noise? To address this, single-unit recordings were made from $105 \mathrm{CN}$ neurons and $43 \mathrm{AN}$ fibers of 17 anesthetized chinchillas. Results indicate: (1) Depending on a sound's intensity and AM frequency, a continuous wideband background noise decreased, preserved, or enhanced a unit's ability to encode AM relative to quiet; (2) in most cases, background noise reduces the average response to $\mathrm{AM}$; (3) the synchronous response to AM (fundamental frequency response) decreased in units that decreased their AM coding, and generally increased in units that preserved or enhanced encoding of AM in noise; and (4) some of these effects of noise on CN AM processing can be explained by alterations in AN AM coding at moderate sound levels, but enhancement of CN AM coding at high-sound levels appears to be mediated primarily within the CN. [Supported by NIH-NIDCD Grant No. R29 DC00408-05 and the International Center for Hearing \& Speech Research-RICHS.]

\section{1:15}

5aPP13. Spectral representation of envelope beat frequency in the discharge of cochlear nucleus units in the chinchilla. W. P. Shofner and Stanley Sheft (Parmly Hear. Inst., Loyola Univ., $6525 \mathrm{~N}$. Sheridan Rd., Chicago, IL 60626)

Nonlinear processing of stimulus envelope was investigated in the discharge of cochlear nucleus ( $\mathrm{CN}$ ) neurons. A standard method of investigating nonlinearities is to examine the output of a system for distortion products when the input consists of two tones. For a tone that is amplitude modulated by a two-tone complex, there is no spectral energy at the envelope beat frequency for either the stimulus or the half-wave rectified version of the stimulus. Post-stimulus time histograms (PSTHs) were generated for $\mathrm{CN}$ units recorded from barbiturate anesthetized chincillas to amplitude modulated best frequency (BF) tones modulated by a one- or two-tone complex. Modulation frequencies were between 4-64 Hz. Fourier analysis of the PSTHs shows spectral peaks corresponding to envelope beat frequencies. The magnitudes of the spectral peaks at the beat frequencies are less than the magnitudes of the spectral peaks at the primary modulator frequencies. Spectral peaks in neural spectra at envelope beat frequencies presumably arise from a compressive nonlinearity. Psychophysically, low-frequency envelope beating can be an effective masker of AM detection at the beat frequency (Sheft and Yost, in review). The preliminary results suggest that a spectral representation of the envelope beat frequency does exist at the level of the CN. [Work supported by a PPG grant from NIDCD.] 
were obtained for pure AM detection and for pure FM detection separately, and predictions made for the thresholds of the general threecomponent signals. The best fit to the data was obtained when the $d$ 's for AM and FM were added in a linear manner, j.e., $d^{\prime}{ }_{\text {TOT }}=d^{\prime}{ }_{\mathrm{AM}}$ $+d^{\prime}{ }_{\text {FM }}$. Thresholds for discrimination were then obtained for both AM and FM signals. The standard was a three-component signal with fixed carrier phase and modulation depth set to the detection threshold measured in the first experiment. Discrimination thresholds were elevated and were independent of the standard's carrier phase, i.e., the ratio of AM to FM in the standard. This implies a common decision statistic for both AM and FM signals near threshold. [Work supported by NIDCD Grant No. DC00683.]

\title{
12:00
} rier, in the presence of low-pass, high-pass, or notched noise. Results indicated that high-pass and notched noise produced a nearly identical increase in thresholds, while low-pass noise had little effect. The effect of the noise was similar for all carrier frequencies, suggesting that listeners were not listening at high frequencies in all conditions. The similarity of the effects of high-pass and notched noise indicate that listeners may use spread of excitation, when available, to aid in detecting modulation of narrow-band signals. [Work supported by NIDCD Grant No. DC00683.]

\section{$11: 45$}

5aPP15. Detection and discrimination of mixed modulation signals at low-modulation frequencies. Brent $W$. Edwards and Neal $F$. Viemeister (Dept. of Psychol., Univ. of Minnesota, Minneapolis, MN 55455)

Modulation detection thresholds were obtained for a fixed phase difference between the carrier and sidebands and for a fixed amplitude ratio between the lower and upper sidebands. These are threecomponent signals that, in general, contain both amplitude and instantaneous frequency fluctuations. In a manner similar to Moore and Sek [J. Acoust. Soc. Am. 92, 3119-3131 (1992)], psychometric functions

FRIDAY MORNING, 21 MAY 1993

DRAWING ROOM, 8:30 A.M. TO 12:00 NOON

5aPP16. Detection of mixed modulation at $6 \mathrm{kHz}$. Aleksander Sek and Brian C. J. Moore (Dept. of Exp. Psychol., Univ. of Cambridge, Downing St., Cambridge CB2 3EB, England)

Previously [B. C. J. Moore and A. Sek, J. Acoust. Soc. Am. 92, 3119-3131 (1992)], psychometric functions were measured for the detection of amplitude modulation (AM) alone and frequency modulation (FM) alone, using a $1-\mathrm{kHz}$ carrier and a modulation rate of $10 \mathrm{~Hz}$. Detectability $\left(d^{\prime}\right)$ was then measured for combined AM and FM, with modulation depths selected so that each type of modulation would be equally detectable if presented alone. Significant effects of relative modulator phase were found. Further, $d^{\prime}$ for combined AM and FM was better than would be predicted if the two types of modulation were coded completely independently. However, the phase effects were not correctly predicted by excitation pattern models. Moore and Sek suggested that the good performance for combined AM and FM may have occurred because FM is coded by changes both in excitation level and in phase locking. This idea was tested by repeating the earlier experiment with a carrier frequency of $6 \mathrm{kHz}$, a frequency where phase locking does not occur. The pattern of results was similar to that obtained for the $1-\mathrm{kHz}$ carrier. [Work supported by The Royal Society and the MRC, UK.]

\section{Session 5aSA}

\section{Structural Acoustics and Vibration: Scattering}

\author{
Guillermo C. Gaunaurd, Cochair \\ Naval Surface Warfare Center, Research Department, White Oak Laboratory, Code R-42, Silver Spring, Maryland 20908
}

Geoffrey L. Main, Cochair

Office of Naval Research, Code 1222, Arlington, Virginia 22031

\section{Contributed Papers}

\section{8:30}

5aSA1. Fresnel width of the coupling regions of generalized leaky Lamb waves and Fermat's principle. Philip L. Marston (Dept. of Phys., Washington State Univ., Pullman, WA 99164-2814)

Ray methods describe guided-wave scattering contributions based on local coupling processes. To estimate the effective width of coupling regions for launching and radiation of sound by leaky guided waves of phase velocity $c_{b}$, Fresnel volume concepts of $Y$. A. Kravtsov and others [see, e.g., P. L. Marston, Physical Acoustics (Academic, New York, 1992), Vol. 21, pp. 1-234] were generalized. First, the usual coupling condition on the angle of incidence $\theta_{l}$ for a sphere or cylinder, $\sin \theta_{l}$ $=c / c_{l}$, is derived by Fermat's principle. Then the angular shift $\Delta \theta_{l}$ is derived for a $\pi$ radian phase deviation from the Fermat ray. For a far-field source or observer, $\left|\Delta \theta_{t}\right| \approx \sqrt{2 \pi /\left(k a \cos \theta_{l}\right)}$ is a measure of the ray width. Fermat's principle is also examined for a prompt backward wave mechanism [D. H. Hughes, Ph.D. thesis, Washington State Univ. (1992)] for the high-frequency scattering enhancement by shells. [Work supported by ONR.]

\section{8:45}

5aSA2. Scattering from a thin spherical shell: Higher-order Lamb waves. Gary Steven Sammelmann (Coastal Syst. Station, Dahlgren Div., NSWC, Code 130B, Panama City, FL 32407-5000), Roger H. Hackman (Lockheed, Res. \& Develop. Div., Palo Alto, CA 94304), and Michael F. Werby (NRL Stennis Space Center, Stennis, MS) 
The acoustic scattering from thin spherical shells is marked by the existence of the thickness quasiresonance in the high-frequency regime. This quasiresonance is due to the existence of a region of negative group velocity surrounding the first symmetric Lamb wave. This article presents results on the existence of higher-order quasiresonancelike phenomena, and it considers the effects of Poisson's ratio on the quasiresonance. Pole trajectories in the complex $k a$ plane are exhibited for the first dozen modes on a spherical shell. This analysis reveals the existence of regions of negative group velocity surrounding higher-order Lamb waves. A time domain representation of the contributions of higherorder Lamb waves to the scattered field is presented. This analysis reveals the early time arrival nature of the thickness quasiresonance as reconstructed from the poles in the complex $k a$ plane.

\section{9:00}

5aSA3. Scattering from submerged elastic shells and the characterization of resonances at coincidence frequency: $A$ consensus view. Michael F. Werby (Code 7181, NRL, Stennis Space Center, MS 39529-5004), Roger H. Hackman (Palo Alto Res. Lab., Palo Alto, CA 94304-1191), H. Überall (Catholic Univ. of Am., Washington, DC 20064), and Gary Steven Sammelmann (CSS, Code 130B, Panama City Beach, FL 32407)

In the neighborhood of coincidence frequency for submerged shells (the frequency at which the flexural phase velocity for a plate or shell equals the phase velocity of the speed of sound in the ambient fluid), very large returns are observed which typically include large amplitude narrow spiked returns superimposed on a broad Gaussian shaped return. Each of us as well as the French group (M. Talmant and G. Quentin, University of Paris, 7) have ventured an interpretation of these events for which the interpretations are fairly consistent with some minor variations in detail. As in any new study, differing language was employed. This presentation offers a consensus view of the event along with proposed language for future use.

\section{9:15}

5aSA4. Time-frequency domain processing of acoustic echoes from elastic shells in water. G. C. Gaunaurd (Naval Surface Warfare Ctr., White Oak Detachment (R-14), Silver Spring, MD 20903-5000) and H. C. Strifors (National Defense Research Establishment, Sundbyberg, Sweden)

Acoustic echoes have been studied from submerged elastic shells in either the frequency or the time domains in a number of earlier works. They are now studied and processed in the combined time-frequency $(t-f)$ domain, using both idealized and realistic incident pulses. In a first analysis, (Gaussian) time-windowed Fourier transforms are used, and later, pseudo-Wigner distributions (PWD) are used, and applied to the cases of both ideal and realistic incident pulses. These echo processing techniques extract in various ways the time evolution of the resonances that the target in question may contain within the chosen (broad) operational band. Suitable choice of viewing windows and carrier frequencies serves to generate $(t-f)$ domain displays in which clearly recognizable resonance features become evident. These can then be linked to particular target characteristics. The adjustable widths of the time windows control the amount of detail in the displays. The resulting three-dimensional plots will be shown in an informative colorized format for various cases dealing with elastic shells in water. This will illustrate the target-ID or target characterization merits of this approach, over those obtainable from the usual (monostatic) sonar cross sections.

\section{$9: 30$}

5aSA5. Ray approach for analyzing wave scattering by a submerged vessel with internal structures. I-Tai Lu and Yuqi Yao (Weber Res. Inst., Dept. of Electr. Eng., Polytech. Univ., Rte. 110, Farmingdale, NY 11735)

This paper discusses a ray approach to analyze wave scattering by fluid-loaded targets (submarinelike structures). The approach consists of the following four elements: (1) modal analysis in separable structures (e.g., flat plate, cylindrical shell, spherical shell, etc.); (2) coupling of plate and shell modes at joints; (3) ray asymptotics and spectral approaches for separable structures; and (4) combination of the results in (2) and (3) for nonseparable structures. The model consisting of a cylindrical pipe with hemispherical endcaps, a bulk head and an internal rib, have been considered. Some computed results are compared with experimental data derived by a tank test at the NUSC facility using Mark 0 model. [Work supported by ONR.]

\section{9:45}

5aSA6. Array measurements of backscattered data from internally loaded cylindrical shells. David P. Mackovjak and Arthur B. Baggeroer (Dept. of Ocean Eng., Rm. 204, MIT, Cambridge, MA 02139)

The structural acoustic interaction of model cylindrical shells with different structural discontinuities and internal loading configurations is studied using the maximum-likelihood method (MLM). Three different finite fluid-loaded cylindrical shells are insonified with a midfrequency broadband pulse. The original MLM algorithm is modified using time windowing and slowness steering to exploit the transient nature of the backscattered field. This allows for a detailed investigation of different propagating waves along the shell surface and internal subsystem. Results show that propagating helical membrane waves are important contributors to the scattered field and are frequency dependent. Frequencies below $k a=5.5$ result in a coupled system where the shell and the internal structures interact dispersively. Above this frequency, the membrane waves interact with the shell's discontinuities creating almost continuously radiating localized point scatters. The source location proves to be an important parameter in the creation of these localized radiation fields. The MLM array processing appears to identify weak flexural resonances established by the shell's physical geometry. [Work supported by ONR and Charles Stark Draper Laboratory, Inc.]

\section{0:00}

5aSA7. Acoustic radiation from a coupled planar semicomplex structure in heavy fluid. Noureddine Atalla and Alain Berry (GAUS, Mech. Eng., Univ. of Sherbrooke, Sherbrooke, PQ J1K 2RI, Canada)

A mathematical model is derived to address the vibrations and sound radiations into a dense fluid, of a coupled system consisting of two semicomplex plates (i.e., supporting added mass, stiffeners, and having arbitrary elastic boundary conditions) linked in four points through multistage suspensions elements. The top plate is subjected to point, line, or surface harmonic excitation, while the radiating plate is excited through the suspensions. Both plates are assumed to be baffled, and the radiating plate is fluid loaded. The model is based on a variational approach for the plates, and a matrix transfer approach is used to handle the coupling between the two plates. The solution is sought using a Rayleigh-Ritz expansion in terms of polynomial trial functions which are shown to allow for the arbitrary elastic boundary conditions and to facilitate the calculation of the radiation impedance matrix. The vibrations and noise design of the system is discussed. The main design indicators are the force transmissibilities between the different excitation and attachment points, the ratio of mean-square velocities of the two plates, the mean-square velocity, the radiated power, and the radiation efficiency of the radiating plate. Numerical examples are presented to show the effects of different design parameters (plates thickness, boundary conditions, added mass, stiffeners,...) on the radiated sound. [Work supported by DREA.]

\section{0:15-10:30 Break}

10:30

5aSA8. Scattering from a finite width panel with attached internal resonances in an infinite baffle. Daniel $A$. Russell, Judith $L$. Rochat, Allan D. Pierce, and Victor W. Sparrow (Grad. Prog. in Acoust., Penn State Univ., 117 Appl. Sci. Bldg., University Park, PA 16802) 
kecent research has shown that the vibration response of a finite panel in an infinite baffle may be strongly affected by attaching structures which have internal resonances. In another study ongoing at Penn State, it has been demonstrated that the mass of a fuzzy attachment has a significant effect on the response of a system to an incident acoustic plane wave. In the present research a formalism is developed for predicting the acoustic scattering from such panels with attached masses. A plane wave is incident on a panel, finite in one direction and infinite in the other, with light fluid loading assumed. The backscattering cross section and target strength are calculated for the panel alone. Attachments, treated as lumped element masses, are then added to the panel producing internal resonances of the system. In this paper, the attachments are assumed as well known, they are not fuzzy. The backscattering and target strength are calculated and compared to those of the panel without attachments. An effective scattering cross-section length then may be determined. [Work supported by ONR.]

\section{0:45}

5aSA9. Stop-band and passhand behavior of ribbed cylinders. Joseph A. Clark and Michael A. Sartori (Carderock Div., Naval Surface Warfare Ctr., Bethesda, MD 20084-5000)

As observed through an experimental analysis, the presence of ribs in a cylinder produces two types of stop-band and passband behavior. One type of stop-band and passband behavior concerns the passage of waves along the cylinder. Depending on frequency, energy from a vibrational source will either pass along a ribbed cylinder or be stopped by the ribs. A second type of stop-band and passband behavior involves the radiation of vibrational energy to the far field, which is also dependent upon frequency. These two types of behavior have been observed experimentally on an immersed cylinder $5 \mathrm{ft}$. in length, $1 \mathrm{ft}$. in diameter, and with 30 ribs. Exciting the cylinder in an $n=0$ circumferential mode with an internal array of shakers, the cylinder's radiation was measured at 180 positions along the cylinder's length with a hydrophone located in the cylinder's evanescent near field. Measurements of two cylinders (one with ribs and one without ribs) were made and were processed to obtain position-frequency and wave number-frequency plots. Using these plots, the stop-band and passband behavior that can be attributed to the ribs will be presented as features of wave propagation along the cylinder and as radiation effects in the far field. The experimental results will also be compared with a numerical analysis of an infinite cylinder with periodically spaced ribs. [Work supported by ONT.]

\section{1:00}

5aSA10. Experimental investigation of enhancements in scattered pressure field of fluid-loaded shell with periodic structure. Douglas M. Photiadis, Brian H. Houston (Naval Res., Code 7131, Washington, DC 20375-5000), and Jonathan E. Spanier (SFA Inc., Landover, MD 20785 and NRL Code 7136)

The scattered field from an irregularly ribbed, fluid-loaded plate has been the subject of several recent investigations; one has proposed the existence of dispersive wave phenomena that is due to interference between Bragg diffraction and locally propagating Bloch waves [D. M. Photiadis, J. Acoust. Soc. Am. 91, 1897-1903 (1992)]. Recent measurements at NRL have confirmed the existence of such interference patterns in the scattered pressure field of a fluid-loaded cylinder having nearly periodically spaced ribs in the frequency region of $4.0<k a<30$. It is shown that the theory for the two-dimensional case can be applied to the three-dimensional cylinder problem with moderate first-order agreement with experimental data. Possible approaches to better approximations of solving the cylinder problem are proposed and discussed, including using the developed (2-D) plate theory with a derived analytic form of the bending wave dispersion relation from the thin shell equations. The far-field behavior is shown to be determined by the dispersion relation of the free waves on the structure, the rib spacing, the transmission $(T)$ and reflection $(R)$ coefficients of a flexural wave incident upon a rib, and the deviation from periodicity of the ribs. The feasibility of applying this theory to experimental data for the purpose of determining $T$ and $R$ is also considered.
5aSA11. Influence of endcaps on mid-frequency scattering from finite cylindrical shells at axial incidence. Matthew Conti, Y. P. Guo, and Ira Dyer (Dept. of Ocean Eng., MIT, Cambridge, MA 02139)

Experimental studies of mid-frequency scattering from cylindrical shell models at axial incidence have demonstrated the excitation of axisymmetric compressional and flexural waves within the endcap. The endcap discussed here consists of spherical and conical sections connected to the cylindrical portion of the shell model via slope discontinuities. Phase-matched coupling to compressional waves occurs on the spherical portion of the endcap. Scattering to both wave types occurs at the slope discontinuities. Conical shell dispersion equations developed by Guo are shown to match experimental observations. The Wigner transform is used to demonstrate these phenomena. Amplitude and group delay effects are discussed. The bistatic measurements were conducted over a frequency range of $2.5<k a<10.5$, corresponding to $3 / 4$ to 3 times ring frequency of the shells. [The authors acknowledge assistance of NRL for acquisition of data. Work supported by ONR.]

\section{$11: 30$}

5aSA12. A dual surface approach to near-field measurements of target strength. Michael A. Sartori and Joseph A. Clark (Carderock Div., Naval Surface Warfare Ctr., Bethesda, MD 20084-5000)

Using near-field measurements of the pressure field over dual surfaces surrounding an immersed and insonified structure, an estimate of the structure's target strength can be made. Choosing a cylindrical shaped surface, two measurement arrays enclosing the structure are formed with one array slightly larger than the other. At discrete points over the dual cylindrical shaped surfaces, two pressures are measured, and the pressure and the pressure gradient at these locations result. With the pressure and the pressure gradient, a discretized solution of the exterior Helmholtz integral is solved for the far-field pressure. Using the near-field measurements and this approach, far-field estimations of both radiation problems and target strength problems are possible. Using a rigid sphere as the insonified structure, the target strength problem is modeled and numerically solved using MATLAB. In this paper, an error analysis of this target strength application is presented. The effects on the estimated target strength due to different variables of the dual surface approach will be discussed, some of which include the following: open and closed dual surfaces; differences in the size of the dual surface; and variations on the density of the scanned dual surface. [Work supported by DARPA.]

\section{1:45}

5aSA13. Determination of structural impedance from scattering data. Charles F. Gaumond, Angie Sarkissian, Earl G. Williams (Naval Res. Lab., Washington, DC 20375-5000), and Timothy J. Yoder (SFA, Inc., Landover, MD 20785)

Knowledge of the structural impedance is significantly important in the computation of acoustic scattering and radiation because it contains all the information about the structure that is necessary for the evaluation of the scattered or radiated field in any fluid medium. The inverse problem of determining the structural impedence from scattering measurements is treated. Based on the determination of surface field quantities from acoustical holography measurements, an algorithm is developed for the reconstruction of the structural impedence from knowledge of the scattered field for a set of incident plane-wave directions. The resolution of this reconstruction is treated. Higher resolution may be obtained with near-field measurements. 


\title{
Session 5aSP
}

\section{Speech Communication and Education in Acoustics: Methods and Instrumentation in Speech Production}

\author{
Maureen L. Stone, Chair \\ Department of Electrical and Computer Engineering and Department of Cognitive Science, Johns Hopkins University, \\ 105 Barton Hall, Baltimore, Maryland 21218
}

Chair's Introduction-8:00

Invited Papers

$\mathbf{8 5 0 2}$

5aSP1. Optoelectronic measurement of orofacial motions during speech production. Eric Vatikiotis-Bateson (ATR Human Information Process. Res. Labs., 2-2 Hikaridai, Seika-cho, Soraku-gun, Kyoto, 619-02 Japan), Kevin Munhall (Queens Univ., Kingston, ON, Canada), and David Ostry (McGill Univ., Montreal, PQ, Canada)

Optoelectronic position sensing devices have been important in the study of speech articulation. While limited to the kinematics of externally visible structures such as the lips and the jaw, such systems can be used easily with a wide range of subjects. This video shows how an innovative marker-tracking system, OPTOTRAK (Northern Digital, Inc.), is currently being used in three areas of experimentation: (1) rigid body analysis in which 3-D motion of the jaw is decomposed into its three orientation and three translation components; (2) modeling of the relation between muscle activity (EMG) and 3-D motion of the lips and jaw using artificial neural networks; and (3) mapping facial motions' for possible use in orofacial modeling and audiovisual recognition. The first experiment shows how technical developments, resulting in accurate measurement of 3-D motion, now make it possible to calculate all orientation angles and positions necessary to fully describe structures such as the jaw. The second area demonstrates a more traditional paradigm in which several types of data must be acquired simultaneously. The third area illustrates the growing interest in mapping speech events to facial motion as well as to changes in vocal tract shape.

\section{8:19}

5aSP2. Video-tape presentation of aerodynamic and respiratory kinematic measures during speech. Elaine T. Stathopoulos (Dept. of Commun. Disord. and Sci., State Univ. at Buffalo, North Campus, Buffalo, NY 14260)

A videotape of aerodynamic techniques for making measurements during speech production will be presented. Equipment shown will be: (1) a circumferentially vented pneumotachograph mask for sensing oral air flow [M. Rothenberg, J. Speech Hear. Res. 20, 155-176 (1977)], (2) a pitot tube for sensing intraoral air pressure, (3) a respirometer for air volume displacements, (4) a rotameter for calibrating airflow, and (5) a U-tube water manometer for calibrating air pressure. Discussion of calibration techniques and possible measurements during speech tasks will be displayed. A short segment of the tape will also demonstrate making simultaneous respiratory kinematic measures with linearized magnetometers using a child subject. Measures of lung volume, ribcage volume, and abdominal volume displacements will be shown. [Research supported by NIH.]

\section{$8: 36$}

5aSP3. Laboratory and clinical use of the electroglottograph. James J. Mahshie (Speech Commun. Lab., Dept. of Audiol. and Speech-Lang. Pathol., Gallaudet Univ., 800 Florida Ave., NE, Washington, DC 20002)

The electroglottograph is a device that enables monitoring of variations of vocal fold contact by measuring motion induced variations in impedance of neck tissue in the area of the vocal folds. Electroglottography represents one of the few noninvasive methods available for obtaining useful information about the vibratory patterns of the vocal folds. Since its initial description [Fabre, Bull. Acad. Med. (1957)], the electroglottograph has become an increasingly popular clinical and research tool, providing insight into numerous phonatory characteristics including vocal fundamental frequency $\left(f_{0}\right)$, the extent of vocal fold abduction during phonation, and alterations in laryngeal height during voicing. The purpose of this video presentation is to describe and demonstrate electroglottography, and to examine its use in the clinic and laboratory. The video will: (1) explain the principle of operation of the electroglottograph; (2) demonstrate setup and use of the device; (3) describe attributes of the electroglottograph signal; (4) demonstrate commercially available hardware and software designed to analyze the electroglottograph signal; and (5) describe clinical and rescarch applications of the electroglottograph. The relative strengths and limitations of the device will also be described.

\section{$8: 53$}

5aSP4. Endoscopy, stroboscopy, and transillumination in speech research. Anders Löfquist and Kiyoshi Oshima (Haskins Labs., 270 Crown St., New Haven, CT 06511-6695)

This tape demonstrates the application of endoscopy, stroboscopy, and transillumination to the study of laryngeal function in speech. Stroboscopy involves intermittent illumination of the vibrating glottis using short flashes of light with a frequency of slightly less than that of the glottal vibrations. Thus it has the effect of apparently slowing down the vibrations so that they can be visualized and measured. It has to be remembered, however, that one vibratory cycle recorded using stroboscopy is the composite of several actual glottal cycles. Recordings can be made using either a rigid endoscope or a flexible fiberscope; the latter 
allows analysis of running speech. Images are recorded on video tape for subsequent processing. Transillumination records the amount of light passing through the glottis and thus variations in the size of the glottal opening. The light is provided by a fiberscope and a transducer is placed on the neck. Transillumination is particularly useful for recording glottal abduction and adduction for voiceless consonants. Stroboscopic recordings using both rigid and flexible endoscopes are demonstrated. Applications are discussed, and the usefulness of simultaneously recording transillumination with other signals is exemplified. [Work supported by NIH.]

\section{9:10}

5aSP5. Electromyography in speech research. Kiyoshi Oshima (Haskins Labs., 270 Crown St., New Haven, CT 06511-6695), Katherine S. Harris (CUNY Graduate School, New York, NY 10036 and Haskins Labs., New Haven, CT 06511-6695), and Fredericka Bell-Berti (St. John's Univ., Jamaica, NY 11439 and Haskins Labs., New Haven, CT 06511-6695)

This video demonstrates the application of electromyography (EMG) to the study of speech production. The origin of the electromyographic signal is the passage of the depolarization wave when a muscle fiber or nerve is stimulated. The particular merit of EMG in speech research is that it can provide information about the speech gesture in its natural units and that it directly reflects the motor command from the central nervous system carried by neural impulses. In other words, it is not merely a substitute for direct-viewing techniques but carries one a step farther back in the chain of speech events. Three general types of electrodes have been used in speech research: surface, needle, and hooked wire electrodes. Of these, surface and hooked wire electrodes have been the most useful for recording from the human articulatory muscles. A number of steps must be taken to deal with such signals, including rectification and integration. Applications and instrumentation are discussed, and the simultaneous recording of the EMG with other signals will be presented. [Work supported by NIH Grants DC-00121 and DC-00865 to the Haskins Labs.]

5aSP6. Measuring articulatory movements with an electro-magnetic midsagittal articulometer (EMMA) system. J. S. Perkell, M. A. Svirsky, M. L. Matthies, and J. Manzella (Rm. 36-511, Speech Commun. Group, Res. Lab. of Electron., MIT, Cambridge, MA 02139)

It is difficult to find experimental evidence for underlying mechanisms in speech production, largely because speech acoustics and kinematics are very variable, within and across speakers. Thus one approach to exploring speech production is with statistical analyses of large quantities of articulatory and acoustic data, gathered in a systematic and efficient fashion. If used carefully, EMMA systems can transduce large amounts of accurate movement data simultaneously from midsagittal points on the maxilla, mandible, tongue, lips, and possibly the velum. The use of such a system in combination with appropriate analysis techniques can make it possible to investigate speech kinematics while taking variability into account. This presentation will cover a number of aspects of the use of an EMMA system including: the theory of operation, subject safety, calibrations, preparing for and running an experiment, signal processing, data extraction, checking and using the data, and limitations of this kind of data. [Work supported by N.I.D.C.D.]

5aSP7. Magnetic resonance imaging (MRI) in speech research. Carol Gracco (Yale School of Medicine, Dept. of Surgery, Otolaryngol., 800 YPB, New Haven, CT 06511 and Haskins Labs., 270 Crown St., New Haven, CT 06511), Mark Tiede, Cathe Browman (Haskins Labs., New Haven, CT 06511-6695), Louis Goldstein (Haskins Labs. and Yale Univ., New Haven, CT 06511), and John Gore (Yale School of Medicine, New Haven, CT 06511)

Data on vocal tract shape and dimensions acquired using magnetic resonance imaging techniques have been useful in understanding the articulatory and acoustical processes involved in speech production. This video demonstrates the application of magnetic resonance imaging (MRI) to the study of vocal tract anatomy and application to the investigation of normal and abnormal aspects of the speech production. MRI techniques used to gather basic data to apply in computational models of speech articulation will be illustrated. Recent studies of the vocal tract in normal subjects used MRI to examine changes in vocal tract shape during production of different vowels and illustrated the correspondence between vocal tract shape and the acoustic product (Baer et al., 1990). Subsequent studies have correlated pre- and post-operative assessment of vocal tract dimensions with acoustic data to determine the effects of surgical alteration of specific vocal tract cites on speech production. Other studies have examined the effects of neurological disease on speech and voice production. Images will be used to illustrate nature of these changes on the speech mechanism. Applications of basic MRI techniques as well as specific limitations of this instrumentation will be discussed. [Work supported by NIH Grants Nos. DC-000444, DC-00121 to the Haskins Labs.]

\section{0:01-10:17 Break}

\section{0:17}

5aSP8. Imaging the tongue: Ultrasound. Maureen Stone (Dept. of Electr. and Comput. Eng. and Dept. of Cognitive Sci., 105 Barton Hall, Johns Hopkins Univ., Baltimore, MD 21218)

This videotaped presentation will explain the fundamentals of using ultrasound imaging to study the vocal tract and vocal folds during speech and swallowing. The demonstration will focus on three areas. First, the placement and use of the ultrasound transducer for different types of measurements, including the use of a transducer/head holder to allow reliable, repeatable measurements. Second, a sampling of data will show the tongue in motion in several planes, and the fluidity and complexity of its movement. Vocal fold movement will also be presented. Finally, the tape will demonstrate the use of the instrument on several normal and patient subjects, including profoundly deaf speakers and swallowing-disordered patients. 
5aSP9. Inferring articulatory movements from acoustic data. Kenneth N. Stevens (Res. Lab. of Electron. and Dept. of Electr. Eng. and Computer Sci., MIT, Cambridge, MA 02139)

In recent years, there have been significant improvements in models for predicting the properties of the radiated sound when the time-varying configurations of the vocal tract and laryngeal structures are known. These advances have been especially evident for models of consonant production, for which there are rapid movements of the articulators and consequent rapid changes in the properties of the sound. Experience gained with these models has made it possible to infer articulatory and laryngeal configurations and movements from appropriate measurements of the sound. Several examples are given to illustrate how these inferences can be made. These include: (1) detailed measurements of the changing spectra of frication and aspiration noise at the release of a stop consonant to infer the position and rate of release of the major articulator and the changes in the laryngeal configuration; (2) measurements to indicate the time course of the velopharyngeal opening; and (3) measurements from which the glottal configuration can be estimated. [Research supported in part by the NIH.]

\section{0:51}

5aSP10. The rise and fall of the soft palate: The Velotrace. Fredericka Bell-Berti (Dept. of Speech, Commun. Sci. \& Theatre, St. John's Univ., Jamaica, NY 11439, and Haskins Labs., 270 Crown St., New Haven, CT 06511), Rena A. Krakow (Temple Univ., Philadelphia, PA 19122, and Haskins Labs., New Haven, CT 06511), Dorothy Ross (CUNY Grad. School, New York, NY 10036, and Haskins Labs., New Haven, CT 06511), and Satoshi Horiguchi (National Ohji Hospital, Tokyo, 115 Japan)

The Velotrace is a mechanical device that makes possible the collection of analog data on velar position [S. Horiguchi and $F$. Bell-Berti, Clin. Pal. J. 24, 104-111 (1987)]. The device consists of two levers connected through a push rod, carried on a support rod. The Velotrace is positioned with the support rod resting on the floor of the nasal cavity, with one lever resting on the nasal surface of the velum. When this internal lever is deflected upward, the second (or external) lever is deflected toward the speaker; i.e., changes in the position of the lever resting on the velum are directly reflected in the changes in position of the external lever. The position of an LED fixed to the end of the external lever is monitored with an optoelectric camera, and recorded with an FM tape recorder. [Supported by NIDCD Grant No. DC-00121 to the Haskins Labs.]

11:08

5aSP11. The whole body plethysmograph in speech research. John J. Ohala (Dept. of Linguist., Univ. of California, Berkeley, CA 94720)

The whole body plethysmograph is used to monitor a subject's pulmonic activity and consists of an air-tight box just big enough to contain the subject. The subject breathes and speaks through a tube or facemask which vents to the atmosphere. Changes in thoracic or abdominal volume during breathing cause proportional changes in the volume of the air inside the box. Either an air pressure or an air flow transducer may be mounted in a second vent in the wall of the box in order to measure the aerodynamic variations associated with respiration including those in the expiratory phase on which virtually all voice production is superimposed. The device, which can be constructed fairly simply, is as accurate as the transducer used. If the breathing vent is momentarily closed off, leaving the subject to breathe the air inside the box-which now constitutes a closed system as regards exchange of air between speaker and box-a rough measure of subglottal air pressure may be obtained in a noninvasive way. Its use in phonetic research will be demonstrated by means of a 10-min video.

\section{1:25}

5aSP12. Physiologically based models of phonation. Ingo R. Titze (Nat. Ctr. for Voice and Speech and Dept. of Speech Pathol. and Audiol., Univ. of Iowa, Iowa City, IA 52242)

The process of gathering data from excised larynges, tissue samples, mechanical models, and human subjects is shown. These data are integrated by computer to simulate the physics of air and tissue motion in the larynx and the vocal tract. Voice simulation offers many pedagogical and clinical opportunities in addition to the obvious research applications.

\section{1:42}

5aSP13. Dynamic electropalatography. William J. Hardcastle (Dept. of Linguist. Sci., Univ. of Reading, P.O. Box 218, Reading RG6 2AA, UK)

This videotape will present a demonstration of electropalatography (EPG). EPG uses a thin acrylic pseudopalate embedded with contact-sensitive electrodes to measure tongue-palate contact during speech movements. Its applications are far reaching. Two major applications are its use in examining coarticulatory effects in speech and as a biofeedback tool for speech disorders.

\section{Contributed Papers}

\section{1:56}

5aSP14. Using a new model of vocal-tract midsagittal profile to area-function conversion to constrain an optimization algorithm for the articulatory-acoustic inversion of fricative consonants. Pierre Badin, Denis Beautemps, and Rafael Laboissière (Inst. de la Commun. Parlée, 46 Ave. Félix Viallet, 38031 Grenoble Cedex, France)

An extension of Heinz and Stevens' $\alpha \beta$ model [Proc. of 5th Int. Congr. Acoust. A44 (1965)], where $\alpha$ continuously depends on both midsagittal distance and VT position (along the midline), has been optimized from $x$-ray cineradiographs and formant frequencies obtained on a set of vowels and fricatives uttered by a subject. The purpose of the study was to recover the midsagittal function (a vector of 50 midsagittal distances) of fricative consonants from the measured formant frequencies, in [VCV] contexts. In order to reduce the excess degrees of freedom of this ill-posed problem, the proposed model has been used as a major way to constrain an optimization algorithm based on a gradient descent technique. The gradient of the error between actual and desired formant 
values is computed through a back-propagation network implementing both sagittal-to-area conversion and acoustic wave propagation. Preliminary results are presented and discussed. [Work partly supported by CNRS \& CEC-ESPRIT project SPEECH MAPS.]

\section{2:08}

5aSP15. Modified two-mass model for the vocal cords. X. Pelorson, A. Hirschberg (Vakgroep Transportfysica, TU Eindhoven, P.O. Box 513, $5600 \mathrm{MB}$ Eindhoven, The Netherlands), and Y. Auregan (Univ. du Maine, Le Mans, France)

The two-mass approximation is often presented as a simple but efficient model for the purpose of numerical synthesis of voiced sounds. However, the quality and the naturainess of the generated signal is still to be improved. A simple modification of the two-mass model is proposed in which the mechanical dynamics of that of two point masses. The movement of these point masses drives a two-parameter curve describing the smooth surface of the vocal folds. This representation allows description of unsteady flow separation within the glottis. A semiempirical boundary layer theory is used to calculate the position of flow separation. This theory is experimentally tested using stationary flow measurements and unsteady flow visualization. Furthermore, computed volume flux and Bernoulli force are presented and compared with classical models. The description also includes an inelastic collision which yields a prediction of an additional volume flux due to the deformation of the vocal folds after the through flux has been stopped by the closure of the glottis. This additional deformation appears to have a significant influence on the voiced sound production.

FRIDAY MORNING, 21 MAY 1993

BANQUET ROOM, 7:55 TO 11:45 A.M.

\title{
Session 5aUW
}

\section{Underwater Acoustics: Noise and Arctic Acoustics}

\author{
Barbara J. Sotirin, Chair \\ Ocean and Atmospheric Sciences Division, Code 541, NRaD, San Diego, California 92152-5000
}

Chair's Introduction-7:55

\section{Contributed Papers}

\section{8:00}

5aUW1. Recent work on biological choruses in Australian waters. Douglas H. Cato (Maritime Operations Div., Mater. Res. Lab., Defence Sci. and Technol. Organisation, P.O. Box 44, Pyrmont, NSW 2009, Australia) and Robert D. McCauley (James Cook Univ., Townsville, Queensland 4811, Australia)

Earlier studies of ambient noise in waters near Australia showed that diurnally varying biological choruses were widespread, especially in shallow tropical waters. The most common time of occurrence was between sunset and midnight and the choruses contributed to the frequency band $400 \mathrm{~Hz}$ to $5 \mathrm{kHz}$. Recent work has involved long-term measurements at a fixed location in the Great Barrier Reef with the view to identifying sources and determining the diurnal and seasonal patterns, with shorter measurements at other locations. The measurements show high level choruses over the frequency band $50 \mathrm{~Hz}$ to $2 \mathrm{kHz}$. The substantial lower frequency appears to result from the predominance of fish sources which involve the swimbladder in sound production. The diurnal and seasonal variation is complex, and differs significantly from species to species. There is some evidence of interaction between sources.

\section{8:15}

5aUW2. Analysis and modeling of the results of ECONOMEX. D. Keith Wilson and George V. Frisk (Dept. of Appl. Ocean Phys. and Eng., Woods Hole Oceanogr. Inst., Woods Hole, MA 02543)

ECONOMEX was a study of ULF/VLF noise along an eastern US continental shelf/slope region, conducted between January and April 1991. Concurrent measurements of surface wave directional spectra and meteorological parameters were collected as a part of the SWADE experiment. Extended time series of pressure and bottom displacement have now been analyzed, plotted, and compared with predictions based on the local wave spectra. In most cases, the contribution from local sources appears far more significant than distant sources, such as storms. D. H. Cato's dipole source mechanism [J. Acoust. Soc. Am. 57, 1076-1095 (1975)] agrees well with data from sensors ranging in depth between 450 and $2500 \mathrm{~m}$, but the monopole mechanism is also significant at the shallowest sensor $(100 \mathrm{~m})$. A new method for evaluating Cato's theory is also discussed. The method, since it involves direct evaluation of the dipole source convolution integral, does not require separability of the magnitude and directional dependence of the surface wave spectrum.

8:30

5aUW3. Rapid calculation of distant shipping noise in an underwater environment. Christopher J. Burkhalter (Naval Res. Lab., Code 7181, Stennis Space Center, MS 39529-5004)

Various methods were explored and developed to simulate distant shipping ambient noise without executing very time-consuming sound propagation models for each surface ship contributing to the overall noise levels. The methods were developed with certain things in mind: the effects of various environments between source and receiver, the proximity of the surface ships to the receiver, horizontal directionality, source levels, vertical arrival structure, overall precision and accuracy, and substantial reductions in time for most runs. There was, of course, a direct trade-off between run times and the precision and accuracy of the answer, but because of the inherent randomness of ambient noise, in many cases the control case (i.e., a noise model with propagation model runs for each ship) differed only slightly from the much speedier alternatives developed here. Run times and results for the two methods were compared and analyzed. [Work supported by ONR, NRL, and the Univ. of New Orleans.]

\section{$8: 45$}

5aUW4. Bubble clouds as sources of underwater noise. Hasan $\mathbf{N}$. Oguz (Dept. of Mech. Eng., The Johns Hopkins Univ., Baltimore, MD 21218)

Wave breaking and subsequent formation of whitecaps are known to be the major contributor to the wind-dependent ambient noise levels in the ocean in the low-frequency range $(100 \mathrm{~Hz}-1 \mathrm{kHz})$. A theoretical model that can account for the noise emissions from randomly distrib- 
uted bubble clouds is developed in this study. The model assumes that individual whitecaps produce bubble plumes that grow as a result of air entrainment at the ocean surface. The injection of bubbles at the base of this plume excites the bubble cloud. With the only experimental input of the whitecap coverage ratio, the underwater ambient noise level is calculated, without the need of empirical constants, by integrating contributions from bubble plumes of all sizes. The results are found to be in good agreement with the available field measurements. [Work supported by the ONR.]

\section{9:00}

5aUW5. The correlation between ocean wave energy dissipation and ambient noise. Francis C. Felizardo (R. M. Parsons Lab., MIT, Cambridge, MA 02139) and W. Kendall Melville (Scripps Inst. of Oceanogr., U.C.S.D., La Jolla, CA 92093-0213)

An experiment that measured the NSL between 2 to $25 \mathrm{kHz}$, the wind speed $U$, and the directional wave spectrum was conducted off the coast of Oregon to examine the correlation between the ambient noise spectrum level (NSL) and surface wave parameters. These measurements show that although the correlation between the ambient NSL and the rms wave height is poor, the NSL and wave height spectrum energy levels at frequencies somewhat higher than the spectral peak give correlation coefficients that are comparable to that between the well-known correlation between $U$ and NSL. Estimates of wave energy dissipation due to breaking waves using models proposed by Komen et al. [J. Phys. Ocean. 14, 1271-1285 (1984)] and Phillips [J. Fluid Mech. 156, 505531 (1985)] likewise give comparable correlation coefficients. These results are consistent with the laboratory experiments [M. R. Loewen and W. K. Melville, J. Fluid Mech. 224, 601-623 (1991)] which show that the energy dissipated by wave breaking scales with the acoustic energy radiated. The implications of these results for acoustic remote sensing of breaking waves and associated processes of air-sea interaction are discussed.

\section{9:15}

5aUW6. Influence of bathymetry on the spatial characteristics of ambient noise. J. Newcomb (Naval Res. Lab., SSC, MS 39529-5004)

The ambient noise field is a complex and highly variable phenomena with many components, including contributions due to shipping. Spatial characteristics (e.g., the horizontal directionality) of the ambient noise field have been measured with towed line arrays in many of the major water masses of the world. This paper will address the effects of bathymetry on the propagation and measurement of ambient noise. It is shown that, in many cases, the horizontal directionality of the ambient noise can be fully understood only when the bathymetry along the propagation paths has been considered. Examples of measured data will be presented illustrating the effects of bathymetric stripping and bathymetric shielding on the spatial character of the ambient noise. Other examples of measured data will be shown demonstrating that bathymetric influences are not limited to a narrow frequency range. In addition, modeled data will be used to illustrate the effects of the surrounding bathymetry on noise and signal propagation. [Work supported by the Office of Naval Research (ONR) and the ASW Environmental Acoustic Support (AEAS) Project.]

\section{9:30}

5aUW7. Estimation of the spatial (vertical and horizontal) arrival structure of the undersea ambient noise from towed line array data. R. A. Wagstaff (Naval Res. Lab., Stennis Space Center, MS 39529-5004)

Towed horizontal line arrays have been used very successfully in the past to acquire data to estimate the horizontal directionality of the ambient noise. Those estimates are commonly called noise roses. A more comprehensive algorithm which estimates the horizontal and vertical arrival structure of the ambient noise from towed line array data has been developed at NRL. The algorithm has been exercised with both simulated and measured data, and the results have boen compared to noise roses that have been obtained from the same beam noise data using previous algorithms. The agreement has been good. In addition, the increased dimensionality of the new results has permitted the identification and quantification of spatial "holes" in the noise field that could be exploited by multidimensional arrays to achieve gains well in excess of that which one might expect from adding the independent gains for vertical and horizontal aperture. The algorithm is presented, and some results are discussed. [Work supported by the Office of Naval Research (ONR) and the ASW Environmental Acoustic Support (AEAS) Project.]

\section{9:45}

5aUW8. Ambient noise measurements in the southern Norwegian Sea. S. F. Kooney (Naval Res. Lab., Stennis Space Center, MS 39529-5004)

Ambient noise horizontal directionality measurements were made in the southern Norwegian Sea by a towed horizontal line array during March 1992. The measurements were taken at three sites near an oceanographic front. The first site was north of the front, the second site was within the front, and the last site was south of the front. The ambient noise results, omnidirectional levels, and various beam noise statistics are discussed. In general, the low-frequency ambient noise showed approximately $15 \mathrm{~dB}$ more noise coming within a broad sector to the east and decreased with increased frequency. Along other azimuths, high levels in the horizontal directionality patterns correlated well with directions in which the bathymetry provided windows for the noise to propagate to the site from distant ships. The beam noise statistics at all three sites showed that the ambient noise possessed a high degree of spatial anisotropy at frequencies below $150 \mathrm{~Hz}$ and very little at frequencies of $300 \mathrm{~Hz}$ and above. [Work sponsored by the Office of Naval Research.]

\section{0:00-10:15 Break}

\section{0:15}

5aUw9. Gaussian mixture statistical model of Aretic noise. $R$. Myers and B. Sotirin (Ocean and Atmospheric Sci. Div., Code 541, NRaD, San Diego, CA 92152-5000)

Arctic ambient noise is often stationary and Gaussian for significant periods of time. However, during periods of local ice activity, the ambient noise can become highly non-Gaussian, nonstationary, and exhibit substantial variability in spectral shape. During these periods, the task of characterizing the statistics of the ambient noise is greatly complicated. In many cases, however, the distribution of the noise during the periods of intense ice activity can be modeled as a two-state Gaussian mixture, where the mixture parameters are estimated from the data by searching for the maximum of the likelihood function. A statistical analysis of selected ambient noise collected over a 10-day period during which long-range ambient noise was interspersed with intense, brief periods of local ice noise indicates that the ambient noise was highly Gaussian except during the periods of ice activity. During the periods of iee aetivity, the distribution of the ambient noise was modeled quite well as a two-state Gaussian mixture.

10:30

5aUW10. Acoustic characterization of ice vibrations. G. R. Giellis and T. C. Yang (Naval Res. Lab., Washington, DC 20375)

Ice cracking/breaking induces ice vibrations, hence measurements of acoustic waves in ice can be used to probe the forcing function which cracks/breaks the ice. Previous measurements using ice-implanted geophone sensors (array) have revealed the existence of longitudinal (plate), flexural, and horizontally polarized shear waves at low $(<100$ $\mathbf{H z}$ ) frequencies. Applying frequency-wave number analysis to hammer blow data received on a line array of three-axis geophones, the phase velocities of these waves were measured. As expected, the longitudinal 
and horizontal-shear waves travel with constant phase velocities whereas the phase velocity of the flexural wave is frequency dependent. The frequency wave number analysis also reveals the existence of a new wave, heretofore not noticed, with a different frequency dispersion characteristic compared with the flexural wave. This wave travels with a phase velocity comparable to the flexural wave, consequently identifying it in the time series data is difficult. This wave is hypothesized as the forced wave excited by a point force. Measurements of group velocities and attenuation for each type of wave are also discussed.

\section{0:45}

5aUW11. Free and forced vibrations in a floating ice sheet: Modeling and comparison with data. T. C. Yang (Naval Res. Lab., Washington, DC 20375) and T. Yates (Vector Res. Co., Rockville, MD 20852)

Time domain waveforms excited by an impulse, point force are modeled using fluid-loaded thin plate theory and compared with measured data collected on ice-implanted geophones. In the frequency domain, the solution, expressed as an integral over wave number, can be expressed in terms of poles (free waves) and branch cuts (forced waves). The time domain solution is obtained by taking a Fourier transform of the frequency response of the plate, weighted by the (measured) source spectrum. The modeled time domain waveform compared favorably with the received time series data. A simple direct time domain solution for the forced wave in a thin plate in air also approximates the data well. It is shown that the forced wave solution produces the "abnormal" dispersion relation observed in recent geophone data. The free-wave solutions, which have been extensively studied before, also agree with the geophone data.

\section{1:00}

5aUW12. Matched-field estimation of scattering from Arctic ice-Analysis of the CEAREX 89 field experiments. Tarun $K$. Kapoor and Henrik Schmidt (Dept. of Ocean Eng., MIT, Cambridge, MA 02139)

Scattering experiments were conducted in 1989 in the NorwegianIceland Sea using explosive charges detonated at varying depths. The scattered field was received at a crossed horizontal hydrophone array positioned at a depth of $60.0 \mathrm{~m}$. In this paper, the scattering of lowfrequency acoustic waves from the Arctic Ice sheet will be evaluated via matched-field processing of short-range returns. Using near-field beamforming (focusing), which assumes a point scatterer, it was possible to isolate the scattering hot spots under the ice sheet. Essential inputs to this processing include the source location and shot times which were determined using an adaptive least-squares estimator in a matched-field approach. [Work supported by ONR.]

\section{1:15}

5aUW13. Optimal array configurations for a Bartlett beamformer in an Arctic environment. R. D. Huston (Defence Res. Establ. Pacific, FMO Victoria, BC VOS 1B0, Canada)

Optimal or near-optimal array configurations for a vertical array in a shallow-water Arctic environment were determined using a Bartlett matched-field beamformer. A normal mode model was employed to calculate the acoustic field for a range-independent waveguide, and a simulated annealing (SA) algorithm was implimented to solve the combinitorial aspect of this problem. The cost function was designed to minimize sidelobes and the mainlobe width in the ambiguity surface, with the objective of optimizing the localization accuracy of the Bartlett beamformer. Exhaustive searches, which are possible for arrays with up to five hydrophones, confirmed the accuracy of array configurations from the SA method. An analysis of the range dependence of these solutions compares the localization accuracy from optimal and conventional uniform arrays for 8,16 , and 32 hydrophones at source ranges between 10 and $40 \mathrm{~km}$.

\section{1:30}

5aUW14. Measurement of ocean-bottom compressional and shear speeds in the Canadian high Arctic. S. E. Dosso and G. H. Brooke (Defence Res. Establ. Pacific, FMO Victoria, BC VOS 1B0, Canada)

Geoacoustic properties of the ocean bottom can be a significant factor influencing acoustic propagation in shallow-water environments. Knowledge of these properties is required for reliable acoustic propagation modeling and matched-field processing. This paper presents the preliminary analysis of a sea-floor seismic experiment carried out on the continental shelf of the Lincoln Sea north of Ellesmere Island, Canada, to determine compressional- and shear-speed models for the ocean bottom. A three-component ocean-bottom seismometer (OBS) was deployed through $\sim 4 \mathrm{~m}$ of multiyear sea ice in $540 \mathrm{~m}$ of water. Broadband explosive sources were detonated on the sea floor at ranges from 50 to $900 \mathrm{~m}$. Source-receiver ranges and shot instants were verified by timing the direct and surface-reflected arrivals through the water. Subsequently, a layered compressional-speed model for the ocean bottom was determined from a first-break analysis of the head-wave arrivals. Also, interface (Sholte) wave arrivals have been identified on many of the seismograms and exhibit characteristic elliptical particle motions. Waveform modeling of these interface waves is discussed with a view to extracting shear-speed properties in the uppermost bottom layers.

FRIDAY AFTERNOON, 21 MAY 1993

SALON RENAISSANCE, 1:00 TO 4:45 P.M.

\title{
Session 5pPA
}

\section{Physical Acoustics: General II}

\author{
Quan Qi, Chair \\ Theoretical and Applied Mechanics, University of Illinois, Urbana, Illinois 61801
}

\section{Contributed Papers}

\section{1:00}

5pPA1. The influence of boundary layer on elastic wave scattering by spherical inclusions. Daniel Lévesque and Luc Piché (Indust. Mater. Inst., Natl. Res. Council, 75 De Mortagne Blvd., Boucherville, PQ J4B 6Y4, Canada)

The influence of a boundary layer on the scattering of elastic waves by spherical inclusions is investigated. Based on the exact transition matrix ( $T$ ) developed for layered obstacles [A. Boström, J. Acoust. Soc. Am. 67, 399-413 (1980)], a numerical study is performed for all values of $h / a$, where $h$ is the layer thickness and $a$ is the effective radius of the layered obstacle. For $h / a<0.1$, the layer behaves like an interface, allowing one to investigate a wide range of boundary conditions. Except for high mass density interfaces, the scattered field amplitude is sensitive mainly to the normal and transverse stiffnesses and classical solutions for the so-called rigid and slip boundary conditions are recovered. For 
$h / a<0.1$, the layer acts as an interface region and property changes of the host near the obstacle can be modeled. Ultrasonic measurements with charge-filled polymers present evidence for such a layer. Finally, for $h / a \approx 0.1$, the layer behaves as a coating or a shell depending on the mass density. In this case, full interactions are included in the formalism.

\section{$1: 15$}

5pPA2. Scattering from an elastic shell and a doubly infinite fluid-solid interface with random surface roughness. Garner $C$. Bishop and Judy Smith (Naval Undersea Warfare Ctr. Div. Newport, Newport, RI 02840)

A null field $T$-matrix formalism similar to that of Kristensson and Strom [J. Acoust. Soc. Am. 64, 917-936 (1978)], is used to obtain a formal solution for scattering from a stationary elastic shell immersed in a homogeneous and isotropic fluid half-space and in the vicinity of a doubly infinite fluid-solid interface with random surface roughness. The full elastic tensor boundary conditions are applied at each fluid-solid interface and equations that follow from the application of the Helmholtz-Kirchhoff integral and the null hypothesis to the various regions are used to construct the $T$ matrix for the shell-interface system and the free-field $T$ matrices for the elastic shell and the randomly rough fluid-solid interface. Spherical basis functions are used to construct the conventional free-field $T$ matrix for the elastic shell. However, rectangular vector basis functions are used to construct a formal representation of the $T$ matrix for the randomly rough fluid-solid interface. The free-field $T$ matrices are introduced into the Helmholtz-Kirchhoff and the null field equations for the shell-interface system and it is shown that the $T$ matrix for the system is simply related to the free-field $T$ matrices for the shell and the randomly rough fluid-solid interface. Therefore, a perturbation theory $T$ matrix for the randomly rough surface can be introduced in a relatively simple manner. An explicit representation for the scattered pressure field in the fluid is constructed. It is also shown that the formalism contains multiple-scattering effects between the surface and the shell and also on the rough interface.

\section{1:30}

5pPA3. Increased backscattering from an inhomogeneous liquid-solid interface at the Rayleigh angle. Peter B. Nagy and J aszlo Adler (Dept. of Welding Eng., Ohio State Univ., Columbus, OH 43210)

It is shown that the experimentally observed peak in the incoherent backscattering from a liquid-solid interface at the Rayleigh angle is caused by the sharp maxima of the longitudinal and shear transmission coefficients. Of course, both refracted waves are evanescent in this region, i.e., they do not carry energy away from the interface but rather keep the vibration within approximately one wavelength of the surface. Nevertheless, the resulting vibration in the solid is much stronger than at other angles of incidence. The incoherent scattering can be caused by either geometrical irregularity or material inhomogeneity (e.g., surface roughness or polycrystalline grain structure). This paper presents analytical and experimental results showing that, regardless of the physical nature of the scattering inhomogeneity, there is a distinct maximum in the backscattered intensity around the Rayleigh angle. The width of the peak is determined by the density ratio between the solid and the liquid while the maximum backscattering at the peak is essentially independent of the average material properties and is determined solely by the scattering inhomogeneity.

\section{$1: 45$}

5pPA4. Wave scattering from a thin random fluid layer. Yuan Zhang and Richard L. Weaver (Dept. of Theor. and Appl. Mech., Univ. of Illinois, Talbot Lab., 104 South Wright St., Urbana, IL 61801)

The problem of a plane harmonic wave obliquely incident from a homogeneous ideal fluid space upon a random fluid layer is considered. The thin layer is taken to have properties that vary randomly only in the in-plane directions. A first Born approximation is used and the average intensity of the incoherent part of the scattered wave is found to be proportional to the two-dimensional spatial Fourier transform of the auto- and cross-covariance functions of the layer, within the confines of the validity of the first Born approximation. Therefore, the inverse scattering problems may be straightforward, provided the necessary experimental data of the incoherent wave can be found. A sufficient condition is also given to estimate the range in which the second-order term in the Born series can be ignored. [Work supported by the National Science Foundation Solid Mechanics Program, Grant No. MSM-91-14360.]

\section{2:00}

5pPA5. Scattering from partially contacting interfaces. J. G. Harris and E. Yogeswaren (Theoret. Appl. Mech., UIUC, 216 Talbot Lab., 104 S. Wright St., Urbana, IL 61801)

Fatigue cracks and diffusion bonds are two common objects of nondestructive inspection. Both are characterized by extensive regions of rough, partially contacting interfaces, where the characteristic size of a void or asperity is much smaller than the compressional wavelength in the material, at frequencies on the order of $100 \mathrm{MHz}$. Work describing efforts to characterize scattering from such an interface, including the effects of multiple scattering, is described. The multiply scattered wave fields are calculated by solving scalar or vector integral equations. Two formulations will be discussed. One uses a specific interface model consisting of multiple, small cracks having arbitrary lengths and spacings, while the other uses a more general formulation called a polarization theory [G. Wickham, J. Nondestr. Eval. (to appear in 1993)]. The scattered wave fields are then used in a measurement model, whose purpose is to emulate the effects that the emitting and receiving transducers have upon the incident and received signals. Both scattering by an incident plane wave and by a focused beam are discussed. [Work supported by NSF.]

\section{2:15}

5pPA6. On the scattering of elastic waves in inhomogeneous material. Eveline J. Aymé-Bellegarda (IBM T. J. Watson Res. Ctr., P.O. Box 704, Yorktown Heights, NY 10598) and Tarek M. Habashy (Schlumberger-Doll Res., Ridgefield, CT 06877-4108)

This work is concerned with the imaging and quantitative characterization of objects embedded in inhomogeneous material, using acoustic or elastic waves as the interrogating sources. Such problems are recurrent in many areas such as, for instance, geophysics, oceanography, and medical imaging. The interest here hinges on the determination of high contrast channels embedded in multilayered elastic structures. A general framework is presented, based on vector integral equations. In addition to being suitable for both the forward and inverse problem, this framework is appropriate for any geometry and transmitter-receiver characteristics. Tractability issues are discussed, leading to the consideration of the Born approximation for inhomogeneous elastic background. To go beyond the inherent limitations of this approximation, an iterative inversion scheme is introduced. The method is illustrated in the case of a 2-D problem. First, the forward model is validated against an independent means based on a finite-difference approach. Then, constrained least-square reconstruction is performed, aiming at recovering the density distribution of a 2-D fluid-filled object buried in high contrast layers.

\section{2:30}

5pPA7. Acoustic diffraction by multiple two-dimensional objects; its calculation using an approximate integral method. T. $R$. T. Nightingale (Bldg. M-27, Acoust. Lab., Inst. for Res. in Construction, Natl. Res. Council, Ottawa, ON K1A OR6, Canada)

A method for determining the diffracted or scattered components of an acoustical wave by multiple two-dimensional objects is given. The method presented uses approximate boundary conditions to simplify the cumbersome set of integral equations associated with nonapproximate analytic solutions of the Helmholtz integral. The approximate boundary 
conditions are used to calculate the insertion loss for multiple twodimensional abjects of various sizes. The predictions are compared to measured results and limitations are discussed.

\section{2:45-3:00 Break}

\section{3:00}

5pPA8. Elastic normal modes in multilayered heterostructures. $R$. Esquivel-Sirvent and G. H. Cocoletzi (Dept. of Phys., Ohio Univ., Athens, OH 45701)

A simple matrix approach is presented to study the elastic properties of multilayered systems. Periodic superlattices are considered to calculate the dispersion relations of the collective normal modes, and finite and semi-infinite structures to obtain the reflectivity of longitudinal modes. The dispersion relations for a two layer period superlattice show band structure. For transverse waves, it is considered that the single layer may support surface modes and found that their interaction with those of the adjacent layers yield also a band structure. The reflectivity of normal incident longitudinal waves for the semi-infinite superlattices resembles the band and gap regions of the dispersion relations. The comparisons between theory and experiment of the reflectivity of sound waves for the three layer systems show good agreement.

\section{3:15}

5pPA9. Variational methods and boundary conditions for Bloch waves in composite materials. B. L. Jiao and R. Siems (Theoret. Phys., Univ. des Saarlandes, D-6600 Saarbrücken, Germany)

A modified variational procedure is presented for an efficient determination of dispersion curves and especially of normal modes of phonons in periodic, inhomogeneous materials with abrupt changes of material constants: The Bloch wave eigenvalue problem is solved by a Ritz method using expansion functions with discontinuous first derivatives for the displacements, which satisfy the boundary conditions for displacements and stresses. Explicit expressions are given for a threefold periodic arrangement of inclusions in a matrix. Questions of convergency and completeness of the expansion are discussed. A numerical comparison with results obtained with customary Fourier trial functions for structures inhomogeneous in one and two dimensions and with exact results for one dimensional inhomogeneities shows the great accuracy of the method.

\section{3:30}

5pPA10. The effect of domainons on the energy band in ferroelectric $180^{\circ}$ multilayered domain superlattice. Xingjiao $\mathrm{Li}$, Zhiming $\mathrm{Wu}$, Jeffery Chen, Dingguo Cai (Dept. of Solid State Electron., Huazhong Univ. of Sci. and Technol., Wuhan 430074, People's Republic of China), and Shaopin Li (Penn State Univ., University Park, PA 16802)

Ferroelectric domain layer wave (FDLW) [X. Li, J. Appl. Phys. 56, 88 (1984)] would be induced by the ultrasonic wave in the ferroelectric $180^{\circ}$ domain superlattice. There exist many new phenomena under the action of FDLW. Its characteristics sum up an elementary excitation "domainon" [X. Li et al., J. Phys.: Condensed Matter 2, 9577 (1990)]. It has been shown that the domainons subband can be formed by the interaction between domainons and electrons at the conductive band or holes at the valence band of ferroelectric $180^{\circ}$ domain superlattice, in other words, under the action of the ultrasonic wave, the domainons would change the distribution of electrons or holes and affect the electric properties of ferroclectries. In addition, the variation of the subband generated by ultrasonic wave with the vector $k$ of FDLW is similar to the variation of the subband with periodic width of superlattice. The characteristics of the symmetry domainons and asymmetry domainons have been discussed. Further analysis above that the periodic potential fields have the symmetry vibration modes and asymmetry vibration modes corresponding to the same dispersion relation of FDLW, while the displacements of the two opposite domains are asymmetry and sym. metry, respectively.

5pPA11. Ferroelectric domain layer wave in ABA type $180^{\circ}$ domain sandwich structure. Xingjiao $\mathrm{Li}$, Zhiming Wu, Jeffery Chen, Dingguo Cai, Shaopin Li (Dept. of Solid State Electron., Huazhong Univ. of Sci. and Technol., Wuhan 430074, People's Republic of China), and Zhuohui Chen (Univ. of Toronto M5S 1A4, Canada)

The ferroelectric domain layer wave (FDLW) in the ABA type $180^{\circ}$ domain sandwich has been discussed. The polarized direction of A material is opposite to that of $\mathbf{B}$ material. The results show that there exist two types of ferroelectric domain layer waves in the structure. One is the FDLW of symmetric vibration [X. Li, J. Appl. Phys. 56, 88 (1984)], another is the FDLW of asymmetric vibration. The asymmetric vibration FDLW is a new FDLW, it has a cutoff frequency. From the dispersion relation of the two modes, it is shown that the frequency of the asymmetry mode is higher than the frequency of the symmetry mode. The amplitudes of displacement fields and potential fields of the asymmetry mode are smaller than that of the symmetry mode in the $B$ layer. The system that is composed of the different A, B materials have been analyzed and the general dispersion relation is given; it has a very simple formation, and the relations between the parameter of $A, B$ material and dispersion relation have been discussed.

5pPA12. Electroacoustic waves in a piezoelectric-layered structure under periodic metal gratings. Youxin Zhu, Zhiming Wu, Dingguo Cai, and Xingjiao Li (Dept. of Solid State Electron., Huazhong Univ. of Sci. and Technol., Wuhan 430074, People's Republic of China)

The propagation of electroacoustic waves in a piezoelectric-layered structure under periodic metal gratings is investigated. A general theory that fits both the elastic and electric periodic boundary conditions has been developed and is applicable to a grating with an arbitrary electrical connection between adjacent metal strips. Numerical works are given. It is found that the considered electroacoustic waves, which penetrate deep into the substrate in the absence of the metal grating, become trapped at the interface between the substrate and the surface layer when the grating is presented. It is noted that the penetrate depth is heavily dependent on the grating thickness. The electroacoustic wave penetration depth can be adjusted by a proper design of the grating structure to minimize the acoustic power density in the layered structure. The relevant attributes of the wave such as particle displacement electrical potential, and dispersion are also computed and analyzed. Some practical application in acoustoelectronics are suggested. [Work supported by NNSF.]

\section{$4: 15$}

5pPA13. Shear horizontal waves in layered structure with $180^{\circ}$ ferroelectric domain. Dingguo Cai, Youxin Zhu, Zhiming Wu, and Xingjiao Li (Dept. of Solid State Electron., Huazhong Univ. of Sci. and Technol., Wuhan 430074, People's Republic of China)

Theoretical analyses and numerical results describing the propagation characteristics of shear horizontal interface waves in layered structure with $180^{\circ}$ ferroelectric domain are presented. A sandwich structure and a multilayered structure with finite layers are considered. It is found that there exist universally strong dispersion and multimode in a multilayered structure. There are $N-1$ dispersion curves in a structure with $N 180^{\circ}$ domain layers. For the multimode, some symmetry properties are found. The fundamental mode is a symmetrical one and the higherorder modes are symmetry or antisymmetry. The multimodes are arranged as symmetrical-antisymmetrical alternatively. There exist cutoff frequencies. It is noted that the $\mathrm{B}-\mathrm{G}$ wave and the $\mathrm{M}-\mathrm{T}$ wave may be 
obtained as special cases of the present theory. Three kinds of dispersion curves are found: ferroelectric domain layer wave, ferroelectric domain layer flexure wave, and lovelike wave. The dispersion relations and the multimode characteristics of the SH interface waves in $180^{\circ}$ domain finite multilayered structure were verified by experiments. [Work supported by NNSF.]

\section{4:30}

5pPA14. The subband in ferroelectric $180^{\circ}$ multilayered domain structure. Zhiming Wu, Dingguo Cai, Youxin Zhu, Jeffery Chen, and Xingjiao Li (Dept. of Solid State Electron., Huazhong Univ. of Sci. and Technol., Wuhan 430074, People's Republic of China)

In a ferroelectric $180^{\circ}$ multilayered domain structure, the ferroelectric domain layer wave (FDLW) propagating along the domain inter- face would cause a periodic electric potential field [X. J. Li et aL, J. Phys. C 2, 9577-9588 (1991)]. The periodic potential field would split the energy band of electrons into subbands called the "minigap." The feature of the minigap is that it depends on the existence of FDLW because the wave vector and amplitude of FDLW would affect the minigap structure. This is different from the normal superlattice in which the minigap is determined. In addition, the periodic potential fields have the symmetry vibration modes and asymmetry vibration modes corresponding to the same dispersion relation of FDLW, while the displacements of the two opposite domains are asymmetry and symmetry, respectively. The calculating results of the subband in ferroelectric $180^{\circ}$ domain superlattice have shown that the subband variation with the vector $k$ of FDLW is similar to the subband variation with periodic width of superlattice.

FRIDAY AFTERNOON, 21 MAY 1993

BALLROOM, 1:00 TO 4:00 P.M.

\title{
Session 5pSP
}

\section{Speech Communication: Issues in Vowel Perception}

\author{
Linda Polka, Chair \\ School of Human Communication Disorders, McGill University, 1266 Pine Avenue West, Montreal, Quebec H3G 1A8, \\ Canada
}

\section{Contributed Papers}

1:00

5pSP1. Modeling format frequency discrimination. Mitchell S. Sommers and Diane Kewley-Port (Depts. of Psychol. and Speech and Hear. Sci., Indiana Univ., Bloomington, IN 47405)

Changes in the formant frequencies of steady-state vowels can produce level changes in harmonic components throughout the bandwidth of the stimulus. To successfully model thresholds of formant frequency discrimination, however, it is necessary to determine whether level changes in components spectrally remote from the altered formant affect discrimination performance. Therefore, the present experiments investigate which harmonics affect performance in order to evaluate excitation-pattern models of formant frequency discrimination. Synthetic tokens of the vowels / $\mathrm{U} /$ and /e/ were generated by digitally summing the first 24 harmonics of a $200-\mathrm{Hz}$ fundamental. Minimal uncertainty thresholds were obtained for $F 1$ increments in /e/ and $F 2$ decrements in /U/ when: (1) all harmonics were allowed to vary; (2) harmonic level variations were restricted to a subset of components spectrally close to the altered formant; and (3) harmonics remote from the shifted formant were excluded from the stimulus. Results indicated that thresholds for conditions (1) and (2) did not differ until level variations were restricted to between two and three components. Thresholds for condition (3) were not significantly different than those obtained for vowels with a full complement of harmonics. Excitationpattern models of the results will be discussed. [Research supported by NIH.]

\section{1:12}

5pSP2. Perception of vowel height: Effects of varying F1-FO difference. Kathryn A. Hoemeke (Scott Instrum., 111 Willow Springs Dr., Denton, TX 76205) and Randy L. Diehl (Univ. of Texas, Austin, TX 78712)

Perceived vowel height has been reported to vary inversely with the difference (in Bark) between the first formant frequency $(F 1)$ and the fundamental frequency $(F)$ ) [H. Traunmüller, J. Acoust. Soc. Am. 69, 1465-1475 (1981)]. Syrdal and Gopal [J. Acoust. Soc. Am. 79, 10861100 (1986)] observed that naturally produced [+high] and [-high] vowels tend to divide at a critical F1-F0 difference of 3-3.5 Bark, corresponding to the range of the spectral center of gravity effect [L. Chistovich and V. Lublinskaja, Hear. Res. 1, 185-195 (1979)]. In the present study, listeners identified three sets of synthetic vowels varying orthogonally in $F 1$ and $F 0$ and ranging from $/ \mathrm{i} /-/ 1 /, / 1 /-/ \varepsilon /$, and $/ \varepsilon /-/ x /$. For the $/ I /-/ \varepsilon /$ set, which spanned the $[+$ high] $/[-$ high] distinction, there was a steep identification boundary located at an $F 1-F 0$ difference of 3-3.5 Bark. However, for the $/ \varepsilon /-/ 2 /$ and $/ \mathrm{i} /-/ \mathrm{l} /$ sets, which occupied regions where $F \mathbf{1}-F 0$ was always greater than or less than 3 Bark, vowel labeling varied more gradually as a function of $F 1-F 0$. In fact, for the $/ \mathrm{i} /-/ \mathrm{l} / \mathrm{set}, F 1-F 0$ was actually a poorer predictor of identification performance than $F 1$ alone. [Work supported by NIDCD.]

\section{1:24}

5pSP3. Formant transitions and the identification of concurrent vowels. Peter Assmann (School of Human Devel., Univ. of Texas at Dallas, Box 830688, GR4.1, Richardson, TX 75083)

Listeners exploit several sources of information to segregate the speech sounds produced by two competing talkers. When two synthetic vowels are presented simultaneously and monaurally, listeners can take advantage of a difference in fundamental frequency $\left(f_{0}\right)$ to identify the vowels. This study compared the contributions of $f_{0}$ differences and formant-frequency transitions to the identification of concurrent vowels. Accuracy improved when a two-semitone difference in $f_{0}$ was introduced, compared to the case where both $f_{0}$ 's were the same. In some conditions a small, independent improvement was observed when one of the vowels was preceded by a 100 -ms gliding formant pattern. However, the presence of the glide did not help to identify the vowel which is preceded; its presence helped by making the competing vowel more identifiable. One explanation for improvement in the glide condition is a formant-tracking process which groups together the formants of each voice on the basis of good continuity. However, this account predicts improvement for both vowels which was not observed. An alternative account is provided by a model that (i) performs a series of analyses using a brief, sliding temporal window, and (ii) determines which re- 
gion of the signal provides the strongest evidence of each vowel constituent. [Work supported by NIDCD.]

\section{$1: 36$}

5pSP4. On the distinctive pitch of vowels: Perceptual prototypes for sinewave analogs? Philip E. Rubin (Haskins Labs., 270 Crown St., New Haven, CT 06511), Robert E. Remez, Jennifer S. Pardo, Jennifer M. Fellowes, Eva Y. Blumenthal, Danielle A. Warren, and Bella Schanzer (Barnard College, 3009 Broadway, New York, NY 10027)

A recent study by Kuhl et al. (1991) found striking perceptual correspondences between vowels and steady tones. Whether subjects experienced spoken vowels, visually presented images of articulating faces producing vowels, or imaginary vowels, they matched the vowel / $a$ / with a low-pitch tone, and / i/ with a high-pitch tone. However, tonal analogs of vowels were matched in the opposite manner, with low pitch associated with the vowel $/ i$ / and high with the vowel $/ a /$. These sine-wave vowels were therefore excluded from hypothesized recognition mechanisms employing distinctive vowel pitches as perceptual prototypes. This finding is counterevidence to claims that tonal analogs of utterances are perceived through ordinary means. The present study employed sinewave realizations of several words differing solely in the nuclear vowel, $/ a /$ or $/ \mathrm{i} /$, in an attempt to replicate and extend this finding. Subjects were asked to match the predominant pitch or vowel quality of these medial sine-wave vowels to the pitch of a single tone. The results will be discussed with respect to claims about the ordinariness of the perception of sinewave replicas and hypothetically prototypic distinctive vowel pitches. [Research supported by NIDCD and NICHD.]

$1: 48$

5pSP5. The curious case of English $/ w /$ and $/ y /$, Leigh Lisker (Haskins Labs., 270 Crown St., New Haven, CT 06511-6695 and Univ. of Pennsylvania, Philadelphia, PA 19104)

English $/ w /$ and $/ y /$ are often identified phonetically with $/ u /$ and $/ i /: / w /$ and $/ w /$ involve dorso-velar constriction and lip rounding, $/ y /$ and $/ i$ / raising of the tongue toward the palate without rounding. Although the glides are thus more or less equated with the vowels, most linguists keep /w, $y /$ apart from $/ u, i /$. The justification is that $/ w, y /$ are nonsyllabic "glides," whereas / $u, i /$ are not. As a phonetic basis for keeping / $w, y /$ and / $u, i /$ apart, this is not very compelling. Aside from the fact that the articulatory postures for $/ w, y /$ may not always be identical with those for $/ \mathrm{u}, \mathrm{i} /$, the phonological argument advanced here is that $/ w /$ differs from $/ u /$ in being distinctively rounded, but not distinctively dorso-velar, while $/ y /$ is distinctively palatal, but not distinctively unrounded. In fact, the same articulatory configuration, that of a rounded palatal $[\mathrm{H}]$, is differently interpreted in the contexts $/ \mathrm{i}-\mathrm{i}$ / and /u-u/, so that [iui] is /iwi/, while [uyu] is /uyu/. Thus the same vocal tract configuration, reflected in an identical $F$ pattem, is differently interpreted in the two vocalic contexts. It would seem that the listener knows that it was achieved by two quite different articulatory maneuvers. [Work supported by NIH Grant HD-01994 to Haskins Labs.]

\section{2:00}

5pSP6. An examination of the perceptual magnet efiect. Scott E Lively (Speech Res. Lab., Indiana Univ., Bloomington, IN 47405)

Recently, Kuhl [Pecept. Psychophys. 50, 93-107 (1991)] has argued that speech perception is guided by phonetic prototypes that are assumed to be stored in long-term memory. This claim is based on the finding of a "perceptual magnet effect." According to Kuhl, speech prototypes act like "magnets" because they attract similar stimuli to themselves. Furthermore, prototypes attract other stimuli more strongly than nonprototypes. Thus vowels that surround a prototypical stimulus are more difficult to discriminate than vowels that surround a nonprototype. Data are presented from vowel "goodness" rating experiments that suggest that listeners' prototypes for the vowel /i/ may be more extreme and variable than Kuhl's data indicate. Data are also presented from a categorization experiment that indicates that all of Kuhl's vowels may not be from the same phonetic category. These findings call into question the basis of the perceptual magnet effect. An alternative interpretation of both sets of findings is given. [Work supported by NIH.]

\section{$2 \cdot 12$}

5pSP7. Identification and change/no-change discrimination of [i] stimuli: Further tests of the "magnet" effect. Valerie J. Lauckner-Morano and Joan E. Sussman (Dept. of Commun. Disord. and Sci., State Univ. of New York at Buffalo, Buffalo, NY 14260)

In original work Kuhl [Percept. Psychophys. 50, 93-107 (1991)] used a stimulus that was 120 mels away from the prototype (P) stimulus for demonstration of the "magnet" effect. Listeners in the current study who phonetically transcribed $P$ and nonprototype (NP) [i] stimuli showed that $92 \%$ of responses for the 120 -mel $P$ token were not [i]. Further, another group of listeners rated the 64 stimuli as either the [i] in "peep" or "not [i]." Results showed that only $20 \%$ of responses rated the 120-mel token as [i] suggesting that this token along the -1 diagonal was not considreed an [i] member. Thus three other groups of listeners participated in "change/no-change" discrimination testing with 500 -ms interstimulus intervals for stimuli along the -1 diagonal common to the $P$ and NP distributions. Each group had a different fixed standard in each four-item trial: the Kuhl P token, the 120-mel token, and an 85-mel token. Results will be discussed according to the effects of different standards on discrimination sensitivity and prototype theories in phonetic perception.

\section{2:24}

5pSP8. Influence of dynamic spectral information on rate-dependent vowel perception. Terry L. Gottfried and Susanne K. Triesch (Dept. of Psychol., Lawrence Univ., Appleton, WI 54912)

Earlier research has shown that listeners adjust their identification of spectrally adjacent tense and lax vowels according to the speaking rate of the surrounding sentence. This study attempted to determine whether these rate effects could be reduced if test stimuli more closely resembled natural speech. Two synthetic $/ \mathfrak{x} /-/ \varepsilon /$ series were created: (1) covarying $F 1$ and $F 2$ with syllable duration (i.e., stimuli with more /a/-like formants were also longer in duration); (2) covarying formant frequencies with syllable duration and with formant frequency contours (i.e., stimuli with more /æ/-like formants and duration had more symmetrical formant contours.) Stimuli from these series were embedded in fast and slow sentence contexts. In series (1), listeners identified more stimuli as $/ \mathfrak{x} /$ in fast sentences, showing rate-dependent perception of this tense-lax contrast, replicating Gottfried et al. [Phonetica 47, 155-172 (1990)]. However, in series (2) (with appropriate formant contourssymmetrical for $/ \mathfrak{x} /$ and longer offglide for $/ \varepsilon /$ ), listeners showed no significant effect of rate. These results suggest that sentence rate may primarily affect vowel identification when important dynamic acoustic information is absent from the syllable itself.

5pSP9. Perception of synthetic silent-center syllables: A replication and extension. William D. Clarke, III (Dept. of Commun. Sci. and Disord., Univ. of South Florida, Tampa, FL 33620)

The purpose of this study was to replicate and extend the synthetic silent-center vowel perception study of Miranda and Strange [J. Acoust. Soc. Am. Suppl. 1 88, S55 (1990)]. The goal was to generate synthetic syllables that adequately captured the dynamic spectral information in syllable onsets and offsets. Natural and synthetic /dVd/ syllables were presented to subjects in two forms: full syllable and silent center. Natural silent centers were constructed ty retaining the burst and first three and final four pitch periods in order to achieve a desired vowel identification error rate of about $10 \%$. Natural duration was not altered. From ILS analysis of the first three formants, synthetic syllables were 
generated with Haskins Labs SYN software synthesis. Natural and synthetic full syllable error rates were $2 \%$. Natural silent center error rates were $11 \%$. Synthetic silent centers were $23 \%$, far less than the $60 \%$ reported by Miranda and Strange. Thus these synthetic syllables appear to have captured some of the dynamic spectral information in the syllable onsets and offsets. [Work supported by NIDCD.]

\section{2:48}

5pSP10. Categorical perception of silent-center syllables. Heather McLeod and Allard Jongman (Dept. of Modern Lang. and Linguist., Cornell Univ., Ithaca, NY 14853)

Work by Strange and colleagues [e.g., Strange et al, J. Acoust. Soc. Am. 74, 695-705 (1983)] has shown that CVC syllables without the steady-state vowel ("silent-center syllables") are as accurately recognized as CVC syllables containing the steady-state vowel, suggesting that dynamic rather than static properties are more important in vowel perception. The present study expands this line of research by studying the identification of silent-center syllables as members of a vowel continuum. Two synthetic continua were presented to listeners for identification: A [bIb-beb] continuum and a silent-center [blb-beb] continuum. The silent-center version was created by excising the steady-state portion of the original continuum, leaving the formant transitions intact. A comparison of the two identification functions revealed a significant shift, suggesting that full and silent-center syllables are not perceptually equivalent in this type of identification task. The results will be discussed in terms of theories of vowel perception.

\section{3:00}

5pSP11. Compensation abilities to lip perturbations of rounded vowel [u]. Christophe Savariaux, Pascal Perrier, and Jean-Pierre Orliaguet (ICP, INPG \& Univ. Stendhal, URA CNRS 368, 46 Ave. Félix Viallet, 38031 Grenoble Cedex, France)

An experiment is described involving lip perturbation, using a large $(20 \mathrm{~mm})$ Plexiglas tube, during the production of the rounded french vowel $[u]$. Ten naive speakers pronounced isolated repetitions of $[u]$ in three conditions: (1) without perturbation, (2) with the lip-tube after a learning period of 20 repetitions, and (3) with the lip-tube after 20 repetitions with information on the way to deal with the perturbation. $\mathrm{X}$-ray recordings were made at the end of each phase, and formants were extracted from the acoustical record. Compensation abilities were evaluated by considering the first three formants, in reference to the difference limens [Mermelstein, J. Acoust. Soc. Am. 63, 572-580 (1978)]. At the end of the second phase, one speaker was able to compensate for the perturbation. For all other speakers, we observed a strong increase in $F 2$. For the third phase, the speakers were divided into three groups who were given different information in order to help them compensate for the perturbation (first group: articulatory information, second group: acoustic information, third group: both types of information). Speakers in the first group all produced a fairly good [u]. Speakers in the second group showed some enhancement, but the F1-F2 targets were not reached. In the third group, no reliable compensation was observed. Some interpretations are proposed concerning compensation strategies and speech space control.

\section{3:12}

5pSP12. An acoustic and perceptual comparison of English and Spanish rowels. Ann R. Bradlow (Dept. Modern Lang. \& Linguist., Cornell Univ., Ithaca, NY 14853)

This paper addresses three issues. First, the effect of inventory size on the acoustic realization of vowels in English and Spanish is examined. Results indicate that the English vowel space differs from the Spanish vowel space in range, but not in tightness of within-category clustering. The second experiment tests the hypothesis that the perceptual categories coincide with the produced categories within a given language. Indeed, the data suggest that the perceptual and acoustic vowel spaces within English and Spanish correspond closely to each other. Finally, this study explores cross-language differences in vowel perception. These data suggest that there are universal, perhaps psychophysical, boundaries which are independent of inventory size: in both languages, the boundaries between $/ \mathrm{i} / \mathrm{and} / \mathrm{e} /$, and between $/ \mathrm{u} /$ and /o/ are primarily in the $F 1$ dimension, and are in the 400 - to 500 -mels region. This suggests that the observed regularities in vowel inventories across languages may stem, in part, from universal, auditory-perceptual mechanisms. Results of experiments in which English listeners responded to Spanish-based stimuli, and vice-versa, will also be discussed.

\section{3:24}

5pSP13. Developmental changes in cross-language vowel perception. Linda Polka (School of Human Commun. Disord., McGill Univ., 1266 Pine Ave. West, Montreal, PQ H3G 1A8, Canada) and Janet F. Werker (University of British Columbia, Vancouver, BC V6T 124, Canada)

There is considerable evidence for a reorganization in speech perception during infancy that reflects the influence of specific language experience. Infants of 6-8 months of age reliably discriminate certain non-native consonant contrasts that 10 - to 12 -month-olds fail to discriminate unless they have experience with those categories in their everyday language. This recent research also shows a decline in English infants' discrimination of two German vowel contrasts, $/ \mathrm{y}-\mathrm{u} /$ and $/ \mathrm{Y}-$ $\mathrm{U} /$, but discrimination at 6-8 months was below levels that have been observed for non-native consonant contrasts. The present experiment further investigated the timing and nature of developmental changes in cross-language vowel perception. Discrimination of German $/ v-y /$ and $/ Y-U /$ and English $/ \mathrm{i} /-/ \mathrm{a} /$ was examined in English-learning infants aged 4 and 6 months using a habituation procedure. The 4-month-old infants discriminated all three contrasts, whereas 6-month-olds failed to discriminate both German vowel contrasts. The results indicate that developmental changes in discrimination of phonetic contrasts occur earlier for vowels than for consonants. Evidence for age-related changes in the internal structure of vowel categories will also be discussed.

\section{3:36}

5pSP14. Vowel normalization by articulatory normalization: Preliminary results. Yohan Payan and Pascal Perrier (ICP, INPG \& Univ. Stendhal, 46 Ave. Félix Viallet, 38031 Grenoble Cedex, France)

This paper presents an approach to vowel normalization based on the exloitation of the concept of formant to cavity affiliation as described in the vocalic theory of speech production [G. Fant, Acoustic Theory of Speech Production (Mouton, La Hague, 1960)]. For closed vowels, this concept allows an estimation of the ratio of the respective back and front cavities lengths of two speakers, by the ratio of the associated formants. In order to propose a normalization of the vocalic space of different speakers, a "reference speaker" was used whose articulatory and acoustic properties are well defined: the articulatory model as proposed by Maeda [J. Acoust. Soc. Am. Suppl. 1 81, S146 (1988)]. Vowel normalization consists then in the projection of the vocalic space of one given speaker in the vocalic space of this model. Geometrical relations between the studied speaker and the "reference speaker" are extracted from [i] and [u] for front and velopalatal vowels, and from [a] and [u] for back and velopharyngeal vowels. Here, [y] and [u] give some indications about the lip shapes. This method is here tested by her ability to predict the vowels of the studied speaker starting from the vowels of this model.

\section{$3: 48$}

5pSP15. Integrality of nasalization and $F 1$ in vorels in isolation and before oral and nasal consonants. John Kingston (Linguist. Dept., South College, Univ. of Massachusetts, Amherst, MA 01003) and Neil A. Macmillan (Univ. of Massachusetts, Amherst, MA 01003)

Vowels vary multidimensionally, and the perceptual effects of the dimensions may combine independently or may interact. The dimensions examined in these experiments were the acoustic correlates of soft 
palate height and tongue height, which covary directly in natural speech. A modified Garner paradigm was used in which accuracy replaced speed as the dependent measure; a detection-theory analysis permitted inferences about perceptual representation. For isolated vowels, nasalization and $F 1$ were integral, in the sense that corresponding perceptual dimensions were correlated, replicating Kingston [Phonetica 47, 149-179 (1991)]. The degree of integrality was yet stronger for vowels before consonants, whether the consonant was nasal or oral. These results suggest that soft palate height and tongue height are covaried by speakers so that their acoustic correlates contribute to a single perceptual property, the distribution of energy at the low end of the vowel's spectrum. The lack of a context effect is unexpected given the finding of Krakow et al. [J. Acoust. Soc. Am. 83, 1146-1158 (1988)] that nasalization in a vowel does not affect judgment of its height if there is a neighboring nasal to which that nasalization can be attributed. This discrepancy may occur because listeners in this experiment made judgments of nasalization as well as vowel quality.

FRIDAY AFTERNOON, 21 MAY 1993

BANQUET ROOM, 12:40 TO 4:30 P.M.

\title{
Session 5pUW
}

\section{Underwater Acoustics: Propagation}

\author{
Nicholas P. Chotiros, Chair \\ Applied Research Laboratories, University of Texas at Austin, P.O. Box 8029, Austin, Texas 78713-8029
}

Chair's Introduction-12:40

\section{Contributed Papers}

\section{$12: 45$}

5pUW1. Finding eigenrays by optimization with application to tomography and overcoming chaos. W. A. Kuperman and Michael D. Collins (Naval Res. Lab., Washington, DC 20375)

Eigenrays may be determined with the initial-value approach of shooting. The eikonal equation is solved repeatedly, with the initial conditions being the position and direction of the ray at one of the end points, until the correct initial conditions are found (i.e., until the ray nearly intersects the other end point). It has been demonstrated that this approach is relatively inefficient [B. R. Julian and D. Gubbins, J. Geophys. 43, 95-113 (1977)] and prone to chaos [Smith et al., J. Acoust. Soc. Am. 91, 1939-1949 (1992)]. When formulated in terms of Fermat's principle, finding eigenrays is a boundary-value problem that should be free of the ill effects of chaos. If the index of refraction is perturbed, the perturbed eigenrays may be determined efficiently from the unperturbed eigenrays. This fact is exploited to perform tomography efficiently with an optimization procedure that alternates between attempting to satisfy Fermat's principle and attempting to match travel time. After a sequence of iterations, both the index of refraction and the eigenrays are obtained.

\section{$1: 00$}

5pUW2. Sound-pressure level calculations using the RRA algorithm for depth-dependent speeds of sound valid at turning points and focal points. Lawrence J. Ziomek (Dept. of Electr. and Computer Eng., Code EC/Zm, Naval Postgrad. School, Monterey, CA 93943)

Sound-pressure level (SPL) calculations are made along individual ray paths for one-dimensional, depth-dependent, speeds of sound using an enhanced version of the recursive ray acoustics (RRA) algorithm. The SPL calculations are valid (i.e., finite) at turning points and focal points and do not require the use of Airy functions. The SPL calculations include the effects of frequency-dependent volume attenuation and frequency-dependent attenuation due to surface and bottom reflections. The ocean surface and bottom are treated as boundaries between viscous fluid media. Although the ocean surface is modeled as a planar boundary, the bathymetry is an arbitrary function of horizontal range. Sound speed versus depth and bathymetric data are represented by orthogonal function expansions. Computer simulation results from preliminary test cases are presented. [Work supported by NAVSEA.]
$1: 15$

5pUW3. Prufer transforms and numerical solutions to the Helmholtz equation. Arthur B. Baggercer (MIT, Rm. 5-204, Cambridge, MA 02139)

The Prufer transformation maps the second-order, linear Helmholtz differential equation to two first-order, nonlinear differential equations. The coupling between the two separates such that the equation for the eigenvalue is a single nonlinear first-order equation. This transformation has long been useful in theoretical studies of Strum-Liouville where many properties of the eigenvalues have been derived using it. It turns out also to have some useful numerical properties especially for problems involving waveguides where the coupling between the boundaries is poorly conditioned. It also identifies explicitly the both stability points in the evanescent sections of the modes and the local oscillation frequency in the ducted section. The numerical aspects of using the Prufer transform and some examples are discussed in the presentation.

\section{$1: 30$}

5pUW4. Exact mode representation of sound propagation in a shallow-water wedge with a penetrable bottom. Dezhang Chu (Dept. of Appl. Ocean Phys. and Eng., Woods Hole Oceanogr. Inst., Woods Hole, MA 02543)

A new solution for the acoustic wave propagating in a wedge-shaped shallow-water waveguide with a penetrable bottom $\left(\rho_{1} \neq \rho_{2}, c_{1} \neq c_{2}\right)$ is presented. By using a special transformation of range function (Bessel function), the inherent nonseparable problem can be converted to a pseudoseparable one. The solution is exact and is expressed as a summation of wedge mode sets, i.e., each eigenvalue corresponds to a set of eigenfunctions. There are several advantages of the present method over the conventional coupling mode method: (1) since the cylindrical coordinates corresponding to wedge modes are used, the media are bounded in angular direction $0 \Leftarrow \theta \Leftarrow \pi$, therefore, the mode spectrum of the exact solution only contains discrete modes. (2) The diffracted wave from wedge apex is included automatically. The contribution of the diffracted wave to the total field is important when $k r \ll 1$, where $k$ is the wave number and $r$ is the range from the wedge apex. (3) The mode coefficients are obtained through recursive formulas instead of solving $M$ linear equations simultaneously, where $M$ is the number of modes. When $c_{1}=c_{2}$, the problem becomes separable and for each eigenvalue, only the primary eigenfunction corresponding to a conventional wedge mode remains nonzero. Hence, the solution smoothly reduces to 
that for an isovelocity/density contrast wedge [D. Chu, J. Acoust. Soc. Am. 87, 2442-2450 (1990)]. Numerical computations are presented to show the effect of mode coupling in terms of different geometrical and physical parameters.

1:45

5pUW5. Multipath structures of pulse signals in shallow water with a thermocline. Renhe Zhang and Ye Zhu (State Key Lab. of Acoust., Chinese Acad. of Sci., Beijing, 100080, People's Republic of China)

It was observed that the pulse signals in shallow water with a thermocline have regular multipath structures, which are strongly dependent on the depths of source and receiver. When both the source and receiver are located above the thermocline, the received signal consists of a train of almost equispaced wave packets and the time interval $T$ between packets is related to the speed-profile parameters. When the source is below the thermocline and the receiver is above, the received signal consists of a train of wave packets with time intervals $r$ and $(T-\tau)$, where $\tau$ is dependent on the distance from the source to the bottom. The formulas of intervals $T$ and $\tau$ were deduced by the ray theory, and the calculated values of $T$ and $\tau$ are consistent with measured ones. The normal-mode theory was used to model the waveforms of pulse signals, and the effects of speed-profile, geoacoustic parameters, signal parameters, and source and receiver depths on waveform structure were studied.

\section{2:00}

5pUW6. Simulation studies of narrow-band and ew acoustic propagation through a shallow-water internal wave. Stanley $\mathbf{A}$. Chin-Bing, David B. King, and Robert W. McGirr (Naval Res. Lab., Code 7181, Stennis Space Center, MS 39529-5004)

The effects of a shallow-water, solitary internal wave on $\mathrm{cw}$ and narrow-band acoustic propagation have been studied using computer simulations. Numerous observations have been made of solitary internal waves in shallow water. These internal waves travel along the nearsurface thermocline for some distance without loss of coherence. Due to their prevalence, they have been cited as the likely cause of the anomalous effects observed in some recent shallow-water acoustic measurements. In order to test this hypothesis, computer simulations of acoustic propagation through an internal wave have been made using an oceanacoustic PE propagation computer model (EFEPE), fast Fourier transformations (FFTs), and beamforming algorithms. A narrow band of frequencies was used with the center frequency at $250 \mathrm{~Hz}$. The resulting frequency-dependent fields were then converted into the time domain via an FFT. Beamforming algorithms were used to analyze the field structure. Examples will be shown that contrast the internal wave effects on cw propagation and on narrow-band propagation. [Work supported by ONR and NRL.]

\section{2:15}

5pUW7. PE modal decomposition of VLA data. David J. Thomson and Gordon R. Ebbeson (Defence Res. Establishment Pacific, FMO Victoria, BC VOS IBO, Canada)

The first step in any matched-mode processing application is the decomposition of the measured acoustic field into its horizontal wavenumber (modal) components. Previously, a PE-based method was described for carrying out the modal decomposition of the pressure field received on a vertical line array (VLA) [D. J. Thomson et al., J. Acoust. Soc. Am. 89, 2000 (1991)]. For that method, the VLA field was first interpolated between hydrophones and then extrapolated into the ocean bottom. This interpolation/extrapolation stage was needed to construct a suitable starting field on the fine computational grid used by the PE algorithm. In this paper, a modified PE model is presented that eliminates the computational grid in the bottom by applying an exact nonlocal boundary condition along the ocean-bottom interface. Consequently, it is no longer necessary to extrapolate the VLA field prior to propagating it with PE. To demonstrate the effectiveness of the bound- ary condition, PE-based modal decomposition is carried out using simulated VLA data generated for a shallow-water environment.

\section{2:30-2:45 Break}

\section{2:45}

5pUW8. Comparison of data and parabolic equation solutions for long-range pulse propagation. Kevin D. Heaney (Planning Systems, Inc., McLean, VA 22102), w. A. Kuperman, B. Edward McDonald, and Michael D. Collins (Naval Res. Lab., Washington, DC 20375)

A propagation model that combines the horizontal refraction of the adiabatic mode solution and the accurate field computation of the parabolic equation (PE) method is applicable to long-range problems. In a previous application of this model, qualitative agreement was achieved for narrow-band data from the Heard Island Feasibility Test (HIFT). The broad-band extension of this model has been applied to two longrange propagation paths. Data have been analyzed from a single-slice tomography experiment that involved a $1000-\mathrm{km}$ propagation path in deep water and a source centered at $250 \mathrm{~Hz}$. These data were previously modeled with a WKB solution [Duda et al., J. Acoust. Soc. Am. 92, 939-955 (1992)], which breaks down for the lower modes in the sound channel. These results are in agreement with these data even for the arrivals associated with the lower modes. The Heard Island to Christmas Island propagation path of the HIFT has also been analyzed. Several features along this path cause significant mode coupling, including a sloping bottom near the source, the Antarctic Circumpolar Convergence, and a sea mount. Although there is variability in this data due to fluctuations, the observed dispersion and arrival structure is consistent with these results.

3:00

5pUW9. Modeling of the acoustic propagation through the Gulf Stream. F. Desharnais (SACLANTCEN, 19026 Viale S. Bartolomeo 400 , La Spezia, Italy)

Propagation loss modeling has been done in support of the experimental cruise "Maple Gelato" conducted in an area of the Gulf Stream, east of Cape May, New Jersey (by the SACLANTCEN, Italy, and the Defence Research Establishment Atlantic, Canada). The experiment was conducted in summer conditions (September 1992), and seamounts were also present in the area. Although the data will not be presented at this time, the modeling of the propagation through the front, done with the SACLANT parabolic equation model, will be presented for frequencies ranging from 10 to $300 \mathrm{~Hz}$, and for sensors distributed along a $300-\mathrm{m}$ vertical array. Preliminary results show that the effect of the front is to redistribute the energy in space, and to shift convergence zones. The acoustic levels within the two first convergence zones however are not affected substantially, although this depends on source and receiver depths. Seamounts, depending on their locations relative to the source, can counteract the effects of the fronts. The modeling results will be compared with results obtained in other frontal areas, to emphasize the particularities of the Gulf Stream.

\section{$3: 15$}

5pUW10. Measurements of propagation loss in isothermal surface ducts. F. D. Cotaras, P. D. Morash, and J. B. Franklin (Defence Res Establishment Allantic, P.O. Box 1012, Dartmouth, NS B2Y 3Z7, Canada)

Over the past several years, the Defence Research Establishment Atlantic has made measurements of propagation loss in surface ducts. The experiments were conducted at deep-water locations, and both the source (SUS charges) and receiver were placed in the duct. At the sites where the surface duct was well defined and constant with range, the data are in good agreement with a simple propagation-loss model. Inputs to the model inelude the sound absorption coefficient cited by Scheifele, Mellen, and Browning as well as the reflection-loss predic- 
tions of Brekhovskikh and Lysanov's method of small perturbations. The sea-surface was assumed to be described by a Pierson-Moskowitz frequency spectrum.

5pUW11. VLF ew signal analysis. Scott J. Levinson, Nancy $R$ Bedford, Stephen K. Mitchell, and Joan C. Lange (Appl. Res. Labs., Univ. of Texas at Austin, Austin, TX 78713)

Very-low-frequency (VLF) sound waves transmitted at 7 and $10 \mathrm{~Hz}$ from a source towed at a depth of $122 \mathrm{~m}$ were sampled over the bottom half of the water column by an 18 -element vertical hydrophone array anchored on the bottom ( $3170 \mathrm{~m}$ deep). Analysis included the comparison of measured beam levels with corresponding eigenray and adiabatic normal mode models at short $(0.7-20 \mathrm{~km})$ and long (35-60 km) ranges. At short ranges, the VLF measurements and both models were consistent in indicating easily distinguishable direct, bottom, bottomsurface, and bottom-surface-bottom interacting arrivals. At longer range, the arrivals typically consisted of three from the surface and three from the bottom, corresponding to two, three, and four bottom interactions. The continuous range over which a particular arrival order dominated was $5 \mathrm{~km}$ or less. An anomalous bottom loss observed at $30^{\circ}-34^{\circ}$ at $7 \mathrm{~Hz}$ appears to be the result of a slow hydrate layer in the sediment. Except for these angles, VLF bottom loss was low compared to typical values at higher frequency, and it is consistent with conventional modeled loss calculations for the pre-assessed geoacoustic parameters of the sediment. [Work supported by the ASW Environmental Acoustic Support (AEAS) Program of the Office of Naval Research.]

\section{$3: 45$}

5pUW12. Comparison of time-domain parabolic equation and measured ocean impulse responses in a range-dependent environment. James H. Leclere and Robert L. Field (Naval Res. Lab., Stennis Space Center, MS 39529)

Ocean impulse response functions computed with the time-domain parabolic equation model are shown to be consistent with measured response functions in a shallow-water range-dependent environment off the east coast of the United States. Impulse response functions are measured by correlating the source signature of a 20 - to $150-\mathrm{Hz}$ linear, modulated signal with signals received on a 15 element vertical line array. Environmental inputs to the model are derived from Ocean Drilling Project (ODP) sites near the experiment location. Correlation coefficients as a function of depth are used to compare measured and modeled responses at three separate ranges. [Work supported by The Office of Naval Research under the Naval Research Laboratory/Stennis Space Center Acoustic Transients Project.]

\section{4:00}

5pUW13. Improvement in the shallow-water prediction of sonobuoy detection ranges for ASW aircraft using on-site acoustic measurements. Charles Wiseman (Lockheed Missiles and Space Co. P.O. Box 3504, Sunnyvale, CA 94088-3504)

Sonobuoy detection ranges predicted at shore stations for use by antisubmarine warfare (ASW) aircraft are based on historical data and on expendable bathythermograph (XBT) data that are typically at least 6 to $12 \mathrm{~h}$ old. The accuracy of such predictions is questionable as shown by measurements and analysis. Prediction accuracy needs to be improved to increase the effectiveness and lower the costs of antisubmarine search and barriers using sonobuoys dropped from ASW aircraft. These predictions can be improved during the mission by measuring the onsite sound propagation loss between a specially modified air-dropped electronic sound source, similar in concept to a long-lived MK 84 SUS sound projector, and the acoustic receivers in the air-dropped sonobuoys. The derived propagation loss, used in conjunction with the XBT-based predictions, can be used to more effectively select sonobuoy depths and patterns. The existing MK 84 SUS projector could be modified to be neutrally buoyant and provide 140 ping seconds of preprogrammed transmissions over a $6 \mathrm{~h}$ life. The transmissions can be shaped for low probability of intercept or mimicry. Similar applications to improve sonar range prediction for surface ships are also addressed.

\section{4:15}

5pUW14. Exact reflection and transmission operator symbols in two-dimensional, invariant imbedding-based, propagation modeling. Louis Fishman (Dept. of Math. and Computer Sci., Colorado School of Mines, Golden, CO 80401)

Recognizing that typical ocean propagation problems are essentially scattering problems in terms of a transition region and transversely inhomogeneous asymptotic half-spaces, wave-field splitting, invariant imbedding, and phase space methods have reformulated the problem in terms of an operator scattering matrix characteristic of the transition region. This approach solves the elliptic (fixed-frequency) scattering problem by well-posed marching (one-way) methods, and is centered on the reflection and transmission operator symbol equations. For several nontrivial, two-dimensional, refractive index profiles, these equations (and the wave-field equations) are solved exactly and analyzed. These results provide benchmark cases to test both the scattering operator and subsequent wave-field calculations, providing a severe test of the stiff nonlinear solvers employed in the numerical implementation of the method. The exactly solved models incorporate symmetric and asymmetric wells, trapped modes, and sharp-gradient features. Numerical results will be presented. [Work supported by NSF, AFOSR, ONR, ASEE, JEWC, and the $\mathrm{S}$. N. Bose Centre for Basic Sciences.] 